\title{
Foragers on the Frontiers The IXam Bushmen of the Northern Cape, South Africa, in the Nineteenth Century
}

\author{
Mark McGranaghan \\ Hertford College \\ University of Oxford
}

Submitted in partial fulfilment of the degree of

Doctor of Philosophy

Hilary Term, 2012 


\title{
Abstract
}

\section{Foragers on the Frontiers: the IXam Bushmen of the Northern Cape, South Africa, in the Nineteenth Century}

\author{
Mark McGranaghan, Hertford College, University of Oxford
}

Hilary 2012

This thesis constructs an ethnography for the nineteenth century IXam Bushmen of the Northern Cape Province of South Africa, known primarily through a nineteenth century manuscript collection of oral narrative (the Bleek-Lloyd archive), which has, over the past twenty-five years, increasingly become the focus of scholarly attention, mined for insights about the cultural world of southern Bushman societies. It draws on the Bleek-Lloyd archive to produce a detailed ethnographic case study, focusing on the ideological and ontological concepts that underpinned the differentiation of IXam society. Firstly, the thesis situates the archive and IXam society within their particular environmental and historical contexts, providing valuable supplementary information that informs readings of the narratives. By producing a fully searchable transcription of the entirety of the archive, paying close attention to emic terminology, and examining the recurrence of thematic associations of this phraseology throughout the narratives, the analysis explores the constitution of IXam 'personhood' and examines the extent to which the 'hunter-gatherer' category forms a useful heuristic for understanding IXam society, with a particular focus on models of the 'animic ontology'. The IXam deployed a series of positively and negatively evaluated traits in the creation of dimensions of authority, obligation, and social responsibility, embedded in particular social identities; central to these constructions and to the differentiation of these identities were the techniques and resources of IXam subsistence practices, salient in the production of admirable (socially-responsible hunters), reprehensible (antagonistic 'beasts of prey'), and more ambiguous (!gi:tən ritual specialists) identities. Recognising this internal differentiation, the thesis outlines IXam 'subsistence strategies' and suggests they should be defined broadly to include their contacts and interactions with nonIXam groups, with domesticated animals, and with the novel material culture of the colonial period; these interactions were a consequence of their 'huntergatherer' strategies rather than a negation of them. Such strategies generated experiences that reinforced and reconstituted IXam ideological frameworks, incorporating the dynamics of the nineteenth century 'frontier' scenario and provided avenues for social change that ultimately led to the collapse of independent hunter-gatherer lifeways, and to the adoption of strategies that incorporated IXam individuals within rural and urban 'Coloured' populations of the Northern Cape; placing the IXam in a comparative colonial context, the thesis stresses the wider relevance of this particular ethnography for understanding hunter-gatherer engagements with food-producing, state-level societies. 


\section{Acknowledgements}

It would not have been possible to write this doctoral thesis without the assistance and support of a great many people, to only some of whom particular acknowledgement can be given here.

Firstly, thanks must go to my supervisor Professor Peter Mitchell, who introduced me to the Bleek and Lloyd archive, and whose enthusiasm and support over the years has been fundamental in shaping my academic development. His advice, and encyclopaedic knowledge of Southern African prehistory, has been indispensible, and our discussions underpin the ideas and issues explored in this thesis. The academic and financial support provided by the Department of Archaeology, University of Oxford, has been an invaluable aid; I gratefully acknowledge their Doctoral Scholarship, without which I should not have been able to undertake this research.

This thesis should also not have been possible without the assistance of a number of people in South Africa, amongst whom I would like particularly to thank the Mallen family in Johannesburg, who kindly put up with my staying with them for some weeks. The staff of the MacGregor Museum, Kimberley, too were unfailing helpful and welcoming to me during my time there. My thanks go particularly to Mr. David Morris (Head of Archaeology), whose willingness to facilitate my visits to Kimberley went far beyond the bounds of professional courtesy into the realms of friendship; the many stimulating discussions with which we whiled 
away long drives around the Karoo and Northern Cape helped bring my own ideas into focus, though any errors and inaccuracies of course remain my own.

Friends and colleagues in Oxford, Wales, and South Africa have provided the social support that has kept me sane throughout the writing process, and helped ensure that my postgraduate studies have been not only academically engaging but also incredibly enjoyable; particular thanks must go to Liam Isaac, for accompanying me to the Karoo on extremely short notice, to Nathan and Jamie for their much-needed camaraderie during the final push to write up, to my housemates for providing delicious meals, great company, and many hours of board-gaming, and to Brian Stewart and Genevieve Dewar, whose 'AMEMSA' field seasons provided very many memorable experiences.

Finally, I should like to express my gratitude for the unstinting support of my family, without which I would never have had the opportunity to begin my studies and should never have been able to reach this culmination; my thanks and my love go with them. 


\section{Contents}

Chapter 1: Introduction............................................................11

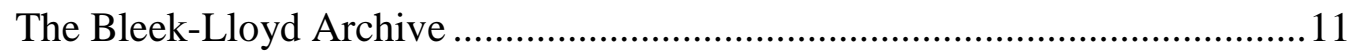

Chapter 2: Theorising Foraging in a Colonial Context...23

Chapter 3: Exploring Definitions of the IXam-ka-!xoe ....45

Chapter 3a: Physical and climatological parameters of the Karoo environment

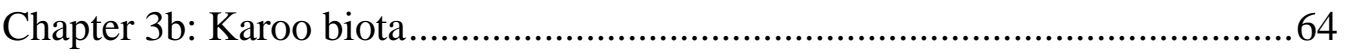

Chapter 4: Historical Contexts ......................................82

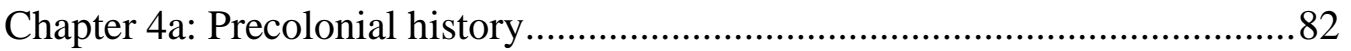

Chapter 4b: The Cape Colony in the eighteenth century .................................99

Chapter 4c: Encapsulation on the northern borders .....................................110

Chapter 5: Historical Ethnography of the IXam............132

Chapter 5a: Odours, winds, and the construction of $\mid$ Xam personhood...........132

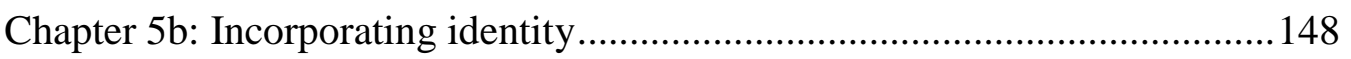

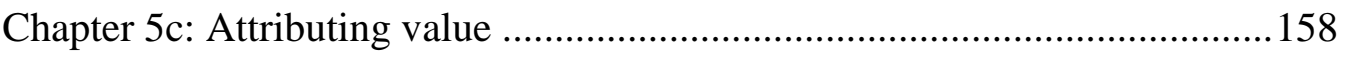

Chapter 6: Social Responsibility, Authority, and

Obligation ..................................................................168

Chapter 6a: 'Understanding people' and foolish actions................................168

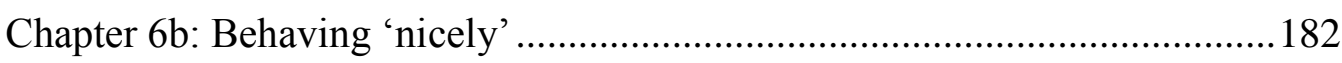

Chapter 6c: Behaving 'differently' .........................................................201

Chapter 7: |Xam Practice ..............................................213

Chapter 7a: The people who shoot springbok ..............................................213

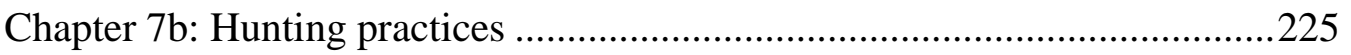

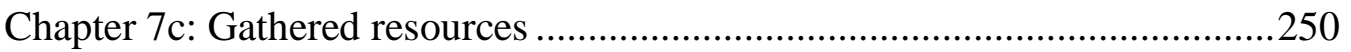

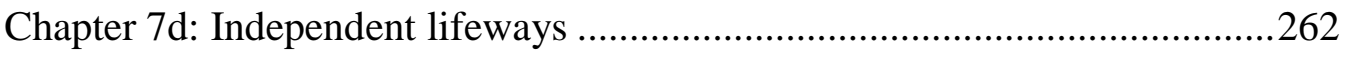

Chapter 8: IXam Interactions..............................................282 
Chapter 8a: Engagement with domesticated species ...................................282

Chapter 8b: The people who were 'different' ..............................................298

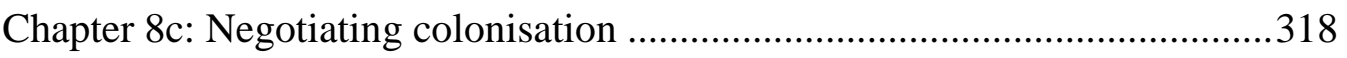

Chapter 9: Conclusions...............................................337

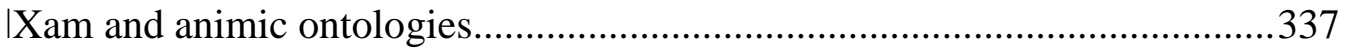

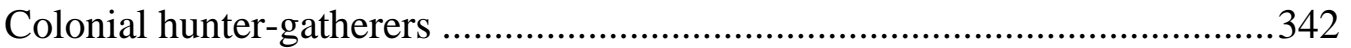

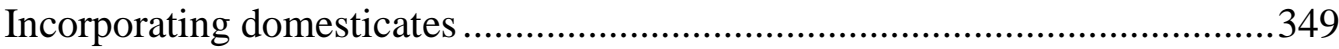

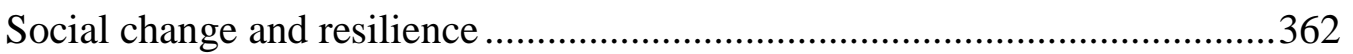

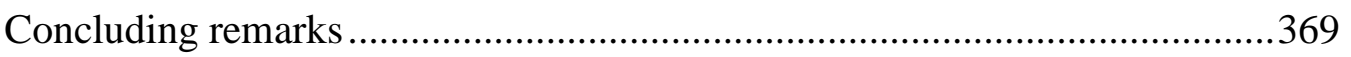

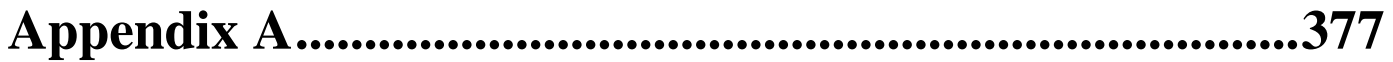

A Note on Orthography and Pronunciation .................................................377

Appendix B ............................................................................381

Referencing the Bleek-Lloyd Archive ….........................................................381

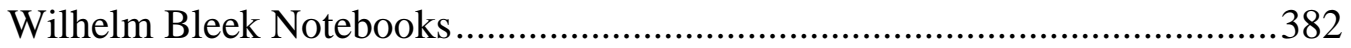

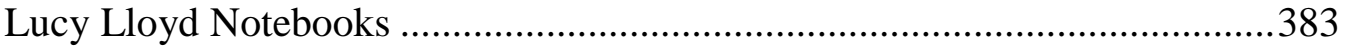

Appendix C.............................................................387

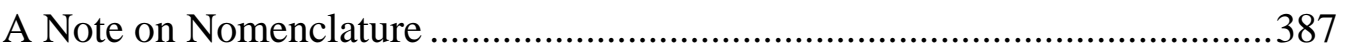

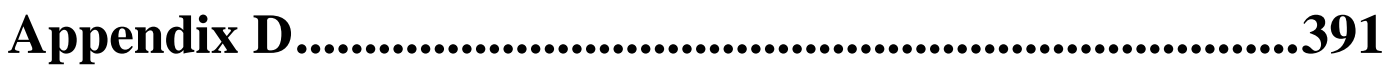

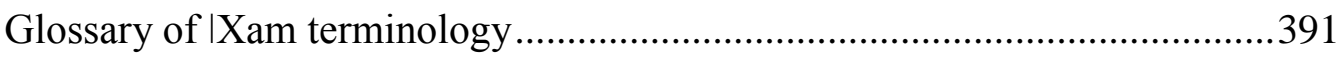

Appendix E ................................................................................455

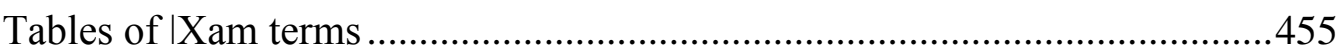

Table 1: IXam terms for mammals, with scientific and common names.........456

Table 2: |Xam terms for birds, with common and scientific names ...............463

Table 3: IXam terms for reptiles and amphibians, with common and scientific

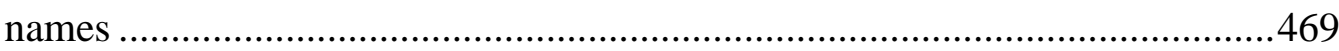

Table 4: IXam terms for invertebrates, with common and scientific names....474

Table 5: IXam terms for plants, their uses, and identifications.......................483

Table 6: IXam lithic terminology, translation and uses ................................488

Bibliography ...........................................................................491 


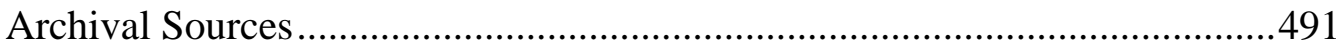

Published Historical Sources (Eighteenth and Nineteenth Century) ................494

Unpublished Theses and Reports.....................................................................496

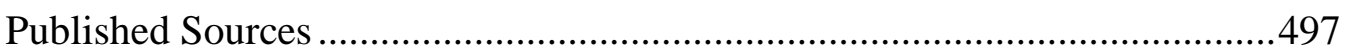

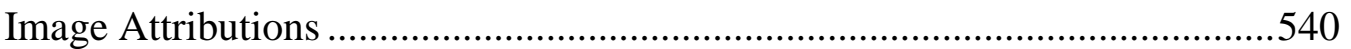




\section{Figures}

\section{Chapter 3}

Figure 3.1: Succulent Karoo (green outline) and Nama-Karoo (yellow outline) biomes, with the Kenhardt-Brandvlei-Vanwyksvlei red triangle (home territories of the Bleek-Lloyd IXam informants).

Figure 3.2: Rainfall zones of the Cape (modified after Hoffmann and Cowling, 1987:3, and Watkeys, 1999:25) .....

Figure 3.3: Distribution of summer (Oct-Mar) rainfall as percentage of mean annual (after Venter, Mocke and de Jager, 1986:40).

Figure 3.4: Isohyets of mean annual rainfall $(\mathrm{mm})$ in Karoo biomes (after Venter, Mocke and de Jager, 1986:40).

Figure 3.5: Reliability of rainfall; percentage years with rainfall $\geq 85 \%$ mean annual (after Venter, Mocke and de Jager, 1986:42).

Figure 3.6: Mean annual temperatures $\left({ }^{\circ} \mathrm{C}\right)$ in the Karoo biome (after Venter, Mocke and de Jager, 1986:46)

Figure 3.7: Isotherms of mean annual summer temperatures $\left({ }^{\circ} \mathrm{C}\right.$ ) (after Venter, Mocke and de Jager, 1986:47)

Figure 3.8: Mean annual frequency of days with maximum temperatures above $30^{\circ} \mathrm{C}$ (after Venter, Mocke and de Jager, 1986:47)

Figure 3.9: Mean annual frequency of days with minimum temperature below $0^{\circ} \mathrm{C}$ (after Venter, Mocke and de Jager, 1986:48) .55

Figure 3.10: Simplified geological map of the Nama-Karoo (after Visser, 1986:2)

Figure 3.11: Soil regions of the Nama-Karoo; region 2 (yellow) and region 3

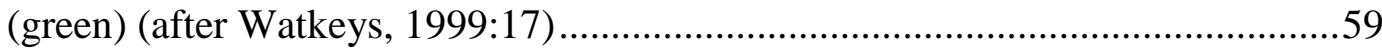

Figure 3.12: Simplified diagram of Acocks' (1953) veld types ............................67

Figure 3.13: Hoffman's (1996, 1996a, 1996b) Nama-Karoo veld types ...............69

\section{Chapter 4}

Figure 4.1: Stone walling associated with European colonisation, Northern Cape

Figure 4.2: Aerial view of Karoo farm walling (from figure 4.1) (GoogleMaps image)

Figure 4.3: Bushmanland (Kenhardt-Brandvlei-Vanwyksvlei triangle) showing places mentioned on the Bleek map and in manuscripts (after J. Deacon, 1986). 98 Figure 4.4: Map of the boundaries of the Cape Colony (1795) 


\section{Chapter 5}

Figure 5.1: Haasfontein (łkoaxa) in the Kenhardt-Brandvlei-Vanwyksvlei triangle

Figure 5.2: Characteristic 'hairy' coat of brown hyena (Hyena brunnea)...........160

Figure 5.3: African lion (Panthera leo), showing 'hairiness' characteristic of $\|k e\| k e$ status. .160

\section{Chapter 7}

Figure 7.1: Hartog's Kloof in the Kenhardt-Brandvlei-Vanwyksvlei triangle....223

Figure 7.2: Comparison of mouthparts of Bombylius major and Glossina

morsitans.

Figure 7.3: IXam terms for springbok markings and colour varients (modified

after Hetem et al. (2009)

Figure 7.4: Arragement of ostrich-feather brushes in hunting springbok

(LL.VIII.23.8066-8068; and accompanying sketch)

Figure 7.5: "Bushmen Hottentots Armed for an Expedition" (Daniell, 1804-1805)

266

Figure 7.6: The Strandberg in the Kendhardt-Brandvlei-Vanwyksvlei triangle .276

\section{Chapter 8}

Figure 8.1: Shepherding route; łni-tye-łxi; 'Jang ora' .....................................291

Figure 8.2: Bastard Puts in the Kenhardt-Brandvlei-Vanwyksvlei triangle .........313

Figure 8.3: 'Witberg' in the Kenhardt-Brandvlei-Vanwyksvlei triangle .............317

Figure 8.4: "Halt of a Boors Family” (Daniell, 1804-1805:No.11).....................321

Figure 8.5: Riet Kolk in the Kenhardt-Brandvlei-Vanwyksvlei triangle..............323 


\section{Chapter 1: Introduction}

\section{The Bleek-Lloyd Archive}

This thesis owes its existence to a remarkable project of late nineteenth century South Africa. In 1862, the German linguist Wilhelm Bleek became the curator of the Grey Collection at the South African Library in Cape Town. Bleek was interested in the IXam language (Appendix A, orthography and pronunciation guide:377) spoken by southern African 'Bushman' populations, seeing it both as in imminent danger of extinction and (guided by contemporary social theory) as illustrative of primitive stages of language evolution (Moran, 2009:125). Beginning in the late 1850 s and continuing throughout the 1860s, Bleek interviewed Khoekhoe and Bushman (Appendix C, notes on nomenclature:387) convicts in the Breakwater prison, eventually gaining permission to have convicts transferred to his house. The information collected from the $\mid$ Xam individuals ${ }^{1}$

\footnotetext{
${ }^{1}$ The main informants were five men, la!kunta, \|kabbo, $\neq$ kasin, Dia!kwain, Ihanłkass'o , and one

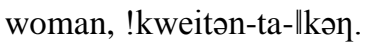


released to his custody constitutes what is now known as the Bleek-Lloyd archive, a collection of traditional narrative, personal histories and ethnographic information. The production of a IXam language dictionary was central to Bleek's project, and as part of this process narratives were taken down verbatim in IXam, with English translations subsequently pencilled in alongside. Bleek was assisted by his sister-in-law Lucy Lloyd, who came to play an increasingly central role in the project, continuing it and bringing it to fruition after his death in August 1875. In total, over eleven thousand notebook pages or approximately half a million (translated) words were recorded, around eighty-five percent of them collected by Lloyd. This archive continued to have an impact on southern African scholarship throughout the early decades of the twentieth century; Bleek's daughter Dorothea oversaw the production of a comparative Bushman vocabulary (Bleek, 2011 [1929]) and the Bushman Dictionary (Bleek, 1956) ${ }^{2}$, the publication of notebook material $^{3}$ in a series of Bantu Studies papers (Bleek, 1931, 1932, 1932a, 1932b, 1933, 1933a, 1935, 1936, 1936a), and undertook substantial research on extant Bushman communities herself (Bleek, 1924; 1927; 1936b). The archive sank into relative obscurity during the middle decades of the twentieth century as a new wave of anthropologists came to focus on Kalahari hunter-gatherers (Lee and deVore, 1968, 1976), but from the mid-1970s, Hewitt's presentation of his doctoral thesis focused attention once more on the IXam Bushmen. The subsequent publication of his research (Hewitt, 2008 [1986]) helped cement the archive's significance for a wider audience.

\footnotetext{
${ }^{2}$ Hereafter, the Dictionary.

3 Bleek $(1874$; 1874a 1875; 1875a) and Lloyd (1880; 1889) both published reports and summaries of material.
} 
Throughout this thesis I rely upon digital scans of the primary texts, first published alongside Skotnes (2007) and now available online ${ }^{4}$. Previously published portions of the archive alter the information contained in the notebooks to varying degrees; Dorothea Bleek's Bantu Studies papers (Bleek 1931, 1932, $1933,1934,1935,1936)$ attempted to minimise the loss of information, preserving the bilingual format and the complex suite of diacritics and nonstandard orthographic symbols employed by Bleek and Lloyd. Even here, the standardisation of publication removes the 'fluidity' and ambiguities of the notebooks, which record not only the stories themselves but also the process of their collection (Bank, 2006:83-87). Recent presentations of IXam material vary in their editing approach (Lewis-Williams, 2000:35-41), but can be separated into two strands: 'academic' works emphasising fidelity to the notebooks (e.g. Guenther, 1989; Hollman, 2004), and more 'artistic' works drawing inspiration from IXam narratives (e.g. Watson, 1991; James, 2001; Krog, 2004). Even scholarly works require considerable editing, and the simplification of diacritics, elimination of the marginalia, and even the removal of the IXam language component altogether is common. More 'artistic' collections attribute authorship to the IXam informants in varying degrees, discussing with very different emphases the 'authenticity' or faithfulness of the texts presented, and often are situated firmly in post-apartheid contemporary South Africa. Moran (2001) discusses Watson's (1991) presentation of Bleek-Lloyd material as an attempt to assure readers of a similarity between themselves and a non-racist, indigenous

\footnotetext{
${ }^{4}$ http://lloydbleekcollection.cs.uct.ac.za/
} 
Bushman population, for example by eliding descriptions of white and black men (Moran 2001:48). Given these issues, I rely primarily on my own transcriptions throughout (Appendix B, Bleek-Lloyd archive references:381), with comparison between these and published extracts where appropriate. I pay particular attention to the original IXam terminology. Although Bleek and Lloyd's work constitutes the fundamental basis of our understanding of IXam, close reading of terms in the wider context of the whole corpus allows for reassessment of some translations, particularly in earlier notebooks; these often mistranslate words, a fact appreciated by the original translators themselves (e.g. Lloyd's corrections of her confusing \|gaue, to seek, with \|gaua, to lie in the shade, LL.II.11.1142')

Within contemporary South Africa, the texts have taken on a special meaning as a rare historical moment of collaboration and cooperation rather than the more usual colonial relationships of conflict and oppression. Brought to the attention of a new audience in the 1980s, by 1997 the collection was inscribed in the UNESCO 'Memory of the World' programme. Both Bleek and Lloyd have been characterised as people out of their time, a prescient foreshadowing of the liberal white academic in later twentieth century South Africa. While the verbatim texts do mark the collection as atypical, allowing us to revisit the translations and introduce IXam voices (however distorted), such a reading of the project and the relationships unfolding within it has not passed unchallenged (Bank, 2000). Bleek and Lloyd collected the narratives under conditions that, although less overtly destructive than many colonial relationships, included marked power discrepancies between the informants and the collectors; their collection therefore 
is necessarily partial, and subsequent commentators have remarked upon the lack of emphasis on documenting nineteenth century conditions (Hewitt, 2008 [1986]:32).

However tempting it may be, the archive cannot then be viewed as 'primary' ethnographic contact, transcribing IXam ideas, beliefs, and practices. The creation of the texts represents a particular form of European writing (Wessels, 2010:188), and issues of translation are key. The stylistic choices employed by the translators impart a specific 'flavour' to the narratives; the accuracy of their convoluted syntax and quasi-Elizabethan English (Hewitt, 2008 [1986]:165) in mirroring IXam idioms and expressions is unclear. Literal translations from IXam often have a 'poetic' cadence in English (WB.IV.554-555), but this does not necessarily mean that the IXam intended them as poetry. While the narratives were part of a creative aesthetic tradition, valorisation of this tradition may easily lead to the imposition of tones and cadences considered appropriate for a 'primitive' huntergatherer literature. While distortion in translation is inevitable, examining the application of this distortion (with particular concern for information loss) is crucial. Even single word translations illustrate this point well: the term ! $k$ "wakka, rendered variously as decayed, putrid, and ill-smelling (WB.IV.540), incorporates notions of personal smell that were part of a complex suite of ideas relating to identity (Chapter 5a:134; see glossary ${ }^{5}$ !k"wakkən-\|kũ, !k"wakka-tukən:436). It is clear from these wider contexts of its use that $! k^{\prime \prime} w a k k a$ was a complex term

\footnotetext{
${ }^{5}$ |Xam terminology is used extensively throughout Chapters 5-8; the glossary (Appendix D:390) provides definitions and notes for key terms, highlighted in bold when first encountered and thereafter italicised. Where explicit reference to the glossary is required, the relevant term is again highlighted in bold and underlined.
} 
connoting stinginess or incompetence in meat acquisition and distribution, both of which had social implications (Chapter 6b:183). The difficulty of accurately conveying such concepts in English translations is obvious.

Furthermore, the collection of the texts should not be viewed as a single ahistorical event. It represents a series of relationships, developing over time and varying with the informants and collectors involved, situated in the broader context of colonial contact. The project was dependent on the support of colonial government, necessitating the production of official publications for specific audiences, with reports presented to the Cape Parliament (Bleek, 1873; 1875a; Lloyd, 1880) betraying different sentiments to those evident in the marginalia of the notebooks. Intellectual tools employed by Bleek and Lloyd structure the archive, woven throughout the texts with pasted-in newspaper articles and notes from correspondence with various specialists. Both collected information under the aegis of 'folklore' (Bleek and Lloyd, 1911), a central category for emergent social science disciplines in the late nineteenth century, particularly in British, German, and Scandinavian approaches (Georges and Owen Jones, 1995:32; Roper, 2007). Beginning 'at home' in Europe (where early folklorists were concerned with demonstrating the coherence and antiquity of 'national character') and founded on a division between urban, industrialised modernity and the primitive, the discipline was transferred easily to the study of colonised populations. For Bleek, Bushman folklore and the medium through which it was transmitted (his own specialist field, language) revealed the true character of this nation, a nation associated with a suite of biological characteristics (i.e. 'race'), 
theoretical underpinnings that guided his approach to the narratives (Bank, 2000; Moran, 2001; Hewitt, 2008[1986]). Study of the archive as 'narrative' (or folklore) has remained central in contemporary scholarship, with shifts in aims and theoretical underpinnings. Both Guenther $(1989,1999)$ and Wessels (2010) point out the key role of the audience, with the performative nature of storytelling and the implications of the circumstances of collection for the types and styles of tales included becoming an increasingly important theme. Wessels (2010) also stresses the need to 'entangle' the stories by focusing on the total semantic content of the signifiers within each tale. Close reading of many tales (preferably all of the archive) can help approximate the cultural world of the late nineteenth century IXam, elucidating the complex web of interrelated ideas that underlay the deployment of individual signifiers, the implications of specific tales, and the meanings fore-grounded or inferences left for the audience to draw.

The IXam had only one word for their narratives, kum (pl. kukumm-i, Bleek, 1956:106), differentiated in Bleek and Lloyd's translations as stories, histories, talk, and news; these kukumm-i incorporate topics and narrative styles ranging from accounts of personal experiences of arrests and imprisonment (LL.II.1.266272; LL.II.1.242-250) to formal narration of the creation of the IXam world by various non-human agents (LL.II.3.394-475). Scholarship (including that by the original researchers) has dealt with this variety by employing a series of analytical categories (Hewitt, 2008 [1986]:33-47), which have at one extreme 'myths' dealing with ontological themes and, at the other, anecdotes drawn from the informants' life histories: in between are a series of 'folkloric' narrations, 
displaying various degrees of stereotypical, formalised structure. Such divisions clearly misrepresent emic narrative differentiation; indeed, Guenther's (1986, 1999) characterisation of Bushman narrative traditions undermines any approach heavily based on categorisation, as he considers Bushman narrative traditions as highly fluid and ambiguous, and characterised by a willingness to entertain alternative experiences and explanations. However, even discounting the analytical difficulties attendant on removing categorisation, approaching the archive as a homogeneous unit would equally misrepresent the information contained within it, ignoring the contextual information specific to each story that houses much of its meaning. Without suggesting that these categories reflect emic divisions, I employ the basic tripartite separation outlined above: narratives concerning the Early-Race (see Glossary, !xwe-\|na-s'o-!k'e:440) times, information drawn from first-hand experience, and a vaguer interstitial category (legends, memorates, fabulates, \&c.).

Rather than providing detailed analyses of specific stories, this thesis extrapolates information about IXam ways of understanding the world, applying this to our understandings of their interactions with the colonial frontier. As stories from all three categories can reveal important information about IXam conceptions, the tripartite division is often peripheral to this aim but remains a useful tool facilitating discussion. One point that it is important to stress here, though, is that the relationship between memorates (Honko, 1964; Dégh, 2001:2-4) and 'IXam belief' is a problematic one. Unlike more stereotyped narrative genres that may be assigned to a cultural tradition despite the idiosyncrasies of any specific 
manifestation, the memorate records personal experience. Although this experience will be shaped by socio-cultural factors, it may also record a highly idiomatic 'reading', a problem exacerbated by the small number of IXam informants, and memorates claimed to pertain to 'IXam thought' in general must therefore be treated with caution. A good example of this is the \|khã:-ka-mumu (Glossary:447; translated by W. Bleek as 'spectre lion', WB.XIII.2190'), a phenomenon mentioned only once in the archive; "kabbo's note appears to relate to his 'making sense' of a particular, unusual experience he had, and it would be misleading to attribute it to a general belief the IXam had about 'lion ghosts', though the idea was obviously not nonsensical to at least one informant.

Contemporary exploration of the archive also requires careful consideration of one's own theoretical approach. In this thesis, I discuss the relationship of the archive to one of the major debates in southern Africa hunter-gatherer scholarship of the later twentieth century: the extent to which ethnographically documented hunter-gatherers can be used as analogues for hunter-gatherer populations in the past (i.e. the 'Kalahari Debate'). The degree to which the 'classic' ethnographies of central Kalahari groups can be used as proxies for hunter-gatherers geographically or temporally separated from them is still contested, but since the rise of revisionist critiques (Wilmsen, 1989; R. Gordon, 1992), the influence of particular historical circumstances on specific ethnographic observations has become an important theme. The eclipsing of the archive during the rise of Kalahari ethnography as the source of dominant models for hunter-gatherer societies has meant the IXam Bushman have not significantly contributed to the 
Kalahari Debate, although such major dialogues have had impacts on contemporary study of the Bleek-Lloyd archives: there now exists a corpus of historical work focusing on elucidating the circumstances existing in the Karoo over the colonial period (Penn, 2005). Missing from this discussion is an exploration of the ways in which the Bleek-Lloyd archive should be viewed in light of the wider study of hunter-gatherers in contact scenarios. The apparent paradox of "Later Stone Age hunter-gatherers" using metal and keeping livestock can only be resolved with careful consideration of the way in which we are using the term 'hunter-gatherer' and what this means in relation to other, non- 'huntergatherer' groups; exploring anthropological approaches that deconstruct and reexamine the use of this term, I ask whether looking at the IXam as exemplars of a series of processes occurring in the context of hunter-gatherer interactions with colonising populations is a useful approach (Chapter 2:31; Chapter 9:342).

A thorough understanding of the wider situation in the nineteen century Cape Colony is necessary before we can hope to properly understand the archive. The oldest informant (\|kabbo) was born around 1815 (Bank, 2006:129), and the youngest (la!kunta) in the early 1850s (Bank, 2006:74). Firsthand accounts therefore date approximately from the third decade of the nineteenth century onwards, while second-hand narratives (attributed to parents or grandparents) record the experiences of Karoo Bushmen from the late eighteenth century. Penn $(1995,2005)$ has synthesised in detail the primary literature for the eighteenth century (Chapter 4b:82), but there is no comparable overview for the nineteenth century. Authors (Strauss, 1979; Kallaway 1982; Anderson, 1987) focusing on 
specific issues or groups for the region during the period, and wider syntheses of southern African history (Elphick and Giliomee, 1979; Newton-King, 1999; Etherington, 2001; Elbourne, 2002; Legassick, 2010 [1969]), are relevant for understanding the position of the IXam at this time; Chapter 4c (110) introduces archival evidence from the nineteenth century in the light of this scholarship. Building on the idea of contextualisation, other forms of evidence including the material culture and built environment accessible through archaeology (Chapter 4a:82), as well as environmental considerations, climate, biota and geology (Chapter 3:45, 64), are introduced in the initial sections of the thesis, defining the parameters of the nineteenth century Karoo.

Although references in the archive to IXam lifeways predominantly take the form of brief, scattered notes (J. Deacon, 1996a:253), the records contain a considerable amount of information that has never been collated, or discussed with an overarching focus on IXam strategies. Hewitt (2008 [1986]) provided a brief ethnographic sketch, but was concerned primarily with the structure of the texts themselves; subsequent works have continued this trend or focused on 'mining' the archive for specific kinds of information (particularly, the 'mental' or religious world of the $\mid \mathrm{Xam})$. Chapters 5, 6, 7, and 8 are concerned with the detailed reconstruction of IXam practice during the nineteenth century. I investigate the extent to which Bushman participation in hunting and gathering practices was connected to a specific identity that we might characterise as distinctively 'hunter-gatherer'. Focusing on concepts of agency, I examine IXam understandings of the fundamental attributes of personhood, before moving on to 
consider the way in which this structured interactions between the various identities of the nineteenth century IXam world. Differentiating between various 'internal' |Xam identities, and introducing a range of non-Bushman groups, I consider the ways in which IXam understandings influenced their relationships with these individuals and their material culture, and particularly whether behaviours inherent in these contacts represent the disaggregation of any 'huntergatherer' identity and practice. These themes are then placed within a broader global context in a concluding chapter (Chapter 9:337), which draws upon examples of encounters between colonial populations and arid-zone 'huntergatherer' societies elsewhere in the world and examines the degree to which this contextualisation improves our understanding of the IXam situation. 


\section{Chapter 2: Theorising Foraging in a Colonial Context}

\section{The IXam as 'Hunter-Gatherers'}

The following discussion introduces the central anthropological constructs employed throughout the thesis. If analyses of the specific situation of the nineteenth century IXam are to have wider applicability, it is crucial that a theoretical justification for extending any insights obtained is explored; this exploration also attempts to challenge the limitations of the nineteenth century theoretical framework (Chapter 1:16; cf. Bank, 2000; Moran, 2001, 2009) and access the 'ethnographic' data of the archive. Beginning with contemporary characterisations of 'Bushman' populations, we can see that at least one component of nineteenth century characterisations has remained a central point of interest. Societies practising hunting and gathering subsistence strategies have come to play an increasingly significant role for social science disciplines over the 
course of the twentieth century, and while this is not the place for an extensive review of this development (for 'hunter-gatherer studies' historiography, see Ingold, Riches and Washburn, 1988, 1988a; Bettinger, 1991; Barnard, 2004), the placement of IXam populations within this category renders pertinent some of its themes.

Starting from the basic observation that subsistence-led group definitions simplify real-world complexities, it is imperative for us to examine the types of differences that are elided and the sorts of commonalities that are stressed, returning to the premises and assumptions embedded in such definitions. In a southern African context, the IXam must be situated relative to hunter-gatherer populations further north in Namibia and Botswana, groups that have become dominant examples of southern African hunter-gatherer strategies; their more complete ethnographic record has favoured an emphasis on the similarities between these Kalahari populations and no longer extant Karoo hunter-gatherers, overshadowing potential differences between northern populations and the IXam (and other southern groups). While this assumption has been justified through its role in generating insights regarding southern Bushmen practices not documented ethnographically (see Glossary, !gi:xa:415), it deserves critical scrutiny if we are to avoid essentialising features of both northern and southern Bushman populations, removing them from their specific contexts. It is with this overall aim that I introduce three thematic strands: outlining some of the long-term impacts of eighteenth and nineteenth century conceptualisations of the 'hunter-gatherer', moving on to consider issues of historicity in hunter-gatherer societies that arose 
in later twentieth century scholarship (the 'Kalahari debate'), and concluding with an examination of the impact of these debates on redefining the category, with a particular focus on the increasing significance of the relationship between practice and ideology.

The development of the 'hunter-gatherer' as a discrete category of human sociality has a considerable pedigree in Western thought as part of subsistencebased schemes for classifying and understanding societies in a broad, comparative manner (c.f. Barnard, 2005:375-380). While interest in identifying differences and similarities between human societies was stimulated by colonial expansions that brought about increased contacts between European and non-European populations, attempts to define the 'hunter-gatherer society' began not as a result of ethnographic observation but rather derived from political philosophies emergent in late eighteenth century European revolutionary thought, for which the notion of the 'natural man' took on a particular salience. Although the social and intellectual upheavals of this time drew heavily on and reacted to Renaissance and mediæval philosophy (Pluciennik, 2004), the codification of 'hunter-gatherer society' is most appropriately situated within the milieu of 'Enlightenment' Europe and its colonial encounters. A key theme of this milieu was an increasing concern with 'rationality', a preoccupation that had significant impacts on a diverse range of social phenomena including religious institutions (Haakonssen, 1996), notions of criminality (Foucault, 1977), and theories of illness (Foucault, 2001 [1964]). When defining hunter-gatherer societies, this stress on rationality manifested itself in the belief that reliance on hunted or gathered resources was 
'irrational' or indolent, demonstrating 'animal' modes of interaction with the environment. In John Locke's (1764 [1689]) link between legitimate control of territory and 'industrious and rational' use of land, this belief was tied intimately to the colonial experience, forming a cornerstone of 'terra nullius' doctrines attempting to legalise settler land claims (Claeys, 2010:15-20). Such 'irrationality' grouped hunter-gatherers with a range of other ungoverned (or ungovernable) groups, represented in Enlightenment terms as those who could not properly regulate themselves in accordance with the norms of civilised society; those who consequently had to be made subject to the coercive and violent elements of state power to prevent their disruption of these norms. In the eighteenth and nineteenth century Cape Colony, this amalgamation was very much in evidence, and 'Bushman' groups formed part of a heterogeneous 'anti-social' population including criminal fugitives, escaped slaves, and marginalised indigenes fleeing colonial society.

The expansion of colonial holdings in geographic spread and settler population density created a concomitant intensification of interaction with indigenous societies, and over time European (and colonial) intellectuals became increasingly aware of variability in the societies they encountered. Attempting to make sense of this variability, Enlightenment scholars situated these societies within classificatory systems that inevitably assigned hunter-gatherers a 'primitive' status, differentiated least from animal societies. As the nineteenth century progressed, this position was strengthened, particularly with the widespread 
dissemination of evolutionary theory ${ }^{6}$, and the increasing popularity of this model (a convenient locus uniting theories of biological differences between human populations with the aforementioned social theories) for explaining human variability. For nineteenth century audiences, hunter-gatherers became 'contemporary primitives', snapshots of the human past (or even 'missing links' between humans and animals) isolated either by geographical remoteness or biological incapacity from the transformative historical processes that farming and state-level societies had undergone.

As the developing notion of the significance of this subsistence category was tied intimately to colonial encounters, the next salient question becomes whether the term has any contemporary utility, if we reject nineteenth (and early twentieth) century models of universal social evolution. Of central relevance is the putative link between 'primitiveness' and pristineness, and whether 'authentic' huntergatherers must maintain a status as isolated, autonomous, and 'uncontaminated' groups with no (or at least, very little) contact with societies employing alternate modes of subsistence. Despite shifts in emphasis following the consensus rejection of social evolutionary models, this contention has remained significant through to the present. As the explanatory power of evolutionary theory in modern biology became increasingly apparent, the 'hunter-gatherer' category retained its vital importance in attempts to understand the theory as applied to the human species. When conceptualising humans as the result of evolutionary trajectories, hunting and gathering practices acquire a special significance, as they

\footnotetext{
${ }^{6}$ Darwin himself famously regarded Yaghan hunter-gatherer populations as straddling the gap between humans and animals (Bettinger, 1991:3; Browne, 2009:10)
} 
must in some form have been important dimensions of the human evolutionary environment; even recent approaches exploring hunter-gatherer historicity often begin with observations emphasising the time-depth of hunter-gatherer strategies relative to food production (Barnard, 2004:1). For African groups, the issue is compounded by the fact that our hominin lineage evolved on this continent, as it is here that we might expect to find the closest parallels for the environmental conditions that influenced this evolution (Lee, 1976). This observation lies at the core of a range of mid-twentieth century approaches in hunter-gatherer studies, proposing various kinds of 'ecologies', cultural, evolutionary and behavioural (e.g. Winterhalder, 2001) as methods for understanding hunter-gatherer practice. Our information about hunter-gatherer subsistence practices stems only from contemporary or historically-documented hunter-gatherer populations; the question becomes whether they represent privileged sources of analogical information as (in modern evolutionary terms) groups with less derived characteristics. Crucial within this inquiry is exactly the aforementioned issue of 'pristineness', as the impacts that contacts with farming or pastoralist societies in shaping the 'ecologies' of ethnographically-documented hunter-gatherer societies represent one very obvious reason to doubt the appropriateness of privileging such societies as analogues for the 'deep' evolutionary past.

Analyses of hunter-gatherer societies drawn from the evolutionary tradition have tended to characterise them as (relatively) isolated social units whose subsistence practices were, being well-suited to their particular ecological setting, in some form of stable equilibrium with their environment. Revisionist critiques suggested 
that such analyses rendered hunter-gatherers 'people without history', subject to change through shifting environmental conditions rather than any agency of their own; such analyses were theoretically situated in anthropological attempts to reconcile the notions socio-cultural 'structure' with the exercise of agency and individual practice, and politically located in 'post-colonial' revisions of intellectual categories developed under the aegis of colonial milieus (Lee and Guenther, 1993:186-187). For scholars of southern African hunter-gatherers, this is succinctly encapsulated by application of these critiques to the 'classic' Kalahari ethnographies (Jul'hoansi, and, to a perhaps lesser extent, the Glwi and Gllana). Constraints of space prohibit in-depth discussion of the specifics of this 'Kalahari debate', which has produced an extensive (and often polemic) literature: Barnard (1992a) provides an overview of the considerable body of work pertinent to the topic that had developed even twenty years ago.

For my purposes, the debate can be separated into two major facets. Firstly, the revisionist critique stressed that particular ethnographies down-played the interaction of Kalahari foragers with food-producing societies. Anticipated to a degree by other authors (e.g. Schrire 1980), this critique coalesced around Wilmsen's (1989) premise that many of the features observed by the Harvard Research Group for the NyaeNyae and Dobe Jul'hoansi were consequent on their incorporation as a low status community within the wider society of their Bantuspeaking farmer neighbours. Although the 'founding' ethnographic texts (Lee and deVore, 1968, 1976) had from the outset recognised an element of historicity in these groups, it is certainly true that such elements were muted in favour the 
aforementioned equilibrium model. Revisionist approaches attempted to demonstrate that this muting was an understatement of the degree to which Bushman institutions and practices were consequent on their interactions, by exploration of the temporal extent of this interaction. Specific applications of this approach have been criticised on empirical (Lee and Guenther, 1993) and interpretive (Solway and Lee, $1990^{7}$ ) grounds, and the imperfect and partial nature of our archaeological understandings of precolonial interactions in the Kalahari (Sadr, 1997:109-111) is a considerable obstacle in attempts to reconstruct longerterm trajectories for the region. However, at least one positive outcome of Kalahari revisionism was an increased awareness that NyaeNyae and Dobe groups should not necessarily be considered "the" paradigmatic group within a variable set of Kalahari Bushman strategies ( $c f$. Kent, 1992), and that the specific histories of these diverse populations were central to the inferences that might be drawn from their study.

This historicising trend is well-supported in the case of eighteenth and nineteenth century IXam populations in the Cape Colony (Chapter 4b:99; Chapter 4c:110); even a cursory historical overview demonstrates that they were not only merely 'in contact' with food-producing populations, but that a great deal of their subsistence behaviour was oriented towards species that could not be termed 'wild' by any definition (Chapter 8a:282). Although they seem not to have

\footnotetext{
7 These authors suggest revisionist approaches tend to elide issues of autonomy and isolation, conflating contact with domination and subordination.
} 
incorporated horses $^{8}$, there are many instances of IXam individuals obtaining stock (through raiding or intermarriage with herders), or engaging in labour relations that allowed them access to domesticated resources (Chapter 8c:318). Colonial populations considered certain forms of IXam engagement with their livestock as not just coherent with, but almost as emblematic of, their huntergatherer identity. Subsistence was always a significant factor in colonial characterisations of 'hunter-gatherers', but it was not always structured around the exploitation of wild versus domesticated resources; raiding practices were classified as an interaction that was in many ways the premier activity of huntergatherer groups. Although other groups (Khoekhoe herders, agro-pastoralist Xhosa) were associated with raiding, 'Bushman' involvement in the same practices was characterised in a very particular manner, as ahistorical, 'passive' extensions of hunter-gatherer relationships with wild fauna rather than as trajectories emergent from the engagement of active agents with their specific historical circumstances; not recognising the concept of private property, the hunter-gatherer was supposed to treat the colonists' stock simply as any other form of wild resource. Later in the nineteenth century, after decades of violence had sapped the capacity for Bushman populations to resist large-scale encroachment of their territories, other populations (Korana, Chapter 4c:116) became the 'quintessential' raiders, and hunter-gatherers were characterised as the 'harmless' people (Thomas, 1959) unwisely exploiting a precarious and unpredictable suite of wild resources, destined to vanish before the vigorous

\footnotetext{
${ }^{8}$ Some Bushman populations in the Drakensberg-Maluti ranges used horses as the basis of raiding subsistence strategies (Challis, 2009:104-106; Wright, 1971; 2007:127-128), demonstrating the variable trajectories emergent from hunter-gatherer engagements with colonial societies.
} 
expansion of herding or farming societies; where such societies were themselves indigenous, the characterisation of such expansions as 'colonisation' was politically useful for European settlers ${ }^{9}$.

One consequence of the Kalahari debate has been an increasing acceptance that hunter-gatherer groups of the region experienced variable historical trajectories, and that, for some at least, interactions with farming groups have a considerable time depth. The issue now becomes a more theoretical one, of whether the relative isolation of these groups means they remain acceptable analogues for prehistoric hunter-gatherers, or whether historicity represents an insurmountable problem for extending insights into the pre-farming past. This debate is far from resolved, and, although it no longer provokes the (often acrimonious) dialogue of the early 1990s, its themes continue to arise: Humphreys' (2007) recent suggestion that Australian Aboriginal hunter-gatherer groups may provide better analogies (than the Kalahari ethnographies) for pre-pastoralism Later Stone Age southern Africa is based on the absence of indigenous pastoralism in the former areas. Recognising varying degrees of contact between hunter-gatherer and foodproducing societies, the question of why this interaction is to be considered such a profound moment in hunter-gatherer historical trajectories requires a reexamination of why subsistence is valorised as the primary factor in defining differences between societies, and what might replace or modify this factor.

\footnotetext{
${ }^{9}$ Stow's (1905) subtitle for his history of South Africa casts the 'intrusion of the Hottentots and Bantu on the hunting-grounds of the Bushmen' in this light.
} 
Definitions for hunted and gathered resources often seem initially straightforward, as with Winterhalder's (2001:12) restriction of them to species that are 'not actively managed' by people. This simplicity is swiftly complicated by the fact that definitions revolve not only around the classification of subsistence resources themselves, but must incorporate the suite of human behaviours and practices directed toward these resources. Focusing solely on exploitation in terms of domesticated versus wild resources ${ }^{10}$ is not without problems, as it neglects the specifics of what people actually do in their subsistence strategy and how they think about doing it. For example, the distinction between foraging and subsistence production as it relates to wild versus domesticated resources depends on a definition for 'growing'; Ingold (2000) suggests that employing economic metaphors drawn from the production of material culture (items made from raw materials) misrepresents farming and herding practices. As farmers or herders at best exert an imperfect control over the reproduction of their herd or crop, a more appropriate definition would stress the "relative scope of human involvement in establishing the conditions for growth" (Ingold, 2000:86). Ingold sees this involvement as having more similarities with the production of socialised human individuals (bringing up children) than manufacturing processes; with such a definition, hunter-gatherer concern with the resource 'socialisation' (see below) overlaps with food production.

\footnotetext{
${ }^{10}$ Standard criterion for domestication emphasise genetic distinctness from wild progenitors, through evolutionary selection pressures determined by human aims and desires or (if species can become 'self-domesticated') by close association with humans.
} 
Control over reproduction is a crucial determinant of domestication in a genetic sense, but is only incidental to plant cultivation and animal husbandry in experiential terms. Ecological differences between species mean that human engagements with them will be highly differentiated depending on the demands of the resources exploited; large, long-lived plant domesticates (e.g. olive trees) demand different relations to annual grass species, and human involvement in honeybee reproduction is very different to human control of breeding cattle. Resources accessed by hunter-gatherer populations are also highly differentiated from one another, and may in practice require relationships more similar to certain domesticated resources than to other wild resources; interactions with wild honeybee nests are markedly more similar to apiculture than to hunting large game, while relationships between man and reindeer in Siberian populations often blur the lines between hunting and herding (Willerslev, 2007:3-4).

The impacts of resource differentiation on hunter-gatherer subsistence strategies became well-established in anthropological research by the early twentieth century; Steward's (1936) definition of the 'band' society stressed influences of ecological constraints (e.g. scattered resources) on social organisation and subsistence ${ }^{11}$. Although defining 'hunter-gatherer' society a priori focuses on subsistence issues, the recognition of socio-cultural dimensions as integral components within 'subsistence strategies' was incorporated in Steward's 'band' society; patrilineal, patrilocal, exogamous groups of related males with their wives

\footnotetext{
${ }^{11}$ Although Steward's model recognised specific development sequences within certain areas, he focus on ecological variability rather than 'social evolution' as a source of differentiation in sociocultural forms.
} 
and children residing in a communally-owned territory were considered characteristically 'hunter-gatherer'. Ecological differentiation and socio-cultural organisation were incorporated in the formulation of later twentieth century models dividing the 'hunter-gatherer' society into a number of subcategories, recognising and attempting to systematise hunter-gatherer diversity. Woodburn's (1982) separation of immediate-return and delayed-return populations and Binford's (1980) division between 'foragers' and 'collectors' both extrapolate many socio-cultural features not proximally related to subsistence practices within the broad ecological factors that dictate the parameters within which any given socio-cultural unit must operate (e.g. attributing food-sharing practices to riskaversion in subsistence strategies). Other models (Testart, 1982; T. Price and Brown, 1985; Arnold, 1996) focused more particularly on a continuum of sociocultural 'complexity' (social differentiation, the emergence of 'rank') as separating types of hunter-gatherer society. Expanding on this notion of rank and relations between people, issues of resource-sharing and exchange relationships (situated within Marxist economic theory that emphasises social and political dimensions of modes of production) were incorporated into these subdivisions, with reciprocity, kin relationships and notions of egalitarianism (Ingold, 1999:400-404) valorised as distinctive 'economic' features of hunter-gatherer lifeways.

Ecological and (at least partly correlated) sociological variability in huntergatherer society is an integral component of contemporary definitions: while the details of such variability are contested, the basic premise of diversity in hunter- 
gatherer lifeways is recognised widely. This recognition provides a convenient point of introduction for the final issues I wish to discuss, located in the relatively small step from the incorporation of socio-cultural features to the adoption of 'ideological' attributes (which re/create socio-cultural phenomena) into definitions; this development relates also to intellectual dissatisfaction with the relegation of more abstract cultural components as largely epiphenomenal to concrete subsistence realities, a portrayal often framed as a basic disconnection between humanistic and scientific approaches to the study of human societies (Jordan, 2008:455). The identification of a 'foraging mode of thought' (Barnard, 2002) has become a major preoccupation of later twentieth century anthropological hunter-gatherer scholarship, emerging from a re-conceptualisation of nineteenth century definitions of ideological and ontological components in hunter-gatherer cultures, including one of the earliest theoretical constructs of the anthropological discipline, Tylor's concept of animism (Tylor, 1871:377-453; for a review, see Bird-David, 1999). The revisiting and redefining of animism arose primarily from ethnographies of lowland South American societies (Descola, 1992, 1996), where it has found much purchase within the literature specific to the region and been subject numerous modifications. De Castro's (1998) interpretations of their cosmological systems as a particular form of 'perspectivist' animism characterises Amazonian hunter-gatherer notions of personhood as ones in which sentient beings (possessed of a soul or vital force) engage in relationships with one other, sharing a common subjective experience with entities occupying similar bodies to themselves (i.e. bodily form shapes 
perspectives) but embedded in networks of communication and interaction that transcend their bodily form. Descola $(2010: 218)$ characterises this interaction as an exchange of signs, with a basic contiguity in subjective experience allowing beings to comprehend one another's intentions in practice; the capacity to exchange perspectives in such societies is a 'special' ability, and humans who can perform such exchanges are usually ritual specialists for these groups.

Several approaches have explored the insights of these models beyond Lowland South America (e.g. Bird-David's 'animistic epistemology', 1999:S77-S79), perhaps becoming most widely known in Ingold's (2000) deployment of similar models when characterising the ontological underpinnings of relationships between humans and their environment in various subsistence strategies, providing idealised models derived from ethnographic examples. Suggesting that a pervasive 'Enlightenment ${ }^{12}$, division between the human and natural worlds continues to underlie definitions of 'hunter-gathererness', he sees this as an imposition undermining the most basic emic premise: that the quality of being animated is a principle shared amongst human and non-human communities. $\mathrm{He}$ contrasts totemic and animic ontological systems as alternative forms of huntergatherer ideologies, situating the former among Australian Aboriginal societies; an apposite reminder, given the origins of the word totem (derived from the North American Ojibwa), that his models are tools for understanding rather than attempts to produce accurate reifications of particular emic perspectives. The

${ }^{12}$ Ingold uses 'Enlightenment' and 'Western' as shorthand for the 'rational' worldview that (loosely) became codified during the Enlightenment, while recognising variability in these societies; Ingold (2000:176-178) himself develops approaches based upon the work of 'Western' zoologists, such as Seboek (1963) and von Uexküll (1957 [1934]). 
notion of the Dreamtime (the creation of the landscape by ancestral beings, who imbued it with their creative essence) is the prototype of his model for totemic systems: the particular forms that living beings manifest are drawn from their relationship with their landscape, which (if 'looked after' in the correct manner) continues to engender new life derived from these creative essences.

Form in animic systems is generated by life processes and interactions between agents, and the movement of animating principles between ontologically similar inhabitants is vital for the continuity of the living world. Ingold draws on ethnographies of indigenous American societies ${ }^{13}$, for whom notions of circulation and reciprocity in exchanges of 'vital force' are salient in ontological understandings: "the powers that bring forth life ... [are] ... distributed among the manifold beings that inhabit it" (Ingold 2000:113). Agents share ontologically equivalent vitality but live in a manner consistent with their form, relying for their continued existence on sharing their vitality though interactions with a range of different forms. Where in totemic systems, hunting practices form part of a preestablished order that constitutes the ancestrally mandated 'proper' way of living $^{14}$, animic interactions between different types of 'body' are necessary for the vital force to circulate; these interactions are vital for the reproduction of both human and non-human societies (Ingold, 2000:113-114). Having significant consequences for the construction of relationships between human and non-

\footnotetext{
${ }^{13}$ Ingold draws on ethnographies from the circumpolar North, but there are clear resonances with the South American models.

${ }^{14}$ Resource acquisition is therefore subsumed under the rubric of relationships with ancestral beings, and is not of particular cosmological significance in and of itself.
} 
human agents, these ontological models are implicated fundamentally in subsistence strategies.

Ingold (2000) discusses another heuristic tool pertinent to this theme of relationships between human and non-human entities, defining trust and domination as two dimensions that allow us to contrast 'hunter-gatherer' and 'pastoralist' orientations. He derives these from a more general premise involving the establishment of social relationships with non-human agents, and places them along a continuum; 'hunter-gatherer' and 'pastoralist' here represent convenient labels for the kind of practice involved, rather than particular 'categories' of society $^{15}$. Although Ingold's definition of 'domination' (suggesting that masterslave relationships obtain between pastoralists and their livestock) has been criticised as neglecting dimensions of care and co-operation in interactions with domesticated animals (Armstrong Oma, 2010:176, 180-181), such critiques serve to emphasise his more general point that a shift between hunter-gatherer and pastoralist activities is a "transition [that] involves a change in the terms of engagement" (Ingold, 2000:75, emphasis in original).

Trust, the 'hunter-gatherer' mode of interaction, represents a combination of autonomy and dependency, requiring a particular response from another person, but not attempting to force this response. This dimension can be located in any relationship between persons; Ingold suggests that resolves a contraction of Woodburn's immediate-return model by accounting for the co-occurrence of

\footnotetext{
${ }^{15}$ (Subsistence) pastoralists might have 'trust' relationships with some non-human agents, and vice versa.
} 
strong emphases on personal autonomy with enduring relationships. Proposing that characterisations of hunter-gatherer ontologies as analogical extensions of human sociality misrepresent emic ontologies by imposing an Enlightenment division between social-cultural and biological-natural worlds, Ingold unites the cultivation of relationships with hunted and gathered species with the maintenance of connections between human agents; that is, hunter-gatherers attempt to make non-humans 'behave properly'. From an emic perspective, the characterisation of hunter-gatherers as focusing on wild, 'out of control' (Ingold, 2000:62, 67) resources is inappropriate: these groups focus on making game ${ }^{16}$ act appropriately and on controlling prey species by cultivating correct relationships with them (Ingold, 2000:69).

Emergent in a range of 'ontologically' oriented approaches to hunter-gatherer societies is a concern with the relationship between ideology and practice, particularly how the 'working through' of ontological understandings shapes and is shaped by experience: Willerslev (2007) discusses at length the relationship between the encounters between hunters and their quarry, and the strengthening of their animic understandings that arise from their interpretations of these. A clear implication is that if the terms of the encounters changes, we should expect alterations in the ontological model (and vice versa). These theories of huntergatherer identity are therefore situated in humanistic interpretations that

\footnotetext{
${ }^{16}$ Ingold's models focus on animals, but themes discussed echo the approaches of authors looking at hunter-gatherer groups occupying forest environments, including the Nayaka of south India (Bird-David, 1990) and the Huaorani in Amazonian Ecuador (Rival, 1993, 2002) for whom the notion of 'trust' is expanded to include the inculcation of relationships of 'unconditional giving' on the part of the forest.
} 
emphasise individual agency within socio-cultural contexts, a manifestation of practice theory (Bourdieu, 1977 [1972]) and located at the intersection of structural models for human societies and behaviour, and those relying on experiential agent-led approaches. Performance theory (Schieffelin, 1996, 1998:201-204) is concerned specifically with its expression of practice theory in 'presentation', affective action, and relationships between agents; traditionally formulated for structured performative contexts (theatre, ritual contexts, storytelling ${ }^{17}$ ), a wider definition can incorporate the performative dimensions of everyday actions. It contributes a crucial component when introducing nonsubsistence components to 'hunter-gatherer' definitions, as the performative dimension of identity remains manifest in relationships with other identities and is enacted with material culture, both in terms of bodily action (if one understands the human body as a form of material culture; Sofaer, 2006) and use of specific items ${ }^{18}$. As participation in certain lifestyles requires the employment of specific forms of material culture, and identities based around such participation are linked to this material culture, performance theory forces us to return to the actual practices involved in the construction of more abstract components of identity.

Landscape, the largest domain of material culture, has long been considered a central element in hunter-gatherer lifestyles, due in large part to its salience in the cultural world of Australian Aboriginal societies (cf. Morphy, 1993:206; Head, 2000:17-18) occupying an important role in hunter-gatherer ethnography since the

\footnotetext{
${ }^{17}$ Well suited to the exploration of the Bleek-Lloyd archive ( $c f$. Bauman, 1992)

${ }^{18}$ Employed not only when undertaking specific actions, but also participating as a social agent in and of itself (Appadurai, 1986; Knappett and Malafouris, 2008).
} 
emergence of the anthropological discipline. Although unusual in the degree to which they emphasise people-to-landscape relations, themes made obvious in Australian ethnographies have found widespread resonance outside this continent. In colonial contexts, the necessity of incorporating these components of huntergatherer worldviews is evident: colonial projects are tied intimately to issues of landscape as attempts to incorporate new territory under the aegis of a particular polity. Such incorporation is shaped by the pre-existing landscape and requires substantial modification of it, as colonial populations arrive with established modes of interacting with land. Both indigenous and incoming populations are forced to re-negotiate these established relations: map-making and boundary delineation in the European colonies was crucial to the development and consolidations of issues of nationhood and sovereignty (D. Martin, 2011:146-151; Sparke, 2011:430-433). In the Cape Colony, the maps that accompanied early traveller accounts of their journeys in the subcontinent illustrate processes of interaction, reflecting their dependence on local knowledge and guides for the successful completion of these journeys, and demonstrating what D. Martin (2011:160) characterises as colonial agents' simultaneous desire to obtain (and take advantage of) and to eradicate indigenous systems of knowledge. Allowing that space has a fundamentally social dimension ( $c f$. Lefebvre, 1991 [1974]), this re-construction of 'space' (farm portions, official maps, borders) production can be seen as intrinsically colonial, manifesting and attempting to impose a particular definition of 'authentic' places. 
When attempting to formulate an understanding of hunter-gatherers in colonial situations, the relationship between practice and identity becomes increasingly significant. The 'structure' (pre-existing identities possessed by indigenous and settler individuals and groups) influences substantially the nature of the colonising process in terms of abilities and desires for compromise or conflict, while the interaction of groups of people previously unknown to one another allows for the radical transformation of previous identities: colonial experiences must therefore be regarded as simultaneously destructive, constructive, and transformative of identities, for both indigenes and incomers. If material culture is a constitutive element of the performance of identity, there can be considerable interaction between hunter-gatherer and non hunter-gatherer groups, even in the absence of settled populations of colonisers. This links well to the notion of an increasingly 'closed' (Chapter 4b:107) frontier scenario: when down-the-line introduction of material culture represents the extent of colonial intrusion, indigenous groups selectively incorporating new 'identities' (material culture) exert considerable power in their interactions.

I have discussed here some of the limitations of 'strict' subsistence-led definitions for "hunter-gatherer" societies. Although retaining a focus on subsistence as a major component of everyday experience and practice is crucial, the debate emerging from attempts to pin down the 'essential' characteristics of populations defined by this factor uncovers a range of variability such that even where we have a situation of 'hunters in a world of hunters' (Lee and deVore, 1968a:4), issues of 'pristineness' and contamination remain important concerns. The scope 
for hunter-gatherers behaving in ways similar to agriculture-intensive groups is limited in some ways, but it should not be dismissed out of hand; ethnographies for extant and historical hunter-gatherer groups presumably do not document the full range of past hunter-gatherer variability. As forms of farming such as intensive agriculture or stock-keeping represent only one method of food production, there might be a greater degree of similarity between 'wild' and 'domestic' resource exploitation than is at first obvious. With a spectrum-based view of subsistence practice, the concept of contact-as-contamination becomes less of a stark either/or division. Although contact and interaction may lead to considerable shifts in subsistence practice, at least as important is the manner in which people conceptualise their interaction with the resources incorporated in their subsistence strategies. Models of 'ontological orientation' overlain onto more subsistence-based constructs provide a useful adjunct that remind us that shifts in practice need to be understood: novel material culture, practices, and ideas can all be incorporated within existing ideologies, and this incorporation leads to change. The relevance of any given ideological system is grounded in experience situated within life histories; this fundamentally temporal component ensures ideology and practices are not shifting concurrently, and are differentiated between individuals with their own particular histories. It is with these thoughts in mind that I shall generate an historical ethnography of the IXam, focusing on the ways in which their socio-cultural world incorporated their colonial context. 


\title{
Chapter 3: Exploring Definitions of
}

\author{
the IXam-ka-!xoe
}

\section{Chapter 3a: Physical and climatological}

\section{parameters of the Karoo environment}

This chapter outlines the geographical parameters for the groups referred to in this thesis, drawing together relevant environmental, ecological, climatological and geological aspects to contextualise IXam hunter-gatherers in space, and providing the requisite background for understanding of some of the factors with which their subsistence and social strategies had to engage. The area that these groups inhabited lies within the Karoo regions of South Africa: this is a complex term encompassing substantial environmental variance, used extensively in twentieth century zoological and botanical studies (Acocks, 1953, 1975), with many authors creating subtly different definitions. It developed out of colonial experiences in 
the late eighteenth and early nineteenth centuries, where it was used to refer to the regions into which trekboer settlers moved as they expanded from the SouthWestern Cape (Chapter 4b:100). Moving from environments characterised by the distinctive floral kingdom of the Cape (a unique flora that represents one of the richest areas for plant diversity in the world) and away from the winter-rainfall zone that facilitated European agricultural practices, these settlers came to refer to the complex range of environments they encountered to the north and east as "Karoo" and subdivided it based on local variation: in South Africa today, regional toponyms identify several 'Karoos', including the Tanqua (Tankwa), Moordenaar's, Groot, and Klein Karoo.

Derived from now-extinct languages spoken by Cape Khoekhoen, the precise etymology of the term is uncertain. Early writers translated it as 'dry' (Sparrman, 1785:197; Burchell, 1822:75), while comparisons with Korana suggest 'hard' as another possibility (Wuras, 1920; Meinhof, 1930). Nienaber (1963) concludes that the term primarily signifies aridity, an observation backed up by the fact that the IXam term !karru relates to concepts of dryness and heat (Bleek, 1956:454): although they did not apply it to the IXam-ka-!xoe (Glossary:414), the IXam (speakers)'s country, Karoo and its cognates likely constitute part of a general Khoisan linguistic complex. The Karoo represents an extensive inland plateau separated from the coastal regions by the broken country and mountain ranges of the southern- and westernmost extensions of the Great Escarpment, and separated into two distinct biomes: a western Succulent Karoo that spreads from the coast over the Escarpment and its foothills, and the Nama-Karoo typifying the interior 


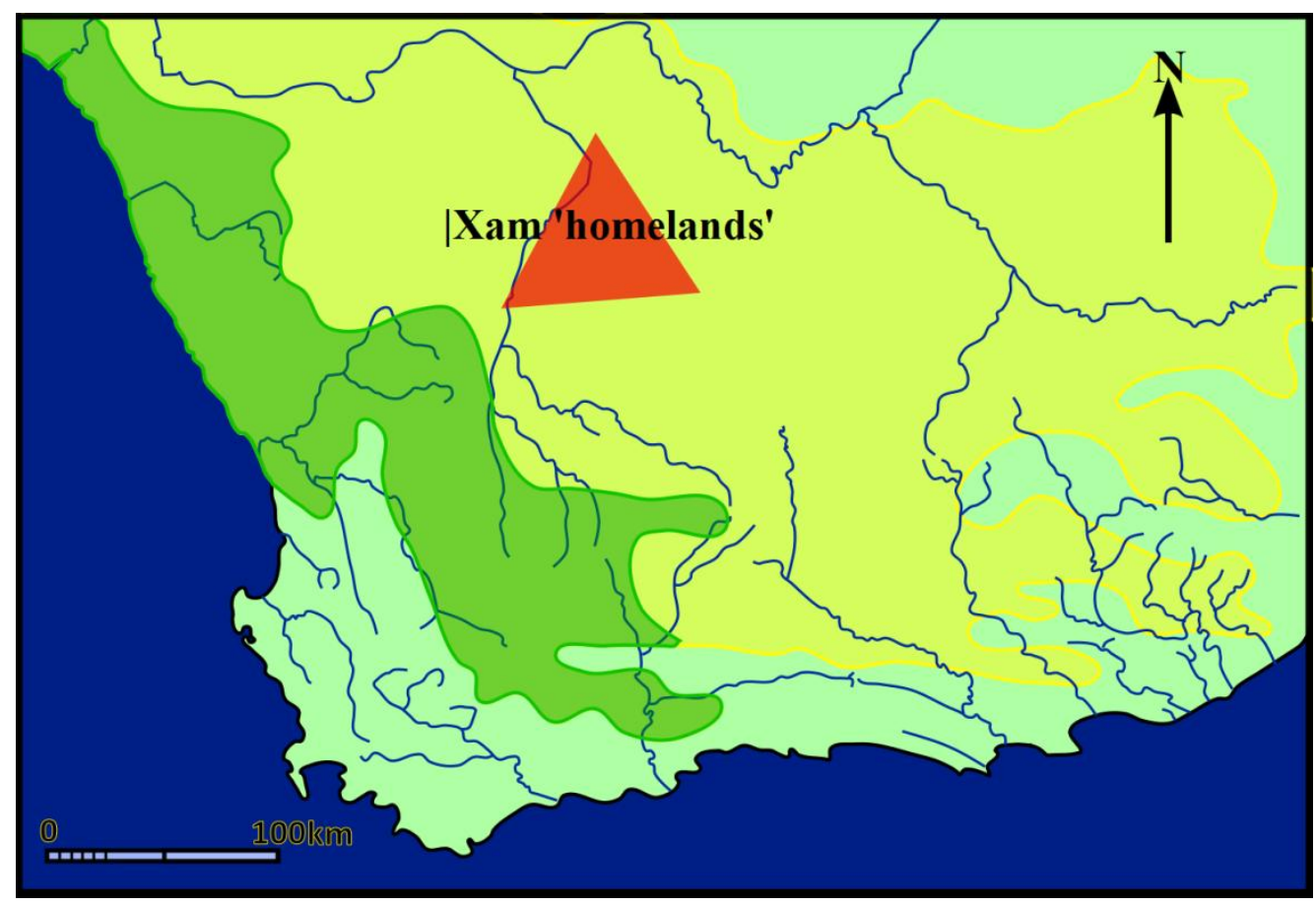

Figure 3.1: Succulent Karoo (green outline) and Nama-Karoo (yellow outline) biomes, with the Kenhardt-Brandvlei-Vanwyksvlei red triangle (home territories of the Bleek-Lloyd IXam informants)

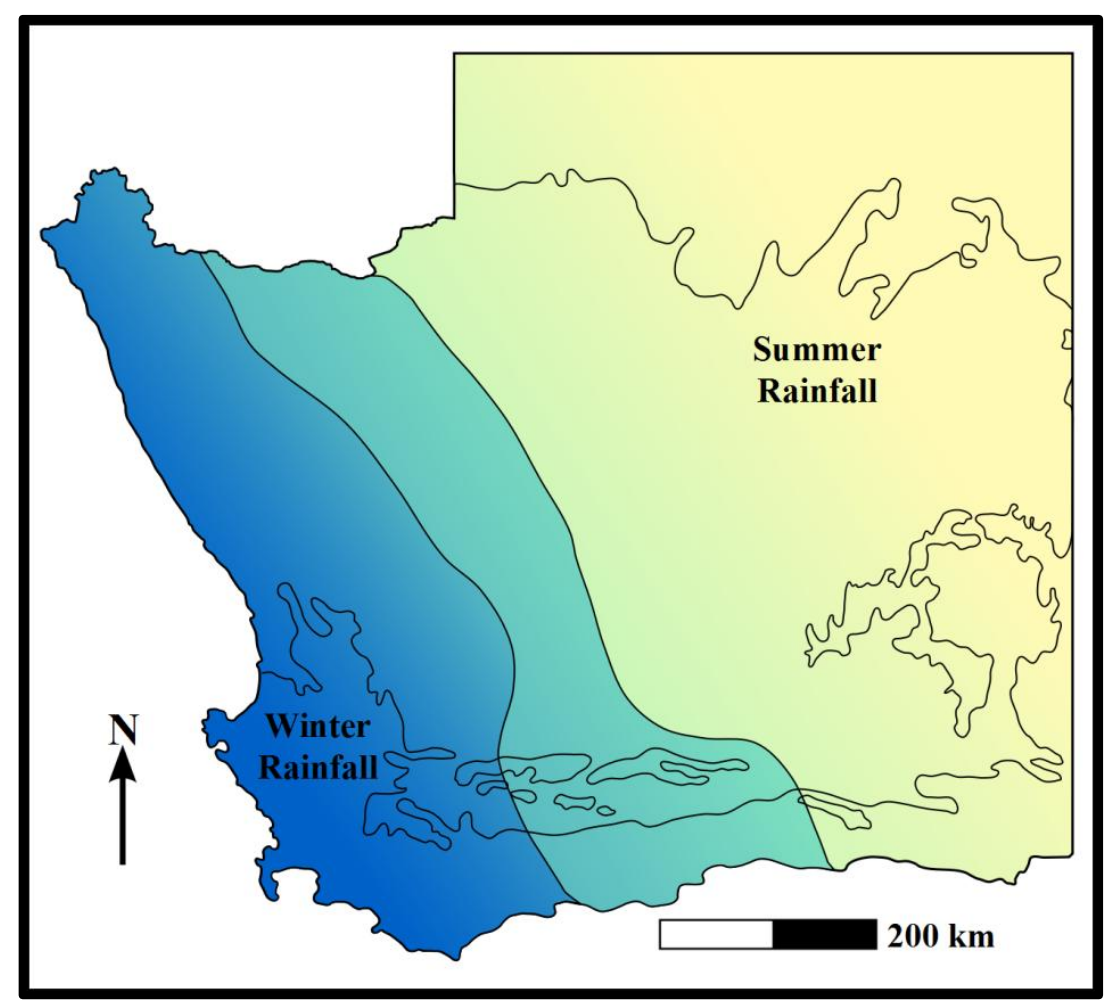

Figure 3.2: Rainfall zones of the Cape (modified after Hoffmann and Cowling, 1987:3, and Watkeys, 1999:25) 
plateau proper (Rutherford and Westfall, 1986; Low and Rebelo, 1996; Rutherford, 1997; Mucina and Rutherford, 2006; Figure 3.1:47). This chapter does not consider in detail the Succulent Karoo: although the area was inhabited by hunter-gatherers who almost certainly spoke languages (or even dialects) related to IXam, it was outside the experiences of the Bleek-Lloyd informants and had a different colonial (as well as prehistoric) trajectory (Rohde, Hoffmann and Allsopp, 2003:v-vi).

I begin by considering the Karoo's climate, the key structuring principle for Karoo environments and ecology. Like other arid environments, the infrequency and stochastic nature of water input influences the Nama-Karoo biome strongly (Lovegrove, 1993:18). Water availability and, to a lesser extent, temperature stress are major limiting factors for plant growth and faunal strategies, and substantially influence soil formation. Climate within the region is the result of the intersection of a wide range of weather systems (Figure 3.2:47), notably the shift between the two major rainfall zones of the subcontinent (Desmet and Cowling, 1999:3). Rainfall in most of southern Africa is governed by subtropical monsoonal systems of the Indian Ocean, falling maximally in summer, but the extreme South-West Cape has a 'Mediterranean' climate with hot dry summers and wet winters, determined by Atlantic and Antarctic systems (Reason and Rouault, 2005). The Nama-Karoo is interstitial between these two rainfall regimes, falling at the margins of one or other of the systems or in the overlap between the two. The shift from winter-rainfall dominated to summer-rainfall dominated zones determines the boundaries of Karoo biomes, with the Succulent 
Karoo experiencing rainfall from Atlantic weather-systems and the Nama-Karoo receiving mainly summer rains (Desmet and Cowling, 1999:4). Moving northeast, summer-rainfall becomes increasingly dominant; the reverse is true for south-westerly movement, and central regions are influenced by a subtle mix of the two (Figure 3.3:51). Marginal to both systems, the volume of rain experienced is low, between the $100-200 \mathrm{~mm}$ and $200-300 \mathrm{~mm}$ isohyets for mean annual rainfall (Figure 3.4:51), and orographic rainfall associated with the Escarpment ensures that systems approaching the Karoo from any direction lose a large portion of their moisture before they arrive.

The weather systems of both regions are predominantly anticyclonic, with strongly subsiding air masses that result in clear, dry conditions prevailing for weeks at a time. These are more common in winter but can also occur in summer, where they manifest as extremely hot and desiccating conditions (Desmet and Cowling, 1999:10) contributing significant heat stresses in an already arid environment. Rainfall from convective thunderstorms is rare in the Karoo, relative to elsewhere in South Africa, though it does occur. Winds are for the most part dry, and in the central Nama-Karoo blow mainly from the south-west in summer and the north in winter; although small whirlwinds and dust devils are common, larger dust storms are rare (Desmet and Cowling, 1999:10). Topographic relief, such as valley wind channels, plays an important role on a local scale (Werger, 1986). 
The unpredictability of rainfall is as important as its generally low overall volume. Broadly, the pattern for rainfall reliability follows that of volume of rainfall, and areas that receive less rain also receive their rain less regularly (Figure 3.5:52).

Comparing areas with the same annual rainfall in the two different biomes suggests that the winter-rainfall Succulent Karoo experiences more reliable rainfall than the central Nama-Karoo (Desmet and Cowling, 1999:9), where droughts $^{19}$ are common (Venter, Mocke and de Jager, 1986:41). There are indications of periodicity in the form of wetter and drier cycles over 15-20 year intervals, though the complexity of rainfall regimes and lack of temporal depth for detailed climate records prevent the construction of comprehensive models (Rouault and Richard, 2003:499-500); although periodic global climate events such as the southern oscillation of El Niño may influence South African regimes, there is no simple correlation between particular events and observed climatic data (Kane, 2009:206).

Fog and dew provide additional sources of moisture. The former is associated predominantly with coastal regions, where its significance has long been appreciated despite difficulties of quantification: adaptations of Namibian fauna (e.g. Hamilton and Seely, 1976:284) to the acquisition of moisture from coastal fog are well-known. Although the Orange (Gariep) River allows for the penetration of fogs some distance inland, they do not play a major role in the central interior, though heavy dews may partly replicate their effects (Desmet and Cowling, 1999:5). Dewfall responds to air temperature, humidity, and turbulence:

${ }^{19} 12$ month periods in which rainfall is less than $60 \%$ of average annual rainfall. 


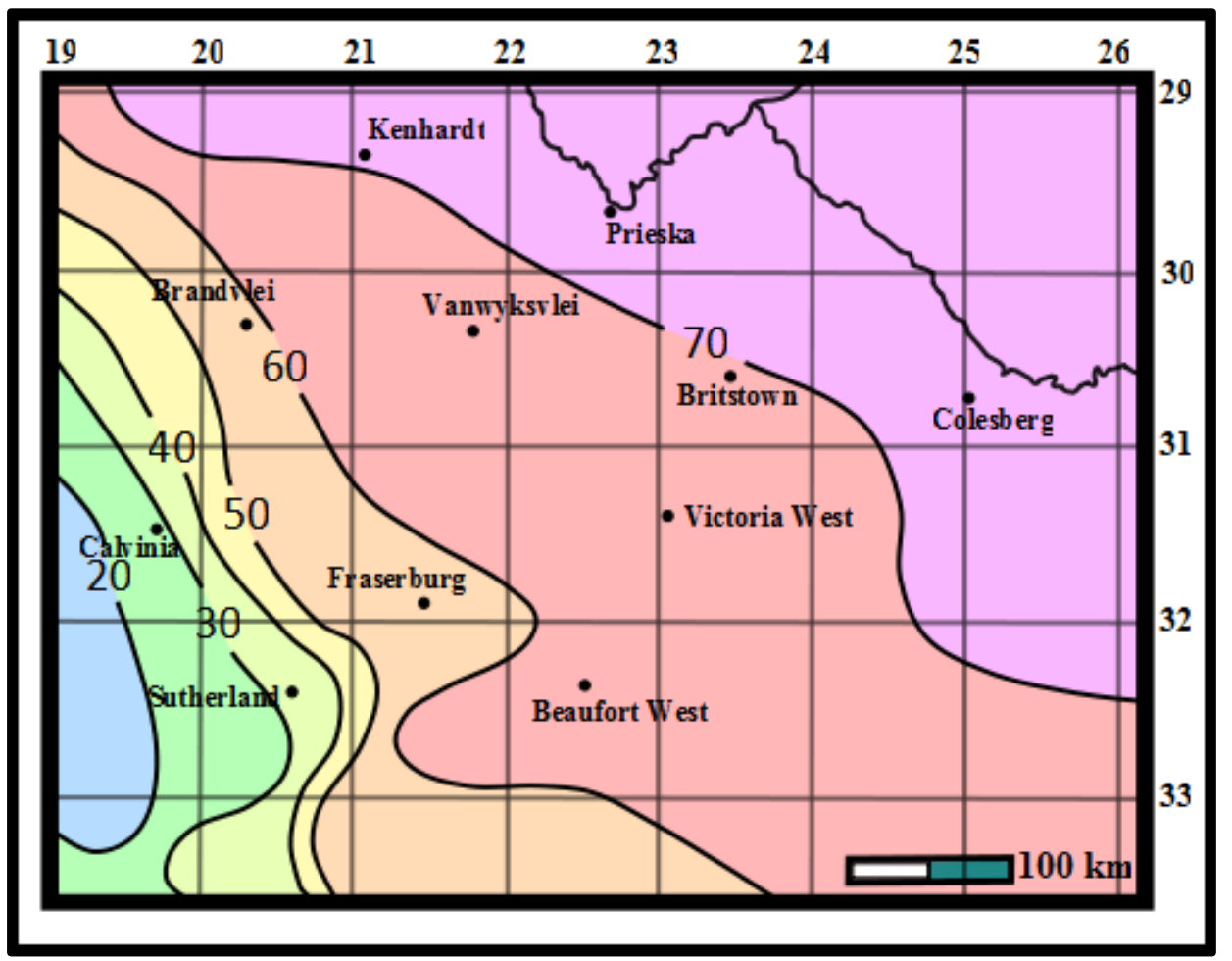

Figure 3.3: Distribution of summer (Oct-Mar) rainfall as percentage of mean annual (after Venter, Mocke and de Jager, 1986:40)

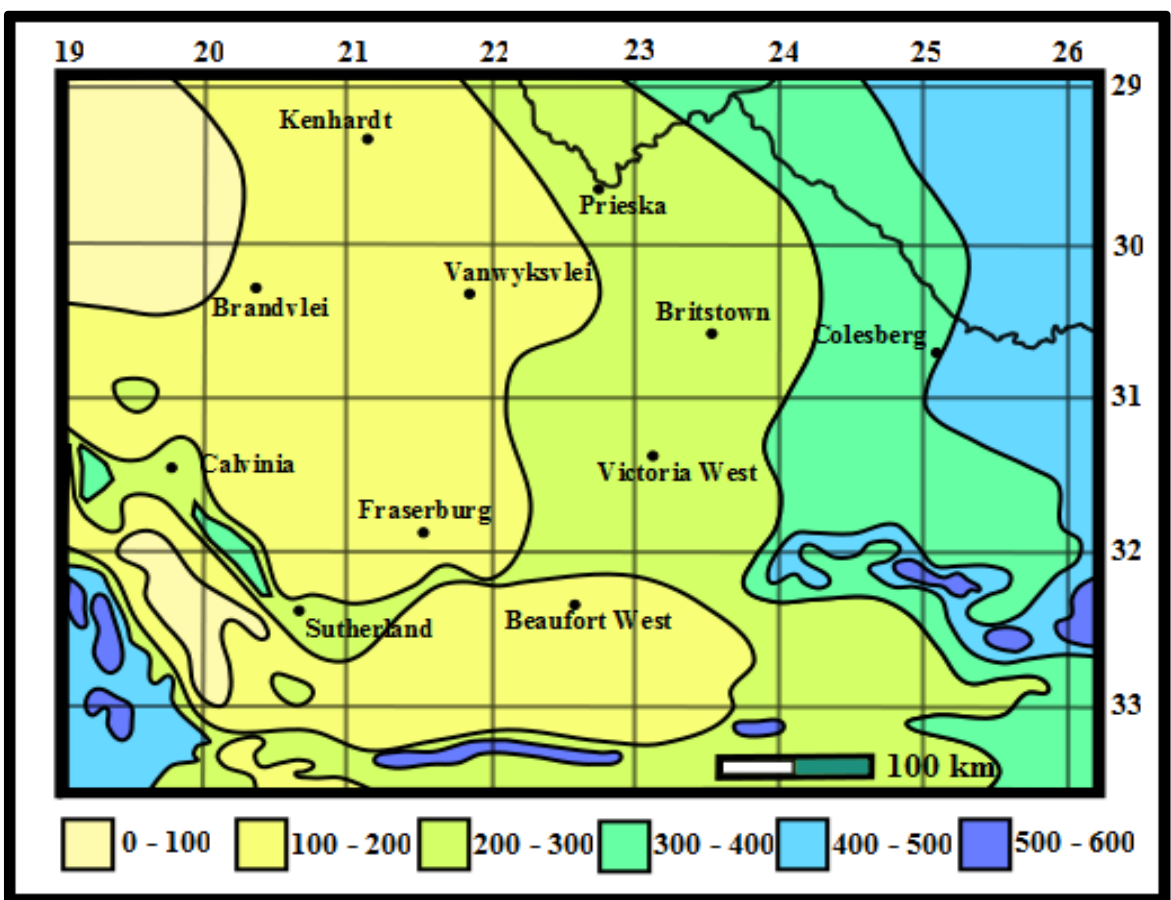

Figure 3.4: Isohyets of mean annual rainfall $(\mathrm{mm})$ in Karoo biomes (after Venter, Mocke and de Jager, 1986:40) 


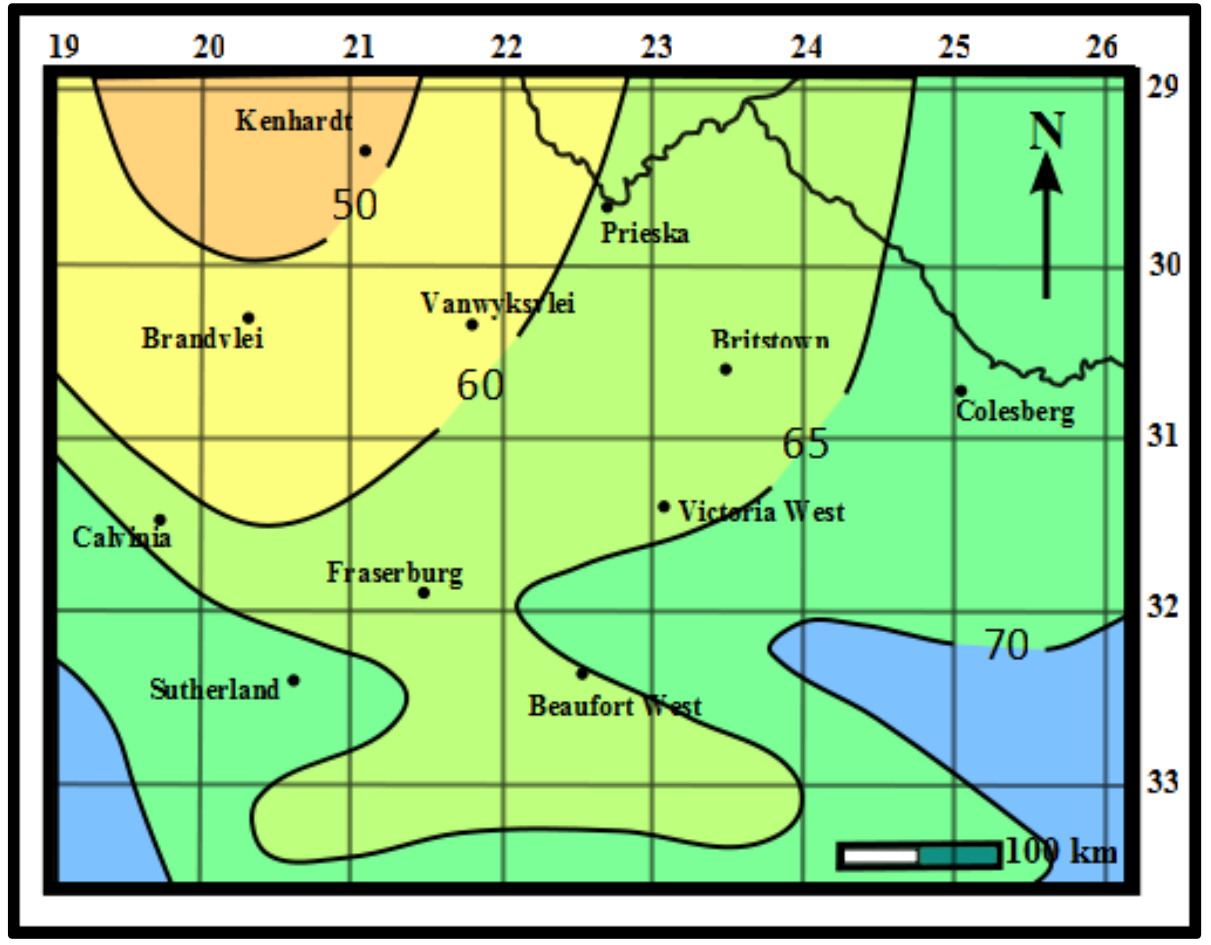

Figure 3.5: Reliability of rainfall; percentage years with rainfall $\geq 85 \%$ mean annual (after Venter, Mocke and de Jager, 1986:42)

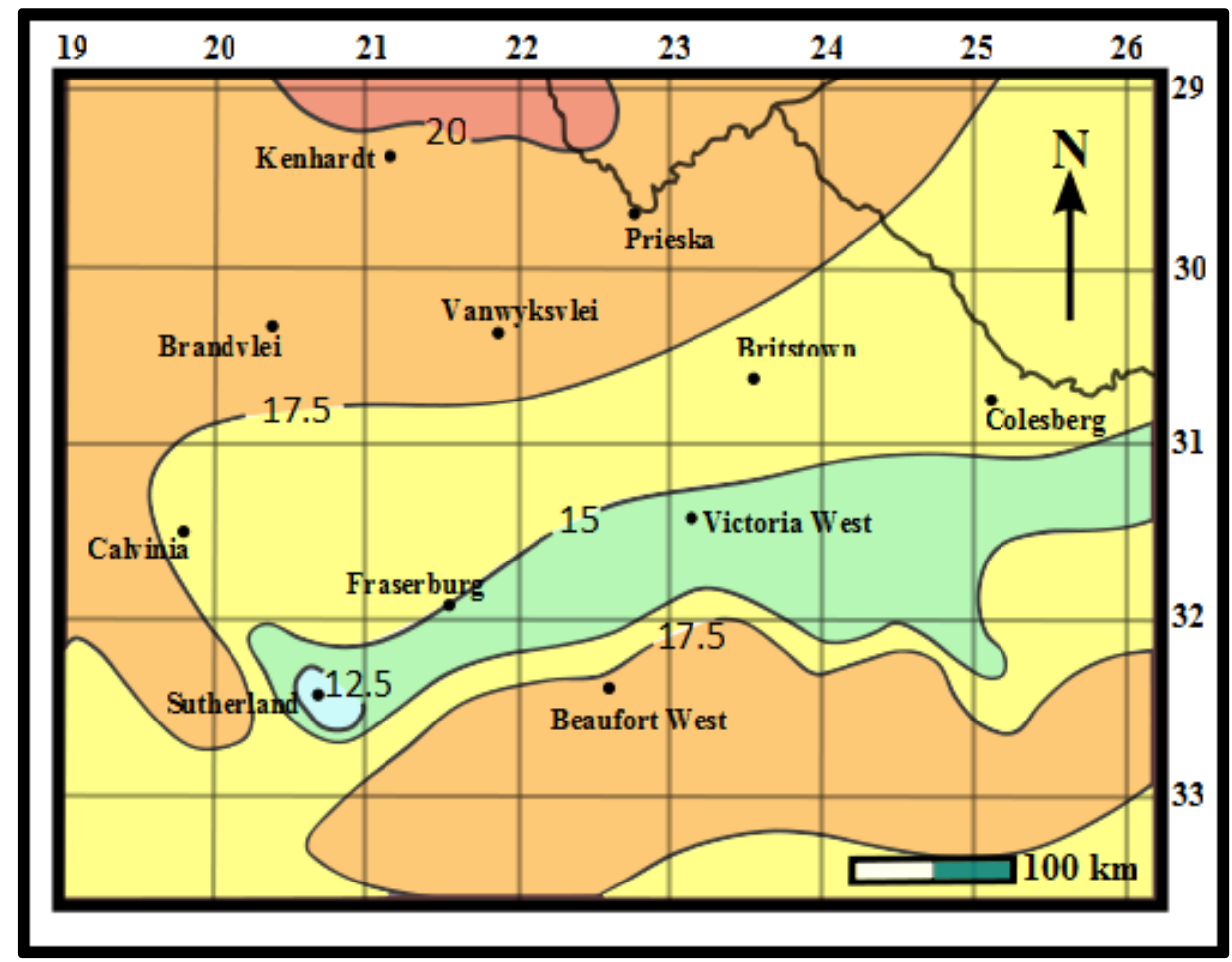

Figure 3.6: Mean annual temperatures $\left({ }^{\circ} \mathrm{C}\right)$ in the Karoo biome (after Venter, Mocke and de Jager, 1986:46) 
the low night-time temperatures of the arid interior during April-May (summerrainfall) and July-August (winter-rainfall) favour this form of precipitation. Both fog and dewfall likely provide important counterpoints to rainfall by providing relatively reliable and widespread sources of moisture; although the volume of water made available in this way is unlikely to be considerable, its regularity over large areas may be significant in maintaining basic water requirements for an overall low level of plant cover.

Temperature, another key factor, is influenced by latitude, continentality and topographic variation. Movement northwards towards the equator, and inland onto the central plateau, combines to render the IXam territories some of the hottest areas of the Karoo (Figures 3.6:52, 3.7:54, and 3.8:54). The Orange River valley experiences consistently higher temperatures than its surroundings. In summer the central areas of the Karoo experience large temperature ranges combining the effects of continentality with the absence of cloud cover, and in some areas dayto-night shifts of over $25^{\circ} \mathrm{C}$ are not uncommon (Venter, Mocke and de Jager, 1986:44). In winter, compounding effects of altitude and continentality ensure that the high interior plateau experiences some of the coldest temperatures of the subcontinent (Desmet and Cowling, 1999:9). Frosts are not uncommon, and snow may even fall at higher altitudes (more commonly to the south of the IXam homelands, but not impossible further north, Figure 3:9:55). Disturbances in the westerly airstream (winter-rainfall system) produce characteristic cold snaps that can penetrate well into the Karoo, associated with precipitation and determining 


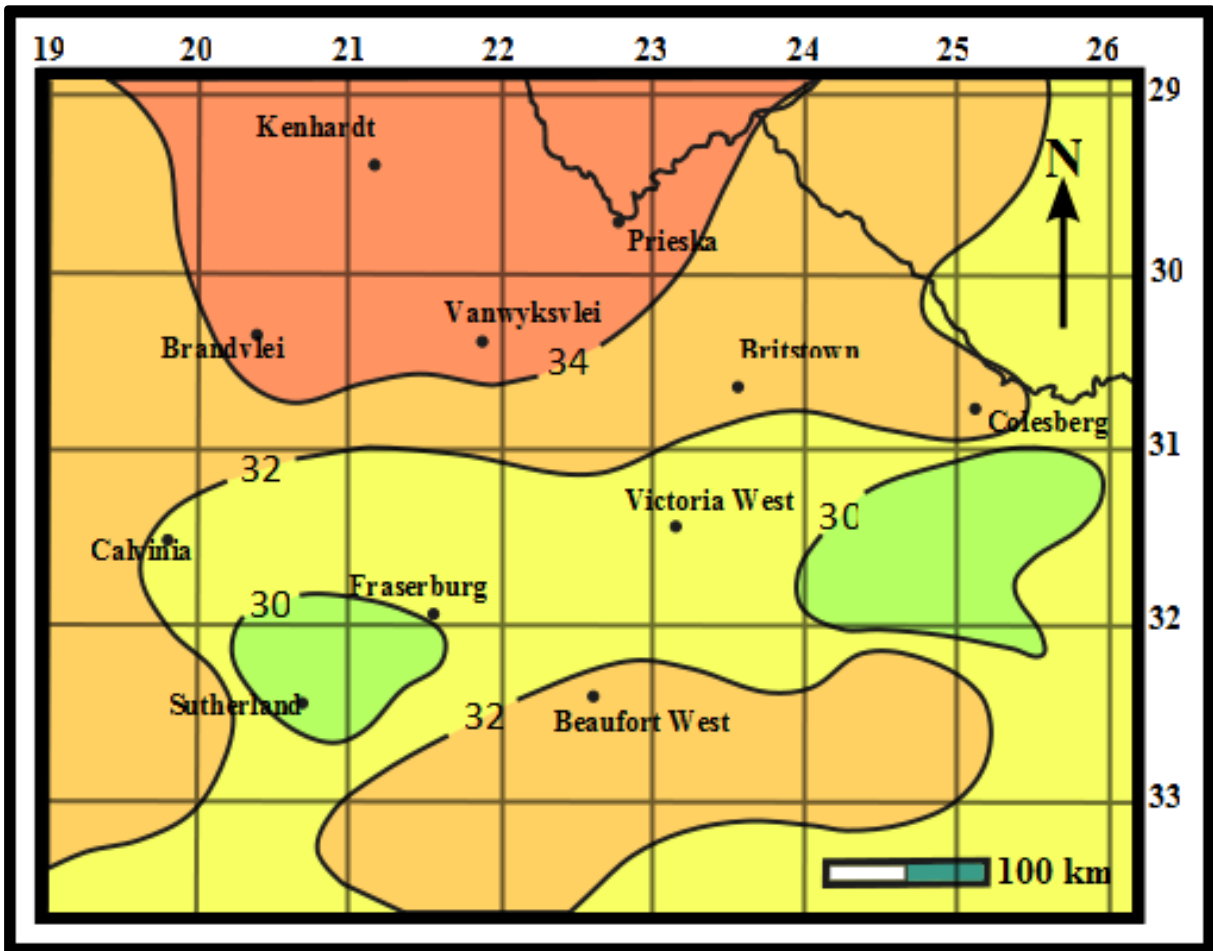

Figure 3.7: Isotherms of mean annual summer temperatures $\left({ }^{\circ} \mathrm{C}\right)$ (after Venter, Mocke and de Jager, 1986:47)

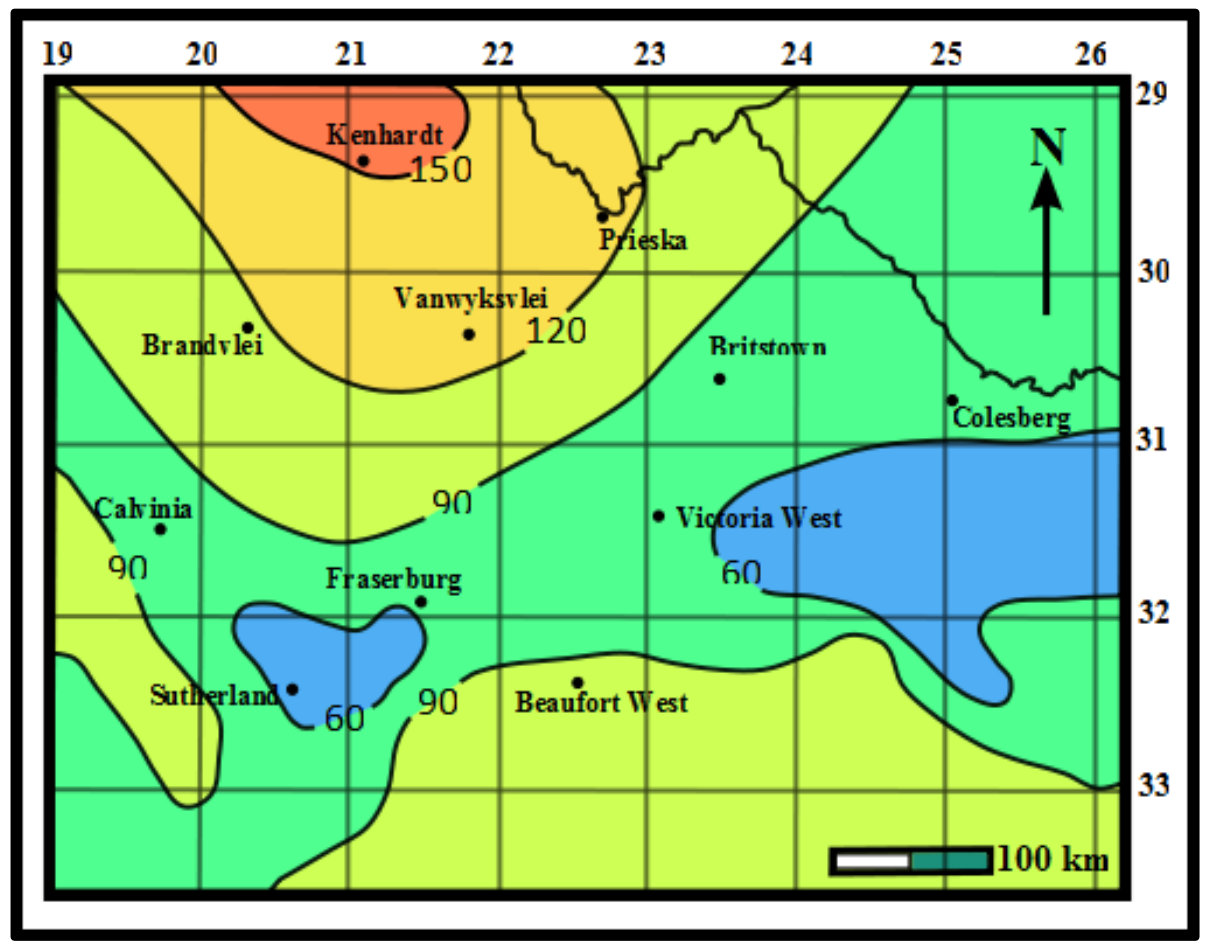

Figure 3.8: Mean annual frequency of days with maximum temperatures above $30^{\circ} \mathrm{C}$ (after Venter, Mocke and de Jager, 1986:47) 


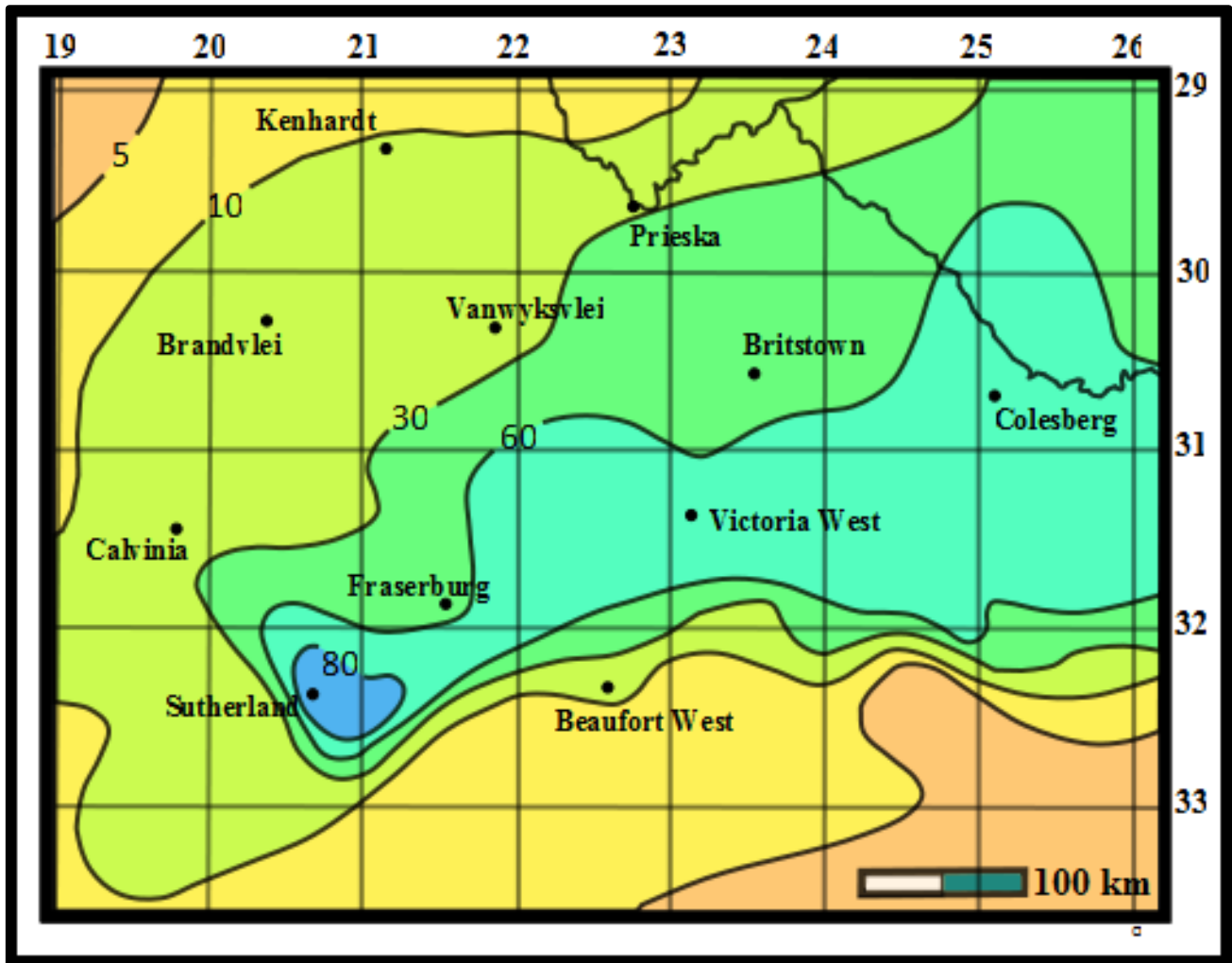

Figure 3.9: Mean annual frequency of days with minimum temperature below $0^{\circ} \mathrm{C}$ (after Venter, Mocke and de Jager, 1986:48)

snowfall for higher-lying areas: the IXam homelands experience some of both the greatest annual and daily ranges of temperature on the subcontinent.

With the increasing interest in the geology of the interior during the later part of the nineteenth century, the term Karoo was also adopted as a label for the Karoo Sequence geological formations. Although our geologic understanding of the region at more local levels continues to be refined, the area as a whole is fairly well documented (Figure 3:10:58). Much of the current surface of the NamaKaroo is dominated by sedimentary deposits, with numerous igneous intrusions and contact metamorphoses associated with them (Watkeys, 1999:19). The Karoo Sequence (Carboniferous to Jurassic in age) overlies the Cape Supergroup, 
exposed in areas to the south and west of the central Nama-Karoo: the interface between these two formations corresponds to specific types of Karoo (Succulent Karoo in the west; Klein Karoo in the south), and is partly responsible for this variation. The IXam homelands lie entirely within the exposure of Karoo Sequence rocks. The oldest rocks of this sequence represent the deposition of glacial tillite (Dwyka tillite), associated with the retreat of the continental icesheet, during the late Carboniferous-early Permian period. It is exposed only in a narrow band abutting the mountains of the Cape Folded Belt, and to the north along some stretches of the Orange River, with larger areas of exposure to the north in Gordonia. In terms of its impact for stone tool-using hunter-gatherers, this element is important chiefly because of the glacial erratics it deposited, providing varied sources of high-quality lithic material. Above the Dwyka tillite are the shales of the Ecca and Beaufort Series, deposits formed under a vast inland sea (Ecca) or in poorly-drained alluvial plains (Beaufort). These series include the mudstones, sandstones, and siltstones that constitute the bedrock of the Karoo landscape; their horizontal erosion planes are responsible both for the flat aspect of the country, and (where capped by harder rocks) for its distinctive low flattopped hills or koppies (Visser, 1986:7-8). Large horizontal beds of sedimentary rocks create a gently sloping plain, around 900-1000masl, running from the Escarpment in the south to the incised channel of the Orange River in the north. Combined with low levels of rainfall, this creates extensive pans (periodic shallow lakes, often extensive in area) and determines the endorheic catchment of 
several periodic rivers in the central Karoo ${ }^{20}$. Not present in the interior Karoo, the final sedimentary rocks of the Karoo Sequence are the Cape Sandstones (Molteno and Clarens Formations), which in the mountains of the Escarpment erode to form rockshelters and the 'canvas' for much of the painted rock art of South Africa.

The Karoo Sequence terminates with an igneous component (Stormberg and Drakensberg basalts), a result of disturbances associated with the early Jurassic break up of Gondwanaland and manifest in a range of forms largely responsible for the vastly different landforms incorporated within area covered by rocks of the Karoo Sequence. In the mountainous uKhahlamba-Drakensberg/Maluti region, the extrusion of Drakensberg lavas created basaltic caps that cover the sedimentary element to depths of up to $1400 \mathrm{~m}$, contributing to a dramatic vertical topography (Haskins and Bell, 1995:287-288). In the interior, however, these igneous deposits did not extrude and instead form numerous vertical dykes and horizontal sills (Visser, 1986:12), leading to considerable contact metamorphosis (primarily, the induration of shales) and converting the mudstones and siltstones into harder rocks such as hornfels (lydianite). These harder igneous and metamorphic deposits form lenses within their sedimentary contexts, responsible for such vertical topography as exists in the central Karoo; their differential susceptibility to erosion leaves them 'capping' softer sedimentary rocks as koppies (Visser, 1986:12). Harder metamorphosed shales also provided a ready and widespread source of fine-grained raw materials for lithic production,

${ }^{20}$ Only the larger rivers in the region drain into the Orange River. 


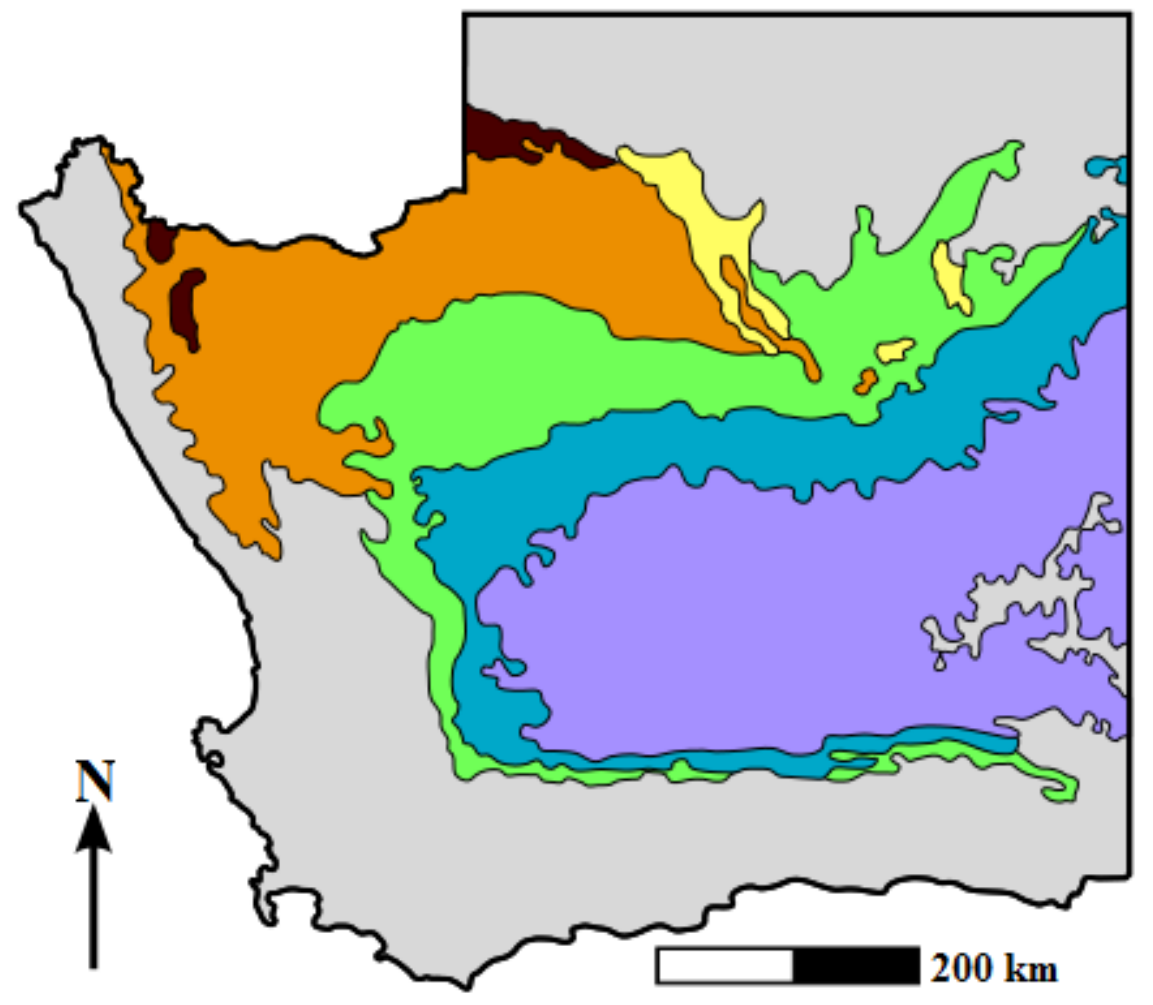

$\square$ Nama Sequence

$\square$ Namaqualand Complex

$\square$ Proterozoic-Archaean Sequence

$\square$ Dwyka Formation

$\square$ Ecca Group

Beaufort Group

Figure 3.10: Simplified geological map of the Nama-Karoo (after Visser, 1986:2) 


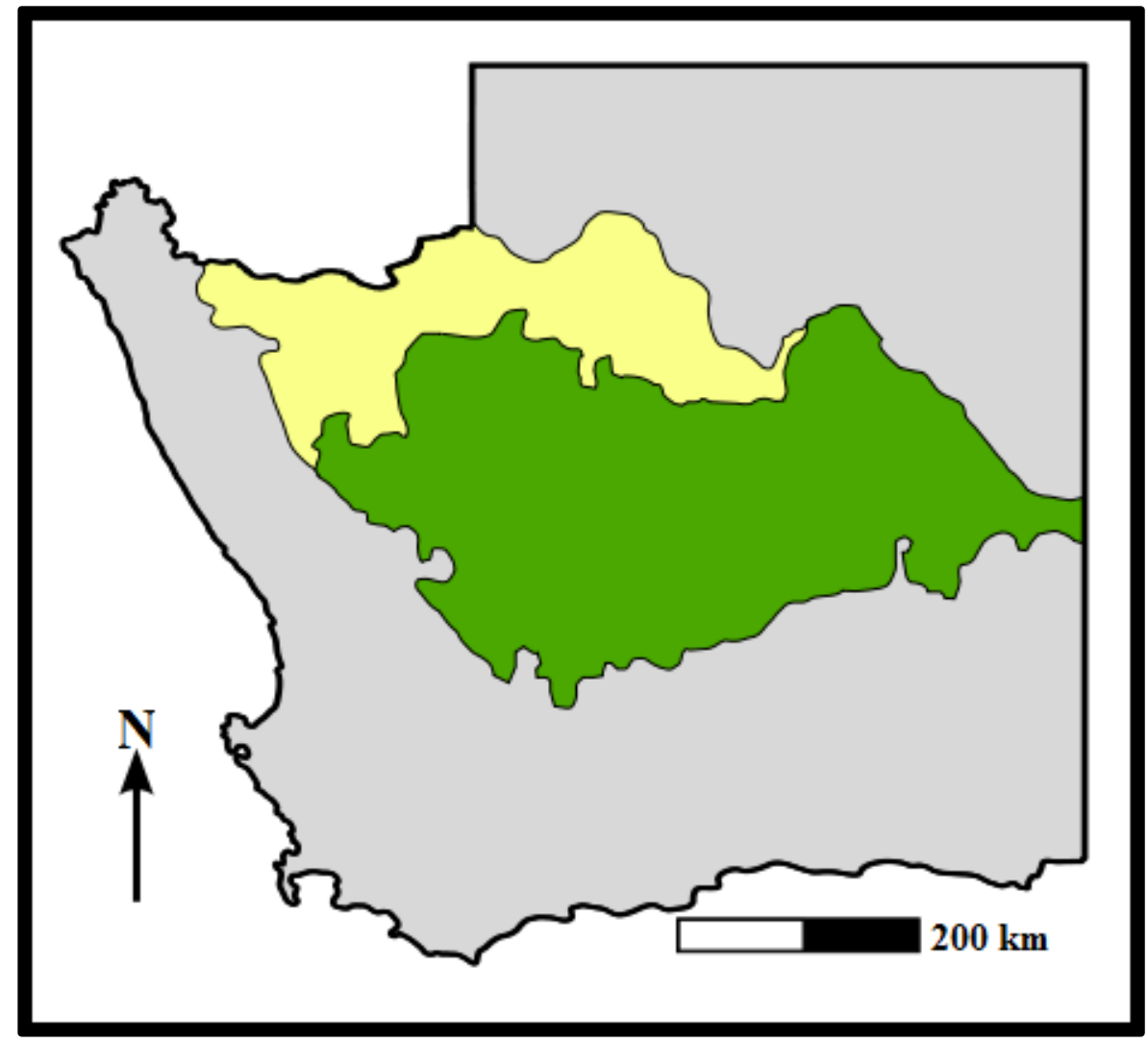

Figure 3.11: Soil regions of the Nama-Karoo; region 2 (yellow) and region 3 (green) (after Watkeys, 1999:17)

dominating some of the archaeological lithic assemblages from the central interior Karoo (Beaumont, Smith and Vogel, 1995).

In the colonial period, 'Karoo' came also to refer to soil types associated with Karoo regions: Lichtenstein (1812:122) reports that 'yellow-tinted soils throughout the colony' were referred to as Karoo ground. Two of Watkeys' (1999:17) Karoo soil regions cut across the IXam homelands (Figure 3.11:59): region 2, 'Namaqualand, Bushmanland and Korannaland', covering a band to the south of the Orange River, and region 3, 'Great Karoo (North)', in the central interior. Region 2 soils are commonly red or yellow, reflecting the well-draining 
and oxidising environment in which they are generated. Granite and dolerite are important parent materials, and these soils are common where Dwyka tillite is the underlying substrate. They tend to have an impoverished organic component, or to lack one altogether, are not water-logged, and range from very sandy (less than $6 \%$ clay) in the west, where dune areas can form, to sandy (6-15\% clay) in the east. The distinctive geology around the Orange River itself gives rise to local and restricted soil variations (e.g. infertile soils derived from the weathering of siliceous quartzite hills), and in granitic environments north of Kenhardt shallow (less than $400 \mathrm{~mm}$ ) sandy loam soils develop, overlaying calcareous or calcreted hardpans (Ellis and Lambrechts, 1986:26). Region 3 represents a pedologically young landscape with a range of calcareous, medium-texture soils, from sandy loams to sandy-clay loams. Soil variation here corresponds with floral divisions, dwarf shrublands developing on saline or calcareous soils and low grasslands on acidic Aeolian sands (Palmer, Novellie and Lloyd, 1999:210). These soils are the most widespread of all Karoo soils; shallow upland soils or recent alluvium interspersed with patches of bare rock. In some low rainfall areas (e.g. between Brandvlei and Carnarvon) lime is abundant. Along larger river channels (the Orange, Zak and Hartebeest Rivers), deep unconsolidated deposits form in alluvial terraces that can be over $1000 \mathrm{~mm}$ in depth, and are calcareous throughout (Ellis and Lambrechts, 1986:35).

Low rainfall and large temperature ranges favour mechanical rather than chemical weathering of the bedrock, tending to produce weakly structured, shallow, but fairly fertile soils, with high mineral contents (Burke, 2002). Formed by in situ 
weathering, variations in local geology can have considerable impacts upon the soils present. Dwyka tillite substrates supply large amounts of sodium chloride to the groundwater (Hodgson, 1986:88); this is registered toponymically in a large number of place names and landscape features (Humphreys and Thackeray, 1983:27-31) incorporating the Afrikaans Sout- (salt-) and Brak- (brackish-). Burke's (2002) study of soil variations associated with koppies in Namibia suggests that the localised geology and topography of these mountains is mirrored in the physical and chemical composition of their soils. Areas covered by Karoo Sequence rocks show considerable variations in groundwater (Hodgson, 1986:88), and doleritic intrusions (especially dykes) are influential in determining its local availability. These intrusions often form landform features (such as koppies), creating small 'islands' of environmental variation, with special floral communities, water supplies, and soils.

The Karoo produces less than $6.5 \%$ of South Africa's mean annual surface water runoff despite covering around $45 \%$ of its surface area, as large shallow pans contribute to large evaporative losses in an already-low precipitation region (Görgens and Hughes, 1986:54-55). The contribution of rainfall to erosion and soil redistribution is generally low; wind represents the primary agent of erosion, as the karroid bushes here seldom provide a protective closed canopy, leaving substantial bare patches on which it can act (Roux and Opperman, 1986:97). Sporadic but often violent rainfall can cause large scale erosion and redistribution of Karoo soils, as their weak structure and the absence of substantial vegetal cover mean that occasional flash floods (e.g. the 1981 Laingsburg floods) can cause 
massive movements of sediment and soil loss into river drainage systems. The extensive pans are dominated by transported soils, accumulating as adequate rainfall occurs until a sufficiently large rainfall event causes substantial erosion (Ellis and Lambrechts, 1986:33). Water features, even though seldom flowing, therefore create special dynamic environmental locales with particular successional flora communities.

Karoo soils are vulnerable to anthropogenic or zoogenic forms of erosion, such as that associated with overstocking. Detrimental impacts of colonial sheep farming on Karoo vegetation were noted as early as 1864 (Roux and Opperman, 1986:94); the effects of trampling (soil compaction) and path formation (opening erosion channels) remain current issues for livestock farmers. Once the Karoo was divided into private farms in the late nineteenth century (Chapter 4c:119), owners engaged in substantial efforts to 'improve' the land by burning the veld, draining or watering lands by breaking down embankments and digging furrows, and controlling access to pasture around springs. These changes significantly undermined the ability of wild fauna to subsist in competition with livestock, leading to substantial alterations of the veld (Acocks, 1975), and contributing to the difficulties faced by the IXam in maintaining a hunter-gatherer lifestyle at this time (Chapter 4c:121). However, it should not be imagined that anthropogenic erosion impacts on Karoo soils were initiated with colonisation; indigenous herders utilised these landscapes prior to European arrival and, while their strategies of exploitation were doubtless not identical, the impact their herds had on vulnerable soils must have been comparable. Large scale burning of the veld, 
as part of the IXam exploitation of swarming locusts (LL.VIII.7.6627-6633), must also have had similar effects on the landscape. Zoogenic factors on the part of wild species can also be considerable; springbok (Antidorcas marsupialis) and other migrant species were formerly responsible for veld deterioration and subsequent secondary succession, forming very dense populations far beyond that of the normal carrying capacities of the region and causing large-scale floral change through trampling and grazing, creating bare patches then subject to further erosion (Roux and Opperman, 1986:96). Smaller indigenous fauna contribute to erosion on local scales, through the burrowing actions of suricates (Suricata suricatta), springhare (Pedetes capensis), ground squirrels (Xerus inauris), and hyraxes (Procavia capensis). 


\section{Chapter 3b: Karoo biota}

The two Karoo biomes are partly defined by vegetation differences between, and similarities within, them. Two basic forms typify Karoo vegetation, and the form and development of both is determined by water stress: dwarfed woody shrubs with ericoid (or otherwise xeromorphic) leaves, and leaf or stem succulents (Werger, 1978:236). Unsurprisingly, the latter dominate the Succulent Karoo while the former are more characteristic of the Nama-Karoo, but both forms can be found in abundance in both of the Karoo biomes. A similar suite of flora, divisible based on species proportions, can be identified across much of the Nama-Karoo, highly endemic at a species level with numerous advanced adaptations to the aridity of the region. Werger (1978:242) suggests Karoo flora is the result of long-term adaptation of fynbos and tropical floras to the conditions of the interior plateau and that arid conditions have persisted (not necessarily as a stable system) in the region for a considerable period; Acocks (1953) too sees karroid vegetation as modifications of the fynbos of the temperate southwest and the tropical Sudano-Zambezian grass- and bush-velds. Typical Nama-Karoo flora in IXam territories consists of evergreen shrublands with an understory of grasses. Common Karoo shrub bushes include Pentzia spp., Eriocephalus spp., Rosenia spp., Salsola spp., Galenia spp., Pteronia spp., Ruschia spp., and Zygophyllum 
spp. (Vorster and Roux, 1983:19), and in some (especially sandier) areas spiny caespitose desert grasses of Stipagrostis spp. and Aristida spp. (Vorster and Roux, 1983:19), can dominate. These shrublands vary with moisture availability, underlying soils, and intensity of grazing, from being extremely open to relatively closed, with larger shrubs and trees (Acacia spp., Rhigozum spp., Rhus spp. and Lycium spp.) confined to water courses and springs (Lovegrove, 1993:25).

Variation between biomes does not exhaust the total floral variation of the region, as geological and pedological factors, landform features such as the Orange River channel and the rain shadow of the Escarpment, altitude, and topography modify substantially, at more local levels, the climatically-driven biome division. The 'bleeding over' of components from biomes in close physical proximity (savannah and grasslands to the north and east, fynbos to the southwest) creates atypical floral assemblages around the margins of biomes, partaking of two or more biome communities. In terms of the floral environment, three major zones exist in the central areas of the Nama-Karoo: an area to the north focusing on the Orange River and (through its major tributaries) extending some distance to the south; the 'core' IXam area; and areas along the southern, south-western and south-eastern margins of this centre, characterised by a transition to more broken country as one approaches the Escarpment. Variation in South African vegetation has been the focus of several classificatory schemes, the first major one being Acocks' (1953). Subsequent authors (Low and Rebelo, 1996) modify his model by amalgamating or splitting his 'veld types', grouping together some of Acocks' 'deteriorated' veld types as variation within particular parent veld types rather than providing a 
major re-working of the basic units (for the central interior Karoo, at least). For the purposes of this summary, I largely follow Acocks' discussion with references for both classifications (Figure 3:12:67; Figure 3:13:69).

Acocks' (1953) work was prompted by the impression that veld deterioration was a reality, one that would have severe economic consequences. His organisation of floral variation and delineation of veld types was designed with farming in mind, specifically defining a veld type as a vegetal unit with the same 'farming potentialities' (Acocks, 1953:2). His classification was not designed to record and emphasise variation that might have been of significance to hunter-gatherer groups, formulated on a much larger scale than would have been relevant to everyday gathering activities. However, for general understandings of the ecology of the region, and for the capacity of areas to support medium to large bovids, his model has considerable utility. Six of his categories overlap with IXam-ka-!xoe; Orange River Broken Veld (Type 32), Central Upper Karoo (Type 27), False Arid Karoo (Type 35), Arid Karoo (Type 29), False Succulent Karoo (Type 39) and Western Mountain Karoo (Type 28). Arid Karoo and the Central Upper Karoo represent the most typical, widespread systems, and others are associated with specific landforms, proximity to other veld types, or veld deterioration.

The Orange River Broken Veld revolves around the Orange River channel, with boundaries more-or-less identical to Hoffman's (1996b) Type 51, Orange River Nama Karoo. It is derived mainly from tropical flora exploiting a favourable channel into the Karoo, and is separated from other Karoo veld types by the incorporation of larger plants. Along the broken topography of the river, quiver 


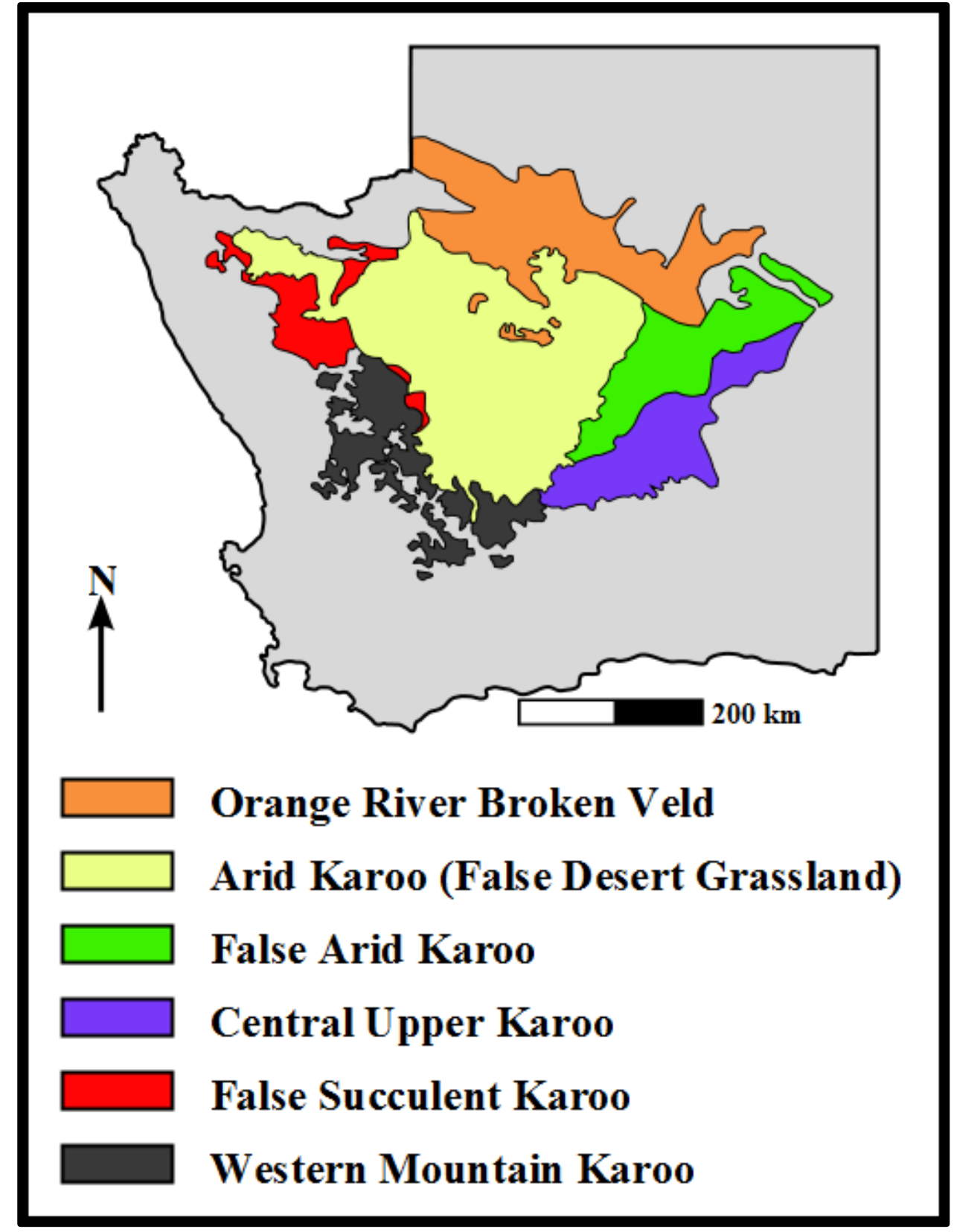

Figure 3.12: Simplified diagram of Acocks' (1953) veld types 
trees (Aloe dichotoma) and Bushman poison trees (Euphorbia avasmontana) are common, and thickets of trees including acacias (Acacia mellifera, A. eriolaba), wild tamarisk (Tamarix usneoides) and buffalo thorn (Ziziphus mucronata) are abundant along the Orange River channel and the (usually dry) channels of its tributaries. Dependent on higher moisture levels than are typical, this veld type does not extend far into the central interior: the Hartebeest River channel represents the major incursion of this veld type into IXam territories.

The False Succulent Karoo represents deteriorating, overgrazed veld (Acocks, 1953:116) in which winter-rainfall adapted Succulent Karoo species (e.g. Mesembryanthemum sp.) sparsely colonise autumn-rainfall areas formerly characterised by Arid Karoo veld ${ }^{21}$. Coupled with impoverishment of the Arid Karoo substrate, this gives the veld type almost the character of a true desert. The Arid Karoo, to the east, covers the vast majority of the IXam-inhabited areas, as close to a 'typical' vegetation as should be imagined for the region. Perennial grasses and geophytes are well represented, and while the Karoo bush species are abundant, they are less dominant than in regions further south. Grasses are represented chiefly by silvery-white desert species (Aristida, Eragostis and Stipagostis spp.), particularly prevalent in the sandier western areas: the IXam term for Bushmen from this area (see Glossary, Ikhe:-\|en:408, Chapter 7a:220) reflected this ecological division. In the north, the Arid Karoo is represented by blomkool (Afr. cauliflower) ganna (Salsola tuberculata) veld, consisting of small karroid bushes and grasses: Acocks (1953) suggested that this was the

${ }^{21}$ Hoffman (1996) combines the two as Bushmanland Upper Nama Karoo (Type 49). 


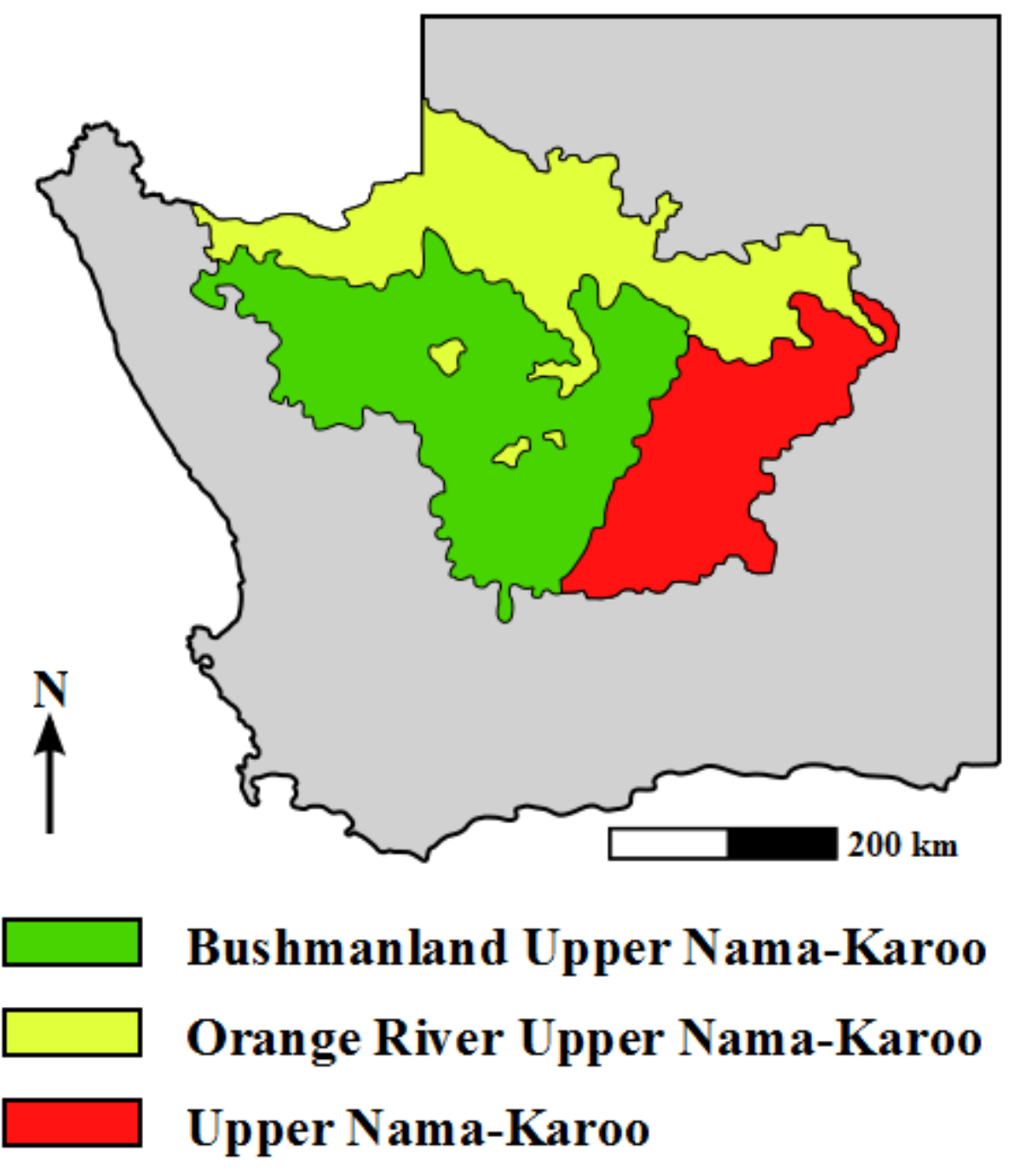

Figure 3.13: Hoffman's (1996, 1996a, 1996b) Nama-Karoo veld types

climax community for the Arid Karoo, and that before overgrazing it would have been much more widespread. In the central parts of the Arid Karoo, a transition to driedoring (Afr. three-thorn, Rhigozum trichotomum) veld, a degraded, semidesert, stony veld, represents the combination of overgrazing and subsequent erosion; the exposure of the dark Dwyka tillite or Ecca shales overheats surrounding soils, retarding floral colonisation and creating extensive denuded plains. Along the southern margins of the Arid Karoo (more broken and less arid 
areas closer to winter-rainfall systems), a more mixed veld has developed, incorporating a considerable number of Mesembryanthemum species with blomkoolganna veld.

The Central Upper and False Arid Karoo mark the easternmost extensions of IXam territories, the best watered velds of their homelands. Acocks (1953:113) notes that the False Arid Karoo is an invasion (a 'thickening up' of Arid Karoo species) within his Central Upper Karoo ${ }^{22}$; the two are so thoroughly mixed that it is impossible to pick them apart, as overgrazing and erosion have facilitated the spread of Arid Karoo species. While a False Arid Karoo 'border zone' between the two veld types probably always existed, it is impossible to reconstruct the dynamics of this margin. Within the Central Upper Karoo, well-watered areas display dense coverings of Karoo bushes with large numbers of grass species, and may reflect conditions prior to the impact of over-grazing (Acocks, 1953:94). At its eastern margins, the Central Upper Karoo merges into the more broken country of the Western Mountain Karoo (Acocks' Veld Type 28), which is both cooler and wetter than previously discussed regions. The bushes in this more mesic area grow considerably larger than commonly observed elsewhere in the Karoo (Acocks, 1953:95), though taller evergreen shrubs do penetrate into the Arid Karoo along dolerite ridges (Hoffmann and Cowling, 1987:9), reflecting variation in water availability.

Temperature, and the timing or volume of rainfall, determines floral phenological responses, which in the Nama-Karoo means that this occurs at any time of year if

${ }^{22}$ Hoffman (1996a) links them as Upper Nama Karoo (Type 50). 
sufficient precipitation occurs. Although autumn and spring (outside of temperature extremes) represent period of greatest activity, highly localised precipitation commonly creates a 'mosaic' of resource patches in any given area, displaying diversity in floral composition and in growth stages (Hoffmann and Cowling, 1987:11-12). Trees, Karoo shrubs and bushes, and grasses have different growth cycles, and while these often overlap or are stimulated by the same phenological events, this creates a 'staggered' availability for higher quality browse (Vorster and Roux, 1983). Much variation in floral communities occurs at a local level, reflecting patchiness of certain resources within any given area over time or shifts in relative frequencies of species within a specific veld 'type.' As the key determining factor of plant communities (rainfall) is highly stochastic, floral resource predictability is low; aridity adaptations of Karoo plants enable them to survive water-stressed periods at a low density or in diapauses, until large 'rainfall events' provoke responses that can alter veld composition for years (Hoffmann and Cowling, 1987:2). Zoogenic impacts on karroid flora composition can be considerable, chiefly in the context of fenced farming or in periodic highdensity population irruptions of indigenous species (Acocks, 1975). Irruptions of arthropods may defoliate particular species (e.g. Karoo caterpillar, Loxostege frustalis, favours Pentzia sp. bushes), with successional effects (Vorster and Roux, 1983:20). Depictions of Karoo flora are therefore fairly coarse, framing a range of possibilities or general character; certain patterns, such as the distinct resource 'islands' associated with vertical topography or the role of water sources as local refugia, can be observed across the whole region. Specific veld 
composition exhibits small-scale variation that, for people relying heavily on these resources (farmers and their livestock as much as hunter-gatherers), plays important roles in determining the specifics of day-to-day choices and movements around the landscape.

Unfortunately, our understanding of the capacity of Nama-Karoo flora to support human populations remains limited. There are sound nutritional reasons (e.g. the generally low fat content of African bovids) to suspect that, as with ethnographically documented Kalahari hunter-gatherers, plant foods would have represented key caloric resources (H.J. Deacon, 1993:86). A large number of Karoo plants have nutritional value (Wehmeyer, 1986), many of which have passed into Afrikaans vernacular plant knowledge as veldkos (wild plant foods), presumably in part from Khoekhoe or Bushman sources (Youngblood, 2008:1). Floral palatability and productivity with respect to livestock requirements have been well examined (e.g. Vorster and Roux, 1983), but there have been few detailed studies of the productivity, distributions, and life histories of plants suited for human consumption; the detailed information recorded for Kalahari huntergatherer plant resource strategies (Silberbauer, 1981:198-204) was not collected by observers of Karoo hunter-gatherers. The nutritive potential of species likely to have been significant can be assessed, but the integration of this potential into overall subsistence strategies is poorly understood. Ethnohistorical records give some indication of the plants eaten by Bushmen (Chapter 7c:257), and an understanding of the dynamics of Karoo floral systems allows us to expand on this, by looking at categories of resources and exploring the ecological 
implications contingent on the exploitation of certain types. Fleshy-fruited plants requiring significant water inputs are rare in arid environments (Bronstein et al, 2007:148), but they do occur, providing irruptions of resources circumscribed in time and space. In the Karoo, cucurbits are a major fruit resource, well represented in IXam subsistence (Appendix E:484). As this fruit production relies upon often unreliable rainfall cues, it generates irregular 'pulses' of productivity, important not only for the nutritional value of the fruit but as key components in faunal subsistence strategies, especially those of birds (Bronstein et al. 2007:151) and some reptiles (S. Milton, 1992). Faunal dispersal of seeds is an important factor in maintaining a heterogeneous resource distribution crucial for Karoo occupation; species move across the landscape in a non-random fashion, creating 'patches' (Bronstein et al. 2007:154-155).

In the relatively treeless Karoo biomes, geophytes represent an obvious source of carbohydrates (H.J. Deacon, 1993:87). Well represented in the ethnohistorical accounts, geophytes are reliable food sources that provide year-round caloric supplies, though their nutritive value and palatability does vary with the season. They often produce distinct 'patches' of resources, as many propagate vegetatively, forming clusters in restricted areas, thereby contributing to a heterogeneous resource distribution. Youngblood $(2004,2008)$ examines the nutritive content and productivity of a range of geophytic species in the Upper Seacow Valley; although located to the east of the IXam-ka-!xoe (in a slightly wetter form of Karoo veld), her study provides a useful reference point for considering the significance of geophytes, giving some indication of their 
densities (Youngblood, 2004:56; 2008:23-24) and highlighting their productivity. In the summer-to-autumn rainfall zone (the eastern portions of $\mid X a m-k a-! x o e)$, geophytes would ideally be harvested in winter (Youngblood, 2004:61), rendering them complementary to other Karoo flora (undergoing productivity bursts in the spring and autumn).

The Karoo's fauna displays a low level of endemism, with larger species (particularly mammals) primarily derived from other biomes. Differences in the reliability of seasonal rainfall in Nama-Karoo versus Succulent Karoo biomes form effective barriers between the two for many Succulent Karoo species: NamaKaroo birds and larger mammals stem from savannah and grassland faunas, with species adopting nomadic or partly migratory strategies utilising resources that are patchy in time and/or space (Vernon, 1999:79). Much of the Karoo fauna has been severely affected by anthropogenic factors associated with colonial expansion, many species becoming locally extinct or restricted to reduced ranges. I refer primarily to the eighteenth- and nineteenth- century fauna, insofar as this can be reconstructed from historical sources (Skead, 1980, 1987). Relying on the unsystematic and impressionistic observations of sporadic travellers and hunters for an accurate picture of the population dynamics of Karoo ecosystems is problematic ( $c f$. Boshoff and Kerley, 2010), but the low level of endemism of Karoo species allows us to combine the historical record with analogical data from behavioural and ecological studies of these species elsewhere in Africa to glean useful information. Combining these studies with our knowledge of arid 
environments in general allows the reconstruction of some dimensions of this ecosystem as it existed in the colonial and immediately precolonial past.

The non-endemic nature of many Nama-Karoo species entails several important ethological consequences that structure potentials and constraints for huntergatherer strategies. For some species, Karoo exploitation represents an opportunistic phase within a larger subsistence pattern; species not particularly adapted to aridity find the Karoo a challenging environment, and successful expansion into it requires certain behavioural modifications, such as the adoption of highly mobile 'retreating' cycles of resource acquisition (Lovegrove, 1999:156). Species utilise favourable variations of the Karoo (e.g. river channels) to reach resources in an area ordinarily unable to support them, retreating to refugia zones in stressed periods. Species can also be sensitive to climatic variation on intra-annual and inter-annual scales, entering the Karoo when its aridity is most ameliorated. While all the variants of the Karoo represent arid environments, there is considerable variation in how this aridity is spatially and temporally distributed, facilitating short-term expansions of range $\mathrm{e}^{23}$.

All water-dependent herbivores in the Karoo utilised these adaptations to varying degrees; equids, preferential (not necessarily obligate) grazers, are more reliant on water than many savannah bovids, and some species (Equus zebra zebra) will modify their environment by digging to expose water in dry riverbeds, where possible (Estes, 1991:235). Two equid species formerly dwelt in the Nama-Karoo;

\footnotetext{
${ }^{23}$ Both strategies were important for pastoralist occupation. Cattle and sheep require open water, forcing pastoralists to control access to permanent sources, and/or to rely on movement to favourable areas.
} 
the extinct quagga (Equus quagga quagga ${ }^{24}$ ), over much of the region, and the Cape mountain zebra (Equus zebra zebra), along its southern fringes. The early date for the quagga's extinction (Skead, 1980) throughout its range perhaps relates to increased stress on its subsistence strategies (relative to less water reliant species), incurred by increasing farmer monopolisation of water sources. Alcelaphines, represented in the Nama-Karoo by black wildebeest (Connochates gnou), blue wildebeest (Connochoetes taurinus) and hartebeest (Alcelaphus buselaphus), are also fairly water-dependent, retreating to water sources in the dry season (in savannah environments). This suggests they were probably vagrants in the Nama-Karoo, utilising channels (e.g. the Hartebeest River) to reach graze and forage deeper into this area. Blue wildebeest are the most water-dependent of the three (drinking at least every other day, Estes, 1991:150), almost certainly limiting their Nama-Karoo range to regions bordering the Orange River, while black wildebeest can incorporate a greater degree of browse (i.e. karroid bushes) in their diet, increasing tolerance of arid conditions. Hartebeest will drink regularly if water is available, but in the Kalahari will incorporate tsamma melons (Citrullus lanatus) and geophytes to alleviate water stress (Estes, 1991:138), a possible strategy for Karoo environments. Other species pursue similar tactics; the willingness of brown hyenas (Hyaena brunnea) to incorporate tsamma melons and gemsbok cucumbers (Acanthosicyas naudinianus) into their dry carrion-based diet likely contributes to their successful colonisation of arid zones (Lovegrove, 1993:60). This constitutes a second key adaptation to aridity; dietary modification,

${ }^{24}$ The local subspecies of plains zebra, Equus quagga (Hack and Lorenzen, 2008; Harley et al. 2009:155-156). 
and extraction of sufficient water from food to permit survival in the absence of standing water. Many bovids achieve a degree of water-independence with these strategies, and some extract sufficient water from their ordinary diet to permit year-round Karoo occupation without recourse to migration or dependence on specific plants (with unusually high-water content). Springbok employ selective grazing strategies but primarily are browse specialists of the dwarf shrubs (especially Pentzia sp.) and succulents characteristic of the Nama-Karoo biome (Lovegrove, 1993:100-101), allowing them water-independence ${ }^{25}$. The combination of selective grazing, exploitation of key areas of environmental variation, and large territories maintained low-density populations of a range of bovid species.

For larger mammals and birds with low locomotive costs, long distance movements become viable mechanisms for avoiding the extremes of Karoo environments: smaller or less mobile species are forced to adopt different strategies to cope with these extremes. Physiological strategies of diapause followed by rapid florescences are crucial in the lifecycles of a range of algae, bacteria and protozoa species: the ability of these species to survive long desiccating periods is the foundation of the short-lived ecosystems of Karoo water-courses. In effect only for brief stretches, these systems introduce temporally- and spatially-confined variation providing crucial resources many species; quite dramatic population aggregations, such as those of sandgrouse and flamingos, develop around shallow temporary water sources (Lovegrove,

${ }^{25}$ They will drink, tolerating a high mineral content (Estes, 1991:80). 
1993:159). Fauna employing xeromorphic physiological mechanisms (e.g. retraherence, Lovegrove, 1993:152) allowing them to tolerate arid conditions are prominent among Karoo fauna; reptiles are diverse and abundant and tortoises are notably common, with species endemic to both biomes. Short-term retreat from the extremes of Karoo environments to the shade reduces heat stresses and water consumption, while full-scale nocturnalism represents a more extreme form of this avoidance strategy (Lovegrove, 1999:154): both are widespread strategies for small fauna, particularly among rodents. Burrows and nest structures represent the creation of favourable micro-environments for retreat when external conditions become intolerable (Lovegrove, 1999:154-155): they are employed by invertebrates and rodents as well as larger mammals such as aardvarks (Orycteropus afer), aardwolves (Proteles cristatus) and bat-eared foxes (Otocyon megalotis). The success of these strategies for smaller fauna, facilitating continual occupation of the Karoo, maintains relatively stable, local faunal populations: combined with floral resources, the species constitute the basic carrying capacity of Karoo environments for hunter-gatherer strategies.

Social insects play a crucial role in the maintenance of the Karoo environment: their tolerance for its extremes is considerable, with some ant species withstanding body temperatures of over $50^{\circ} \mathrm{C}$ (Curtis, 1985). They contribute a vital organic component to the shallow soils and increase the availability of soil nutrients: old termite nests creating distinctive micro-habitats that contribute significantly to plant diversity (Lovegrove, 1993:40,106). Physiological (Formicidae) or behavioural (Termitoidae) adaptations allow social insects to 
thrive where competitors struggle, allowing for high population densities. They therefore constitute significant resources for Karoo insectivores; several large mammals in the Nama-Karoo focus more-or-less exclusively on these species. Aardvarks (Shoshani, Goldman and Thewissen, 1988), aardwolves (Estes, 1991:344) and bat-eared foxes (Estes, 1991:392) specialise in these insects, and chacma baboons (Papio ursinus) utilise them when in arid areas (Estes, 1991:509).

The other invertebrate of major significance to Karoo biomes is the brown locust (Locustana pardalina), which remains a contemporary economic concern $(\mathrm{R}$. Price and Brown, 2000:37-38). Lovegrove (1999:162) suggest this species personifies reproductive adaptation with respect to the unpredictability of Karoo environments; in its cryptic solitaria phase it behaves much like other Karoo orthoptera, with a low density occupation that can survive through drought periods. The characteristic swarming of the gregaria phase (stimulated by overcrowded solitaria hoppers) initiates massive colonisation of new environments once conditions improve, and longer periods of low density occupation are punctuated by large, but sporadic and short-lived, irruptions. The Afrikaans term for migratory gregaria locusts, treksprinkane, echoes the term for the famous large-scale springbok population movements, trekbokke. Prior to the fencing of Karoo farms (Roche, 2008), springbok undertook large scale population movements in response to rainfall conditions, and herds comprising many thousands of animals are well-documented (Dunn, 1873). These nomadic movements were primarily oriented along north-south and northeast-southwest 
axes, crossing from summer-rainfall dominated to winter-rainfall zones (Lovegrove, 1993:163) and responding to localised good rains. Springbok treks were not seasonal migratory movements of stable populations from one area to another, but rather represented population irruptions with subsequent movement to favourable conditions: relatively wet years allowing large increases in population, and subsequent drought provided the catalyst for movement, creating periodically abundant resources that represented significant components of IXam subsistence strategies (Chapter 7b:237).

Although Karoo environments can appear homogeneous, even monotonous, there is considerable variation at the local level: Venter and Watson (2008) identify seven habitats even within a small study area, demonstrating the significance of this variation in determining forage strategies and population growth among the bovid population. Highlighting the importance of the small-scale and of detailed information in understanding ecosystem dynamics, this study recalls the difficulties for understanding IXam hunter-gatherer strategies: for the nineteenth century Karoo this information is largely irrecoverable, though such reconstruction as we can attempt outlines the broad issues facing arid-zone hunter-gatherer groups, and the impacts of certain variables. Periodicity rather than seasonality is central to understanding many elements of the Nama-Karoo biome; compared with surrounding biomes, it is difficult to place temporal variation in a strong bimodal pattern. Although many plants have phenological lifecycles, the absence of consistently marked seasons means that this potential for 'seasonality' is unevenly distributed across the landscape. This suggests that 
aggregation-dispersal residence patterns observed for Kalahari Bushman groups (Lee, 1979:360; Barnard, 1992:43-45) are unlikely to have played a major role in IXam life; while there are references to seasonal variation in resources on the part of the Bleek-Lloyd informants (LL.II.23.2044-2045; Chapter 7c:259), there is little indication that this was accompanied by shifts in population density ${ }^{26}$. References to activities employing larger numbers of people in resource collection, such as some forms of springbok hunting (LL.VIII.23.8028-8029; LL.VIII.32.8841'; Dewar et al., 2006), the collection of locusts, and the large groups noted in the late eighteenth century (Chapter 4b:103), suggest that IXam populations did occasionally come together: the maintenance of low population densities with the fluidity to mobilise larger units during population irruptions of key resources represents a common Nama-Karoo strategy.

\footnotetext{
${ }^{26}$ Taking one's wife and children to live in |ho (translated as 'solitude' by Lloyd, LL.II.30.2711'), rather than 'being with one's 'fellows', perhaps broadly corresponded with aggregation-dispersal models; little detail is available about this practice, unfortunately.
} 


\section{Chapter 4: Historical Contexts}

\section{Chapter 4a: Precolonial history}

Archaeological interest in the interior Karoo is long-standing: in the latter decades of the nineteenth century, the emergent discipline considered Bushman groups to be useful sources of information regarding the human past. As in other colonial settings (Finzsch, 2005), the persistence in the arid interior of hunter-gatherer lifestyles was seen as an opportunity to observe, through the actions of 'primitive' groups in the present, the prehistory of peoples elsewhere. Paralleling Bleek's interest in Bushman languages, interest in the material culture of stone-tool using populations reflected a belief that this information would construct the overarching narrative of prehistoric social development. Material signatures of hunter-gatherer practices were employed to establish the supposed lack of innovation and change these societies had undergone, representing them as long- 
standing, 'static' occupants of their current territories. Dunn (1872) characterised ostrich eggshell fragments and lithic debris in the Karoo as evidence of long-term Bushman occupation; the evidence of materials 'serviceable to primitive man' was linked with contemporary Bushman populations though ethnographic vignettes (Dunn, 1873:34), and earlier authors (Stow, 1905) drew heavily upon archaeological material to construct culture history models for the region. With the cessation of hunter-gatherer lifestyles in the Karoo, the region became less central to the construction of archaeological narratives. Isolated from major population centres and lacking the deep rock shelter deposits found elsewhere in the country, the interior Nama-Karoo remains a relatively neglected contributor to our understanding of southern African prehistory.

The Early (ESA) and Middle Stone Age (MSA) occupation of what would become the IXam territories in the central Nama-Karoo is poorly understood, with only a few sites identified and excavated; the extent to which this paucity reflects a real absence rather than taphonomic (and research history) issues is debatable. The aridity of the region implies a challenging environment for colonisation on the part of early hominins. Surface lithic scatters typologically assignable to Pleistocene industries can be found across much of the area (Beaumont, Smith and Vogel, 1995), but more securely dated material from the late Holocene periods recording abandonment during climatic deterioration suggests a 'patchy' occupation history. The only major rock shelter site in the central interior with deep stratified Pleistocene deposits is Wonderwerk cave (Beaumont and Vogel, 2006), north of the Orange and Vaal rivers; confirming long-term hominin 
exploitation of arid interior biomes (Chazan et al. 2009), it has little to say about the central Nama-Karoo biome directly. Characteristic pans (e.g. Bundu Pan, Beaumont, Smith and Vogel, 1995:241) of the Nama-Karoo provide stratified sequences and therefore partly replicate rock shelters in their potential for dating. Analysis of Bundu Pan faunal assemblages suggests that hominin occupation during the Middle Pleistocene was strongly influenced by climate, associated with a grassland biome faunal suite implying increased water availability (Kiberd, 2001, 2006:200); this (admittedly limited) evidence is consistent with patterns noted for MSA occupation elsewhere in the Nama-Karoo (Sampson, 1985).

Later Stone Age (LSA) industries of the late Pleistocene and Holocene across much of southern Africa are characterised by increasing diversity in both time and space (P. Mitchell, 2002:161-191), and the LSA archaeology of the Nama-Karoo corresponds well with these trends, shifting away from prepared core (mode 3) lithic technologies towards a formal component dominated by microlithic tools and a range of scraper forms, a formal component displaying considerable variation (J. Deacon, 1984) that has been the basis for separating the period into several key industries (see McKay, 2009 for a critique). For the purposes of this discussion, I focus on the local manifestations of the later Holocene industries that presumably segue into the material record of antecedent IXam populations.

Precise dynamics for interior Karoo climate change at a local scale remain elusive, but this factor appears to have been a significance component in a human occupation characterised by repeated movements in to and out of the region. While a paucity of dated material charting Late Pleistocene occupation may 
reflect a relative dearth of research rather than a 'real' gap in occupation, even the well-surveyed Seacow Valley (Sampson, 1985) lacks material typologically assignable to Robberg industries (Last Glacial Maximum until c. 12-10 000 BP; P. Mitchell, 2002:120) ${ }^{27}$. 'Oakhurst Complex ${ }^{28}$, assemblages of the terminal Pleistocene and early Holocene are also poorly documented for Bushmanland itself; Beaumont and Vogel (1989:75) report an 'undoubted Oakhurst aggregate' on the far eastern margins of the Karoo, and more tentatively assign material from Springbokoog 1 (Bushmanland) to this industry. As assemblages with less microlithic tendencies are known from surrounding regions (e.g. the Lockshoek, Sampson, 1974), we might imagine these hints of occupation to represent lowdensity and sporadic utilisation of Bushmanland proper during the early Holocene.

The period approximately 7000 BP-4000 BP appears to have been marked by a transition to a drier environment than exists in the Nama-Karoo today (Meadows and Watkeys, 1999:33); a period corresponding to spread of the Wilton (Classic Wilton) assemblages in South Africa. Wilton assemblages are characterised by high incidences of backed artefacts, particularly in the form of segments ( $\mathrm{P}$. Mitchell, 2002:144) and a wide range of other microlithic tools including borers and small scrapers; Beaumont, Smith and Vogel, (1995) identify the Springbokoog industry as the manifestation of the Wilton in the central interior

\footnotetext{
${ }^{27}$ Bousman (2005) notes Robberg assemblages at Blydefontein, on the extreme eastern margins of the biome and Orton (2008) identifies a similar Late Pleistocene microlithic industry at a site in coastal Namaqualand, on the western extremes of the Succulent Karoo.

${ }^{28}$ Less microlithic in character with a formal tool element incorporating large D-shaped and sidescrapers on coarser-grained rocks (P. Mitchell, 2002:141).
} 
Nama-Karoo. Microlithic in overall character, the formal component of this industry is dominated (50-80\%) by backed blades (in contrast to contemporaneous scraper-dominated assemblages from the south and east; J. Deacon, 1984) and a preference for hornfels (indurated shales or 'lydianite') raw material. Consistent with climate data, this Springbokoog industry reflects highly 'pulsed' occupations, concentrated in two phases (4500-4300 BP and 2600-2300 BP, Beaumont, Smith and Vogel, 1995), with some hints of earlier occupation perhaps associated with fine-line engravings that depict fauna more typically associated with Savannah biomes (Beaumont and Vogel, 1989) and therefore reflecting periods of climatic amelioration and shifts to increased summer rainfall. Later manifestations of Wilton assemblages blend into a range of 'post-Classic' industries retaining a general microlithic character, but with fewer segments; referred to under various headings (Interior or Inland Wilton, Late Wilton, PostWilton and Post-Climax Wilton; Deacon and Deacon, 1999), these industries are of most relevance for the archaeology of the IXam. For the Ceramic LSA (approximating the last two thousand years) of the central Nama-Karoo, Beaumont, Smith and Vogel, (1995) propose two distinct assemblages, incorporating differences in lithic industry, pottery manufacture, bead sizes and settlement pattern, designated as the Swartkop and Doornfontein.

The lithic component of the Swartkop industry is very similar to the Springbokoog, with a high percentage of blades and bladelets, predominantly on hornfels: the retouched component primarily consists of backed bladelets (up to sixty percent of formal tools). More recent manifestations of the Swartkop have 
fewer bladelets, more ceramics, and rely more heavily on local raw materials for lithic sources. 'Coarse' Swartkop ceramics are undecorated and often (not exclusively) contain grass-temper, and iron is occasionally present in the assemblages, which are usually found close to periodic water sources, on low koppies, or in deflation hollows. The presence of iron suggests that, at the historic end of the scale, the Swartkop industry reflects the material signature of IXam populations (Chapter 7d:267), and that its earlier manifestations relate to the ancestors of these groups.

By contrast, Doornfontein lithic assemblages are dominated by larger, irregular flakes, most often on quartz: the formal element is extremely impoverished (and may be completely absent), but includes backed pieces and a range of scraper forms (Parsons, 2007:5). Doornfontein pottery is thin-walled and thick-based, with grit temper and often incorporating structural features including lugs, spouts, and decorated rim and neck sherds. A degree of temporal variation is observed, with assemblages older than $\mathrm{AD} 700$ having no lugs and a larger formal lithic component and later manifestations incorporating grass-tempered wares, more iron and copper objects, and larger ostrich eggshell beads. Restricted to major, reliable water sources (especially the Middle Orange River; A. Smith, 1999:251), these sites have (primarily by contrast with the Swartkop, but also through the presence of distinctive features such as lugged pottery) been linked with a pastoralist occupation assumed to segue into historically documented Khoekhoe populations (Beaumont, Smith and Vogel, 1995). 
Surface scatters found on both sides of the Middle Orange River may document LSA groups employing different socio-economic strategies occupying the same environment (Beaumont, Smith and Vogel, 1995:263); substantial herding encampments have been identified within the floodplain of this river (Morris and Beaumont, 1991), though intensive farming of these terraces likely obscures much precolonial exploitation (P. Mitchell, 2002:246). Highly productive, well-watered zones encapsulated by aridity, these floodplain terraces would have constituted attractive environments for both hunter-gatherers and herders: processes of exclusion demonstrated by the separation between herder sites along the river and purportedly hunter-gatherer sites in its hinterland may therefore reflect the increasing marginalisation of groups pursuing foraging subsistence modes (Beaumont, Smith and Vogel, 1995). Such interpretations accord well with those applied elsewhere in southern Africa, where increased presence of food-producing societies has been associated with hunter-gatherer retreat to more marginal mountainous or arid zones (e.g. Parkington and Hall, 1987:10, for the southwest Cape).

The supposed correlation of Swartkop/Doornfontein material signatures with hunter and herder 'ethnic' identities situates the archaeology of central NamaKaroo within investigations of the emergence and spread of pastoralism, a longstanding and on-going debate in the prehistory of the subcontinent, combining evidence from archaeology, linguistics and documentary sources ( $c f$. P. Mitchell, 2002:227-258). As the spread of livestock (and/or people) to the southwest Cape necessitates a negotiation of some form of Karoo veld, Karoo sites (outside the 
central Nama-Karoo) have provided important information for this debate: early dates confirm the presence of sheep in Namaqualand (a hyper-arid area of the western Succulent Karoo) from c. 2100 BP (at Spoegrivier; Sealy and Yates, 1994), while sheep-dominated faunal sequences from 1400-1300 BP at Jakkalsberg A (Webley, 1997) demonstrate the consolidation of herding practices in the region. On the opposite margins of the Karoo, the ongoing Zeekoe Valley ${ }^{29}$ Archaeological Project (instigated by Garth Sampson in the late 1970s) provides a detail case study of contact scenarios between hunters and herders during the latter part of the LSA, plotting thousands of open-air sites and excavating eleven rock shelters (Sampson, 2010:843). This extensive study (Sampson, 1984, 1986, 1988, 1996, 2010) explores the dynamics of interactions between pastoralists and hunter-gatherers, with a particular focus on the extent to which their ceramics may reflect cultural identities (Sampson et al. 1989; Sampson and Vogel, 1995, 1996; Bollong, Sampson and Smith, 1997; Sampson, Bailiff and Barnett, 1997; Sadr and Sampson, 1999; Sampson and Sadr, 1999).

In light of this 'pastoralist' debate, the theoretical and methodological basis for the Swartkop/Doornfontein separation has been subject to scrutiny, with Orton (2003) noting a lack of clarity in published descriptions. Parsons' (2003, 2006, 2007) re-examined the original sites, revising her earlier acceptance of the distinctiveness of the two industries (Parsons, 2007:2), with assemblages attributed to these industries subject to considerable diachronic and synchronic variation that creates a 'fuzzy boundary' between the two (Parsons, 2007:9). The

\footnotetext{
${ }^{29}$ A valley running approximately north-south to the Orange River from the northern side of the Sneeuberg Mountains.
} 
'pastoralist' debate has suggested increasingly that insistence on rigid huntergatherer versus pastoralist identities misrepresents the variability of the archaeological record (Sadr, 2003), obscuring the processes whereby domesticated animals spread into the region; although the adoption of pastoralist lifestyles on the part of indigenous hunter-gatherer populations remains a contentious issue, it is clear that the intrusion of domesticated species cannot be represented (only) as the movement of discrete culturally and ethnically distinct populations. Returning to Parsons' work, we can see that while the division represents a convenient starting point (Parsons, 2007:8), the recognition of areas of overlap between the two suggests that hunter-gatherer and herder practices are not isolated or completely dissimilar. 'Fuzzy' examples such as Vlermuisgat (a 'Swartkop' site with sheep remains) recall Sadr's (2004) notion of 'hunters-withsheep', reminding us that foraging-to-pastoralism is a transition rather than an 'all-or-nothing' proposition.

No discussion of Northern Cape archaeology would be complete without mention of its engraved rock art. The Bleek-Lloyd material has contributed to our understanding of rock art on a global scale; the seminal work of David LewisWilliams $(1981 ; 1983)$, combining this corpus with data from extant southern African hunter-gatherer populations and applying his 'shamanic' rock art interpretation (Chapter 9:337; Glossary, !gi:xa:415), cements the archive's wider relevance for archaeology. Perhaps the one downside of the richness of southern Africa's painted imagery is that engraved sites have remained relatively neglected. Although engraved imagery is the most common form of 
representation in the IXam homelands, the painted imagery has proven more amenable to detailed analyses, leading the way in refining understandings: Dowson's (1992) application of the 'shamanic' model to the engraved imagery was informed by earlier work on painted imagery (e.g. Lewis-Williams and Dowson, 1988; Dowson, 1989; Lewis-Williams and Dowson, 1989).

Historically, the engravings have been the focus of considerable archaeological attention. Initiated in 1906, Maria Wilman's (1933) work on Northern Cape engravings coincided with Dorothea Bleek's 'Bushman researches' (Parkington, Morris and Rusch, 2008:33), directly linking emergent archaeology, ongoing ethnographic work, and the Bleek-Lloyd project. Despite this, there was little attempt to employ Bleek-Lloyd accounts in interpretation: early- and midtwentieth century South African archaeologists focused primarily on documentation of 'style' and typological seriation, rather than substantial interpretation of the images (Parkington, Morris and Rusch, 2008:34-36). Recent refinements of this approach (Beaumont and Vogel, 1989) outline three 'styles' for Karoo rock engravings: fine-line incised, scraped, and pecked engravings (Beaumont and Vogel, 1989:73). Comparing these styles with the dating of spatially associated surface scatters, these authors conclude the latter two styles were contemporaneous, antedating earlier fine-line imagery. The spatial distribution of scraped engravings (Beaumont and Vogel, 1989:79) corresponds broadly with the territory occupied by IXam groups, and that hunter-gatherer communities of the region were responsible for the execution of a considerable portion of the engravings is well accepted. 
However, the nature of the nineteenth century informants' relationship with the engravings is less secure (J. Deacon, 1988:132). Bleek and Lloyd's use of rock art copies (LL.V.22.5739-5742,5755-5775; LL.VIII.19.7639-7641; LL.VIII.22.79697975,7983-7993; LL.VIII.23.7999-8004) obtained information evoked by the informants interpretation of the content of the copies (LL.V.22.5755-5775), rather than exploration of the contexts, practices and meanings associated with the production or use of 'rock art' itself. The lack of direct commentaries on rock art practices per se may reflect 'real' absence of practice or the effects of the unusual context of the imagery shown ( $c f$. Bank, 2006:94-95). The narrators do not mention engraving as part of personal histories and daily activities, excepting in two ambiguous annotations; a lack of clarity in Lloyd's handwriting (LL.V.24.5963') implies that Dia!kwain's father himself engraved, and in publication Lloyd very strongly alludes to this being the case (Lloyd, 1889:19). If Lewis-Williams' model is an accurate representation of rock art in Bushman culture, this seeming lack of interest ${ }^{30}$ in discussing it is rather surprising, implying that (even if Dia!kwain recognised his 'fathers' role $^{31}$ in its production) it had relatively little significance in contemporary life.

The archive documents the strong affective attachments IXam individuals felt towards particular places in the landscape ('topophilia'; Tuan, 1974). Deacon (1988:136) examines the way in which these narrative themes can inform our reading of engraved sites, connecting the story of the Agama lizard (incorporating

\footnotetext{
${ }^{30}$ Recalling Lewis-Williams' (1983:13-14) discussion of Jul'hoansi perspectives on rock art in Tsodilo hills, Botswana.

31 The connection between his father/ancestors and rock art incorporated a water source; other references to fathers and water (Chapter 6b:187) suggest this may state 'ownership'.
} 
an aetiological 'explanation' for two hills), this animal's link to rainfall, and the imagery of engravings located on these hills. Morris' (2002, 2008) work discussing Driekopseiland as a significant 'place', crossing cultural boundaries just as easily other more mobile forms of material culture, provides a useful and innovative perspective on issues of shared cultural practice across a spectrum of hunter-gatherer and herder identities: many of the beliefs connecting water, serpents and female menarcheal rituals (likely informing the placement of engravings) had resonance beyond a single 'ethnic' or cultural identity.

Potentially valuable in providing context for understanding of historical sources, research exploring the archaeology of colonial expansion has been patchy (Malan, 2002:510) and often directed by specific links with the historical record, as with rockshelter excavations that provide an alternative form of 'documentation' for sites (Humphreys, 1975; Wadley, 2001). The South-western Cape region has received the most sustained work ( $c f$. Hall and Markell, 1993), focusing on vernacular architecture (Brink, 1990), local foodways, and material culture as a signature of non-literate experiences (e.g. Malan, 1998) in the development of Cape Town itself; outside of the city, sites such as Oudepost 1 (Schrire, 1988:218222; Schrire, Cruz-Uribe and Klose, 1993) and Vergelegen (Markell, Hall and Schrire, 1995) record the initial expansion of European populations into the Cape interior, but little archaeology of the northern expansion of colonial frontier districts has been attempted. Here, vernacular architecture remains a central concern, exploring the production of distinctive forms of 'corbelled houses' (Kramer and Malan, 2007; Maguire, 2007, Walton, 2007 [1989]; Kramer et al., 
2008), and other stone built structures such as wolwehokke (hyena traps, Walton, 1975); these structures are discussed as material signatures of the intrusion and impositions of colonial encroachment, relating to issues of settlement, livestock control, and predator eradication. Seacow Valley material pertinent to historical period hunter-gatherers (combined with written and cartographic sources) has yielded observations on the acquisition of new forms of material culture (Saitowitz and Sampson, 1992; Moir and Sampson, 1993; Westbury and Sampson, 1993) and livestock (Sampson, 1995; Voigt, Plug and Sampson, 1995), on the settlement patterns and mobility infrastructure of colonial settlers (Neville, Sampson and Sampson, 1994; Sampson, Sampson and Neville,. 1994), and on emergent inequalities in relationships between settlers and indigenes, negotiated through control of access to certain resources (Sampson, 1993; Plug and Sampson, 1996).

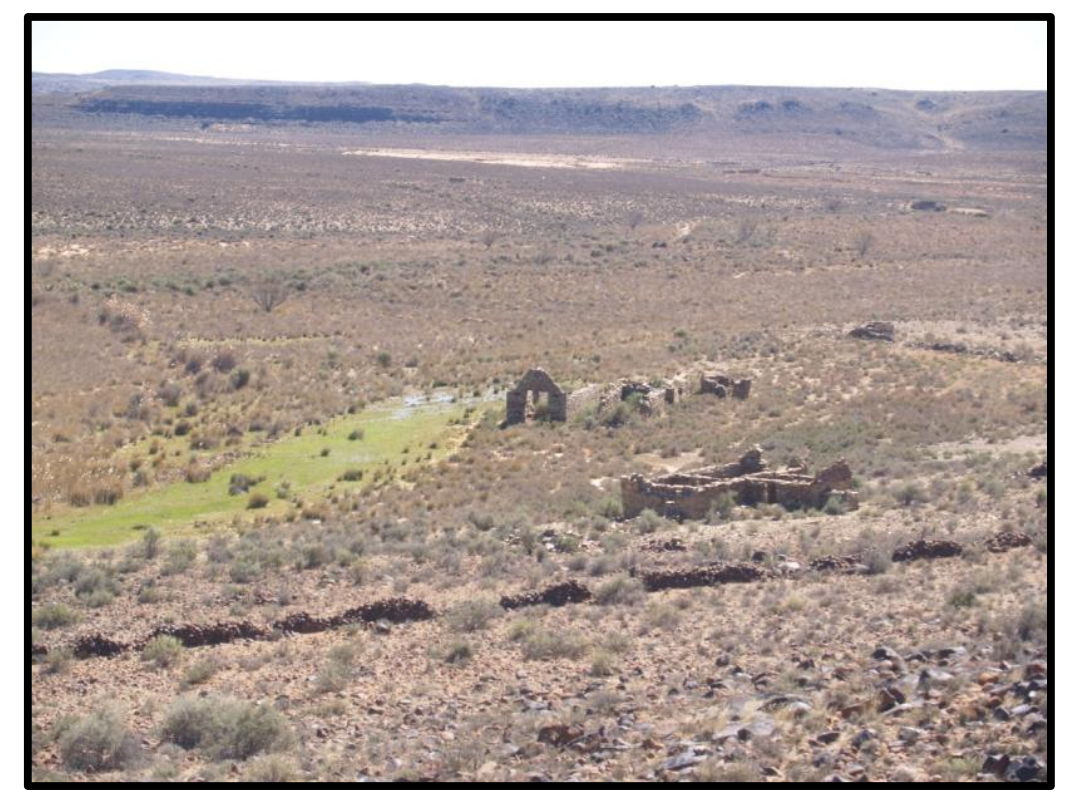

Figure 4.1: Stone walling associated with European colonisation, Northern Cape 


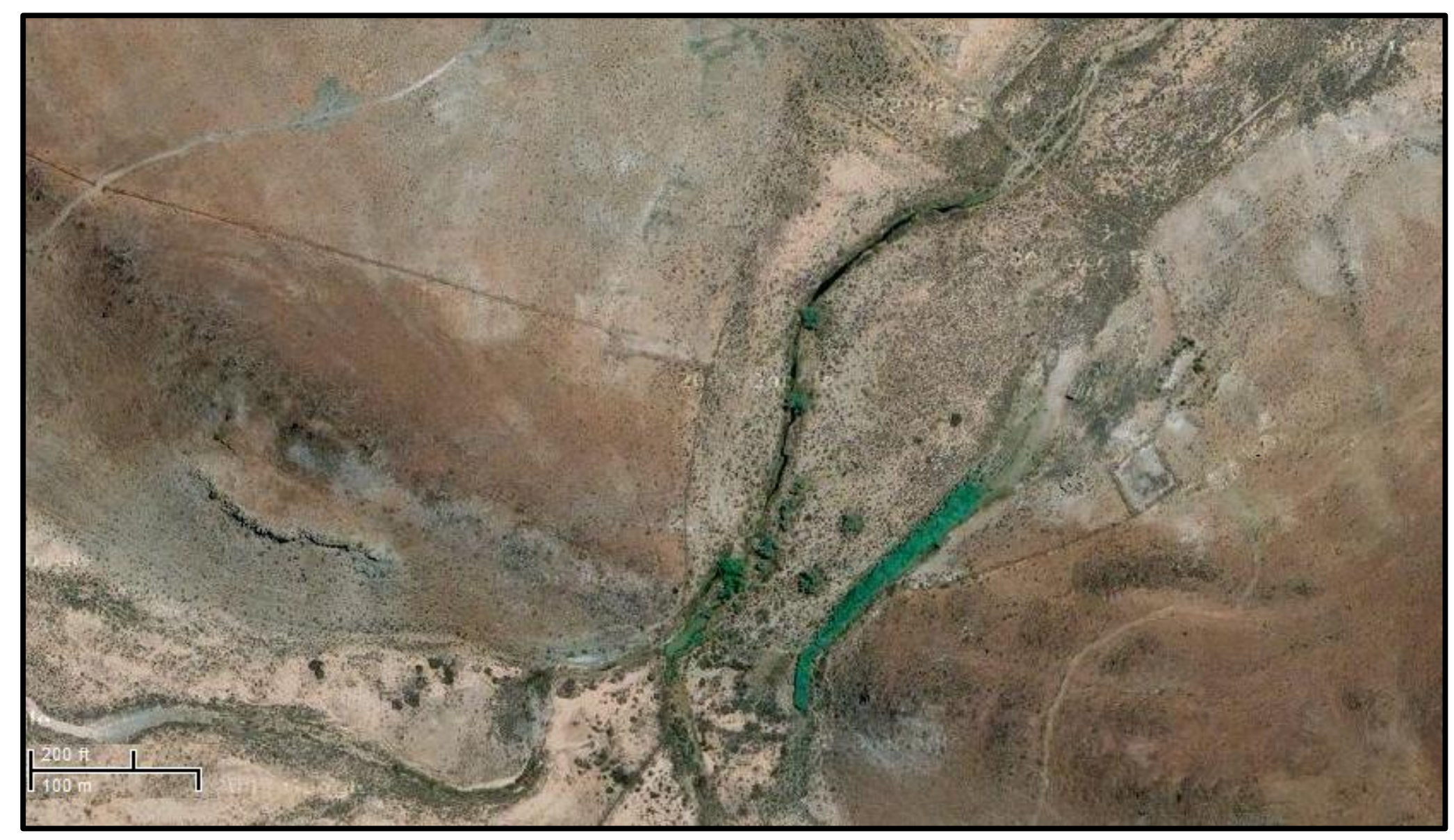

Figure 4.2: Aerial view of Karoo farm walling (from figure 4.1) (GoogleMaps image) 
The generally low incidence of large-scale redistribution of sediment in the Karoo environments means that 'structural' site features, such as stone walling reflecting stock-keeping, residence, and larger-scale works associated with the 'improvement' of farmland (Figure 4.1:94; Figure 4.2:95), can remain highly visible. Living surfaces are unlikely to be buried and preserved, and there is a continually accumulating palimpsest of human and faunal activity; Swartkop (and Doornfontein) assemblages likely incorporate IXam material, but in the absence of chronologically-sensitive colonial material culture it is difficult to separate this from an LSA background. Substantial historical information is necessary to identify sites with (fairly) certain IXam contributions to the assemblage: Deacon's $(1986 ; 1988 ; 1995)$ work represents the major application of this to IXam sites, while parallel approaches by Kinahan (1996) and Humphreys (1989) also combine extensive documentation with their archaeological analyses of non-|Xam historical period sites.

Connecting contemporary locales with places mentioned on a map produced by Bleek (Figure 4.3:98), Deacon excavated sites corresponding to known places in the IXam world, one at Arbeidsvreugd and another at Gifvlei. She concludes that the archaeology contributes relatively little ethnographic evidence, limited to 'ethnographic snap' confirming specific observations in the archive (Deacon, 1995), but that despite the allusive nature of IXam references to their practices, the archive greatly enriches archaeological interpretations, imbuing obscure or apparently mundane elements of the material record with wider social and cultural meaning. The absence of a comprehensive ethnological analysis of the IXam 
material is problematic for this use of Bleek-Lloyd material (or its deployment in the interpretation of other archaeological evidence such as rock art). The sheer scope of the archive facilitates the 'mining' of narratives for quotes to support particular perspectives, but treating the corpus piecemeal (without considering the IXam language component and examining the translation process) fails to integrate these quotes within a framework explicating IXam beliefs and practice: Chapters 5 (132), 6 (168), 7 (213), and 8 (282) return to the original Bleek-Lloyd notebooks in an attempt to develop such a framework. 


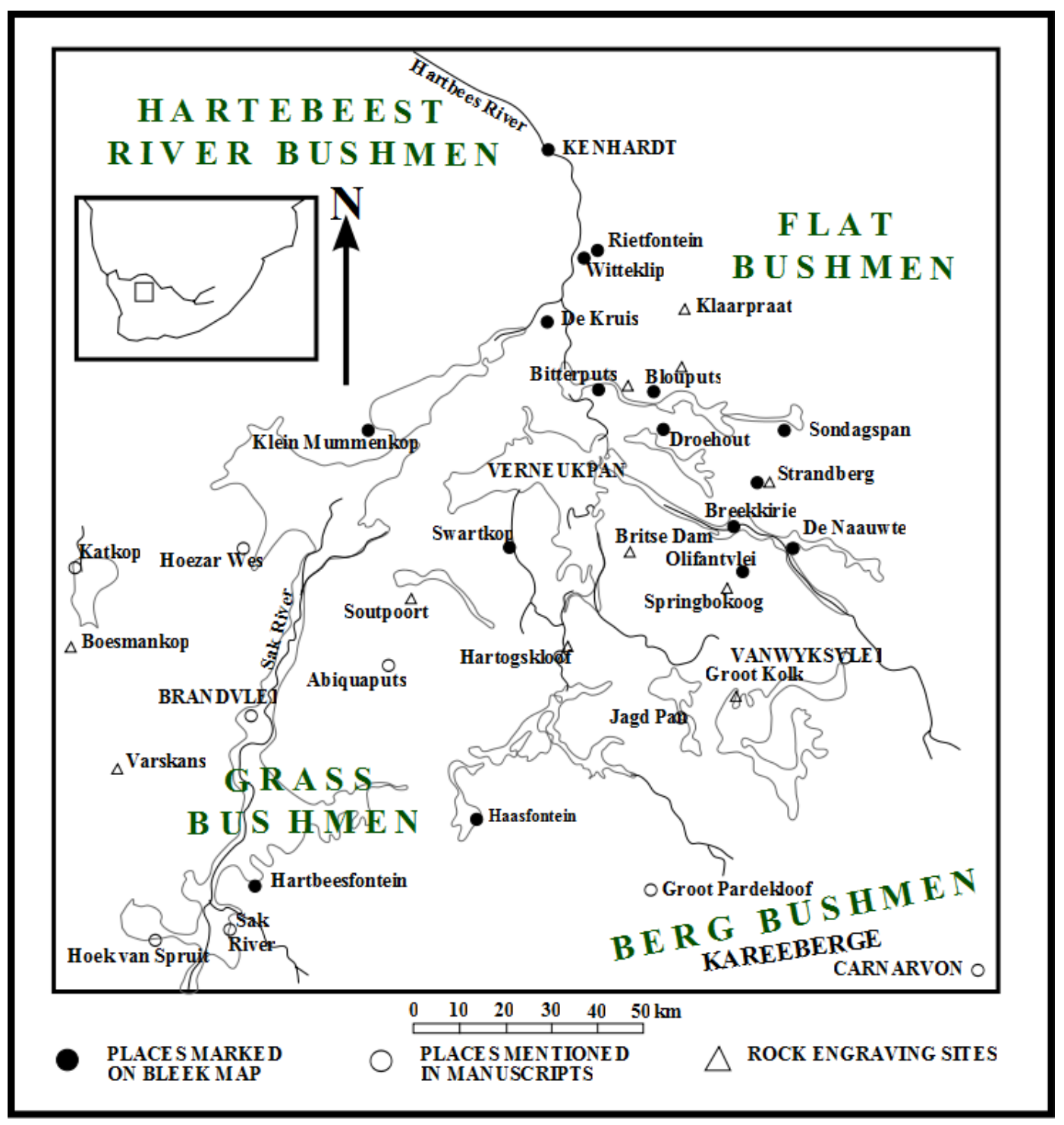

Figure 4.3: Bushmanland (Kenhardt-Brandvlei-Vanwyksvlei triangle) showing places mentioned on the Bleek map and in manuscripts (after J. Deacon, 1986). 


\section{Chapter 4b: The Cape Colony in the eighteenth}

\section{century}

Significant as they are, the Bleek-Lloyd archives do not exist in a vacuum, forming merely a single component in the web of colonial documents referring to the IXam Bushmen. Missionaries, explorers, colonial military officials and constabulary, farmers, and traders of the northern border districts all left accounts alluding to IXam demographics, subsistence strategies, and cultural practices, highlighting components absent from the archive, outside the aims of the project and the specific life experiences of the informants. This provides an important balance for the relatively small number of informants, allowing us to judge the wider applicability of their experiences. Additionally, it forces us to consider IXam society of the Bleek-Lloyd archive as a product of the nineteenth century context within which the informants grew up and dwelt.

This context originates in seventeenth and eighteenth century interactions between Europeans and indigenous populations in the fynbos regions of the south-western corner of the subcontinent. As in other colonial situations (Chapter 9:342), exported attitudes and perspectives interacted with indigenous counterparts producing a complex shared trajectory; numerous syntheses describe the overall 
course of this trajectory (Beck, 2000; Feinstein, 2005; Ross, 2008). Here, I provide a brief sketch of the processes whereby colonial society and IXam Bushmen came into contact, drawing out some central themes in their interaction. In 1652, the Dutch East India Company (Vereenigde Oost-Indische Compagnie, VOC) established a supply post at Table Bay, on the spice trade route to Batavia (Jakarta). Supplies were traded from indigenous Khoekhoe pastoralists, whose large herds of cattle and sheep were a major incentive dictating the placement of the station. The VOC, having little interest in creating a potentially expensive colonial state, attempted to maintain strict control of trade and territorial expansion, regulating the interactions of colonists with indigenous populations. However, as local Khoekhoen became reluctant to trade their increasingly depleted herds, settlers began to farm for themselves to produce the goods the company required (Ross, 2008:22-23). Around Cape Town, settlers developed farming strategies incorporating European crops (vines and wheat), relying on slave labour brought from VOC territories in the East Indies, or elsewhere in Africa. With an increasing population, farmers moved further into the interior, where environmental differences undermined strategies based on European crops: as settlers moved into drier Karoo regions a transhumant pastoralist system, relying on indigenous labour and incorporating considerable mobility, developed. Close contact between settlers and indigenous societies (coupled with considerable gender imbalances in initial European immigrants) favoured the formation of creolised populations, including the Bastaards (descendants of Europeans and Khoekhoen) and Oorlams (descendants of Khoekhoen and slaves), 
that would in the nineteenth century influence considerably the trajectory of the northern border districts (Legassick, 1979).

A prominent institution arising in the early contact period was the commando system, a major structuring principle constructing 'dominating' and 'dominated' classes in the Northern Cape (Penn, 2005:110). Requiring access to firearms and horses, the commando responded to the demands of conflict with widely-scattered hunter-gatherer populations, in areas themselves only sparsely populated by colonists, revolving around rapid, local mobilisation of armed forces to retrieve stolen livestock and exact punitive retribution. Late eighteenth century Bushman resistance along the north-eastern border beyond the Sneeuberg Mountains catalysed an escalation of the commando system; the scale of resistance in this area provoked a withdrawal of settlers, and in response the colonial authorities organised the 'Great Commando' in 1774, increasing markedly the scale and centralised control of the system. These two forms of the commando (local and large-scale) continued throughout the nineteenth century, a source of tension between colonial authorities (finding commandos prohibitively expensive) and individual farmers (resenting colonial appropriation of their labour).

Commandos captured large number of indigenous individuals, primarily women and children, distributing them amongst commando participants and establishing them as a rural labour force crucial for settler farming strategies. These individuals had numerous options for resistance; Newton-King (1999) sees the violence of the system as a coercive attempt to maintain unequal labour relations. 
While commandos were not slave raids in the sense that they supplied a market ${ }^{32}$, the labour relations set up and maintained by the commando were substantially similar to slavery; these unequal labour relations represented the major constraint imposed upon Bushmen in their relationship with colonial agents, requiring their successful engagement with an often violently coercive regime. However, commandos also formed a major locus for 'concerted' action between colonists and indigenes, incorporating both European and Khoekhoe/Bushman participants. Large-scale commandos could be numerically dominated by Khoekhoe and Bushman-descended individuals ${ }^{33}$, despite official concerns regarding provisioning these individuals with firearms (Marks, 1972:76); 'Tame Hottentot' participation was a (highly constrained) choice, measured against their other options (e.g. flight from the colony), demonstrating a reliance on indigenous communities even in creating fundamental colonial institutions.

The eighteenth century Northern Cape was often violent, but no single group had a monopoly on the production of this violence. VOC authorities struggled to exert their influence on populations nominally under their control, as geographical isolation and limited infrastructure hampered effective communication (Penn, 2005:41). Diffuse central political control attracted a disparate group of people wishing to avoid the colonial authorities, from criminals fleeing the judicial system, to marginalised populations seeking to escape colonial society, to individuals attempting to access lucrative trading opportunities. Some individuals

\footnotetext{
${ }^{32}$ Official discourse represented the captives as 'rewards' for participation in punitive, anti-stock theft measures.

${ }^{33}$ Farmers were loathe to leave livestock unattended for extended periods.
} 
came to have considerable influence forming loci of new social groups; Stephanos, a forger fleeing the colonial authorities, amassed a considerable following preaching among Khoekhoe populations of the middle Orange River (Kicherer, 1804), and leaders of creolised groups shaped polities integral to the historical trajectories of the interior throughout the nineteenth century. Droster gangs (Penn, 1999:147-166), originally formed by escaped slaves but increasingly incorporating disaffected Khoekhoe and Bushman farm servants fleeing the colony, represented a different form of (often violent) colonial intrusion, attempting to set up viable strategies that would allow them to exist outside of colonial control. Some Bushman populations in the Nama-Karoo began to aggregate into large units; the presence of the Bushman 'Groot Kraal' (supposedly comprising several hundred individuals) along the Zak River boundary was a matter of considerable concern for colonists exploiting the same region (Kicherer, 1804; Burchell, 1822); large aggregations would have demanded very different subsistence strategies to those discussed by Bleek-Lloyd informants (Chapter 7b:225; Chapter 7c:250), and the incorporation of livestock (as raiders, pastoralists, or both) seems likely.

Bantu-speaking groups expanded their range into the Nama-Karoo during the late eighteenth century, as power struggles among Xhosa chiefs in the Eastern Cape resulted in the movement of some groups westwards towards the colony. The consolidated eastern frontier of the colony effectively curtailed this movement, leaving Xhosa polities resisting suzerainty under larger chiefdoms with decreasing space for traditional strategies of fission. These groups adopted new strategies 
facilitated by the colonial presence; raiding stock, 'begging' from, trading with, and labouring for colonial farmers. Several groups comprising hundreds of individuals moved into the arid interior, travelling extensively across Bushmanland to the Orange River; initial inexperience with the Karoo environment $^{34}$ coupled with Bushman hostility (Burchell, 1822:268) presented considerable challenges for these groups (Lichtenstein, 1815:225-226). By employing a new suite of practices based on hunting, raiding, and stock-keeping, these groups became increasingly adept in managing their new environment (Kallaway, 1982); upon securing access to firearms, the Xhosa groups became foci for multi-ethnic coalitions vying with the pastoralists of the Orange River valley (Anderson, 1987) for control of access to the large cattle markets of southern Tswana towns. Just as colonial populations were coming to know them as 'Bushmanland', then, the IXam homelands had become newly incorporated into the subsistence strategies of a wide range of groups. Though permanent pastoralist occupation remained impractical, given water limitations, the increased mobility of horses and ox-wagons, coupled with opportunities for participation in new trading networks, strengthened links between the South West Cape and the Orange River.

The British arrived at the Cape in 1795, enforcing a claim to the colony motivated primarily by a desire to prevent Napoleonic France from acquiring a station for naval forces (Ross, 2008:37). Creating increasingly close ties between European and colonial societies, this encapsulates the essence of early British presence at

\footnotetext{
${ }^{34}$ Karoo rainfall regimes could not support the cereal crops employed by Bantu-speaking groups.
} 
the Cape. Symptomatic of these ties, this period also saw the arrival of evangelical missionaries, importing novel attitudes towards the Bushmen. Proselytising efforts at the Cape began with the short-lived Baviaanskloof (Genadendal) Moravian mission, running 1737-1742 (Humphreys, 1989), but it was not until the evangelical revivals of the late eighteenth century that missionary practice became widespread (Elbourne, 2002). Although British colonial and missionary 'Bushman policy' co-opted livestock subscriptions aimed at curtailing Bushman stockraiding (developed among farmers after the conflicts of the 1770s; Sampson, 1995), differences between British notions of appropriate colonist-indigene relationships and those generated over the past one hundred and fifty years at the Cape, soon became a source of tension between the colonial government and the farmers.

With the transition to British governance, the London Missionary Society (LMS) became interested in the Cape; Bushmen (and other hunter-gatherers) were important to late eighteenth century Evangelical $^{35}$ missionary organisations relying on public subscriptions for the maintenance of missionaries. Mission propaganda stressed the moral duty of Christians to support attempts to minister to 'benighted' individuals ignorant of the Bible, and the Bushmen were emblematic of those most in need of conversion (Kicherer, 1804:6-7). Attention was directed to the northern colonial boundary through the presence in Cape Town of Bushman 'kapteins' asking for missionaries to be sent to them: missionaries were necessary to establish the claims to Christianity and 'civilised' status that facilitated a

\footnotetext{
${ }^{35}$ A popular movement founded on opposition to established church hierarchies, and not receiving funding from them.
} 
privileged access to imported resources (especially gunpowder), powerful tools in the northern border regions ( $c f$. Legassick, 2010:77).

Kicherer's (a Dutch LMS missionary; Schoeman, 1996a; Penn, 2005:237) first mission to the Bushmen was not a resounding success. The mobility strategies and scattered populations of Bushman groups were difficult to incorporate into missionary visions of residential missions with a strong focus on cultivation, and Kicherer himself was often away from the station visiting pastoralist and creolised communities of the Orange River, whose zeal and numbers swiftly made them the major focus of missionary work; Tswana agro-pastoralists north of the Orange River also became increasingly important targets as Europeans became aware of them (Comaroff and Comaroff, 1991). After the perceived failures of this first mission, Bushman-focused endeavours in the central Nama-Karoo were reduced and drifted eastwards through a series of short-lived stations (Schoeman, 1993, 1993a), with out-stations manned by indigenous converts (generally more successful in maintaining Bushman stations) coming to dominate the LMS strategy for hunter-gatherer populations. Bushmen who elected to visit stations set up among creolised (Bastaards, Oorlams) and pastoralist (Namaqua, Korana) populations continued to be accepted, and mission stations developed into early examples of multi-ethnic aggregation sites (similar to nascent urban centres in the nineteenth century), locales whereby Bushman individuals could incorporate themselves into the mixed-heritage populations underpinning emergent 'Coloured' identities in the Northern Cape (Sales, 1975). 
Although the 'long nineteenth century' (Hobsbawm 1962; 1975; 1987) was developed as a coherent temporal unit of analysis for European societies, it has considerable relevance for approaching the incorporation of Karoo societies within the Cape Colony, corresponding to a period in the colonial history of South Africa running from the beginnings of British interest in the area (1790s) to the formation of the Union of South Africa (1910), and approximates the chronological period shaping the bulk of the first- and second-hand experiences of Bleek and Lloyd's informants (Bank, 2006). It was over the long nineteenth century that Bushman society underwent its most radical transformations. In the closing decades of the eighteenth century, largely independent IXam groups interacted with the various groups exploiting their territories; colonial farmers consolidating their positions along the southern fringes these areas (moving seasonally to exploit more arid regions; Penn, 2005:81-87), and creolised groups, criminals, escaped slaves, hunters, and traders spreading the colony's influence beyond its official geographical frontier.

The concept of the 'frontier' in South African historiography is complex ( $c f$. Penn, 2005:10-14; Legassick, 2010:3-10), but one dimension that has remained important, despite challenges ${ }^{36}$ and refinements, is Giliomee's (1979) distinction between 'open' frontiers (no single group exercising hegemonic control) and 'closed' frontiers (balance of power favouring one group). I use 'frontier' primarily as shorthand for the geographical area of the 'northern frontier': at the

36 L. Mitchell (2009) suggests the model assumes rather than explains colonial conquest; she favours Guelke's (1985) notion of 'orthodoxy' (the construction of colonial authority), with both indigenous and incoming communities participating in the construction of new orthodox (supporting) and heterodox (challenging) strategies for dealing with this authority. 
end of eighteenth century this was officially delineated by the Zak River (Figure 4.4:109), and by the mid-to-late nineteenth century the 'northern border districts' more-or-less coincided with the |Xam-ka-!xoe. Physical and environmental features (especially river channels) channelled the expansion and contraction of these frontiers, tending towards encapsulation of sporadically-exploited IXam territories, but even geographically definitions cannot escape a social dimension. In practice the nominal boundary did not limit the movements and impact of colonial society, and as a frontier must be an area of contact between populations, we must therefore identify a series of overlapping frontiers incorporating Xhosa moving in from the east, Tswana-speaking groups to the north-east, Khoekhoen and non-IXam Bushmen to the north, and Europeans and Bastaards from the south.

Though faced with considerable destructive violence in the course of their interactions with incoming colonial populations, hunting and gathering strategies remained viable subsistence alternatives for Bushman populations. By the early twentieth century, however, they were incorporated as an 'underclass' of rural labourers together with other dispossessed indigenous groups; 'the IXam' as a linguistically and culturally distinct entity no longer existed. The process of this disintegration, explored in the following section, is crucial in attempts to understand the production of the Bleek-Lloyd texts, speaking to global themes in hunter-gatherer interactions with colonial states (Chapter 9:342, 362). 


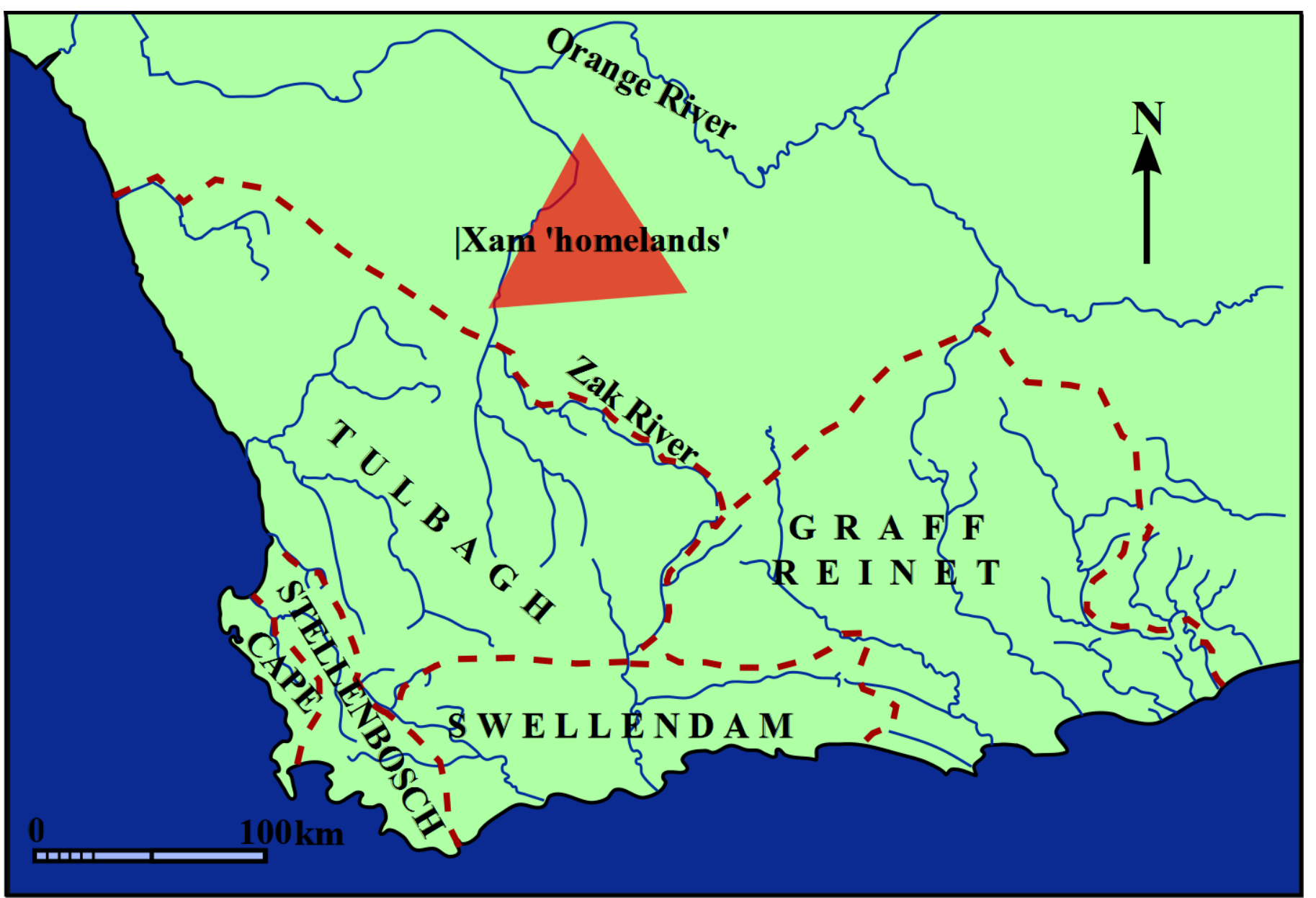

Figure 4.4: Map of the boundaries of the Cape Colony (1795) 


\section{Chapter 4c: Encapsulation on the northern borders}

Official interest in the interior waned from the first half of the nineteenth century until the closing years of the 1860 s, when the discovery of diamonds along the Vaal River attracted government attention toward the mineral wealth of the region; the transformation of the Transvaal into a major gold producing region in the 1880s (Ross, 2008:75) cemented this interest. The first decades of the nineteenth century saw considerable continuity in the colonial exploitation of the arid Karoo, with white and Bastaard farmers maintaining their eighteenth century strategies (Penn, 2005:81-87) and a relative lack of government intrusion facilitating the persistence of hunter-gatherer lifestyles, retarding the arrival of the apparatus of colonial government that finally 'closed' the Karoo frontier to these strategies.

Governor Sir Harry Smith's proclamation at the end of the Seventh Xhosa War (1846-1847) extended the official colony boundary to the Orange River, redefining the nebulous northern boundaries of the colony as the 'natural' boundary of the Orange River and incorporating land eastwards to the Keiskamma River into the dependency of British Kaffraria (Peires, 1981). Annexation to the Orange River in theory incorporated the entirety of the IXam-ka-!xoe, but there 
was little incentive for government intrusion; at the time, the incorporation of this largely unprofitable and potentially expensive land (in terms of demands for government protection) attracted censure (Wodehouse, 1868). Although on paper these regions became part of the colony, little changed in practice. Two decades after annexation, the region was still sparsely populated by a heterogeneous set of mobile communities (Wodehouse, 1868); Moffat (1858:155), travelling in 1854, noted that the region remained poorly understood by surrounding European settlers.

The mobile strategies of indigenous herders and hunter-gatherers caused colonial farmers a great deal of consternation, undermining their ability to command the labour of Khoekhoe and Bushman servants, largely acquired as captives commando raids (Chapter 4b:101). This de facto 'slave raiding' placed farmers in opposition to an early nineteenth century British government concerned with the abolition of slavery; in the colony this manifested with the passing of the 'Hottentot Proclamation' (Harlow and Madden, 1953) in 1835, aimed at codifying the rights and obligations of these farm labourers. However, colonial authorities' habit of emphasising that their jurisdiction ended at the official border and their lack of interest in the interior meant that commando acquisition of Khoekhoe and Bushman servants in the border regions persisted throughout the nineteenth century, with Bastaards often playing a 'middle man' role (Smit, 1823; Moffat, 1858:155). Although the 'open' (or heterodox) frontier undermined colonial farming strategies of controlling labour by facilitating independent huntergatherer existence or the adoption of raiding lifestyles (see below), the 
unregulated violence that persisted in consequence of attempts to 'close' it contributed in a major way to the eventual collapse of IXam society.

In 1863, Louis Anthing presented a report to the Cape Parliament (Anthing, 1863) detailing the results of his investigation of allegations of ongoing violence towards Bushman groups. His involvement as an advocate for the IXam (J. Deacon, 1996:13) is well established in the literature ${ }^{37}$, and it will suffice here to summarise his report (J. Deacon, 1996:13-16). Interviewing traders and inhabitants of the Karoo, Anthing concluded that the Bushmen were subject to a 'wholesale system of extermination' in northern districts (Anthing, 1863:447), the structure of which was predicated upon eighteenth century patterns, with Bushmen being killed in 'opportunistic' encounters and by commandos with this express purpose (Anthing, 1863:449). Noting the role of European colonists in violent encounters, he also emphasised that indigenous or creolised populations played a significant parts, paralleling the Bleek-Lloyd accounts that stress conflict between Bushmen and Bantu-speakers and between IXam and Korana pastoralists as much as they do violence in relationships with European farmers (LL.VIII.27.8436'; LL.II.34.2960'; LL.II.36.3244', Chapter 8b:303, 315).

'Wholesale extermination' does not exhaust the range of interactions that existed between hunter-gatherers and colonial agents. Contrary to characterisations of IXam interaction with other indigenes, the literature has under-emphasised less antagonistic dimensions of European and IXam relationships. Although the IXam

\footnotetext{
37 His reports concerning the Koranna betray rather more typical colonial attitudes towards indigenous groups (Anthing, 1868; 1868a).
} 
are not generally considered 'specialist producer' hunter-gatherers ${ }^{38}$, they traded extensively with the colony, and their specialised environmental knowledge underpinned their roles as guides, employed by travellers beyond the borders on hunting and trading expeditions. Faunal resources were important to all groups occupying the arid interior: pastoralists hunted for subsistence, preserving their stock and acquiring the raw materials for a range of products otherwise only attainable in distant markets, while 'commercial' hunting extracting lucrative resources (ivory, ostrich feathers, and hides) was also common. The specialised prey selection of semi-professional hunters was accompanied by slaughter of game animals; minimising provisioning costs, they relied on wild fauna for subsistence. This provided new resources for Bushman groups attaching themselves to such parties, as hunters discarded the offal of game shot and would not eat the flesh of elephant and zebra they shot. IXam groups also participated directly in the trade in faunal products; ostrich feathers became a lucrative export item in the mid-nineteenth century (offering greater potential returns than ivory; van Sittert, 2005:274), and IXam acquisition of these feathers for trade is noted in the Bleek-Lloyd archive (LL.II.7:771-776; Chapter 8c:335).

Nineteenth century IXam also engaged in relationships of labour exchange or appropriation, the foundation of their incorporation into a rural underclass ( $R$. Gordon, 1992) in the early twentieth century. A major motivation for colonising populations to interact with indigenous hunter-gatherers, these relationships represent arguably some of the most enduring, stable accommodations made by

\footnotetext{
${ }^{38}$ Hunter-gatherer societies where exchange relationships with food-producers have an important subsistence role (Chapter 9:359).
} 
Bushmen with their colonial situation; it is within this stability that we should situate linguistic interactions between the two groups. Traill (2007:146) notes that frontier farmer bilingualism in |Xam and Afrikaans implies settler-|Xam social relationships moved beyond an 'exclusive' hostility; \|kabbo's list of IXamspeaking farmers (WB.XI.1120) provides evidence for linguistic exchange covering at least sixty years, presumably in the context of working relationships that induced strong motivations for mutual comprehension. Afrikaans (contemporaneously known as Cape Dutch) was certainly dominant, persisting in the long term, but at a more local level there was scope for the continuance and even spread of the IXam language into new socio-cultural contexts.

'Successful' Bushman assimilation into colonial society is often largely invisible in official discourse concerned with policies dealing with 'the Bushmen', but legal documentation such as death notices and wills can give an occasional glimpse into the lives of these Bushmen. These Death Notices $(1849 ; 1849 \mathrm{a} ; 1857)$ record the acquisition of livestock herds of around 50-100 animals, and colonial material culture (firearms) by individuals, who, while undoubtedly represented the colonial poor (the estates being described as 'trifling'), negotiated the colonial scenario successfully in the face of increasingly untenable foraging strategies and widespread commando violence. Assimilation moved individuals into a 'Colonial (or 'tame') Hottentot' category, as colonial definitions of Bushmen as inveterate raiders (incapable of civilised practice) or as primitive (thus unchanging) allotted little space for 'successful' hunter-gatherer engagement with the frontier as 
labourers; individuals who did so moved out of the Bushman category, obscuring the extent to which Bushmen became part of the creolised societies of the colony.

Although colonial policy was tailored around constructions of indigenous ethnicities, responses to raiding practices were fairly uniform; multi-ethnic participation in these practices helped create new forms of shared experience between indigenous groups. Direct subsistence competition and settler appropriation of reliable water sources (Chapter 3a:61; Chapter 3b:71) meant that indigenous herding groups faced more immediate crises than hunter-gatherer populations. Deprived of livestock, herder societies could not by definition continue; opportunities for pursing 'interstitial' independent subsistence are more constrained for pastoralists than hunter-gatherers, and issues of incorporation versus flight or resistance more acute. However, the potential for losing stock did not arrive with European settlers, and responses to stock dispossession were substantially prefigured by indigenous conceptions. Familiarity with hunting and gathering strategies or links with hunter-gatherer groups were therefore necessary components of Khoekhoe subsistence strategies, as fallbacks for times of resource stress ( $c f$. Marks, 1972; Elphick, 1977). Raiding to replenish herds was endemic among Khoekhoe groups of the middle Orange River (Penn, 1995:39), as were 'clientship' strategies drawing on kinship or affinal ties to build up new herds. Khoekhoe interactions with settlers extended these strategies to the colonial situation; working as shepherds for European farmers within their original territories represented a form of clientship, though one that offered little chance of regaining subsistence independence. Colonial stresses on livestock populations 
increased interactions with hunter-gatherer populations (or promoted increasingly similar Khoekhoe and Bushman practice), and for the Northern Cape, intensive interaction between IXam and Korana populations is confirmed in the life histories of Bleek and Lloyd's informants (Chapter 8b:299). Small-scale stock raiding of colonial farmers was incorporated in Korana strategies from the early nineteenth century, taking place as farmers took their stock to graze beyond the colonial boundary in territories claimed by the Korana (Wade, 1832; Redelinghuis, 1834; Ryneveld, 1846, 1846a), and increasingly employing colonial material culture in the form of horse and firearms (Ouzman, 2005) $)^{39}$.

As colonists came to place greater strains on the resources of the middle Orange River, the Korana became the quintessential enemy of colonial settlement; farmer depictions of their raiding in Memorials Received (1846) by Government demonstrate the use of this 'enemy' to convey the relative competences of frontier officials, to excuse themselves from government demands on their labour, and to make claims on Government resources (munitions; Redelinghuis, 1834). At the end of $1860 \mathrm{~s}$, the government responded to their representations with large-scale efforts, in a series of conflicts known contemporaneously as the Korana Wars (Ross, 1975; Strauss, 1979). With the 1847 border shift, Korana territories became potential targets for permanent colonial settlement; Bastaard and Oorlams farmers (marginalised within colonial society), whose movement was facilitated by the same colonial material culture that supported European settlers, were initially the primary agents of this settlement. Permanent occupation increased competition for

\footnotetext{
${ }^{39}$ These became sufficiently well established in Koranna strategies to present significant problems for colonists during the Koranna Wars (Anthing, 1868).
} 
resources, with some Korana groups losing large numbers of stock in internecine warfare, which they then sought to replace through raiding practices (Strauss, 1979). Colonial farmers (now major livestock-owning groups in Korana territories) became major targets for these raiders, who moved along the Hartebeest River channel deep into the northern districts (Anthing, 1868a) and retreated to formidable redoubts on the islands of the Middle Orange River to secure their stolen livestock (Anthing, 1868). Combined with drought conditions, these raids prompted widespread farmer retreat, leaving IXam and Korana groups largely in sole possession of the region in mid-1868 (Strauss, 1979:37).

Although the major combatants were Korana, these conflicts also mark the last example of IXam participation in large-scale violent resistance to colonial occupation, and the nature of this participation was symptomatic of the changes IXam society experienced during the nineteenth century. The large Bushmen aggregations reported by travellers and missionaries during the opening years of the nineteenth century (Collins, 1808; Penn, 2005:225) were no longer an option, as deaths associated with commando raiding undermined the demographic capacity for independent IXam resistance; a denser population and relatively late contact with the colonial frontier meant that the Korana retained this capacity, and Bushmen pursuing these strategies amalgamated with the Korana, either though election or coercion. Official reports grouped the Korana and Bushmen together as the contiguous 'restless Tribes' of the northern border (with Bushman playing a subsidiary role), but emphasised Bushman participation as the result of Korana antagonism, with Bushmen being compelled or 'employed' by Korana to serve as 
members of raiding parties (Jackson, 1879:98). These reports suggest a range of different forms of Bushman involvement in Korana activity, and it is likely that the degree of compulsion versus election varied a great deal with geographical location (Chapter 7a:222). Although stock-raiding parties were composed predominantly of Korana, linked to Korana political leaders, and originated in Korana territories, there was a general impression at the time that raiding groups were largely multi-ethnic in composition (Jackson, 1879); Xhosa groups settled around mission stations in the Kareeberg were heavily implicated in their actions, for example (Layton, 1879), and it seems likely that they would attract those Bushmen unable to pursue such tactics independently.

Border conflicts also continued practices more typically associated with the unregulated commando practices reported by Anthing. The Korana Wars suffered from a considerable bad press, with emergent metropolitan perceptions of Bushman as 'a poor unarmed lot' (Jackson, 1879) creating an impression in colonial government and the wider public that the colonial agents involved were applying an excessive amount of force. Attacks such as the engagement of van Niekerk's commando against a group of 'Bushmen ${ }^{40}$, killing forty-six people (Jackson, 1879), contributed to this impression and to the realities of the border conflicts; the presence of children among the victims of this commando suggests that the group was not (only) a raiding party, and that, for Bushmen groups, officially delineated 'wars' must be placed within the continuum of violent relationships that persisted throughout the nineteenth century.

\footnotetext{
${ }^{40}$ Historical accounts refer to this both as a mixed band of Xhosa, Khoekhoen and Bushmen raiders, and as a Bushman group.
} 
After the Second Korana War, colonial authorities collected testimonies from farmers regarding their stock losses, assessing their claims for compensation from the government. These accounts reveal another dimension of Bushman involvement in the raiding, recounting small-scale opportunistic thefts of large and small stock (Upington, 1879:xxxv-xxxvi; House of Assembly, 1880). These instances appear to represent highly individualised responses, part of an on-going modification of subsistence strategies to deal with an environment in which the medium-to-large bovid fauna was beginning to be dominated by domesticated species: tellingly, this type of small-scale raiding did not end with the border conflicts, but rather came to be defined differently by the government, provoking a different response. It is in this process of shifting definitions that we see the impact of border conflicts in consolidating the processes of encapsulation that ensured, within a generation, the destruction both of the viability of huntergatherer subsistence in the Northern Cape, and of IXam cultural identities.

The final three decades of the nineteenth century saw the Crown Lands of the northern districts surveyed and divided into farms. After the Second Korana War, a report commissioned by the colonial authorities (Upington, 1879) found the northern border districts largely un-surveyed. Some attractive, well-watered areas (along the Orange River) had been divided into large farms occupied on one year leases $^{41}$ and used for grazing livestock, but much (certainly in the IXam homelands) was still used communally, as scarce water supplies away from the river itself meant transhumant strategies remained essential. Once land survey and

${ }^{41}$ Some held by Europeans, but most by Bastaards (Upington, 1879:iv). 
sale began, however, it progressed with remarkable rapidity: the equivalent report at the end of 1880s (House of Assembly, 1888) found the whole region occupied by settlers living in permanent structures on privately-owned land or by farmers on lease lands utilising more ephemeral housing. This marked change was facilitated by the widespread adoption of two new forms of technology (wire fencing and borehole windmills), spreading as a result of a confluence of economic, political, social and technological factors. Private ownership of land promoted the installation of 'improvements', particularly with the expanding markets of large urban mining settlements in the interior that drove up the price of mutton and increased the farmers' investment capital. Borehole and windmill (Archer, 2000:683) technology allowed easier access to water, and tapping artesian water sources allowed settlers to occupy the Karoo on a more permanent basis, by creating new locales from which grazing resources could be reached. These long-term occupations increased both the settler population and intensified its exploitation of resources, encouraging private ownership of land that could now be delineated with cheap wire fencing. By impeding the free movement of fauna across the landscape and creating static, alienated areas where mobile strategies had once flourished, this constituted a practically and symbolically significant step in the imposition of settler conceptions of land and resource ownership; wire fences inscribed the landscape with the processes whereby the colonial authorities took possession of the land, representing concretely the surveying and commercial sale of the rights to access designated areas of land 
In addition to alienating hunter-gatherers from the resources necessary for independent subsistence, these phenomena of colonial expansion had considerable impact upon the resources themselves. Attitudes of European colonists toward faunal resources had, in combination with the arrival of new forms of material culture, devastating impacts on the ecology of already-fragile arid regions (Chapter 3b:74). The collapse of the trekbokke phenomenon at the end of nineteenth century was a crucial component in establishing modern land use patterns in the arid interior; severe drought, over-hunting during the springbok trek of 1895-1896, and a reduction of range through the spread of wire fencing triggered the sudden and cataclysmic collapse of mass springbok migrations, dependent upon critical population densities as a trigger (Roche, 2008). Doubtless in part a consequence of the influence of large-scale ecological processes in the trekbokke phenomenon, springbok persisted at periodically high densities long after other game species had suffered dramatic losses (Dunn, 1873:39). The emphasis on this species in IXam hunting narratives (LL.VIII.7:6604-6605, 66076621; LL.VIII.23:8067-8073; Chapter 7b:237) points to its salience in nineteenth century IXam subsistence; as with the over-hunting of Great Plains bison herds in North America, the disappearance of the trekbokke undermined one of the last substantial resources (and a key cultural referent) of the IXam, greatly diminishing their ability to sustain themselves without recourse to stock-raiding or employment with farmers, even before land alienation.

Fencing also influenced the social world of the emergent Khoekhoe and Bushman rural underclass. Increased expansion into the arid interior coincided broadly with 
an economic incentive for land claims. In the middle of the nineteenth century, decreases in wool output from Britain, and increased capacities for woollen good production, created a large demand for wool imports from the colonies (Anderson, 1987). Introduced breeds of wool-producing sheep, such as the merino, cemented the rise of wool as an important element of the colony's export economy (Beck, 2000:50). Although Nama-Karoo farms remained relatively unproductive for nonnative breeds, if large enough they could be used to graze sheep for wool. The shift to wool production for export helped 'close' the northern frontier (MacKenzie, 1988), as it left little space for the more flexible transhumant systems that had co-opted indigenous strategies (Kinahan, 1991:76-86), or for competing wild fauna. With borehole technology overcoming water demands, shifts to wool production became comprehensive, and by the 1870 s farmers for the most part focused on merino breeds (Dunn, 1873:31-32). Wire fencing allowed the large farms of the region to be cheaply and quickly secured, reducing the demand for continual shepherding labour. The commercialisation of sheep farming geared toward wool production required large labour forces at specific times of the year (for shearing), supporting an emergent class of rural labourers travelling between farms to take advantage of this seasonal work, the peripatetic Karretjiemense ('Donkey-cart People'; de Jongh, 2002). A creolised identity recalling those developing around population centres and on farms, this lifestyle incorporated Khoekhoe and Bushman individuals, representing another niche and strategy available within the constraints of an increasingly rigid colonial order. 
Throughout the nineteenth century, stock-raiding was an important dimension of interethnic relationships in the Karoo, and a considerable disincentive for its incorporation into the purview of colonial government, which feared inhabitants would make expensive demands ${ }^{42}$ on it for protection from this raiding: prior to the discovery of mineral wealth, the expenses likely to be incurred by annexation outweighed the benefits of any revenue that would be generated. After the costly Korana campaigns, the government adopted new strategies for dealing with 'disturbances' on the northern border, significant for the ultimate demise of hunter-gatherer subsistence. Bushman (and Korana) opposition to colonial appropriation of their labour were increasingly defined as criminal rather than military resistance. The cumulative impact of a century of 'wholesale extermination' on hunter-gatherer populations and the recent disruption of relatively powerful Korana polities removed obvious loci for aggregation and large-scale raiding, but in spite of this demographic collapse, colonists were not able to exert an uncontested hegemonic control. Definitions of criminality constituted an attempt to exert colonial authority over the remaining heterodox space that allowed individuals to manoeuvre for continued recourse to huntergatherer strategies (including livestock raiding).

The application of legal frameworks and colonial constabularies to the northern districts imposed standards that left little space for interstitial or peripatetic hunter-gatherer existence. Vagrancy laws undermined mobility strategies, poaching legislation restricted access to key resources, and private land ownership

${ }^{42}$ The Second Koranna War cost the government over $£ 100000$ by the end of 1879 (Garcia, 1879). 
sequestered Bushman populations from their traditional homelands, a 'bureaucratisation' that marked the true encapsulation of the northern border regions. A regular police force and defined magistracies, land survey, farm portions delineation, road and rail construction, and improved communication networks (telegraph) dramatically reduced the 'space' available for non-orthodox participation in colonial strategies. While communal exploitation of Crown Lands continued, IXam groups could access resources despite their increasing depletion through over-exploitation; colonial authorities recognised the difficulty of forcing Bushmen to accept employment without permanent farmer settlements, as they retired to unoccupied hinterlands for their subsistence (Legassick, 2006). Although official metropolitan discourse increasingly viewed Bushmen as harmless, their stock theft represented a problem for farmers even in the closing decades of the nineteenth century. Local magistrates were vital in incorporating these practical difficulties into authoritative discourse; Scott (Special Magistrate for the region from 1880) became increasingly sympathetic to the attitudes of the farmers during his residency (Legassick, 2006). While there was no systematic stock-raiding, domestic stock was occasionally killed for food on an opportunistic basis: Special Commissioner (and Magistrate) Jackson (1879:xxiv) explicitly characterised this in terms of criminality. The precarious economic situation of many frontier farmers in the more marginal areas of the Karoo meant that these practices could be ruinous on a personal level, despite their inability to affect colonial expansion generally. 
Scott's shift in attitude points to an underlying similarity of purpose in commando tactics and more 'humane' apparatus of the colonial state. Employing vastly different means and rhetoric, Scott's approach was motivated by similar aims to earlier and more overtly violent systems, in attempting to control Bushman labour. Late nineteenth century colonial metropolitan definitions of primitive, pitiable Bushmen were echoed in academic discourse as emergent anthropological concerns for Bushman 'survivals': Bushmen emerged as scientific curiosities just as they ceased to be a serious threat to colonial settlement (Legassick, 2006:65). Attitudes developed in newly 'policed' border districts were fundamental in this re-definition of Bushmen as scientific objects to be researched: Scott himself suggested a Bushman camp be created on Robben Island, to facilitate 'Bushman Researches'. The significance of colonial internment to Bleek and Lloyd's research is well known (J. Deacon, 1996:16-17; Hewitt, 2008 [1986]:8), and Robben Island too was a long-standing component of this disciplinary system (Peires, 1989: 300-301). This confluence of imprisonment, policing and research suggests that the Cape Colony saw the concomitant emergence of penal and scientific 'discipline' in mechanisms of colonial control, common elsewhere in the nineteenth century world (Foucault, 1975, 1977).

By the end of the long nineteenth century, a distinctive IXam identity was swiftly fragmenting. Dorothea Bleek’s (Bleek, 1936b) interactions with IXam Bushmen in 1910 and 1911 highlight this dissolution, providing a snap-shot of the final stages of IXam transformation into a component of the rural underclass. The older generation interviewed and photographed here represented the end of IXam 
Bushman cultural identity, the last generation to have firsthand childhood memories of hunting and gathering lifestyles. Their life histories illustrate the issues the fragmentation this cultural identity; language loss had become severe, and while some could speak IXam fluently ${ }^{43}$, others lacked even IXam names. Even individuals who both spoke the language and maintained aspects of IXam culture, including songs, music and dances, could not provide D. Bleek with examples of the tales recorded less than forty years ago, attributing this to disruption caused by their being 'driven about' while young. The photographs ${ }^{44}$ document the shifting parameters of Bushman life as they moved to squat around urban settlements, displaying the incorporation of elements of European clothing and the use of cast-off colonial materials in the construction of matjieshuis ${ }^{45}$ dwellings in combination with the continued use of 'traditional' items and materials. With the sequestration of the traditional IXam homelands into privately owned farms, and consequent relocation of IXam populations, the imposition of settler landscapes on the prior Bushman cultural landscape (J. Deacon, 1988) was complete; 'topophilic' (Tuan, 1974) attachments to specific owned places (Glossary !xoe-s'o-!k'e:439; Chapter 6b:188) were not replicated, as colonists exercised increasing control over land.

Displacement and encapsulation in the colonial 'underclass' were not processes restricted solely to IXam populations, contributing to the dissolution of distinctive

\footnotetext{
43 Perhaps more significantly, some had only a poor grasp of Afrikaans, suggesting limited contacts with settlers.

${ }^{44}$ The implication of photography in creating a colonial 'gaze' (Foucault, 1975:89) in Bushmen representations has been widely recognised (Skotnes, 2001; Bank, 2006:105-107).

${ }^{45}$ A traditional housing style of Northern Cape herders and hunter-gatherers consisting of reed or animal hide mats cast over a wooden frame (Boonzaier et al. 1996:37).
} 
IXam identities. Bleek's (1936b) interviews document the integration of IXam descendents with other impoverished individuals; while she excluded 'mixed descent' (Korana and IXam) from her more detailed notes, their presence points to increasing contiguousness of these populations. Emergent population centres (paralleling the earlier mission stations) attracted individuals from a range of backgrounds, creating new locales for interaction such as Kakamas on the Orange River, occupied by 'Bastards, Kafirs, Korannas, and Bushmen’ (Dunn, 1873:57). Loss of access to graze and water, as more land became alienated as private farms, made it difficult for indigenous herders to maintain livestock, and with reprisals for resistance to colonial encroachment that served to increase the rate of dispossession, they moved to increasingly-large population centres. After the Korana Wars ended the capacity for large-scale raiding (Nightingale, 1888) to replenish depleted herds, pastoralists who lost stock were in much the same position as IXam groups severed from the wild resources of their lands: both groups now relied upon colonial populations for their subsistence. Through a combination of a colonial tendency to treat them in similar fashion and similar experiences of dispossession, Khoekhoe and Bushman populations came to inhabit increasingly similar worlds. The rural communities that emerged from this shared experience may be best discussed as 'Khoisan' in a cultural ${ }^{46}$ sense, retaining elements of IXam and Khoekhoe beliefs that resonated among individuals drawn from both populations; even in the late twentieth century, a complex of beliefs centring on the relationships between water, snakes, and

\footnotetext{
${ }^{46}$ I.e. in addition to their shared genetic heritage.
} 
'rainbulls', paralleling themes in the IXam narratives and in Khoekhoe ethnography, was extant among members of rural 'Coloured' communities, descended from these early twentieth century groups (Hoff, 1997, 1998; $c f$. Chapter 8a:293).

In terms of actual practice, living conditions would not have been conducive to the survival of detailed beliefs centring on hunting traditions. The IXam in the early twentieth century relied upon government rations distributed by local magistrates, or laboured as shepherds (if male) or maids (if female). Employment by farmers revolved around livestock, and diminishing game populations in combination with increasingly strict and well-enforced hunting laws (van Sittert, 2005), offered little opportunity for experiencing hunting, certainly not of the kind described in the Bleek-Lloyd narratives (Chapter 7b:225). Those individuals who joined the urban underclass were even further removed from this possibility. If hunter-gatherer orientations need to be continually re-created through experience (Chapter 2:40), severe disruption in hunting practices cannot but have had considerable impacts on the meaningfulness of their transmission.

Although circumstances militated against continuation of hunter-gatherer subsistence practices and attitudes, this does not entail a complete discontinuity. For individuals residing on large Northern Cape farms, hunting and (more usually) gathering continued to play a supplementary subsistence role: one of Bleek's (1936b) informants recalled subsisting on 'ant's eggs' (termites), wild tubers, tortoises, springhare, porcupines (Hystrix africaeaustralis) and springbok, even when in service, and combined working for settlers with gathering, using 
their own tools. Although complete subsistence independence using these resources was no longer practicable, and cultural traditions and knowledge were increasingly shared between people from different cultural backgrounds, such practices went some way to preserving knowledge: contributions of Khoekhoe and Bushman lore to South African vernacular medicines based on wild plants are well documented (e.g. Musselman, 2003; van Wyk, 2008).

The terminal decades of the nineteenth century continued established trends in IXam Bushman communities, but ultimately led to a dramatically altered situation. The violent frontier relationships initiated in the eighteenth century continued into the nineteenth, and hunter-gatherer groups began to lose the war of attrition. Increasing incorporation into international markets (the result of trends established with British occupation), and a transition from a heterodox, relatively lawless Karoo, with many different groups competing for access to resources, to a Karoo that was fully integrated into the colonial state, promoted an increasingly hegemonic colonial government presence that began to influence the behaviour of both colonists and IXam. From the 1850s onwards, ostrich feather and wool trades created a potential for profits that rendered the area more attractive to settlers, who nonetheless struggled with the lack of open water sources, until borehole technologies became available in the late 1860s-1870s. For colonial government, neither of these goods was as significant as the fact that the northern districts represented a route through which the mineral wealth of the interior flowed toward international markets; resources invested to incorporate mining towns into the colonial state were crucial in allowing farming settlement to become dominant 
in the region. The establishment of a police force, magistracy, railways, roads, and the other paraphernalia of colonial bureaucracy cemented colonial presence in a new and enduring fashion. Government resources effectively imposed a colonial 'landscape' over the Karoo, one that no longer included the necessary space for the pursuit of hunter-gatherer or nomadic pastoral lifeways, now defined as illegitimate and illegal uses of land.

This increased level of interest in the interior shaped considerably a new relationship between Bushmen and the colony: hunting and gathering practices, surviving over a century of contact with colonial populations and a much longer prehistoric contact with pastoralists, finally ceased. As Bushman populations were considered as quintessential hunter-gatherers, the end of these practices has traditionally been seen as the end of the Bushmen. Though changes in practice are significant (indeed, the central theme of this thesis), it is important to emphasise that equating 'Bushman' and 'hunter-gatherer' is a theoretical imposition; IXamspeaking populations, though diminished, did not vanish when they stopped hunting and gathering. In dealing with a situation that differed markedly from those previously experienced, the IXam adopted new strategies with varying degrees of success. Bushman strategies of violent resistance to land alienation manifested with considerable variation, from leading multi-ethnic, horse-riding raiding groups as postulated for the south-eastern mountain ranges of southern Africa (Challis, 2009) to large-scale aggregations in the early nineteenth century Karoo. In the later nineteenth century, IXam participated in raiding activities by attaching themselves (or being attached) to powerful Korana chieftaincies along 
the middle Orange River. IXam individuals also engaged in small-scale raiding, taking advantage of periods of chaotic irruption that masked their often opportunistic forays (or assigned them to other raiding parties).

Ultimately, Korana and Bushman successes in driving back settler populations did not allow IXam groups to claim exclusive rights to their homelands, and they made (or were compelled to make) accommodations with the political realities of the closing decades of the long nineteenth century. Moving to urban centres to access wage labour and government rations, settling on farms to provide labour, or becoming peripatetic sheep-shears, such accommodations were highly individualised and flexible, traits that Guenther (1999) characterises as part of hunter-gatherer ideologies. Over time, the reproduction of hunter-gatherer subsistence practices and ideologies became increasingly irrelevant or impractical in the face of increasing colonial intrusion into their territory. As a result, the IXam entered the rural underclass of the Northern Cape, a creolised group (with considerable variation across the vast areas of this province) incorporating Khoekhoe, Bushman, Tswana, Xhosa and European elements, in terms of people, language, and culture. Though by the end of the long nineteenth century, the IXam language was all but extinct and their hunting and gathering practices curtailed, through their contribution to this emergent group they continued to contribute to the dynamics of Northern Cape history. 


\title{
Chapter 5: Historical Ethnography
}

\author{
of the IXam
}

\section{Chapter 5a: Odours, winds, and the construction of}

\section{IXam personhood}

The following discussion centres on the extent to which Bushman participation in hunting and gathering practices was connected with a distinctively 'huntergatherer' identity (Chapter 2:36). Nineteenth century colonial populations applied wide classificatory labels to the peoples with whom they interacted (Appendix C:387), structuring their responses to complex scenarios by reducing local and contingent variability. As historical sources are predicated upon this categorisation process, disaggregation and re-evaluation of colonial constructs is a challenging prospect; the Bleek-Lloyd archive's major strength is its verbatim record of IXam narrative, allowing for reconstruction founded on principles 
articulated in their own words. Focusing initially on IXam understandings of the fundamental attributes of personhood, I then consider the impact of these understandings in interactions between the various identities encountered in nineteenth century contact experiences. IXam terminology is employed where appropriate, and the reader is directed to the accompanying glossary (Appendix D:391) for definitions of key concepts.

Direct application of the animic ontology model (Chapter 2:37) is not appropriate for southern African IXam Bushman groups; the narratives contain little evidence for notions corresponding to the 'cycling' of vital forces ${ }^{47}$ between humans and non-humans, and their ritual specialists consequently did not focus on regulating this cycle. However, the model was never intended to be applied in a straightforward, universalised manner to hunter-gatherer societies worldwide; Ingold (2000:112-113) describes both differences between hunter-gatherer populations, as well as similarities between hunter-gatherers and pastoralists (Ingold, 2000:69). I use it as a starting point for thinking through the narratives, suggesting that it has value for considering IXam conceptions of the nature of personhood (particularly relationships between persons). Bleek and Lloyd were not attempting to explore this heuristic construct, and IXam ideas regarding 'personhood' are therefore only revealed piecemeal through narratives that recount the interaction of historical, legendary, and mythological persons (Chapter 1:17). The Bleek-Lloyd informants came from different IXam groups (Chapter

\footnotetext{
${ }^{47}$ There is little evidence for any explicit, emic terminology reflecting this concept. This may partly reflect the difficulties Bleek and Lloyd had with developing translations for 'spiritual' dimensions of IXam life (see glossary, !gi:xa:414); there certainly are similarities between 'animic' systems and IXam ideologies (cf. Dowson, 2007, 2009; Chapter 9:336).
} 
7a:213) and had substantially different personal experiences of the northern frontier (Bank, 2006), but the archive presents an underlying 'cultural logic', informing both the formalised narratives themselves (Hewitt, 2008 [1986]) and a more nebulous collection of attitudes and beliefs, that transcends these differences. Later narratives (from different informants) often reiterate earlier concepts and Lloyd elicited direct commentary on earlier narratives from later informants (e.g. LL.II.26.2374'); we can thus speak of overarching '|Xam' notions despite variation in informants' preoccupations and knowledge. The IXam valorised several attributes in the construction of personal identity, ranging from concepts structuring generic 'personhood' to traits imbued with specific moral dimensions. I firstly examine more general structures before moving on to consider more specific traits, which must be placed in context (especially, their association with particular agents) to develop definitions of morally appropriate behaviour, necessary for understanding IXam evaluation of non-|Xam populations. Obviously, this discussion is far from exhaustive and many complex dimensions have, for reasons of brevity, to be elided; I concentrate on aspects reiterated in numerous contexts (personal histories, and more stereotyped narratives), themes with resonance for 'IXam society' as a whole.

In terms of general structure, the salient notion was one of 'odour', an attribute that remains significant for many Khoisan populations today (Low, 2004; 2007; 2007a). Scent and smelling were integral to many different kinds of IXam 'personhood', constructing social roles in human society and embodying the specific traits or abilities of non-humans. The flesh of animals manifested 
particular smells: caracal meat was smelt 'strongly' (LL.VIII.12.7103-7105), while the ostrich and the Kori bustard (Afr. gompou, Ardeotis kori) ${ }^{48}$ had a pleasant scent (LL.II.3.461). The characteristic odours of game animals reflected the smell of their 'stomachs' (LL.II.11.1127'), which signalled their dietary habits: smells of their preferred food plants were perceptible in their rumen, with gemsbok (Oryx gazella) scent linked explicitly to grass in their stomachs (LL.II.11.1134'), and the springbok scent (LL.II.3.466) to the bushes they ate (both scents were twai:ĩ, Glossary:400, or sweet, comfortable, nice, pleasant, Bleek, 1956:243). By way of contrast, the quagga and the baboon smelled like people (LL.V.23.5881') because their (digestive) 'organs' resembled those of humans. As omnivorous primates, baboon and human digestive tracts share many traits (K. Milton, 1987:103), and while equid digestion (hindgut fermentation) necessitates differences in the size and complexity of their large intestines (Stevens and Hume, 1995:89) when compared to primate systems, in contrast with bovid ruminants (foregut fermentation) quagga might be considered as broadly similar to primates (Stevens and Hume, 1995:93). Perceptions of animal identities were therefore based (partly) on opportunities for close observation of the dietary habits, scat, and anatomy of the fauna that shared their environment, feeding back into the wider cultural context: the 'human-ness' of baboons and quagga demonstrated their continuing connection with the !xwe-\|na-s'o-!k'e (Glossary:440, Early-Race).

${ }^{48}$ See Appendix E (454) for species lists. 
Protagonists in Early-Race stories consist primarily of animals, but also celestial bodies, meteorological phenomena, and a series of Rabelaisian 'grotesques' including \|khwai-hem (Glossary:448), !gwe-!kweitən-tu (Glossary:419), and !k"wakka-tukən (Glossary:435). Early-Race characters often displayed their identities in their scents: the young man who raised a lion cub as a dog let the cub smell his sweat, so that it would 'know' him (LL.II.29.2609), and the Lizard's daughter (LL.VIII.30.8671-8702; LL.VIII.31.8703-8736) identified a visitor as the striped mouse (Rhabdomys pumlico), rather than the round-eared elephantshrew (Macroscelides proboscideus), through his smell. The Early-Race narrative (LL.II.15.1432-1499, LL.II.16.1500-1548; LL.VIII.27.8393-8432; Hewitt， 2008 [1986]:71-82) of the Caracal (|ku-g-!nwin, Glossary:409) and the Day's Heart Star ('gaue-li:-ta-|kwakttən, Glossary:414) illustrates with clarity the link between smell and identity, with most of the major personae involved manifesting distinctive odours. The Caracal's (LL.II.15.1443) 'sweet' odour rendered her attractive, while the hyena (!gwaĩ, Glossary:418, Hyena brunnea) and jackal (koro, Glossary:396, Canis mesomelas) had a 'different' odour that smelt 'badly' (LL.II.15.1442) $)^{49}$ and constructed their shared, antagonist role in the narrative.

This concept of personal scent resonated in daily life, as human scent became salient in their interactions with animal species (gemsbok, LL.II.9.930-931; porcupines, LL.V.7.4449-4450; jackals; LL.II.21.1897) that could readily perceive it; other non-human entities, such as !khwa: (Glossary:424, 'water/rain') when he discovered a 'cursed' person (LL.V.13.4987-4988) or identified people

${ }^{49}$ Difference was a negative attribute for the IXam (Chapter 8b:297; Chapter 6a:182). 
that he intended to 'stealthily kill' (LL.V.22.5804-5807), also demonstrated this ability. As with animal scents, much of this was based in experience, encoding serious practical considerations for animal encounters. The movement of scent on the wind was important when hunting (LL.II.21.1899); 'walking about' careless of one's smell would make the quarry wary, ruining the chance for successful ambushes (LL.VIII.16.7407-7408). Concrete demonstrations of the significance of scent in hunting and other interactions with wild fauna provided an experiential basis that underscored a wider employment of these traits: after hunting smell remained an important concern, for the hunter's scent (if he behaved inappropriately, Chapter 5c:162) had potentially negative consequences for the meat of the animal (LL.V.18.5358-5359). Continuing hunting practices, illustrating faunal olfactory abilities, demonstrated the truth of such beliefs and underpinned their continuing relevance.

Specific IXam ritual identities were connected with distinctive odours. For the !kwi-la-\|ka:n (Glossary:433; New Maiden; cf. Hewitt, 2008 [1986]:205-211), a 'provocative' scent was paramount in cementing the relationship with !khwa: that was the foundation of the role. Her odour attracted !khwa:'s attention or anger both in Early-Race narratives (LL.V.2.3869) and contemporary practice, where much focus was placed on palliating the negative effects of her scent (LL.V.6.4393'-4394'). Jo-|õä-ka-!kwi (Glossary:399), ritual specialists in the use of 'medicinal' plants (de Prada-Samper, 2007), also displayed characteristic odours. Medicinal plants worked primarily through this medium: the !gauë-taOho (lit. morning's plant) was smelt when ill in the morning (LL.IV.1.3434- 
3435), and the effective component of buchu (LL.V.3.4120'; sã:, Glossary:398, Bleek, 1956:162) was its placating scent. By incorporating plant scents, the personal odours of $\int o-\mid \tilde{o} \ddot{a}-k a-! k w i$ embodied their special relationships with the plants they used. The process of becoming proficient with these medicines was one of being 'introduced' to the plants under the guidance of an established expert, amalgamating the potentially-dangerous plant scent with the neophyte's own scent. Intimate identification of $\int o$-|õ $\ddot{a}-k a-! k w i$ with dangerous $\int o$-|ö $\ddot{a}$ scent meant they had to take care to 'inoculate' their close relatives; if they did not, these relatives were in danger just as if they had come into contact with the $\int o-\mid \tilde{o} \ddot{a}$ plant itself (LL.II.36.3281), a compelling illustration of the extension of the plant's identity/smell. !gi:tən (Glossary:415, sing. !gi:xa), premier ritual specialists of the IXam world, had scents that manifested their 'coldness' (LL.V.22.5763-5766), a peculiarity serving as a categorical ${ }^{50}$ marker of their difference vis-à-vis ordinary people; they were, for example, supposed to continue wearing their karosses (skin cloaks, with hair left on the skin) while everyone else was hot (LL.V.22.5766).

!gi:tan scent was not confined to their persons: their blood was in and of itself 'cold' (LL.V.22.5765), retaining something of the essential identity of the !gi:xa. It was believed to continue acting on their behalf even when separated from them, combating the antagonistic 'magic power' (!gi:, Glossary:414; |ko: $\xi_{\mathbf{o}} \xi_{\text {-de, }}$ Glossary:408; or \|ken, Glossary:445) that caused sickness (LL.V.19.5529). It was one of the !gi:tən's 'possessions' (i.e. things with which they 'worked sorcery')

50 !gi:tən also possessed individual odours, through which other !gi:tən could identify them (LL.V.19.5509). 
and acted 'as if alive' because it partook of its possessor's odour (LL.V.19.55005501). This living magic power manifesting !gi:tən-scent could even continue to act after their death (LL.V.22.5735'), at which time it constituted an entity that could be beseeched and interacted with in its own right (LL.V.11.4804'-4806'). This relationship parallels one present in numerous Early-Race narratives between Ikaggən (Glossary:406, Mantis) and his 'hartebeest children' (quiver, shoes, arrows, kaross, \&c.), which were imbued with marvellous abilities of speech and flight through their involvement in his identity (LL.II.6.674-675). These dimensions of |kaggən's and !gi:tən scent introduce several concepts crucial for understanding the wider implications of the significance of 'odour' to general ontological constructions: odours were mobile (even motile), not confined to a single embodiment, and transferable. In particular, we shall see below that they were linked to bodily secretions, readily incorporated in other identities, and moved around on winds.

The potential for 'identities' to blend and mix was often a problematic complication, in IXam conceptions. In stalking and shooting an animal, hunters necessarily became increasingly entwined with its identity, closing an ontological 'gap' that was potentially detrimental to hunting success, mirroring concerns seen amongst many hunting-oriented populations elsewhere (Willerslev, 2004:364). This state of shared identity underpinned many of the !nanna-se (Glossary:437) behavioural prescripts fundamental to successful hunting performance, most comprehensively illustrated in eland (Taurotragus oryx) 'hunting' narratives (LL.V.6.4411-4434), which focus almost exclusively on these regulations 
(Chapter 7b:230). Some rules revolved around the establishment of 'correct' relations with the game (Chapter 6b:192), but others stemmed from basic connections between the hunters and hunted: urination rules for the hunter, for example, were supposed to prevent the animal similarly voiding the poison. Smelling the scent of cooking food (incorporating it within one's scent) was presumably not normally an issue, but when sharing identities with the wounded eland it became a problem: if the eland also incorporated this food smell, it could escape the effects of the poison (LL.V.6.4421-4422). After shooting gemsbok, hunters were forbidden from picking up springbok meat or smelling the scent of their viscera (LL.V.17.5312-5316); transgression of this prohibition would allow the gemsbok to escape, by incorporating (via the hunter) something of the identity of the springbok that were 'wont to walk about' (LL.V.17.5305) at night. Valorising identity 'contaminations' while linked with game animals, !nanna-se rules emphasise general notions about the transferability and entanglement of identities.

The blending of identity could also be an active component in causing illness and death. I noted earlier that the potency of $\int o$-|õ $\ddot{a}$ entailed negative consequences for people establishing an 'incorrect' relationship with its scent; if the smell got into meat, it would cause peoples' stomachs to swell and kill them (LL.II.36.3274'), if women inhaled the smell of the plant, it would make their hearts 'lean' (LL.II.36.3284-3286), and the problems encountered in digging the plant without expert guidance resulted from incorrect scent incorporation (LL.II.36.3309, 3328). This detrimental incorporation of $\int o-\mid \tilde{o} \ddot{a}$ scent formed a component of broader 
notions of foreign identities having negative consequences for people who amalgamated them with their own 'scents'. A common vector for the transfer of these identities was bodily fluids; the dogs became afraid of Ttai-tchuen after her close encounter with a lioness, because its 'milking' on her had combined their scents (LL.V.12.4969). In the Caracal and Day's-Heart story, the Caracal transformed itself into an anti-social 'beast-of-prey' (\|ke\|ke, Glossary:443) when she had incorporated the smell of the Hyena's sweat, severing her from the relationships and obligations that constituted her former social identity

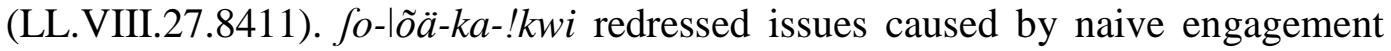
with the plant by using their bodily fluids to transfer their 'correct' incorporation of its scent to the victim, spitting in their eyes (LL.II.36.3305) or rubbing them with their perspiration (LL.II.36.3264').

Notions of negative incorporation were distilled in Igwaiz̧n (Glossary:403, getting into flesh, taking possession, Bleek, 1956:285), a form of 'magical' action which invariably connoted illness and death. Addressing it represented the primary function of the curative !gi:tan, who rubbed their blood (transferring their identities) on people to prevent their incorporation of negative agents (LL.V.3.4135) from a range of 'foreign' forces. Antagonistic !gi:tən might Igwaiz̧n someone with their 'things' (imbued with their scent) to cause sickness or death (LL.V.19.5500-5501), springbok would Igwaişn people who did not comport themselves correctly towards the animals (causing illness, LL.VIII.14.7264'), and when !khwa: was angry with people he would send objects (!k"abbe, Glossary:434) to Igwaiz̧n and kill them (LL.V.20.5609-5611). People 


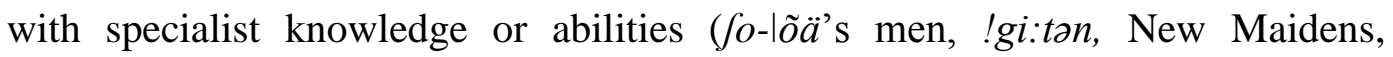
hunters) were able to moderate the detrimental effects of overlapping identities or channelled them in socially-beneficial ways, a quality of knowing how to regulate interactions to which I return below when discussing competence (\|kwakka, Glossary:448, 'understanding', Bleek, 1956:596) in IXam constructions of social responsibility.

Beyond the direct application of materials embodying them, smells and identities could travel through more nebulous mechanisms, such as the creation and movement of smoke to effect changes on physically distant phenomena and agencies: the unpleasant smell of burning springbok horns, for example, caused

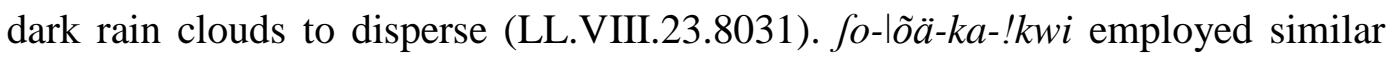
tactics when they burnt plants in restorative practices (LL.II.36.3305) or when activating medicine that made springbok run 'gently' towards hunters (LL.II.36.3252), as did New Maidens when burning buchu to 'fumigate' their relatives in protections against !khwa: (LL.V.6.4393'). A more significant longdistance mechanism, however, was movement on winds. In hunting contexts, humans and animals interacted primarily through the medium of winds (Low, 2007a:S75), which had to be incorporated in successful hunting strategies. Although humans rely only peripherally on olfaction, for many mammals it is a major channel for inter- and intra-specific communication. Their consequent perceptual acuity empowered them with privileged access to the information contained in scents, a powerful trait emphasising the relatively poor sense of smell of humans: the olfactory superiority of non-human species endowed them with 
supra-human abilities. Wind carried sensory information to game (LL.II.9.930; LL.V.4454) and mediated interactions between predator and prey (LL.II.21.1903), demonstrating their ability to 'understand the wind' (LL.II.11.1110). This concept of 'understanding wind' was widely employed in IXam characterisations of animal abilities, underlying (to give one illustration) their belief that certain game animals had privileged knowledge about events passing at a distance (LL.V.9.4652-4673; Wessels, 2007:319).

The relationship between wind and smell directs attention toward a 'meteorological complex' (Guenther, 1999:79; Low, 2007a:S80) in IXam thoughts on personal identity. The wind and the 'son of the wind' were themselves EarlyRace characters, present only in a single narrative (LL.VIII.8.6687). This 'son of the wind' was supposed to have turned into a bird at the end of the Early-Race times (LL.VIII.8.6694'), and one short account (LL.VIII.8.6709-6724) described his interactions with contemporaneous |Xam individuals. His actions in this interaction strongly recall those of antagonistic !gi:tən ${ }^{51}$; on being treated in a disrespectful manner, he 'blew up dust' and forced ॥go-ka-!kwi ("lkabbo's son) to take shelter. As dust was a mechanism whereby ill-intentioned individuals could cause sickness (LL.V.20.5537-5546), the parallels between this transformed Early-Race figure and anti-social !gi:tan (Chapter 6c:209) become striking.

Winds in contemporary experience were differentiated through their connections with other meteorological phenomena; the south wind was cold (LL.II.33.3034), the north wind linked to rain (J. Deacon, 1988:134) and the west wind turned back

${ }^{51}$ Like antagonistic non-living !gi:tan (LL.VIII.14.7275-7276), he lived in a hole in the mountain. 
clouds (LL.VIII.1.6097). Winds and clouds were essentially contiguous in observations of precipitation, as the wind blew 'strengthening' ('hardening') the rain's 'hair' until it became (rain) clouds (LL.II.25.2246-2247). Together, they constituted another dimension of IXam personhood: individuals 'possessed' winds (LL.V.15.5141; LL.VIII.8.6727; LL.VIII.18.7566; LL.VIII.18.7590-7591), and 'made clouds' when they died (LL.V.15.5147). As with personal smells, not only humans, but also predators (LL.V.23.5854-5856) and game animals (LL.VIII.28.8459') were possessed of winds. It is implied that this 'possession' of winds represented particular connections with one of the types of wind differentiated by the IXam (LL.VIII.8.6727), rather than a personal, unique attribute. This connection was particularly noticeable at time of death, for both animals $^{52}$ and humans, when the wind they had possessed blew, taking away their footprints (LL.V.15.5147). This belief should be read in light of IXam notions of ownership, which incorporated the ability to influence or control certain forces (e.g. animals 'possessed' by !gi:tən; Lewis-Williams and Pearce, 2004:190); manifestations of winds at death reflected dissipation of this influence, and the 'making of clouds' said to accompany it, a natural consequence of the 'hardening' associated with winds blowing.

Some winds belonged to supernatural agents, such as the serre: wind that enacted !khwa:'s anger (LL.VIII.16.7419), or the !ho whirlwind that brought illness or knocked people down if they transgressed certain behaviour rules (LL.V.20.5559'5568'). These winds manifested the desires of the agents with whom they were

52 If killed by a person, their cold winds would make the hunter's (own) wind cold (LL.VIII.28.8459'), demonstrating again the contiguities of identity established while hunting. 
associated, valuable tools for their enactment of the roles they were supposed to fulfil; lions, for example, used their winds to facilitate their hunting (of people), making their winds blow to hide their approach the huts from dogs (LL.V.23.5854-5856). As winds were necessary for game animals accessing olfactory information, they also could be employed usefully in human hunting tactics: Dia!kwain's father engaged in this practice (LL.V.15.5141-5142), and lhanłkass'o noted that the north wind could be useful deployed when hunting springbok (LL.VIII.8.6725), making the springboks' superior perception work against them by inducing them to run into an ambush (LL.VIII.8.6730-6731). The connection between winds and clouds meant that !khwa:-ka-!gi:tən (Glossary:425, specialising in rain-making) had the greatest opportunity and motivation to demonstrate their influence over meteorological phenomena (controlling the winds that made rain clouds approach LL.V.14.5076'-5077'), but others too used such influence to claim a special status: |xarran|xarran was entreated to employ her wind to blow away mosquitoes (LL.V.23.5842'-5844'), and also engaged her wind control to blow dust about, demonstrating her displeasure at being scolded $^{53}$. Winds might then equally be influenced by responsible human persons in achieving socially significant ends, or by anti-social forces working antagonistically toward human communities.

These dimensions of the IXam person locate 'identity' as a mutable and shifting property not solely within a single individual: though not identical, this is markedly similar to 'identities' outlined in the animic ontology model. Labile

${ }^{53}$ Though not described as a ! $g i: x a$, her supposed influence over winds after death (LL.V.23.5864$5865)$ is reminiscent of their 'sorcery's things'. 
aspects of identity point to fundamental equivalences between persons, with humans and non-humans 'infecting' one another equally with their identities. The ordering of relationships at the end of the Early-Race times (Chapter 5b:148) established rules for consumption and marriage (thereby forcing Early-Race characters into their animal forms), but attributes of personhood remained substantially similar for both animals and humans. The Early-Race times were less resolved than characterisations of Early-Race narratives as 'creation' stories would suggest, with certain categories of animals emerging as similar actors in both Early-Race and more anecdotal narratives. Baboons retained fluid identities, and were confusing animals that blended human and non-human identities: contemporary baboons were supposed to speak IXam (LL.V.24.5923') and exhibit violent desires (LL.V.24.5930-5947) that paralleled their actions in Early-Race stories (LL.VIII.11.6978-7014; LL.VIII.12.7065-7094). The ambiguous status of some Early-Race people was sufficiently entrenched in IXam thought as to offer viable explanations of unusual events, as in the memorate of $\| \mathrm{go}-\mathrm{ka}$-!kwi 'seeing the wind' in the form of a bird at łkoaxu (Haasfontein, Figure 5.1:147). Ongoing engagements with non-human agents were important in IXam life; !khwa: played an identical role in Bushman and Early-Race communities, and |kaggən remained a potent force in hunting beliefs. While different perspectives and aims ensured 'persons' acted very differently from one another, these actions often paralleled one another; lions would utilise similar strategies to those deployed by human hunters, for example. After death, differentiations of the second creation became blurred, with humans and game believed to 'walk one path' (LL.II.6.669'), and at 
this time even ordinary Bushmen transitioned into non-human communities by entering into largely antagonistic relationships with their living counterparts. Between responsible adults displaying 'proper' behaviour and the anti-social activities of the dead and of predators (Chapter 6c:204), a series of liminal individuals engaged in what IXam considered species-inappropriate behaviour or demonstrated proficiencies in non-ordinary skills. The examination of the dimensions of this propriety versus anti-sociality forms the focus of chapters $6 \mathrm{~b}$ (182) and 6c (201).

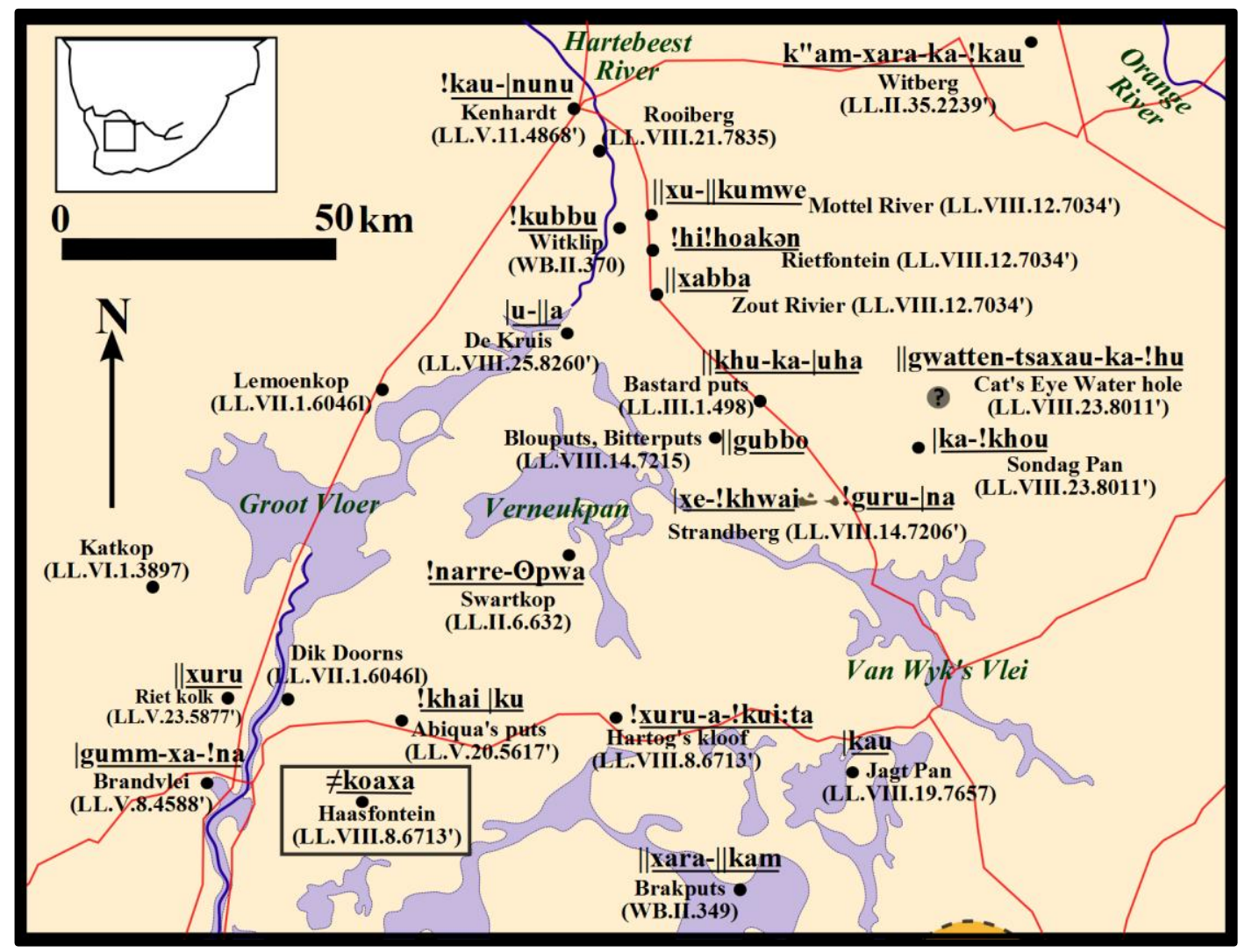

Figure 5.1: Haasfontein (łkoaxa) in the Kenhardt-Brandvlei-Vanwyksvlei triangle 


\section{Chapter 5b: Incorporating identity}

Rules regarding consumption were integral to the construction of identity: as the concept of transferral of identities through incorporation of foreign substances had widespread currency in IXam belief, this comes as no surprise. Food proscriptions revolved around two basic axioms: avoiding the transferral of 'dangerous' identities to others, and avoiding the incorporation of similarly dangerous identities oneself. Their delineation constituted part of the 'Anteater's [aardvark] Laws', an episode of secondary creation establishing proper relationships between species (Hewitt, 2008 [1986]:92-95), and helped define identities both within and between Bushman groups; distributed along age and gender lines, they embodied major axes of social differentiation. Although institutionalised social hierarchies are notably lacking among 'egalitarian' northern Bushman groups (and, by extension, are assumed absent for southern Bushman populations), this clearly does not entail monolithic societies. Numerous authors (L. Marshall, 1961; Lee, 1969; Draper, 1978) have emphasised this egalitarianism in contemporary Bushman societies as requiring strongly coercive social mechanisms working (ideally) to prevent the formation of persistent hierarchies through complex distribution rules and systematic 'belittling' of performances that acquire desirable resources. The presence of such mechanisms points to the presence of 
'fracture' lines in northern Bushman societies, and to axes along which tensions can potentially develop; for the IXam, food proscriptions marked differentiation between people, and constructed roles subject to normative social mechanisms.

Some consumption was incompatible with human identity in an ontological sense. The comestible species list dictated to humans as part of the aetiological component of the 'Anteater's laws' was much broader than for other animals (at least thirty-four species, LL.II.3.454-457), and the rules for humans emphasised rather the manner in which these species should be eaten, cooked instead of raw (LL.II.19.1705). Certain prohibitions did apply to humans in general. Cannibalism was forbidden: hare (Lepus sp.) thigh-meat (LL.VIII.27.8398') was rejected because it was considered a piece of human flesh incompletely transformed by the Anteater's Laws. Wild dogs were excluded, resembling domesticated dogs too closely (LL.II.3.460; Hewitt, 2008 [1986]:95): this may represent an extension of the cannibalism prohibition, with domesticated dogs participating ambiguously in their owner's identities (Chapter 8a:283). This 'ontological' consumption reflected continuing affiliation with a 'human' in-group, and ambiguous agents who had opportunities to shift allegiances might reflect this by altering their dietary preferences: antagonistic !gi:tan were supposed to consume similarly to lions (i.e. to eat people, LL.VIII.15.7304).

The IXam avoided pigs, fish, shellfish and fowl (LL.VIII.30.8649; LL.IV.3.3736'), not under the rubric of the Anteater's Laws but as part of the production of a Bushman identity in contrast to 'others'. łkasin juxtaposed his (Korana) father's willingness to eat fish with his (|Xam) mother's avoidance of the same, while 
\|kabbo used dietary habits in discriminating between !kaukən-ka-!k'e (Glossary:422, Mountain Bushman) who ate baboons, and Swa:-ka-!k'e (Glossary:399, Flat Bushman), who did not (LL.II.7.798; Chapter 7a:219). This avoidance was not attributed to a fundamental ontological 'law', being instead presented as the result of baboon habitat preferences; it may thus be considered analogous to Korana (living along the Middle Orange River) consumption of aquatic resources. Not all dietary habits need be considered as reflecting deepseated ontological constructions; adult avoidance of smaller birds and animals (LL.VIII.30.8649-8650) was probably more a reflection of the relatively low return obtained from these food sources, than a categorical statement about adult and child identity.

In general, IXam food proscriptions were framed as avoiding potential dangers (similar to soxa among the Haillom, Widlok, 1999:213-221), including foods inducing unwanted effects (including poisonous species) and those that attracted the destructive attentions of supra-human agencies. This variation existed in a single overlapping spectrum, with giant centipedes (Scolopendra sp.) avoided both because of their poison and because they belonged to !khwa: (LL.VIII.1.6074-6076). In the former category, we find toxic plants that could not be safely consumed by humans (LL.II.7.783'; LL.VIII.8.6687'). These were avoided by everyone, though a few of them were considered poisonous only for specific people: gambro (Fockea edulis) was supposed to kill children if they ate it unaccompanied by animal products (LL.II.13.1254-1255). Other species were palatable only at specific times of the year: !kerri roots were avoided during the 
summer, because they were not 'sweet' (twai: $\tilde{\text {, }}$ LL.VIII.18.7599-7600). 'Sweetness' was used to delineate other undesirable foodstuffs (such as lion's flesh, WB.I.263), making assessments of relative attractiveness, and classifying non-a:kən (Glossary:392; Chapter 5c:162, 165) foods; briefly, this included bitterness, dryness, and associations with predatory species possessed of traits that one hardly wanted to incorporate though consumption.

Among the Flat Bushmen, women did not eat caracal (LL.VIII.12.7105'), caama fox (Vulpes chama), cheetah (Acinonyx jubata) (LL.II.1.235'), or jackal (WB.I.303). Ihanłkass'o imputed this avoidance to the animals' smell, stating that women 'dislike' it, but that men in general do not (he noted two men who did 'dislike' it). The word used was $\|$ karitan (Bleek, 1956:559), an unusual term (only found in this fragment) that D. Bleek suggests may mean 'to abstain from'. This construction was not a common form; displeasure (LL.II.25.2304) and disinclination (LL.V.4.4211) were usually expressed by combining an appropriate verb with a $k^{\prime \prime} a u k i$ negation (Bleek, 1956:121). Although Lloyd's translation at first evokes a simple (if culturally conditioned) personal preference based on affective qualities, the 'smell' of meat can hardly be considered a neutral, physical trait (Chapter 5a:134). Combined with unusual word choice, this 'dislike' of the women appears to imply something of a desire to avoid incorporating caracalidentity. The specific contrast of male and female habits in lhanłkass'o's presentation of the avoidance suggests that he saw it as a gendered performance, and considering the wider connotations of the 'Caracal' signifier, this division makes much sense: for hunters, the caracal (although a 'beast-of-prey') was $a: k \partial n$ 
(LL.II.3.419; Chapter 6b:183; Chapter 7d:277) because it did not eat immoderately, was not particularly 'anti-social' (did not eat people, LL.II.3.425426), and behaved 'understandingly' (LL.II.35.3240). From a female perspective, the most obvious referent is the transformation of the Early-Race she-Caracal into an anti-social being. In this light, the masculine avoidance (unfortunately lacking in detailed information) may point to the fact that masculinity and femininity themselves were not enacted identically by all people, representing contested domains.

Children were particularly vulnerable to the dangers of consumption, especially the incorporation of inappropriate foods; their food rules attempted to prevent these associations developing. Poorly considered consumption generated undesirable personal characteristics: ostrich gizzards caused disfigurement (LL.V.20.5573') and springbok tongues induced clumsiness (LL.V.25.60266028). Leopard hearts were given preferentially to children, while jackal hearts were denied them (LL.VIII.16.7457-7458), in attempts to avoid them becoming li:-sa ('cowards', Chapter 6a:175). Adult identity was moulded by identities incorporated in childhood, alternatively manifest in the link between incorrect childhood consumption and the attentions of antisocial forces (lions) in later life, forces that attacked these individuals in retribution for earlier transgressions (LL.V.8.4576-4577). Negative developmental effects could also be inculcated by the consumption habits of individuals closely associated with the child: mothers 
with young children avoided hartebeest ${ }^{54}$ meat to prevent Ikaggan from harming the child (LL.V.6.4415'). This regulation of diet held considerable potential for establishing moral dimensions, and negative consequences were stressed throughout; mothers were told they must behave in certain ways ${ }^{55}$, in instructions from older individuals and, while children were supposed to be self-regulating in their diet (becoming 'afraid' of inappropriate foods, LL.V.8.4577), this was largely controlled by adults who were supposed to create and sustain childhood consumption habits.

This hartebeest example falls into the highly-variable category of food avoided because of its links with dangerous non-humans: a range of non-human agents (!khwa:, |kaggən, Inu-ka-!k'e, Glossary:413) guarded different resources, behaved differently depending on the transgressor's identity, and effected different punishments for transgressions. I focus here on the 'rain's things', perhaps the most heavily circumscribed component of the dietary laws. Animals standing in a relationship with !khwa: were subject to consumption rules differentiated by age and gender. They included some birds (swallows, LL.V.21.5698) and arthropods (LL.VIII.1.6074; LL.VIII.17.7463) not eaten by anyone, but mainly involved reptiles and amphibians; frogs and toads (LL.VIII.16.7449) and snakes and tortoises (LL.V.6.4383'-4384') ${ }^{56}$. These associations incorporate ethological resemblances, with leopard tortoises

\footnotetext{
${ }^{54}$ Strongly associated with $\mid$ kaggən, perhaps manifest visibly in the similarity of hartebeest horns to mantis antennae.

${ }^{55}$ Broadly reminiscent of New Maiden proscriptions that curtailed consumption and movement.

56 The connection between waterslang and tortoises in Griqua hokmeisie rituals (Waldman, 2003:665) links snakes, tortoises, and water for contemporary groups descended from Khoekhoe and Bushman populations.
} 
(Stigomochelys pardalis) ${ }^{57}$ and puffadders (Bitis arietans), the quintessential 'rain's animals', producing very similar warning hisses (Boycott and Bourquin, 1988:17). Some of the 'rain's things' were permissible for general consumption (tortoises, LL.V.11.4813), but this remained risky enough to inspire fear, and for the young this risk was much increased. Particularly vulnerable, New Maidens were not even supposed to touch tortoises (LL.V.6.4381'-4382') in case the tortoise 'soiled' (ts'erre, Glossary:400, bewitch, Bleek, 1956:215) them. Even in arid environments, urination and defecation are common chelonian responses to stress (Mann, O'Riain and Hofmeyer, 2006:374); the ability to eject forcibly water stored in the cloacal bursa is one of the few 'active' defensive mechanisms employed by these species (Boycott and Bourquin, 1988:19), a physiological capacity that may have reinforced (in a region with little surface water) a privileged connection between tortoises and !khwa. Tails of porcupines (also 'rain's things') were not eaten by young men or girls lest hail strike and kill them (LL.V.13.5036), a proscription that applied men old enough to hunt porcupines (LL.VIII.16.7405). Only older people ate these tails (LL.VIII.16.7405, 7408'), recalling a restriction on consumption of Ikappəm water tortoises (Psammobates tentorius), eaten only by old women (LL.VIII.26.8303). The connection between !khwa: and fertility that underpinned his interest in youth in general (New Maidens in particular) rendered their consumption of his animals enough to attract

\footnotetext{
${ }^{57}$ A good candidate for the 'water tortoise', being more active during or after rain (Boycott and Bourquin, 1988:88). The only true waterskilpad (Afr. terrapin, lit. 'water tortoise') of the region is the Cape Terrapin (Pelomedusa subrufa), a species name not recorded by Bleek and Lloyd (Appendix E:468).
} 
his wrath (LL.VIII.26.8308) where older individuals could eat with relative impunity.

Consumption was central to the New Maiden role, regulating not only the type of food consumed, but also the manner of its ingestion. She was supposed to consume and imbibe 'moderately' (LL.V.2.3874-3881), given water to drink in a manner comparable to practices with small children (LL.V.2.3874'), and fed small pieces of food. This moderation of intake was structured by specially designed 'restrictive' material culture in the form of a xabbu (ostrich eggshell drinking vessel, LL.VIII.15.7360) with a small aperture and narrow reed straw (LL.V.2.3879'). Other restrictions of the role were facilitated by material culture; the New Maiden's built environment, a small hut (the 'illness's house', LL.VI.2.3997) with an equally small entrance (LL.II.28.2520') occupied for a month following her first menses, was crucial to constructing limitations on her movements. These restrictions were enacted to inculcate similarly moderate habits in all people $\mathrm{e}^{58}$ and to control the potentially disastrous consequences of her unrestrained fertility. Her performance incorporated various human agents, and a series of nonhumans (springbok, LL.II.28.2523; baboons, LL.V.24.5921; dogs, LL.V.20.5596; and of course !khwa:), all of whom had a stake in the role. Her emotions, glances, and 'snapping of fingers' became channels directing !khwa:'s negative attentions (LL.VI.2.4000; LL.V.20.5619), almost against her will. The food she collected became imbued with danger, placed in the same category as foods associated with !khwa: (LL.V.6.4392'); $k h w a$ 's: attraction effected her

58 'Small' consumption was highly valued (Chapter 6b:182). 
transformation into a 'rain's thing', and her productions were as jealously guarded as the tortoises, porcupines, and other resources representing his normal jurisdiction, eaten only by older people. Her kin (especially males) and residence group (LL.V.6.4393'-4397') had to be treated with buchu to render her gathered food safe for their consumption. The rules followed by the New Maiden, then, manifested both the mechanisms by which she attracted the attention of !khwa: (the cultural 'logic' of the role), and the wider social context of this identity, making statements about the contestable definitions of femininity, fertility, and moderation.

Numerous food rules enacted structural impositions with similarly significant social content. Women forbore from eating meat from springbok shoulderblades to !nanna-se their husbands' arrows (LL.VIII.14.7263'), a restriction clearly applied only to a specific category of women. This behaviour was supposed to contribute to the relationship between arrows and springbok, inculcating correct (socially desirable) outcomes for the hunt and loaded with the same suite of exhortations applied to successful hunters (Chapter 6b:191, 192-193), combining implications of propriety and skill. Consumption rules of this 'proper behaviour' type were applied to both men and women. Flat Bushman men were supposed to bring porcupine meat back to camp before eating (LL.VIII.6.6590'-6594'), in a 'gendered' performance: men brought it to camp, women cooked it, men divided it, and, in conclusion, each gender consumed it differently (women eating the flesh, men 'gnawing' the backbone). Ihanłkass'o contrasted this with the behaviour of |khe-\|en (Grass Bushmen, Chapter 7a:221) who 'ate up' porcupines on the veld; 
the negative connotations of unrestrained consumption implied by 'eating up' (Chapter 6b:182) make it clear he considered Flat Bushman behaviour more 'proper'. A similar logic structured consumption rules for ritual roles. !gi:tən were not allowed to consume freely (LL.V.22.5768), and had to maintain certain standards that supported their particular skills. Their abilities were weakened through incorrect consumption (LL.V.3.4151'); if they consumed ashes together with food, their curative 'snoring power' (lit. their 'nose', Bleek, 1956:352) would leave them, and contaminating identities of 'unclean' food incorporated in their healing practices would render them ineffective, even harmful (LL.V.4.4177). The narrative dealing with !kwarra-an's (a !gi:xa) loss of potency presents this 'weakening' as an area of social contestation, in which she attempted to characterise her actions as congruent with dominant notions of how a (sociallyadmirable) !gi:xa should behave (LL.V.3.4132-4161; LL.V.4.4162-4199). In all of these examples, a connection between identity and 'correct' behaviour is valorised; the men giving women porcupine meat, the women restraining themselves from eating certain portions, and !gi:tən regulating their incorporation of (potentially detrimental) substances; all used consumption to demonstrate participation in 'proper' Bushman social norms. 


\section{Chapter 5c: Attributing value}

Before discussing propriety, morality, and their instantiation in social roles, it is important to consider the attributes that the IXam used in evaluating differences between persons. I outline some of the physical traits deployed in the construction of IXam identities, with the familiar caveat that this does not represent the entirety of a complex dimension in IXam life. Starting with an exploration of 'colour', located in visually obvious traits loaded universally with cultural significance ${ }^{59}$, I consider a series of interwoven traits that constructed admirable or condemnable persons. IXam colour terminology is quite extensive, the result of deliberate solicitation by Bleek and Lloyd to further the production of a comprehensive dictionary; some terms collected are encountered extremely rarely, such as 'dead brownish green' (Bleek, 1956:291), appearing only in a wordlist (LL.VIII.7.66016606). I focus on terms used with greatest regularity in the archive, and on the manner in which their connotations were mobilised in judgemental and evaluative processes underlying social roles: Ikai:nja (Glossary:407, yellow, green or ‘shining', Bleek, 1956:297), Iko:乡wa (Glossary:409, red, pale, Bleek, 1956:321), !khi:ja (Glossary:423, red, Bleek, 1956:427, 434), Ihoake (Glossary:405, black or

\footnotetext{
59 Anthropological approaches focusing on colour are extensive; ( $c f$. MacLaury, Paramei and Dedrick, 2007) and long-standing (Conklin, 1955; Turner, 1967; Berlin and Kay, 1969).
} 
dark, Bleek, 1956:289), and !kúi:ta (Glossary:430, white, Bleek, 1956:450), terms that could be combined to describe other colours ${ }^{60}$. One less frequently employed term I shall touch upon is kerru (Glossary:395), to be or become green, or to sprout (as a noun, vegetation, Bleek, 1956:87).

Ikai:nja had strong negative connotations, characteristic of the $\|k e\| k e$, 'beast-ofprey', epitomes of anti-social agency. A prime trait of 'beastly' identities was hairiness: lions (\|khã:, Glossary:446) and brown hyenas (!gwaĩ), the two species the IXam considered as beasts-of-prey par excellence, display notably 'hairy' physiognomies, in the mane of the adult male lion and the voluminous coat of brown hyenas (Figure 5.2:160; Figure 5.3:160; LL.II.20.1793'). Lions, the quintessential predators, were quintessentially hairy, referred to by children with the respect name 'Hair' (LL.V.8.4563-4564). In the Early-Race times, when characters displayed features foreshadowing their future animality, lions had hair which was 'different' (LL.II.1.276), revealing their desire to eat people (LL.II.16.1553). Hair growth and predation were causally linked in hyenas, which began to eat springbok when they 'got hair' (LL.II.3.445), and the division between domesticated predators (dogs) and wild ones was replicated in their hairiness, with dogs feet being considered less hairy than those of lions (LL.II.26.2339). |kai:nja went alongside hairiness: the Early-Race Caracal became both yellow and hairy in her transformation into an anti-social 'beast-of-prey' (LL.II.18. 1654-1657). Reinforcing the connection, lions' faces similarly were described as yellow

60 'Blue' (LL.VIII.7.6601), for example, being defined with a phrase literally translating as “'shining/green-yellow'-and-seems-dark". 


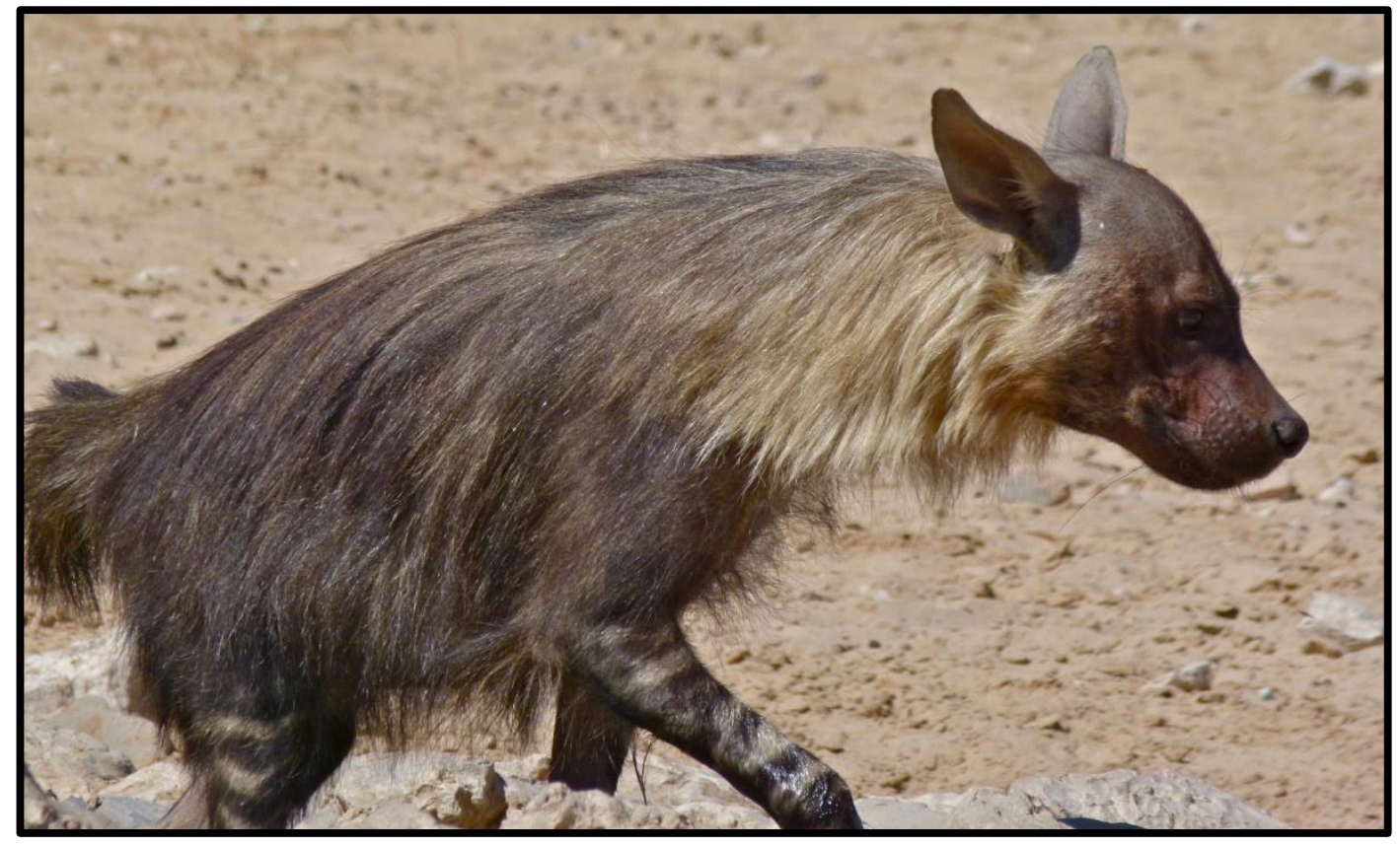

Figure 5.2: Characteristic 'hairy' coat of brown hyena (Hyena brunnea). From 'Brown hyena, Parahyaena brunnea' by Berniedup, 2011, http://www.flickr.com/photos/berniedup/6472939035/sizes/o/in/photostream/ Copyright 2011 by Berniedup (CC BY-NC-SA 2.0).

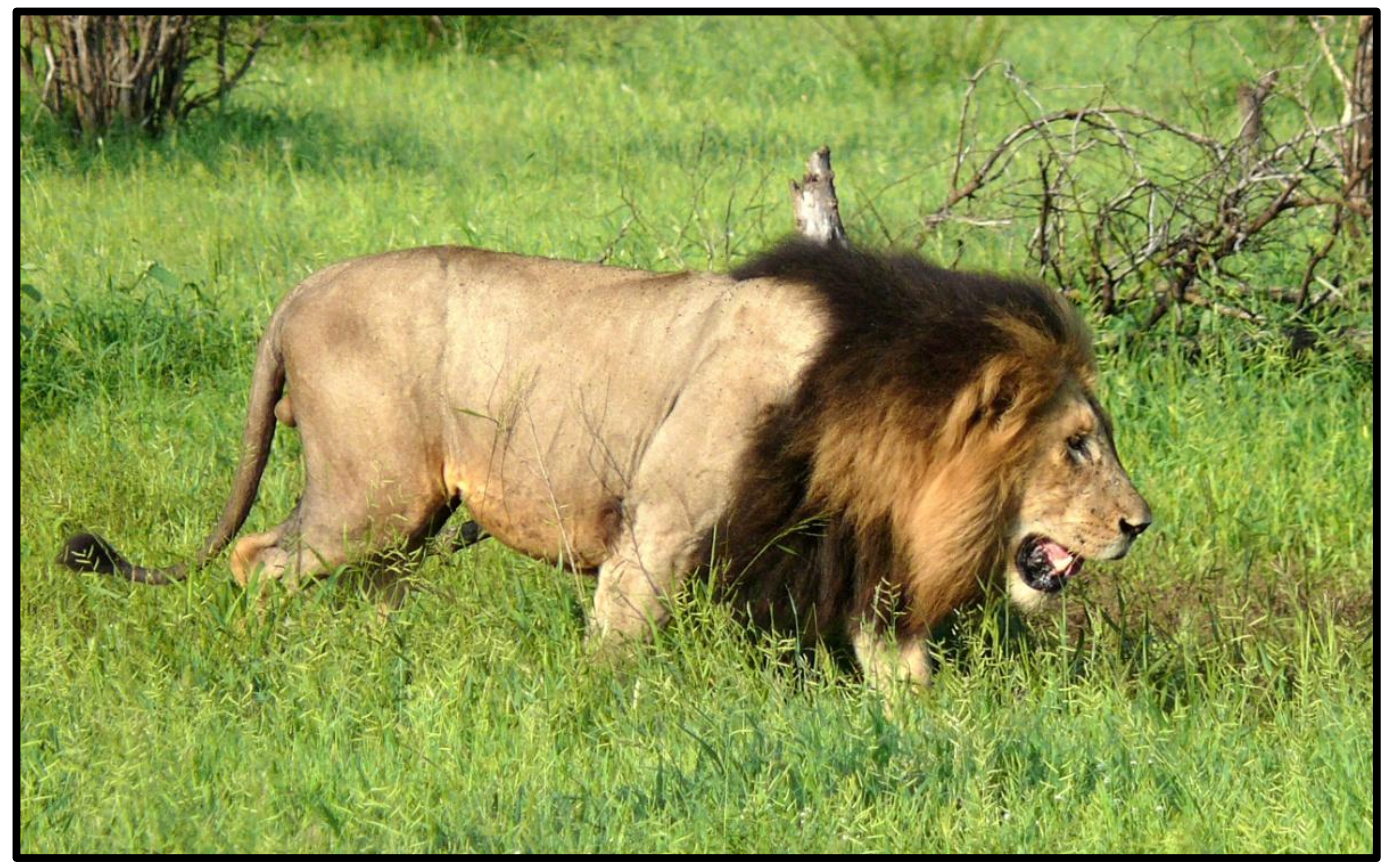

Figure 5.3: African lion (Panthera leo), showing 'hairiness' characteristic of $\|k e\| k e$ status. From 'African lion, Panthera leo' by Berniedup, 2009, http://www.flickr.com/photos/berniedup/6041671298/sizes/o/in/photostream/. Copyright 2009 by Berniedup (CC BY-NC-SA 2.0). 
(LL.II.26.2379), with eyes that 'resembled fire' and shone (LL.II.3.421) ${ }^{61}$, likely making reference to tapeta lucida particularly characteristic of nocturnal predators (Vaughan, Ryan and Czaplewski, 2011:27, 298). Concordant with this, shining yellow eyes 'rolled with foolishness' (WB.XV.1408) or connoted 'angry actions' (WB.XXIII.2204): both foolishness and anger went along with the inappropriate social behaviour expected from predators (Chapter 6a:173).

Connections with predatory forces lent a dangerous aspect to |kai:nja, mobilised in association with a more generic anti-sociality. |kaggən, often antagonistic towards human societies and usually behaving inappropriately even with his peers, was described as yellow (LL.VIII.7.6600'-6601') and, in an oft-quoted passage (Guenther, 2002:67; Lewis-Williams and Pearce, 2004:113), transformed into a kai:nja-@pua', or 'little green thing'. Things belonging to the unpredictable !khwa: were described with the term: puffadders (LL.V.6.4384') were this colour, and the (Early-Race) New Maiden (LL.VIII.16.7434-7448) became |kai:nja when smelling of a certain fungus that belong to !khwa: (i.e., when she had become intimately identified with him). Consistent with its antisociality, |kai:nja manifested the more destructive elements of !khwa:, including lightning strikes (LL.V.22.5722-5723), abduction, and transformation; in the Early-Race story referred to above, the New Maiden's 'green' scent must be removed to prevent !khwa: transforming her family into frogs.

\footnotetext{
${ }^{61}$ Hyenas are also mentioned here.

${ }^{62}$ Often cited as evidence for direct transformation into a mantis, this is not necessarily implied by the IXam phrase: Ikaggən also became |kai:nja after leaving water (WB.XX.1894), suggesting he may have been 'shiny' rather than green/yellow.
} 
Personal association with dangerous identities might induce 'yellowness': incorrect relationships with $\int o$-| $\tilde{o} \ddot{a}$ 's scent (LL.II.36.3285-3286) manifested in yellowed eyes, part of a larger suite of ideas connecting dangerous, unpleasant, or inappropriate conditions. The yellow eyes of women improperly introduced to fo|̃̃ä reflected their 'becoming lean', an undesirable quality for a culture that placed a high value on fat in general (and fat in meat in particular, Chapter 7b:227).

Leanness in meat was commonly conceptualised as |hoake, blackness or darkness: after smelling $\int o-\mid \tilde{o} \ddot{a}$, flesh became black through loss of fat (LL.II.36.3284-3285), and transgressions of !nanna-se behaviours caused the moon to 'swallow the game's fat', making its flesh black (LL.V.16.5225-5226) ${ }^{63}$. Strengthening its undesirability, aetiological dimensions of Early-Race narratives (LL.II.3.417; WB.VIII.894) almost invariably link 'blackness' with being burnt and becoming ugly, largely (though not exclusively) in association with beast-of-prey identities and linked prototypically with the hyena, which (even before being burnt black on its hindquarters) 'blackened' its perspiration to contaminate the Caracal (LL.VIII.27.8397). As with |kai:nja, this underscored connections between ugliness and inappropriate or antisocial action, with burning/blackening representing a common punishment for transgressing norms or antagonising other Early-Race characters. Links between transgressive/antagonistic acts and predators (hyenas, LL.II.20.1835) or other anti-social creatures (baboons, LL.V.24.5986), further characterised blackness as a terrifying attribute: lhanłkass'o spoke of terror making the mouth 'black' (when coming on a lion

\footnotetext{
${ }^{63}$ 'Blackness' here connoting undesirability of consumption: after hunting ostrich, the 'blackened' meat around the poisoned wound had to be thrown away (LL.II.24.2203-2204).
} 
unexpectedly, LL.VIII.23.8079), while the darkness associated with heavy rainfall made people afraid (LL.II.25.2233), a fear motivated by its potential for violence. The 'black' lightning (which killed people, LL.VIII.30.8644') that accompanied such rain embodied !khwa:'s destructive capacity ${ }^{64}$, and lhoake also described the !ku!ku:itən (Glossary:431) or Early-Race Ticks (LL.II.32.2929), 'black people' with a propensity for 'anger's fighting' (WB.XXIII.2171) and 'bloodyhanded[ness]' (LL.II.32.2960'). Leanness and blackness, then, were 'bad' traits, linked with destructive violence and ugliness.

By contrast to the general negativity of non-human 'yellowness', $\mid k o: \xi w a$ (red/pale) held consistently positive associations, used primarily to describe people: rubbing fat onto the skin made a person $\mid k o: \xi w a$ (LL.II.13.1290) and 'handsome'. This 'handsomeness' described physical appearance (LL.II.15.1446) but also competent performance and appropriate management of social relations (Chapter 6a:170), united in the IXam a:kən (Bleek, 1956:6-7) that referred to handsome appearance, the ability to perform tasks 'nicely' ${ }^{65}$, and a general sense of moral rectitude; these connotations were connected explicitly in descriptions of people as $\mid k o: \xi w a$ (LL.II.13.1245). In a physical sense, a:kan combined with $\mid k o: \xi w a$ in a complex of notions concerning fat, anointing, and rubbing. Flesh anointed or rubbed with fat became handsome (LL.II.12.1232), and eating fat, 'handsome' food (LL.II.14.1343) transferred this trait to the consumer. Heads

${ }^{64}$ Black lightning was compared to gunfire (LL.VIII.30.8644'), surely a powerful evocation of violence and murder for populations living in the northern frontier regions of the Cape Colony (Chapter 4b:100; Chapter 4c:115).

${ }^{65}$ Often paired with ttwai, an adjective describing competent (or beguiling) performance (LL.V.6.4345-4356; Bleek, 1956:244). 
anointed with a mixture of specularite (llhara, Glossary:442) and fat (LL.VIII.14.7275') were the apogee of physical handsomeness, creating a state of being sparkling (wawaitən, Bleek, 1956:252) or shimmering (!k"arrakən, Bleek, $1956: 507)^{66}$ that could even overcome the negative connotations of 'blackness': |hoake specularite made hair 'grow downwards' abundantly (LL.VIII.14.7272'7273'), as part of the positively evaluated component of 'youthfulness' (Chapter 5c:165; Chapter 7d:278).

Despite general themes in negative or positive connotations, there was some ambiguity in the use of colour referents: this is best illustrated in notions of 'redness'. Although positive evaluations of $\mid k o: \xi w a$ were applied consistently, !khi:ja ${ }^{67}$ had both positive and negative valence. !khi:ja was, in the 'new' plumage of the female ostrich (LL.II.23.2130) or the 'fat' male ostrich (LL.II.24.2149), a handsome, positive trait displaying youthfulness and resource surfeits, and in some contexts it signified humanity in contrast with 'yellow', predatory species (LL.II.18.1656-1657): descriptions of hare's flesh as red (LL.II.18.1667) here reference its 'human' identity (Chapter 5b:149). However, it could also become emblematic of the very opposite of human sociality in descriptions of beasts-of-prey: the red hair of !kúi-se-!khwi-|ku (Glossary:428, Early-Race lion cub raised as a dog) revealed its improper, bestial status (LL.II.26.2323). When linked to these beasts-of-prey, !khi:ja was an ugly characteristic (e.g. LL.II.3.418), clearly participating in the 'negative' suite

\footnotetext{
${ }^{66}$ In contrast to 'shininess' (|kai:nja)

${ }^{67}$ Most instances of 'red' (in translation) refer to this term.
} 
outlined for |kai:nja and |hoake. Like |kai:nja, it was associated with !khwa: ${ }^{68}$, as the colour of certain animals belonging to him (LL.VIII.16.7464). The more ambiguous redness did not stress his destructive aspect, and was mobilised in practices aimed at diffusing !khwa:'s dangerous attentions: to: (Glossary:400) and $\| \mathbf{k a :} \xi$ (Glossary:442) were ground to produce red pigments (LL.VIII.14.7272'; LL.VI.1.3970) strewn, along with sweet-smelling buchu, by the New Maiden to placate !khwa: (LL.VIII.16.7426). Red $\| k a: \xi$ was also used to paint stripes on the backs of (young) men (LL.VI.1.3970-3973), uniting two traits linked to !khwa: ${ }^{69}$, and in applying this combination New Maidens shielded young men from his antagonism.

'Ugliness' in meat was connected the absence of fat, but also with age, the unappetising meat of old game animals being described as such (LL.V.11.4802); flesh that was rested, well-watered, and not 'exhausted' by heat was handsome (LL.II.13.1255-1256). Issues of age, aridity, and heat were important determinates of the positive and negative associations of !kúi:ta (whiteness), which revolved around connections between whiteness and excessive heat (LL.II.4.485), and between whiteness and age (LL.VIII.22.7981): !kúi:ta-Ina ('white-head') was employed as an epithet for elders (LL.II.4.523). These two elements were themselves connected, as heat was supposed to age things; springbok had 'old white hair' in summer but grew young, red hair in winter (LL.II.25.2262). An opposition in IXam thought between youthful foolishness and adult understanding

\footnotetext{
68 This figure was highly ambiguous, intensely destructive but providing vital rainfall (Hewitt, 2008 [1986]:59).

69 His !khwa:-ka-!kaukən (Glossary:425, 'rain's-children') were said to be striped (LL.VI.1.3942).
} 
(Chapter 6a:168) was incorporated into signification of this colour term: people were supposed to leave off foolish k"e:nk"e:n (Glossary:397, teasing) when their hair became white (LL.VIII.3.6255-6256). Negative dimensions of 'whiteness' centred on links with high temperatures and desiccation; excessive heat was a potentially-dangerous condition that could 'devour' a person (LL.V.20.5538) $)^{70}$, linked with debilitation through heat exhaustion (LL.VIII.22.7961) and the disease-causing actions of anti-social !gi:tan (LL.V.20.5549). High temperatures contributed to the desiccation of vegetation (LL.II.22.2038), and things as they dried became !kúi:ta, whether flesh (LL.II.33.3019'), bushes (LL.VIII.7.6640'), or whole regions (LL.VIII.16.7448'). In contrast to dried objects, new or fresh things were $a$ :kan: new feathers that the ostriches grew after plucking out their 'summer's feathers' (burnt dry by the sun) were handsome (LL.II.24.2137) and springbok hair 'waxed handsome' when it was newly grown (at which time their 'fat' meat became particularly desirable). For both of these species, the coming of 'new rain' instigated processes of renewal, with the whole country becoming a:kan when vegetation was 'sprouting', rather than withered, white, dry, and 'uncomfortable' (LL.VIII.7.6640'-6642'); this plant growth following rainfall was referred to as kerru (applying specifically to the greening of vegetation), a term encapsulating the opposite side of the spectrum to !kúi:ta associations.

Descriptive attributes were not neutral but implied specific moral and ethical evaluations. Concepts introduced above outline a complex of ideas centring on

\footnotetext{
${ }^{70}$ Immoderate consumption was 'monstrous' in IXam thought (Chapter 6b:181).
} 
evaluations of favourable versus unpleasant, undesirable traits: states of being handsome, wet, fat, and fresh on the one hand, and issues of being ugly, dry, lean, and withered on the other. The following chapter examines the employment of these characteristics in the creation of social identities, constructing praiseworthy actions and characters and deployed in critiques of anti-social behaviour. 


\title{
Chapter 6: Social Responsibility,
}

\author{
Authority, and Obligation
}

Chapter 6a: 'Understanding people' and foolish

actions

In discussing the social dimensions of IXam identities, I focus on two key concepts: the characteristics emblematic of authoritative persons, and issues of the entitlements or obligations emerging from participation in certain identities, with particular focus on 'propriety'. Social dimensions of identity are difficult to reconstruct with certainty, simply because they were contested by IXam individuals themselves. Narratives expressing injunctions and proscriptions exhorting people to behave in particular ways, and moral evaluations of this behaviour, may be highly idiosyncratic; the informant was him- $^{71}$ or herself a

${ }^{71}$ The collection was primarily obtained from male informants. 
IXam agent, embedded in the relations they were describing. Some elements of the narrative structure, such as reported speech (Hewitt, 2008 [1986]:198), offer greater opportunities for revealing wider attitudes by presenting specific views and opinions of characters (mythological and otherwise) that may be contrary those of other characters or the narrator; although they may not represent the full range of IXam viewpoints, this presentation reminds us that Bushman groups were not monolithic entities and that (as certain narratives or narrative devices present social commentary quite explicitly) the informants felt some constructions were contestable. The ichneumon's critiques of |kaggən's behaviour have been highlighted (Hollmann, 2001:69; Guenther, 2002) as potentially offering insight into definitions of admirable social identities, and \|kabbo's narratives concerning a man accidentally wounded and killed when hunting springbok (LL.II.12.1181LL.II.13.1268) and his widow's experiences after his death (LL.II.13.1269LL.II.14.1396) reveal much about IXam notions of social responsibility, presenting obligations concerning care for dependent relatives. As in Chapter 5 (134), I focus on dimensions that recur throughout the archive; statements about 'IXam attitudes' rendered here should not be taken as definitive structures obeyed unthinkingly by all, but as facets within complex and contested presentations of social acceptability.

At the centre of both authority and propriety was a notion of behaving 'understandingly' ( $\| k w a k k a)$, an issue that combined two strands of thought, measuring competency, and governing the acceptable channels along which competence was directed. The concepts of $a: k a n$ and twai: $\tilde{\imath}$ encountered in 
Chapter 5c (163), in addition to evaluating the attractiveness of traits, were used to discuss aptitude and performance. Evaluations of actions (done 'nicely' or 'sweetly') or of traits (that were good or handsome) made reference to expertise and skill, as with pots (LL.VIII.23.8081) or karosses (LL.II.14.1336-1337) that had been made or worked 'nicely'. They also denoted competence in subsistence practices; the husband in the widow's story lost his life because the hunters did not shoot 'nicely' (twai:ĩ) or look 'well' (a:kan, LL.II.13.1309), while adults enjoined children to treat locusts 'nicely' or well (instead of $\| k$ "werre, 'playing' with them, see glossary k"e:nk"e:n:397) when processing them (LL.V.22.5720; LL.VIII.7.6630-6638). These evaluations described a wide range of activities: the Early-Race Caracal played the !kımmi ${ }^{72}$ 'touchingly’ (łho, Glossary:452) or sweetly, and even more outré abilities, such as (the Early-Race character) Korotuitən's (Glossary:396) magical strategies of obtaining Bushman rice (\|xe:, Glossary:451; LL.VIII.10.6885-6940), were described in these terms. A:kən and twai: $\tilde{\imath}$ were implicated in the construction of amicable and desirable social relationships, in contemporary situations and Early-Race times. The Caracal's skilled, sweet !kımmi playing was the reason the anteater considered her as a 'heart's child' (LL.V.6.4345-4356), and the love of the widow for her deceased husband was tied to his 'sweet' behaviour (LL.II.14.1315). Similar concepts structured relationships with non-humans: springbok would run 'nicely' between the hunters (LL.II.36.3255) if the latter treated them correctly, while inappropriate relationships set up by transgressions of !nanna-se behaviours would not be 'nice'

\footnotetext{
${ }^{72}$ A musical bow played by women (LL.V.6.4348), striking it with a feather or the thumb and finger (LL.V.6.4376')
} 
(LL.V.6.4380'). Kin relations, ritual identities, and subsistence roles all demanded performances involving skills that could be described in these terms, marking out people who fulfilled them in an 'understanding' fashion, and referring directly to positively valued relationships this created.

However, capacities for successful, competent action cannot in isolation define 'understanding': other attributes also connoted efficacy. 'Cunningness' (!khwi, Glossary:426) provides a useful example here, as while the two IXam terms may sometimes be glossed with the same English translation (clever), the contexts of their use suggest that their emic connotations were distinct. Superficially, the two both denoted individuals displaying their mastery of certain beneficial techniques. Thus, in one of the many narratives in which |kaggan instigates conflict (LL.II.9.966-978), the cat (\|gwattən, Glossary:442, Felis sylvestris cafra or F. nigrepes) triumphs in his fight with |kaggən by displaying 'cunning' behaviour; drawing on his virtuoso skills in flight and transformation, |kaggən then escaped from the very paws of his captor, demonstrating his own 'cunning' (LL.II.10.1046). Animal hunting behaviours also manifested this trait; the lynx evaded the hunter by doubling back 'cunningly' (LL.II.21.1911) and the jackal's 'cunning' behaviour allowed him to escape the dogs, overcoming even their superior perceptive skills (LL.II.15.1481-1482).

These displays of competence grouped 'cunningness' with understanding, and they were occasionally directly linked with one another (e.g. LL.II.35.3239-3240). However, while not mutually exclusive, there was a crucial difference between them; where 'understanding' behaviour was associated directly with a:kən and 
twai:ĩ (LL.II.4.487'; LL.II.11.1111; LL.V.11.4857) evaluations， 'cunning' performances were not. 'Cunningness' was not a wholly negative attribute, as its deployment allowed agents to achieve their aims successfully, and it could just as well be present in people as beasts-of-prey (LL.V.12.4964). However, it certainly was not unambiguous, demonstrated succinctly by its strong ties with the jackal, a nocturnal thief (whose victims included its 'fellows', other beasts-of-prey, LL.II.19.1711-1712) and a glutton who loved fat (LL.II.15.1477-1478), both of which violated the correct treatment of comestible resources (Chapter 6b:183). Cunningness was consistently connected with interactions between agents that involved contestation and antagonism, specifically those involving deception; attempts to 'cheat' were highly antagonistic in IXam thought, as with Ikaggən's attempts to cheat or deceive the man who shot an eland (LL.V.6.4429-4430). Although deception was implicated in certain aspects of human hunting (daudáuka-!k'e, Glossary:393), it was generally not considered as a characteristic of 'nice' actions: lying, in particular, was explicitly contrasted with talking 'nicely' (LL.V.18.5383-5384), and in the Early-Race narrative of !ko $\xi \mathbf{m}:-\mathbf{t a}-\mathbf{h i}:$ (Glossary:428), a deception regarding his 'nice' treatment led directly to the game become 'fearful' and difficult to hunt (LL.V.19.5457-5477). These more general negative associations of deception contributed to the ambiguity of 'cunningness' in comparison with understanding.

More crucially, the two terms differed in their relationship with Igebbi (Glossary:403), or 'foolishness'. Cunningness did not preclude foolishness: a man who was cunning 'on his other side' might still think foolish thoughts 
(LL.II.30.2743). Understanding was antithetical to foolishness: a foolish person was one who did not understand (LL.II.36.3293; LL.V.8.4602-4603). Referring occasionally to inappropriate choices in the performance of some task (e.g. the Early-Race brothers who used 'soft' ostrich bones for clubs, LL.VIII.18.7582), foolishness related more commonly to moral rectitude and social responsibility than to competence. Thus, the Early-Race youth who used !kúi-se-!khwi-|ku as a dog was behaving foolishly (despite the fact that it allowed him to hunt successfully) because he lied to his relatives about how he obtained him (LL.II.30.2743); invoking 'respectful' behaviour (Chapter 6b:193), this youth placed his acquisition of the 'dog' in the context of his social relationships ${ }^{73}$, misrepresenting them as he did so (LL.II.30.2757). More extreme types of morally and socially abhorrent behaviour, such as the willingness of lions to kill and eat one another (LL.II.16.1552), also manifested a lack of understanding; just like !gu: and !haue-ta-łhou (Glossary:417, 421; their predecessors in Early-Race narratives), lions that did not lack cunningness in hunting disregarded social norms and were consequently foolish. These actions resulted from having 'closed thinking-strings' (LL.II.30.2754). "\|kaulkaugən (Glossary:443), or 'thinking strings' represented the IXam idiom for thoughts in general (Skotnes, 1991; LL.II.6.710), and, more particularly, the thoughts of people (LL.V.10.4783) or animals (LL.V.13.5038-5039) that understood, and were integral to, the production of 'ordered' persons, acting appropriately and in accordance with the norms of their identities: when the Early-Race Caracal transformed into a beast-

\footnotetext{
${ }^{73}$ Dogs were exchanged as part of on-going reciprocal relationships (Chapter 7d:278).
} 
of-prey, she described her alienation from her previous social relationships as the 'falling down' of her thinking-strings (LL.VIII.27.8407). This alienation stemmed from an increasing violence directed towards her kin, a common consequence of this loss of propriety: people linked |xarran|xarran's desire to cause harm with her thinking-strings 'going astray' (LL.V.23.5869-5871) and the illness-causing actions of the $\mid n u-k a-! k^{\prime} e$ were motivated by their loss of their thinking strings (LL.VIII.26.8310'-8311).

Propensities for harming people, then, were bound up with poor 'understanding': lions that killed people were ones that 'did not understand' (LL.VIII.23.8074'). Fighting (or its motivation, anger) was an apt demonstration of foolishness, seen in the criticisms made by the ichneumon regarding |kaggan's foolishly seeking of fights, or 'teasing' (LL.II.33.3028; LL.VIII.3.6246-6247), and conflict in general was portrayed as non-a:kan in the opposition between asking 'nicely' and quarrelling in the story of !gwa $\xi$-!nuntu (Glossary:419, LL.VIII.5.6425-6438). Anger was a more nuanced construct than this statement suggests, and itself was not fundamentally negative; it was inappropriate anger that was a problem. Propriety here referred to the target of the anger, which if directed towards antisocial forces was not censured. Indeed, the concept of sswatt-sswatten (LL.II.26.2352') or 'speaking angrily" ${ }^{74}$, demonstrates that it could even represent a meritorious response; this angry, rough speech was employed to deal with lions, frightening them off (LL.II.17.1567'), a skill very much associated with 'understanding' behaviour. Anger directed towards one's fellows, however, was

\footnotetext{
${ }^{74}$ Not in the Dictionary.
} 
equated with childishness, and consequently with poorly developed understandings (WB.XXIII.2204; Chapter 6a:178). Negative connotations of anger complicated the relationship between cowardice and bravery, which for the IXam was not a simple duality. Hunting success was partly attributable to a lack of fear: a brave man was a literal lhou-!kwi (Glossary:405) or 'bow-man' (LL.VIII.5.6481; LL.VIII.25.8247'; pl. !houkan!houkan-!k'e), proficient in the acquisition of game, while a Ikerre-gwai (Glossary:408) or 'unsuccessfully catching male' (LL.II.21.1889) was timid (Bleek, 1956:309). Proficiency in bow and arrow use was highly valued, determining the ability to fulfil the provisioning obligations (Chapter 6b:185, 186) associated with masculine social responsibility: the man who shot well would live, while he who shot badly would die (LL.II.20.1177'). Unsurprisingly then, bravery often manifested itself as a positive trait: the Early-Race Leopard was successful in his suite for the Rhinoceros' daughter's hand in marriage because he was a 'bow-man' rather than a $\mid i$ :-sa (coward, LL.VIII.5.6481-6482). It was a trait that people hoped to inculcate, refusing to feed children jackal hearts specifically because this might cause them to become cowards (Chapter 5b:152; LL.VIII.16.7457). A similar term was łkwerre (Glossary,453; Bleek, 1956:667), for a person who was a bad shot or was not 'strong' (LL.II.35.3183'); those who did not know how to deal properly with subsistence resources (LL.VIII.7.6671-6672) were weaklings or 'feebleminded' ("swach" LL.VIII.7.6670'; Afr. swak, weak, infirm, delicate, feeble) rather than sensible, or strong. On the basis of skill in resource acquisition, a marked, strong 
opposition between the understanding, successful, brave !hou-!kwi and the $\mid i:-s a$ or $¥ k w e r r e$ appears to have dominated the construction of 'bravery’.

However, 'bow-men' were not presented in an unequivocally positive light, as their prototypical masculinity encompassed intensely negative attributes that had to be controlled. Anger was, generically, a masculine trait, with tukan-di, often glossed as 'angry actions' more literally translating as 'male actions' (Bleek, 1956:241). An alternative translation for !hou-!kwi was 'angry man' (LL.II.21.1920), and Tsatsi (Ihanłkass'o's maternal grandfather, and a !hou-!kwi, LL.VIII.15.73751/2') embodied these elements of the role. He was an impatient and 'passionate' man (LL.VIII.28.8464'-8465), both traits connected with anger: !kwã :-'a (the word translated as passionate here) commonly referred to wrath (Bleek, 1956:457, see glossary la::402), and the antithesis of the patient man was one who became 'quickly angry' (LL.V.14.5083'). lhanłkass'o described him as a man who 'talked to the lion', reiterated and strengthened links both with violence and with foolishness; lions that lacked understanding (i.e. killed and ate people) were also described as 'angry men' (LL.II.17.1598). He shot and killed his third wife Igai-an, a stereotyped form of violent 'angry action' in IXam communities, with informants on several occasions referring to men who shot at their wives as 'angry' husbands (LL.III.1.485'): this characterisation introduced parallel ambiguities in the status of bows and arrows that, though vital in subsistence, could become deadly when misused by people displaying inappropriate social attitudes. Despite his shortcomings, the coward was emphatically a man whose heart was 'mild' rather than angry (LL.II.34.3130'). As inappropriate social 
behaviour demonstrated 'foolishness', and inappropriate anger was the quintessence of inappropriate sociality, the connection between bravery and anger (though crucial in effective hunting) entailed that, from another perspective, the opposite of bravery was also the opposite of foolishness: a man who was afraid, though not particularly admirable, might be considered wise (WB.XXIII.2203').

Socially desirable states of understanding were inculcated through narrative transmission. Talking could drive away foolishness (LL.II.30.2764), and even 'foolish' Early-Race lions talked to their children to inculcate the understanding necessary for them to hunt springbok (LL.II.11.1111). Giving and receiving stories had strong positive connotations in general (LL.II.22.2001), linked with the markedly social 'visiting' practices undertaken after successful hunts; people met 'laughing', recounting their hunts, bringing meat, and dancing. In this context, narratives were sought, with people questioning each other to acquire stories they had thus far 'avoided' (LL.V.18.5373'). Similarly, when a man brought back many ostrich eggs, people would sit together and talk 'in peace' (LL.II.22.2028). Being 'at peace' had a wider positive applicability, connected with the desirable 'greenness' of new growth after rain and the shade of the clouds that brought this vital resource (LL.II.24.2226), and opposed to conditions of fear, darkness, and solitude (LL.II.30.2711). Sitting in company and talking was linked with surfeits of desirable foodstuffs and an absence of quarrelling, mirroring at a larger scale the 'cooling down' in the aftermath of fights, when antagonists went to sit among their own people and talk (LL.II.36.3268). Narrative transfer in these calm, non-discordant settings reinforced an association between them and the 
proper, 'understanding' sociality they inculcated, clearly opposing the foolish anger of inappropriate social behaviour: telling inappropriate stories, as with |kaggən's 'story which was foul' (LL.VIII.2.6195; kwai-i, unpleasant, weak, nasty, Bleek, 1956:329), provoked the opposite of this pleasant atmosphere and was explicitly marked as one of his 'tricks' (i.e. a foolish action).

Issues of comprehension defined emic classifications of narrative types, with specific information defining correct action obtained in everyday intercourse and in the 'getting' of more formal narratives. Information was sought from individuals who had already mastered the particular skills desired: !nui-|kuitən (a !gi:xa) could teach his 'doings' and songs (LL.V.15.5099) and Natta, 'understanding' the calls of partridges and springbok ewes, could teach the accompaniments sung in the $\ddagger g e b b i-g u$ dance (LL.V.25.6006-6007). Although narratives were referenced with a single term, at least two categories were differentiated based on their content; old/great and little stories ${ }^{75}$. The former were instructive stories: |hanłkass'o spoke about receiving stories in educative circumstances when he was a child (LL.VIII.14.7251), and teaching through narratives produced responsible, adult members of society (LL.VIII.26.8308'). Where 'cleverness' connoted acting as a 'grown-up' person (LL.V.2.3848-3849; LL.V.11.4857), rather than pursuing 'foolish actions', the term used is $\| k w a k k a$ rather than !khwi:, creating a connection becoming understanding, and 'growing up' through internalising requisite information from stories over time

\footnotetext{
${ }^{75}$ Cross-cutting the tripartite division I have been employing: Early-Race, legendary and personal accounts all potentially contained 'great' information. 'Great stories' were not a specific corpus, but rather represented attempts to present certain information or interpretations as authoritative.
} 
(LL.II.9.989-990); this implies a link between youth and foolishness to be rectified by education, accepting definitions of authoritative stories from elders.

'Great' stories combined ethological observations required for successful foraging, information about dangers and potential pitfalls, and advice regarding correct !nanna-se behaviours: a person might be equally exhorted to avoid calling the lion's name in a 'disrespectful' manner (LL.II.21.1916-1917) as to lie watching for the lion that came at night (LL.II.17.1566-1568). Sometimes, narratives combined these elements, describing the behavioural and physiological traits that structured appropriate hunting actions and delineating safe consumption rules (e.g. LL.V.6.4404-4405). Dia!kwain's discussion of the !kwe: state (Glossary:432; orphan; LL.V.6.4410'-4413') provides a compelling illustration of the relationship between narrative transmission and the development of vital skills. He classified orphancy as a 'great thing' (i.e. an important concern), a state of potentially deadly deprivation: ! $k w e$ itself translated literally as poor or lean person (Bleek, 1956:463). Dia!kwain stressed that it was unlikely that other relations would be able or willing to support them (LL.V.6.4410'), thus emphasising the need for children to develop independent subsistence skills by paying attention to 'great' stories. The transmission of these stories was bound up with notions of authority, suggesting that information conveyed by certain people ('understanding' people) was to be accepted: $\| k h o u$ (to instruct, Bleek, 1956:576), used in the context of transmission of information from an old woman (negating the 'stupidity' of childhood, LL.VI.2.4032), had in its Afrikaans gloss ('orden', Afr. to ordain, regulate) authoritative connotations. Understanding was developed 
not only from having heard the requisite information, but by 'agreement' with it: this agreement (an implicit acceptance of the authority of her teachers) allowed Ttai-tchuen to deploy her understanding successfully, and escape from a lion (LL.V.12.4937-4938).

Such acceptance was not uncontested, and Dia!kwain's mother's reprimand for his treatment of her stories as 'small' (he had disobeyed her injunction on 'playing with' locusts, LL.V.22.5725-5726) demonstrates this disputed presentation. Locusts were an important component of subsistence strategies, and also potential sources of danger (as mechanisms whereby anti-social !gi:tən could send illness, LL.V.22.5719): Dia!kwain's mother emphasised that ill-treatment of them would have negative effects both for Dia!kwain himself and for those with whom he shared social relationships, a theme much in evidence in other narratives detailing the acceptance of information, such as those relating to the behaviour of the New Maiden (e.g. LL.V.20.5613-5614). IXam socialisation of children revolved around the inculcation of competence within the context of correct relationships with others. Salient in this socialisation were issues relating to resources, the acquisition of food, its distribution, and consumption. It is sometimes difficult to reach IXam judgement through translator input ${ }^{76}$, but these concerns were returned to repeatedly, by the whole range of narrators. Understanding was linked fundamentally with food acquisition: even infants were 'understanding' insofar as they looked to their mothers' breasts for milk, and children grew up as they

\footnotetext{
${ }^{76}$ Notes on people who 'threw away' their wives (LL.III.1.506), or commentary on brother-sister incest given with 'disapprobation' (LL.III.1.488'), are suggestive of moral judgement, but without accompanying |Xam translations, it is difficult to assess their significance.
} 
became 'understanding' in seeking flesh (LL.II.9.991), and eventually developed appropriate adult identities, bring food to their own children. Issues of consumption underpinned a general sense of responsible adulthood, more specific roles linked with age and gender, and agency in 'ritual' domains, and it is with these issues that the following section is concerned. 


\section{Chapter 6b: Behaving 'nicely'}

Issues of consumption were integral to IXam identities (Chapter 5b:148): equally, regulation of appetite and control of food intake was of prime social concern. 'Monstrous' consumption, recurrent in Early-Race stories, portrayed dramatically the negative consequences of inappropriate indulgence of appetite. \|khwai-hem, the consummate, indiscriminate 'devourer', represented the most overt example, with his visits ending in disaster as he ate up all the available food, personal possessions, and (eventually) entire communities (LL.II.34.3137-3138). !nu!numma-!kwiton (Glossary:437; White-Mouth, LL.VIII.29.8554'), a bogeyman used to frighten crying children into silence, was portrayed as a monster that would 'swallow them down' (LL.VIII.29.8559-8560). At a less exaggerated level, Early-Race beasts-of-prey were connected with overconsumption, swallowing down their food or choosing to eat inappropriate things: the !kúi-se-!khwi-|ku wanted to 'swallow down' people (LL.II.30.2695-2696), and !gu: and !haue-ta-łhou would also eat people if the opportunity presented itself (LL.VIII.32.8797'). Ikaggən's inability to control his appetites provided the tension that drove several narratives and was emblematic of his foolishness, as seen in his disastrous repeated consumption of the eggs of the struthious Early- 
Race character !kakən-!kaka-!k'aui (Glossary:421; LL.II.6.667-615; LL.II.7.716-737).

Conversely, displays of control and restraint when eating were commendable: the desirability of the Early-Race Caracal was enhanced by her moderate habits of consumption as a handsome !xau: (Glossary:438), or 'small-eater' (LL.V.3.4351) ${ }^{77}$. Avoiding monopolising access to fat (LL.II.3.419), the 'smalleater' existed in diametric opposition to the 'gwunni (Glossary:421; greedy person, Bleek, 1956:393), applied to animals (e.g. jackals, LL.II.15.1477) and people (LL.II.14.1319) noted for generally excessive consumption. In the widow's story, the !gwunni represented a rhetorical construct embodying her potential fate (after re-marriage), and exhibiting a suite of undesirable social traits (anger, ugliness, and selfishness) that contrasted with her deceased husband, who was sweet (twai:ĩ) to her. This !kwi-a-|xarra, (Glossary:432; 'man that (was) different') or !kõ!koin (Glossary:427; 'bad man'), she emphasised, would beat her children, as they were not his own (LL.II.14.1317), and would eat up the desirable a:kən food (especially fat) on the hunting ground, bringing only hard, ugly flesh to the house (LL.II.14.1319-1322).

Issues of the regulation of consumption were thus implicated as necessary for proper food distribution: by limiting one's own consumption one was able to fulfil obligations to distribute or provision. People who did not properly distribute were

\footnotetext{
${ }^{77}$ Based partly on ethological observation; Dia!kwain noted that an antelope would decay before the caracal could completely consume it (LL.V.3.4349'; Estes, 1991:365)
} 
called 'hyenas $^{78,}($ !gwaĩ), or if they did not share meat, 'decayed-arms' (!k"wakkən-\|kũn, Glossary:436; LL.II.20.1793'; Bleek and Lloyd, 1911:125; Lewis-Williams and Biesele, 1978:130): this latter also applied to !gi:tən who did not allow free access to resources they controlled (LL.VIII.7.6638). After Korotuiton taught |kaggan 'nicely' about gathering tactics (LL.VIII.10.6901), he enjoined lkaggan to divide the resources he acquired as a result of the teachings (LL.VIII.10.6923); |kaggən's inevitable refusal incurred Koro-tuitən's anger, a narrative dynamic pointing to wider tensions in IXam thought about obligations to share resources. Improper distribution was characteristic of the foolish, aggressive Early-Race predators, particularly in their relationships with other predators: the hyena's attempt to kill the lion was motivated by the lion's refusal to distribute (LL.II.2.362), and the Early-Race lion that 'refused to give' (!koe) food to his companion was punished for it (WB.XXVI.2487-2492). An associated reverso note (WB.XXVI.2490') comments that this !koe (not in the Dictionary) also denoted refusals to accept pieces of food that were too small. The sharing of food thus appears as a contested domain, in which it was equally possible to attempt to secure exclusive access to food and to comment on a person's 'stinginess' in distribution.

Sharing was the habit of the socially responsible Bushman, who 'did nicely' with food: even 'poor people' rendered down little pieces of fat to share them with their fellows (LL.VIII.12.7108'-7109'). The !gwunni's marked preference for fat (LL.II.14.1319), then, represented a severe breach of proper sociality,

\footnotetext{
${ }^{78}$ Given the characterisation of hyenas (Chapter 5a:135), a strongly negative evaluation of insufficient distribution.
} 
underpinning the negative depictions of such characters. Distribution of food was governed by complex rules, incorporating respect behaviours (Chapter 6b:192193), and influenced by social relationships: female porcupines were given to the father-in-law of the hunter and (once cooked) divided by affinal, consanguinal, and residency ties (LL.VIII.16.7399, 7399'-7401'). Sharing rules applied primarily (but not only) to meat: main roots (LL.VIII.18.7554') were shared with |ka:gan ‘mates' (lit. plural of woman, Bleek, 1956:296, but commonly applied by married individuals to one other). The widow's depiction of the 'greedy man' presented food distribution as a fundamental component of conjugal (and parental) relationships, provisioning one's wife and children with meat and fat. Parallel ideas in Northern Bushman groups are well-known; Jul'hoansi women demand meat as a 'social right' (Biesele, 1993:187-188), and may leave their husbands to marry elsewhere if it is not forthcoming. For the IXam, this shared food was mobilised as integral to other familial relationships; $k^{\prime \prime}$ aise, commonly translated as 'to bring up, look after' (Bleek, 1956:118), might also be translated as to share food (LL.II.14.1327).

However, the importance of meat sharing in conjugal relationships can be seen clearly in the fnuturu (Glossary:453) narrative (LL.VIII.9.6786-6857). In this Early-Race story, the beautiful ‡nuturu-weevil was given springbok meat to eat by men, who thereby neglected their wives (LL.VIII.9.6802-6805). Feeling that they 'married' the łnuturu, the men dipped up water for her, brought her firewood, and gave her the fattest portions of their kills: the custom of giving shares of the 'best part of the meat' was considered (by Flat Bushmen) as a sine qua non for being 
married (LL.VIII.6.6590'-6594'). Given the connotations of similar exchanges of meat in Northern Bushman societies (Biesele, 1993), it is tempting to read this 'marriage' as the imposition of Victorian reticence (Guenther, 1989, 1999) over a description of sexual relationships; whether the apparent disinclination to discuss matters sexual is the result of translator editing, the relationship between informant and translator, or even differences between Northern and Southern Bushman societies, the narrative themes make clear the connection between 'giving meat' and 'being married'. The story concludes with the men being chastised for their foolish doings, cementing an association between displaying 'understanding', the successful acquisition of resources, and the deployment of these resources to construct specific relationships.

Notions concerning the appropriate sharing of food between other related individuals arose above, in discussing the 'orphan state'; Dia!kwain believed that relatives would drive away orphans, who should not hope to rely on their assistance. The widow's story reinforces this theme, emphasising the importance of this provisioning: her mother-in-law stressed that she must not (from grief) neglect her obligation to acquire food resources for her children (LL.II.13.1254). This story did not, however, present extended family assistance as unfeasible, suggesting instead that skilled, moral persons (i.e. understanding people) might accept responsibility for care, when confronted with an eloquent plea for aid ${ }^{79}$. Such dependencies were not characterised as specifically 'female' problems, with loss of either parent necessitating the mobilisation of 'social aid' networks: after

\footnotetext{
${ }^{79}$ These children were not orphans, however; the effort their mother expended acquiring aid underscores Dia!kwain's message.
} 
the death of his wife, Dia!kwain called on his niece $\|$ kuan to wean his child (LL.V.9.4671'). In some cases, sufficiently skilled providers might take on these responsibilities even in the absence of such pleas; "kabbo, when he was a young man who was 'quick to run shooting' (LL.II.32.2902), was able to raise his orphaned niece after the death of his brother and sister-in-law. Having returned to her father's place (LL.II.13.1259), the widow's !kwi-a-|xarra rhetoric was designed as an appeal to her brothers to assist her. She exhorted them to fulfil the role of her husband in feeding her children a:kan meat (LL.II.14.1322-1323), stressing their relatedness (LL.II.14.1329) and telling them that she considered them to be the children's !kõin-gu (Glossary:427). This relationship was invoked to obtain access to food and to the knowledge and material culture necessary for the children's long-term ability to acquire resources (for their mother, as well as themselves): she asked that her brothers make !gwattan-ta-!kaukan ${ }^{80}$ for her children. The widow emphasised that as part of this education the children could also provide valuable assistance for their uncles, in driving the springbok for them (LL.II.14.1325), introducing a negotiated element, and at least some conception of 'exchange' and of mutuality of responsibilities in the establishment of social relationships.

Themes of responsibility were fundamental to relationships in general, not only in issues of provisioning and not only between human persons. A good example of this can be seen in the role of the !xoe-s'o-!k'e (Glossary:439; LL.II.32.2890'), or

\footnotetext{
${ }^{80}$ Literally "arrowhead's children": 'children of' was used to denote superlative diminutiveness (LL.VIII.22.7980). This may be connected with !gwara-@pwa, arrows designed for hunting springbok (Chapter 7d:269)
} 
'owners' of places. This term referred to their 'possession' of certain locales but did not employ the usual $-k a$ possessive, using instead a distinctive form recalling

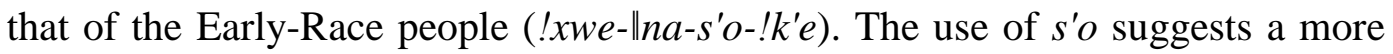
literal translation as people 'who sit/remain at a place' (Bleek, 1956:171), or more idiomatically, 'dwellers' or 'inhabitants' (Ingold, 1993, 2008:1808). These individuals inherited rights to use water sources and to occupy the 'old houses' near the water, as well as rights of access to 'emplaced' food resources such as bees' nests (LL.II.14.1360-1363) and ostrich eggs (LL.II.22.2029'). "kabbo (LL.II.32.2894-2896) described a desire to 'work nicely' ('putting in order') the water-pits that he possessed, which contrasted with the current occupation of his water-pits by 'different people'. His own inhabitation of the pits was part of the proper social order, and transfers of this ownership along consanguineal family lines transmitted this occupation 'properly' (LL.II.13.1300): while he lived there, his (adult, married) children came to live with him, allowing his grandchildren to grow up understandingly (LL.II.32.2897-2898). Not made explicit in translation, the terms with which $\|$ kabbo discussed his relationship with these 'owned' places suggest a conceptual connection between understanding behaviours and 'responsibilities' in maintaining this appropriate form of occupation (transferred to him from his brother).

The brother with whom the widow went to live had stayed with his mother (bringing his wife with him), and cast the widow's choice to live with her in-laws 
in a negative light ${ }^{81}$. He portrayed his (and his wife's) choice of residence as a result of their possession of several positively-evaluated traits. Unlike her relatives, his wife was łgou (Glossary:452, 'still' or peaceful) rather than da'̧rrakən (Glossary:392, 'wild'). She did not eat 'strongly' (excessively), leaving food even when still hungry, and worked 'things' (made karosses) nicely. These traits underpinned their ability to help raise additional dependants (LL.II.14.1336-1338), and the widow's misfortunes were thus presented as the result (in part) of moral failings: this same brother criticised the widow's deceased husband's unwillingness to remain 'quietly' with his in-laws as a contributing factor in his death (LL.II.13.1289). The adoption emerges as a negotiation between increased hunting demands on the brother, and the duties demanded of his female relations in return. He stressed the extra burden placed upon him, now initiating his hunting early in the morning because he knew the children were 'crying with hunger' (LL.II.14.1339-1343). As a proviso to this comment, he placed an obligation on his sister to work in exchange for his provisioning, asking her to assist his wife in making karosses. For her part, the widow reversed this obligation, casting clothing (and other equipment) manufacture as an entitlement to portions of 'good things' (LL.II.14.1347). She emphasised hunting success as dependent on an alliance between masculine and feminine activity, with even indirect participation being vital to the success of the operation, as women by dressing skins 'nicely' (making arrow-sacks) kept the fletching dry and thus allowed arrows to fly 'nicely' and kill springbok (LL.II.14.1348).

\footnotetext{
${ }^{81}$ Not a general admonition for matrilocal residence, though there was a preference for this (immediately following marriage) for a period that varied with personal choice (LL.II.13.1290', 1292; Hewitt, 2008 [1986]).
} 
'Nice' behaviour was, then, implicated in relationships, used in critical statements aimed at motivating or compelling people to act in certain ways by portraying specific actions as coherent with (or central to) the performance of certain roles. Positive traits could be mobilised to achieve desirable ends; a man with a 'good' heart could address the rain (LL.VIII.26.8305), invoking his moral superiority to prevent it from shooting lightning at people, by telling the rain that it should be 'ashamed' (Chapter 6b:193) of letting women see its !khweitan ${ }^{82}$. In other instances, 'nice' behaviour determined successful action, as when exhorting women to shave 'nicely' their husbands' heads to make the springbok come straight up to them (LL.VIII.14.7286), given in consequence of lhanłkass'o's evaluation of their failure in their duty to scold the children for lapses in !nannase behaviour: the 'nice' treatment was aimed at expiating their lapse. When opposing the 'nicety' of the behaviour of children versus women in driving the springbok toward hunters (LL.VIII.14.7231), Ihanłkass'o similarly combined notions of 'nice' behaviour, successful action, and the integration of this action within a positively-valued social identity. Stating that women would sit talking until the sun was warm (when the springboks disperse) while children would go out in the cold, he suggested that the children (usually associated with poor understanding) behaved 'nicely', attempting to induce the women to behave in a similar fashion (LL.VIII.14.7234). These !nanna-se strictures (Chapter 5c:162; see below, 191-193) formed one of the major avenues whereby people attempted to exert proscriptive control over others' actions, especially those of hunters and

${ }^{82}$ Left untranslated in the text; probably 'penis' (Bleek, 1956:431) 
New Maidens. Adherence to !nanna-se was portrayed as fundamental to the successful enactment of these roles, and, as both had considerable impacts on society in general, this had significance for all: hunter failures in compliance resulted in game animal escaping (LL.V.17.5270) or becoming lean (LL.V.18.5360; Chapter 5c:162). The transgressions of other members of IXam society could have similarly far-reaching effects; if girls (LL.V.6.4377') failed to show the porcupine sufficient respect (calling it $\|$ gauxu, rather than using the respect-name $\mid$ khogən), the animal would not leave its hole, and the treatment of animal bones in accordance with !nanna-se rules (followed by all members of IXam society) was necessary for hunting success (LL.VIII.14.7257'). Widespread imposition of !nanna-se strictures forced all group members to participate in this success, thus subjecting them all (to differing degrees) to similar pressures for compliance.

Respect behaviour was applicable in establishing favourable terms for hunting as part of a more general regulation of interactions between non-human and human persons: people addressed the moon in accordance with these rules (LL.VIII.28.8442), and the New Maiden's placatory interactions with !khwa: were also phrased with the term (LL.V.13.4986). If children transgressed the strictures, lions were believed to attack them when they were grown-up (LL.II.21.1920-1923), waiting to see whether the children had become 'angry folk' (!houkan!houkan-!k'e) like the lions themselves. Here, !nanna-se beliefs directed not only childhood behaviours, but also acted as a continuing exhortation to behave in particular ways as a 'grown-up' person, a theme repeated in the 
deployment of respectful attitudes as coherent with general 'understanding' personae. Understanding behaviour displayed by Ttai-tchuen (LL.V.12.49374938) in her encounter with a lioness (remaining still and not struggling) meant that, although the lioness sought an excuse to kill her, she escaped unharmed. This 'still', quiet behaviour avoided angering the lioness with disrespectful actions, setting up a 'proper' social relationship that facilitated her escape. Although this adherence to respectful behaviour was not discussed in terms of widespread social impacts (as with hunter or New Maidens), the framing of the narrative embedded her actions in a wider context, attributing her success to her obedience to her parents' instructions, reinforcing the opposition between children and elders in the construction of 'understanding people'.

In dealing with non-human forces, close relationships were maintained between notions of 'fearing' and those of 'respecting': avoidance of the name of the lion was described with both !hımmi (to fear, Bleek, 1956:400) and !nanna-se (LL.V.7.4488'). This link with fear had a basis in the consequences of transgression: Xwerri-k"au's eating of prohibited portions of food and not 'respecting' (here translating !hımmi) the lion's name (LL.V.8.4574-4610) led to severe injuries in a lion attack, while other destructive 'magic power' might cause deadly illnesses if not respected or 'feared' (LL.V.20.5544-5545). Xwerri-k"au's transgressions set up inappropriate relationships between him and other animals; the lion that lay in wait for him and the game that led him 'obstinately' toward this ambushing predator were reciprocating his own improper behaviour. Conversely, non-human persons might themselves act inappropriately by 
transgressing respectful norms: |hanłkass'o (LL.VIII.16.7376'-7377') described a porcupine that dug its way out of the burrow to escape the hunters as $! k^{\prime \prime}$ wakkan$\| k u n$ (i.e. failing an obligation to distribute), implying that in behaving correctly toward the porcupine, the hunters required it to 'give' itself to them. The failure of the porcupine to do so marked a transgression somewhere in the relationship; lhanłkass'o chastised the women at home for allowing children to break porcupine bones, on the principle that some incorrect (human) behaviour may have entitled the porcupine to an escape attempt. |kaggən's involvements in !nanna-se practices regarding his 'beloved' antelope (eland, LL.V.6.4411-4434; red hartebeest, LL.V.17.5289-5290) were also based on this principle. Taking the form of a louse or a puffadder, Ikaggən would attempt (by pinching or frightening) to get the hunter to stir, a form of disrespectful action that would allow the game to escape. Two major themes are apparent in the deployment of !nanna-se beliefs; firstly, that they represented a means whereby humans could maintain desirable social relations with non-human populations, acquiring resources and avoiding debilitating or deadly accidents, and secondly (given the desirability of these correct relationships) that they could be deployed in a prescriptive way in the production of specific roles (successful hunters, responsible New Maidens).

!nanna-se 'respect' behaviours dealt primarily with non-human contacts, but !k"werri:tən (Glossary:436, show respect, or be ashamed, Bleek, 1956:510) was a common alternative. It appeared primarily in reference to relationships with human persons, especially kin, being applied to mothers- and fathers-in-law, to brothers-in-law (LL.II.30.2756), and to affinal kin in general (LL.VIII.27.8435'). 
As with 'non-human' respect behaviours ${ }^{83}$, it manifested itself in avoidance practices: Ihanłkass'o noted that (sometimes) men might not address their fathersin-law (LL.VIII.18.7615'; LL.VIII.32.8844), or conversely that they sometimes should address their fathers-in-law rather than their mothers-in-law (LL.VIII.32.8843). This connection is reiterated in the occasional use of ! $k^{\prime \prime}$ werri:tan in a manner reminiscent of !nanna-se. !gi:tan who sought to test whether children had been taught well (i.e. were acting 'understandingly') would look to see if they showed respect (! $\left.k^{\prime \prime} w e r r i: t a n\right)$, by not laughing at the 'odd' costume they wore when performing the $\| k a n$ dance (LL.V.10.4757); the terminology (particularly the emphasis on not laughing) was identical to that employed when referencing !nanna-se practices (e.g. those involving the moon, LL.VIII.28.8442). This form of respect or 'shame' could also be a powerful tool in encounters with non-humans; Xwerri-k"au was able to escape his lion attack, having remembered that he had been taught that lions are 'not a little ashamed', by kicking it between the legs and shamed the predator (LL.V.8.4593'). Dia!kwain reported that if a baboon pulled out the arrows with which it had been shot (LL.V.20.5920-5922), or if it seized a hunting dog (LL.V.24.5953-5956), the hunters informed it that the arrows/dog belonged to a girl, causing the baboon to treat them with respect ('become ashamed'). These practices emphasise the importance of establishing correct 'relatedness' between human and non-human: addressing them with terminology commonly employed in referring to kin, human

${ }^{83}$ Where avoidance of names was important in avoiding unwelcome attentions. 
hunters attempted to avoid potentially negative repercussions of engagement with non-human individuals.

Food acquisition and consumption were central facets in constructing the IXam social world, and nowhere more so (at least for its masculine component) than in role of the 'hunter'. Two of the primary traits of the socially successfully hunter were the acquisition of resources through demonstrations of skill, and the ability to restrain and control oneself (the two key themes of social responsibility in IXam society outlined above), while the products of hunting themselves were the foci of redistribution strictures essential to the 'performance' of relationships. Surprisingly, given the number of narratives recounting hunting experiences, there is little reference to a term translated specifically as 'hunters': the epithet Lloyd

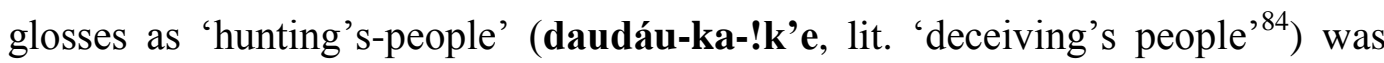
used infrequently, being mentioned only in one story ('The łnuturu', LL.VIII.9.6786-6857). The two words IXam commonly employed in defining masculine hunting activity, (\|koe: and !hen. Bleek, 1956:397, 400, 584) were both related to the idea of coming in, moving among or approaching close to something; several additional terms related to ambush hunting, lying in wait or stealthily approaching, may underpin the literal translation of daudáu-ka-!k' $e^{85}$. Coupled with the different techniques of shooting or aiming that might be employed by the hunter (Bleek, 1956:415, 468), the range of IXam hunting terminology implies that hunting represented a domain offering the opportunity

\footnotetext{
${ }^{84}$ See this glossary entry (392) for IXam hunting terminology.

85 Congruent with the idea that cheating, cunningness, and deception represented effective techniques for dealing with antagonistic forces (which included prey species).
} 
for demonstrations of diverse talents, and of various iterations of technical and behavioural expertise. The IXam made these talents the targets of deliberate assessments; a game involving shooting arrows at a rolling ball of resin, LL.VIII.8.6687-6687') demonstrated archery skill, and lhanłkass'o evaluated $\|$ kabbo's pitfall traps (that could capture even ostriches, LL.VIII.14.7217) as 'surpassingly good'.

Additionally, it is worth considering similar judgements of other predators' hunting techniques, which formed an integral part of their evaluation of these species. The cheetah was said to !Ahatton (to stalk, head, go round, Bleek, 1956:494) the springbok 'like a person' (LL.V.21.5637) and the leopard that 'stole up to' or 'lay in wait for' prey (LL.V.21.5638'; |xwerri, Bleek, 1956:367), just as men did when lying in wait behind bush screens (LL.II.24.2145). Both these animals were considered handsome, behaving 'nicely' in their hunts (LL.II.3.447). By contrast, hyenas were thought to kill in a cowardly or cunning fashion (LL.II.3.446-448), stealing from other predators rather than 'running [to] catch' prey (LL.II.9.926). The strategies of positively evaluated predators were similar to those pursued by human hunters, and, combined with a reverso note (LL.VIII.7.6610') that clarifies terms used to describe 'handsome' people alongside a discussion about hunting springbok, this provides further support for the notion that human hunting strategies were characteristically 'handsome' forms of appropriate action. Even allowing that the latter connection is rather speculative, it is clear that hunting practices represented a domain of IXam society eminently suitable for the demonstration of 'understanding', one in which specific 
skills, recognised as being unequally distributed in individuals, were highly valued.

IXam notions of 'skill' in the fulfilment of hunting practice were not limited to tactical strategies or physical abilities, as a wide range of behaviours embedded hunting within the context of maintaining sufficient inter-personal relationships between humans and non-humans. The Bushman hunter partook of the identity of his prey (Chapter 5a:139), an identification initiated well before beginning the physical hunting process in the form of 'presentiments' ( $\mid k^{\prime \prime} \wedge m m a$, Bleek, 1956:340) that created stirring, quivering, or tapping in the flesh. These presentiments manifested shared identity in an intimate, embodied fashion, being based in behavioural and physical attributes: black stripes along the necks and flanks of springbok were felt bodily in anticipation of the hunt (LL.II.28.2536'), when an ostrich approached scratching its neck they 'beat' in the corresponding place (LL.II.28.2532'), and (when foreshadowing the butchering of springbok) they echoed the blood that would run into the hollows of their knees and down their calves as the meat was carried home (LL.II.28.2540). The ability to receive information from presentiments was not reserved for hunters and hunting contexts alone. Although some applications of 'beatings' were connected with this field (e.g. guiding arrow choices, LL.II.28.2530'), many were of more general utility (avoiding lions or people with wagons, finding people), where they were felt in a similar 'embodied' way: a man might become aware of his wife returning from collecting veldkos by feeling the child slung on her back, or recognise an imminent visit from a friend by a tapping corresponding to a distinctive wound 
(LL.II.28.2533'-2533). Another kind of presentiment called hounhoun ${ }^{86}$ (presumably from houn, to perceive or be aware of, Bleek, 1956:64) was also embodied, making a person start up as if afraid and informing them about hidden dangers or about things happening at a distance (LL.V.19.5504').

Ways of gathering information about things that were happening at a distance (or at some later date) were not therefore restricted particularly to hunters; dreams, another method of acquiring such information, were experienced by all (e.g. dreaming of 'evil things' foreshadowing issues in gathering resources, LL.V.15.5160'-5163'). Information from dreams could be deceptive and therefore needed to be discussed and debated: Dia!kwain's dialogue with his mother (after his dream of his father's death LL.V.15.5131-5140) demonstrates the way in which this discussion made dream content meaningful, describing a joint decision that the dream had been a truthful one (a common pattern in memorates; Honko, 1964:15). In all of these contexts, abilities relating to long-distance or prophetic acquisition of information demonstrated understanding; "kabbo believed that presentiments needed to be heard and obeyed, using the same terminology as applied for listening to and understanding stories (LL.II.28.2530'), and in the dream memorate above Dia!kwain's mother chided him for 'disbelieving' its information, a phrase also connected with refusals to accept 'understanding' interpretations (LL.V.17.5275). Although (unlike dreams) presentiments would always yield accurate information if correctly interpreted, this interpretative ability was not shared amongst all people equally, and they thereby formed

\footnotetext{
${ }^{86}$ These were not even restricted to humans, as Dia!kwain noted that lions might use hounhoun to set up ambushes for hunters (LL.V.8.4616').
} 
another skill that could be used to evaluate individuals' contributions to the hunt. The ability to perceive and interpret these presentiments entailed the valorisation of certain traits in the construction of an 'understanding' hunter identity: in order to hear the beatings of the 'Bushman letters' the hunter had be a calm, patient 'still' man (LL.II.28.2531; Chapter 6b:189), and possession of this trait presumably added weight to presentiment interpretations.

In the widest sense, presentiments tracked the interaction of autonomous agents, whether animal or human. The mutual exploitation of presentiments in interactions that crossed species boundaries underpinned a social idiom for the interaction of hunter and prey, a characteristic that in turn demanded the rules of restraint and control necessary for hunting success. While presentiments were alerting hunters, their quarry used identity-sharing in evasion attempts: many !nanna-se practices were designed to minimise any beneficent components of identity hunters might inadvertently transfer to their prey (Chapter 5a:140). As with Northern Bushman groups ( $c f$. Lee, 1969; L. Marshall, 1976:287-312; Wiessner, 1982), IXam hunters were warned that dealing immodestly ${ }^{87}$ with the kill endangered it, even after it had been killed (LL.V.18.5368-5373): adoption of these character traits demonstrated the on-going significance of links between hunter and prey identities. A successful and skilful hunter participated in the identity of his prey (experiencing the useful physical connection with his potential victim), but maintained the ontological distance that preventing its escape. Hunters thus became a special kind of 'controlled' therianthrope, balancing the

${ }^{87}$ Demonstrated by peculiar ways of walking and handling food, as well as avoidance phrasings (LL.V.21.5686-5697). 
advantage of becoming like (and understanding) prey, with the danger of losing their hunter's perspective by becoming too closely identified with their prey (Willerslev, 2007:97) 


\section{Chapter 6c: Behaving 'differently'}

The hunter's sphere of action was the !kau:xu (Glossary:422), translated in the Dictionary as 'hunting-ground' (Bleek, 1956:417). Lewis-Williams (1997:199) opposes the 'hunting-ground' with the camp, describing it as a dangerous, unpredictable 'anti-social' place, while Riley (2007:292) defines it as 'a frontier and theatre' for constant struggles for existence, involving super-human agents: both definitions emphasise the hunting-ground as a contact zone, the setting for interactions and negotiations between human and non-human agents. The status of hunting as the primary form of these interactions highlights the integrated nature of the IXam world, with more numinous and seemingly extra-ordinary components of the IXam narratives being interwoven thoroughly with mundane practices and 'pragmatic' considerations. In the course of day-to-day life, this contiguity generated experiences reinforcing their ontological understandings: when \|goo-ka-!kwi 'saw the wind' (in the form of a bird), he was busy herding sheep for his 'master', a Bastaard (LL.VIII.8.6713-6715), and hunting experiences were fundamental in constructing cultural understandings concerning the abilities of non-humans (Chapter 5a:137).

Although the notion of the 'spirit world' is almost universally deployed as a heuristic device when discussing IXam ritual and religious life, the archive 
provides little direct evidence that this reflects an emic categorisation (see Glossary, !gi:xa:415). All of the informants gave examples of encounters with a supra-normal component occurring while hunting, visiting relatives, and searching for plant foods; experiences involving agents who were encountered in !gi:tən 'ritual' action ${ }^{88}$ occurred most commonly in the context of the many subsistence practices that necessitated or occasioned interaction with them, and all members of society (including children) were required to participate in these practices, everyone engaged with non-human forces in some manner. Although !gi:tən were powerful because of their unique abilities to influence these entities (or their abilities to influence them in unique ways), their 'spirit world' had relevance precisely because it manifested itself in day-to-day life: the IXam knew !gi:tan could influence! khwa: and go about in animal forms because their experiences of rainfall or encounters with animals demonstrated this (e.g. jackals behaving in unusual ways, similarly to domesticated dogs, were potentially transformed !gi:tan, LL.V.14.5060-5066). The contiguity of subsistence practices and 'spiritual' action was demonstrated in a substantial overlap in characteristics of the key personae involved, with the similarities between hunters and !gi:tan being particularly striking; both interacted with non-human forces central to subsistence strategies and wellbeing, both were differentiated by their possession of certain skills, and both suffered from potentially problematic identifications with the agents with whom they interacted.

${ }^{88}$ Or who played important roles in 'mythological' narratives. 
Bleek and Lloyd appear to have found !gi:tan a complex and difficult term to translate (see glossary entry, !gi:), but two mistranslations associated with the 'magic power' of this role are suggestive of its dimension; firstly, in a vague association between magic and moral turpitude $(\mid k \ddot{o} \xi o \xi$-de being given first as 'evil things', LL.V.19.5528) and, secondly, a more convincing one between sorcery and 'thinking' ( $\| k \partial \eta$ given as 'memories' or 'thoughts ${ }^{89}$, LL.V.19.5502). The connection between thoughts and 'understanding' creates an association that portrays !gi:tən behaviour as a domain of skilful performance. Maintaining the basic contiguity between ritual and mundane activity, this skilful behaviour attracted the same terminology used to describe hunting or material culture production: !khwa:-ka-!gi:tən succeeded in rain making by approaching the water bull 'nicely' (stealthily), having worked the thong 'nicely' with buchu (LL.V.3.4093, 4119'-4120); fo-löa-ka-!kwi specialists treated those who 'foolishly' dug the plant by working their hearts 'nicely' (LL.II.36.3323); and New Maidens placated !khwa: with 'nice' actions (LL.VIII.16,7427-7428). Behaving 'nicely' to achieve goals successfully was not restricted to a single domain, but resonated throughout the spheres of action associated with hunters, fo-|ö̈̈’s men, New Maidens, and !gi:ton. Some terminological parallels made associations between hunting and 'spiritual' action even more explicit. 'Shooting' (and its material culture) provided referents used extensively in connection with !gi:tan and the entities they hoped to engage or combat (J. Deacon, 1992): the term Ixau ('shooting with magic arrows', Bleek 1956:363) was used only with

\footnotetext{
${ }^{89}$ Links between 'sorcery' and thoughts were maintained elsewhere; Dia!kwain spoke of sorcery being effected by 'thoughts' (LL.V.11.4801').
} 
respect to 'magical' activities ${ }^{90}$ and $\neq x i$ : (to shoot, Bleek, 1956:679) was also definitely associated with 'sorcerers' shooting (LL.V.21.5719) ${ }^{91}$. !gi:tən defended human groups against anti-social forces included other !gi:tan and $\mid n u-k a-! k^{\prime} e^{92}$ (LL.V.19.5516-5522; LL.V.20.5543; Chapter 6c:209), and game animals such as springbok (LL.VIII.14.7263'). These forces attempted to cause illnesses in human communities, primarily through shooting magic arrows or 'sorcery's things' (transferring undesirable 'identity') at people: when such items 'got into the flesh' (Igwaisn), they caused sickness and death. In mediating IXam theories of disease and theories about other forms of violent death, hunters and !gi:tən were broadly equivalent; hunters (who shot deadly, poisoned arrows) represented a species of anti-social !gi:tən, at least as far as their prey was concerned, thus 'mirroring' relationships between IXam and anti-social agents ${ }^{93}$.

!gi:tan formed key links between notions of 'correct' human personhood, and issues relating to non-human communities. Ritual specialists who displayed nontypical human abilities had much in common with animal species that could perform tasks people could not, and their motives (like those of non-human communities) might not always coincide with and could even oppose the sociallysanctioned behaviours considered ideal for IXam individuals. The most typical exemplars of persons who did not act in accordance with these norms and aims of IXam society were to be found (unsurprisingly) in non-human communities such

\footnotetext{
${ }^{90}$ It also referred to!gi:tan to leaving their bodies to travel on a 'magical expedition.'

91 If txi: (to shoot, Bleek, 1956:245) represents an incorrect transliteration (or variant pronunciation) of $\neq x i$, it was used also to refer to ! $k h w a$ :'s 'shooting at young men who angered New Maidens (LL.V.20.5622, 5624).

${ }_{92}$ Possibly the same group (Chapter 6c:208)

93 The !gi:tan's actions thus parallel the transformations, 'shooting', and alliances with suprahuman forces (|kaggan) that might allow prey to escape the hunter or recover from arrow poisons.
} 
as those of lions (or 'beasts-of-prey' generally) or the lnu-ka-!k'e (see below, 209), and these communities formed a key referent for the potential ambiguities the !gi:tən. In the animic model, certain species (or individual members of species) are marked out by unusual modes of interacting with people, which emphasise their agency; where these might be polar bears in the circumpolar north (Saladin d'Anglure, 1994) or jaguars in South America (Reichel-Dolmatoff, 1975), for the IXam such species were best exemplified by the $\|k e\| k e$ 'beasts-of-prey'. These species were marked with the physical attributes of bestial nature, hairiness and the colour yellow, and displayed characteristic angry, greedy, and selfish personae $^{94}$. They also possessed particular non-human abilities: lions might transform themselves into antelope (e.g hartebeest, LL.VIII.23.8075-8077) and fool a person into hunting them, and the abilities of lions to become aware of people not showing respect for their names, through the medium of flies or the wind (e.g. LL.V.11.4875'), demonstrated their abilities in gathering and correctly interpreting knowledge from afar. Their 'angry' and violent tendencies were made most obvious in personal attacks, which, although most often recounted in stereotyped 'legends', were a real danger in everyday life; genealogical information as well as several personal history narratives indicate that trauma or mortality from big cat encounters was not uncommon. This included individuals killed by lions in camp (LL.III.1.485') and people killed when out on the hunting-

\footnotetext{
${ }^{94}$ Colonel Gordon's accounts (Raper and Boucher, 1988:194) of his journeys in the 1770s note of the 'Sunei' Bushmen that "they say we [colonists] are evil and come in the night like wolves [hyenas], and have hair like lions." Placed in relation to the Bleek-Lloyd archive, the cultural logic underpinning this statement becomes apparent. Nocturnal activity (commandoes would often attack in the early morning, LL.V.16.5199) and hirsuteness were consistent with both the destructive actions of commando raiding (k"a:o, Glossary:396) and the attitudes of anti-social beasts-of-prey, providing the basis for the connection between the two.
} 
ground (LL.III.1.488'): łkasin himself recounted his own violent encounter with a leopard (LL.IV.1.3461-3464), which left him seriously injured and unable to hunt for a time. Beasts-of-prey, then, formed a very real concern for IXam groups, and 'understanding' methods of dealing with them are prominent themes in many narratives (e.g. where to walk safely in a Karoo environment, LL.II.18.16821683).

It was imagined that ritual specialists could become alienated from their true communities and blend with non-human populations; particularly anti-social individuals took the form of lions or went about by night to attack people (LL.V.19.55361/2). Although individuals with the requisite characters were more susceptible than others, even well-disposed !gi:tən might potentially become antisocial while enacting their roles. This was evoked through 'beast-of-prey' performances, commonly in violent, 'biting' (LL.II.1.273-274; LL.V.3.4128) leonine behaviour: people rubbed the !gi:xa with fat (i.e. a 'handsome' substance) to take away the 'lion hair' (i.e. embodiment of a bestial character) that came out at this time. Just as successful hunting depended on potentially-problematic identifications with game species, !gi:tan curative practices necessitated the incorporation of the essence of objects that caused illness. IXam definitions of illness as the influence of foreign, antagonistic forces meant this incorporation in turn necessitated the specialist identifying him or herself with an anti-social entity. When her abilities as a !gi:xa weakened, !kwarra-an was no longer able to cure 'nicely' (LL.V.3.4149) and consequently people no longer paid her. This led to her magic power 'eating at' people (LL.V.3.4150), an idiom recalling associations 
between monstrous consumption and non-human identity, and related to a more general notion that !gi:tən would think evil and 'work death' to others (LL.V.4.4182-4184), if their hearts were not made 'cool' or 'comfortable' (twai:ĩ) by payment. The rarity of !gi:tən in Early-Race narratives is quite striking when compared to the prominence they held in contemporary |Xam society (see LL.IV.1.3484' and LL.VIII.8202 for exceptions); these Early-Race communities contained most of the normal social roles of nineteenth century IXam groups, having hunters, New Maidens, xoakən-gu (Glossary:402; 'old women'), !kõi $\eta$-gu ('uncles'), husbands, wives, and so on. Although named 'mythological' characters sometimes behaved in ways consistent with the actions or abilities of !gi: $\tan ^{95}$, these latter figures were notable absent in Early-Race times. It is tempting to read this absence as an index of the habitual lack of understanding demonstrated by Early-Race people (LL.II.28.2505); !gi:tan who acted as if they did not understand (i.e. not directing skills in a socially-responsible fashion) were extremely dangerous. In contemporary IXam society, individuals who displayed inappropriate behaviour were not ideal candidates for these roles: |hanłkass'o contrasted Tsatsi's 'passionate' (angry) nature with his not being a !gi:xa (LL.VIII.31.8764'), and !nuin |kuitən, an angry man who was a !gi:xa, was feared as a person who went about at night (as a lion), potentially to kill people (LL.V.5084-5085).

\footnotetext{
95 Most obvious is Lewis-Williams (1996:126) description of Ikaggan as the prototypical !gi:xa (shaman, in his terminology); !khwa: also exhibited characteristics of (anti-social) !gi:tən; including associations with magic-power (LL.V.6.4400').
} 
The potential for harm that emerged as a fundamental consequence of the role they performed for human societies in incorporating anti-social agents also made reference to illness, another major contributor to Bushman mortality that affected a broad spectrum of IXam individuals including adults, children, and the elderly (LL.III.1.486'). Events that impacted negatively on IXam communities were framed in a personified idiom, with illness and misfortune being seen as the result of agentive action, whether as the unintended consequences of foolish people acting without understanding, or, more seriously, deliberate assaults from antagonistic communities. Although these illnesses could result from interactions with animal species (see above, 204), it was the $\mid n u-k a-! k^{\prime} e$ who represented the most common cause of such afflictions, and the close relationship between !gi:tən and these 'spirit-people' was a further indication of !gi:tən contiguity with antisocial forces. All people became $\mid n u-k a-! k^{\prime} e$ after death, bringing illness when they came as a $k^{\prime \prime} a: o$ ('fighting-party'; LL.VIII.26.8310-8311, 8310'). This Inu-ka$! k^{\prime} e$ category was diffuse and lacking in individuality, although some extraordinary abilities continued to manifest after death (e.g. |xarran|xarran's control of winds, Chapter 5a:145). In an ontological sense, a person was transformed into a non-human '!kwi-a-|xarra' upon death, becoming the subject of avoidance behaviours (not speaking the 'old people's names', LL.VIII.26.8310) ${ }^{96}$ commonly deployed in !nanna-se behaviours that maintained non-destructive relationships with non-human forces.

\footnotetext{
${ }^{96}$ The extent to which 'old people's names' referred to individual names of specific people rather than the name for the category as a whole is unclear.
} 
Closely associated with extra-ordinary abilities, it was !gi:tzn who embodied the continuum between these non-human and human agents. !gi:tən could be individual, living members of IXam social groups, subject to social pressures and engaging in the normal range of social relationships with other individuals (while occasionally succumbing to leonine behaviour). This potential to become identified with non-human agency while alive, however, also allotted them special positions in non-human communities after their deaths, when their 'sorcery's doings' (!gi-ka-ddi-ddi) would go about embodying their will. Living IXam interacted with these dead sorcerers as they had when they were alive, making appeals to remind them of their social obligations: Dia!kwain's father called on !nuin-|kuitən to send rain after the latter's death, recalling a promise that he had tendered (LL.V.14.5073'), and his mother used to beg dead @pwaitən-ka-!gi:ten (Glossary:454; !gitzn-of-the-game) to act as if they continued to 'really love' her and to send game (LL.V.11.4805'). This difference was marked linguistically by the manner of calling upon this power of the !gi:tan (LL.VIII.26.8326'); living !gi:tən were called or 'given orders' (|kwen, Bleek, 1956:332) while equivalent demands made of dead ! gi:tən were phrased with the more placatory Igaukən (to beg, pray, call on, Bleek, 1956:276).

Dead !gi:tən might attempt to enact social claims of their own, longing for those people who were close to them, and even when well-disposed were potentially dangerous as they might lead their friends and relatives to death (LL.V.19.54855486). Dia!kwain described an illness he experienced as an attempt by !gi:tan to take him away (LL.V.4137-4139), motivated by their desire for him live with and 
seek food for them. Although not explicated in the text, this likely referred to dead !gi:tən; these 'other sorcerers' wished to take him away by means of an illness (caused by sorcery), which would presumably have killed him $^{97}$. When cross referenced with an Early-Race narrative in which a lioness gathers up children to live with and work for her (LL.VIII.8178), this represents another link to leonine (anti-social) behaviour and casts the establishment of 'incorrect' relatedness (with a special emphasis on labour appropriation) with non-IXam modes of life. Despite similarities in means and motivations, !gi:ten who were acting to kill people with sorcery were not completely assimilated into the general |nu-ka-!k'e (LL.V.19.5520) category; Dia!kwain spoke of a (living, curing) !gi:xa sending a Ihõa-gau (Glossary:404; 'rascally') ${ }^{98}$ sorcerer that had 'possessed' (|gwaiz̧n) a person to the Inu-ka-!k'e. The relationship between living and 'dead' !gi:tən, and between both of these and the $\mid n u-k a-! k$ 'e, was evidently complex (Solomon, 2011:103) and appears to have been rather inchoate. In the examples mentioned above, 'dead !gi:tən' were in some instances specific individuals who had died, with idiosyncratic powers, but equally there were also more categorical 'spirit !gi:tən' separated into game-controlling, rain-making, and 'curing' (i.e. illnesscausing) forms just as with living !gi:tən. Although Solomon (2011:102) has suggested that Opwaiton-ta-!gi:tən were spirits, a reverso note given by Dia!kwain (LL.V.11.4801') speaking of 'spirit people who had been' these

\footnotetext{
${ }^{97}$ Alternatively, living !gi:tən wanted to take Dia!kwain with them but (having been refused by his parents) they became angry, consequently making him ill. This seems a less likely interpretation, primarily because of the clear link made between being 'taken away' by the illness and leaving to go with the !gi:tən, but the ambiguity emphasises the contiguity of living/dead !gi:tən.

${ }_{98}$ Here referring to a disembodied (probably deceased) !gi:xa, the term could also be applied to living individuals (LL.V.4.4181)
} 
!gi:tan-of-the-game rather implies that there were both living and non-living examples of these specialists; this division also obtained with rain-making !khwa:ka-!gi:tən ('dead people who [were] with the rain', LL.VIII.26.8304'). Similarly, though Solomon (2011:105) suggests that the $\| x i-k a-\mid n u(c f$. glossary entry Inu-ka!k'e:413; 'illness's departed people', LL.V.20.5543) represented a category of spirit-being that caused illness, an earlier note by Dia!kwain making reference to \|xi-ka-!gi:xa ('illness's-!gi:tan', LL.V.19.5488-5489) who were alive (the narrative concerns the stars that fall at the time of their death), at least places them in the same vague, ambiguous state as other specific 'types' of !gi:tən.

This chapter has discussed issues of propriety and responsibility that were central concerns for IXam individuals, used to construct general definitions of 'human' behaviour and to delineate more specific forms of identity. Crucially, concepts of agency and personhood were not restricted to humans but were expansive enough to incorporate various faunal, meteorological, or metaphysical entities and communities. These 'societies' provided examples of non-|Xam lifeways, about which the IXam made moral judgements. These identities were not inalienable, and could be incorporated and transferred between people (Chapter 5a:145); internal differentiations of IXam society were largely dependent on the different relationships that individuals developed with this range of non-|Xam societies. Issues of anti-sociality were implicated in IXam experiences of non-IXam populations. Anti-social agents were for the IXam fundamentally 'different', violent and angry 'beasts-of-prey' who went about by night to kill people (LL.II.3.421-422) and eat them (LL.II.16.1550). Characterisations of anti-sociality 
found much play in IXam interactions with non-|Xam groups, which often incorporated issues of labour appropriation, extreme violence, and drastically alien forms of resource redistribution. It is with such conceptions that the IXam understood their interactions with the various groups that populated their world, and the following two chapters deal with this interaction, examining IXam practice and placing it in the context of 'dealing with' the various non-IXam agents of their nineteenth century situation. 


\section{Chapter 7: IXam Practice}

\section{Chapter 7a: The people who shoot springbok}

If one employs strict subsistence definitions for 'hunter-gatherer' societies, insisting on exclusive reliance on wild (however defined) food sources, nineteenth century IXam cannot be considered 'pristine' foragers. Historical records (Chapter 4b:99; Chapter 4c:110) demonstrate the presence of a diverse set of human groups in the Northern Cape at this time, groups that can be categorised according to a number of criteria, self-imposed or otherwise. Colonial European classifications imposed onto the complex suite of populations occupying the interior Karoo developed out of a long trajectory of intellectual, political and economic considerations (Appendix C:387). Particular forms of action, social organisation, and physical appearance were reified as essentially 'Bushman', constructing a category that structured the 'appropriate' European modes of interaction with them. Although these classifications revolved around subsistence, they wavered between depicting Bushmen as inveterate raiders and portraying them as 
benighted primitives doomed to extinction, a construction overlain on indigenous notions of similarity and difference, and encompassing (at times) populations distinct at least in some measure linguistically, culturally, and socio-economically from the IXam informants; I begin this chapter by discussing the relevance of the 'Bushman' category in emic definitions of group identity.

While interaction between the (emically and etically defined) populations in the southern African interior had a long precolonial history (Sadr, 2005; Chapter 4a:82), the late eighteenth and nineteenth centuries saw both the intrusion of new (and unprecedented) populations, and some radical shifts in practice on the part of indigenous groups as they became intertwined with new populations, new modes of production, and new forms of material culture; their interaction necessitated or facilitated shifts to new forms of behaviour that employed these new resources or took into account the motivations of the new incoming groups. If we consider that behaviour exists in a recursive relationship with identity (Chapter 2:40), then the constitution of IXam identities in their historical context becomes central to understanding the Bleek-Lloyd archive as a document of wider, global historical processes. Crucial to this understanding is the reconstruction of IXam practice; this chapter documents the range of material culture employed and resources exploited within IXam subsistence strategies, discussing (and providing species identifications where possible) the faunal, floral, and mineral resources referenced in the Bleek-Lloyd archive and considering the ways in which their utilisation informed, and was informed by, |Xam ideology. 
The Bleek-Lloyd archive can be used to tease apart IXam notions of similarities and differences between groups, asking to what extent they viewed themselves as part of a 'hunter-gatherer' population. Perhaps most compelling is the fact that (some of) the informants used hunting as an explicit component of their distinctive identity, defining the IXam as 'people who shoot springbok' (LL.II.36.3244'-3245'). Unfortunately, this reference does not permit clarification of whether the definition referred to Bushmen in general, or to the Flat Bushmen in particular: the phrase 'people who kill springbok' was annotated by Lloyd (in English only) with the statement that it meant 'Bushmen'. While hunting was a significant component of many frontier identities, their explicit contrasting of this practice with the habits of Khoekhoe groups (Chapter 8b:303) suggests that for the informants themselves engagements with wild fauna were considered distinctive components of their identities vis-á-vis 'others'; these engagements were linked with occupation of specific locales, familiarity with which would allow people to acquire resources (for $\|$ kabbo, 'his' place was a place where he ate springbok (LL.II.1.242). For divisions between |Xam-speaking groups the assignation of identity based on dwelling was central; informants linked people to specific places in the landscape at a variety of scales. One form of personal affiliation was with particular 'owned' places, important in the construction of 'responsible' |Xam adults (Chapter 6b:188), and another more general connection was referenced in descriptions of people as 'belonging' to localities; "kabbo described his mother as a !nwa-tsaxáu-ka-!kwi or an 'arrow/reed-eye's-person', with 'eye' in this case referring to a water source (WB.II.349; LL.VIII.31.8747'). 
Larger-scale divisions noted by the informants (outlined below) all make some reference to geographical features.

The bulk of the archive was collected from Swa:-ka-!k'e (Flat Bushmen) informants; "kabbo and Ihanłkass'o, both from this group, provided approximately two thirds of the pages recorded by Lloyd. This group thus emerges as the default from which other groups vary, the 'regte' (Afr. right, correct; used as 'true' here) Bushmen, in \|kabbo's phrase (LL.II.19.1704'). This 'default' status often makes judgements about the reasoning behind specific deployments of the term difficult, though it presumably had some special significance as the alternative term for 'Bushmen' in general (|Xam-ka-!k'e, IXam-speakers) was employed by informants who were themselves Flat Bushmen (LL.II.3.453). When \|kabbo or Ihanłkass'o refer to 'Flat Bushmen' practices and material culture, we must consider whether this reflects conceptions of distinctive 'Flat Bushman' ways of behaving in contrast to other groups, or whether it transposes the narrators' sense of participation in this group ${ }^{99}$ (or simply their ignorance about how other groups behaved). In some instances, the terms are used more-or-less interchangeably: recounting an Early-Race story (LL.II.18.1653-1691; LL.II.19.1692-1710), \|kabbo described the establishment of human and animal identities with the consecutive statements 'Bushmen (|Xam-ka-!k'e) say, we are the Day-Hearts' and 'the Flat Bushmen (are) who must speak' (LL.II.18.1663). Like many groups worldwide, the IXam valorised (their own) speech as a distinctively human trait (LL.II.8.1663-1664; LL.II.19.1705), emerging at the end of the Early-Race times:

\footnotetext{
${ }^{99}$ I.e. whether Bushmen from different groups would refer to the same practices as characteristic of their own group.
} 
although $\|$ kabbo discussed this using the term for his own group, such beliefs were probably significant for all Bushmen. Many of the practices described for 'Flat Bushmen' were presumably widespread among |Xam-speaking populations; drying flesh (LL.II.16.1522), dipping up fat (LL.II.16.1535), visiting, smoking, and 'getting stories' (LL.II.32.2875, 2880), all described on occasion as things that 'Flat Bushmen' did, were concordant with ideologies also expressed by nonFlat Bushman informants and should therefore be seen as congruent with a more generic Bushman identity.

In some cases, however, the 'Flat Bushman' epithet did refer to practices that the informants clearly considered as markers of their identities in contrast to those of other Bushmen; Chapter 5b (149) mentioned some consumption habits used in this manner, where the ability to access geographically-localised resources was mobilised as a source of differentiation. These practices extended beyond foodstuffs: when talking about $\int o-\mid \tilde{o} \ddot{a}, \|$ kabbo noted that Flat Bushmen used a distinctive phrase (\|karruken\|karruken) to refer to it, and that (as with certain types of poison, Chapter 7d:271) they could not acquire it themselves (buying it from groups living near the Orange River, LL.II.36.3242, 3269). While \|kabbo's observation that Flat Bushmen made 'bowls' from ostrich breast bones might be taken as a generic one that happened to use the more specific epithet, his emphasis on the use of ostrich breastbones in contrast with a 'bucket' (correcting Lloyd's misunderstanding, LL.II.34.3072') by those engaging in exchange relationships with European farmers (LL.II.34.3087'), transforms it into a statement of differentiation, as different groups participated in these relationships to varying 
degrees ${ }^{100}$. Comments made by Dia!kwain (a Grass Bushman) on Flat Bushmen idiosyncrasies noted their relatively less intensive interactions with Europeans. Their use of $\ddagger$ oro (tools shaped from springbok leg bones) when sewing karosses (LL.V.10.4765') revealed a lack of familiarity with colonial material culture: they did not know 'needle's sewing' (LL.V.10.4765') or possess (metal) knives (LL.V.10.4769), and they made springbok-horn tinderboxes rather than using 'real' (i.e. European) ones (LL.V.10.4763). Where \|kabbo and Ihanłkass'o emphasised this as coherent with 'regte' Bushmen practice, Dia!kwain cast their distinctiveness in a more negative light, as symptomatic of their poverty, ignorance, and pitiable situation.

Flat Bushman informants identified several populations inhabiting regions bordering their own territories as closely related to their own groups, including the Hartebeest River Bushmen (|k'a-ka-!k'e, Glossary:411, $\| k a i t ə n-s^{\prime} o-! k^{\prime} e$ ) and Mountain Bushmen (!kauken-ka-!k'e,!kaugən-s'o-!k'e). The first of these referred to populations living along the Hartebeest River ( $\mid k^{\prime \prime} a$, WB.IV.547'), otherwise called !khou-ka-!k'e or Doornboom (Afr. thorn-tree, Acacia sp.) people from the large thorn trees that grew in and along this (usually dry) river channel. The latter term did not refer to a specific, single group of people, but rather to those who inhabited more broken country; two commonly mentioned populations were those living in the Kareeberg to the south of Flat Bushman territory, and those living to the north in the more mountainous country bordering the Orange River

\footnotetext{
${ }^{100}$ Grass Bushmen lived near the official colony boundary from the beginning of the nineteenth century.
} 
(LL.VIII.19.7672-7673) ${ }^{101}$. The language of these groups was considered by the Flat Bushmen to be similar to their own speech; despite their dialectal peculiarities (WB.IV.546', LL.VIII.26.8281'), they could be 'understood nicely' (LL.VIII.19.7672-7674). Flat and Mountain groups readily intermarried (LL.VIII.20.7746; LL.VIII.1.6052): Ihanłkass'o’s father was a Mountain Bushman and, although his mother (|xabbi-an) identified as Flat Bushman, her father (Tsatsi) appears also to have been a Mountain Bushman (at least, some of his relatives were, LL.VIII.20.7746').

Mountain Bushman distinctiveness was associated primarily with the consumption of animal species that favoured more broken country (LL.II.7.798, LL.VIII.22.7982'): baboons, klipspringer (Oreotragus oreotragus), steenbok (Raphicerus campestris), duiker (Sylvicapra grimmia), and hyraxes. The notable fondness of Hartebeest River Bushmen for gum arabic (LL.VIII.18.7551') may be an equivalent for these populations as this substance was derived from acacias that grew along the river channels (thus more readily available to them). References to the material culture of Mountain and Hartebeest River groups strongly suggest that they were contiguous in Flat Bushman eyes. Mountain Bushmen wore cat skins (presumably as front-aprons, Chapter 7d:276), which Ihanłkass'o clearly considered an indicative marker of their Mountain Bushman identity (LL.VIII.22.7970), and those from the Orange River area were said to barter beads made of ostrich eggshell (LL.VIII.22.7971') and bone (LL.VIII.22.7985);

\footnotetext{
${ }^{101}$ This population may itself represent two groups, one living in the mountains themselves (the !kaugen-!k'e, whom Flat Bushman 'did not know'), and the other group (!kaugan-ss'o-!k'e) living in less elevated regions to the south of those mountains (LL.III.1.483'; LL.VIII.26.8281')
} 
Hartebeest River Bushmen (LL.VIII.22.7970, 7984) also wore cat skins ${ }^{102}$ and made beads ${ }^{103}$. These 'Mountain and Kenhardt' Bushmen populations were further associated (LL.VIII.21.7828) in their use of particular tortoise species (Igolgokan, or Chersina angulata $)^{104}$, presumably as containers: tortoise shell containers were commonly used as 'powder boxes' for processed plant medicines such as buchu (LL.V.25.6007', 6008). There is a hint that one of these groups were distinguished by the production of distinct styles of arrow, called !keitan-ta!nwa (Hartebeest River arrow, WB.I.276); however, this is an early translation and !nwa may refer to 'reed' rather than arrow (Bleek, 1956:487). The archive confirms the marking of arrows with incisions and red pigment (LL.VIII.26.82898292) in practices analogous to Wiessner's (1983:258) 'assertive style', and, if accepted, this !keitan-ta-!nwa suggests regional differences in arrow styles parallel to the variation that Wiessner (1983:257) terms 'emblematic' style in the arrows of Kalahari Bushman groups. Such potential differences notwithstanding, however, these two groups appear from their material culture at least to have been firmly tied together in the minds of the Flat Bushmen informants.

The Grass Bushmen (|khe:-\|en, LL.V.25.6005') can be considered the most 'different' of the IXam-speaking populations, although intermarriage between Flat and Grass Bushmen was still noted (LL.V.3.4132'). They were associated with specific forms of hunting technology, favouring particular white raw materials for

\footnotetext{
102 They also wore jackal and hyrax skin karosses (LL.VIII.22.7971).

103 While they appear to have made both ostrich eggshell (LL.VIII.22.7971-7972) and bone (LL.VIII.22.7984) beads, only the Mountain Bushmen were explicitly said to give or barter them. ${ }^{104}$ There is little indication that this might reflect ecological factors, as ranges for this species accord best with Grass Bushman territory (Boycott and Bourquin, 1988:103; Hofmeyr, 2009).
} 
their lithic arrow points (WB.XXIII.2156'; J. Deacon, 1996a:266-267) and manufacturing a particular 'gilt-coloured' poison (LL.II.7.780') ${ }^{105}$; the importance of hunting practices for IXam identity (Chapter 7a:215) suggests that these distinctive technologies (with presumably distinctive practical consequences) would be powerful markers of alterity. Extending this, the Flat Bushmen linked the Grass Bushmen with Khoekhoe populations: Korana (WB.II.376) and Nama (LL.VIII.19.7675) associations were believed close enough to have affected their language, which |hanłkass'o characterised as 'stammering' (LL.VIII.22.7969). Linguistic oddity was compounded by physical distinctiveness, with lhanłkass'o also commenting on perceived physical differences between the Flat and Grass Bushmen, sometimes describing the latter as short (LL.VIII.22.7980), and sometimes as tall and 'yellow-' $(\mid k o: \xi w a)$ or 'different-' faced (LL.VIII.21.7981').

This use of $\mid k o: \xi w a$ indicates a perceived physical dimension to their ties with the Korana, who were described by Dia!kwain (himself a Grass Bushman) as 'white' (LL.V.9.4626'), probably using the same term ${ }^{106}$. Dia!kwain also connected certain aspects of Grass Bushman material culture to the Korana, with both favouring sieves constructed from skins rather than matting (LL.V.25.5993). Material cultural, physical, and linguistic connections with the Korana underpinned the increased 'social' distance between Flat and Grass Bushmen, with the latter incorporating Korana traits considered inappropriate by IXam standards (Chapter 8b:303); Ihanłkass'o suggested that they treated their women

\footnotetext{
${ }^{105}$ Presumably the $\mid k a i-k a-!$ gaokan made from insect larvae (LL.IV.1.inset) that Grass Bushmen traded with other Bushman groups (LL.IV.2.inset).

${ }^{106}$ This note is in English only, but the use of 'white' to translate $\mid k o: w a$ in physical descriptions elsewhere in the archive suggests that it was the term employed by Dia!kwain.
} 
inappropriately by refusing them the better portions of meat (LL.VIII.6.6590'), thus behaving as 'decayed-arms'. Ihanłkass'o considered Grass Bushman groups encountered when he travelled to their country while working for Jakob Kotze (LL.VIII.22.7969') ${ }^{107}$ to be foolish (Chapter 6a:172) and consequently afraid, moving away from areas exploited by Europeans to water sources not yet appropriated. Even Dia!kwain noted that the Grass Bushmen were angry people (LL.V.20.5537-5539), who would raise dusts to cause illness (i.e. behave in an anti-social manner). All of these traits (selfishness, foolishness, anger, fear) formed part of IXam constructions of non-|Xam behaviours, manifesting Flat Bushman beliefs that geographically distant groups were also socially alien.

This social 'distance' increased with physical distance; moving towards the Orange River and beyond, there existed a series of groups of whom the informants were aware but about whom they knew little, groups that might accurately be characterised in IXam idioms as 'Bushmen who were different'. They included a group of people called the $\left\|k^{\prime \prime} i-\right\| e n$ ('Orange River Dwellers', Bleek, 1956:339, 519), Bushmen who lived on the colonial side of the river, speaking both IXam and Korana and strongly linked with the latter (LL.II.36.3309'). Further away, on the far side of the Orange River, dwelt the Inu-sa (LL.VIII.10.6892'; also referred to with $\mid n u-k a-! k^{\prime} e$, LL.II.36.3242). Even these most distant groups of Bushmen had customs recognisable to the Flat Bushmen who described them; $\|$ kabbo

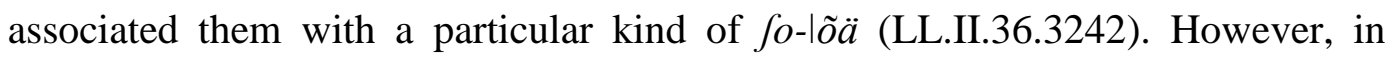
coming from the wrong side of the river, they were fundamentally anti-social:

\footnotetext{
${ }^{107}$ The route of this journey was not given, but presumably ran approximately north-west from Jacob Kotze's place at 'Hartus Kloof' (Figure 7.1:222).
} 


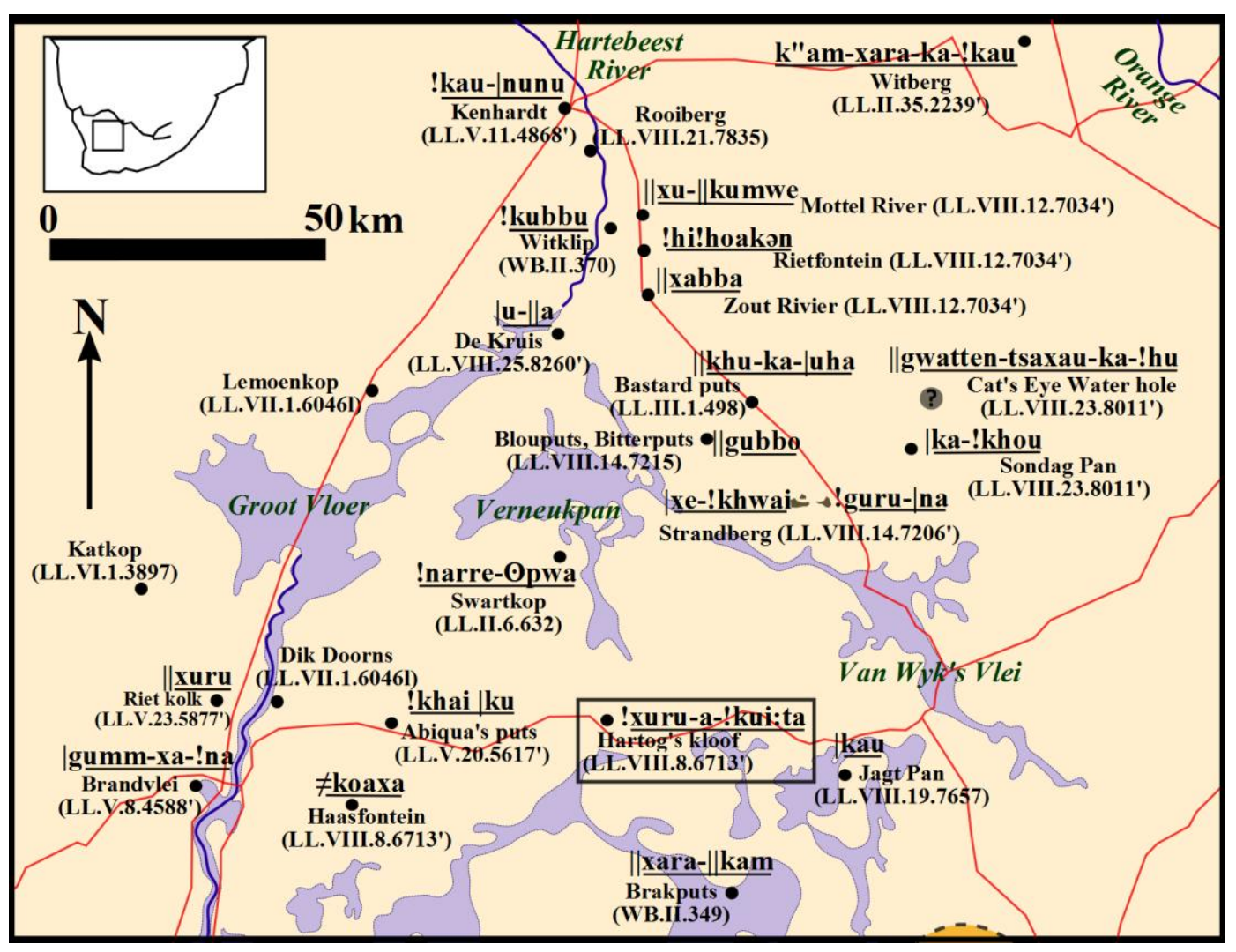

Figure 7.1: Hartog's Kloof in the Kenhardt-Brandvlei-Vanwyksvlei triangle

they cut their hands with $|n u-k a-| a \mid a$ ('cursing/fighting'; $c f$. Glossary entry, la::402; LL.II.36.3309') and were prone to stabbing people with assegais. Although he had never seen them, Ihanłkass'o feared them by reputation (LL.VIII.22.7968') as they were supposed to shoot arrows up to the sky to fall down upon the IXam (LL.VIII.22.7972-7974). Such arrows recall the arrows of sickness fired by the 'spirit people' (who shared their name with these transOrange River groups), creating a connection between geographical distance (and unfamiliarity), physical and spiritual violence, and the dangers of interacting with 'non-|Xam' societies. Informants defined variation between Bushman groups as a combination of location and perceived intensity of interaction with 'non- 
Bushman' populations, reinforcing the observation that colonial categorisations of populations did not necessarily track emic identity claims (especially for these widely dispersed, low population density groups); the IXam recognised a continuum of populations, with behaviours that differed in their degree of similarity to their own practices. Having explored ideological and social components of this identity in the previous two chapters, the rest of this chapter examines the material cultural and practical components of ' $\mid$ Xam Bushman' identity. 


\section{Chapter 7b: Hunting practices}

IXam terms relating to the classification of fauna are extensive: Bleek and Lloyd were keen to elicit as many names for species as possible, utilising the nearby South African Museum collections as a source of inspiration for vocabulary (Bank, 2006:205). The species lists (Appendix E, species tables 1-4:455) therefore represent the interaction of research methodology, Bushman modes of classification, and the scientific schema of the museum collection itself, as Bleek and Lloyd attempted to force IXam folk taxonomy into Western scientific classificatory models. This is particularly evident in the invertebrate terminology (Appendix E:474), where many more 'species names' were elicited than the IXam are likely to have habitually employed in practice; IXam terms crosscutting species names are readily apparent. Some of these categories reflect morphological similarities (e.g. !kum Omui, applied to the tsetse fly, longproboscid fly, and beefly, Figure 7.2:226) and others less tangible attributes (e.g. $\| k h \tilde{a}$ :-ka-|goro (lion's moth), which likely referenced a belief that certain insects conveyed information to lions, Chapter 6c:205). Discrepancies in the narrative significance of the species terms highlight the mismatch between the intersecting modes of classification, and a set group of species dominated the IXam cultural 


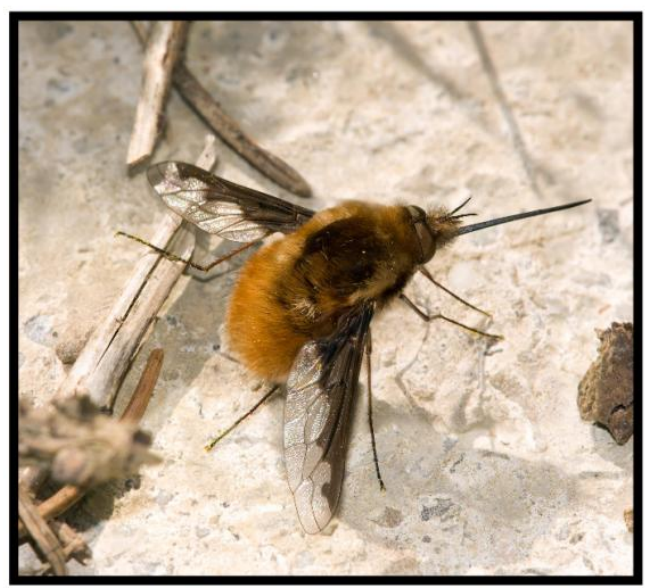

Beefly (Bombylius major)

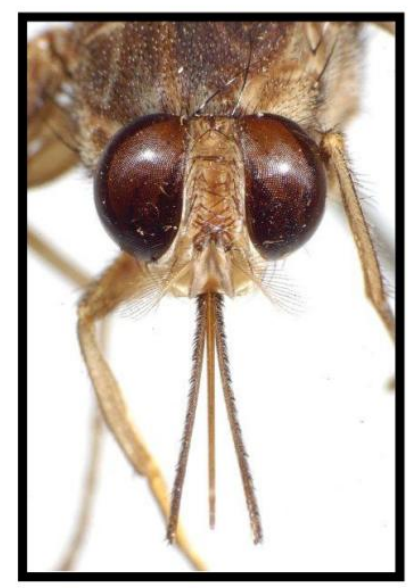

Tsetse Fly (Glossina morsitans)

Figure 7.2: Comparison of mouthparts of Bombylius major and Glossina morsitans. From 'Tsetse fly (Glossina morsitans) head and proboscis' by A. Custer, 2006, http://commons.wikimedia.org/wiki/File:Tsetse_headproboscis.jpeg. Copyright 2006 by A. Custer (CC BY-SA 3.0) and 'Beefly (Bombylius major)' by R. http://commons.wikimedia.org/wiki/File:Grosser_Wollschweber_Bombylius_ major.jpg. Copyright 2008 by R. Barz (CC BY-SA 2.5).

sphere: this narrative dominance is a complex issue, combining IXam preoccupations, with Bleek and Lloyd's intellectual framework. For animals such as the Ini (Glossary:412; ichneumon mongoose, Herpestes ichneumon) or mantis, narrative presence is almost entirely attributable to participation in 'mythological' narratives, while other species (e.g. porcupine, springbok, and lions) were important in 'mythological' contexts and feature prominently in accounts drawn from personal experience; it is with these latter accounts that I am primarily concerned here. 
As with contemporary northern Bushman societies (Biesele, 1993), meat derived from the larger herbivorous mammals (primarily bovids) represented for the IXam the culturally salient foodstuff: the single most positively valued component ${ }^{108}$ of the IXam diet was animal fat, a resource integrated into the suite of positive connotations linking moisture and ease of consumption (WB.XXIII.2175; Chapter 5c: 162-165). In a more general sense, meat was considered 'proper' consumption; in the narrative of the man who killed his pregnant wife (LL.VI.2.4064-4070) ${ }^{109}$, !kweitən-ta-\|kən contrasted the eating of !kúise (an underground storage organ) with that of meat. The belief of the husband that his wife had 'filled herself with meat' versus her contention that she had only been eating roots formed the central conflict of this narrative, giving some indication of the relative values of the two foodstuffs. Not all meat, however, was highly valued; that derived from very small vertebrates was habitually consumed only by the children who hunted them. Adults and children overlapped in participating in general subsistence through acquisition of slightly larger species such as hares (LL.III.1.503), but even these (for adult male hunters) were not ideal, desirable game species, becoming important only in the absence of bovid prey (especially when springbok were not numerous, LL.II.32.2906-2907).

Starvation was a real concern for the IXam, and several references record foods consumed in extremis, such as the chasing off of vultures from the skin of a springbok that could then be consumed (LL.II.14.1370'; LL.II.25.2301); although

\footnotetext{
${ }^{108}$ With the possible exception of honey (Chapter 7c:249).

${ }^{109}$ Not explicated in the text, this is probably an Early-Race story covering the familiar theme of the inappropriate actions of the foolish or uneducated man.
} 
the northern Kalahari ethnographies have stressed the calorific significance of plant foods, the (male) IXam informants of the Karoo emphasised that meat was vital for survival. Persistence hunting ( $c f$. Liebenberg, 2006) was a major tactic for acquiring the smaller game during periods of diminished larger game populations; this was done with steenbok by forcing them to flee in the sun until the point of collapse (LL.VIII.17.7524-7526), and hares too were run to exhaustion in the midday heat (LL.II.33.2907). Even unwounded springbok (not normally hunted in this fashion) might be run down by people who could endure this (LL.VIII.22.7965'), guiding the springbok back towards the house until the animals collapsed vomiting from exhaustion. This was seen as a less desirable and potentially hazardous form of hunting, which might lead to a man being overcome with heat ${ }^{110}$ or becoming stranded in the veld (LL.VIII.22.7964), but one that was necessary in straitened circumstances: a man who could hunt in this fashion could fulfil provisioning responsibilities with aplomb. The inculcation of these effective hunting abilities was therefore paramount (Chapter 6b:195), and impairment of these facilities could have dire consequences: !gwara-!nwa starved to death after a lion attack rendered his arm non-functional (LL.III.1.486'). Precautionary measures for avoiding injury were part of the hunters' material culture repertoire: lhanłkass'o used a special animal skin cover for the bow finger (index finger) to avoid injuries that would make hunting more difficult, and, equally, some !nannase ritual observances (e.g. disposal practices that ensured dogs would not eat

${ }^{110}$ Mean summer temperatures in the Nama-Karoo exceed $30^{\circ} \mathrm{C}$ (Desmet and Cowling, 1999:7), and the potential dangers of heatstroke resulting from strenuous midday exertion are obvious. IXam treatment involved people fanning and pouring water over hunters (LL.VIII.22.7964-7965): evaporative cooling of this type remains the most common 'field' treatment of hyperthermic conditions (Glazer, 2005:2138). 
certain bones) were designed to minimise the risk of injuries that would limit hunting efficacy (LL.VIII.7269'-7270').

Also characteristic of IXam subsistence strategies were practices of food storage demanded by the periodicity of their arid environment. Pulses in faunal populations (and nomadic or migratory strategies) meant that hunters would periodically encounter large populations of certain resource species; at these times they had opportunities to acquire surpluses (Dewar et al., 2006). When men acquired several animals over the course of a hunting trip, these would be stored in a porcupine nest (LL.II.14.1387) and covered over with bushes (LL.IV.2.35473571 ) or marked with a "scarecrow' ${ }^{111}$ to prevent scavengers ${ }^{112}$ finding them in the course of the day. After this initial expedient storage, surplus meat was dried and hidden (LL.II.25.2301) or processed into a type of 'meal' (LL.VIII.32.8828'). Similar practices obtained with other sporadically abundant resources. The legs of locusts were prepared ('made soft', LL.VIII.7.6678') and reserved for winter, when they could be eaten together with plant foodstuffs that would otherwise 'fight' people at this time (LL.VIII.7.6680), facilitating year-round occupation. Resources, including $\| x e$ : or Bushman Rice (LL.VI.2.4008; Chapter 7c:254) and water (LL.II.13.1267), were commonly stored in ostrich eggshells, and when buried $^{113}$ (Henderson, 2002; Morris, 2002a, 2005) represented crucial modifications of the Karoo landscape, relatively small-scale alterations of key

\footnotetext{
${ }^{111} \neq$ kasin placed his hat on a bush to discourage scavengers (LL.IV.2.3561'); this 'hat' was referred to as $h h u$ (not in the Dictionary), perhaps some form of headscarf (as it was described as a 'flag').

${ }^{112}$ In other cases scavengers could provide useful information, with children being exhorted to watch for crows, which could guide them to springbok carcasses (LL.VIII.2.6158-6162).

${ }^{113}$ Mats (and presumably other material culture) were also buried in anticipation of future needs (LL.V.12.4927-4928)
} 
locales that facilitated the exploitation of larger regions; shifts in their ability to execute these modifications would have large effects on the viability of overall subsistence strategies.

Despite the evident cultural importance of meat from large antelope, the archive contains relatively little information regarding the acquisition of this resource. Accounts dealing directly with the hunting of large antelope are scarce, although some narratives do provide relevant information. Such large-game hunting narratives as are present (e.g. LL.II.36.inset; LL.V.17.5317-5353; LL.VIII.23.8033-8039) emphasise the uncertainty of the endeavour, focusing on the proscriptions the hunter had to follow rather than the actual hunting practices (Chapter 5c:162; Chapter 6b:191-193); this is particularly noticeable in narratives concerning the hartebeest and the eland but holds true for other large game, and there are no detailed descriptions of Bushmen hunting wildebeest or kudu (although they apparently did so, LL.II.3.454-455). The most detailed examples of the practicalities of this type of hunting make reference to gemsbok, in discussions of behaviours relevant to or emergent in hunting attempts (LL.II.9.930-931； LL.II.16.1524； LL.V.10.4745; LL.VIII.31.8775-8788). The greater detail provided for this species may be related to the fact that adherence to proscriptions was largely based on relationships with |kaggan (Chapter 6b:193), who (although he did protect them) did not 'make his heart of' the gemsbok (LL.VIII.23.8036'-8037') as he did with the eland and hartebeest. This accords well with the dominance of springbok in 'antelope hunting' narratives, as |kaggən's link with this species was not developed beyond his generalised 
connection with game species. Dia!kwain's encounter with gemsbok (LL.V.9.4662-4679) ${ }^{114}$ suggests that people 'stealthily approached' them (rather than 'lying in wait', Chapter 6b:196), while notes on the material culture considered suitable for large-game hunts reveals further dimensions; the use of poison (LL.II.19.1704) and barbed arrows (LL.VIII.31.8770) implies that this hunting involved the stalking, shooting, and tracking of individual animals. Hunters attempted to get wounded gemsbok to run towards the huts (LL.II.24.2158-2171) ${ }^{115}$, tracking the animal overnight and sleeping upon its spoor; it was a 'great thing', which only died when the sun set (LL.II.36.3291). These details accord well with typical 'Kalahari' models for large bovid hunting practices, though this may partly be the result of the allusive nature of the IXam narratives, which provide insufficient information to confirm this similarity.

Without under-emphasising the significance of larger antelope, narratives concerned specifically with hunting outline a specific restricted set of species that were dominant within IXam subsistence strategies; namely, ostriches, springbok, and porcupines. These narratives took the form of both personal experiences and information integrated with Early-Race narratives; \|kabbo provided a considerable body of information within the context of an Early-Race narrative relating the Ichneumon's advice to |kaggən (beginning at LL.II.22.2015), which segued into a description detailing strategies for dealing with various resources (bees, ostrich,

\footnotetext{
${ }^{114}$ A narrative discussing their supra-human abilities; the gemsbok ran away 'for nothing' because they knew that his wife was going to die.

115 Knowledge about gemsbok habits of running upwind allowed them to drive them closer to home and saving themselves the labour of carrying it (also done with quagga, LL.V.8.4574-4617). If hunters 'running turned' an ostrich (LL.II.25.2288-2289) it might collapse (from poison) near its nest, allowing them to take home both meat and eggs (LL.II.23.2092).
} 
springbok), firmly embedded in contemporary practices and even including references to IXam relationships with Europeans (LL.II.23.2096). Informants displayed clear differences in their species preferences and hunting knowledge, with $\|$ kabbo focusing on ostriches and springbok, lhanłkass'o on springbok and porcupines, and Dia!kwain on porcupines. Although these narratives centre on concerted attempts to acquire a single species, relationships between species mean other species are referenced 'opportunistically': on a nocturnal expedition to acquire porcupines ${ }^{116}$, |hanłkass'o discovered an aardvark in the hole (LL.II.6.632') and proceeded to dig it out and shoot it. These 'opportunistic' incorporations remain important reminders that narratives were not designed to systematically investigate subsistence strategies, and that reconstructions of these strategies from the Bleek-Lloyd collection are necessarily incomplete.

Descriptions of ostrich hunting demonstrate an appreciation for small-scale environmental variation in strategic decisions regarding hunting routes or places for ambushes (LL.II.24.2151-2152): the verdure of river-beds governed the routes that ostriches took while foraging, a predictable element allowing hunters to overcome the problems of hunting wary prey species that looked 'afar' (LL.II.28.2551-2553). The relationship between local landscapes and ostrich acquisition underpinned the incorporation of ostriches into a suite of resources that 'belonged' to the territories (see Glossary, !xoe-s'o-!k'e:439) that the IXam associated with particular individuals (Chapter 6b:188); ostriches themselves form territories during the breeding season (Deeming and Bubier, 1999:86), and could

${ }^{116}$ Often occupying aardvark holes (LL.VIII.16.7428) 
therefore be associated with !xoe in a semi-permanent fashion. Like other resources that were tied to specific geographical locales (e.g. bee hives), ostrich meat and eggs were forms of 'property', with people holding rights that entitled access to the resources. Men ${ }^{117}$ collected eggs belonging to ostriches that dwelt at 'their' place, which were then distributed, dispersing the labour requirements and pressures on local ostrich populations (LL.II.22.2032). Embedded in issues of redistribution, access to territorial resources was a potential source of tension; people hunted ostriches associated with 'their' places in deliberate attempts to avoid angering other people by targeting ostriches from elsewhere (LL.II.21.20302031). Such proscriptions were in effect even in Early-Race times; |kaggən's justification for fighting !gwe-!kweitan-tu was that he had been hunting ostriches on |kaggan's territory (WB.XXIII.2238-2243). This potential for tension referred to an awareness that ostriches had to be properly managed, or 'taken care of' (LL.II.22.2035). By 'taking care' of the ostriches associated with a place, individuals ensured their access to food when alternative sources were absent: ostrich eggs were collected when the springboks had gone to the 'springbok's place' (i.e. migrated). Other resources that were tied to specific places were treated similarly; when springbok returned to an area, bees' hives were left alone (honey was 'closed up') to allow the insects to recuperate (LL.II.23.2045), a regulation (both of honey and of ostrich egg collection) marked by timings of the appearance and disappearance of specific stars. Hunters took advantage of

\footnotetext{
117 There were gendered elements in the exploitation of ostrich resources, just as with the acquisition of large bovids. Although the collection of ostrich meat and eggs emerges as a masculine activity in Ikabbo's narration, the latter at least were processed and distributed by women (LL.II.7.753; LL.II.22.2025-2028).
} 
opportunistic strategies to ameliorate their effects on local populations; having observed ostriches travelling along a riverbed, they decided to hunt these instead of the nesting ostriches (LL.II.7.795-804), 'controlling' their demands on resources that could easily be over-exploited. This was integral to survival during lean times, and threats to these management practices would clearly have been problematic for long-term occupation of episodic Karoo environments.

The 'localised' nature of ostrich resources coupled with the importance of correct exploitation necessitated a nuanced understanding of ostrich behaviour, revealed by the detailed ethological information contained in the narratives; the shading of eggs, the sharing of incubation labour between (several) female and male birds, the time of year at which they laid (LL.II.22.2037-2039), symbiotic relationships between springbok and ostriches (LL.II.23.2062-2063), the fact that the male rather than female ostriches guarded the nest at night (LL.II.23.2077), and the habit of dominant females for pushing subordinate females' eggs to the outside of the nest (LL.V.10.4788') ${ }^{118}$ were all recorded in the narratives. Ostrich acquisition was an 'understanding' behaviour, discussed in familiar terms; success required that the hunter 'lie nicely' (LL.II.7.746) and use a new (mooi, Afr. pretty) arrow (LL.II.7.747), for example. This understanding incorporated anatomical awareness, informing the hunter that the thigh rather than the chest should be shot at (the latter was 'bones' LL.II.23.2090), but it was in the mobilisation of ethological information that 'understanding' behaviour was most clearly manifest: after killing one ostrich, knowing that ostriches do not incubate their eggs alone,

${ }^{118}$ Ostriches hatched from these eggs were called !kaui-!kwa, or 'dwarf' (LL.V.10.4789) 
hunters returned to the bush screen to await the partner(s) of the dead bird (LL.II.24.2155). The penchant of the ostrich for distraction displays (LL.II.23.2070; Cooper et al. 2010:369) and flight from the nest (LL.II.22.20202021) was exploited to reveal nest locations, allowing people to acquire eggs with ease. Hunters took advantage of interactions between animals, waiting for the ostrich to chase away crows from its nest (taking this opportunity to shoot, LL.II.23.2090) or watching for birds-of-prey that dropped stones upon ostrich eggs (LL.II.23.2083). If they had already discovered the nest, hunters would build bush screens and lie in wait there for the returning ostrich (LL.II.7.739; LL.II.23.2072), but when encountering an ostrich otherwise, 'stealing up to' tactics (crawling along the ground) were employed (LL.II.24.2218). If they observed during the day where the ostrich was feeding, the diurnal nature of these birds allowed them to creep by night into these bushes (LL.II.24.2144-2146), setting themselves to shoot it when it returned the next day; close anatomical and ethological observations were crucial influences in determining choices in hunting strategies, ensuring that sufficient opportunities for experiencing varied hunting strategies were essential if these resources were to provide significant subsistence contributions.

The ostrich carcass was processed on the veld; the liver and heart were immediately sliced, roasted, and eaten (LL.II.24.2193-2196), while the rest was skinned, cleaned, cooked and placed into arrow sacks to be taken home (LL.II.7.760-765). The meat of male ostriches was highly desirable, occasioning 'rejoicing' as a 'fat' resource used to soften and make palatable dried antelope 
meat (LL.II.23.2106-2107). Unusually, the hunters praised the ostrich that had been shot, calling it fat, handsome, and red (LL.II.24.2149); this behaviour was discouraged in bovid hunting as it might affect the quality of the meat (Chapter 5c:162), but for ostriches it was another of their products that was subject to these restrictions. In the nineteenth century, (male) ostrich feathers were an important trade item for the IXam, providing access to highly desirable tobacco resources (LL.II.23.2096; Chapter 8c:333). These feathers needed to be plucked and stored separately if they were to be traded; upon killing a bird, they immediately began plucking the white feathers, binding them into bundles using the shorter feathers (LL.II.7.784). When the wife of the hunter asked him about the feathers, he would reply that they were 'old' and ugly feathers that had been torn and broken by the nesting ostrich as it lay in the dust (LL.II.23.2108-2109), congruent with modesty behaviours enacted towards bovid meat, and some indication of the relative value the nineteenth century IXam assigned to ostrich resources.

The IXam noted a mutualistic relationship between ostriches and springbok, commenting that the two animals travelled together (LL.II.28.2251) and contrasting the springbok that looked to the ground with the ostrich that might perceive a man from afar (LL.II.28.2552-2553). Although this compounded the difficulties of approaching prey unseen, it also meant that hunting ostrich and springbok was complementary; \|kabbo interspersed his descriptions of ostrich egg acquisition with tactics for hunting springbok (LL.II.24.2155-2157). Both were 


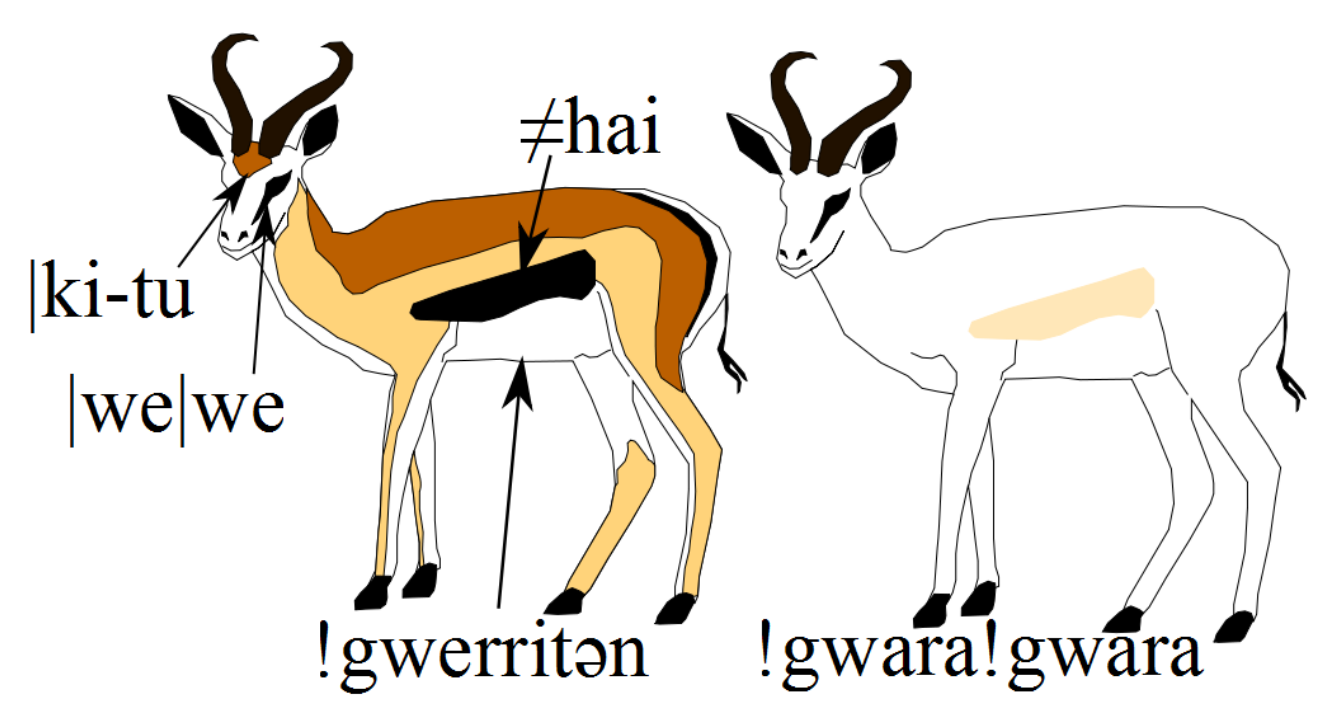

Figure 7.3: IXam terms for springbok markings and colour varients (modified after Hetem et al. (2009)

hunted at night, though this required different approaches, as they 'stole up to' the springbok but 'lay in wait for' the ostrich at this time (LL.II.24.2141-2143). Hunters took advantage of the relationship to acquire resources opportunistically; when they panicked the springbok into running, so too would the ostriches flee, revealing their nesting sites and leaving them unprotected (LL.II.23.2061-2063). The mutualism existing between the ostrich and springbok is an apt metaphor for the complementary role they played in IXam strategies, with ostriches forming the low-density residential resources facilitating long-term occupation, and springbok a highly productive but sporadic and unpredictable counterpoint that created potentials for resource surplus, food storage, and larger-scale communal action. Springbok (whai, Glossary:401), the quintessential Karoo antelope, were vital to IXam subsistence, and their population movements were of paramount 
importance; if they did not arrive when expected, death from starvation became increasingly possible (LL.III.1.485'). The IXam consequently relied on a range of strategies of exploitation, employed techniques indicated by specialised terminology, differentiating, for example, between $\|$ kauwin (Bleek, 1956:563) for running and shooting simultaneously, and !uhattakən (Bleek, 1956:494), or shooting at springbok that had not yet perceived the hunters (LL.VIII.14.7224'). IXam specialisation and reliance on springbok forms the most relevant caveat for any attempt to extend analogies of IXam practice to other southern African huntergatherer groups; the absence of springbok from the southwest Cape and the eastern Escarpment necessitates hunter-gatherer groups in these regions having employed alternative subsistence strategies. Terminological manifestations of springbok significance continued in the identification of a range of physical traits specific to this species, primarily those of coat colour and markings (Figure $7.3: 237)^{119}$; if the terminological profusion of cattle-terms in Nguni cultures is seen as a reflection of the significance of these animals in their socio-cultural world (Poland and Hammond-Tooke, 2003), then the IXam can justly be termed a 'springbok culture'. Their differentiation also included peculiarities such as the Igou-gun (a springbok with horns that turned forwards, LL.VIII.22.7993') or the !gwara!gwara (a white springbok, Bleek, 1956:392); also called |hi-gusa (LL.VIII.14.7242'), these latter appeared when the 'number of springbok resemble[d] the stars' and Moffat (1858:155), observing one in a trekking herd, remarked that Bastaards also considered them precursors of unusually large treks.

\footnotetext{
119 ! gwerritan, white hairs on the underside (Bleek, 1956:392); łhai, dark stripes along the flanks (Bleek, 1956:650); |ki-tu, black facial stripes (Bleek, 1956:316); |we:|we:, black marks on the eyes (Bleek, 1956:362).
} 
Springbok exist in three colour morphs, and white varieties are rare but naturallyoccurring sports (Hetem et al., 2009:379) that might be expected in association with large herds purely on statistical grounds. The IXam would not kill a white springbok, as doing so might make other springbok reluctant to visit a place (LL.VIII.22.7993'), while the short-horned springbok (|kwi-sa, Bleek, 1956:334) was not a 'food's springbok' (LL.V.10.4716-4718) but rather the avatar of a whaika-!gi:xa (a type of Opwaitən-ka-!gi:tən, LL.V.10.4740): killing it attracted the illness-causing antipathy of its 'owner', or made these owners less inclined to bring springbok to the hunters (LL.V.10.4726-4727). These avoidances of unusual springbok expressed desires to maintain 'proper' relationships with unpredictable, large springbok herds, thereby ensuring they would continue to visit IXam territories.

By contrast, evaluations of other traits allowed hunters to make discerning judgements between targets they wanted to acquire. Springbok prey was chosen on the basis of 'beauty': Ihanłkass'o left older springboks to shoot at a beautiful young springbok ewe ${ }^{120}$ (LL.VIII.14.7246-7248), \|kabbo emphasised the shooting of female springboks once their hair became 'handsome' (indicating their 'fat' condition, LL.II.25.2259), and male springbok with 'beautiful' fur were also sought after (referred to as does, LL.VIII.3.6265). Femininity and youth (assumed to correlate with fatness) thus underpinned this identification of desirable prey. Other aspects of terminology encoded information about behavioural qualities;

${ }^{120}$ Whai-\|khou, young springbok without offspring (LL.VIII.14.7247') 
when the springbok came from the east they would call $\left(! k o u k a n^{121}\right)$, when coming from the west they would sneeze (LL.II.25.2317'), and the IXam also registered differences between springbok calls (!karro, Bleek, 1956; 440) and the cries of springbok kids (me:, Bleek, 1956:136). These behavioural nuances had implications for hunting, indicating the condition of the animals (which would sneeze when they were fat) and predicting subsequent movements of the herd (LL.VIII.14.7238). Most significantly, mimicry skills allowed hunters to interact with the herd directly, giving a 'peculiar kind of liquid call ${ }^{122}$, that induced the springbok to approach and lie down for the hunter (LL.VIII.14.7256'; LL.VIII.26.8286-8288); these skills again had to be refined in practice, through actual hunting experience and contact with the relevant species.

Hunting springbok made particular physical demands on people. Both lhanłkass'o and $\|$ kabbo stressed that people who ran to shoot springbok had to be young, fast and 'masculine" ${ }^{123}$, (LL.II.25.2287-2288; LL.VIII.14.7225). These tactics were characterised in a strongly positive fashion, as running well/handsomely to hunt the springbok nicely (LL.II.13.1245'), and springbok hunting formed a cornerstone of successful, 'understanding' masculine hunter identities (Chapter 6b:195). Springbok hunting narratives also incorporate a political element, through deliberate attempts to incorporate the skills of different individuals, bringing different domains of 'understanding' relevant to the task. Men who could

\footnotetext{
${ }^{121}$ Probably an alternative pronunciation of !kau (call, bark or roar, Bleek, 1956:412), as the reference coupled it with !gum (roar, bellow or call, Bleek, 1956:388).

${ }^{122}$ Evoking the 'tame' state of Early-Race game animals (LL.V.19.5473); the same noise was used by lkaggan to make the eland he created to come to him (LL.VIII.6.6508').

${ }^{123}$ Ihanłkass'o explicitly excluded women from these 'chasing' activities (LL.VIII.22.7966).
} 
not run fast sat behind the children who were driving the springbok, telling them that rather than 'shooting in among' springbok they should let the animals approach their ambush sites (LL.VIII.7.6609-6617). Expertise in archery (a 'grown-up' skill) and knowledge of springbok behaviour and anatomy were presented as better options for achieving their goals: waiting until the startled springbok turned sideways on and shooting at the dark stripe along its body, waiting hunters would successful wound the animals. Often, arrows used for springbok were not poisoned ${ }^{124}$ (LL.VIII.1.6087) and these animals were instead killed by trauma; if accidentally wounded when hunting springbok, people died not because of poison ${ }^{125}$ but because the arrow left a wound that did not heal (LL.II.12.1231). Tactics for shooting springbok maximised the potential for trauma, as people were advised to shoot the animal broadside (LL.VIII.7.6617) and to aim at the ribs behind the shoulder, sufficiently injuring it but allowing it to extricate the arrow from the wound (LL.VIII.14.7255-7257). These tactics took advantage of springbok behaviour: springbok would continue to flee if they could not remove the projectile, and this latter strategy was designed to counterbalance this tendency (decreasing the labour associated with tracking and transporting the carcass).

Unlike ostriches, springbok did not belong to 'owned' places: when the rain did not fall the springbok went to their own place, where they became lean

\footnotetext{
${ }^{124}$ Alternative strategies using poisoned arrows were also employed (LL.V.3.4133'); Ihanłkass'o observed that when letting arrows fly 'singly' at springbok, people would use featherless arrows (!khau), which were poisoned (LL.VIII.10.6923').

${ }^{125}$ By contrast, the man who trod on his arrow when out hunting gemsbok was poisoned (LL.VIII.31.8780-8781)
} 
(LL.II.23.2050). They travelled and came to the !xoe (LL.II.13.1304) when the 'nice' (twai:ĩ) rain's wind blew, and drank at waterholes close to the huts (LL.II.25.2269-2279). Their arrival was thereby integrated into a suite of positively evaluated events, representing a season in which people could enjoy a surfeit of water (which itself had become sweet rather than bitter) and food, obtained without the labour of travelling long distances. This strong connection between the arrival of springbok and rain meant that hunting this animal was dependent on knowledge of the likely timings and distribution of rainfall and other weather conditions, combined with detailed information regarding springbok responses to these factors. As with management of ostrich, this knowledge was often encoded in observations of stars (LL.II.25.2319), and of other resources that responded to the meteorological phenomena at different rates: when Bushman rice 'got feathers' 126 the springbok would arrive, and the blossoming of certain flowers $(\ddagger k u-k " o m m-k a-\mid k a g ə n$, women’s daisies Bleek, 1956:296, 665) attracted the male springboks, who would eat them and become fat (LL.II.33.3023'). Knowledge of this kind enabled rapid mobilisation, taking advantage of sporadic and short-lived influxes of springbok populations.

Small resident populations of springbok could be hunted with tactics broadly similar to those encountered for ostriches and larger game, though with some characteristic performances such as the use of odiferous substances: men sewed a

\footnotetext{
${ }^{126}$ The nuptial flights of the eusocial insects that constituted 'Bushman rice'; rainfall is a common stimulus for such flights, especially in arid areas (Wilson, 1971:115)
} 
band into which they could put $\int o$-|̃̃̈ when hunting (LL.II.36.3276-3278) ${ }^{127}$. Rubbed on the bodies of the hunters, this strongly smelling plant made the game 'foolishly afraid' (running in among the people, rather than fleeing in a 'sensible' fashion), paralleling the use of ostrich feather brushes in other forms springbok hunting (see below, 244). Other methods were identical to those employed for ostriches, and involved constructing $\|$ kuitzn (bush-screens) or digging out holes in which to lie (LL.VIII.8.6768) and waiting for grazing springbok to approach (in the evening, LL.VIII.8.6751-6769'). Again as with ostriches, springbok ambushes could be set up after dark; moving to temporary water sources and setting up camp nearby, men used higher ground as a vantage point from which to observe the springbok (presumably travelling to the water, LL.II.6.629) and set up their ambush to intersect their movements the following day (LL.II.14.1375).

However, it was the large migratory or nomadic population influxes that determined the most unusual (and salient) manifestations of springbok hunting. When the springbok came in such numbers that they hid the bushes with the bodies (LL.VIII.8.6726), the IXam engaged in large-scale, communal game drives. This whai-ka-!k'iton (springbok's 'beating', LL.VIII.23.8028') was a complex procedure requiring the participation of many people, dividing the herd and separating the 'advance guard' ( $x u$-\|kei, lit. surface-edge, Bleek, 1956:261, 568) from the numerous part (|kwobbe, probably 'massed', Bleek, 1956:335). Some people went round in front of the game and 'shot in among' them, using special arrows (with long feathers) and tightly bound bows (which would

${ }^{127}$ Used here when hunting 'quietly' (i.e. stalking game), and also when 'running in among' springbok. 


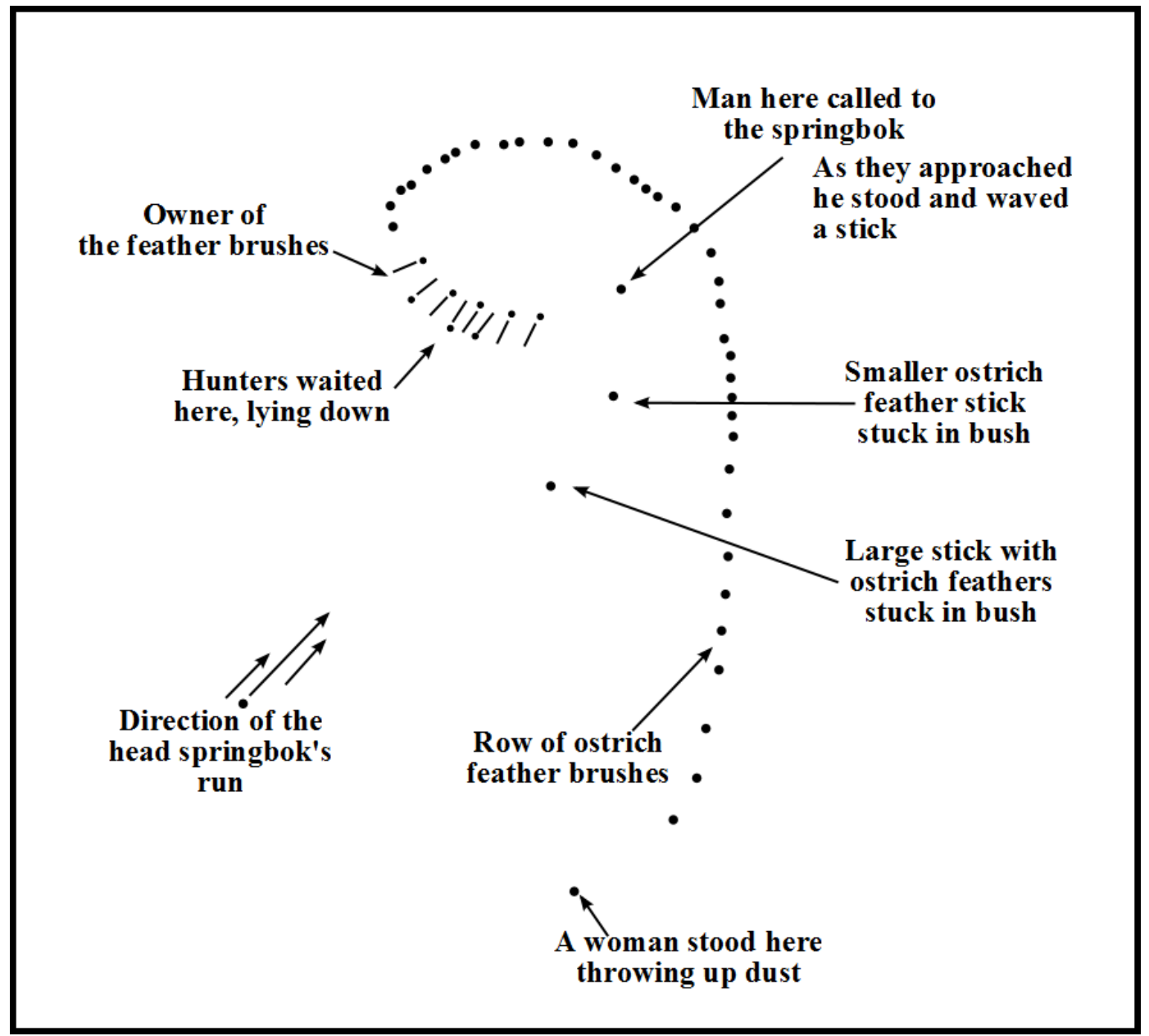

Figure 7.4: Arragement of ostrich-feather brushes in hunting springbok (LL.VIII.23.8066-8068; and accompanying sketch)

reverberate strongly) to induce the herd to flee (LL.VIII.8.6728-6735) ${ }^{128}$. The men who startled the herd had to be able to run quickly, aiming to panic the springbok and spend them 'leaping' towards other hunters lying lie in wait for them (LL.VIII.8.6740). Once the herd was moving, people attempted to channel it along desired routes by using (male) ostrich feather brushes (!koa:kən, Bleek,

\footnotetext{
${ }^{128}$ Ihanłkass'o criticised this tactic as wasteful of arrows (breaking against the springboks’ heads), and this 'wastefulness' was a major motivation for the placement of marks ( $¥ k e t t \partial n$, LL.VIII.26.8288') designed to facilitate identification (LL.VIII.26.8290) on the arrows.
} 
1956:438), treated with buchu and red pigment ( $\| k a$, LL.VIII.23.8072') that made the game foolish or afraid (LL.VIII.23.8029). Ihanłkass'o made several references to this practice (LL.VIII.23.8027'-8029, 8066-8068), recording the patterns according to which the brushes were stuck in the ground (Figure 7.4:244) to get the springbok to run between two hunters in a wide curve. Women (LL.VIII.14.7241-7242), children (LL.VIII.7.6604), and more dynamic uses of feather brushes (standing and waving them, LL.VIII.23.8067) also induced the herd to flight.

Children ran behind and along the sides of the herd, keeping it from dispersing, and threw up dust (LL.II.36.3256) to make the springbok run closely together, while older men ran across in front to shoot game (LL.II.14.1392-1393). One account (LL.II.14.7227) suggests that children would fire arrows to drive springbok but probably in reference to adult children (the speaker, an old man, refers to the children as his own, LL.VIII.14.7224), though other narratives do provide evidence that younger children assisted in killing springbok (LL.II.14.1389) $^{129}$. This participation of women and children was significant in determining success, and equally, if they behaved in a non-understanding fashion by letting the springbok perceive them too early on in the hunt, the prey would escape. When hunting springbok alone, $ł$ kasin was only able to shoot them successfully because of an inadvertent partnership with a lion, which startled them into flight (LL.IV.2.3547-3571), and $\|$ kabbo also noted that solitary hunters had difficulties getting close enough to shoot springbok (LL.II.14.1332'). Springbok

${ }^{129}$ This account may present a 'first-kill' rite: the 'young boy' (by killing the springbok) caused the women to dance for joy at his demonstration of an unexpected level of 'understanding' 
hunting thus emerges as somewhat 'non-typical', often involving large-scale cooperation between individuals from the whole spectrum of IXam society, taking advantage the potential to acquire surplus from these periodic population irruptuons; archaeological evidence for this form of exploitation is available from a springbok mass kill site in Namaqualand, on the western extremes of the Succulent Karoo (Dewar et al., 2006) ${ }^{130}$.

Springbok and ostrich thus dominated the larger game resources, and it it is porcupine-hunting that stands out as by far the most significant for the smaller mammals. Hunting porcupines required markedly different approaches to those employed for the large bovids or ostrich and springbok. There was little danger that porcupines could outrun hunters, but their nocturnal habits and acute senses posed serious problems: the porcupine was an 'understanding' animal that, perceiving with its thinking-strings the dangers of its walking abroad in daylight, could only be acquired after dark (LL.V.15.5049; LL.II.6.632) ${ }^{131}$. The porcupine 'made its eyes' of its nose (LL.V.6.4449), using its olfactory prowess to find food and (more significantly) perceive danger. Being able to smell predators from afar, the porcupine would avoid the hunter (LL.VIII.16.7407-7408). In Dia!kwain's account (LL.V.7.4436-4456), it is the combination of these factors that determines porcupine-hunting as a test of endurance; the hunter needed to resist tiredness, take extreme care with movement (to prevent rustling), and remain aware of wind direction. Its ability to evade notice imbued the porcupine (and aardwolf,

\footnotetext{
130 These authors also note bone damage consistent with death by trauma (Dewar et al. 2006:1269), recalling IXam practices of using arrows without poison, when hunting springbok.

131 Hunting at night was facilitated by (LL.VIII.6.6582), but not dependent upon (LL.VIII.16.7384), the full moon.
} 
LL.VIII.16.7381') with superhuman potency as a kwa $\xi \mathbf{k w a} \xi$ sikuitən (Glossary:396; 'elusive thing', Bleek, 1956:110). Although they were not 'real' $k w a \xi k w a \xi s i k u i t o n$ (which could not be perceived at all), the IXam applied this term to these animals because they often found their spoor without seeing the animal itself, demonstrating superlative evasive prowess, and the porcupine displayed further skills in its supposed ability to make men sleep against their will while they waited at the hole for it to return (LL.V.6.4381-4384). These superhuman capacities necessitated !nanna-se respect behaviours towards the species (LL.V.6.4379'; Chapter 5c:162; Chapter 6b:191, 193) to generate favourable outcomes when hunting them; underscoring its significance, the products of porcupine hunts were heavily governed by redistribution rules crucial in the maintenance of correct social relations (Chapter 6b:183-186). Young porcupines would be cooked on the veld, and the others left raw; on returning to camp, the meat was given to the wife of the hunter, and she became responsible for its distribution according to kinship (female porcupines were given to her father) and residence (young porcupines were distributed around the camp, and male porcupines to immediate neighbours, LL.VIII.16.7396-7400).

Porcupines were thus incorporated within the category of meats suitable for the construction of masculine provisioning responsibilities, and informants ${ }^{132}$ presented this hunting as a masculine activity. Congruent with this, porcupines were acquired in an 'understanding' fashion: Dia!kwain strove to emulate Xatin (his father), who was a skilful hunter of porcupines, watching 'well' (a:kən) at the

\footnotetext{
${ }^{132}$ Feminine participation in this hunting (though not direct) was not inconsiderable, as the animals were 'water's things' and thereby implicated in the relationship between New Maiden and !khwa:
} 
burrow without succumbing to its soporific abilities (LL.V.6.4390). As with other species, 'understanding' required detailed ethological data; Xatin (LL.V.6.43994400) told Dia!kwain about the fact that the species walked 'across the wind' rather than into it (implicated in the placement of ambushes), and stressed that the time they returned to their holes was predictable (judged by watching stars). When they discovered them at a distance from their home, the hunters would camp at porcupine holes (LL.V.7.4438-4441). By observing the behaviour of inquiline bats ${ }^{133}$ (LL.V.6.4378), watching for when they returned to porcupine holes, they could ascertain whether the porcupine was inside. Blocking the holes with bushes (LL.VIII.6.6583) placed a little way into the hole, they manipulated the behaviour of the animal, knowing it would (unable to see the bushes) run for the hole and get trapped there. Incorrect interpretation of the spoor around the burrow would be disastrous for the hunt, as if they stopped up a hole that the porcupine was inside, it would escape and leave the area (LL.VIII.16.7376'). When making the kill, the hunter would simply run to the animal and strike it. This represented the easiest element of the hunt, although the quills of the porcupine were feared and governed the techniques employed: the animal was killed with a long stick that could avert its attack (LL.VIII.6.6586; LL.VIII.16.7388-7389). As with ostriches, after killing one porcupine they waited for the second to return to the burrow, knowing that there were usually at least two inhabitants of any hole (LL.V.7.4440') and that female porcupines tended to spend longer foraging (LL.VIII.16.7392). Seasonal differences in behaviour were

\footnotetext{
133 Probably a Nycteridae species (Vaughan, Ryan and Czaplewski, 2011:277); the likeliest candidate for the region is Nycteris thebaica (Monadjem et al., 2009).
} 
also registered: in the summer, porcupines returned to their nest towards morning, while in winter they returned earlier, in the middle of the night (LL.VIII.16.7391'). Detailed 'natural history' knowledge was thus encoded in porcupine hunting narratives, generated in on-going experiences that represented, to judge from the emphasis on the physical discomfort encountered and mental fortitude required, highly affective 'embodied' encounters with the species. The prevalence of porcupine hunting narratives was thus at least partly determined by their importance in the hunting experiences of the informants themselves. Although Northern Cape animal population were undergoing rapid change in the nineteenth century, this was not distributed equally across all species. Porcupines were more resilient than larger bovids, remaining available even after the imposition of fenced, bounded farms: porcupine hunting continues today on South African farms (Chevallier and Ashton, 2006:13-14). Hunting porcupines both demanded and demonstrated experiential knowledge of non-humans, thoroughly integrated into the IXam worldview; their resistance to novel anthropogenic pressures was a key factor in ensuring (perhaps increasing) their continuing relevance within the production of IXam cultural knowledge. 


\section{Chapter 7c: Gathered resources}

Arthropods (mainly insects) were important nutritional and cultural resources in IXam life. Non-comestible species tended to produce reactions of disgust or fear; $\neq$ natta-xam ${ }^{134}$ insects were described having an unpleasant scent (LL.VIII.9.6770), $\| k^{\prime \prime}$ ururu $^{135}$ were feared for being poisonous (LL.IV.2.3518'), while Ihanłkass'o’s reaction ${ }^{136}$ on being shown a large solifuge combined the two (LL.VIII.12.7072'). Social insects, including several eusocial species and gregaria phase locusts, were of paramount significance as central components of IXam subsistence, though the range of insect resources consumed was increased by species gathered opportunistically when encountered: these insects were primarily larger, solitary species such as the bara-xam ${ }^{137}$ (Bleek, 1956:15) or low-density resources such as (solitaria phase) locusts that were collected ad hoc and carried about in karosses (LL.VIII.7.6667).

In cultural terms, several bee (Apoidea; Anthophila) species attained especial prominence; Lewis-Williams (1981a:8; 2002:81-82; 2010:8-9) has drawn

${ }^{134}$ Derivation is unclear, but the category broadly incorporated the Hemiptera (true bugs; see Appendix E:473)

${ }^{135}$ An Orthoptera species; Lloyd identified as Kritje (Afr. cricket, LL.IV.2.3518-3519). łkasin’s description of its strong bite and poison suggests it may represent an Anostostomatidae or Bradyporidae species: although these 'King Crickets' and koringkrieke are not venomous, they can bite and often emit foul odours (Picker, Griffiths and Weaving, 2004:74-78)

${ }^{136}$ Ttui-ttui ttuappem!; approximately 'get away, let go (of it)!' (Bleek, 1956:240).

${ }^{137}$ Various Buprestidae beetles. 
attention repeatedly to the significance of these insects (and their products) as referents for 'shamanic potency'. In subsistence terms, they were valued for their larvae and (particularly) for their honey. The former, eaten while still in their cells (LL.V.3.4073'), were referred to as the 'honey-liquid's fat' (LL.II.14.1365) or 'honey's liquid fat' (LL.II.23.2057) ${ }^{138}$ : the word given for fat here was very similar to that for 'maggot' (suenssuen, Bleek, 1956:174). This passage, used by Lewis-Williams (2002:81) to support a connection between honey, fat, and anomalous but potent 'liquid' foods, probably represents a mistranslation: Lloyd was unsure of her translation (LL.II.23.2057), and her evident error in later referring to the 'fats' of termites, which had feet and could fly (see below, 255; LL.II.35.3224') suggests that her gloss for ssuen here may similarly be incorrect. It more likely makes reference to bee brood, specifically to the earlier larval instars fed with brood food derived from nectar that has been insufficiently dehydrated to form honey proper (Kilani, 1999). The connection between fat and honey was therefore indirect ('maggot'-like instars were properly linked with fat, which makes nutritional sense ${ }^{139}$ ), and the 'liquid' honey may refer directly to the consistency of the transitional nectar-honey stored around these larvae, rather than to a categorically anomalous foodstuff (both 'eaten' and 'drunk'), as with northern Bushman populations (Lewis-Williams, 2010:9).

\footnotetext{
$138\|k h a u-\| k i-k a-s s u e n$ and $\|k h a u-k a-s s u e n-\| k i$, respectively. $\| k i$ (juice, saliva spittle, Bleek, 1956:580) locates this substance within the assignation of potency and 'identity' to bodily fluids (Chapter 5a:131).

${ }^{139}$ Mature bee larvae can contain around thirty percent more fat per unit weight than beef (Crane, 2009:72)
} 
As with other African hunter-gatherer groups (Turnbull, 1965:168-173; Blackburn, 1982:291), the IXam practised a sophisticated management of bee colonies, governing their exploitation by observations of plant resources and stars, and the availability of other food sources (LL.II.23.2051-2062) and paying particular attention to swarming behaviours (LL.II.23.2059; 7033') that founded new colonies. They would smoke the nests to stultify the bees before cutting out portions of the comb, carefully leaving enough for the hive to regenerate ('become fat'), and closed off with large stones hives to which they wished to return, preventing ratels (Mellivora capensis) from robbing them (LL.II.14.1363$1364)^{140}$. Bees provided desirable components of IXam diet: honey had strong connections with masculine faunal resources (containing 'fat' larvae), imbuing the game animals with their colours (when fed by |kaggən in the Early-Race times, LL.V.3.4071-4074). In keeping with this status, it was one of the few gathered resources that was acquired by men (LL.II.14.1368) as well as women (LL.II.23.2058). These women were beseeched to give honey to their husbands, paralleling meat-sharing demands (Chapter 6b:185-186) and emphasising the importance of honey-sharing within conjugal relationships. The collection of honey (from Apis mellifera nests) ${ }^{141}$ formed another component of the 'rights of access' associated with !xoe territories, and nests were sometimes passed along patrilines (LL.II.14.1363-1366). While some early commentators (Stow, 1905:86)

\footnotetext{
${ }^{140}$ Other resources could be 'closed up': a waterhole called Ikaggan-tsaxau (Mantis-Eye) was blocked with a stone after the IXam drank there (LL.II.14.1383')

${ }^{141}$ Productions of other bee species were distinguished with specific terms: łkauakən, made by 'small bees', and !akka !gaua, the sweet food of the 'blenner vlieg' (Opwai:, Bleek, 1956:685). This latter may represent blindevlieg (Afr. blind-fly; biting clegs/horseflies, Haematopota sp), reflecting IXam appropriation and re-application of the Afrikaans term.
} 
remarked upon violent defence of these resources, this is not confirmed directly in the Bleek-Lloyd archive. Narratives recounting |kaggən' misappropriation of honey (LL.V.1.3612') do indicate a potential for social conflict in honey distribution, and, as other !xoe-bound resources (Chapter 7b:233) were a source of tension, honey can be situated with a suite of 'managed' and (to an extent) guarded resources firmly 'placed' in the IXam landscape.

Another key invertebrate resource was the locust. Like springbok, it displayed dramatic periodic increases in resource density ${ }^{142}$, creating gluts of food that raised problems of competition; jackals and birds finished off the locusts while people slept (LL.VIII.7.6671). As with springbok, the IXam employed a large number of specialised terms referring to them, derived from close observation of their lifecycle stages (LL.VIII.31.8746-8748) and charting their growth from hatchlings (toetoe) to larger instars ( $|k i| k i)$, the first appearance of wing buds around the fifth instar $\left(\| u k w a b b a^{143}\right.$ ), metamorphosis into winged forms (berre), and final emergence as locusts (!hou) when they were strong enough to 'fly against the wind' (LL.VIII.31.8752) and received the epithet $\| k h o u$ ('flier', Bleek, 1956:576). Like game animals, locusts could cause illness, though they were deployed by anti-social !gi:tan rather than acting of their own volition (LL.V.21.5707'-5709'). This association appears to have encompassed the periodicity of locust irruptions: they were 'shut up' by !gi:tan that 'possessed' them and only these 'owners' could release them. This represented a form of resource control equivalent to that exerted by Opwaitən-ka-!gi:tən, and living

\footnotetext{
${ }^{142}$ Coinciding with plentiful rains and an abundance of plant resources (LL.VIII.16.7388')

${ }^{143}$ Possibly 'fatigue-unfolds/unwinds' (Bleek, 1956: 109, 627).
} 
!gi:tan certainly did involve themselves in the arrival of locusts (Ikarru owned locusts, LL.VIII.7.6639'). However, the role of locusts as dangerous 'fighting's things' (see Glossary entry la::402; LL.V.21.5709') and their incorporation into a triad of foods devoured by anti-social !gi:tan (flies, locusts and human beings, LL.VIII.15.7305) suggests control of this resource was often the province of these non-living, antagonistic forces. Consequently, 'taking care' of locusts involved avoiding the destructive 'magical' attention manifest in these short-lived resource surpluses (rather than the 'care' applied to !xoe, Chapter 6b:188). Locusts 'flying massed' necessitated large-scale participation in subsistence activities; children were told to go and build fires to cook the swarm, while others drove them forward (LL.VIII.7.6623-6640). Requiring people to remain awake for much of the night, feeding the fires and preventing the locusts from burning up as they were roasted (LL.VIII.7.6656-6663), this treatment was seen as rather onerous and discussion of it (and the storage of locust-products) emphasised terms associated with responsibility; people should work the excess food 'nicely' instead of behaving like jackals by gorging themselves while food was plentiful (LL.VIII.16.7449'), and children were instructed to go picking up locusts 'properly' for their elders (LL.VIII.7.6671). Combining these instructions with requirement for 'taking care' of their potentially hazardous nature, these insect resources belonged in cultural terms firmly with 'masculine' antelope products (Chapter 7b:227).

A more 'feminine' and no less significant insect resource was that represented by the epithet 'Bushman rice' or 'ants' chrysalides (or eggs)', a phrase used to 
translate $\| x e$ : but also to describe closely related resources, including !hakan (which 'resembled' Bushman rice, LL.VIII.9.6789') and !kuin ('other' Bushman rice, LL.II.3.470'). These were species of eusocial insects, either ants or (more likely) termites (see Glossary entry, \|xe::451): "kabbo's identification of !kwan and ssuenssuen manifestations of $\| x e$ : (LL.II.35.3224') and !kuin (LL.II.37.33453346) strongly suggest a hemimetabolous lifecycle. !kwan ('Great Rice', LL.IV.4.3905) was motile and 'alive', ssuenssuen 'got feathers' and flew away, and both had 'feet': it is therefore unlikely that either represented a pupal stage. \|xe probably referred to a harvester termite (Hodotermitidae); the informants noted that it was eaten by aardvarks (LL.II.2.338-339) and bat-eared foxes ${ }^{144}$ (LL.II.3.471), which favour such species ${ }^{145}$. !hakən can be identified with some certainty, as the aardwolf ate only this 'ground's rice' and did not consume $\| x e$ : (LL.II.3.426'-427; LL.VIII.28.8494'): aardwolves subsist almost exclusively on 'snouted termites', Trinervitermes sp. (Richardson, 1987). Further confirmation is provided by the observation that a 'beacon'-like fungus (!gu!gu, Glossary:418, or |ku kwai; LL.VIII.11.6945'-6946') grew out of the !hakan, probably a Podaxis sp. fungus: worldwide, these are associated with termite mounds, growing out of Trinervitermes sp. mounds after rains (Bottomley and Fuller, 1921:140; Morse, 1933; Priest and Lenz, 1999). !kuin, mentioned only infrequently, is more difficult to identify, but descriptions of its behaviour emphasise similarities with $\| x e$; it

\footnotetext{
${ }^{144}$ Bat-eared foxes are more generalist in feeding habits than aardvarks and aardwolves (Kuntzsch and Nel, 1992:47; Clark, 2005:2-3); "kabbo noted these more catholic tastes (LL.II.3.470-471).

${ }^{145}$ It is not possible to exclude ant species on these grounds, as these mammals are not exclusive termite specialists (Taylor, Lindsey and Skinner, 2002; Clark, 2005)
} 
emerged in a winged adult form, had 'maggots' (ssuenssuen) and !kwan, and was 'dug out' (LL.II.37.3345-3347).

This 'digging out' (|k"wã:, Glossary:411), connected with a range of resources, including insects and medicinal-ritual or food plants, was a 'feminine' contribution to subsistence, sometimes being translated as 'women's hunting' (Bleek, 1956:340): Bushman rice was dug out using digging-sticks weighted with stones (LL.VIII.10.6889-6893; artefacts themselves strongly associated with women, Chapter 7d:275) or with springbok-horn points (see below, 257). Despite this connection with feminine practice, many of the associations of 'digging out Bushman rice' overlapped with those applied to masculine resources. Like game species, these insects could be affected by the condition of the 'hunter': before going to dig Bushman rice, Dia!kwain's mother had to remove the influence of 'evil things' that would otherwise have caused the insects to act unfavourably (LL.V.15.5161'-5163'). As with 'masculine' honey, ostriches, porcupines, and springbok, the exploitation of Bushman rice was regulated through observation of stars, used to judge when the alates were about to emerge in their nuptial flights (LL.II.37.3343'-3347): the arrival of \|xohai stars (Alpha and Gamma Aquilae, Altair, Bleek, 1956:638) was associated with rainfall, which motivated the winged adults of the $\|_{x e}$ : and !kuin to leave their holes. Bushman rice 'products' were explicitly equated with those acquired in hunting: dry rice being comparable to dried flesh, and 'wet' rice to raw meat (LL.II.16.1521-1528), with these two resources combining to construct palatability. 'Wet' rice made the liver ache (LL.II.35.3151) just as 'wet' meat did, with the high protein and (relatively) high 
fat content of termite species (Phelps, Struthers and Movo, 1975:131; de Foliart, 1992:395-396) ${ }^{146}$ rendering it nutritionally similar to meat: Associated material culture also existed in 'masculinised' forms, with horned-tipped digging-sticks being used by Flat Bushman men (LL.II.1.262'; LL.VIII.23.8083'-80831/2). W. Bleek also recorded a horn-tipped 'Berg Bushman woman's digging stick' (WB.XII.1123') ${ }^{147}$ used to dig for insect resources; this differentiation of diggingstick types (horn-tipped versus stone-weighted) may thus reflect the demands of resource acquisition, with horn-tipped sticks being used exclusively for insects and weighted sticks encompassing both insect and plant resources (the former undertaken by both genders and the latter associated more exclusively with women). Bushman rice was processed by roasting, then dried on mat-sieves (LL.VIII.19.7640-7641) and sifted to separate earth (and overly chitinous elements) from the components that would be ground up for storage (LL.VIII.28.8507-8510; 8506'-8508'): this processing was also undertaken ‘jointly' by men and women. Bushman rice insects, then, were 'feminine', dugout resources that overlapped considerable (in cultural evaluations) with 'masculine' game: !hakən was particularly desirable, one of the 'nicest' of foodstuffs (LL.VI.1.3929'; LL.VIII.9.6789’).

In contrast to the fauna, many plant species ('dug-out' or otherwise) are known only by vague descriptions, even where they played significant roles in narratives or where informants alluded to their subsistence and economic importance.

\footnotetext{
${ }^{146}$ It must be noted, however, that these studies were not undertaken on Karoo species.

${ }^{147}$ In the absence of any explicit commentary, it is difficult to be certain whether this represented a distinctive ‘Berg Bushman’ material culture.
} 
Consequently, it is difficult to ascertain which particular Karoo species were most important for Bushmen subsistence. As with the fauna, the availability of information likely reflects the preoccupations of their informants ${ }^{148}$ with research interests: neither Bleek nor Lloyd appears to have been particularly knowledgeable about or interested in plants, recording them with general descriptions that render identifications extremely difficult ${ }^{149}$. Nonetheless, the general role of plant-gathering in IXam subsistence can be elucidated, even if nutritional contributions are obscure. As with Kalahari hunter-gatherers (L. Marshall, 1976a), meat was subject to rules of wider distribution than those applied to plants. People would kwai:i meat ('to distribute', Bleek, 1956:110); Ihanłkass'o's stressed that this was to 'distribute to the other people' (LL.VIII.16.7399', emphasis added), and contrasted with 'sharing' plant foods within the family (|ken, Bleek, 1956:309; LL.VIII.18.7554'). However, certain plants were prized as excellent sources of food (e.g. the !hwin root, LL.VIII.27.8395'), and the use of en-en (flesh, meat, Bleek, 1956:39) to refer to bulbs (LL.VIII.18.7553') implies underground storage organs (USOs) were recognised as significant elements within the plant resource category.

In a biological sense, most comestible plant resources represented either these USOs or seed dispersal organs ${ }^{150}$, though there were additional sources such as

\footnotetext{
${ }^{148}$ Another remainder of the fact that only one of the major informants was female.

149 This is compounded by the extraordinary diversity of the southern African flora: this diversity (especially for interior regions) was only poorly understood when Bleek and Lloyd were writing.

${ }^{150}$ Tsaxaitzn was used for both berries and seeds (Bleek, 1956:213; LL.V.25.5997')
} 
acacia gums ${ }^{151}$. These categories were highly differentiated in emic classifications; at least fourteen distinct named types of comestible roots and tubers were mentioned (Appendix E:483). Few have secure species identifications associated with them, but Cunningham and Davis (1997:488) identify !kaui (Glossary:422; wild onion, Bleek, 1956:414), as a probable reference to Cyanella hyacinthoides, and the "food called by the Boers bitter bulle" (!kauru, LL.V.25.5997') may be a wild bitter watermelon, Citrullus lanatus (Afr. bitterboela $)^{152}$. Wild southern African curcurbit species usually exist in bitter and sweet forms (Livingstone, 1857:48-49; Dane and Liu, 2007): distinctions between $\| k w a k k \partial n$ ('axoort ${ }^{, 53}$ ) and !nwanna (bitter versus sweet gherkins, Appendix E:484) may reflect within-species variation rather than different species. If the relevant criteria are issues of edibility and palatability, taxonomies that cross-cut phylogenetic schemes are likely and classifications of bitterness versus sweetness were common criteria in IXam judgements of palatability: brackish 'old' water was sour, bitter and 'bad' where fresh rainwater was sweet (LL.II.25.2304-2307). People feared that the 'sweetness' of Igara (Glossary:402) berries would make their teeth unfit to chew meat (WB.XXIII.2155') and the !koa root was described as bitter (LL.V.13.5006'); although this undoubtedly made reference to gustatory qualities, themes of bitterness versus sweetness were integrated into wider evaluative schemes of 'good' versus 'bad' traits (Chapter 5c:158). For some

\footnotetext{
${ }^{151}$ Providing valuable nutritional supplements: Acacia eriolaba gum has a protein content of up to 43\% (Wehmeyer, 1986:14).

152 The Dictionary describes this as eaten by Bushmen (Bleek, 1956:416), but Ihanłkass'o suggested it was eaten by porcupines, not people (LL.VIII.6.6560'); consumption of its berries or seeds was noted only in Early-Race contexts (LL.V.25.5997').

${ }^{153}$ Probably agurk or argukjie (Afr. gherkin), commonly referring to Cucumis africanus (Bosch, 2004:237-238)
} 
plants, this bitter versus sweet palatability was a guide for seasonal exploitation; łkasin noted that !kouwi (see !kaui, Glossary:422) was bitter and watery in winter, but sweet in summer (LL.IV.4.3903') while Ihanłkass'o described the opposite (LL.VIII.10.6869') ${ }^{154}$ and !kerri roots were eaten only in winter, when sweet (LL.VIII.18.7598-7601).

Another identifiable resource is Ikui (Glossary:410; Bleek, 1956:324) or gambro (Fockea edulis; C.A. Smith, 1966:218, 272, 273, 481), a large rootstock. An important component of IXam diet, prioritised by people when choosing campsites (LL.VIII.1.6106), it too illustrates the centrality of seasonal variations for plant resource exploitation as (although consumed throughout the year) it was processed and consumed differently according to the season, requiring access to other seasonally available resources. Gambro became increasingly significant when !kouwi bulbs were not available (LL.VIII.16.7450-7451;7450'), and women focused on it when men were collecting Bushman rice, hares and birds (i.e., when the springbok had left, LL.VIII.10.6888'). At these times, gambro had to be eaten in combination with preserved locusts, lest it 'fight' them (LL.VIII.7.6679'-6681) and make their heads split and ears sing ${ }^{155}$. Plant foods underwent similar processing treatments to those employed in producing stored faunal products: in an Early-Race narrative, !kauru seeds were roasted, sifted (to removed dust) and ground between stones (after removing the husks) to produce a fine meal

\footnotetext{
${ }^{154}$ Potentially reflecting environmental differences: łkasin lived closer to the influence of the Cape winter rainfall zone, while lhanłkass'o dwelt in a region more firmly associated with summer rainfall.

${ }^{155}$ This description strongly suggests a mildly toxic biochemical composition, though resources that 'fought' had a wider cultural relevance (Chapter 7c:253).
} 
(LL.V.25.5997'-6001'; 'flour', ttamberre or !kun!kun, Bleek, 1956:243, 543), analogous to that produced from xarru-ssi bulbs in contemporary Bushman practice (LL.VIII.26.7552'). This treatment recalls practices (removing dust and 'husks' before grinding up) associated with insect resources; !kúise roots too were treated in a similar manner to locusts, placed in a pre-heated hole in the ground and covered over to bake (LL.V.7.4498'). Similar themes thus emerge in faunal exploitation and plant resource strategies; seasonal variation in particular plant resources over the year was vital to the production of coherent subsistence strategies, requiring intimate knowledge of the range and characteristics of the species present. While plant foods constituted the stable, consistent basis of these subsistence strategies, the necessity for incorporating particular combinations of resources within a 'palatable' diet meant that they too were highly dependent on 'integrated' socio-economic systems, accessing a wide range of resources over the year. 


\section{Chapter 7d: Independent lifeways}

The independent existence of IXam groups was not only sustained by their knowledge of animal behaviour, hunting strategies, and gathering techniques, but also depended upon their ability to produce the wide range of goods that facilitated these practices; as J. Marshall (1973:116) observed for NyaeNyae Jul'hoansi, horns, sinews, skins, and other by-products of hunting were perhaps even more important for subsistence than the nutritive contribution of meat. Shifts in abilities to acquire these resources had implications for the sustainability of wider socio-economic strategies, as it is quite clear that resource access was interrelated: after killing a gemsbok, hunters 'rejoiced' to be able to make tough 'gemsbok-shoes ${ }^{156}$, that facilitated long-distance expeditions to acquire desirable but patchily distributed resources, including reeds for arrow production and certain types of wood for bows (LL.II.24.2176). IXam material culture demonstrated their keen awareness and assessment of subtle differences in the resources available to them, as evinced in descriptions of brushes used as 'handkerchiefs' for wiping away sweat. Made from ostrich-feathers (LL.VIII.4.6359') or the tails of caama fox, jackal, or bat-eared fox, inserted into a

\footnotetext{
${ }^{156}$ Shoes were made from the skins of a number of antelope species (LL.II.3.453-454, 458, 460), but Bushmen favoured gemsbok (LL.II.24.2171); although inter-related, resources were not precisely interchangeable.
} 
handle made from a 'driedoorn ${ }^{157}$ 's twig', and bound together with sinews from the springbok necks (WB.XII.facing 1121-1121'), the construction of this artefact required access to a range of resources. This underpinned functional differentiation, with ostrich-feather brushes being used specifically for wiping the eyes and aardwolf-hair brushes of identical form (called $\mid \mathrm{ku}{ }^{158}$ ) used as cooking and eating utensils, and allowed for assessments of skill in resource acquisition and artefact construction; the tail of the bat-eared fox was preferred for crafting a !nabbe ${ }^{159}$ (handkerchief) that had 'no equal in beauty' (LL.VIII.11.7005'-7007).

Although processes of production must have been crucial for IXam abilities to occupy their territories, complete descriptions of manufacturing processes are rare; they did not form a specific focus for Bleek and Lloyd, although Lloyd later developed more interest in technological processes (Bank, 2006:355-357). One major process that was described is that associated with pottery construction, linked to arrow production (see below, 267) in a gendered division of labour; women made pots while men made arrows for shooting springbok (LL.VIII.23.8065'), shaping the omission of the firing stage from the descriptions (J. Deacon, 1996a:259) and highlighting both the limitations of the male-

157 Afr. three-thorn, applied today to several species; one likely candidate is Rhigozum trichotomum, a woody Karoo shrub first classified by Burchell (1822:298-299).

${ }^{158}$ Aardwolf (Bleek, 1956:323).

159 The 'driedoorn' used for the handle was !nabba (Bleek, 1956:472); names of IXam artefacts commonly reflected raw materials used in their construction. Reeds gave their name to the arrow, sinew was used for bowstring, and 'metal' additionally referred to arrowheads and knives. In a wider context, objects transforming back into their original material is a common narrative feature (Hewitt, 2008 [1986]:60; Guenther, 2002). 
dominated corpus and the importance of gender for structuring |Xam society ${ }^{160}$. It required logistical trips to acquire preferred clays ${ }^{161}$ and specific grasses, which were pounded soft, moistened, and worked by hand into small, 'handsome' pots (LL.VIII.23.8054-8059), which were rubbed with fat to prevent them splitting. This rubbing of the interior and exterior surfaces with fat, blood, and other substances was described as doing 'nicely' (a:kən) with the pot, recalling the treatment of 'handsome' flesh (Chapter 5c:163; LL.VIII.23.8061'-8063') and establishing pottery manufacture as an 'understanding' domain. It required a specialised knowledge of (and access to) a range of raw materials, relayed by older relations (Suobbakən learnt from her aunt, LL.VIII.23.8059'). Assimilation of this knowledge allowed people to achieve the status of a $\mid k u n$-\|en (see glossary, a:kən:392), or person who worked nicely (LL.VIII.23.8056'), a positive epithet repeated elsewhere in connection with feminine activities; karosses could be 'made nicely' (Chapter 6b:189) and dancing rattles (springbok ears filled with seeds and sewn together, LL.VIII.1.6129') would only sound 'sweetly' if made by women who worked 'nicely' (LL.VIII.1.6131-6133). Manufacturing processes, then, were as integral part of the construction of adult, responsible, and socially meritorious identities as were strategies of food procurement.

Presumably also reflecting masculine bias, some of the most detailed information relating to material culture pertains to the production of hunting equipment, particularly bows, arrows, and their associated paraphernalia (LL.VIII.26.8290-

\footnotetext{
${ }^{160}$ Other 'gendered' constructions included the $\mid k u$ brush (made by men, LL.VIII.1.6082'), mat sieves (made by women LL.VIII.19.7640'-7641), and perhaps, as Ihanłkass'o spoke of traded beads as not being made by women (LL.VIII.22.7984), some types of bead.

${ }^{161}$ Dug out using sticks, a 'feminine' activity (Chapter 7c:255).
} 
$8302,8315-8334)$. This material was paramount in emic definitions of identity and in non-|Xam perceptions of these populations: arrow poisons were central to colonial presentations of Bushman hunting, livestock raiding and homicidal resistance (Voss, 1987), with more specific items were also being incorporated, such as a distinctive headband considered emblematic of Bushmen identity during the eighteenth and nineteenth centuries (Daniell, 1804-1805; Stow, 1905:71) and commonly featuring in visual depictions (Figure 7.5:266) ${ }^{162}$.

Arrow-shafts were made from reeds (!nwa:, Bleek, 1956:488) harvested on logistical forays, as water-dependent species were not distributed homogeneously across the arid Karoo environment. They were straightened using repurposed broken digging-stick stone weights (!kui LL.VIII.26.8295'), which imparted a 'smoothness' that allowed the arrow to fly 'nicely' (LL.VIII.14.7235) in the performance of understanding actions: as with pots, 'understanding' was implicated in hunting skills and knowledge regarding appropriate strategies, and in the manufacture of their material culture, providing another dimension of specialisation though which people might participate in resource acquisition. Unsurprisingly, the material culture of archery played particularly significant roles in the construction of masculine identities, social and individual. Bows were metaphorically employed to delineate a social identity as successful, brave, though possibly angry, men (Chapter 6a:175), but there was a more individualised component in their production, which was founded on the careful, personal selection of particular woods that were favoured as raw materials

${ }^{162}$ Probably the xaui (not given in the Dictionary); the notebooks referenced a xaui-ta-!nwa, referring to an arrow stuck into a band (LL.II.12.1215') 


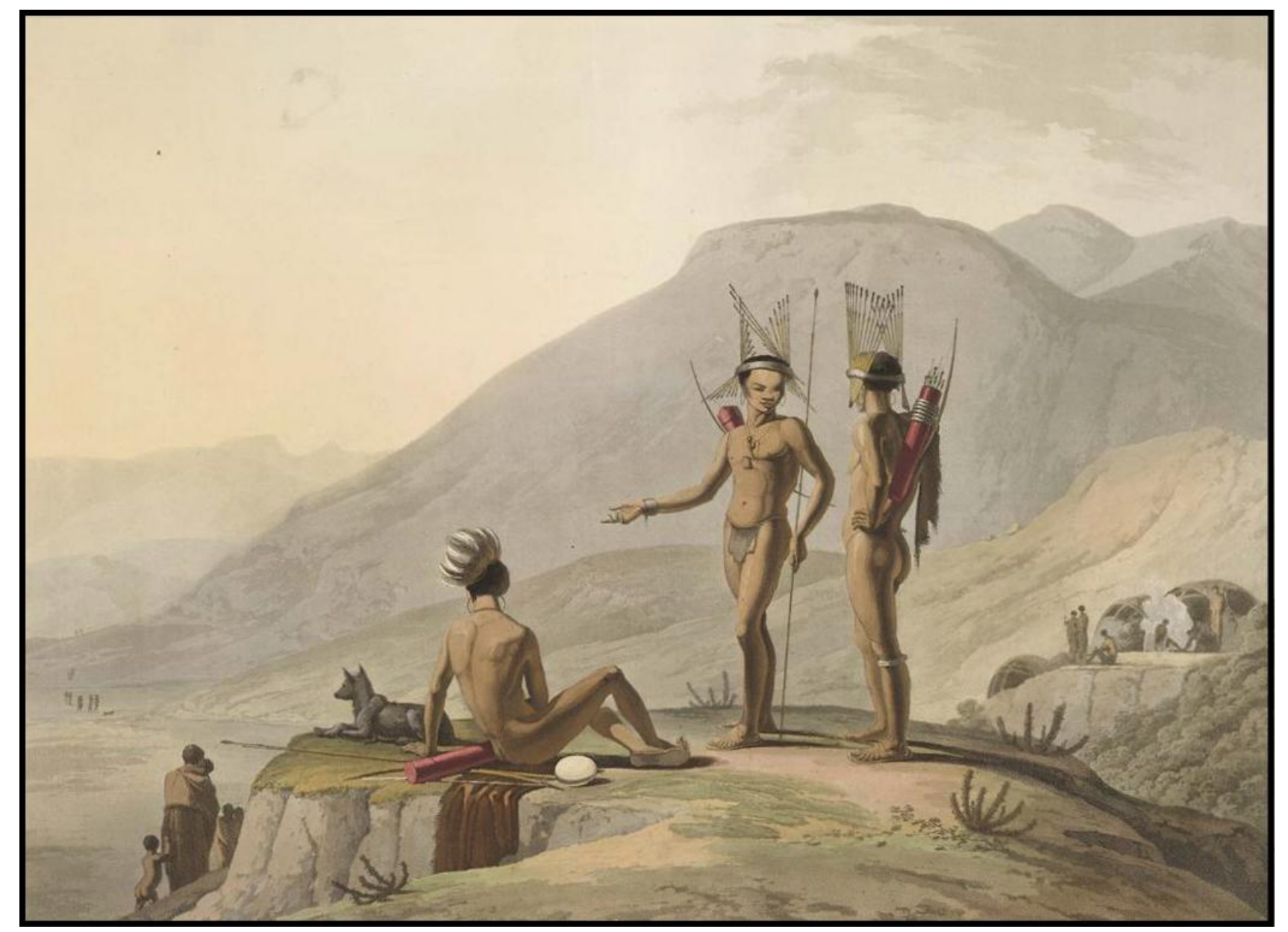

Figure 7.5: "Bushmen Hottentots Armed for an Expedition" (Daniell, 1804-1805) 
(LL.VIII.23.8059'). \|na: (Searsia sp., see glossary Igara:402) was used rather than !nabba (driedoorn) or łkarro (probably haakdoring, Acacia mellifera detinens), and this necessitated specific trips, in which men selected sticks, cleaned them and carried them back to camp to dry (LL.II.24.2178-2180). Bows (and arrows) were taken by male relatives after death (LL.II.13.1295), suggesting (through the salience of individual relationships in determining their disposal) a strong personal affiliation between hunters and their material culture. A link between personalisation and incisions was a recurrent feature of IXam material culture; the !au: (rib, Bleek, 1956:372; a spoon used to eat gambro), was inscribed with personalised markings identifying its owner (WB.XII.1121'). Just as arrows were marked individually (Chapter 7d:279), so too were bows personalised with fine lines incisions around the points of the bow ('bow's ear', LL.VIII.10.6891'; LL.V.24.5911), applied after the owner killed a baboon or hyena and thereby tracking his hunting experiences.

Arrows, perhaps the premier 'artefact of the mind' (J. Deacon, 1992:1) for nineteenth century Bushman informants, were typically regarded as being metaltipped, in an succinct illustration of the socio-economic importance of their interactions with non-Bushman populations. The most common term used to refer to arrowheads was !gwara (Bleek, 1956:391), denoting metallic objects in general and especially other edged weapons and tools; these were further distinguished with descriptive epithets such as łkarro-ka-!gwara (lit. haakdoorn's knife, 
LL.II.12.1175') ${ }^{163}$. More detailed descriptions of arrow manufacture deal primarily with metal arrowheads, which were beaten thin with stones (LL.II.19.1703) and sharpened or polished ${ }^{164}$ with the slate-like $|k o u| k o u$ stone (or |xo, LL.II.19.1702'; LL.VIII.21.8319'-8320). In his assessment of certain irontipped arrows (LL.VIII.1.6086) as 'ugly', |hanłkass'o located these items within familiar 'understanding' evaluative systems, but the widespread use (and presumed advantages) of metal arrowheads should not distract us from the fact that they formed only one manifestation of IXam projectile technologies; metal arrowheads were not necessarily functionally distinct from other forms ( $\mathrm{J}$. Deacon, 1992). IXam Bushman populations employed them as vectors for introducing poison ${ }^{165}$ just as they did with stone- or bone-tipped arrows, and the "introduction of metal arrowheads was as much a stylistic change as it was an exploitation of new raw materials" (J. Deacon, 1992:11). While iron was the de facto material for edged implements, its use was not universal: both $\|$ kabbo (WB.XX.1872) and Ihanłkass'o (LL.VIII.27.8434) noted that stone flakes might be used as knives ${ }^{166}$ when processing game. The knives were described (by $\|$ kabbo) as Flat Bushman productions made on 'Flat Place's stones' (in contrast to Grass

\footnotetext{
163 !gwara also referred to 'spears', often translated as 'assegai' (WB.IV.561): the 'exotic' connotations of this somewhat unfamiliar term arguably render it an apposite translation for the types of !gwara associated with 'different', non-IXam populations (Chapter 8b:302, 316). This translation was almost exclusively reserved for tools used in interpersonal conflicts: spears used to drive off or kill anti-social lions (LL.IV.2.3575) are better considered weapons rather than hunting tools (lions were not eaten, WB.1.263).

${ }^{164}$ Lexically differentiated as $\mid$ kou (sharpening, Bleek, 1956:321) and Inanan (polishing, not in Dictionary), though the $|k o u| k o u$ stone was used in both activities.

165 Arrows were identified closely with poison; !gao (poison) by extension referred to poisoned arrows (LL.VIII.14.7274-7275).

${ }^{166}$ Particularly in Early-Race stories (WB.XXXIV.2282; LL.V.1.3613; LL.VIII.32.8827'). If $\| k u r u$ ('stone knife', Bleek, 1956:593) is related to \|xauru ('knife of a certain kind', Bleek, 1956:634), the use of stone tools was fairly widespread, as these latter were used to shape bone tools in contemporary practice (LL.V.10.4765').
} 
Bushmen practices), and Ihanłkass'o also specifically stated that 'we' (presumably, the Flat Bushman group to which he belonged) used stone in the production of projectiles (LL.VIII.1.6086); variations in projectile technologies were therefore implicated in the production of group identity (Chapter 7a:221).

Much of the diversity of the material culture associated with hunting was coincident with the range of techniques employed. Arrows were carried in quivers, made either from kokerboom (Afr. quiver-trees, Aloe dichotoma, LL.II.26.2354') or from animal skins (LL.VIII.16.7423), or in the !nwa-ka-\|ho (arrow's bag, Bleek, 1956:542), the latter used only in fine conditions and when few arrows were needed (LL.VIII.30.8663'). When 'lying-in-wait' techniques were employed, head-coverings were worn to disguise the hunter (LL.II.23.2081), replacing the arrow-band (xaui) worn when running after prey (LL.II.12.1216). Arrows, especially, displayed this diversity, varying in terms of raw material choice for the link-shaft and point, presence of barbs, use of poison, and presence of fletching, all designed with particular prey species and their hunting strategies ${ }^{167}$ in mind: when hunting springbok 'singly' the !khau, an alternative form of springbok-arrow (without feathers and bone-tipped) was used. Pieces of wood called $\|$ kau\|kautan (LL.II.14.1330', 1388; LL.II.17.1602') usually formed the link-shaft of the completed arrow, allowing the reed body of the arrow to fall away from the wounded animal, and leaving the arrowhead embedded in the wound. The $\| k a b b a$ (a piece of eland bone) performed the same function for arrows that were intended for shooting eland (LL.V.17.5317-5320); although

${ }^{167}$ Burchell (1824:62) reported arrows designed specifically for use in raiding, extending this specialisation to interpersonal conflict. 
Dia!kwain did not make their purpose entirely clear, they were designed to fall off the arrow when it struck prey (people examined them to discover the prey species, so the hunter did not break !nanna-se by speaking of it, LL.V.17.5329'-5330'). Used for hunting ostrich and gemsbok, arrows with barbs (\|kukən, constructed from an ostrich wing-feather calamus ${ }^{168}$ and bound with sinew on to the linkshaft) were coated in poison (LL.VIII.31.8767', 8769', 8771). By contrast, arrows for springbok that were not poisoned (LL.VIII.1.6807) did not require barbs ${ }^{169}$ : they were called !gwara-@pwa (lit. arrowhead-child; Chapter 6b:187), and positively evaluated as 'clean' or handsome items (LL.VIII.31.8769'-8770'). Fletched with the feathers of large birds (Kori bustards, Karoo bustards, Eupodotis vigorsii, blue cranes, Anthropoides paradiseus, and vultures ${ }^{170}$ ), IXam assessment of well-made, handsome arrows incorporated an auditory component; the feathers of arrows that flew well made 'tearing' noises, while badly made arrows whistled or hissed (LL.II.36.3250'). The presentation of hunting material culture highlights the degree to which it was consequent on the practices deployed. IXam hunting strategies required particular tools, and, conversely, effective deployment of these tools required the specialised ethological knowledge arising in hunting experiences; material culture emerges as a domain subject to 'understanding' evaluations.

\footnotetext{
${ }^{168}$ Assuming 'wing-feather root' describes the thickened end of the rachis (LL.VIII.31.8767')

${ }^{169}$ It is unclear whether they possessed link-shafts: notes on picking up arrows when tracking the wounded springbok (probably fallen shafts) suggest they did (LL.VIII.26.8287-8292).

170 Applied to the lammergeier (Gypaetus barbatus), a montane species, in the South African Museum, this was mostly likely a reference to the widespread Cape vulture (Gyps coprotheres).
} 
Diversity in arrow type was matched by diversity of arrow poisons, which were associated with particular animal prey and with human populations from whom they were traded. Poison production was a critical component of IXam socioeconomies, and it was one that was not distributed equally between individuals and groups: Lichtenstein (1815:248) reported that Northern Cape Bushmen considered poison production an expert skill, which few people ever perfected. Arrow poisons commonly derived from plant sources, and blended masculine and feminine contributions to subsistence. Commonly collected by men (LL.IV.1.3474), they often required feminine 'digging out' (Chapter 7c:256) practices: !gwe: (malkopgif, Afr. madness-poison, or Boöphane disticha, Williams et al. 2008) and !kanna (not identified) were both extracted from bulbs. Use of insect species in arrow poisons amongst extant Bushman populations is well documented (Shaw, Woolley and Rae, 1963). The IXam also drew on these sources, using |kai (insect larvae that resembled the 'children of bees') and the abdomen of the $\| k^{\prime \prime}$ ururu (L.IV.2.3518-3519; Chapter 7c:250) ${ }^{171}$ in poison production, as well as other faunal resources such as Cape cobra (Naja nivea) and puffadder venom (LL.VIII.26.8328; LL.IV.1.3477-3479).

Although familiar with the requisite processing techniques, $\|$ kabbo did not know the 'parents' of $\mid k a i$ insects, obtaining them in trade with Grass Bushmen (LL.IV.1.3425). !gauukan poison or 'boom gift' (Afr. 'tree poison',

\footnotetext{
${ }^{171}$ Many Orthoptera accumulate toxins from their diet; Pyrgomorphidae, for example, incorporate cardiac glycosides from milkweeds, Asclepius spp. (Steyn, 1962)
} 
LL.IV.2.inset) ${ }^{172}$ was also acquired in long distance trade; łkasin bought it from Bushmen who lived farther away (from the colony), relying upon his increased contact with the colonial economy to acquire goods (sheepskins karosses, handkerchiefs, European tinderboxes, metal knives) that these more distant populations desired (LL.IV.1.3476). That such a vital component of IXam strategies was acquired through trade with geographically distant groups is a powerful reminder that IXam communities were not 'isolationist', instead depending upon their links with others: trading relationships with non-|Xam thus appear as highly congruent with (rather than an imposition upon) IXam subsistence practice (Chapter 8c:335). Local and specific knowledge was, however, valorised in the complex poison manufacturing processes; $\neq k u$ poison was produced by mixing a 'vegetable juice' plant with venom glands, the number of which was determined by whether the snakes had been taken from a 'strong' place or a 'bad' one (LL.VIII.26.8329-8330). The ability to produce this poison was therefore determined by knowledge of local conditions (where to find 'powerful' places), and possession of the requisite understanding concerning the snakes themselves. This was deployed in the familiar context of constructing 'understanding' hunting personae, as (for example) in contestations debating whether enough cobra and puffadder 'heads' (venom glands) had been incorporated to kill ostrich prey (LL.II.7.781-782).

172 Probably a poisonous Euphorbia: Gifboom today refers to Euphorbia virosa and Boesmansgifboom (Afr. Bushman's poison tree) to E. avasmontana. Descriptions of the poison as blackish or dark brown in colour are puzzling, as Euphorbias exude a milky sap; IXam poisons were commonly admixtures of various substances, which may have altered the colour of the latex. 
It is difficult from the texts to ascertain IXam investment in lithic production for projectile points, as descriptions of arrow manufacture do not focus these variants. Bleek and Lloyd collected only limited terminology relating to lithic production; the only obvious examples are $\mid k w e:$, to strike with a stone (Bleek, 1956:331) and !xu:, to collect stones (Bleek, 1956:502). Dunn's (1873) broadly contemporaneous account of Bushman knapping in the Northern Cape, however, implies the production of standardised formal stone tools. Although the informants stressed masculine labour in arrow production, Dunn observed the work of a female knapper ${ }^{173}$ and the description he obtained thereby again recalls the dangers of relying solely on these few individuals, and (particularly in this case) the limitations of the interview context that structures the archive. These limitations are also evident in the absence of detailed information regarding raw material selection practices: Bleek and Lloyd, without access to rock samples from their informants' territories, recorded little geological information. Several emic categories were outlined, the most secure of which was !goa (Bleek, 1956:383), usually translated as quartz or 'witklip' (Afr. 'white stone'). Deacon's (1996:266267) archaeological work in Grass Bushman territory identified a 'distinctive white chalcedony' that may represent a local manifestation of this category: the informants themselves applied this term to several samples shown to them, sharing properties of whiteness (LL.VIII.31.8728'; LL.VIII.1.6086) and lustre (LL.VIII.31.8728'; LL.V.22.5574-5575). Deacon (1996) suggests that variation in lithic raw material choice was an important component in differentiating between

\footnotetext{
${ }^{173}$ If these formal artefacts were the standardised microlithic insets usually assumed to represent arrow armatures (LL.VIII.1.6088-6091), then Dunn's account directly contradicts 'masculine' production of arrows (LL.VIII.23.8065').
} 
Grass and Flat Bushman identity, based on \|kabbo's (WB.II.375; WB.XXIII.2156') emphasis on Grass Bushmen production of witklip-tipped arrows (versus Flat Bushmen reliance on metal). Other lithic material types are more ambiguously described (Appendix E:488), though ssa-!nwa-|ka, (lit. 'Elandarrow-stone') a black stone presumably used in the manufacture of arrows, and related etymologically to $\mid$ kalkagən (blue stone, slate) can be tentatively assigned as hornfels (metamorphic alteration of a range of parent rocks that includes slate): certainly this raw material is common in the archaeological assemblages of the region (Beaumont, Smith and Vogel, 1995).

A recurring theme emerging from references to flaked lithic technologies is the largely ad hoc nature of these practices, and expedient production is noted throughout: 'stone knives' used to process meat were thrown away after use (LL.VIII.27.8434), these knives were used when they did not have a (metal) knife to hand (WB.XX.1872), and Bushmen who did not possess an axe (!k'o, LL.VIII.23.8065') ${ }^{174}$ used stones to split bones and break open tortoise shells (WB.XXVI.2487). Expediency in lithic use was mirrored in other domains of IXam material culture, and the paraphernalia of cooking techniques largely took this form: sticks were used as utensils, homogenising ostrich eggs before they were cooked or scooping out honey and insect larvae (Bleek, 1956:489, 670), stones and pits were used to regulate heat ${ }^{175}$, and tortoise shells, ostrich breastbones, and ox horns were all used as bowls, dishes, or 'shovels' (\|goro,

\footnotetext{
${ }^{174}$ The ! $k^{\prime} o$ is only mentioned in this note relating practices of Bushmen who did not possess one. ${ }^{175}$ Associated particularly with processing locusts; ! gwirri referred specifically to a hole used for roasting locusts (Bleek, 1956:393).
} 
LL.II.26.2374'). Musical instruments too were often ad hoc modifications of other types of material culture: sinews were used to tie wet springbok skin over the mouth of a pot, creating a drum as it dried and tightened (LL.VIII.1.6126'), and at least one type of musical bow (\|ha: or goura) was an ordinary bow with a quill insert attached near one end of the bowstring (Stow, 1905:109). Bags made of stomachs (!gwa:na and !kabba, used for carrying water, LL.V.11.4870'; !khoukan used to hold tobacco, WB.XXV.2355) were also used expediently; when a springbok was killed, its stomach, fastened by running a stick through the opening (LL.VIII.28.8502'), formed a vessel for transporting its blood back to camp (LL.VIII.23.8064). These expedient aspects of their material culture point to fundamental flexibilities in IXam subsistence strategies and socio-cultural practices, a significant dimension that would be largely invisible in the absence of the Bleek-Lloyd archive.

The use of material culture to construct masculine subsistence roles had a counterpart in associations between women's particular mode of acquisition $\left(\mid k^{\prime \prime} w a\right.$, Chapter 7c:256) and the production of digging-tools as a similarly feminine practice: women manufactured the digging-stick stones (!kwe:, also referring to the implement as a whole, Bleek, 1956:463) that provided heft for excavation (LL.VII.1.6046,5), grinding them out of a soft white stone ${ }^{176}$ from the Strandberg (WB.IX.906'; Figure 7.6:276), and attaching them to sticks collected

\footnotetext{
${ }^{176}$ W. Bleek's note was given only in English, but the use of 'soft white stone' as a phrase implies it was the translation offered for a particular, specific IXam term; !kue!kuerritan, which Ihanłkass'o described as a soft white stone not used in the production of 'stone knives', seems a likely candidate (LL.VIII.27.8433').
} 


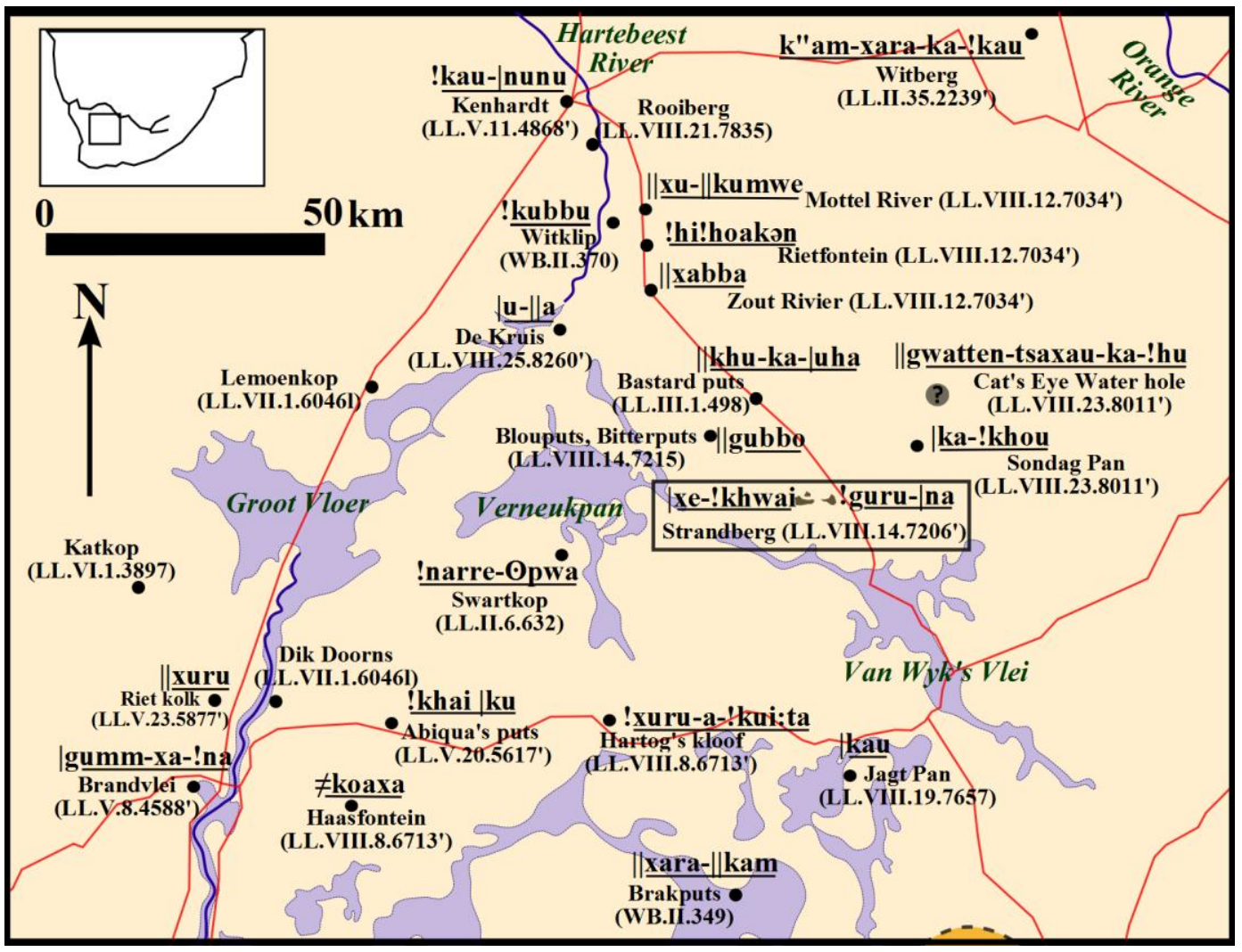

Figure 7.6: The Strandberg in the Kendhardt-Brandvlei-Vanwyksvlei triangle

(as with bows) from particular tree species. Gender identities were also marked through clothing; although larger karosses were worn by both men and women, 'aprons' or loincloths took different forms. Women wore the !kwobbo (LL.II.6.652) on the back and the t'wari in front, while men worn the $\|_{4} h i$ : ('forekaross', LL.IV.3.3709'), commonly made from jackals' skin (often referred to as a 'jackal'). Smaller karosses had, in general, a gendered aspect, as a whole range ("henni, !k'ausi and !e-si WB.XXVI.2457') were employed by women as carrying devices, usually for children: men used nets made of sinew for carrying objects (LL.VIII.23.8000-8001). Much IXam ornamentation, by contrast, did not 
denote gender, although seed-pod necklaces (LL.IV.1.3436) and $¥ k h w i$, (metal ornaments constructed from cut-up brass tinderboxes, tied into the hair with thread, LL.II.15.1440-1441) were both made and worn by women. $\neq k h w i$ were part of a suite of head ornaments tied into the hair and hanging over the face that connoted positively valued visual attributes; the 'brightly shining' round metal ornament called !uhi-!hoa ${ }^{177}$ (LL.II.15.1445'), which was fastened to hang down in the middle of the forehead, and the ! $k$ 'anni or 'beautiful' black and white head ornaments (LL.VIII.25.8230'), worn by men and women.

The ! $k^{\prime}$ anni sat in a collection of patterned, bicoloured objects, such as the striped Igi lizard (LL.VIII.32.8858'), the water's children (LL.VI.1.3941'), and the zebra (LL.II.3.417), that were classified as beautiful. Beads made of ostrich eggshell (or bone, LL.VIII.22.7984) may also have evoked this patterned beauty, as they were evidently coloured either by heat treatment or the application of ochreous pigments: Ihanłkass'o's commentary on beads was prompted by 'red and white things' seen in a rock art copy Lloyd had shown him. Worn as necklaces (LL.VIII.26.7826-7827) or in piercings (WB.1.304), there is little indication that they were associated with a particular gender: ear and nose piercings were 'handsome' traits for both genders (LL.II.2.360-361). Ornamentation, then, primarily referenced 'beauty', and descriptions of the Caracal in the Day-Heart story (LL.II.15.1440-1447) make it clear that distinctive, personalised ornaments were integral to her 'handsomeness'. Beauty was, in many contexts, associated with youthfulness, 'newness' and freshness (Chapter 5c:163, 165): youth was

\footnotetext{
177 This term is not given in the Dictionary, but may combine ! 4 hi (to cross, precede, pass, be upon, over, Bleek, 1956:495) with !hoa (to put, lay, Bleek, 1956:398).
} 
explicitly emphasised as a trait contributing to handsomeness in people (LL.II.13.1245-1246). Ornamentation that made people handsome may therefore have reflected age rather than gender divisions, and some ornamentation practices (especially the application of pigments) deliberately amalgamated handsomeness, youth, and masculinity (LL.VIII.15.7275'). By contrast with the lithic materials used in tool production, the soft ferruginous minerals used by the IXam as pigments are well documented. To: and $\| k a: \xi$ referred to red haematite (Afr. rooiklip, red stone), and \|hara most likely designated specularite. Precolonial specularite mining is known archaeologically from regions north of the Orange River (Humphreys and Thackeray, 1983:100-107), but the exact source of the IXam minerals is obscure, with accounts merely mentioning a 'mountain'. It was apparently collected directly rather than traded, and the pigments were all acquired from the same source (LL.VIII.14.7280): Zoovoobij (Smith, 1983:7) and Nauga (corresponding with 'Nougat, identified by Moffat (1854:158) as a site where Bushmen dug out pigments) are both possibilities. "hara was valued for its cosmetic qualities, eminently suitable for making young men 'handsome', LL.VIII.14.7275') and tied to positively evaluated 'fat' through its lustrous qualities (LL.VIII.14.7272'-7273', Chapter 5c:164). Red pigments were more complex, with a greater range of uses: the two categories overlapped considerably in use, although the linguistic differentiation was maintained (LL.VIII.23.8072'8073). The colour of the material was the primary factor in its deployment, determining its use by the New Maiden, in attempts to attract the rain 
(LL.VI.1.3969'; LL.VIII.16.7424) and to protect young men from !khwa:'s anger (LL.VI.1.3973; Chapter 5c:165).

The key difference between to: and $\| k a: \xi$ lay in the connection between to: and !gi:tən. 'Sorcerers' (presumably of the anti-social non-living type, Chapter $6 \mathrm{c}: 209)$ lived in holes at the to: mines, and made locusts out of this mineral (LL.VIII.14.7272'-7279'; LL.V.21.5709'): these they sent about as 'fighting things' (see Glossary entry, la::402) to cause illness (LL.V.21.5708') or a form of 'magic arrow' (Chapter 6c:203). Bushmen applied the same pigment to the reed shafts of their own arrows (LL.VIII.26.8288') in the creation of individual identification marks ( $¥ k e t t ə n)$. This association was cemented in exchange relationships that saw to: (and \|hara) being given in return for arrows (LL.VIII.14.7272-7273), as part of a larger series of exchanges between married couples, involving springbok skin bags, mineral pigments, and poisoned arrows. The 'individualisation' of material culture represented a form of diversification in production that underpinned some key areas of exchange between IXam communities. Although the exact structure of these exchange relationships is unclear, the complementary nature of the labour of the conjugal partners in generating them emphasises their dependence on social relationships. Tsatsi's acquisition of dogs from Igappəm-tu (LL.VIII.16.7456'-7458') evoked similar social dimensions, with female relations playing a vital role in his ability to complete the transaction. This exchange implied that delayed reciprocity was a feature of IXam exchange, as Tsatsi waited for the dog to kill a jackal and for the women to dress the skin before returning with a jackal kaross; this delayed 
exchange, when coupled with individual ownership of arrows, suggests that Jul'hoansi hxaro exchange networks (Wiessner, 1977, 1994) may provide broadly appropriate idioms for discussing exchange amongst IXam individuals.

Deacon (1996:269) highlights the dearth of scrapers (a typical tool of Later Stone Age assemblages elsewhere) in LSA assemblages of the interior Karoo. The IXam must either have been using more informal tools ${ }^{178}$ or non-lithic implements to dress their skins, for the archive suggests that the preparation and dressing of skins formed a major component of their material culture, one that moves beyond exchanges involving only |Xam individuals and introduces a triad of concepts that will become significant in Chapter 8 . Karosses were made from the skins of wild fauna, most commonly springbok (LL.II.3.453), but also from sheep-skins and goat-skins (WB.XXIII.2156'), incorporating livestock within a spectrum of subsistence resources rather than dividing wild and domesticated species. Access to livestock resources varied, and, while some IXam did own domesticates (Chapter 8a:282), the production of karosses was an important component in their labour relations with non-Bushman populations (LL.II.6.650). Finally, the use of łoro (implements carved from springbok cannon bones) to sew karosses was taken by the Grass Bushmen as a marker of the relative lack of Flat Bushman knowledge and experience of dealing with European material culture, and by extension of trading with European populations: references to karosses record the incorporation of new resources, the development of new socio-economic

\footnotetext{
${ }^{178}$ Perhaps similar to Nama use of $\| k h o m$ stones (Webley, 2005:159, 169); a powdered white stone called Ixwomma (LL.VIII.21.7834) was used by the IXam for working skins.
} 
strategies based on interaction with the new groups in their territories, and the discontinuation of other forms of practice or material culture.

These three themes are replicated with other forms of material culture. Tinderboxes (doro) employed by nineteenth century IXam were commonly of European manufacture; both Dia!kwain and Ihanłkass'o (i.e. Grass and Flat Bushmen) remarked upon the incorporation of this colonial material culture (LL.V.10.4759-4763; LL.VIII.28.8472'). Other forms of fire-making knowledge were retained, including the use of arrow-shafts to kindle friction-fires (LL.VIII.28.8471-8472) and manufacture of springbok-horn tinderboxes, but were indicative of 'poverty' rather than representative of 'proper' practices. Spoons, used to skim fat from soup (LL.II.14.1354) or scoop blood from pots (LL.VIII.23.8065), might be constructed from wood or the horns of springbok (LL.VIII.20.7811'), but sheep horns were equally typical, and the same word referred also to iron spoons obtained through trade (LL.V.4.4199'-4200). Although 'traditional' material culture (items that could have been utilised in the precolonial past) continued to play an important role in nineteenth century IXam life, it was increasingly being rendered redundant by changing behaviour. Conversely, new resources were increasingly incorporated as raw materials or as novel items within a creolised ' $\mid X a m$ ' suite of material culture, and new or changed practices (underpinning the deployment of material culture) that reflected participation in new relationships with non-IXam populations became widespread. 


\section{Chapter 8: |Xam Interactions}

\section{Chapter 8a: Engagement with domesticated species}

This chapter considers the influence of IXam understandings (Chapter 5 and 6) and subsistence activities (Chapter 7) on their relationships with a range of nonIXam individuals, exploring their emic divisions of Northern Cape populations: I include here relationships with domesticated animals and introduced forms of material culture under the rubric of 'interaction'. This section focuses on whether behaviours inherent in contacts with food-producers represented the disaggregation of IXam 'hunter-gatherer' identity and practice, exploring participation in pastoralism, labouring for immigrant European and mixedheritage groups, raiding practices, and trading engagements. IXam relationships with domesticated fauna were numerous and long-standing, incorporated into the practical exigencies of their everyday experiences, and thoroughly integrated into mythological narratives and significant rituals: moving approximately from greatest to least prominence, I discuss interactions with dogs, herds, and horses. 
Although the details are poorly understood, complicated by difficulties in separating domesticated from wild canids (Horsburgh, 2008), archaeological evidence confirms that interactions with dogs represent the longest-standing connection between southern African hunter-gatherer populations and domesticates (P. Mitchell, 2008). Fittingly then, dogs represent the most prominent domesticated species in the archive, although the distribution of

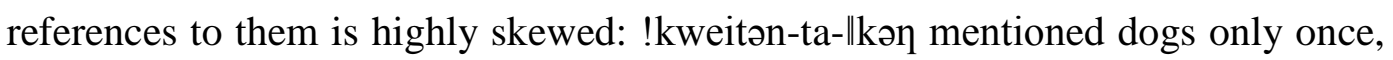
whereas Dia!kwain, łkasin, and $\|$ kabbo all discussed them frequently. Dogs were commonly characterised as extensions of hunter personae, closely associated with their owners (!kuin-ta-!kwi, 'dog's man', WB.XXIII.2158'), and it is in this guise that they emerge in male informants' narratives, a closeness of identity expressed in a manner consistent with the other inter-specific links (Chapter 5a:139). This relationship between the 'dog's man' and his animal was expressed by the incorporation of dogs within the connection between the successful hunter and his quarry: if a 'dog's man' broke the forelegs of prey killed by his dog, corresponding bones would break in his dog's leg when it was next running down game (LL.V.20.5604), while dogs that consumed inappropriately would cause the men to miss their aim (LL.VIII.14.7268) or injure themselves (LL.VIII.14.7269'). Like humans, dogs could develop special abilities or traits by amalgamating other scents and substances with their own identities; as dogs were in large part hunting tools, this often took the form of treatments for undesirable characteristics $(e . g$. the koro-ka-@ho plant made dogs willing to kill jackals, LL.VIII.21.7830). 
Dogs were thus sufficiently associated with humans to be subject to dietary restrictions; some of their rules, such as avoiding the springbok shoulderblades (forbidden to women, as this would cause men to miss their aim, LL.VIII.14.7263'), were identical to human proscriptions. Dogs, exempt from the 'cannibalism' proscription (Chapter 5b:149) on consuming the piece of flesh from the hare's thighs (LL.VIII.28.8398') and otherwise given food unfit (or undesirable) for human consumption (LL.II.25.2304-2305), were not, however, categorically identical with humans. The relationship between dogs and the New Maiden (who 'treated' her father's dog to make it hunt well) mirrored her treatment of his hunting accoutrements; before she could eat meat from game killed by this dog, she had to feed it meat mixed with saliva and dirt from her knee (i.e. familiarise it with her identity). Though dogs possessed their own agency, identities, and unique personal names (LL.VIII.26.8279'), their special status (much like those of |kaggan's 'hartebeest children', Chapter 5a:139) was connected intimately to their deployment as part of a hunting kit 'owned' by human agents. Dogs' actions were discussed in familiar terms: if they were not treated by the New Maiden, they would 'play' with game rather than kill it (LL.V.20.5597-5603) and do 'foolish things'. This inappropriate behaviour highlights the fact that a properly performing, competent dog was displaying 'understanding' behaviour, as part its deployment within 'understanding' hunting practice (itself directed by people).

The extension of identities between humans and dogs could be put to good use by these understanding individuals: if a baboon captured a dog, the hunters would 
misrepresent it as a 'girl's dog ${ }^{179}$, which would cause the baboon to treat the dog with respect and fear rather than injuring it (LL.V.24.5953-5956). This desire to injure dogs was situated in the context of baboons' antipathetic attitudes towards Bushman hunters (Chapter 6b:194), and by obscuring the 'dog's man' relationships the hunters presumably were intimating to the baboons that they had engaged inappropriately in interactions with animals that were not associated with hunting. IXam conceptions of dogs emphasised their superlative olfactory senses that constructed their protective or alerting roles. Dogs defended against intrusions of anti-social beings, paradigmatically lions (LL.VI.2.4020-4021; but also antagonistic !gi:tan, LL.VIII.15.7300-7301), into the domestic sphere, providing early warnings that allowed people time to prepare correct responses. Their relationships with non-human forces on the hunting-ground also facilitated appropriate action ${ }^{180}$ : $ł$ kasin’s dogs held down a hyena by the ears and thereby gave him the opportunity to shoot it (LL.IV.1.3465-3467), and Dia!kwain described them attempting to hold leopards encountered in the !kau:xu (LL.V.18.5442-5443), broadly paralleling the role of dogs in driving off lions in central Kalahari hunting practice (Ikeya, 1994:127-128) ${ }^{181}$.

IXam relationships with dogs were firmly integrated into their ontological understandings of the world; firstly, as tools contributing to hunting success, and secondly, as animals linked intimately to particular individuals. Combined, these

179 Parallel practice were performed with arrows, if the baboons pulled them out after they had been shot (LL.V.24.5920-5921).

${ }^{180}$ Dogs were not equally suited to all hunting interactions: łkasin tied his up to prevent them interfering with attempts to steal up to springbok undetected (LL.IV.2.3549-3550).

${ }^{181}$ Antithetical relationships between lions and dogs were also recognised by the IXam, forming a central dynamic in the Early-Race narrative of the young man who attempted to raise a lion cub as his dog (see glossary entry, !kúi-se-!khwi-|ku:427). 
two factors rendered their position rather different to that occupied by game species or wild carnivores, with their treatment being reminiscent of the strictures that all IXam were exhorted to obey for the good of the community: placed in the light of Ingold's (2000) trust versus domination model for discussing relationships between humans and animals (Chapter 2:39), IXam treatment of dogs fits more comfortably in the latter category, although metaphors drawn from familial obligations and responsibilities (i.e. factors that structured human relations) provide more appropriate referents than the slavery idiom employed by Ingold.

While it took different forms depending on the species, the first of these integrations was repeated in interactions with other domesticated species, as livestock were recognised as components of subsistence strategies: Dia!kwain even explicitly equated sheep with the all-important springbok. Dia!kwain's mother exhorted Ttanno-!kaukən (a !gi:xa) to let the springbok travel to her place because they lacked flocks $(g u-x u)^{182}$, presenting the demand for springbok as a requirement for an equivalent subsistence staple (LL.V.10.4742). Although the total number of domesticated species mentioned by the informants is elevated by occasional notes on animals such as pigs (Iho, Glossary:404; LL.VIII.22.7991; LL.VIII.23.8015) or fowl (denoted by the presumably onomatopoeic term kukuru, LL.I.2.135), sheep overwhelmingly dominate references to livestock, as is only to be expected for individuals used only to encountering Northern Cape farming practices (Chapter 4c:121). Though hunter-gatherer interactions with

\footnotetext{
${ }^{182}$ D. Bleek (1956:52) linked this the Nama $g u: i$ (sheep); it may represent a loanword. Dia!kwain translated it as vee (Afr. cattle, livestock generally), commenting that it referred to sheep or goats in particular (LL.V.10.4741').
} 
livestock and livestock-owning communities in the Nama-Karoo had a considerable precolonial dimension (Chapter 4a:88), experiences recorded in the informants' personal histories are primarily associated with European or Bastaard colonial populations and their domesticates. While this is not surprising given the dramatic consequences of colonial expansion into the region, it does raise the necessary caveat that these relationships are not necessarily appropriate analogues for precolonial interaction with these species.

Colonial populations characterised raiding as the quintessential 'Bushman' form of interaction with livestock (Chapter 2:31), and Bleek and Lloyd's informants were well acquainted with these practices. Ia!kunta, \|kabbo and Ihanłkass'o, and some of the minor informants (Jan Plat, LL.V.19.5454-5456), were all convicted for stock theft or for consuming stolen sheep and goats; their arrests emerged from personal involvement in the widespread raiding of the First Korana War ${ }^{183}$. Familiarity with raiding was reflected in its thorough incorporation into IXam schemes of understanding; "kabbo attributed the desire to steal livestock ('from hunger') to Ikaggən's influence on the 'thinking strings' (LL.II.4.501'; Chapter 6b:173). This link with 'thinking-strings' placed stock theft within the realm of foolish versus understanding action; the derangement of these strings by $\mid$ kaggan (usually 'cunning', often foolish, and frequently in antagonistic relationships with human agents) implies an unfavourable assessment of such action, a perhaps notunsurprising judgment from a man who was ultimately arrested for stock theft.

\footnotetext{
${ }^{183}$ Livestock acquisition was an important component of the Koranna War conflicts: a commission set up after the Second Koranna War recorded over six and a half thousand head lost (House of Assembly, 1880; Chapter 4c:118).
} 
The bokmakierie (Telophonus sp., a bushshrike) informed Bushmen (not involved in the theft) of other Bushmen stealing cattle, when coming to tell them of 'bad things which it knows' (LL.V.25.6021'). This belief too represents an example of 'understanding' behaviour, with sufficiently skilled people correctly interpreting these ethological occurrences to avoid danger and acquire useful information from afar about the status of resources (Chapter 6b:197). Dia!kwain's reference to the 'bad things' it told, and his statement that the bokmakierie should not be mocked ("gwitzn, see glossary entry k"e:nk"e:n:397) lest it cause injury (LL.V.25.6023), cast a negative light on the type of 'understanding' derived from interpretations of its behaviour: potential consequences of engaging in stock raiding (or sharing its spoils) thus undermined attributes and abilities normally considered praiseworthy or demonstrative of unusual skill. Even the premier socially-responsible practice, food-sharing, might have disastrous consequences if it involved stolen livestock; Kuirri-tu was killed by a farmer after he travelled to share in an ox (LL.VIII.11.6978'-6969'). As even displays of extra-ordinary potency (such as !nuin-|kuitən's foray in the form of lion to kill an ox, LL.V.15.5089-5090) did not provide protection from the consequences, participation in livestock-raiding practices becomes a compelling testimony of the degree to which IXam subsistence strategies were undermined by encroaching pastoralists.

Despite their familiarity with raiding, the dominant representations of interactions with sheep are in the context of herding. Although there are no narratives comparable to hunting descriptions that deal with herding experiences specifically, incidental remarks noting that particular events took place while 
herding sheep are scattered throughout; $\|$ kabbo encountered a $\| k h \tilde{a}:-k a-m u m u$ ('lion ghost') when driving sheep home after sunset (WB.XIII.2190'), and \|gooka-!kwi was out herding sheep when he 'saw the wind' (LL.VIII.8.6715-6724). In both cases, shepherding 'framed' events that would not be incongruous in the hunting narratives, presenting as an activity that took people out into the veld: though it did not require interaction with non-human forces to the same extent as hunting, it took people into the !kau:xu where non-human forces might be encountered at any time (Chapter 6c:201). Although interactions with sheep per se did not provide specific support for IXam notions of the motivations and capacities of prey species, they at least provided a space in which IXam beliefs could receive confirmation. This contiguity of herding and hunting or gathering strategies was also seen in their integration into socio-cultural practice. Man-sse received the nickname !kau-Inoan (Stone-Knee, Bleek, 1956:349, 412) after an injury obtained when he fell down running after the sheep. Practices of nicknaming (gen, Glossary:395; Bleek, 1956:46; LL.VIII.32.8808') were widespread, with people receiving additional names that referenced particular events in their lives. For men this typically meant incidents occurring while hunting: !khwai-!kwa (gemsbok-leg, Bleek, 1956:431, 457) was named for a gemsbok that stabbed his leg (LL.V.18.5419) and Dia!kwain's father received the name $\ddagger$ gwai- $\mid \mathrm{k} " \mathrm{a}$ (Clay-hand, Bleek, 1956:336, 649) after dislocating his thumb when stumbling in the clay, running in front of springbok (LL.V.20.5572-5573). Even when involved in shepherding activity, Mansse was embedded in a network of IXam-speakers who continued naming practices widespread among groups 
engaged in more 'traditional' subsistence practices, a succinct demonstration of vitality in IXam culture even in close association with pastoralist activities.

Male informants recounted personal experiences with these activities as part of their interactions with colonial farmers: Dia!kwain and Jan Plat recounted a herding route ranging widely across the south-west Hantam, in the course of their shepherding activities (Figure 8.1:291), as part of Dia!kwain's close association with the van Wyks (Europeans mentioned frequently by him, LL.V.10.4717-4718; LL.V.16.5446), whose farms were situated here. Informants' notes on sheep demonstrate highly pragmatic orientations founded in herding experiences, encountered in these transhumant strategies. Just as with hunting techniques (Chapter 7b:234, 239, 248), this included close observation of the behaviour of the relevant species: different techniques of physical interaction with wild and domesticated species were linguistically distinguished, with sswai describing 'driving' tame animals (which could be approached closely), and Igamme-sse the driving of wild animals by chasing them (LL.V.9.4618'). Sheep breeds were distinguished through their possession of particular physical and behavioural traits. They contrasted the 'Africaander' or 'Kaap' sheep (!gei, Bleek, $1956: 381)^{184}$ with the European-introduced Farland and Mof breeds (! $k^{\prime \prime} o: a$, Bleek, 1956:508), on the basis that the former returned to their kraals without being led, while the latter would not return from the veld unless herded back (LL.VIII.8.6717').

\footnotetext{
${ }^{184}$ Indigenous fat-tailed sheep breeds of the Cape; 'Africaander' presumably represents one of the extant Namaqua or Ronderib Afrikaner breeds, or a closely related extinct variety. All are mutton stock with fat deposits distributed around the tail and hairy rather than woolly coats, likely the result of selection for kaross production (Campbell, 1995; Smith, 2000:223).
} 


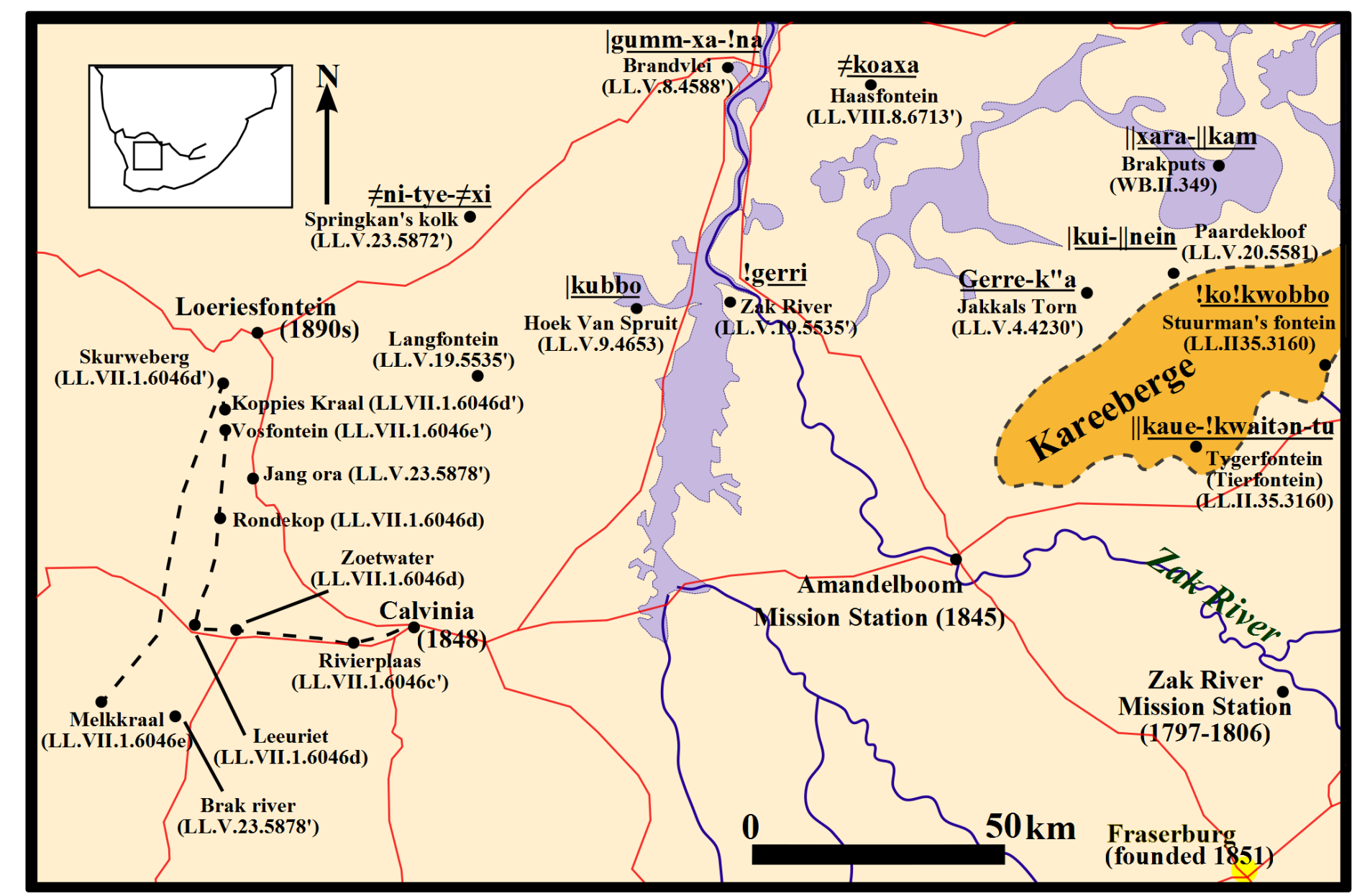

Figure 8.1: Shepherding route; łni-tye-łxi; ‘Jang ora’ 
Introduced breeds were separated into three categories on the basis of their wool, a phenotypically and economically salient feature with the rise of wool-production as the major impetus for sheep farming by the mid-nineteenth century (Chapter 4c:121): they were differentiated into 'Farland schaap' (vaderland skaap, Afr. fatherland-sheep) ${ }^{185}$ with thick hair, Mof sheep with hair that was 'so much and so big' (merinos), and a cross between the two (LL.I.2.184). IXam interactions with sheep did not incorporate these animals in isolation, and were situated in relationships with colonial stock-owning communities. Occurrences of European vocabulary (e.g. hamel, Afr. wether, LL.II.33.3022') for techniques of stock management indicate that much of their more specialised knowledge of domesticates drew heavily upon European or Bastaard sources, and was of fairly recent origin (though it is difficult to be certain that Afrikaans vocabulary did not overlay prior familiarity). These interactions were predominantly framed in a coercive idiom, as farmers attempted to control and direct IXam labour through the abduction of IXam children (WB.I.308) and the subjection of 'their' herders to disciplinary violence; both processes illustrated in a narrative concerning the fate of Ruyter ${ }^{186}$, who died after being beaten by a farmer for perceived deficiencies in his herding work (LL.V.23.5873).

Despite the herding experiences of male informants, however, it was !kweitən-ta-

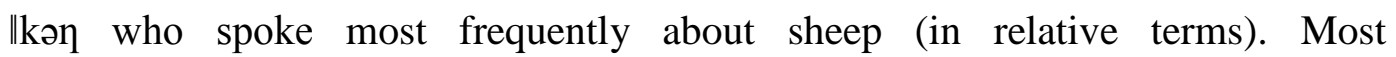

\footnotetext{
185 'Farland' is a corruption of vaderland (Bleek, 1956:508), used in the nineteenth century to refer to stock derived from European breeds (e.g. Bird and Colebrooke, 1823:235)

${ }^{186}$ Ruyter was the brother of Jan Plat; they were Grass Bushmen, living at $¥ n i-t y e-\Varangle x i$ near present day Calvinia. Ruyter grew up on Old Koos Struys' farm at "Young Gra", and was later murdered by this man's son (Figure 8.1:290)
} 
significantly, she recounted that after emerging from her seclusion the New Maiden, together with the xoakan-gu, consumed a sheep (LL.VI.2.4003): young men were specifically excluded from this consumption. This seemingly represents an intersection of Bushman and Khoekhoen beliefs around the locus of domesticated species, strikingly similar to menarcheal rites observed among a range of Khoekhoe groups, including the Nama (Hoernlé, 1918) and the Korana (Maingard, 1932:141-142; Engelbrecht, 1936), and extant among Griqua populations in the Northern Cape today (Waldeman, 2003:662). Their widespread occurrence suggests that such practices draw upon a common 'Khoisan' core (Barnard, 1992:252), and that, despite the obvious differences between Bushmen and Khoekhoe populations, their shared history and overlapping territories may have rendered certain cultural practices mutually 'meaningful'. Such meanings need not have been identical, and deployment of sheep in ritualised New Maiden behaviours likely derived from wider associations of the role in IXam society. The connection probably stemmed from sheep fat deposits, with indigenous southern African sheep breeds having distinctive large concentrations of fat in their tails. !khwa: was attracted to 'fatness' (Chapter 7b:239, Chapter 5b:153) and foodstuffs belonging to him, such as tortoises and porcupine tails, usually displayed this quality: the incorporation of fat-tailed sheep with ritualised treatments of the figure most intimately associated with !khwa: thus situates these domesticates in their logical niche, in terms of IXam criteria for resource evaluation.

Sheep and goats were occasionally employed in Early-Race stories in connection with the establishment of proper 'human' order. In one version of the Day's-Heart 
story, the contents of goats' stomachs were used to rub the (beast-of-prey) hair off the wild Caracal, restoring much of her 'human' (Early-Race person) identity

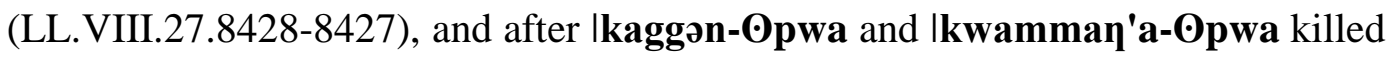
\|khwai-hem (releasing the things he had swallowed, Chapter 6b:182), the restoration of the sheep and their kraal made the place handsome (a:kan) once more (LL.II.34.3140). Small stock appear in Early-Race narratives as notably 'mundane' features, treated as objects belonging to Early-Race characters or groups much as they would belong to any contemporaneous pastoralist group (LL.II.32.2926); their significance comes from their incorporation into the strategies of these groups (e.g. motivating |kaggan's desire to visit the Ticks). Cattle and horses intruded only rarely in these Early-Race stories, being much more closely associated with the colonial situation ${ }^{187}$. As with sheep, brief references to larger livestock outlined concerns regarding their behaviour in the context of labour associated with their care, speaking of the timings for cattle eating, drinking, and sleeping in the context of tending herds (LL.VIII.1.60586059), interactions with domestic livestock at Bleek's house in Mowbray ('taking care' of it, LL.II.22.1956-1964; Chapter 6b:188), practical concerns about livestock diet (LL.IV.1.3473-3474), and evaluations of the relative abilities and value of domesticated species (mules versus horses, LL.VIII.24.8170'). These domesticates were sometimes linked explicitly to colonial scenarios, and horses were often experienced as tools of colonial populations, used to control IXam movement and facilitating violent action taken against them (LL.V.21.5703').

\footnotetext{
${ }^{187}$ Horses and other domesticated equines were only introduced into the subcontinent during the colonial period; derivations of Afrikaans terms (eseletan for mules, LL.VIII.24.8170' and baralpara for horses, Bleek, 1956:156) were commonly used for these species.
} 
Unlike Bushman populations that inhabited the south-eastern portions of the subcontinent (Challis, 2009:104-107) Karoo groups do not appear to have adopted horses: Collins (1808a, $3^{\text {rd }}$ February) recounted that practices of stealing horses were "unknown among the Bosjesmen of Karee Berg and the neighbourhood of Zak River" at the beginning of the nineteenth century. In part, this was related to environmental difficulties in sustaining horse populations there, and even experienced horse-keeping groups found the lack of water and dearth of pasture a major challenge (Jackson, 1879a:xxiii).

Some Bushman individuals by the mid-nineteenth century were well, however, enough acquainted with horse riding to judge distances in these terms (LL.II.35.3161), suggesting that familiarity with these animals on an individual level had developed by this time, either through horse ownership or through the increasing percolation of 'horse-knowledge' into the region as it became incorporated within the colonial purview. One of the few direct confirmations of IXam ownership of livestock (rather than interactions with animals belonging to other people) is present in Dia!kwain's reference to cattle he once owned, given to his wife by his father-in-law. Although his ability to control and look after them was limited, his account demonstrates vividly the benefits of being able to access even small numbers of stock (LL.V.9.4678'). After the death of his wife, he relied heavily upon the assistance of his female relatives, as he needed milk to feed his young child (Chapter 6b:187); Dia!kwain observed that had he maintained his control of the cattle, he would have been able to feed his child without familial assistance, which (given the deployment of constructions of responsibility and 
obligation in mobilising this assistance, Chapter $6 \mathrm{~b}: 187)$ points to the potential significance of domesticates in altering the terms of interpersonal relationships. The importance of secondary dairy products to pastoralist populations in providing subsistence without depleting herds is well known (Sadler et al. 2010); where colonial writers emphasised Bushman interactions with domesticates as 'improvident' (Chapter 2:31), Dia!kwain's discussion of dairy products as a fallback against the sometimes precarious nature of Karoo subsistence suggests emic IXam conceptions recognised secondary products as salient components of their interactions with cattle.

Although mentioned only infrequently, the existence of specialised terminology referring to domestic stock suggests that focusing on narrative appearances alone may misrepresent their wider significance. Recalling springbok terminology (Chapter 7b:238), a number of colour terms related exclusively to horses: $₫ a \neq a t t \partial n$ (Bleek, 1956:642) for 'schimmel' horses (grey and roan horses, Pettman, 1913:429) and !ai:tən (Bleek, 1956:370) for horses with a white blaze. Further cultural elaborations accompied this terminology, with horses presented as 'praise-worthy' animals that 'danced' as they cantered. They were associated particularly with women, who praised them by addressing them with an 'admiratory ejaculation' (a special click, LL.VIII.31.8736') and referenced them in '!kuppam', a women's play or dance. Although these treatments were discussed only cursorily, it is evident that horses were becoming well-integrated into the nineteenth century IXam socio-cultural milieu, even though they were not acquired by these groups. Such integration finds further confirmation in the fact 
that (despite not appearing as characters in Early-Race narratives) livestock formed key referents in these narratives. Oxen were, for example, linked with the superlative size of the eland (LL.II.10.1096'), which |kaggən 'worked' until it grew to become 'like an ox' (LL.VIII.6.6523); in another instance, their contemporary behaviour was used to evoke the actions of game during Early-Race times (LL.V.19.5457), providing a powerful 'experiential' manifestation of this period. The deployment of domestication as a referent for Early-Race animalhuman relationships cast relationships between pastoralists and their herds as 'outside' the correct social order, perhaps most similar to the Opwaitzn-ta-!gi:tən (themselves an 'extra-ordinary' group, Chapter 6c:209) who influenced the movement of game animals. These extra-ordinary connotations of influence over domesticated species can only have been strengthened by references to domesticates in rain-making practices. Both horses and cattle were connected with these practices, perhaps the most important domain of IXam ritual action: baraka-!hau!hau (horse's-thongs Bleek, 1956:156, 396) were evocative of !gi:tən abilities in influencing rain, as they 'rode' and 'bound' it for their own ends (LL.VIII.27.8399'). More commonly, the rain was likened to cattle, with distinctive characteristics based on gender: the rain-bull had to be bound with a thong and coerced out (LL.V.3.4075), bringing an angry rain (LL.II.25.2227), by contrast with rain-cow, which was 'gentle' and could be 'ridden' (LL.II.25.2238'). Contrasting with more 'ordinary' small stock, these larger animals were referents for 'potent', non-ordinary action, a distinction that implies a much greater degree of IXam familiarity with small stock. 


\section{Chapter 8b: The people who were 'different'}

Korana populations, the dominant indigenous nineteenth century pastoralist groups of the middle Orange River (Chapter 4c:116), are by far the most frequently mentioned indigenous pastoralists in the archive. It is likely that nineteenth century Korana groups incorporated immigrant Cape Khoekhoen as well as populations occupying the middle Orange River prior to historical documentation (Strauss, 1979; Penn, 1995); despite their awareness of huntergatherer populations living there (Chapter 7a:222), informants made no reference to the non-Korana pastoralist Khoekhoe populations ${ }^{188}$ occupying the region during the historical period. They did refer to the Namaqua (LL.V.22.5871'; LL.VIII.19.7675) on occasion, but none of the main informants had much contact with this group ${ }^{189}$; where these encounters did occur (on the western 'Grass Bushman' extremes of IXam territories) relationships seem to have been essentially similar to those obtaining between Bushmen and the Korana, with overlapping life histories of intermarriage and linguistic exchange (LL.V.23.5872). Historically recorded Korana (and other trans-colonial Khoekhoe populations) were rapidly reconfiguring their societies to incorporate the new

\footnotetext{
${ }^{188}$ And groups such as the Gyzikoa, incorporating Tswana influence (Penn, 1995:38-42).

189 Flat Bushmen informants suggested strong Nama influences on Grass Bushmen dialects (Chapter 7a:220).
} 
material culture (primarily horses and firearms) presented by the colonial scenario, and attempting to deal with the limitations of a colonial culture that sought to alienate them from their traditional subsistence strategies (Strauss, 1979). It is therefore difficult to judge the extent to which interactions between 'Korana' and Bushmen in the Bleek-Lloyd archive should be characterised as representative of precolonial relationships between IXam and Khoekhoe generally, as a reflection of their interaction with the range of 'Khoekhoe groups' that existed in the colonial period, or as an engagement with specific series of related groups that had recently (and rapidly) expanded their influence over the arid interior.

When considering interactions between IXam and Korana populations, łkasin (providing a relatively small number of narratives, totalling around five hundred pages) represents a salient source of information. With a Korana father and IXam mother (LL.IV.1.3452), he presented a unique 'insider' viewpoint on the intersection of Northern Cape populations: Bank $(2000 ; 2006)$ notes that łkasin’s mixed ethnic origins may have been reason enough for Bleek to focus his main attentions elsewhere (Bank, 2006:207) ${ }^{190}$. The truncated nature of łkasin’s account makes comparison with the other informants difficult, but some pertinent observations emerge from his narratives. Although marriage between pastoralist men and hunter-gatherer women is usually characterised as 'marrying up' (Bailey, 1988; Spielmann and Elder, 1994:308), with pastoralist men unwilling to adopt lower-status subsistence practices and identities, łkasin’s accounts make it clear

\footnotetext{
190 łkasin also focused upon 'practical' anecdotes (poison preparation, etc.) rather than the 'traditional' narratives in which Bleek was interested (Bank, 2006:209; Chapter 1:15).
} 
that individuals such as his father, married into Bushman-identifying groups, were subject to the norms of these societies through attempts to hold non-IXam to the standards of behaviour expected from responsible IXam adults: łkasin spoke of how his father's heart was made 'uncomfortable' because of his relative lack of skill in acquiring food for the children while łkasin himself was lying injured (LL.IV.1.3463'). Although in cultural terms (below, 303) the Korana were primarily represented as 'people who were different', the realities of nineteenth century Karoo life meant that there were many opportunities for personal life histories of individuals from both groups to become intertwined. The Korana had their own traditions of oral narrative (WB.XXV.2372'), and Bleek's first major engagement with Khoisan cultures had been through 'Hottentot fables and tales' (Bleek, 1864). Consequently, he was keen to control for influences of these traditions on IXam material, carefully recording 'overlaps' between these groups. The genealogies and convict records he collected point not only to the shared circumstances of arrest (LL.II.1.243), but also to an underlying similarity of frontier circumstances for 'Hottentot' and Bushman. Despite their brevity, they provide evidence for intermarriage, multilingualism, and shared experiences of colonial violence (WB.XXIV.2337-2338, 2351) and, in some cases, at least two generations of intermarriage are recorded, both in the (more common) form of Khoekhoe men marrying IXam women, and in the reverse, as with a IXam man married to an 'Africander' (a group of Oorlams) woman (WB.I.12').

The prehistory of the linguistic relationship existing between !Ora and IXam is difficult to reconstruct with certainty; assuming a relationship between the spread 
of the Khoe language family (the Khoekhoe branch, or its antecedents) and the spread of at least some form of pastoralism, the connection between the two can be characterised as the most recent phase of a series of linguistic frontiers that may stretch back over two thousand years ${ }^{191}$. Combined with the paucity of detailed linguistic analyses of languages spoken by southern Khoisan populations, this potential time depth for interaction obscures the relationship between the families. In the historic period bilingualism in the two languages was common, although both also formed distinct linguistic communities at least until the early decades of the nineteenth century (Traill, 1995:9-11, 2002). Khoekhoe and southern Tuu languages (in particular the !Ui branch, including IXam) share many features (Güldemann, 2006, 2006a); in some cases, directional borrowing of lexemes may be inferred, and Güldemann (2006, 2006a) identifies several terms that likely moved from Tuu languages into southern Khoe, raising the important point that (just as with marriage) often-implicit assumptions about directionality in influence between pastoralists and hunter-gatherers are not necessarily accurate. Words certainly did move into IXam from pastoralist communities: goat (peri, WB.XXV.2400) was a loanword deriving ultimately from Bantu languages (Haacke, 2008:169), presumably moving via Nama (pirip) or !ora (perip, WB.XXV.2400). Such transfers, however, cannot simply reflect a coincidence between contact with Khoe-speakers and contact with domestic livestock, as terms for livestock (for example) do not appear to expected to be derived

\footnotetext{
${ }^{191}$ Though archaeological evidence confirms the presence of domesticates by this time, opportunities for reconstructing the (potentially corresponding) linguistic entities are limited; Güldeman (2008:120) situates the expansion of Khoe-Kwadi languages through the southern African region a few centuries before the arrival of the Bantu.
} 
exclusively from Khoe languages: xoro (cattle) bears little resemblance to Nama or Korana counterparts (gumap, WB.XXV.2388).

Commonly, the IXam referred to the Korana with the term !kuarra (Bleek, 1956:448), closely resembling a term that was used for Bastaards, !kwa:ra (Bleek, 1956:461). Whether these terms were meaningfully distinguished (rather than pronunciation variants) remains obscure in the absence of more complete phonological analyses of IXam, but the similarity suggested that defining 'Korana' as the nineteenth century manifestation of a more general 'Khoekhoe' category may not be inaccurate. Like other 'outsider' populations, the Korana were conceived of as being physically distinctive: Dia!kwain considered them to have a characteristic 'whiteness' and beauty (LL.V.12.4926'), almost certainly ${ }^{192}$ employing $\mid k o: \xi w a$ (Chapter 5b:163) in an oddly positive choice for a description of the (usually anti-social) Korana ${ }^{193}$. Equally, they engaged in several distinctive habits, some of which were shared with non-Swa-ka-!k'e Bushmen groups (Chapter 7a:219): the eating of baboons (LL.II.7.797') and fish or shellfish (LL.IV.3.3736'), the carrying of items on their heads (LL.II.13.1266'), the making of particular types of sieves (LL.V.25.5993), and the wearing of particular ornaments (made from wood-pigeon tails, LL.VIII.25.8261) were all mentioned as distinguishing practices. Conversely, certain practices were notably for being shared by, or having comparable manifestations in, both IXam and Korana society: "kabbo noted Korana use of ' $\mid u$ fo- $a$ ' ('their term', LL.II.36.3242)

\footnotetext{
${ }^{192}$ This note was in English only.

${ }^{193}$ It may reflect connections between livestock and fat (Chapter 8a:291), and fat and beauty (Chapter 5c:162)
} 
paralleled the |Xam use of $\int o-\mid \tilde{o} \ddot{a}$, while descriptions of $t o$ : imply that Korana and IXam both used this mineral for dressing their heads (LL.VIII.14.7272').

Despite these shared features, and despite the 'biographical' evidence for intermarriage, in 'cultural' terms the Korana were overwhelmingly associated with inappropriately directed or unregulated violence, prototypically antithetical to IXam notions of propriety. The common epithet for the group (xuaken-!ku, 'bloody-browridges', LL.II.32.2960') referenced a homicidal stereotype, and another alternative name, !giri-\|kein (Bleek, 1956:382), appears to connote stabbing or piercing $\left(\|\right.$ kein, Bleek, 1956:568) ${ }^{194}$; the use of spears in conflict being a notable 'Korana' trait (LL.VIII.21.7968') as part of a suite of violent forms in their material culture, including particular kinds of knife (LL.VIII.18.7606), firearms that they acquired from Europeans through cattle trading (LL.VIII.18.7606'), and ornaments that were bound on for 'war' (la:, LL.VIII.25.8261). Even the practices intelligible to the IXam were structured by this violence: where the IXam incised their hands to improve skills in shooting springbok, parallel Korana practices were considered attempts to make their attacks on people efficacious, with incisions on the left hand helping a person aim when shooting at their fellows and those on the right rendering deadly their blows in fist-fighting (LL.II.36.3244'). The virtually ubiquitous role of the Korana in the more formalised narratives was adversarial, whether in the Early-Race times

\footnotetext{
194 !giri is not in the Dictionary: it may represent !gerri, the Orange River (Bleek, 1956:381), the heartland of Koranna territories.
} 
(LL.VIII.28.8494) ${ }^{195}$, or in 'legends' such as Ikarran's escape from the Korana commando (LL.VIII.26.8269). Regardless, Korana 'commandoes' ( $k$ "a:o) were a danger to Early-Race people (LL.VIII.18.7593; LL.VIII.25.8251) just as much as they were to nineteenth century IXam groups, for whom being 'killed by Koranas' was a real danger, of a piece with lion attacks, starvation, and disease as a component of the causes of death collected in the genealogical information (LL.III.1.497).

Integration into more stereotyped forms of narrative creates two distinct presentations of 'Korana' in the archive. Korana individuals had very real impacts on personal histories and genealogies, and increasingly shared with the IXam trajectories of interaction with colonial populations; at the same time, Korana attempts to acquire access to horses and firearms, and their adoption of more aggressive raiding lifestyles (Ouzman, 2005), meant that Korana violence remained significant in their historical relationships. The coding of violent action in their appearance in Early-Race narratives (LL.VIII.18.7593; LL.VIII.25.8251; LL.VIII.28.8486) suggests either that the Korana (unlike other nineteenth century populations) were swiftly associated with a stereotyped cultural role, or that '!kuarra' referenced longer histories of interaction with indigenous herding 'Khoekhoe' populations in addition to nineteenth century interactions. Other nonIXam populations are present only in their nineteenth century colonial context (personal histories and memorates), with little evidence from the Bleek-Lloyd

195 The Early-Race Ticks have also been characterised as a cipher for Khoekhoe populations (Hewitt, 2008 [1986]:188; J. Deacon, 1994), with both groups being prone to violent, angry action, and possessed of domesticated stock. 
informants for the assimilation of European, Bantu-speaking, or creolised populations into more stereotyped narrative forms of narrative. Although when thinking about global trajectories of colonisation these three populations might be considered separately, the Bleek-Lloyd archive presents these European and nonEuropean groups as contiguous components of the colonial situation: a number of non-Europeans populations were themselves colonising IXam territories for the first time in the nineteenth century (Kallaway 1982; Anderson, 1985), while groups undergoing ethnogenesis at this time (particularly those with a 'mixed' socio-cultural heritage, Oorlams and Bastaards) instigated a novel series of encounters with the $\mathrm{Xam}^{196}$.

Hewitt (2008 [1986]:32) remarks on the lack of emphasis in the stereotyped narratives on incorporating contemporary colonial conditions: documenting these conditions was not a primary interest for the translators (who attempted to record 'traditional' folklore). The incorporation of contemporary situations within the narratives, as Guenther $(1989 ; 1999)$ notes for twentieth century 'farm' Bushmen (Nharo and Heillom), does not appear in the Bleek-Lloyd collection. Guenther (1999:103) casts these narrative forms as a mechanism of resistance to colonial relationships, reminiscent of protest literature; the extent to which IXam informants would have been comfortable relating similar tales to Bleek and Lloyd, their baas (Afr. boss, often deployed in contexts of unequal labour relationships)

\footnotetext{
${ }^{196}$ Indigenous engagements with colonial populations could also occasion changes in pre-existing interactions with surrounding or coterminous groups.
} 
and 'mistress' ${ }^{197}$, is unclear. However, while relatively few narratives have interactions with white, black or Bastaard farmers as their primary focus, there are very many allusions to the colonial presence throughout the archive (especially in the supra-narrative framework of reverso notes and working-in-progress translations), and even this skewed account provides much information about the informants' colonial relationships.

Even vocabulary and grammatical work can elucidate these relationships: a sentence contrasting the 'stout' (!kui:ja, big, great, abundant, Bleek, 1956:450) Dutchman with the 'small' (łerri, small, thin, Bleek, 1956:643) Bushman (WB.I.40), establishes particular physical attributes as distinguishing markers of European identities. Incorporating phenotypic variation, these distinctive features were situated in a wider cultural schema, as łerri and !kui:ja were traits associated with concepts evocative of the relationship between IXam and European colonists. Thinness was linked with starvation, ugliness, and hardship (LL.II.14.1321-1322), describing the Moon as it decayed (LL.II.6.658) or the kaross that would not keep one warm (LL.II.14.1341), connoting inadequacy or privation and according well with IXam characterisations of their standing with Europeans as reminiscent of the 'orphan state' (Chapter 6b:186; Chapter 8c:333). However, 'smallness' was also sometimes portrayed as a positive trait: the smallness of the Caracal's head was a constituent of her handsomeness (LL.II.15.1450-1451), the small pot was made 'handsomely' (LL.VIII.23.8059), and the 'small-eater' who controlled his appetite

\footnotetext{
${ }^{197}$ Bleek tended to receive more formal address (Bank, 2006:87, 231), but even the more familiar terms applied to 'Miss Lucy' still connoted a degree of formality; nooi was explicitly translated as 'mistress' (though this may have been a construction of the translator).
} 
(Chapter 6b:183) all place notions of 'smallness' within the realm of the construction of 'appropriate' IXam sociality. This may be contrasted with the negative aspects of 'greatness'. The immoderately consuming $\|$ khwai-hem was not a 'small man' (!kwi-@pua, LL.VIII.20.7812) and the lion with a 'great' body swallowed down even bones to fill his stomach (LL.II.18.1670): leonine characteristics emblematic of censurable social behaviour (Chapter 5b:159) were thus mobilised in depictions of European characteristics.

Consideration of the meta-narrative commentary sets even apparently 'ahistorical' information ${ }^{198}$ within this context. Reverso asides occasionally relate more formulaic narratives to contemporary situations, giving insight into the on-going relevance of the information they contained. By pointing out that lions had become much scarcer in Bushmanland (LL.VI.2.4033'), !kweitən-ta-\|kən placed herself firmly in a context where increased presence of European and Bastaard farmers, and the ready availability of firearms, had cemented processes leading to the local extinction of this species (Beinart, 1998:180). This extirpation had occurred on a scale perceptible within individual lifetimes; !kweitən-ta-\|kəך's note was a comment on her (older) sister's cautionary story about the correct 'understanding' responses to lions that called out to people (LL.VI.2.4026-4033).

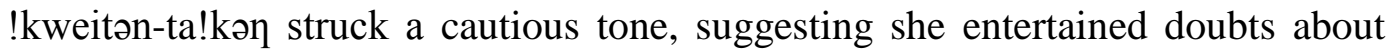
the veracity of the narrative or that her own (limited) experiences of interactions with lions had not reinforced this information. As part of the construction of 'understanding' actions, this story illustrated the utility of the old women's

${ }^{198}$ That is, material that might be (or was) represented as indicative of 'traditional Bushman mythology' or belief. 
teachings, as by listening to these individuals the protagonist was able to escape the lion. !kweitən-ta-\|kən's acknowledgement of the contemporary scarcity of lions therefore provides a cogent example of the manner in which knowledge and belief once reinforced in practical experience was swiftly becoming less relevant to Bushman life; Dia!kwain's note about his elder sister's 'understanding' encounter with an owl was firmly situated in a time before 'the white men's houses' were at Kenhardt (LL.V.11.4868'-4869) and echoes similar themes. Broader patterns in the narratives reflect these same issues in 'passive' fashion. Hunting narratives reflect, in part, the vulnerability of species in the face of pastoralist trekboer encroachments: the dominance of porcupines in Dia!kwain's narratives may reference this differential resilience (Chapter $7 b: 249$ ) and the dearth of large-game (hartebeest, eland, kudu) hunting narratives probably incorporates the rapid depletion of these fauna in the wake of farmer settlement (Beinart, 1998:179). Arid-adapted, fast, and wary animals that even with modern firearms represent challenging quarry (Heath and de Bod, 2000:11), gemsbok survived in the central Nama-Karoo through the entirety of the nineteenth century, albeit at low densities (Roche, 2008:167): this species was the subject of the few detailed IXam hunting narratives that focused on larger bovids (Chapter 7b:231). Though relationships between antelope species and supernatural figures were important factors, the evident importance of personal experience in directing the hunting narratives recounted (each informant focusing on a different suite of species and techniques) suggests that the decreased likelihood of encounters with 
easy or favoured targets of colonial hunters also had an impact on the relevance of these narratives.

This theme is also present in one of the few Korana narratives in which they did not play an antagonistic role, though here it is reversed (with certain beliefs becoming more significant). This fragmentary story discussed the theft of a Korana child by a troop of baboons (WB.I.171-177), and is highly unusual insofar as it involves IXam and Korana protagonists acting in concert against the baboons. Baboons formed one of the more coherent anti-social, non-Bushman collectives in IXam thought (Chapter 5c:162), in some ways quite similar to the Korana: their epithets even referenced the same anatomical structures, with the 'overhanging cliffs' of the baboons' foreheads (LL.V.24.5931') paralleling the 'bloodybrowridged' Korana. Several Early-Race and legendary narratives emphasise conflicts between baboon and human individuals; they killed lkaggən's son and 'played with' his eye (LL.VIII.11.6978-7014; LL.VIII.12.7065-7094), and when |khui-|a ${ }^{199}$ killed a female baboon (while hunting) the male baboon confronted and spoke to him, causing him to abandon the body (LL.V.23.5890-59011/2). \|xabbitən\|xabbitən's anecdote (LL.V.24.5931-5947) about his encounter with baboons was firmly situated in the relationships of the colonial period, with \|xabbitən\|xabbitən returning from acquiring a gift of flour from 'white men'. The baboons here acted much as they do in other narratives, talking to each other and becoming angry with $\|x a b b i t ə n\| x a b b i t ə n$, exhibiting a desire to 'play with' his

\footnotetext{
199 The narrative begins with the phrase 'the baboons used to do as follows' (LL.V.23.5889), reminiscent of phrasing characteristic of Early-Race stories, though (unusually, if this is an EarlyRace fragment) there is no confirmation of this status.
} 
head. "xabbitən\|xabbitən's solution to his problem was tied explicitly to the new resources of the nineteenth century: he called out to the white men, taking advantage of the fear that baboons displayed towards guns, and bluffed his way into escaping without injury. The motivations and actions of baboons were similar in Early-Race, legendary, personal history narratives, which led to fundamentally similar interactions established with human persons in each of these contexts. Contrasting with the declining significance of lion encounters, beliefs about the anti-sociality and special abilities of the baboons remained an experientially significant element of IXam life in the nineteenth century: this was founded partly on the fact that this species, with its catholic subsistence strategy, occupation of relatively inaccessible habitats, and wariness, was well-suited to surviving extermination attempts, despite being designated vermin and maligned by farmers for stock depredation (Beinart, 1998; van Sittert, 1998:341).

Perhaps the most striking feature of references to the historical situation is their depiction of the narrators' concern with the manifestations of unequal power relations and casual violence in the 'border districts' (Chapter 4c:116), manifestations that crossed 'ethnic' boundaries and incorporated the actions of all non-IXam groups. This violence was not one-sided, forming a significance mode of IXam engagement with colonists: the archive documents Bushman violence directed towards colonists ${ }^{200}$, through the well-known murder of Jacob Kruger on the part of Dia!kwain and łkasin (Bank, 2006:218-223), and in references to four men arrested for killing a European farmer and his family (WB.IX.908').

\footnotetext{
${ }^{200}$ Given the status of the informants as convicts, their discussions of their participation in or knowledge of (colonially defined) illegal activity are obviously to be treated cautiously.
} 
Mentions of colonial violence directed toward IXam individuals range from brief annotations to personal accounts describing particular occurrences: vocabulary and grammatical work also referenced it (LL.VIII.14.7246'), with one of the very first sentences Bleek collected (WB.I.1) recording abduction of IXam children. Visceral descriptions in longer narratives give some indication of the impression that beatings (administered even to the point of death, LL.V.23.5874-5880) had on both the observers and recipients of such actions. Although often associated with stock theft (or consumption of stolen stock), homicidal violence of a more arbitrary nature also occurred, described as being shot for 'doing nothing' (LL.V.3.4132'-4133'). Sparse genealogical data reveal the range of violent relationships established, with mentions of children 'taken by the Boers when little' (LL.III.1.488-489), of members of extended families being killed by commandoes for eating stolen livestock (LL.VIII.11.6978'-6979'), and of wives abducted by farmers (LL.II.1.488'-489), while informant accounts of capture and arrest (LL.II.1.269) document 'Weberian' violence, as the colonial government attempted to claim a monopoly on legitimate forms of interaction between the colonial polity and IXam groups ${ }^{201}$. Colonial violence was readily assimilated into structures for understanding violent action more broadly, imbuing it with power and making it 'meaningful' (Scheper-Hughes and Bourgois, 2004:1), but also reconfiguring it for the IXam social world: Korana 'commando' practices were well-integrated into |Xam folklore and equivalent colonial practice must have readily been understandable in these terms. Avoidance of colonial violence

\footnotetext{
${ }^{201}$ Forms of 'commando' violence enacted on IXam populations by colonists in the border regions were often considered illegitimate by the colonial state (Chapter 4b:104).
} 
became incorporated as 'understanding' action: the same presentiments or beatings that warned of impending attacks by beasts-of-prey might also warn people when a wagon was approaching their houses (LL.II.28.2530'), while Dia!kwain recounted a dream concerning a farmer threatening to kill his father and himself (LL.V.15.5110-5111). Both representations assimilated colonial agents as a new danger within the suite of anti-social forces that might afflict |Xam groups.

The role of stock-raiding in promoting conflict was well recognised; genealogical information collected for Jacob Bastard, who lived at "Oud Bastard se pits" (Figure 8.2:313), noted that his habit of killing cattle 'greatly' prompted unsuccessful attempts to catch him (LL.III.1.498). Dia!kwain stressed notions of proportionality in farmer responses to stock theft, emphasising discrepancies between the severity of the crime and colonial responses to it (LL.V.15.51145115), in a succinct illustration of Newton-King's (1999) observation that commando violence reflected the necessity of coercion in establishing essential labour relationships, which the farmers required to maintain large herd surpluses allowing trade with the colony for vital resources. High demands for labour meant that people could mobilise this demand to secure their subsistence: after her husband was killed, !kwarra-an travelled to the south-west of Calvinia, where she was easily able to gain employment with farmers, despite travelling with her (dependent) children and her inability to speak Dutch. The importance of labour also meant that colonial violence was often directed toward attempts to control the movement of people, by assigning them farm residences or employing people in 


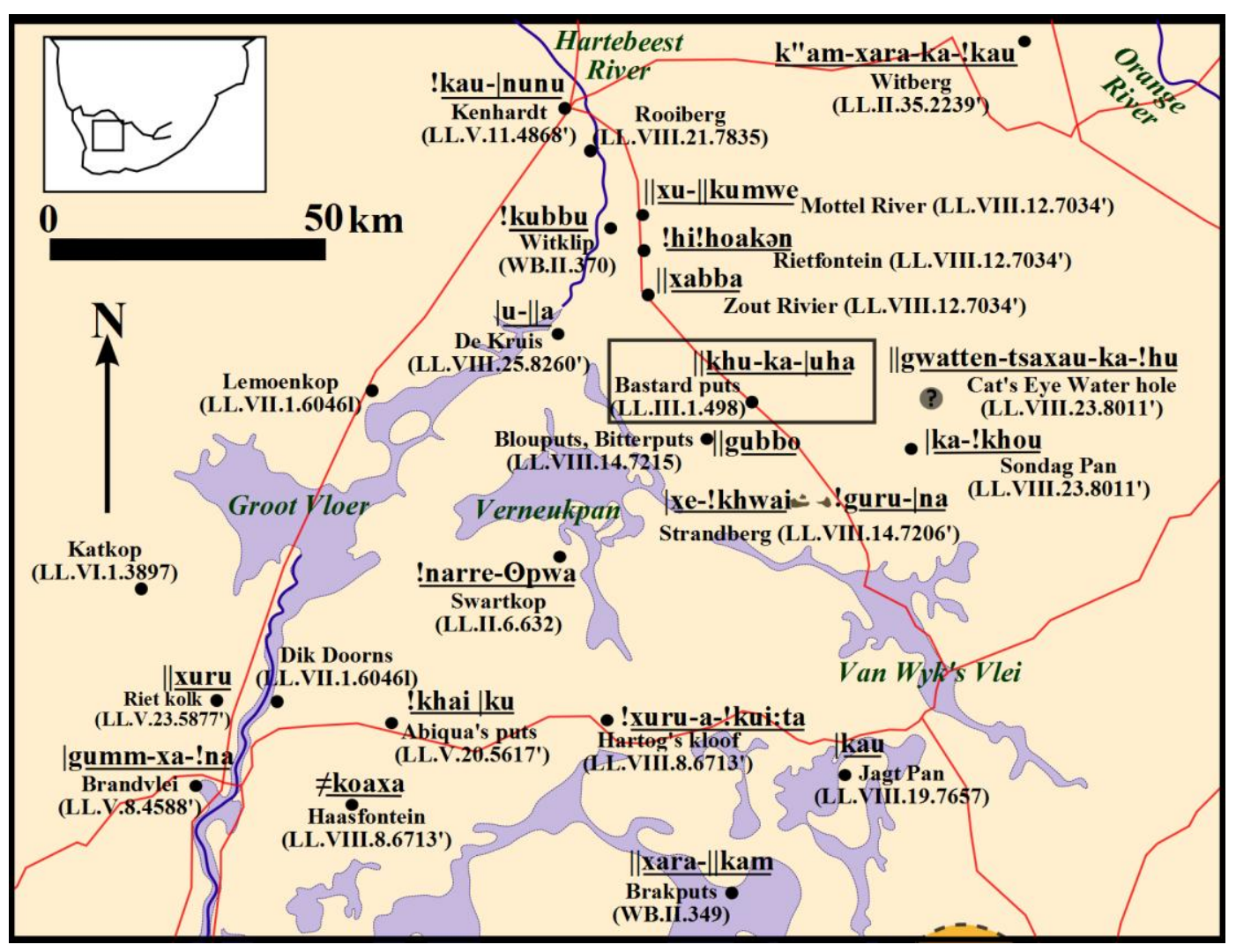

Figure 8.2: Bastard Puts in the Kenhardt-Brandvlei-Vanwyksvlei triangle

transhumant strategies. IXam subsistence strategies demanded a high level of residential mobility as movement in response to key resources was vital (Chapter 7d:264-265), but this took place within well-known territories with local environmental knowledge underpinning resource 'management' (Chapter 6b:188). This mobility was perhaps accompanied by occasional longer-distance travel; there is no indication that la!kunta's visit to a mountain outside of Bushmanland (which $\|$ kabbo had not seen, LL.II.25.2239') was connected with colonial interaction. Colonial translocations did not respect earlier boundaries and often occurred over large distances, with commando practices explicitly aimed at disrupting connections with the land to inhibit resistance opportunities (Penn, 
2005:110-112). Coupled with the unpredictable violence of the border regions, this ensured that Bushmen existed in a state of uncertainty regarding the whereabouts and situation of friends and families. During Dia!kwain's imprisonment, a Bastaard took his wife to Klaarwater (Griquastad) and his mother was supposedly killed by the farmers; although his siblings searched for her, they were unable to uncover any definite information (WB.XXV.2413').

This uncertainty about the condition of family members (particularly children) and friends was a definite source of anxiety for the informants (LL.VII.1.6046,5'), and this separation of social groups contributed significantly to undermining the transmission of cultural knowledge and language: D. Bleek (1936b) attributed loss of narratives among IXam-descended populations to their being 'driven about' while young (Chapter 4c:125). Fragmentation of the population left little opportunity for organised armed resistance, and facilitated violence that was thoroughly interwoven into new IXam lifestyles, by leaving farm and domestic workers dependent upon the temperament of their employers and vulnerable to potential abuse. Igui-an (LL.VIII.19.7657-7668), living with and labouring for the farmer Jacob de Klerk and his wife Trina, was physically assaulted (until she lost consciousness) for a perceived lack of diligence in her herding and domestic labour: the farmer beat her with a $\| h u i-! k a^{202}$ reed, believing that she had taken away (presumably, consumed) one of his flock, while his wife lashed her with a thong for tardiness in washing and drying dishes. Deployment within labour relations represented a 'socialisation' of this form of violence, using it to

${ }^{202}$ Bambush (Afr. bamboes, bamboo), applied to large indigenous reed species with thick, bamboo-like stems (e.g. Cannomois virgata, or bergbamboes). 
perpetuate desired roles: Jacob de Klerk and his wife attempted to entrench these across generations by 'disciplining' Igui-an's child (LL.VIII.19.7658'), and Dia!kwain also noted that even those raised by Europeans on farms might be subject to extreme disciplinary or punitive violence (LL.V.23.5873). This 'socialisation' was successful, insofar as practices demanded by Europeans became intelligible to the informants, as indicated by the extension of a:kan concepts to these practices. Such 'niceness' was elsewhere associated with 'tidying up' and proper, orderly behaviour (LL.VIII.17.7497); Ihanłkass'o described Igui-an's breaking of Trina's cup as a consequence of her failure to take it up 'nicely' (LL.VIII.18.7657'). Given the associations between successful performances and obligations to perform within the context of 'successful' interpersonal relations (Chapter 6a:168), the labour undertaken for farmers (while sometimes accompanied by violence and excessive punishment) appears to have entailed a social relationship: this relationship was founded primarily on the provisioning of labourers that placed them in an 'orphan state' (Chapter 8c:333).

The IXam stressed that violent actions were also typical for non-European colonial populations. Bantu-speaking groups were presented either as destructive agents in their own right or as manifestations of colonial coercive violence; unlike engagements with Koranas, Bastaards and even European colonists, personal interactions between the Bleek-Lloyd informants and Bantu-speakers were almost entirely negative. $\| k o x a i t \partial n$ (Bleek, 1956:589) or |k'ann (LL.VIII.8281') was 
usually translated with 'Kafir' ${ }^{203}$, used by colonial populations primarily as a synonym for Xhosa but by extension referred also to any black, Bantu-speaking, agro-pastoralist group. References to $\|$ koxaiton likely subsumed several of these groups; informants living in the north probably interacted with Tswana-speaking groups, while those from south-western regions of Bushmanland may have engaged with Xhosa populations (Chapter 4b:103). Records of robberies (LL.II.5.624; LL.4203-4204), homicidal violence (LL.VIII.20.7750; LL.VIII.27.8436'), associations with 'poison' (LL.V.4.4207', 4218) and participation in capture and arrests (LL.II.1.242-269) situate IXam interactions with Bantu-speakers as an extension of broadly colonial relationships: indeed, in the Kareeberg, the settlement of Bantu-speaking groups was encouraged by colonial authorities as a buffer against 'Bushman' raids (Anderson, 1987). The narratives document explicit parallels with colonist action: the slaughter of seven IXam by a $\|$ koxaiton man named Meintjes was in essence a small-scale commando raid (LL.VIII.27.8436'), and individuals killed by Bantu-speakers for eating cattle (LL.III.1.499) were discussed with phrases that recall exactly the terminology used for relationships with Europeans, being killed for 'eating cattle from hunger $^{204}$. Like other anti-social groups, Bantu-speakers were linked with regions geographically marginal to the IXam world, connected in particular with the other side of the Orange River (LL.VIII.27.8387). They possessed distinctive styles of assegai (LL.II.32.2900') that manifested the violent identities of their original

${ }^{203}$ Occasionally translated with 'black man', or described as $¥ n u$ (black, Bleek, 1956:674; LL.VIII.22.7985'); these people were not, however, the 'black people' referred to in connection with the Early-Race Ticks (Chapter 5c:162).

${ }^{204}$ Killing things 'from hunger' is a repeated motif in personal history narratives, given as the motivation for actions that led to informants' convictions (LL.V.19.5454-5455). 
owners even when employed by other agents; in one instance of Bushman-onBushman homicide, the murder weapon was a 'Kafir's assegai', brought at Witberg (LL.II.32.2900'). This mountain ( $k^{\prime \prime} a m m-x h a r r a-k a-! k a u^{205}$; Figure 8.3:317) was a 'violent' place ${ }^{206}$, linked with the $\|$ koxaiton, who lived at the water near it (LL.II.25.2239') and 'outside' of Bushmanland ${ }^{207}$. Coherent with their violent identities, their material culture and practices connected them with the Korana (carrying loads on their heads, LL.II.13.1266') and differentiated them from IXam populations.

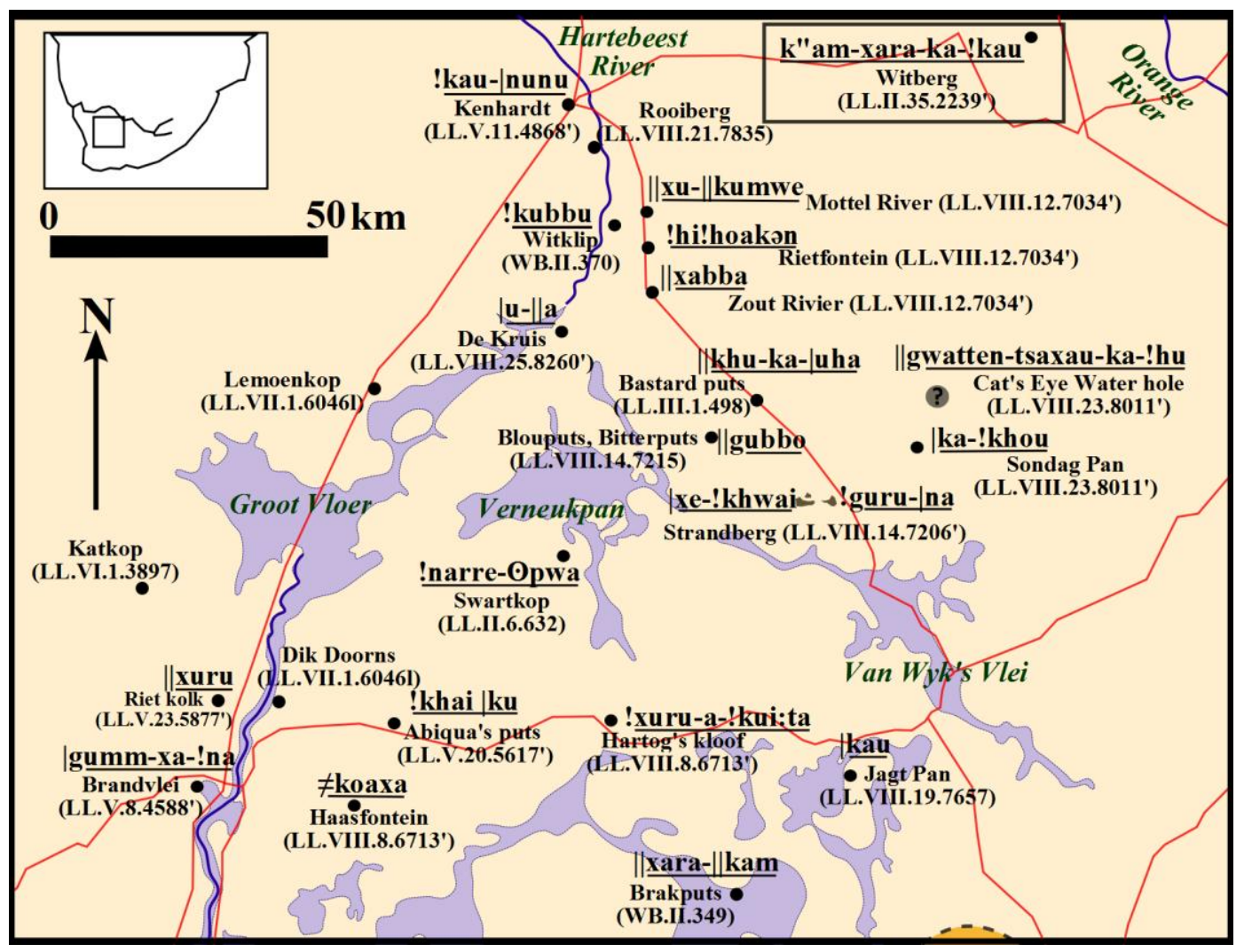

Figure 8.3: 'Witberg' in the Kenhardt-Brandvlei-Vanwyksvlei triangle

\footnotetext{
${ }^{205}$ Loosely translating as "Right-hand Meerkat's mountain" (Bleek, 1956:119, 257, 412)

${ }^{206}$ !kaukən-ta-la, a murderer who killed his grandfather, was a 'Witberg's man' (LL.II.25.2263').

207 Although this site was 'outside' Bushman territories, it was incorporated in the IXam landscape as a rain-making site (LL.II.25.2239').
} 


\section{Chapter 8c: Negotiating colonisation}

Despite their dominant anti-social image, "kaal" Bantu-speaking populations (kaal Afr. naked; they did not wear trousers, LL.VIII.27.8388) engaged in subsistence behaviours that the informants felt linked them to Bushmen, such as using nets to catch locusts; these groups might be referred to with the term ! $k^{\prime}$ etən (people), normally reserved for IXam speakers (LL.VIII.27.8387; Chapter 7a:215), and they almost certainly represented Tswana (or Tswana-influenced) populations on the far side of the Orange river (Penn, 1995). \|koxaitan occupying places (such as Witberg) within the colonial boundary overlapped in territory with more socially distant Bushman populations of the 'Groot Rivier's mountains': although these groups were not 'regte' Bushmen (Chapter 7a:216), similarities in their language and practices (some of which were shared with Bantu-speakers) suggests a continuum of increasingly 'different' identities rather than an abrupt distinctions between groups. While Bleek and Lloyd's informants experienced Bantu-speakers largely as a disruptive force in their lives ${ }^{208}$, other modes of interaction existed: it was a recurrent fear of colonial authorities and border

\footnotetext{
${ }^{208}$ Dia!kwain's account most especially skews the portrayal, with a narrative dedicated to his interactions with a $\|$ koxaitzn man whom he believed had killed his aunt, who stole from his mother, and assaulted him personally (LL.V.4.4200-4211).
} 
farmers that these groups were acting in $\operatorname{concert}^{209}$, and at least one person mentioned in the archive, \|gabbe-|ka, was of mixed Bantu-speaker and IXam heritage (the son of a $\|$ koxaiton man and a IXam woman, LL.VIII.11.6969'). Although the dynamics of this relationship were not explicated, this man was said to speak IXam well; elsewhere this was linked with 'understanding' and moral rectitude (LL.V.19.5446-5447), implying that people who spoke in this fashion had received requisite cultural knowledge and 'properly' internalised IXam notions of appropriate behaviour. Applied to \|gabbe-|ka, this jugdement implied that he was brought up 'correctly', and consequently that he had sustained relationships with his Bushman relatives.

IXam relationships with 'Bastaard' $(\text { Kwobbo })^{210}$ communities were similarly mixed: the extent to which these creolised groups should be characterised as indigenous responses to (rather than agents of) colonial expansion is contentious (Besten, 2006; Waldman, 2007), and it is an obvious distortion to imagine that two were necessarily distinct. In IXam experience, these populations were moreor-less contiguous with Europeans; if frontier farmers constituted a relatively impoverished subset of European colonists expanding into unfamiliar ecosystems and depending upon incorporation of indigenous systems of knowledge (Chapter 4b:100; Chapter 4c:129), then Bastaards arguably represented the extremes of this frontier. Certainly, they participated in the violent dimensions of interaction: the

\footnotetext{
${ }^{209}$ Upington (1879:i-iv) stressed the involvement of Koranna, Bastaard, Tswana, Xhosa and 'other aboriginal natives' in the 'disturbances' of the Second Koranna War

${ }^{210}$ D. Bleek (1956:115) translates it as 'coloured person' and 'black man', but in the narratives it usually denoted creolised colonial populations rather than Bantu-speakers (LL.II.6.645'; LL.V.21.5702')
} 
men who killed Dia!kwain's cousin were described as 'half whites (and half kwobbo's)' (LL.V.21.5702'). Relationships between Bastaard and European farmers were on occasion described with possessives, with Bastaards being 'farmer's men' (LL.II.6.645') either a statement of their parentage in a literal sense or a reference to their acting as farmers' agents in the border regions. Bastaards were the proximate agents of much of the disruption that engulfed Bushmanland in the nineteenth century: the renaming of the IXam landscape with Afrikaans alternatives occurred largely through their agency (LL.VIII.14.7215; Burchell, 1822:286), and \|kabbo noted that two Bastaards (Albert Meintjes and Oud Gert ${ }^{211}$ ) occupied the places he once owned (WB.XII.1182'). When describing their relationships with Bastaards, the IXam employed identical terminology to that used for Europeans, with women referred to as 'mistresses' (LL.II.6.650) and men as 'masters' ( $\mid h u)$. Also translated as white ${ }^{212}$ man, European, or Boer (Bleek, 1956:289), phrases talking about Bastaard 'masters' are (in IXam) indistinguishable from references to Europeans. This similarity is also present in the content of interactions, with the IXam acting as herders and providing other kinds of labour (e.g. tanning sheep and goat hides, LL.II.6.650) for Bastaards (LL.VIII.8.6721') just as they did for Europeans.

|Xam-Bastaard relationships provide a snapshot of the lifestyle of more marginal farmers in the northern border districts. Their settlement patterns, with the Bastaard 'master' occupying a wagon on one side of the flock while the Bushman

\footnotetext{
211 Although Bank (2006:151) associates this figure with Gert van Wyk (a white farmer), the reverso note at WB.XII.1182' explicitly states that these two men were Bastaards.

212 'White' here makes no reference to the colour term !kúi:ta (Chapter 5c:164).
} 


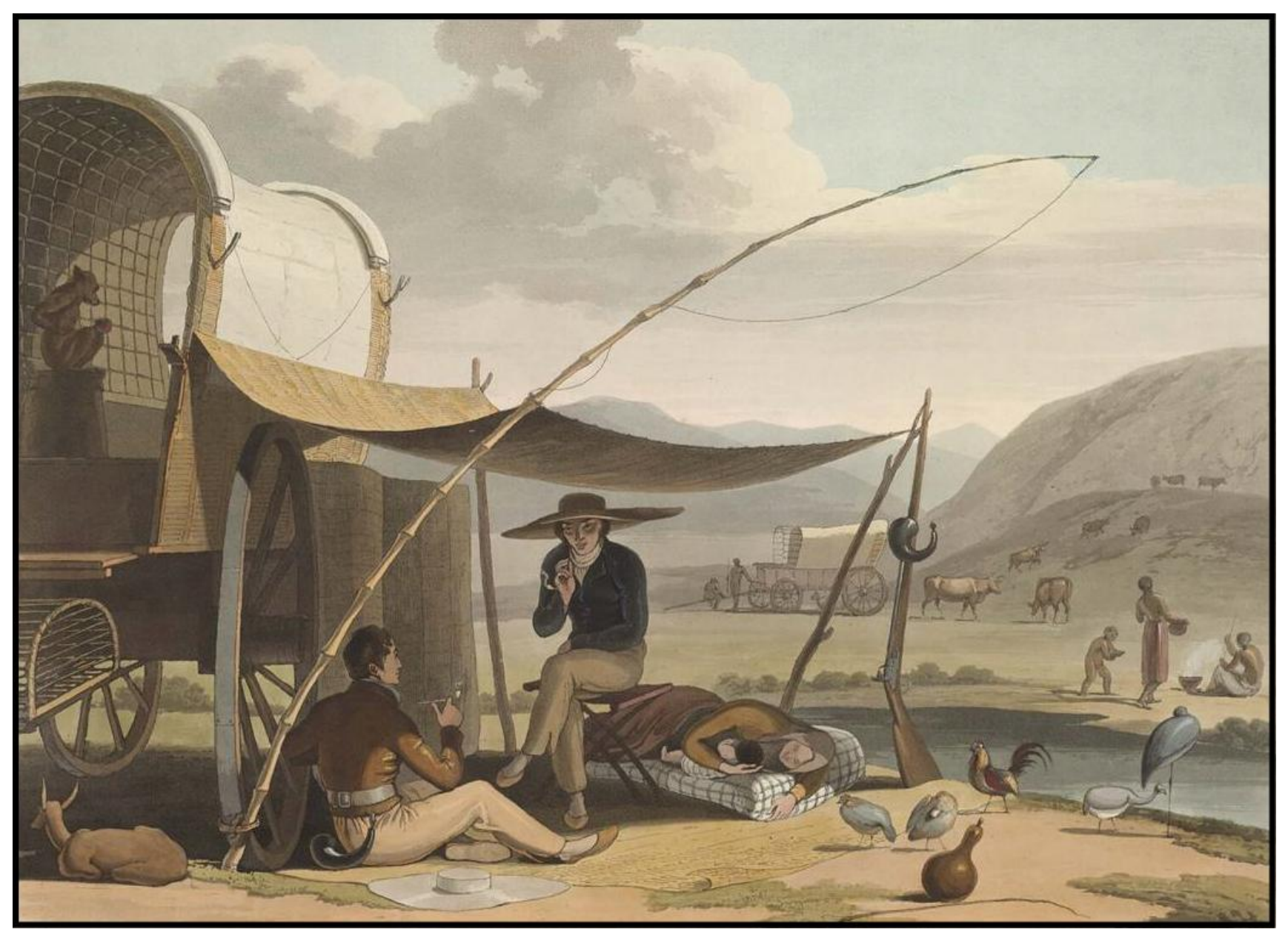

Figure 8.4: "Halt of a Boors Family" (Daniell, 1804-1805:No.11) 
shepherd dwelt opposite (LL.VIII.8.6720), would be equally appropriate for European colonists farmers: early nineteenth century depictions (Figure 8.4:321) of European farmers in regions distant from the Cape consistently emphasise the ephemeral nature of their settlements, and even at the end of nineteenth century the occupation of the northern border districts included considerable numbers living an itinerant lifestyle in tents and wagons (Chapter 4c:120). In these contexts, the lifestyles of the farmers and their labourers (though framed within a highly unequal power dynamic) must have been substantially similar. For the Bastaards, comments on practices that linked them to the Europeans (e.g. making soap from asbossies $^{213}$, LL.VIII.6.6582'; LL.VIII.23.8022) were matched by those connecting them with indigenous communities; Ihanłkass'o recognised similarities between Bushman and Bastaard musical instruments, as well as shared plant knowledge (LL.VIII.21.7833). Differences in the circumstances of colonial farmers had considerable impacts in structuring IXam interaction with them, as illustrated in differences the life histories of Jan Plat and his brother (LL.V.23.5877'). Jan Plat grew up with a farmer named Jan Struys at Riet Kolk (Figure 8.5:323), presumably a relative of Koos Struys, who murdered Jan Plat's brother. By contrast to Koos, Jan had married a coloured woman and lived further into Bushmanland, and exerted a less hegemonic influence over his labourers. Jan Plat retained a degree of control over his labour, shifting employment between farmers (working for at least two additional named farmers), placing his son at service with a different man, and acquiring his own herds of sheep

\footnotetext{
${ }^{213}$ Ash (Afr. as) from several genera of Karoo bush (Psilocaulon sp., Salsola sp.) was used in the production of lye, giving rise to their alternative vernacular names (loogbossies, Afr. lye-bushes).
} 


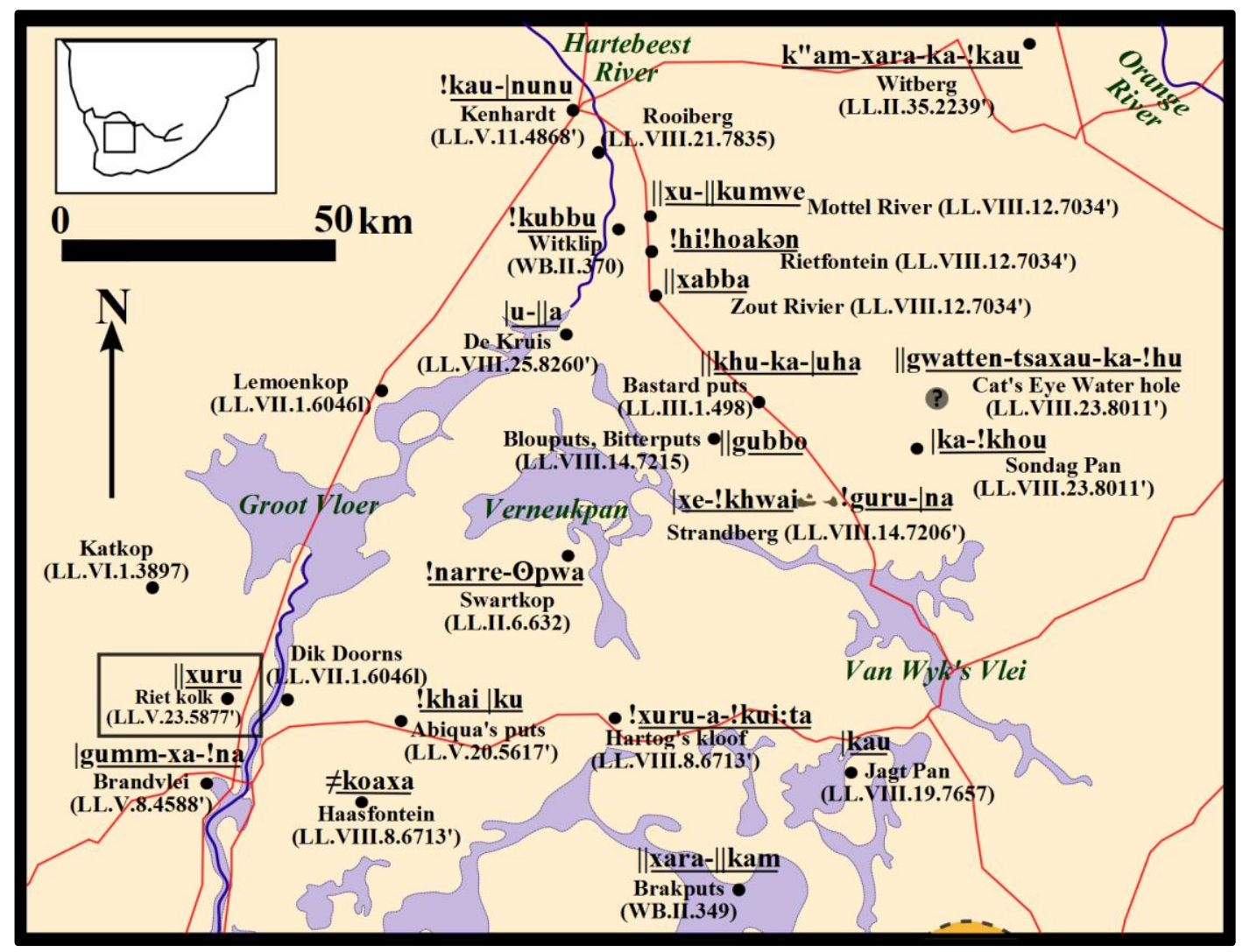

Figure 8.5: Riet Kolk in the Kenhardt-Brandvlei-Vanwyksvlei triangle

(LL.VII.1.6046,5'-6046e'), forms of colonial experience characterised by negotiation, access to new resources, and the selective adoption of alternative lifestyles.

Marriage was a major avenue facilitating these cultural encounters, and while Bastaards were primary agents in dispossessing Bushmen from their territories they also exaggerated the creolised aspects of frontier farmer life, in their adoption of the IXam language (LL.VIII.8080'; WB.XI.1120) and in their conjugal relationships with IXam women ${ }^{214}$. Though there is little evidence regarding the

\footnotetext{
214 Once more raising issues regarding the dearth of female informants; the role of women in crossing frontiers in other colonial contexts is well-documented (Schaffer, 2001:137).
} 
way these women viewed their participation in cross-cultural conjugal relationships, IXam men used their marriages as a means to access farmer resources: Ihanłkass'o went to live at Jacob Kotze's place, because the latter was married to a IXam woman named 'Silla ${ }^{215}$, who supplied |hanłkass'o with food. This account also encapsulates IXam characterisation of Bastaard identity (LL.VIII.8.6723-6724), emphasising their reputation for 'stinginess' with food (a serious criticism in IXam society, Chapter 6b:183), and contrasting this with Silla's Bushman practice in 'liberally giving' (lit. !kou-a:kən, to give well or nicely, Bleek, 1956:7, 444) food. Though wed to social practices alien to Bushman morality, Bastaards could be thus accommodated relatively easily into Bushman sociality through their relationships with IXam individuals. As food distribution was governed by social norms regarding propriety, the subjugation of resources acquired from colonial farmers to redistribution rules demonstrates their thorough incorporation into IXam subsistence, and the continuing relevance of social connections between people accessing these 'farmer' resources and those subsisting outside this domain. Elements of sociality reappear in other instances of Bastaard-|Xam intermarriage, as with the marriage between Albert Meintjes ${ }^{216}$, (Suobbakən's 'master') and !gwarra-an ${ }^{217}$. !gwarra-an was described as Suobbakən's kuobbo-\|kuarra or 'foster mother' (lit. 'Bastaard-ewe', Bleek, 1956:115, 598) and was said to love $\mathrm{e}^{218}$ her. These descriptions (imbued with

\footnotetext{
${ }^{215}$ The common $\mathrm{L}$ and $\mathrm{N}$ substitution (Chapter 8c:325) in IXam Afrikaans suggests this is the same woman referred to elsewhere as 'Sinna'.

${ }^{216}$ Often written as Albert or Ander Neintjes.

${ }^{217}$ Although not directly stated to be a Bushmen, this is a IXam name, and she was associated with Sinna/Silla, who was IXam.

${ }^{218}$ Sinna/Silla also loved Suobbakən (LL.II.6.651)
} 
familial referents) suggest that the IXam occasionally constructed these "masterservant' interactions as 'meaningful' relationships, with marriage drawing incoming farmers into spheres of IXam obligation and food-sharing ${ }^{219}$.

Another major area of negotiation is that represented by linguistic change and, just as with the relationship between Khoekhoe pastoralists and hunter-gatherers, it is erroneous to imagine that linguistic influence between European farmers and Bushmen was unidirectional. Some farmers of the frontier districts incorporated IXam terms, developing their own dialect referred to (in the archive) as 'Boer's Bushman.' Little is recorded, and the dynamics of this exchange remain lamentably obscure; although W. Bleek was evidently interested in IXamspeaking colonists, collecting lists of their names and residences (WB.XXI.1120), he never had the opportunity to interview any of them. This 'Boer's Bushman' appears to have simplified some of the click consonants, (erasing the initial click in ' $₹ k$ "erri-@pua' to form 'kerintte $\odot u a$ ', WB.IV.545') and to have adopted words but assigned them different meanings (using the word $\mid k^{220}$ to refer to the $\neq g e b b i$ $g u$ dance, LL.V.25.6006'). Though some individuals were taught IXam directly from Bushmen (WB.I.12'; $c f$. von Wielligh, 1919, 1920, 1921, 1921a), in other case it was passed on between colonists, even inter-generationally; the van Wyks (Jan, Gert, Stoffel) spoke IXam because their (white) mother taught them (WB.XI.1120), which would, by definition, make this language a creole (Holm, 2000:5-8).

${ }^{219}$ Other |Xam kin terms made reference to exchange relationships; |xwobbe, a genderdifferentiated term (men used it for paternal grandparents, women for maternal), was also a verb meaning 'to lend' (Bleek, 1956:367).

${ }^{220}$ Perhaps from 'to make a thumping sound' (Bleek, 1956:315). 
Unlike lexemes from the Khoekhoe languages, IXam words derived from European languages are very easy to isolate, being the result of recent interaction and deriving from distinctly different language families. While the very nature of Bleek-Lloyd project underemphasises relationships between the informants and European languages, all of the informants were bilingual in Afrikaans; this was not universal among IXam individuals even in the late nineteenth century $(\text { LL.VIII.23.8081') })^{221}$, and its presence among the informants at least partly reflects the significance of bilingualism for the translation process, particular in its early stages (Bank, 2006:87, 180). The Afrikaans ${ }^{222}$ recorded in the archive, (prior to spelling standardisation, Deumert, 2004:1) is highly idiosyncratic, reflecting the initial unfamiliarity of the translators with the language as well as some specific features of IXam-Afrikaans: transpositions of L/N (LL.II.13.1245') are too common to be the result merely of the researcher mishearing a pronunciation, and substituting in the alternative letter often results in a more accurate translation ${ }^{223}$. IXam informants were interested in and highly aware of linguistic nuances: Ihanłkass'o made observations on the parts of the tongue used by IXam and Europeans when they spoke (LL.VIII.8528'). He also discussed linguistic shifts in younger generations, imbuing them with a moral dimension by stating that young

\footnotetext{
${ }^{221}$ Older individuals or those who lived further from the colony understandably tended to be less familiar with it (Dia!kwain's mother understood, but could not speak, the language, WB.XXVI.2486').

${ }^{222}$ English was incorporated only in a limited way, often in place names such as 'Totori-ya' (la!kunta's pronunciation of Victoria West, LL.II.6.643'). This represented the influence of relationships with border farmers on the IXam acquisition of colonial languages: "kabbo informed Lloyd that when speaking of English place names, he spoke 'just as [his] farmer did' (LL.II.6.643').

${ }^{223}$ The ! $k$ 'au or 'sillin xara' (a 'white substance' found along the arms, thighs and spine, LL.VIII.31.8772') for which the Afrikaans silwer garing (silver threads) has been proposed (Goodwin, 1945:441), becomes sinnin xara, or sening garing (Afr. ligament-threads).
} 
people said 'tata' (for father) where they should be using the IXam equivalents (bbo, oa, Bleek). \|kabbo too pointed out the use of 'white man's talk' rather than the correct IXam term (LL.II.36.3261'), an insistence widely seen in concerns that the IXam names for places and people be noted (LL.II.14.1383'; LL.V.19.5448; LL.VIII.9.6801', LL.VIII.12.7034'; LL.VIII.14.7215). There was a definite association between language comprehension and being a 'good' or understanding person (who did not $ł k a k k ə n-\|k w o\| k w o m m a i n$, talk disconnectedly or confusedly, LL.V.19.5448'), but living with farmers (even growing up with them) did not necessarily entail language loss and a lack of understanding (LL.V.16.54465447). Speaking IXam had a strong, largely positive valence for the Bleek-Lloyd informants, and the adoption of Afrikaans terminology was contested; examination of the words that were incorporated provides some sense of the kinds of practices, materials, and ideas that these populations were encountering.

Many of the Afrikaans words scattered through the narratives reference newly introduced material culture and resources, such as baksteen (LL.VIII.21.7834'; Afr. brick) kkoffi (LL.II.22.1960; koffie, Afr. coffee) or bakon (LL.VIII.11.6945'; baken, Afr. beacon) $)^{224}$, and are otherwise unmentioned in the narratives. Conversely, new material culture was incorporated through extensions of preexisting terms; 'cannon’ (\|xwai, Bleek, 1956:638) appears to have referenced the noise of its firing, as the phrase '\|xwai-throat', described the deafening arrival of large springbok herds (LL.VIII.14.7242'). There were also less tangible incorporations into the IXam conceptual world, some of which produced

${ }^{224}$ Often given distinct |Xam pronunciations: kokunmenti ('cups' LL.IV.19.7657') presumably represents kommetje (Afr. bowl). 
synonyms for pre-existing concepts; Lloyd translated Ine as 'help' and herribi as 'assist', but for $\|$ kabbo the former was the IXam and the latter the European term for the same concept (LL.II.36.3262). Others, such as divisions of time into months or days of the week (LL.II.32.2920; Sondag, Afr. Sunday), or words relating to labour control such as ddomminya (LL.V.1.3626, Afr. domineer, to predominate, domineer) and luitan-ddi (LL.VIII.19.7663; lui Afr. laziness), reflect the incorporation of novel ideas into the IXam conceptual world. This last word shows a considerable degree of grammatical (and presumably also conceptual) $\operatorname{assimilation}^{225}$, with the addition of common IXam suffixes tan (creating plural or emphatic forms) and $d d i$ (denoting 'things' or 'doings', Bleek, 1956:24-25): the phrase thus more literally meant 'laziness'-doings'. These brief notes form a reminder that the seemingly simple incorporation of a relatively small number of non-|Xam terms into the IXam vocabulary reflected a much more extensive, longterm interaction with novel ideas and practice, and that the archive itself does not represent the full extent ${ }^{226}$ of this interaction.

Parallel with linguistic change, there were rapid shifts in appellative practices, transitioning in three generations from individuals possessing no Afrikaans name (LL.III.1.480), through those possessing names in two languages, to the youngest generation, some of whom were not receiving IXam names at all (LL.III.1.495), a re-assignment of names that occurred also in the application of a new toponymy.

\footnotetext{
225 'Mama', commonly replacing xoa (mother, Bleek, 1956:258), was also often presented with the appropriate group plural suffix ( $-g g u$, LL.V.20.5568').

${ }_{226}$ |hanłkass'o's Afrikaans translation of the exclamation !khe!khe (Almagtig, Afr. Almighty) LL.VIII.10.6934') is one of only very few references to new religious contexts that would become dominant in the early twentieth century.
} 
However, well-established indigenous traditions of 'nick-naming' both individuals and landscapes (Chapter 8a:289) were also quite literally 'translated' for a new cultural context, by incorporating linguistic change. As familiarity in Afrikaans spread, nicknames could be bestowed in this language: 'Soppie' acquired his nickname from the sop (Afr. soup) that he poured down his front (LL.VIII.32.8808'). Both European and Bastaard farmers were in the habit of giving Afrikaans names to the Bushman labourers working on their farms (and to their families, LL.II.6.649), but there is little sign (other than an insistence that IXam equivalents were also recorded) that informants considered their 'Boer's names' an imposition; in genealogical collections, they were listed as further alternatives to the 'great names', 'little names' and nicknames that individuals already possessed, $\|$ kabbo counted his European name among the names that 'floated along' to his !xoe (LL.II.32.2886), and many Afrikaans names were bestowed by Bushmen themselves (LL.II.35.3162).

Although Afrikaans words highlight the range of 'colonial' material culture with which the informants were familiar, the volume of IXam narrative dedicated to this material culture is not considerable. Once again, this must in part reflect researcher desires to obtain information about 'traditional' practice: contemporary observers (Dunn, 1873) noted the thorough incorporation of colonial material culture into the lifeways of Northern Cape hunter-gatherers. Firearms figure prominently amongst such references: Dia!kwain (LL.V.9.4688) and łkasin (LL.IV.1.3459) recalled their use of guns in hunting, while \|kabbo (LL.II.32.2924'-2925') expressed his desire to possess one for this purpose, 
representing them as new forms of material culture facilitating useful modifications of a central domain of Bushman life, and allowing even older hunters to maintain subsistence independence. As with the 'contextualisation' of stories by mention of herding practices, reverso notes create the impression that depictions of material culture in narratives were not necessarily accurate reflections of familiarity with 'colonial' material culture: "gabbe-|ka received his nickname ('turned-back hand', Bleek, 1956:336, 523) because of an injury sustained in an accident with a gun (LL.VIII.11.6969'), indicating the ready assimilation of new technologies to established socio-cultural practice (i.e. accidents with guns being understood as 'incidents while out hunting' that provided nick-naming material). When lhanłkass'o described hunting practices associated with springbok that arrived en masse (LL.VIII.14.7221-7259), he also noted that these aggregations of springbok led to farmers' expending their supplies of gunpowder and shot (LL.VIII.14.7226'), and that particular kinds of ammunition were used by the farmers for hunting korhaans and hares (LL.VIII.14.7246'); this display of knowledge concerning and familiarity with firearms was entirely absent from the hunting description itself, but evidently associated sufficiently with springbok-hunting as to be recalled in his narrative.

European material was usually evaluated positively in comparisons with indigenous alternatives: continuance of the latter was associated with poverty, as with the use of springbok-horn tinderboxes (LL.V.10.4761-4763; LL.VIII.28.8472'; Chapter 7a:218). European goods also became highly valued for their potential to be re-worked into new objects (brass tinder-boxes 
transformed into head ornaments, LL.II.15.1440'), allowing the construction of superlative example of items already well established in socio-cultural practices. Some traded goods readily found a place in IXam belief systems, creating new resources that could be deployed in the enactment of specific social roles: the 'sparkling' of buttons (toro-bai:tan, Bleek, 1956:209) underpinned their deploying in a demonstration of 'understanding' behaviour, where their visual qualities were used to frighten away lions (LL.V.12.4947). Ongoing pursuance of 'traditional' Bushman practices was not, however, always portrayed as a 'lesser' alternative to new resources: when $\|$ kabbo contrasted the use of an ostrich sternum dish with that of a !kuerre (tub, bowl or vessel, LL.II.34.3072'-3073') bought from Europeans (LL.II.34.3087'), he treated the distinction in a neutral fashion with respect to 'poverty'. Like 'money' (!xoe: Bleek, 1956:500, mentioned only very rarely), this difference was instead taken as an index of contact and personal relationships with colonial agents; $\|$ kabbo (LL.II.6.640) referred to money only in the contexts of his interactions with Bleek, and łkasin associated unfamiliarity with its use with living at a distance from the colony (LL.IV.1.3476). Bushmen closer to the colony used their ability to access European (or livestock-derived) goods, including sheepskin karosses, handkerchiefs, tinderboxes, and (metal) knives, to acquire resources from Bushmen living further away: specifically, they were interested in obtaining a particular poison which these groups monopolised (LL.IV.1.3476). Continued use of 'indigenous' rather than colonial material culture was sometimes portrayed as indicative of less intense contact (LL.V.10.4765'), though many elements of 'traditional' lifestyles were not 
represented in this way: la!kunta lived in a matjieshuis covered with springbok skins, even when directly engaged in colonial relationships and living on a farmer's land (LL.first unnumbered notebook.inset). By the time Bleek and Lloyd were interviewing IXam individuals, many items of colonial material culture had become widespread even among populations that were furthest from Cape. Ihanłkass'o, recounting porcupine hunting by night, noted that when they did not wear trousers (buburuken, Afr. broek), the thorns drew blood on their legs (LL.VIII.14.7220'); incorporation of material of this kind (almost certainly tanned and sewn by the IXam themselves) indicates that 'material culture' incorporations also represent conceptual re-organisations, in this case involving the adoption of European 'styles' within IXam cultural spaces and spheres of action.

The spread of European clothing occurred in the context of labour relations, and $\|$ kabbo recalled 'gifts' of clothing (LL.II.6.636) from farmers to Bushmen. Although Ordinance 50 (Harlow and Maddon, 1953:599) was supposed to introduce a wage economy in farmer-Khoisan relationships, in more remote regions (with limited access to money; Newton-King, 1999:182) older 'clientship' practices were retained, incorporating gifts of clothes, other items of colonially derived material culture, and food. These practices could render groups highly dependent on farmer largesse (LL.VIII.12.7108'), especially when combined with attempts to monopolise access to wild game resources or classify hunter-gatherer subsistence as illegitimate (Chapter 4c:130). Farmer practices of using trekbokke to accumulate large meat surpluses (LL.VIII.14.7226') allowed them to supply their workforce without depleting their herds: although the resilience of springbok 
herds even in the face of these pressures was an important (if not sufficient) component in sustaining hunter-gatherer strategies, it also supported farmers' strategies by increasing their capacity to attract and retain labour. With respect to the Europeans, the IXam described themselves as !kwe!kwetan or 'poor people', drawing upon their conception of the 'orphan' (Chapter 6b:186), as they both needed to beg food from a group of ungenerous people disinclined to support them, often after having undergone some misfortune; after la!kunta's brother died, his wife went to live at a settler's house and depended upon him for her subsistence (LL.III.1.502).

One resource that was both highly desirable and linked closely with the colonists was tobacco; "kabbo suggested that 'love' of tobacco might motivate a person to go to live with a farmer (LL.II.6.648). The IXam differentiated linguistically between different products derived from the plant: łkun ('oil of tobacco’, Bleek, 1956:665) referred to a substance produced either from the distillation of dried tobacco leaves or accumulations (in a pipe bowl) resulting from the process of smoking, and in the nineteenth century was associated with Khoekhoe societies (Thomson, 1832:436). Use of aromatic sã: (buchu) and other plant medicines was a well-established component of both Khoekhoen and Bushman practice (Low, 2007; Chapter 5a:138, 142; Chapter 5b:156), and provided a pre-existing mode for understanding Nicotiana sp. (themselves aromatic herbs and shrubs) that rendered this newly introduced form of material culture familiar. Tobacco was widely valued by Europeans themselves for its medicinal properties (Stewart, 1967; Charlton, 2004), and incorporated in this capacity by colonial populations 
especially in the border regions where access to the medical professionals of urban centres was limited (Digby, 2005:445). Just as indigenous populations reacted to novel, introduced resources, so too did these immigrant populations incorporate and adapt the indigenous resources and knowledge they encountered (H. Deacon, 2004; Low, 2007). Frontier farmer use of herbal remedies drew heavily on their close contact with local populations, modifying them to suit their pre-existing materia medica, by (for example) steeping leaves to form tinctures in brandy, a characteristic feature of 'folk' medicine among colonial European populations (van Wyk, van Oudtshoorn and Gerike, 1997:142; Low, 2007:342). We may then regard this component of tobacco use at least as an instance of reciprocal exchange, even to the point where colonial populations re-incorporated 'colonial' material culture that had been adopted and altered by indigenous populations (H. Deacon, 1998:289).

D. Gordon (1996) postulates that drug dependencies played a significant role in fostering inequalities in relationships between Khoekhoe and European pastoralists, and Sampson (1993) identifies similar processes for Seacow River Valley Bushmen; the IXam term |xuru (Bleek, 1956:367) related particularly to cravings for tobacco, described by la!kunta as the heart's 'thirst' (LL.II.7.771'), suggesting that for them too it was an important motivator in their establishing relationships with European farmers, major suppliers of this substance. Trade played in important role in acquiring the paraphernalia of smoking; while the nineteenth century IXam did make their own pipes using the thigh bones ${ }^{227}$ of

227 'Boot bein' (LL.II.1.247'), probably Afrikaans boud been, buttock/leg-bone. 
sheep (LL.II.1.248) and goats (WB.XII.1123'), European 'trade pipes' (!xoro, !xo LL.II.6.641; LL.VIII.22.7977) were obtained through exchange relationships and appear to have been highly valued ${ }^{228}$. $\|$ kabbo outlined the dynamics of a bartering system (LL.II.7.771-775) in which feathers from male ostriches (and ostrich eggs, LL.II.24.2202) were exchanged in return for tobacco and !gwara-ka-\|gabbe (metal spoons), 'bought' from the Europeans (LL.II.14.1353'). By contrast, lhan‡kass'o described the Grass Bushmen as 'begging' tobacco from white farmers (LL.VIII.22.7976), likely in accordance with his conception of the Grass Bushmen as wild and 'fearing' (avoiding) Europeans.

\|kabbo emphasised the social interaction accompanying these transaction: his recounting of the scenario of deliberating seeking out the farmer's wagon recalls the Bushman institutions of 'visiting' nearby groups for stories. $\|$ kabbo in fact linked this visiting to obtain stories with being a 'smoking's person' (LL.II.32.2880-2881), and the contact associated with bartering tobacco is without doubt one of the most amicable instances of European-|Xam contact (even if rather formal). Asides concerning the 'habits of the Boers' imply close, if not necessarily friendly, relations between the IXam and European colonists, and emphasise that (even in the context of commando raiding and land dispossession) individuals forged working relationships to cope with the demands of the border districts: IXam observations on European development of their own body of

\footnotetext{
${ }^{228} \|$ kabbo dreamt of his wife asking him the details concerning one of these pipes, obtained from Bleek (LL.II.22.1952-1956); dreams often conveyed significant information (Chapter 6b:197).
} 
ecological knowledge (LL.VIII.13.7119') $)^{229}$ or their hunting preferences (LL.I.2.188) are reminders that despite the often bitter and destructive interactions between colonists and IXam, individuals were often living in close proximity to, working alongside, and trekking extensively with one another. While one would hesitate to describe any of the encounters described as particularly amiable, notes present decidedly intimate portrayals of farmers' expressions (LL.VIII.30.8606') and remind us of the closeness of their relationships. A degree of realistic accommodation is implied in this closeness, as in linguistic exchanges and 'provisioning' relations: to neglect such accommodation would be to imbue |Xam strategies with a certain inflexibility that casts them as one of the familiar colonial 'hunter-gatherer' stereotypes, either noble savages attempting to retain their freedom in the face of overwhelming odds, or 'primitives' doomed to passive failure in the wake of pastoralist encroachments (Chapter 2:31).

${ }^{229}$ Noting 'white men' considered places as 'grown up' (i.e. summer's arrival) when certain rain fell. 


\section{Chapter 9: Conclusions}

\section{IXam and animic ontologies}

The previous chapters have examined in detail the Bleek-Lloyd narratives, constructing an understanding of their ontological framework and of the ways this framework was engaged with their historical setting. In order to develop the wider relevance of this study it is necessary to return to theoretical models of 'huntergatherer society' (Chapter 2) and to situate these within a comparative examination of the colonial 'setting' of the archive, examining the engagement of 'hunter-gatherer' ideologies with the expectations and demands of colonial populations and exploring the extent to which the IXam case study fits within such engagements. For the Bleek-Lloyd archive, any discussion of its wider implications must surely make reference to Lewis-Williams' (1981) 'shamanistic' interpretation of southern African rock art, subsequently applied to contexts as diverse as Upper Palaeolithic Europe (Lewis-Williams, 2002a) and precolonial America (Whitley, 1988); the central role of IXam testimonies for the original 
model enshrines their global significance. Fortuitously, it may also serve metonymically for the need to situate the archive within these aforementioned debates concerning 'hunter-gatherer' ideologies, through the strong link between shamanic practices and 'animic' ontologies. The relationship between the two has remained an area of anthropological interest from their introduction as heuristic tools through into recent reformulations of animic (and other relational) epistemologies; Pedersen (2001) has examined this for North Asian populations, and Harvey (2010) situates shamans at the crux of the 'new animisms', as specialists in negotiating relationships with non-human persons.

Outside explicitly shamanic contexts, animic themes are emphasised strongly in hunting practices. These models explore relationships between human and nonhuman persons, and their conspicuous degree of volitional motility ensures animals are prime candidates for this 'personhood': movement is a primary method for inter-specific communication, as physical action represents an immediate and effective means of sharing information that can easily become a sign understood by two species not necessarily sharing an identical 'language' (Hoffmeyer, 2008:15-16; 2010:37). Shamanism and hunting are further linked as highly 'performative' domains of action, representing public demonstrations of positively valued skill and competence ${ }^{230}$; even where virtuoso performances are overtly down-played or criticised they remain testament to individual skill. Linking shamanic practices to interactions experienced by all persons (i.e. animal encounters), animic themes in hunting practice contextualise the role of shamanic

${ }^{230}$ !gi:tan were subject to judgements regarding their performances; people 'depreciated' (llam: Bleek, 1956:516) !kwarra-an for failing to cure a person (LL.V.3.4149). 
specialists, underpinning their relevance and efficacy for all members of the group and stressing the practical, experiential basis of their action within a broad subsistence strategy. The preceding chapters demonstrate that, while IXam beliefs were contextually contingent (often differing from those of South American or circumpolar hunter-gatherer groups), in this hunting domain they closely mirror animic principles; idioms for the construction and transfer of identities through smell and exchanges of bodily fluids (shared among humans and non-humans) and characterisations of the agency and abilities of the species they encountered in hunting practices accord well with Ingold's (2000) models.

The contiguity of shamanism and hunting serves as a reminder of the inter-related nature of ideologies and practice, a connection that becomes significant when considering the incorporation of new practices in subsistence strategies (a key feature of the colonial situation). Ideological dimensions in 'hunter-gatherer' identity create meaningful distinctions between hunter-gatherers who have acquired livestock or started cultivating wild plants, and pastoralists or farmers who happen to hunt or who supplement their diet with wild plants, but there is an underlying assumption that incorporation of novel practices will modify ideologies to a lesser or greater extent (i.e. that subsistence strategy is dynamic). This approach insists that interactions with particular (domestic) species should be situated within the wider context of relationships with non-humans; Rival (1999; 1993) draws attention towards lowland South American groups with broadly 
similar subsistence practices $^{231}$, supported by ideological regimes and sociocultural organisations quite distinct from one another. The ambiguity implied in the debate surrounding the extent to which Khoekhoe and Bushman societies should be considered distinct or as phases in a single cycle (Marks, 1972; Schrire, 1980) demonstrates the relevance of this observation for southern Africa, where there were considerable overlaps in practice between herders (focusing on milk production and large herd sizes) who relied extensively on hunting strategies (Lindholm, 2006:130) and hunter-gatherer groups.

Swart (2007:284) notes that though domesticated animals are often represented as extensions of human agency, interactions with them must incorporate the demands of non-human social structures, dietary requirements, and reproductive strategies: human strategies must become oriented toward the particular species involved. Strategies emerging during the colonial period therefore represent the interaction of at least three parties; indigenes, settlers, and domesticated species themselves. Characterisations of hunter-gatherer interactions accommodating domesticated species can therefore vacillate between looking at the incorporation of new (social) relations, or exploring them as part of the 'material culture' component of Arkush (1990:28) terms the 'proto-historic' period in indigenous engagement with colonial societies. Characterised by 'indirect' colonial presence, this period develops though down-the-line interactions: the movement of material culture and information, changes consequent on other indigenous contacts with

${ }^{231}$ Strategies combining hunting and gathering with mobile horticulture; Rival (1993:646) contrasts the Shuar (for whom cultivation is a 'highly sophisticated art' embedded in 'complex symbolic practice') with the Huaorani ('reluctant gardeners' oriented towards the forest environment). 
the settlers, and sporadic contacts with colonial agents travelling beyond the boundaries of the colonial state. In the IXam case, this period was characterised by an 'inchoate' northern border, facilitating the establishment of labour exchange/appropriation relationships that brought the IXam into contact with settlers and livestock, both within the colony and in the IXam-ka-!xoe. Combined with continued reliance on hunting and gathering subsistence and representing a choice (albeit often constrained) to engage with incoming groups, these shifts in subsistence practices are crucial to understanding the maintenance and modification of 'hunter-gatherer' identities in colonial settings. 


\section{Colonial hunter-gatherers}

Although the historical context of IXam was clearly specific and contingent, it was also located within the complex series of global processes that constituted the European colonialisms of the second millennium AD. Despite Dirks' (1992:7) rather discouraging suggestion that attempts to systemise 'colonialism' deny its fundamental historicity, there is considerable utility in what Willerslev (2011:506) terms the 'distinctive imaginative power' of comparative endeavours; the scope of eighteenth and nineteenth century colonialisms almost demands such an approach, and the integrated nature of the colonial world demands a consideration for this wider context ${ }^{232}$. The historiography of colonialism, empire and imperialism has produced a large corpus devoted to the exploration of a range of definitions (see Wolfe, 1997; Osterhammel, 2005) and heuristic tools for examining the processes whereby these occurred, some of which have already been encountered in course of this thesis (Chapter 4b:107). Common preoccupations of this literature include inequalities in the economic relationships between colonies and metropoles, colonisation as a process of modernisation or Westernisation, and the power dynamics of domination, resistance, and accommodation between indigenes,

\footnotetext{
${ }^{232}$ Ideologies and technologies developed in specific areas (e.g. barbed wire in the south-western United States, Netz, 2004) swiftly attained importance in other colonial contexts, as colonisation was dependent upon links with large-scale networks moving people and goods (Chapter 4c:120).
} 
settlers, and (where present) creolised communities (Pels, 1997); post-colonial scholarship in the later twentieth century has emphasised 'subaltern' experiences (Spivak, 1988). Exploration of colonial scenarios through their material culture has become increasingly important (Lyons and Papadopoulos, 2002; Given, 2004; Gosden, 2004; Stein, 2005); starting from the principle that there can be a large discrepancy between what people ${ }^{233}$ say about what they (and others) do, and what they actually do, such approaches attempt to deconstruct dominant discourse and provide readings of power more sensitive to the 'small voices' of history (Guha, 1996). Material culture perspectives collapse the space between colonised and coloniser, both of whom encounter new practices (with associated material culture) by moving into and through specific geographic areas, and emphasise issues of accommodation and resistance as negotiations between individuals/groups. Inherent qualities of material culture, that can be passed across boundaries and transformed often in highly visible ways, have favoured the application of theories of creolisation and hybridity, casting innovation and creativity in the interactions of indigenous societies with incomers as a form of resistance characteristic of these historical situations (Birmingham, 2000:370).

The agents of late second millennium colonisations were drawn primarily from Western European societies, sharing many cultural practices and ideologies ${ }^{234}$. Though cross-cultural interactions have much potential to become sites of unintended meaning (Sahlins, 1995:247), this cultural similarity ensured that one

\footnotetext{
${ }^{233}$ Those people 'allowed to speak', through a combination of literacy and socio-cultural standing that facilitated the production of authentic narratives.

${ }^{234}$ Some were also part of the same colonial endeavour in a political sense and thus experienced many of the same policies derived from the interests and aims of the metropole.
} 
element of this mutual incomprehension often worked out in strikingly similar ways, as with the widespread impact of notions derived from Protestant Christianity in shaping attitudes towards hunter-gatherers (Pluciennik, 2002). Characterisations of 'Digger Indians' in the western United States (primarily Shoshone groups with subsistence strategies focusing on exploitation of underground storage organs) were a manifestation of a general hunter-gatherer stereotype that recalls dominant colonial representations of the Bushmen; 'savage' in their bestial lifestyle, 'indolent' in their production of a simple material culture, and 'wild' in their resistance to colonial encroachment (Lönnberg, 1981:218), this shared stereotype structured (in both cases) violent colonial commando practices (Hollon, 1974:61-62). Similar tropes recurred commonly in colonial portrayals of Aboriginal Australian populations (Strong, 1986); Mulvaney (1989:1-2) suggests that mutual ignorance (such as that surrounding the norms governing an oftenritualised Aboriginal violence) was a dominant influence shaping interactions between settlers and indigenes in Australia. In the Cape Colony too, representations of particular socio-cultural practices became emblematic of all that separated hunter-gatherers from colonial society; when the IXam engaged in the food-sharing practices vital to their constructions of socially responsible action, they were also (for the Europeans) demonstrating their improvident habits of consumption. However, while issues of mutual incomprehension were widespread (especially in the policies and attitudes of metropolitan centres), there also existed an 'on-the-ground' mediation. Focusing solely on the socio-cultural separateness of 'coloniser' and 'indigene' neglects the blurring consequent on 
their historical interaction; in South Africa, there were intermediate steps between indigenous and colonial societies, ranging from slaves (themselves colonists) escaped and otherwise, to European criminals, to marginalised colonial subjects (Bastaards, 'colonial Hottentots'). In Australia, colonial populations expanding into the arid northwest at the end of the nineteenth century, took with them 'pacified' Aboriginal labourers to work on their ranches; there emerged a division (in both settler and Aboriginal discourse) between those who had 'come in' to pastoral or mission stations and those who were 'wild' (Trigger, 1992:18; Greer, Harrison and McIntyre-Tamwoy, 2002:274; Harrison, 2002a:45), reminiscent of the same term as used by the IXam informants for people who fled from wagons (LL.III.1.503).

Structuring the comparative element of this chapter is that fact that, however defined, colonisation requires a geographic dimension. Incoporating topographical, environmental, and climatological phenomena often largely outside the realm of human control and influence (certainly on 'individual' scales), this dimension can form a convenient basis from which comparative endeavours can begin: while all geographic areas are equal in their specificity, some at least are more equal than others. With this in mind, I focus primarily on comparisons between the interior Karoo and two additional areas: the south-western United States and northern and western Australia, both arid regions ${ }^{235}$ that became the target of European colonial settlement. While non-local dimensions of colonial

\footnotetext{
235 Arid-zone hunter-gatherers have long been central to the anthropological construction of the 'hunter-gatherer' category (M. Smith et al. 2005).
} 
societies $^{236}$ and differences in climate regimes, geologies, and biota were clearly significant factors, similarities emergent from the dominant role of aridity in shaping these environments represent at least one 'controlled' dimension in these comparisons; Paterson's (2005:278) description of arid Australia emphasises water regimes as crucial to the ability of colonial societies to exploit the region and Knack's (1995:151) discussion of the role of 'localised' Great Basin resources (congregated around the few reliable water sources) in shaping the rapid spread of colonial society over the region are both equally applicable to the Karoo. The dominance of water regimes for arid zones, then, is mirrored by key areas of structural similarity in the ways these areas can be occupied, with small zones of resource concentration around water sources playing a vital role in enabling the exploitation of larger regions (M. Smith et al. 2005); European attempts to monopolise access to these key areas were the crucial constituent of the ultimate incompatibility of settler and indigenous lifeways in arid zones (Reynolds, 2006 [1981]: 160).

Aside from their common aridity, these three areas also all represent 'settlement colonies' (Osterhammel, 2005:11-12; see Russell, 2001; Coombes, 2005; Veracini, 2010, 2011 for further discussion of the 'settler colony'); briefly, these colonies saw large-scale immigration of settlers, forming resident communities in the colony itself and exploiting a ready availability of land derived from the

\footnotetext{
${ }^{236}$ Many 'colonial' behaviours and ideologies developed in geographically-distant areas through interactions with indigenous populations of these areas; indigenous communities too initiated contacts with colonisers based on prior engagements with (indigenous) 'Others'.
} 
(militarily backed) alienation of indigenous communities from their territories ${ }^{237}$. These colonies incorporated and attempted to exercise hegemony over large territories, leading to a commensurable intrusion of colonisers into the lifeways of indigenous communities that became a permanent addition to the socio-political landscape. This form of colonisation determined the development of 'the frontier' as an intellectual tool (and cultural motif), describing the geographical parameters of regions delineated by colonial authorities as areas incorporated under their political aegis (Noyes, 2001; Chapter 4b:107); similarities and differences between the development of 'frontier histories' in southern African and North America form the basis of Lamar and Thompson's (1981) edited volume of comparative history.

Although initiated by the arrival of settler populations aimed at exerting a political dominance, like most colonial productions in practice 'frontiers' represented a negotiation between colonial and indigenous populations. This negotiation incorporated the new environments encountered, with European expansions being predicated upon particular subsistence strategies $^{238}$ and thus dependent either upon the capacity of the environment to support the strategies, or of the modification of strategies to the environment; economic motivations for settlement were common to all three areas, with significant inland presence of Europeans in the arid Australia, the interior of southern Africa, and the south-

${ }^{237}$ Osterhammel's 'African' settlement colony type distinguishes South Africa from the 'New England' type in the United States and Australia; treatment of Bushmen in the 'Cape Colony' was substantially closer to his 'New England' model (displacement/annihilation of indigenes).

${ }^{238}$ Taking a broad definition, including the establishment of extractive capitalist strategies (ranching, mining, logging). 
western United States being based on a combination of livestock production and, as the nineteenth century progressed, mineral exploitation. This establishment of settler communities created a novel socio-cultural and environmental milieu, thereby ensuring that indigenous lifeways too had to find space within 'colonial environment', or be modified to suit it. These modifications took many forms, including 'assimilation' within colonial society, seen in the Cape Colony with the emergence of the 'tame Bushman' category as a precursor to a rural 'Coloured' identity. Highly arid areas were amongst the least desirable parts of the colony for incoming European farmers and pastoralists ${ }^{239}$, being the most resistant to their imported suite of domesticates. During the colonial period they thus provided attractive refugia for groups maintaining hunter-gatherer lifeways that required specialised knowledge of arid zone resources, or for those resisting incorporation within the colonial polity; a similar division of strategies occurred among the southern Paiute in the Great Basin, with some groups retreating to remoter and less attractive (for pastoralists/farmers) areas to continue hunting and gathering, and others forming fringe communities around the relatively productive regions that had been appropriated by settler society (Knack, 1995:151).

${ }^{239}$ Colonial authorities characterised the Northern Cape in this way (Chapter 4c:109) 


\section{Incorporating domesticates}

Settler colonisation depended upon the importation of a suite of plants and animals that often underpinned both the motivation for and means of expansion. The transformation of settler colonies through the creation of 'portmanteau biota', scaled-down and simplified versions of Western European biotic communities modified by engagement with local environments and species (Crosby, 2004:89), meant that changing interactions with animal and plant species (indigenous and otherwise) under the aegis of coercive enforcement, appropriation, or some intersection of the two, were essential components of indigenous interactions with colonisation. Crosby portrays portmanteau biota as 'familiarising' novel environments into forms that colonisers could exploit effectively ('Neo-Europes'), but Clark (2007:53) emphasises that these introductions often also represented a 'failure' on the part of colonial populations: attempts to render landscape familiar and controllable were subordinated by exigencies of local environments and by the introduced species' own tendencies to overrun boundaries. Many introductions were accidental, and intentional introductions often instigated unintended consequences. In many colonial settings, the arrival of European livestock was followed swiftly by their naturalisation as they escaped and founded feral communities (Anderson, 2004:108). This phenomenon was particularly 
pronounced on islands that lacked indigenous mammals to compete for ecological niches (e.g. duBois, 2005:17). As colonisation was dependent upon the success, modification, or failure of subsistence strategies, environmental as well as social, cultural and political factors thus played important roles in shaping colonisation. In the Cape Colony itself, the distinctive Western Cape winter-rainfall regime supported an agricultural settlement employing European crops and dependent on imported slave labour (at least, in the immediate hinterland of Cape Town itself), perhaps the only successful 'Neo-Europe' of southern Africa; settlers in the arid interior relied upon the creolised subsistence of trekboer pastoralism, as much the product of local knowledge and environmental factors as it was the result of imported socio-cultural patterns and the market demands of the Dutch East India Company (Beinart, 2003:31).

By contrast with African ecosystems, both Australia and the south-west United States saw rapid and considerable movements of introduced species beyond and ahead of political and settlement frontiers, with important consequences for local ecologies and for the subsistence strategies of indigenous communities. In Australia, introduced carnivore species have been destructive both of indigenous fauna and livestock (Glen and Dickman, 2005), particularly affecting the southern arid zone of spinifex desert (Short and Smith, 1994:288). Such effects influenced subsistence strategies, though not always in a negative fashion: the Mardu of the Western Desert consumed feral cat and rabbit meat, classified as part of a suite of 'bush' foods (Trigger, 2008:632). This spread of introduced species was determined by the environmental and ecological parameters of the continents; 
Late Pleistocene megafaunal extinctions that left both North America and Australia depauperate of large mammalian fauna were significant among these parameters. The extent (and recent date) of these extinctions arguably left 'gaps' in the ecosystems of the regions, with serious consequences for the remaining floral and faunal communities (Janzen and Martin, 1982; Owen-Smith, 1987; Donlan et al. 2006; Rubenstein et al. 2006); the rapid spread of feral horses in North America filling-up grassland niches left empty by the abrupt extinction of native equids from the Americas has been characterised as the 'return of the native' (Catling, 2001; P. Martin, 2005:38). The expansion of native species into recently emptied niches also ensured they did not offer robust competition to introduced species; Geist (1985:356) notes that while big-horned sheep in the Mojave Desert do display adaptations to their desert environment, the fact that these represent relatively recent modifications of an essentially Siberian species undermines their ability to effectively compete with introduced domesticated sheep or goats, with longer trajectories of adaptation to aridity (Geist, 1985:356). Another significant corollary of the absence of indigenous ungulate species in Australia (McKnight, 1976:1) and the low ungulate species diversity in North America (Lott, 1991:136) was that (unlike in Africa) the ability of large 'big game' herds ${ }^{240}$ to act as disease reservoirs impeding the spread of feral livestock (Gifford-Gonzalez, 2000; Grootenhuis, 2000) was limited, if not absent.

\footnotetext{
${ }^{240}$ A major exception is the American Bison (Bison bison), which can harbour bovine diseases (MacCorquodale and DiGiacomo, 1985); the extremely large herds (prior to their collapse through over-exploitation) characteristic of certain regions of colonial North America presumably would have elevated this to a potential limitation on cattle expansion.
} 
The impoverishment of carnivore species in Australia (Wroe, Myers and Gillespie, 1999; Johnson and Wroe, 2003; Johnson, 2006) and North America (P. Martin, 2005:37-38) facilitated the formation of viable feral communities, limiting (though not eliminating) predation pressures that formed in colonial southern Africa (with its numerous sympatric large carnivores; Woodroffe and Gindberg, 2005:157) a major challenge for feral domesticates (McShane and Swart, 2011:213). Malnutrition (or mortality) from the consumption of unfamiliar plants, predation, competition from indigenous wildlife, and a novel suite of pathogens (to which malnutrition increased their vulnerability) all contributed to the challenges faced by introduced equids in this region; perdesiekte (Afr. horsesickness, a viral infection transmitted by Culicoides sp. midges, Brown 2008:30) represented a particularly devastating virgin soil epidemic for these animals ${ }^{241}$. The difficulties of keeping horses alive fundamentally shaped both settler and indigenous relations with these animals (Chapter 8a:295), playing an important role in the establishment of colonial authority in Bushmanland and supporting the rise of Hantam (outside the habitat requirements of the midge vectors) as a horsebreeding area.

Ecological factors should not be taken in isolation from varying modes of human engagement with the biota, which had a considerable influence on the spread of domesticated species. Arid Australian environments did not allow for intensive agricultural production and major settler expansions (in geographical extent) were based on low-density systems of stock-keeping (Harrison, 2002, 2002a); these

${ }^{241}$ A single epidemic in 1854-1855 wiped out forty percent of the Cape's stock, or around 65000 horses (Brown, 2008:35). 
allowed animals to roam large (initially often unfenced) pastures, being mustered once or twice a year and offering ample opportunity for the naturalisation even of putatively 'owned' animals (McKnight, 1976:10). The rapidity and extent of the spread of the horse in North America, even when facilitated by floral biota that had co-evolved with equids, must in part be attributable to the important role this species acquired in emergent indigenous strategies (Woodward and Quinn, 2011:271-275). Unlike Australia and North America, the Cape Colony was occupied by stock-owning groups prior to European arrival, which (while it may have encouraged stock movement of the raiding kind) was as much a barrier to the establishment of independent feral communities of livestock as were the diseases, aridity, predators and competing herds of wild fauna. The long-term presence of pastoralist communities would also have affected the social and cultural context of independent livestock acquisition on the part of hunter-gatherers; if relationships between Khoekhoe and Bushman were cyclical (even if only at the level of shifts in individual practice), then Bushman individuals who wished to acquire stock had alternative avenues open to them than those offered by the incorporation of feral livestock communities or raiding. The presence of Korana horse-riding lifeways (Ouzman, 2005) in the central Karoo and northern frontier regions suggests a socio-cultural dimension to the absence of 'horse culture' Bushmen in the region (versus the south-eastern mountains, Challis, 2008), with Khoekhoe identity forming the nexus around which these types of society developed: Korana and Bastaard/Griqua presence goes some way towards explaining the absence of IXam equivalents for the Apache, Ute, or Comanche 
societies in the south-west United States that created successful, powerful polities through their adoption of the horse, allowing them access to firearms through the trade in hides derived from buffalo hunts (Lamar and Truett, 1996:87).

Returning to theoretical issues relating to hunter-gatherer incorporations of domestic animals, the absence of domestic fauna other than the dog in North America (accompanying initial human settlement; Leonard et al. 2002) and the dingo in Australia (most likely introduced from south east Asia in the mid Holocene; Clutton-Brook, 1995:15; Paterson, 2005:277) entails that any indigenous incorporation of stock in these areas demonstrates the capacity to shift away from reliance on wild fauna, with all the attendant difficulties of incorporating this ideologically. Though lacking domesticated fauna, many North American groups combined a range of subsistence options within their overall strategies, linking hunting and gathering with small-scale horticulture for at least part of the year. For some Southern Paiute groups in the south-west, this combination was itself a development of the colonial period, with Old World and indigenous cultigens being introduced approximately contemporaneously (Kelly and Fowler, 1986); Wallace (1980:271) suggests that the incorporation of smallscale farming represented an 'easy' extension of hunter-gatherer subsistence practices, added on to rather than replacing them, and largely achievable using pre-existing forms of technology. These 'horticultural' strategies of plant exploitation may have been replicated in some areas of pre-colonial Australia $(c f$. Gammage, 2005; Denham, Donohue, and Booth, 2009), particularly in the northeastern regions where tuber replanting (yams, taro) strategies were 
common $^{242}$. Although these resources were less well suited to arid regions, burning strategies were a widespread alternative form of landscape management for these areas: Mardu burning regimes increased both longer-term yields from plant resources and numbers of smaller animal prey in the short-term, thus increasing the calorific return from foraging practices (Bird, Bird and Parker, 2005). Undoubtedly rather different to intensified agricultural production, this represents a significant anthropogenic manipulation of resources, making them more predictable and increasing their productivity (Gammage, 2005:2); though Gammage's (2005:5-6) elision of differences between 'management' and 'farming' obscures the consequences attendant on a shift from a 'hunter-gatherer managed' landscape to a 'stock farmer managed' landscape, it is evident that the strategies of both alter the environment in complex and significant ways.

The appearance of resource management strategies among the Mardu and Southern Paiute suggests that even within a broadly 'egalitarian' ethos, notions of ownership of certain kinds of resource are permissible; for the Mardu, the application of fire-management confers (when the land is newly-burned) a form of limited ownership on the person instigating the burn (obtaining a right of firstaccess that may be defended with violence; Bird, Bird and Parker, 2005:457-458), while springs essential for southern Paiute gardening at Corn Creek in the Las Vegas Valley, Nevada were associated with specific named 'owners' (Von Till Warren, 2007:92-93). The IXam narratives (Chapter 7b:233; Chapter 7c:250) confirm an equivalent ownership of managed resources (honey and ostrich eggs)

\footnotetext{
${ }^{242}$ Human manipulation here took advantage of phenotypic elasticity rather than genetic change; loose friable soils (created by repeated exploitation) yield larger tubers (Denham, 2008:246).
} 
in Nama-Karoo hunter-gatherer strategies, where 'owners' of water sources were obliged to 'correctly' manage their territories. Control of the resources themselves is overlain by a differential access to 'knowledge' that underpins abilities to access particular resources within a given area: among the southern Paiute 'songscapes', encoded knowledge about how to move around the landscape from water source to water source, were subject to ownership (Stoffle, Halmo and Austin, 1997:243), and among the IXam knowledge about how to 'deal with' particular resources was not universally distributed (Chapter 5a:137).

Even egalitarian hunter-gatherer groups with a 'sharing' ethos do not treat resources in an undifferentiated fashion, and different resources are subject to 'sharing' demands that vary in their stringency. Differences between the way plant and animal foods are shared (the latter with a smaller set of kin) are widely recognised, deployed in attempts to examine ideologies of inequality in gender relations within putatively egalitarian groups (Brightman, 1996) ${ }^{243}$. Classifications of livestock as 'large bovids' (hunted prey), resources that for southern African hunter-gatherer groups would typically be subject to obligate sharing rules (A. Smith, 1990), may seem logical, and this certainly was a feature of the IXam conceptual world (linking springbok and sheep, Chapter 8a:286); Trigger (2008:636) notes that for Aboriginal groups on Queensland/Northern Territory border, introduced buffalo are incorporated within a set of cultural traditions linking it explicitly to native, hunted species. However, these classifications do

\footnotetext{
${ }^{243}$ Kent (1993:495-496) suggests that the presence of formally shared plant foods makes attempts to construct this inequality in a simple, dichotomous fashion is a misrepresention of huntergatherer practice.
} 
not represent the only modes of engagement with livestock. Recognising milk production as a form of resource management, by which means an animal that is 'taken care of' produces a regular supply of resources, interactions with livestock can appear as strikingly similar to ostrich eggs and honey as resources that, with the application of 'care', can be relied upon to regenerate themselves; as A. Smith (1990:62) notes for Jul'hoansi in the twentieth century, the sharing of milk rather than the animal itself can allow for the acquisition of stock without transgressing ethical limits.

Whatever their relationships with indigenous fauna and flora, alterations of this biota by the actions of the suite of species brought in with European colonists demanded a reorganisation in indigenous engagement with non-humans. The diversity of this collection of introductions ${ }^{244}$ was matched by a similar diversity in the ways they were incorporated by indigenous societies. This might manifest itself in differential attitudes towards particular species; groups in western Kimberley and in the central Australian deserts considered feral cats as more 'native' (established for longer time periods) than other introduced species such as foxes or rabbits (Trigger, 2008), and the Northern Territory MakMak (Rose, 2002) placed introduced species within a 'mosaic' of assimilation and rejection that classified feral buffalo as animals not 'belonging' to the country, at the same time as they themselves engaged with commercial cattle ranching. The expansion of these feral water buffalo is constrained by their own ecological requirements, and they have remained restricted to suitable wetland habitats in tropical northern

${ }^{244}$ In Australia this included sheep, cattle, goats, pigs, camels, buffaloes, donkeys, horses, rabbits, cats and dogs. 
Australia (Jesser, Markula and Csurhes, 2008:6). The domesticates best suited to more arid areas of the Australian continent were sheep, and the introduction of this species was a fundamental component of European expansion here; in the arid south western US too, sheep became (in the colonial period) thoroughly incorporated into the horticultural, hunting, and raiding subsistence strategies of the Navajo (White and Cronon, 1988:426). These scenarios (which instigated pastoralist strategies) can be seen as rather different to that obtaining in southern Africa, where large scale displacement of one group of (already established) pastoralists occurred. However, as this displacement coincided with a shift between pastoralist practices geared towards subsistence or herd accumulation for political purposes (Khoekhoe strategies) to practices aimed at producing meat as a market commodity ${ }^{245}$ (Lindholm, 2006:130), expansion of colonial sheep farming in the southern African interior created and faced issues similar to those outlined for Australia, in a somewhat ameliorated form (Paterson, 2005:277). In both areas, dependence on herds as a motivation for settlement produced a fluid sheepfarming frontier that fluctuated as farmers attempted to assess the carrying capacity of the environment under shifting conditions, which included the establishment of crops where possible, changes in the composition of grassland with grazing pressure, droughts, and seasonal variability (Paterson, 2005:278279).

Another more significant parallel is manifest in the vital role indigenous communities played in establishing sheep farms, providing the labour-intensive

\footnotetext{
245 Always a dimension of Karoo farming strategies, but one that became more significant during the later nineteenth century, with the expansion of interior markets around mining settlements.
} 
shepherding practices necessary in an unfenced landscape and contributing their detailed knowledge of the environment and geography. In the absence of indigenous herders, much of this knowledge as pertaining to the grazing potential and toxicity of indigenous plants for introduced species had largely to be developed in situ by the settlers in Australia; although less of an issue in southern Africa (Beinart, 2003:29-47), the employment of new breeds of livestock in settler pastoralism (breeds less suited to Karoo environments) meant that farmers needed to develop strategies ensuring the survival of their flocks, in a 'muted' expression of the knowledge acquisition that occurred in Australia. Processes of knowledge transfer occurred both from a settler and indigenous perspective; while European adoption of the requisite local environmental knowledge was matched by indigenous acquisition of knowledge about colonial society, deployed in creating responses suited to the demands of their colonial setting. Beinart (2003:30) considers the category of 'colonial Hottentots' in this fashion, and this might readily be expanded to include the 'tame Bushmen' of the northern frontier; although prior pastoralist subsistence strategies undoubtedly prefigured some of the skills demanded for membership in the mediating communities (such as animal management skills necessary for driving ox-wagons), other skills including tracking, knowledge of flora and their properties, and an understanding of local geography and climate were equally well (or even better) honed by a huntergatherer lifestyle.

Trigger (2008:633) notes that demands for Aboriginal labour in cattle-ranching have generated a strong sense of familiarity with the species (cattle, horses and 
dogs) connected with these practices, as well as a considerable degree of 'bush' knowledge pertinent to keeping and controlling cattle. These developments may have built on indigenous practices of controlling the movement of 'wild' species; Reynolds (2006 [1981]:162) proposes that the employment of Aboriginal populations in livestock industries generated substantial overlaps with extant hunting practices involving the driving of prey with stick and bush constructions. Although these practices were somewhat different to the IXam 'driving' of springbok (Chapter 7b:243), both examples confirm that hunting strategies often portray animals as agents whose movements are to be directed and controlled. Pastoralist labour thus emerges as a practice not qualitatively opposed to hunting and gathering, but rather as part of continuum of interactions with non-human species $^{246}$, especially as one alternative mode of engaging with domesticated species (raiding) forms a logical intermediary between the two: the fact that IXam representations of the 'veld' (Chapter 6c:201) involved both hunting and livestock herding practices implies that the two occupied a contiguous ideological, as well as the same physical, space.

The importance of specialised indigenous knowledge and labour for the establishment of these new subsistence and capitalist regimes in colonial settings places these hunter-gatherers firmly within the realm of 'specialist producers', a point made explicit in Knack's (1995:156) analysis of southern Paiute economic relations with settlers; these hunter-gatherers did not generate material products desired by settler society, other than their labour. This is reminiscent of the

\footnotetext{
${ }^{246}$ According well with Ingold's (2000) characterisation of the continuum between 'trust' and 'domination' modes of relating to non-humans (Chapter 2:38).
} 
economic relationship between central African forest hunter-gatherers and neighbouring farmers, with whom they exchange not only forest products such as meat and honey but also their labour (Köhler, 2005:47). In the Cape Colony, IXam individuals acquired particular products (ostrich feathers) for trade with settlers (Chapter 8c:335), extracting 'commoditised' resources in a manner similar to Irulas snake-catchers in Tamil Nadu, India (Whitaker and Andrews, 1996) and Efe honey-gatherers (Terashima, 1998) or Mbuti net-hunters (Hart, 1978) in the Ituri forest of the Congo. By expanding this to include labour for Europeans and Bastaards, they readily appear as 'specialist producers', a hunter-gatherer strategy that has often been cast in the idiom of contact and contamination (Headland and Reid, 1989; Layton, 2001) and thus connected with the 'Kalahari debate' in defining 'authenticity' in foraging lifeways. IXam subsistence strategies generated the vital constituents of cultural-significant material culture (Chapter 7d:262), and their interactions with food-producing colonial populations were contiguous with relations with other hunting and gathering groups (Chapter 7d:271). Specialisation in their 'productive' activities was not, therefore, a consequence of the colonial setting nor was it attendant on interactions with domesticated livestock: the ability to produce specialised products was not distributed equally among IXam individuals (possessing the cultural knowledge and skill to produce goods 'nicely') and groups ${ }^{247}$ (accessing resources associated with certain areas, such as particular plant poisons), and exchange with other hunter-gatherer groups may thus equally be presented as an instance of cultural contact or 'contamination'.

247 The Selk'nam of Tierra del Fuego (Chapman, n.d.; 1982:35-39) also displayed differential ownership of geographically-circumscribed resources (types of sandstone used to construct arrowstraightening tools), and restricted information to outsiders. 


\section{Social change and resilience}

The incorporation of domesticates thus emerges as a modification of subsistence not only in the sense of techniques of food acquisition, but also in terms of alterations in exchange networks and social relations. The construction of gendered identity represents an important dimension of social relations, one that (along with age) represents the dominant structuring principle of many huntergatherer societies. In a broad sense, Tonkinson's (2000) description of gender relations among the Mardu parallels the pattern evident from the IXam narratives; a strong egalitarian ethos in interpersonal relations on a daily basis (individuals of any gender potentially exerting considerable influence) being experienced through the specific kin relationships within which one is embedded, with age being an important modifier dictating social norms. At the level of 'corporate' identity, this mundane egalitarianism is replaced by 'structural' inequality favouring senior initiated men; products of their labour (meat) are more highly valued, religious institutions stress their vital role in establishing the relations that make the land productive, and social institutions (such as marriage) empower them with rights not accorded women ${ }^{248}$.

\footnotetext{
${ }^{248}$ Markedly distinct from the IXam case: IXam women had the right of divorce, leaving their husbands to remarry (LL.VIII.1.6079) or returning to live with consanguinal kin (LL.II.13.1291)
} 
I have returned several times to the problematic absence of female voices in the Bleek-Lloyd archive; the comparative context highlights the issues of this disparity, with questions of gender becoming increasingly significant components in the later twentieth century scholarship concerning colonial societies. The explicit concern of gender studies with power dynamics (Tonkinson, 2000:345) pre-configures such approaches for the study of colonialism, and conjugal and other gendered relationships ${ }^{249}$ between settlers and indigenes are now emphasised as an integral component of frontier ${ }^{250}$ scenarios. With the encroachment of colonial populations, relationships were established between European and indigenous masculinities and femininities. These became formative influences on the development of gender relations in the new colonial society; Haskin and Maynard (2005) discuss the importance in colonial public discourse of abrogating, or making pathological, sexual relationships between white women and Aboriginal men.

Gendered cross-cultural interactions both constrained and facilitated the kinds of strategies available to indigenous populations; Tonkinson (2000) speaks of both the colonial 'patriarchal' attitudes that shaped the emergence of men as public, political figures in colonial Aboriginal societies (or as 'bosses' in labour relations), as well as the sexual and/or domestic labour relations that provided women with opportunities to acquire knowledge of settler society and colonial

\footnotetext{
${ }^{249}$ This scholarship primarily examines interactions between settler men and indigenous women, though some authors (e.g. Haskin and Maynard, 2005) have focused on alternative relations ${ }^{250}$ Women from indigenous communities often found themselves on both 'sides' of frontier society, with much of the violence of this space consequent on (often abusive) settler relations with Aboriginal women (Schaffer, 2001:135-137)
} 
material culture, and (ultimately) better access to formal education. Similar processes obtained in the transformation of the Southern Paiute from autonomous egalitarian hunter-gatherers into an 'ethnic' class of marginal wage labourers within the colonial state (Knack, 1995:156) ${ }^{251}$. This incorporation forced them to engage with settler notions of gender, as the work offered to labourers was governed by the colonist expectations of appropriate male and female labour; women were hired for domestic work and (especially if accompanied by children) were more successful in soliciting gifts (begging) from Europeans (Knack, 1995:152). This labour allowed women to acquire an independent income, maintaining the significance of their subsistence activities and contributing to the continuance of relatively egalitarian gender relationships of the precolonial period, despite the fact that settler society did not recognise women as community leaders (Knack, 1995:153). Though there are no verbatim accounts from IXam women engaged in similar relations, references to men using their connections with such women to access food (Chapter 8c:323) suggest that similar processes were occurring in the Karoo during the nineteenth century, and marriages documented between IXam and Korana suggest that these types of relationships may have had a deeper precolonial past.

Among the Mardu, the provision of resources (welfare and pensions) by the state has afforded both young unmarried women and more elderly widows, whose 'structural' position formerly circumscribed their actions and made them subject

${ }^{251}$ Southern Paiute groups traditionally practised subsistence strategies rather similar to those of the IXam, employing a gendered division of labour with female gatherers and male hunters. However, this division was fluid; women trapped game, and men participated in piñon nut and mesquite harvests. 
to the dictates of others (usually male elders, but also senior women), a degree of subsistence independence (Tonkinson, 2000:355). This has been mirrored by declining engagements with 'structural' unequal social institutions; polygyny is much reduced, despite older male insistence on its propriety under the 'old law'. The IXam similarly circumscribed their young women with proscriptions derived from an ideological network. Although these prohibitions were contested (at least in oral narratives), it seems likely that marriages to Bastaard farmers (Chapter 8c:323) would have allowed young women opportunities for challenging them further, especially in light of the fact that there was already a gendered incorporation of livestock resources in the ritualised behaviours linked with seclusion practices (see glossary !kwi-|a-\|ka:n:433; Chapter 8a:293). The archive also provides evidence that (relatively expeditious) re-marriage of widows was common among the nineteenth century IXam (LL.V.3.4132'); with this in mind, the decisions made by \|kabbo and lhanłkass'o's wives to stay with a farmer (LL.II.6.648) may be seen as an attempt to ensure secure resource access and to avoid pressures to remarry, as occurred with łkasin's wife during his imprisonment in the Breakwater (WB.XXV.2351').

Although livestock represented a key domain of colonial material culture shaping hunter-gatherer experiences of colonisation, other elements of material culture were also significant. In Australia, narcotics and other novel drugs became a significant component structuring Aboriginal interest in engaging with colonial society, with tobacco and tea becoming seen as essential components of subsistence (Trigger, 1992:27). The more recent nature of European pastoralist 
expansion into arid Australia has facilitated on-going ethnohistorical work that provides detailed accounts of hunter-gatherer experiences of these 'Westernising' desires. Harrison (2002, 2002a, 2004) explores oral traditions emphasising the role of desires for drugs (tea, tobacco) and preferences for European foodstuffs in enticing Aborigines to 'come in' to the pastoral station (Harrison, 2004:116), leaving physical traces (billy cans, tobacco tins) in the archaeological signatures of pastoral workers' camps that chart divisions between people who lived at that station and those who were still 'wild'. The importance of tobacco in IXam life is evident (Chapter 8c:333), and Harrison's observations on the significance of foodstuffs suggests that IXam comments on the abilities of Europeans to give them 'little pieces of fat' (LL.VIII.12.7108') should be accorded a similar recognition as a motivation for interaction.

Looking at the Aboriginal adoption and modification of metal tools, Harrison (2002:67) points out that as contact processes created fundamentally different environments to those obtaining precolonially, new forms of material culture need not represent assimilation or acculturation but may rather arise simply from the new needs of this situation. This notion of material culture as usefully deployed within a shared colonial environment is a useful perspective for examining those practices that cross-cut 'ethnic' or other forms of identity, such as the colonial 'gun cultures' in all three areas I have looked at here: the widespread appeal of guns suggests that they are best viewed as suited to the colonial scenario itself. While the importance of the gun as a formative component of colonial society has sometimes been overstated ( $c f$. Bellesiles, 1996, 2002), from the onset of 
colonisation in the Americas, Australia, and South Africa, the gun took on a new valence for European populations both as a protective device and as a subsistence tool. Guns were very much a cross-cultural obsession of the southern African Karoo (Storey, 2008:17-47, 78-117), and numerous strategies were employed to acquire them, from settlers keen to acquire privileged access to gunpowder supplies by representing their needs to colonial government (Chapter $4 \mathrm{c}: 101$ ), to Bastaard 'respectability' relationships with missionaries that maintained a 'civilised' status that facilitated gun ownership (Legassick, 2010 [1969]), through to acquisition of guns by IXam individuals though labour relationships with farmers. This 'gun culture' of the Karoo thus represented at least in part a strategy of incoming (European, Bastaard, Xhosa) populations of dealing with a novel arid environment, combining subsistence and economic components with the influence of violent interactions; for livestock-keeping groups, hunting with guns allowed the maximisation of stock and the extraction of valuable wild game products. This incoming strategy soon altered the Karoo (and southern African environments generally; Beinart, 1990:167-169) on such a scale that guns were rendered a valuable dimension for coping with what had become a novel environment even for indigenes: by the nineteenth century the IXam were emphasising the importance of guns in subsistence (LL.II.32.2924'-2925'), where they acquired a potentially socially transformative dimension, represented as a more 'individualistic' mode of acquisition in hunting and incorporated in depictions of the actions of 'angry' men (LL.IV.1.3465-3471; Chapter 6a:175). 
As another example, Arkush (1987) suggests that historic-period Paiute winter houses can be taken as indices of the continuance of 'traditional' subsistence strategies, in spite of settler encroachments; the descriptions of these houses are strikingly reminiscent of D. Bleek's (1936) photographs of IXam dwellings in the early twentieth century. In both cases, the material culture of the built environment represents a concrete manifestation of the conflicts and negotiation developing between the 'institutions' of different societies, characteristic of the colonial encounter. The houses constructed by D. Bleek's informants, or by la!kunta while living with a farmer (Chapter 8c:332), are thus indicative of attempts to find the 'space' to accommodate hunting and gathering subsistence within the colonial situation. Conversely, the imposition of a new 'settler' built environment, in terms of the construction of mission stations and farm buildings, represented the physical presence of settlers on the landscape and served metonymically for the arrival of the new forms of religious ideology and sociocultural organisation with which indigenes had to contend; it is no accident that these new-made 'places' have formed the focus for studies of the material dimension of colonial expansion ${ }^{252}$. The occupation of IXam territories at the larger scale was fundamental to the ultimate collapse of their hunter-gatherer ways of life, as with the importance of boreholes and barbed wire for the imposition of a European conceptualisation of land ownership, over negated IXam definitions.

\footnotetext{
252 E.g. Birmingham's (2000) exploration of a Lutheran mission in the Australian interior, mapping the 'falling-off' of colonial material culture with physical proximity to station buildings, and thus implying a link between physical association and cultural accommodation.
} 


\section{Concluding remarks}

The Bleek-Lloyd archive creates an unusual dilemma for the scholar interested in hunter-gatherer relationships with colonial populations, recording an extraordinary 'depth' of information but (initiated as a linguistic programme and not structured as 'ethnography') doing so in a largely ad hoc fashion from a limited number of informants. This creates problems regarding extrapolation from a small and potentially atypical dataset, similar to those Barnard (2008) discusses for Khoekhoe societies of the Eastern and Western Cape. While these issues limit the possibility of developing a social anthropological understandings of their societies in a strict sense, by deploying historical accounts within a comparative context (looking at Khoe populations for which we possess more extensive information), Barnard (2008:62) suggests that partial 'ethnographies' can be reconstructed. Reliance on comparative exercises in the absence of detailed specific ethnographic data for one society may serve to (re-)create more homogeneous identities than truly existed ${ }^{253}$, and assertions must therefore remain cautious; Barnard (2008:68-69) stresses the need to examine 'resilient' social phenomena (such kinship structures) and to employ as broad a comparative dataset as possible (Barnard, 2008:68). In this spirit, the incorporation of insights

${ }^{253}$ A problem faced especially with non-IXam 'Southern Bushmen'. 
from geographically disparate populations employing similar subsistence strategies (Fauvelle-Aymar, 2008) or from groups living under similar ecological, environmental or historical conditions may also prove enlightening (though such groups should exhibit fewer specific resonances with 'Khoekhoe society').

These approaches ring equally true for the IXam, as ethnographic descriptions of other southern Bushman societies are not available, and the archive can no longer be supplemented with information from extant IXam communities; although these hypothetical communities could not provide unproblematic exegeses for the archive, assuming continuity in cultural identity and (at least to some degree) lifeways such communities would be expected to possess useful interpretive insight $^{254}$. The unusual nature of the Bleek-Lloyd material allows us to circumvent this potentially problematic assumption (with its echoes of 'timeless' Bushmen); possessing detailed verbatim accounts where fragmentary documentation from colonial officers, hunters, missionaries, and other travellers would otherwise have to suffice, the archive can be examined for themes that were not at the time of collection considered interesting, and in so doing 'ethnographic' descriptions can be attempted. Tempered by historical, ecological, and environmental contextualisation, and set within a theoretical framework provided by the largescale comparisons of hunter-gatherer studies, production of this IXam 'ethnography' has been the work of this thesis. A key dimension of this work has been the in-depth consideration of IXam terminology, an element hitherto

\footnotetext{
254 Justified to some extent by Hoff's $(1998 ; 1997)$ findings of themes relevant to the archive among IXam-descendants in the Northern Cape
} 
neglected in analyses of the corpus ${ }^{255}$. Although the archive certainly records this terminology comprehensively, the subsequent demise of the IXam language has impeded its treatment according to modern standards of linguistic analysis, a problem compounded by the fact that relatively little is known regarding related Tuu languages. This situation largely reverses that in other areas of huntergatherer interaction with European colonists, where comparatively sparse historical linguistic documentation can be supplemented by ongoing linguistic work $^{256}$.

While the production of such linguistic analyses of IXam is beyond the scope of my thesis, use of the original terminology has been vital to my exploration of emic IXam ideologies, and has proven an invaluable component in the development of fresh insights into Bleek-Lloyd material. Through close analysis of the deployment of specific terminology, I have been able to reconstruct a partial ethnography of the nineteenth century $\mid X a m$, which elucidates the ideological underpinnings of IXam beliefs and practice. By examining the archive in its entirety, I have explored the recurrence of terminology and themes over a range of narrative 'types' collected from all the Bleek-Lloyd informants; this approach allowed me to extract salient cultural dimensions, and highlighted the interwoven and mutually reinforcing nature of these ideological components. In the course of this analyis, one set of themes became particularly significant: issues dealing with the differentiation of IXam society, in terms of the construction of

\footnotetext{
${ }^{255}$ See Lewis-Williams and Challis (2011) for discussion of emic differentiation between types of 'magical' potency.

256 Acknowledging widespread processes of language death, with extant languages representing only a sample of linguistic diversity at the onset of colonisation.
} 
authority, competence, and social responsibility. Building on previous scholarship that has focused particularly on understanding the role of ritual specialists in IXam socio-cultural practice, I have explored the way in which these broader ideological premises supported and made 'meaningful' the performance of a range of specific roles in IXam society; although ritual specialists undoubtedly were culturally salient figures in IXam life, they did not exist in isolation. One major avenue of differentiation, and a salient domain for the construction of IXam identities, was through participation in subsistence activities, where skilful modes of acquiring (and appropriate attitudes in distributing) resources underpinned IXam evaluations of admirable versus reprehensible persons. The development of this ethnographic analysis consequently entailed the reconstruction of a holistic depiction of IXam subsistence strategies, linking these strategies to the underlying ideological framework. Recognition of the relationship of mutual reinforcement existing between this framework and IXam subsistence practices led to a more nuanced understanding of their engagements with and integration into nineteenth century colonial society; by examining IXam strategies as a coherent whole, their engagement with colonial societies, domesticated species, and novel material culture of the colonial period appears not as incongruous with their 'huntergatherer' status but rather becomes a fundamental consequence of it. Contacts and interactions between IXam individuals and the various agents of the nineteenth century Karoo set parameters that were sometimes outside of IXam control, and which (over time) necessitated large-scale modifications in their practices and beliefs; such interactions did not, however, represent an instantaneous and 
complete dissolution of 'IXam practice' or abrogation of their identity, but instead added further complexity to a world that already included a large number of differentiated parties, each with their own aims, desires, and resources.

My detailed ethnographic reconstruction, situated in the specifics of the nineteenth century historical context, provides the necessary foundation for examining the IXam materials in cross-cultural contexts, moving beyond comparisons with Khoe or non-|Xam Bushman societies to include as broad a dataset as possible. In this thesis, I developed further insights by placing IXam ethnography both within a global 'theoretical' situation (allowing me to locate profitably these materials within general models of 'hunter-gatherer' ontologies drawn from circumpolar and South American societies), and by emphasising the status of IXam as 'colonial' hunter-gatherers. Many of the contextually specific manifestations of their interactions in the northern frontier regions of the Cape Colony reiterate thematic issues seen elsewhere in the world. Each of these regions exhibits historical contingencies that have shaped the ways in which their hunter-gatherer communities (and their contemporary descendants) have been conceptualised over the twentieth and into the twenty-first centuries; the North American reservation system, for example, constructed 'official' indigenous identities, and individuals who chose not to participate in this might disappear into more generic rural or urban labour forces (Knack, 2001:183). The different representations thus arising have directed the kinds of research themes emergent in each area, and themes from Australia and North America point to lacunae in southern African research that have the potential to be profitably investigated in 
the future. While the often dramatic transformations required by new colonial environments might be envisioned as an insurmountable separation from earlier cultural forms, Knack's (2001) discussion of Paiute strategies continuing into the twentieth century suggests that even extreme shifts in practice can allow for continuity in some socio-cultural aspects: the traditional kinship obligations were maintained between Paiute individuals who engaged with the reservation system and those who remained outside this system, close to towns or in such 'traditional' spaces as they could still inhabit (Knack, 2001:187-188), underpinning a residential fluidity that, to some extent, perpetuated the shifting residence patterns and rights of resource access acquired by dwelling that characterised precolonial Paiute strategies (Knack, 2001:191).

These survivals of 'hunter-gatherer' attitudes in the face of 'assimilation' to colonial society have particular relevance for South Africa, because the dominance here of twentieth century apartheid, and its role in the formulation of the 'Coloured' identity. This has had important consequences in terms of the disappearance of 'hunter-gatherer' identities from national discourse ${ }^{257}$, and despite post-1994 'Khoisan’ revivals (Lee and Hitchcock, 2001:271-274; Lee, 2003:96-104), this remains largely true in South Africa today, unlike the situation in Australia or North America ${ }^{258}$. Calls from Australia for the development of community-oriented approaches that focus on particular 'places' on the landscape,

\footnotetext{
${ }^{257}$ Excepting two small communities: the 'Schmidsdrift' !Xun and Khwe communities (now living at Platfontein), and the łKhomani 'Kagga Kamma' groups forced out of the Kalahari Gemsbok National Park under apartheid (Lee and Hitchcock, 2001:269-270).

${ }^{258}$ Even in the face of disruption and amalgamation, former hunter-gatherer 'Aboriginal' or 'First Nations' (and more specific) cultural identities have here retained clear continuities into the present.
} 
tracking colonial history not as one of either settler or indigene but as a shared trajectory (Harrison 2002a) represents an approach that would re-configure rural Northern Cape Coloured populations as the result of longer-term trajectories rather than as the product of utterly destructive colonial historical processes. While such a configuration may sometimes demand the amalgamation of 'Khoekhoe', 'Bastaard' and 'Bushman' categories that researchers have preferred to keep distinct, where this amalgamation has been implemented it has yielded profitable insights (Morris, 2002, 2008). In this paradigm, the oral and written histories and archaeologies of Northern Cape farms, of dam and windmill construction, of rural labourer bothies and kraals, would be incorporated within a broader notion of 'IXam' and Khoekhoen adaptations to the colonial environment that have the potential to cast such interactions not only as 'failures' (as undoubtedly was the case for many individuals) but also as successful responses that have ensured their descendants' continuing role in the construction of identities within the 'post-colonial' South African state. 


\section{Appendix A}

\section{A Note on Orthography and Pronunciation}

Through this thesis, extensive reference shall be made to IXam words. The often radical differences between Khoisan and Indo-European languages have necessitated substantial orthographic modifications to express the full range of sounds present in the former; much of the pioneering work in this area was undertaken by Bleek himself. Early efforts focused on the distinctive 'click consonants' of the Khoisan orthography: these developed separately from the IPA (International Phonetic Association) system, whose symbols for clicks are not widely used among past or contemporary scholars of Khoisan languages. As many of these symbols will be unfamiliar, I present here a brief overview of the basic sounds that will be encountered (see Barnard, 1992:xviii-xxv and Hollmann, 2004 for more specific information on IXam pronunciation). The five clicks present in IXam are denoted thus:

I - the dental click. A dental or alveolar affricate (fricative), produced by sucking the tip of the tongue back from the teeth, as in English 'Tsk, tsk' 
\| - the lateral click. A lateral affricate (fricative), produced with the tongue on the roof of the mouth, releasing air between the tongue and one or other cheek, approximating the sound made to urge on a horse.

! - the palatal (cerebral, retroflex) click. An alveopalatal or palatal stop, produced by sharply pulling the tip of the tongue down from the hard palate, sounding much like an imitation of a cork pulled from a bottle.

$\neq$ - the alveolar click. An alveolar stop, produced by sharply pulling the tongue away from the alveolar ridge, behind the teeth.

$\odot$ - the bilabial click. A bilabial stop or affricate, produced by releasing air between the lips, somewhat like a 'kissing' noise but without rounded lips.

Clicks habitually occur in consonant clusters (treated as separate phonemes by linguists) and are often voiced (pronounced with a $d$ or $g$ ) or nasalised (indicated with an $n$ ). Bleek and Lloyd tended to place click symbols in front of any nasalising or voicing letter: although the reverse is now preferred in Khoisan orthography, I follow them in this practice. Letters after click symbols are not capitalised $^{259}$. Remaining consonants and vowels are indicated by the standard IPA phonetic symbols, though $x$ is used to represent a guttural sound similar to the ch in 'loch', or a $g$ in Afrikaans. The cluster denoted $k$ " in Bleek and Lloyd's writing (now $k x^{\prime}$ ) represents the velar ejective affricate. Symbols commonly employed in IXam words include $\int(s h), 3(z h)$, and a (the schwa). Increased vowel length is represented by a colon after the vowel (following Bleek's (1956)

${ }^{259} \mathrm{I}$ have made an exception of 'IXam' itself, due to the widespread use of the capital in related scholarship. 
practice) and glottal stops are indicated by ' rather than ?. A tilde above a letter reflects nasalisation. Bleek (1956:1) uses a spiral symbol to represent what she terms 'pressed' vowels or pharyngeal roughing; I have used a lowercase Xi $(\xi)$ to denote this symbol for consistency of referencing. Tone was also an important feature in IXam, although it is omitted here (following Barnard, 1992:xxiii). The Bleek and Lloyd notebooks (and to some extent, the Dictionary) employ numerous diacritics to convey this dimension of the IXam language, with numerous alternative renditions of the same word. It is often unclear which of these marks represents meaningful information: this is a major challenge for translating obscure passages, and in the absence of a detailed linguistic reconstruction of IXam must remain an ambiguous element (which is indicated where relevant). Personal and group names are not italicised. 


\section{Appendix B}

\section{Referencing the Bleek-Lloyd Archive}

(Centre for Curating the Archive, University of Cape Town, South

Africa)

With respect to the original Bleek and Lloyd notebooks, references take the following form: LL.VIII.22.7906-7908. The first element represents the initials of the collector (Lucy Lloyd or Wilhelm Bleek), the second component a Roman numeral corresponding to a narrator. The remaining numerals refer to the notebook and page number respectively. For Bleek's notebooks, there were no narrator numerals, and the Roman numeral corresponds to the consecutively numbered notebook, followed by a page number (e.g. WB.XIV.1310). For ease of consultation, the following tables provide a list linking these notebook references with the format used for the digitised material: the digitised material can be found in Skotnes (2007), or online at http://lloydbleekcollection.cs.uct.ac.za/books.html. 


\section{Wilhelm Bleek Notebooks}

\begin{tabular}{|l|l|}
\hline Notebook Reference & Digitised Reference \\
\hline WB.I.1-333 & BC_151_A1_4_001 \\
\hline WB.II.334-428 & BC_151_A1_4_002 \\
\hline WB.III.429-514 & BC_151_A1_4_003 \\
\hline WB.IV.515-584 & BC_151_A1_4_004 \\
\hline WB.V.585-679 & BC_151_A1_4_005 \\
\hline WB.VI.680-750 & BC_151_A1_4_006 \\
\hline WB.VII.751-822 & BC_151_A1_4_007 \\
\hline WB.VIII.823-894 & BC_151_A1_4_008 \\
\hline WB.IX.895-964 & BC_151_A1_4_009 \\
\hline WB.X.965-1026 & BC_151_A1_4_010 \\
\hline WB.XI.1027-1120 & BC_151_A1_4_011 \\
\hline WB.XII.1121-1213 & BC_151_A1_4_012 \\
\hline WB.XIII.1214-1306 & BC_151_A1_4_013 \\
\hline WB.XIV.1307-1401 & BC_151_A1_4_014 \\
\hline WB.XV.1402-1495 & BC_151_A1_4_015 \\
\hline WB.XVI.1496-1590 & BC_151_A1_4_016 \\
\hline WB.XVII.1591-1683 & BC_151_A1_4_017 \\
\hline WB.XVIII.1684-1777 & BC_151_A1_4_018 \\
\hline WB.XIX.1778-1871 & BC_151_A1_4_019 \\
\hline WB.XX.1872-1964 & BC_151_A1_4_020 \\
\hline WB.XXI.1965-2058 & BC_151_A1_4_021 \\
\hline WB.XXII.2059-2152 & BC_151_A1_4_022 \\
\hline WB.XXIII.2153-2247 & BC_151_A1_4_023 \\
\hline WB.XXIV.2248-2339 & BC_151_A1_4_024 \\
\hline WB.XXV.2341-2434 & BC_151_A1_4_025 \\
\hline WB.XXVI.2435-2529 & BC_151_A1_4_026 \\
\hline WB.XXVII.2530-2621 & BC_151_A1_4_027 \\
\hline WB.XXVIII.unnumbered & BC_151_A1_4_028_STARS \\
\hline & \\
\hline & \\
\hline
\end{tabular}




\section{Lucy Lloyd Notebooks}

\begin{tabular}{|l|l|}
\hline Notebook Reference & Digitised Reference \\
\hline LL BK I-1 1-77 & BC_151_A2_1_005 \\
\hline LL BK I-2 119-188 & BC_151_A2_1_006 \\
\hline LL BK II-1 189-287 & BC_151_A2_1_007 \\
\hline LL BK II-2 288-382 & BC_151_A2_1_008 \\
\hline LL BK II-3 383-475 & BC_151_A2_1_009 \\
\hline LL BK II-4 476-529 & BC_151_A2_1_010 \\
\hline LL BK II-5 530-624 & BC_151_A2_1_011 \\
\hline LL BK II-6 625-715 & BC_151_A2_1_012 \\
\hline LL BK II-7 716-810 & BC_151_A2_1_013 \\
\hline LL BK II-8 811-907 & BC_151_A2_1_014 \\
\hline LL BK II-9 908-1002 & BC_151_A2_1_015 \\
\hline LL BK II-10 1003-1099 & BC_151_A2_1_016 \\
\hline LL BK II-11 1100-1170 & BC_151_A2_1_017 \\
\hline LL BK II-12 1171-1243 & BC_151_A2_1_018 \\
\hline LL BK II-13 1244-1314 & BC_151_A2_1_019 \\
\hline LL BK II-14 1315-1431 & BC_151_A2_1_020 \\
\hline LL BK II-15 1432-1499 & BC_151_A2_1_021 \\
\hline LL BK II-16 1500-1553 & BC_151_A2_1_022 \\
\hline LL BK II-17 1554-1622 & BC_151_A2_1_023 \\
\hline LL BK II-18 1623-1691 & BC_151_A2_1_024 \\
\hline LL BK II-19 1692-1759 & BC_151_A2_1_025 \\
\hline LL BK II-20 1760-1855 & BC_151_A2_1_026 \\
\hline LL BK II-21 1856-1948 & BC_151_A2_1_027 \\
\hline LL BK II-22 1949-2042 & BC_151_A2_1_028 \\
\hline LL BK II-23 2043-2134 & BC_151_A2_1_029 \\
\hline LL BK II-24 2135-2226 & BC_151_A2_1_030 \\
\hline LL BK II-25 2227-2319 & BC_151_A2_1_031 \\
\hline LL B B II-26 2320-2412 & BC_151_A2_1_032 \\
\hline LL BK II-27 2413-2504 & BC_151_A2_1_033 \\
\hline LL BK II-28 2505-2596 & BC_151_A2_1_034 \\
\hline LL BK II-29 2597-2687 & BC_151_A2_1_035 \\
\hline LL BK II-30 2688-2779 & BC_151_A2_1_036 \\
\hline LL BK II-31 2780-2873 1/2 & BC_151_A2_1_037 \\
\hline LL B B II-32 2874-2965 & BC_151_A2_1_038 \\
\hline LL BK II-33 2966-3057 1/2 & BC_151_A2_1_039 \\
\hline LL BK II-34 3058-3149 1/2 & BC_151_A2_1_040 \\
\hline LL BK II-35 3150-3241 1/2 & BC_151_A2_1_041 \\
\hline LL BK II-36 3242-3332 & BC_151_A2_1_042 \\
\hline & \\
\hline
\end{tabular}




\begin{tabular}{|c|c|}
\hline LL BK II-37 3333-3424 & BC_151_A2_1_043 \\
\hline LL BK IV-1 3425-3515 & BC_151_A2_1_044 \\
\hline LL BK IV-2 3516-3607 & BC_151_A2_1_045 \\
\hline LL BK V-1 3608-3700 & BC_151_A2_1_046 \\
\hline LL BK IV-3 3701-3792 & BC_151_A2_1_047 \\
\hline LL BK V-2 3793-3881 & BC_151_A2_1_048 \\
\hline LL BK IV-4 3882-3968 & BC_151_A2_1_049 \\
\hline LL BK VI-1 3882-3974 & BC_151_A2_1_050 \\
\hline LL BK VI-2 3975-4071 & BC_151_A2_1_051 \\
\hline LL BK V-3 4071-4161 1/2 & BC_151_A2_1_052 \\
\hline LL BK V-4 4162-4252 1/2 & BC_151_A2_1_053 \\
\hline LL BK V-5 4253-4344 1/2 & BC_151_A2_1_054 \\
\hline LL BK V-6 4345-4435 & BC_151_A2_1_055 \\
\hline LL BK V-7 4436-4526 1/2 & BC_151_A2_1_056 \\
\hline LL BK V-8 4527-4617 1/2 & BC_151_A2_1_057 \\
\hline LL BK V-9 4618-4706 1/2 & BC_151_A2_1_058 \\
\hline LL BK V-10 4707-4796 & BC_151_A2_1_059 \\
\hline LL BK V-11 4797-4889 1/2 & BC_151_A2_1_060 \\
\hline LL BK V-12 4890-4980 1/2 & BC_151_A2_1_061 \\
\hline LL BK V-13 4981-5039 & BC_151_A2_1_062 \\
\hline LL BK V-14 5040-5078 1/2 & BC_151_A2_1_063 \\
\hline LL BK V-15 5079-5168 1/2 & BC_151_A2_1_064 \\
\hline LL BK V-16 5169-5260 & BC_151_A2_1_065 \\
\hline LL BK V-17 5261-5353 & BC_151_A2_1_066 \\
\hline LL BK V-18 5354-5444 & BC_151_A2_1_067 \\
\hline LL BK V-19 5445-5536 1/2 & BC_151_A2_1_068 \\
\hline LL BK V-20 5537-5628 & BC_151_A2_1_069 \\
\hline LL BK V-21 5629-5719 1/2 & BC_151_A2_1_070 \\
\hline LL BK V-22 5720-5810 & BC_151_A2_1_071 \\
\hline LL BK V-23 5811-5901 1/2 & BC_151_A2_1_072 \\
\hline LL BK V-24 5902-5992 & BC_151_A2_1_073 \\
\hline LL BK V-25 5993-6030 & BC_151_A2_1_074 \\
\hline LL BK VII-1 6031-6046/5 & BC_151_A2_1_075 \\
\hline LL BK VIII-1 6047-6140 & BC_151_A2_1_076 \\
\hline LL BK VIII-2 6141-6231 & BC_151_A2_1_077 \\
\hline LL BK VIII-3 6232-6322 & BC_151_A2_1_078 \\
\hline LL BK VIII-4 6323-6413 & BC_151_A2_1_079 \\
\hline LL BK VIII-5 6414-6504 & BC_151_A2_1_080 \\
\hline LL BK VIII-6 6505-6595 1/2 & BC_151_A2_1_081 \\
\hline LL BK VIII-7 6596-6686 & BC_151_A2_1_082 \\
\hline LL BK VIII-8 6687-6769 & BC_151_A2_1_083 \\
\hline LL BK VIII-9 6770-6857 & BC_151_A2_1_084 \\
\hline LL BK VIII-10 6858-6944 & BC_151_A2_1_085 \\
\hline LL BK VIII-11 6945-7031 & BC_151_A2_1_086 \\
\hline
\end{tabular}




\begin{tabular}{|l|l|}
\hline LL BK VIII-12 7032-7118 & BC_151_A2_1_087 \\
\hline LL BK VIII-13 7119-7205 & BC_151_A2_1_088 \\
\hline LL BK VIII-14 7206-7288 & BC_151_A2_1_089 \\
\hline LL BK VIII-15 7289-7375 1/2 & BC_151_A2_1_090 \\
\hline LL BK VIII-16 7376-7462 & BC_151_A2_1_091 \\
\hline LL BK VIII-17 7463-7549 & BC_151_A2_1_092 \\
\hline LL BK VIII-18 7550-7638 & BC_151_A2_1_093 \\
\hline LL BK VIII-19 7639-7727 & BC_151_A2_1_094 \\
\hline LL BK VIII-20 7728-7816 & BC_151_A2_1_095 \\
\hline LL BK VIII-21 7817-7905 & BC_151_A2_1_096 \\
\hline LL BK VIII-22 7906-7994 & BC_151_A2_1_097 \\
\hline LL BK VIII-23 7995-8083 3/4 & BC_151_A2_1_098 \\
\hline LL BK VIII-24 8084-8172 & BC_151_A2_1_099 \\
\hline LL BK VIII-25 8173-8261 & BC_151_A2_1_100 \\
\hline LL BK VIII-26 8262-8350 & BC_151_A2_1_101 \\
\hline LL BK VIII-27 8351-8437 & BC_151_A2_1_102 \\
\hline LL BK VIII-28 8438-8525 1/2 & BC_151_A2_1_103 \\
\hline LL BK VIII-29 8526-8614 & BC_151_A2_1_104 \\
\hline LL BK VIII-30 8615-8702 & BC_151_A2_1_105 \\
\hline LL BK VIII-31 8703-8791 & BC_151_A2_1_106 \\
\hline LL BK VIII-32 8792-8878 & BC_151_A2_1_107 \\
\hline LL BK III-1 476-570 & BC_151_A2_1_125 \\
\hline LL.first unnumbered notebook.1-19 & BC_151_A2_1_001 \\
\hline LL.second unnumbered notebook.20-39 & BC_151_A2_1_002 \\
\hline LL.third unnumbered notebook.40-60 & BC_151_A2_1_003 \\
\hline LL.fourth unnumbered notebook.61-118 & BC_151_A2_1_004 \\
\hline Sketch (springbok hunting; LL.VIII.23) & UCTLB_D_h008 \\
\hline
\end{tabular}




\section{Appendix C}

\section{A Note on Nomenclature}

Naming conventions for the groups discussed in this thesis remain a contentious issue in southern African scholarship. Extant hunter-gatherer populations in Botswana and Namibia have historically had little sense of a participation in a pan-southern African hunter-gatherer identity and consequently lack a corresponding autonym ${ }^{260}$. Northern Cape hunter-gatherers also lacked this terminology, although the IXam word ! $k^{\prime} e$ (people, Bleek, 1956:419) was largely reserved for populations linguistically affiliated with them (Chapter 7a:215). From the onset of colonisation, European observers attempted to classify African populations, adopting a mixture of indigenous and imposed ethnonyms. In the south-west Cape, this classification revolved around a perceived difference

\footnotetext{
${ }^{260}$ The existence of organisations such as the Working Group of Indigenous Minorities in Southern Africa (WIMSA, http://www.wimsanet.org/), set up to address the needs of San peoples, suggests that this is changing at least to some extent.
} 
between sheep and cattle pastoralists, and hunter-gatherer populations. Indigenous epithets were used for the autonomous political units (usually referred to at the time as 'tribes') the colonists encountered. The most significant imposed ethnonym was Hottentot: while the etymology of this word is unclear (Nienaber, 1963), it was primarily associated with pastoralist communities. Somewhat confusingly, it was on occasion used in a generic and inclusive sense that encompassed hunter-gatherer populations (as a 'type' of Hottentot), although this usage became increasingly less common in the later eighteenth and nineteenth centuries. It is no longer used in South Africa today, as during the course of the twentieth century it increasingly came to be used as an abusive epithet. Khoekhoe (formerly spelled Khoikhoi; pl. Khoekhoen) is preferred for the pastoralist groups, approximating 'Men of men' or 'true men' in Nama and Korana (Khoe languages): it is used throughout, except where the former term conveys relevant historical information. Specific autonyms (Namaqua, Korana, etc.) are substituted where appropriate.

The appellation Bushman (or in Dutch, Bosjesman) was applied, broadly, to populations with hunter-gatherer subsistence strategies. The concept of hunting and gathering as antithetical to 'civilised' food-production combined with the widespread resistance of these populations to European encroachment meant that during the eighteenth and nineteenth centuries 'Bushman' came also to refer to the quintessentially 'wild' indigene, often participating in raiding practices. During the decades following the founding of the Cape Colony, the Cape Khoekhoe term Soaqua (or Sonqua) was used by European populations to refer to these groups, 
but by the late eighteenth century 'Bushman' or 'Bosjesman' was increasingly replacing this usage. This Khoekhoe term (cognates are known also from Nama and Korana) has become a widely used alternative in scholarship from the later twentieth century onward, where it is generally given as San. The terms are moreor-less synonymous: just as with 'Bushman' in English and Dutch, the Khoekhoe 'San' references occupation of 'wild' places, and carries strong overtones of social inferiority (lack of livestock) and a predatory nature. In the absence of any consensus among extant hunter-gatherer groups or their descendants, I see no reason to favour San over Bushman (indeed the former seems needlessly 'exotic', and emphasises an imposed term, seemingly merely because it is not European). When discussing populations documented in the nineteenth century, the term Bushman is preferred as contextually appropriate. For the Bleek-Lloyd archive in particular, we have the relevant term as provided by the informants: $\mid X a m-k a-! k^{\prime} e$ or IXam (-speaking) people, which I have usually rendered as IXam Bushmen. Perceived linguistic, genetic and cultural similarities have long (Stow, 1905; Schapera, 1930) been used as a basis for separating Khoekhoe and Bushman populations from other African groups. The term Khoisan (Schultze, 1928), first applied as a biological category, refers to this separation.

Broadly similar to creolised groups elsewhere in the colonial world, the Bastaards are the descendents of (for the most part) European men and indigenous Khoekhoe women: in the early nineteenth century, the missionary John Campbell persuaded (at least some of) them to refer to themselves as Griqua (Schoeman, 1996:xi-xii). This latter term primarily distinguishes a particular group of 
Bastaards, who formed a relatively powerful polity in the trans-Orange interior: those who remained independent farmers in the central Nama-Karoo are better referred to by the older term. The Oorlams were similar creolised populations (primarily of Khoekhoe and slave descent), often given specific names based on the identity of their leaders (the Africaanders, $\& c$ ). During the colonial period, Kafir (from the Arabic kâfir, unbeliever) was widely employed as an ethnonym for black agro-pastoralists who spoke Bantu languages. It was most commonly synonymous with Xhosa (the Bantu-speaking group with whom the colony had the longest-standing relationship) and formed the basis of the colonial term for their homelands (Caffraria): it was also, however, widely applied to other Ngunispeakers, and even to Sotho and Tswana populations. Having acquired extremely negative connotations in the twentieth century, it is no longer applied in South African scholarship: I employ indigenous ethnonyms (Xhosa or Tswana for this period and region) where these are required, unless in direct quotation of historical sources. Trekboere (Afr., migrant-farmers) or veeboere (Afr., cattlefarmers) are both used for populations of European or European-descended colonists (primarily of Dutch or German descent), which formed the basis for the emergent colonial Afrikaner identity. 


\section{Appendix D}

\section{Glossary of IXam terminology}

This glossary is arranged alphabetically. Following the Bushman Dictionary (Bleek, 1956), initial 'click consonants' take the order: dental (1), palatal (!), lateral $(\|)$, alveolar $(\ddagger)$, and bilabial $(\odot)$. The velar ejective affricate $\left(k^{\prime \prime}\right)$ is treated as a separate letter, falling between $k$ and $l$. In the notebooks, word transcriptions are not consistent: $W$. Bleek and Lloyd revised their orthography over the course of the project, and became better at discerning phonetic variation. The variation recorded is only partly meaningful (phonemic), and, in the absence of linguistic treatments of IXam to isolate the phonological forms of each word, I have chosen Bleek's (1956) 'standardised' versions. The primary term is written in bold and underlined (variant forms given italicised in brackets). Constituent words with compound terms are bold and italicised, given separately with references to their location in the Dictionary. Terms dealing with related concepts, or those 
necessary for situating the primary terms in their wider context, are italicised and underlined together with their Dictionary reference.

A:kən, (a:kkan); v. to be nice, good, comfortable, handsome, beautiful, to do nicely, well, $n$. beauty (Bleek, 1956:7). This referred to activities that were performed admirably, inculcating desirable states of moral rectitude, visual attractiveness, and physical comfort. A:kan was strongly connected with twai: $\tilde{\imath}$, often being used consecutively: a woman who worked 'nicely' (twai: ̃) made dancing rattles that were 'good' (a:kən), sounding 'sweetly' (twai: l, $^{2}$ LL.VIII.1.6132-6133). A person who behaved in a:kan fashion and who was able to execute tasks competently might be referred to with the specific epithet, |kun\|en, a person who worked 'nicely'. See Twai:ĩ.

Da Yrrakən; $n$. movement, stirring, $v$. to move, quiver, shake, tap (Bleek, 1956:22). When applied to people, the term could be translated as 'wild' (LL.II.14.1335-1336); 'wild' people were those who 'talked much', a translation for the phrase $|k a n| k a \eta-a$ (not in the Dictionary). Comparisons with $\mid k a \xi \eta^{\prime} a$ (to chase, follow, Bleek, 1956:300) and |kan|kan (v. to step along, Bleek, 1956:301) suggests that it connoted restlessness or impatience. These 'wild' people were constructed in opposition to a woman judged to be particularly responsible: this woman was quiet, not a 'strong eater', and worked things well (thus implying that dakrrakən people did not act in this fashion). The good-tempered person was unlikely to be $d a \xi r r a k a n$, as these 'amiable people' were not impatient or quick to become angry (LL.V.15.5083'); anger and the resulting fighting (if among people 
who should be 'fellows') was itself judged negatively. See łgouwa, !gwunni, la:, |xau:.

Daudáu-ka-!k'e; daudáu v. to feign, deceive, lie, be mistaken (Bleek, 1956:22); $-k a$, poss. part. following possessor, preceding the thing possessed, often omitted before words denoting relationship or parts of the body, also written -ga, -ta (Bleek, 1956:74); !k'e noun pl. people, men (sing. !kwi; emph. !k'etən) (Bleek, 1956:419). Daindain, v. to deceive (Bleek, 1956:20). Lloyd translated this epithet as 'hunting's people' (LL.VIII.9.6786-6857); more literally it represented 'deceiving's people' or 'people of deceiving'. This reference was deployed only infrequently and comments on 'hunters' (with an emic equivalent) are therefore rare. However, IXam hunting terminology was extremely complex, with references to the range of techniques varying with the aims of the hunters and the habits of the prey. Some of these demonstrated abilities in careful movement, controlling the sensory information hunters conveyed to game animals. Both words commonly defining masculine hunting activity, $\| k o e$ : $(v$. to hunt, Bleek, 1956:584) and !hen (v. to hunt, Bleek, 1956:397), were connected with notions of coming in, moving among, or approaching close to something: !hein, !he: $n, v$. to approach, be near, !hi:,$v$. to be near, draw near, (Bleek, 1956:397), $\| k o e, v$. to be in, through, among (Bleek, 1956:584), and $\| k o e, v$. to descend, slip down (Bleek, 1956:584). Several additional terms repeated these connotations with an emphasis on stealth, describing the lying in wait and careful approach of ambush hunting: |xorri:, v. to steal up to (Bleek, 1956:366), |xurri, v. to lie in wait for, aim at (Bleek, 1956:366), |xwerri, v. to lie in wait for, steal up to, hunt (Bleek, 
1956:376), and $\|$ kuiton, $v$. to lie in wait for, make a screen of bushes or a watchhouse, $n$. a screen of bushes (Bleek, 1956:591).

Close approaches also necessitated knowledge of animal behaviour, more obviously manifest in a series of techniques attempting to get the animals to move in directions that would allow the hunters to shoot at them: !ku:xe, v. to run, chase (Bleek, 1956:455), $\| k a u k ə n$ ( $\| k a u k a), v$. to chase, give chase to (Bleek, 1956:562), !Ahátta (!ıhátton), v. to head, go round (game), sometimes used as to hunt (Bleek, 1956:494), and Igamma-se, v. to drive, pursue, chase, n. pursuing (Bleek, 1956:526). Tame livestock and wild ungulates were discussed with contrasting terms (LL.V.9.4618'), while the driving of ostriches, with their idiosyncratic bipedal locomotion, was referred to with an additional word, "gauru (not in Dictionary, LL.VIII.26.8287'). These techniques give some hint of the physical capacities that underpinned successful hunting, seen also in the different techniques of shooting or aiming employed by the hunter: !kau: $\xi$ (to shoot from afar, Bleek, 1956:415), !kwo $\xi m m a i n$ (to shoot in among running animals, Bleek, 1956:468; or, to run shooting, LL.II.25.2287), and !kwi:tən (v. to fly singly, let fly at singly (?), Bleek, 1956:467). Other skills such as !gaokən (v. to follow spoor, trail, find spoor, Bleek, 1956:377) and $\| k h w \tilde{i}$ : (v. to follow spoor, track, Bleek, 1956:579, 600), or the ability to recognise the difference between !kurru: (spoor, Bleek, 1956:454), !ku:iton (yesterday’s spoor, Bleek, 1956:450), and Ikwobbokən (spoor of the day before yesterday, Bleek, 1956:335), required keen observations and interpretations based on detailed ethological knowledge ( $c f$. Liebenberg, 1990). This range of terminology thus gives some indication both of the breadth 
of talents required for successful hunting outcomes, and of the potential for 'specialisations' that would allow many different people to contribute meaningfully to these outcomes. See \|kwakka, !gi:xa, !kwi-la-\|ka:n.

Gen; $v$. to give a name, call (Bleek, 1956:46). In the notebooks, this was glossed as 'nicknaming' (LL.VIII.32.8808'). These nicknames, even when denoting physical features, were usually based in particular experiences, and bestowed by friends or relatives; łgwai-|nunu (Clay-Nose, Bleek, 1956:649, 352) received his nickname after he threw a stone in water, causing mud to fly out over his face (LL.VIII.1.6080). IXam nicknames were therefore 'embodied', being situated within particular relationships and personal experiences, a point that becomes more obvious in the wider context of IXam 'naming' practices. Their bestowal of epithets encoded the events transpiring at given locales onto landscape features, both at a 'mythic' level as in the story of the Agama lizard (cf. J. Deacon, 1988) or when |kaggan gave the places their names (LL.VIII.12.7033'), and on more personal scales, as when lhanłkass'o spoke of a waterhole for which he did not know the 'real name' (having recently arrived in the area) by the name of a woman who was killed by a lion at the site (LL.VIII.14.7216).

Kerru; v. to become green, be green, sprout, $n$. grass, foliage, vegetation (Bleek, 1956:87); !kerru:, !kerru, n. blossom, blossomtime (Bleek, 1956:422). As this latter term suggests, this colour term was connected intimately with phenological responses following rainfall. This connection underpinned the positive characterisation of the colour; when the rain arrived, the green foliage caused the 
bad food to 'grow out' and brought the springbok, made the honey plentiful, and led to the ostriches laying the '!kerru's eggs' (LL.II.23.2052-2062).

Koro; $n$. jackal, Canis mesomelas (Bleek, 1956:101); the jackal in day-to-day discourse was considered rather foolish and timid (LL.VIII.14.7229'-7230'; LL.VIII.16.7459), and dealt poorly with its meat, burying it to finish it later rather than sharing it (LL.VIII.16.7449'-7450'). In Early-Race stories, they were one of the 'beast-of-prey' (\|ke\|ke) people, ugly, 'red', greedy, lovers of fat, who would eat people (LL.II.2.351; LL.II.3.418-419); they were particularly associated with hyenas (!gwaĩ), sharing a 'different' odour that smelled badly (LL.II.15.14411442; LL.VIII.27.8395). See !gwaĩ, \|ke\|ke, \|khã:.

Koro-twi:tən; $n$. a certain little bird (Bleek, 1956:101); koro, $n$. jackal, Canis mesomelas (Bleek, 1956:101); twi:, v. to be wounded, $n$. scar, wound, hole (Bleek, 1956:245). The Koro-twi:tan played a role in an Early-Race story (LL.VIII.10.6885-6940), where he inducted |kaggən in his special skills of obtaining $\| x e$ : (Bushman Rice) by rubbing him with perspiration (making him 'become like the other', LL.VIII.10.6887'). In this narrative, |kaggan demonstrated his typical lack of understanding; having learnt the new technique, he destroyed his tools (LL.VIII.10.6891'), refused to share his $\| x e$ : with the person who had 'nicely' instructed him (LL.VIII.10.6901), and ended up getting injured because of his refusal to listen to $\mid k w a m m a \eta^{\prime} a$ (LL.VIII.10.6929-6935).

Kwa $\xi$ kwa $\xi$ sikuitən; $n$. an elusive thing (Bleek, 1956:110). This term is somewhat obscure, but the presence of the related $k w a$ ( $v$. to step softly, steal along, Bleek, 


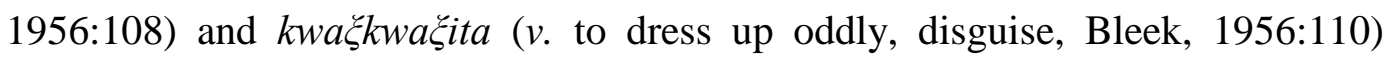
confirms that these figures were associated with superlative skills in misdirection and stealth: the word kwokwaĩ, for a 'sudden disappearance' (Bleek, 1956:115) may also be connected. kwo $k w w a: \xi s i k u: t ə n$, which D. Bleek (1956:115) gives as an 'omniscient person' or 'spirit' is even less well defined; seldom-discussed, Dia!kwain rendered it into 'Boer's language' as perfit (perhaps Afr. profeet, prophet), which W. Bleek translated as 'something like an enchanter or wizard'

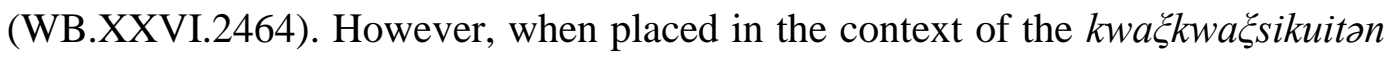
porcupine's ability to make people sleep against their will (|kottan, Bleek, 1956:321; LL.V.11.4381-4832), it is at least evident that these superlative deceptive skills were associated with a more general 'special' status. See !gi:xa.

K"a:o, $\left(k^{\prime \prime} a u\right) ; v$. to advance together, call a commando together, $n$. warparty (Bleek, 1956:120). The form of this word somewhat recalls the anti-social 'cursing' of $\| k^{\prime \prime} a o$ : (though obviously with the addition of a lateral click). See \|k"ao:, la:.

K"e:nk"e:n, (k"eink"ein); v. to tease, make free with, mock, laugh at, deceive (Bleek, 1956:122); k"e:i, (k"eĩ:ja), v. to evade, get away, deceive (Bleek, 1956:122); kurri:tən (kurri:tən, kurri:ta), v. to mock, laugh at (Bleek, 1956:107). $\mid$ kerru, v. to deceive, mock (Bleek, 1956:310); |korru, v. to cheat, mock (Bleek, 1956:320). $\| k^{\prime \prime}$ werre $v$. to play with, tease, annoy, attack (Bleek, 1956:610); $\| k^{\prime \prime}$ worre:, v. to cheat (Bleek, 1956:610); |xorru, v. to tease (Bleek, 1956:366); $k^{\prime \prime} w \tilde{e}^{\prime} a\left(k^{\prime \prime} w e^{\prime} i\right), v$. to laugh at (Bleek, 1956:127); |kaurru, v. to laugh at, mock (Bleek, 1956:304); \|gwitzn, v. to play, n. play (Bleek, 1956:538); kwerre 
(kwerrekwerre), v. to malign, blame, deride (Bleek, 1956:113). This collection of terms linked together issues of mockery, deception, and evasion, uniting them within a domain of antagonistic, inappropriate action that could instigate conflict or harm: if a New Maiden 'played' ( $\| k "$ werre, $\| g w i t \partial n)$ with the young men, she would afflict them with her 'rain's magic power' (LL.V.6.4398'-4400'), the bokmakierie was not to be mocked because it could cause harm (LL.V.25.60146024), and !gi:tən that walked at night would $\| k^{\prime \prime}$ werre people (LL.V.19.55361/2). It was associated with children, either as a characteristic of their behaviour (LL.V.10.4747-4750) or in terms of 'grown-up' attitudes toward their foolish actions (LL.II.33.3029). The term was associated with a specific personal identity; a Inerrisa was a 'teasing person', who 'did not a little' $k^{\prime \prime} e: n k^{\prime \prime} e: n$ (LL.VIII.25.8231'). It was associated particularly with Ikaggən, who 'teased' people to get them to disrespect the eland (LL.V.17.5345-5349): in Early-Race stories, his actions were often criticised as childish 'playing' or 'teasing' (LL.VIII.2.6181-6192), explicitly contrasted with being quiet ( $¥ g o u w a)$ and listening to stories. See \|kwakka, $\neq$ gou, |gebbi, \|k"ao:.

Sã:; $n$. buchu, a fragrant herb, snuff (Bleek, 1956:162). This was a special kind of plant medicine with wider applications than most, working through a placating or attractive scent. Buchu has been discussed extensively in literature pertaining to southern Bushman ritual practice ( $c f$. Low, 2004; 2007a), and was used by a range of ritual practitioners including New Maidens (Hewitt, 2008[1986]) and !gi:tan, who deployed it as a pacifying agent as part of rain-making processes (Lewis- 
Williams, 1977:166, 1983:58; Lewis-Williams and Pearce, 2004). See Jo-|̃̃ä-ka!kwi.

Swa:-ka-!k'e; Flat Bushmen. swa:, n. flat, plain (Bleek, 1956:175); -ka, poss. part. following possessor, preceding the thing possessed, often omitted before words denoting relationship or parts of the body, also written -ga, -ta (Bleek, 1956:74); !k'e noun pl. people, men (sing. !kwi; emph. !k'etən) (Bleek, 1956:419). This was the name for the IXam populations inhabiting central Bushmanland, referring to the characteristic extensive, flat plains of the region.

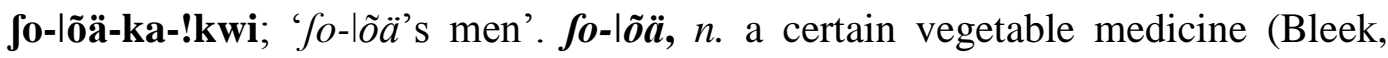
1956:182) -ka, poss. part. following possessor, preceding the thing possessed, often omitted before words denoting relationship or parts of the body, also written -ga, -ta (Bleek, 1956:74); !k'e noun pl. people, men (sing. !kwi; emph. !k'etən) (Bleek, 1956:419); s'o:-|ã, so-|ã, n. a certain vegetable medicine (Bleek, 1956:173). This referred to a category of ritual specialists employing a special category of plant medicine, situated within a wider range of plant medicines deployed by both men and women and working through their particular smells (LL.IV.1.3425-3441). $\|$ kabbo discussed the collection and use of this plant in detail (LL.II.36.3242-3332), although he was not a specialist in its use (LL.II.36.3309'); it was a highly 'masculine' resource employed while hunting, and its' scent when incorporated would make the springbok run 'nicely' to the hunter. It had a potent scent that could $\| k^{\prime \prime}$ werre ('annoy') a person if they dug it without 'understanding' (LL.II.36.3280), and was particularly dangerous for 
women (LL.II.36.3284) despite being 'dug out' using 'feminine' resource acquisition tactics. See k"e:nk"e:n, lk"wã:, sã:.

To:; $n$. red haematite (Bleek, 1956:206). This rock was ground to produce a red pigment that placated !khwa: when strewn on water (LL.VIII.16.7426), and that was rubbed onto the skin (along with fat) as part of a process of making people 'handsome' (a:kan, LL.VIII.14.7272'-7276'). It was implicated in exchange relationships (being given in return for dressed skin bags, LL.VIII.14.7274') and was used to place identifying ‘ $¥ k e t t ə n ’$ marks on arrows (LL.VIII.26.8289). See \|hara, $\|$ ka: $\xi$

Ts'erre; $v$. to bewitch (Bleek, 1956:215). Ts'ammas'e, ts'ammats'e, v. to poison, bewitch (Bleek, 1956:211). !gi:tan (or Inu ka! $k$ 'e) might 'bewitch' game so that it became aware of the stalking hunter (LL.V.11.4802-4811). It was thus linked with a sense of 'being spoilt', ruining the chance of success in a given action, and might be used in antagonistic behaviour (LL.VIII.32.8826'; LL.II.18.1678); Dia!kwain's mother told him that tortoises might ts'erre (which W. Bleek translated as 'avenge', WB.XXVII.2536) themselves upon people who ate them without real necessity. See !k"ao:.

Twai:ĩ, (twa:i'i), v. to be comfortable, well, better, used as adv. well, comfortably, nicely (Bleek, 1956:243); twaita, twaiton, sweet, sweetly, well, pleasant (Bleek,

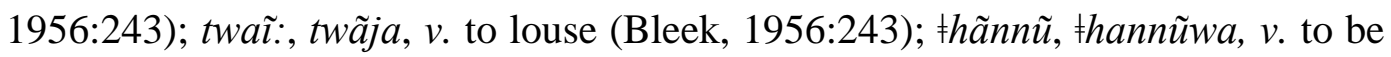
comfortable, happy, good, nice, fortunate (Bleek, 1956:649); !hannu:wa, nicely

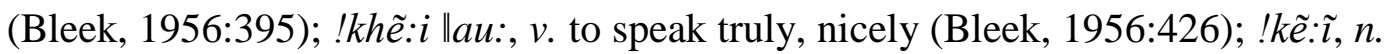




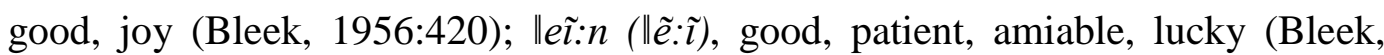
1956:520); !kõ:i, amiable, patient (Bleek, 1956:440). Twai: $\tilde{\imath}$ described states that were pleasant and desirable, usually the result of $a: k a n$ behaviour or skills. The range of terms connected with states of comfort or pleasure made connections between being 'happy' and being 'good', 'fortunate', talking nicely and maintaining amiable social relations (LL.II.14.1316; LL.II.22.2001; LL.VIII.1.6132-6133); this formed a dramatic contrast with notions linked with $\| k^{\prime \prime} a o$ : and $\mid a$ : Being 'comfortable' was associated in particular with possessing a sufficiency of food (\|wã : $\xi, v$. to be full, Bleek, 1956:629; $\ddagger g u m m \neq g u m m$, 'niceness to bite', LL.II.14.1352; LL.VIII.18.7599) and water (\|uã:, v. to be comfortable

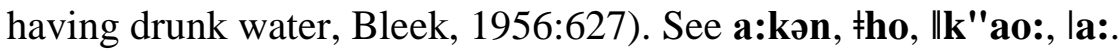

Whai; $n$. springbok, sing. and pl. (Bleek, 1956:251). Springbok formed the primary prey species for |Xam hunters, the 'people who shoot springbok' (LL.II.36.3244'-3245'; Chapter 7a:215; Chapter 7b:238). They were much less dominant in Early-Race stories, but where featured tend to take roles as young (or female) creatures that other agents had strong emotional bonds with: people became 'heart-sore' over them (WB.XXVI.2438') or 'rejoiced' to find them (WB.XXVI.2447-2448). These bonds were based on the attractiveness of the young springbok (WB.XXVI.2449'), which motivated attempts to steal them away and ensured that where they appeared in Early-Race stories, they usually formed a source of conflict (LL.VI.1.3883-3894; LL.VI.1.3916-3930; LL.VIII.29.85618602). 
Xoakən-gu; xoa, $n$. mother, emph. xoakən, $p l$. xoakən-gu (Bleek, 1956:259). This term literally denoted 'mothers', but was used in a more expansive sense as 'old women'. This group of people played a key role in structuring the experiences of the New Maidens, as they were responsible for instructing these girls in the relevant rites and behaviours; consequently, the xoakən-gu also enforced the strictures to which New Maidens were subject (Hewitt, 2008[1986]:18, 58). See

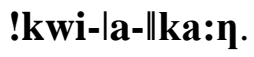

la: (la); $n$. to fight, die, be killed, $n$. fight, harm, curse (Bleek, 1956:267). |shá:, $n$. evil, angry, angrily (Bleek, 1956:358);!kwa: $\xi(! k w \tilde{a}:, ! k w \tilde{a}: ' a)$, v. to be angry, wrathful, also used as $n$. (Bleek, 1956:457). 'Fighting' arose through the attitudes of angry, wrathful persons, and was (as a quality of human relationships) portrayed in a highly negative manner, consequent on 'foolish' attitudes (|gebbi, LL.II.9.974), and opposed to 'stillness’ ( $\ddagger g o u$, LL.II.24.2226). It was linked with the key anti-social communities of the IXam world: the lion that killed people was lakan-se (LL.II.30.2753'-2754'), !gi:tan who could not cure well might 'fight' people (LL.V.4.4165), and the actions of commandos were also described as 'fights' (LL.V.16.5199-5201). Associations with antagonistic communities underpinned its use as a mode of 'cursing' (LL.VIII.31.8741-8743), and a means whereby IXam might violently interact with one another; the $\mid a$-ka-didi (fighting's things, LL.V.21.5709'), sent by anti-social !gi:tan to bring illness, represented an equivalent non-human 'curse'. See \|k"ao:, lhou-!kwi,

Igara; $n$. berries of the 'krieboom' (Bleek, 1956:276); \|na:, n. a tree, 'kareeboom', 'krieboom', 'resinkibos' (Bleek, 1956:612; ‘taaibos', LL.VIII.12.7112). These 
berries were gathered and eaten by both Bushmen and Koranas (WB.XXIII.2154'). The Afrikaans vernacular name kareeboom refers to several Searsia species, primarily S. lancea and S. pendulina: C. Smith (1966:279) notes that the drupes of S. lancea were consumed by Koranas, and its range corresponds more closely with IXam territories than does that of S. pendulina (van Wyk and van Wyk, 1997:402, 406).

Igebbi; $v$. to be foolish, stupid, do foolishly, $n$. foolish person (Bleek, 1956:277); $\| k a: n-d i, n$. foolishness, foolish things (Bleek, 1956:557); $\| k \tilde{a}:$, v. to be foolish (Bleek, 1956:546); $\| k a: \eta$, new, fresh, raw, to eat raw (Bleek, 1956:556); |kaũ:wa, $n$. fool, idiot (Bleek, 1956:304). Doing things 'foolishly' was the converse of understanding ( $\| k w a k k a)$ behaviour (LL.II.22.1984-1986; 2205; 2392), and people whose thoughts were 'closed' to instruction remained foolish (LL.II.30.27632764). The other terms for foolishness suggest connections with 'newness' or 'freshness' that correspond well with the presentation of foolish attitudes as coherent with a 'childish' persona; a grown-up person was not supposed to be cunning, deceitful (!khwi) or foolish (LL.II.5.564-565), and could see through attempts on the parts of others to cheat them (LL.II.27.2422-2423). The EarlyRace people, Ikaggan more than any other, were notable for their 'foolish' or inappropriate actions (LL.VIII.12.7094'), and helped thereby to define 'correct' understanding behaviours for the IXam. See !khwi, \|kwakka.

Igwai $\xi \mathbf{n} ; v$. to get into the flesh, take possession of, used in describing magic (Bleek, 1956:285). This practice represented a 'magical assault', whereby various non-human antagonists would 'get into the flesh' to cause illness in human 
populations (LL.V.3.4135-4136, LL.V.19.5517 for anti-social !gi:tən; LL.V.21.5706 for swallows; LL.VIII.14.7264' for springbok; LL.V.20.5609, for !khwa:). Just as humans would shoot poisoned arrows to sicken and kill prey species, this form of assault often took the form of invisible 'magic arrows' that pierced people and caused them to 'fall dead' (LL.VIII.14.7263'-7264'), by contaminating them with an antagonistic identity.

Iho; $n$. pig, hog (Bleek, 1956:288). The pig featured only in one Early-Race story (LL.VIII.25.8216), in which the !gwiton (translated as 'wild dog' in this story, but usually referring to the Caama fox, Vulpes caama or 'silver jackal') brought the meat of this animal to his wife. The meat of these animals was not eaten either by Grass (LL.IV.3.3736') or Flat (LL.VIII.30.8649) Bushmen (Chapter 5b:149). Unlike with sheep (which even in Early-Race tales were always situated in herding relationships with people), there was little reference to the practice of keeping domesticated pigs. Historically, there was a wild suid present in the Karoo: the range of the now locally-extinct Cape warthog (Phacochoerus aethiopicus aethiopicus) extended into the region (Vercamman and Mason, 1993:78), and the IXam term may have originated with this species.

Ihõa-gau; $n$. rascal (Bleek, 1956:288). When a !gi:xa was not paid for his curing efforts, his or her heart was not made 'comfortable' (LL.4182). This uncomfortable or 'warm' heart meant that his or her angry/evil thoughts would make people ill or 'eat at' them; such a !gi:xa was acting as a Ihõa-gau (LL.V.4.4176-4184). These 'rascals' were not always !gi:tan known to individuals, and the term also applied to the general set of anti-social !gi:tan that 
might afflict communities with illness; in these cases, benevolent !gi:tan would attempt to defend against their incursions by striking them to make them 'come out of' the people whom they had made ill (LL.V.19.5514-5521). Such 'rascally' !gi:tən were connected with the $\mid n u-k a-! k^{\prime} e$, visiting them and displaying similar attitudes to them in wanting to kill or carry off people. See Inu-ka-!k'e, Igwai $\xi_{\mathbf{n}}$.

Ihoake (|hoakan); dark, black, used for any dark colours (Bleek, 1956:289), and usually deployed in descriptions of negative emotions (terror and anger) or in connection with anti-social beasts-of-prey (Chapter 5c:159).

|hou-!kwi; bow-man. |hou, |hau n. bow (Bleek, 1956:287); !kwi, n. man, person, human (Bleek, 1956:466). The |hou-!kwi represented a masculine social role that was largely characterised in a positive manner, though some of the traits associated with it could become extremely negative if improperly directed. The 'bow-man' was brave (LL.VIII.5.6481; LL.VIII.25.8247'), and successful in his attempts at hunting; such a man was a desirable husband (LL.VIII.5.6481). However, his proficiency in the strongly confrontational domain of hunting meant that he could also be an 'angry man' (LL.II.21.1920; LL.VIII.15.73751 1/2') if the traits that underpinned his superlative skills in this domain were extended to his other interpersonal relationships; negative characterisations of 'angry action' as foolish and potentially dangerous ensured that this social role retained a degree of ambiguity (Chapter 6a:175). See la:, li:-sa,

li:-sa; $n$. coward (Bleek, 1956:239); li:, $n$. heart (Bleek, 1956:292); sa, v. to come, become, also found as se, si. It is sometimes used as verb part., shall, will (Bleek, 
1956;1161). As Igebbi formed the opposite of $\| k w a k k a$, the coward or 'runaway' (LL.II.34.3130') represented the converse social role of to that of the $\mid h o u-! k w i$; it was thus characterised as an undesirable identity in masculine roles, linked with insufficient skills in provisioning and typical of the actions of negatively evaluated jackals (LL.VIII.16.7457-7458). It was used to describe Ikaggən and his son |kaggan-@pwa, who were both 'mild' men whose hearts did not stand strong with anger and whose tears came freely (LL.II.34.3131); this characterisation (which contrasted with $\mid k w a m m a \eta^{\prime} a-\odot p w a$ ) was consistent with presentation of |kaggan as a man who did not live up to |Xam expectations of responsible, skilful adulthood. See lhou-!kwi.

Ikaggən; $n$. mantis, both the insect and a demiurge hero of IXam folklore (Bleek, 1956:296). Described variously as a demiurge (Bleek, 1956), a 'southern San trickster-deity' (Lewis-Williams, 1997:195), and as a trickster (Guenther, 1999), |kaggan represented the dominant figure of the Early-Race narratives, and remained a significant factor in contemporary !nanna-se behaviours. His 'trickster-ish' status is based in his ambiguous personality (see li:-sa), his propensity for deception (LL.II.5.533-534), his shape-shifting (LL.II.33.2986), and his transgressions of social norms (LL.II.9.978-982), traits that have been proposed as common features of trickster figures in a large range of cultural contexts (Hynes, 1993:34-42); these features were not, however, unique to |kaggən, and many Early-Race characters displayed 'trickster-ish' qualities. Most stories referring to |kaggən revolved around his conflicts with various individuals and groups of the Early-Race world, and his relationships with members of his 
family, who attempted to limit his foolishness; typically, these included his wife |hun-tu-!ka!katte (|hun, n. rock rabbit, dassie, Procavia capensis, Bleek. 1956:290, otherwise called !kauro-o, LL.VIII.1.6137'), his adopted daughter the Porcupine (!xo, Bleek, 1956:500), her husband $\mid k w a m m a \eta ' a$, and their son, the ichneumon mongoose (Ini, Bleek, 1956:348). |kaggan's actions were for the most part not consistent with his senior status within these relationships, with his young grandson (the $\mid n i$ ) taking a prominent role in correcting and scolding him for his inappropriate, childish, and foolish behaviours (LL.II.5.555-556; LL.II.6.685-687; LL.II.32.2962-2963). See Igebbi, !khwi.

Ikaggən-@pwa; |kaggən; $n$. mantis, both the insect and a demiurge hero of IXam folklore (Bleek, 1956:296); -Opwa, little, young, $n$. child. In IXam it is only used in the singular, changing in the plural to -ta-!kaukən (Bleek, 1956:684). |kaggənOpwa was one of |kaggən's 'biological' children (along with another son, !gaunutsaxau, and k"we-Inan-\|kain-tu, his daughter, LL.VIII.1.6137'); he played a prominent role (along with $\mid k w a m m a \eta^{\prime} a-\odot p w a$ ) in rectifying matters after $\| k h w a i$ hem (invited by |kaggan to partake in sheep he had stolen from the Ticks) had swallowed down all things (LL.II.34.3120-3149). |kaggən-@pwa in this story acted as an extension of his father (though to a socially responsible end), employing his left hand (LL.II.34.3132') and 'fearing greatly' (LL.II.34.3130'). See li:-sa.

|kai:nja (|kai:n); v. to be green, yellow, shining (Bleek, 1956:297); |kai, v. to light, shine (Bleek, 1956:297). The relationship with $\mid k a i$ suggests that the latter of the three translations offered was the primary referent; uses in describing the eyes of 
carnivores, and |kaggən as he emerged from water (Chapter 5c:161) emphasise this 'shining' connection.

|kerre-gwai; |kerre, v. to be timid (Bleek, 1956:309); gwai, n. man, male, husband, used also as great, entirely (Bleek, 1956:52). This was another term that might be translated as 'coward' (LL.II.19.1738; LL.VIII.25.8247'), as an equivalent to $\mid i:-s a$ and contrasting with the brave $\mid h o u-! k w i$. The notion that 'cowardly' figures displayed lacklustre hunting skills was emphasised in an alternative translation that rendered the phrase |kerre-gwai as an 'unsuccessfully catching male' (LL.II.21.1889, referring to an Early-Race hyena). See li:-sa.

|khe:-\|en; ‘Grass Bushmen'. |khe:, n. grass (Bleek, 1956:312); \|en:; v. to dwell, inhabit, live (Bleek, 1956:521). Unlike other Bushman groups, these 'Grass-

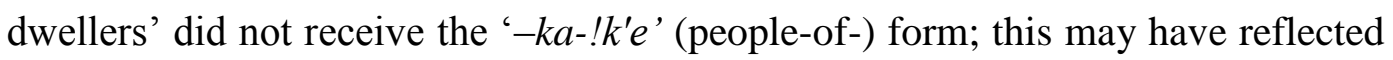
an impression on the part of Flat Bushman informants that the Grass Bushman dialect was distinctly different from their own, and that they did not talk IXam 'well' (Chapter 7a:221). They inhabited the western regions of Bushmanland, a region characterised by a transition to a veld dominated by silvery-white desert grasses (Chapter 3b:68).

$\mid \mathbf{k o}: \xi_{\mathbf{o}} \xi$-de, $n ., p l$. magic things, magic doings, magic power, sing. $\mid$ ko: $\xi_{\mathrm{o}} \xi_{\text {-ti }}$ (Bleek, 1956:320); !kokan-de (!k'okan-tikan), n. magic sticks (Bleek, 1956:441); |kokan-de, n. magic arrows (invisible), magic sticks (Bleek, 1956:318). This form of 'magic power' primarily manifested the agency of anti-social forces; as the 'rain's magic power' of the New Maiden (LL.V.6.4400'; LL.V.13.4989); as the 
'magic power' of the eland and hartebeest that let them escape the effects of the poison (LL.V.6.4411-4413; LL.V.18.5358); as the 'magic power' of a dead !gi:xa, which 'turned back' to take away (make ill) people (LL.V.19.5484-5485); and as 'magic power' (mistranslated originally as 'evil things') that visited a person in the night to kill them (LL.V.19.5528-5529). See !gi:, Igwaikn, !gi:xa.

Iko: $\xi \mathbf{w a} ; v$. to be pale, red (Bleek, 1956:321). This term was commonly used to describe flesh (of people), where it described a 'handsome' trait. This 'paleness' of flesh referred primarily to Koranas, Grass Bushmen, and those whose flesh had been rubbed with to: and fat (Chapter 5c:163); where the colour of European skin was described (though it was also noted as 'handsome') the word used was !khi:ja (WB.II.356).

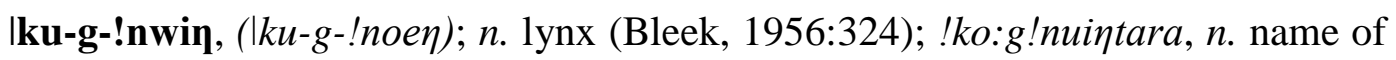
the female lynx (Bleek, 1956:439). The original notebooks and the Dictionary translate this as 'Lynx'. It referred to the caracal (Caracal caracal; Afr. rooikat, red-cat), a species that, despite superficial physical resemblances, does not have a close phylogenetic relationship with Eurasian and American lynx species (Johnson et al., 2006); I have consequently preferred to translate the term as 'Caracal' throughout. The Early-Race Caracal was married to !gaue-li:-ta$\| k w a \xi t t a n$ and was characterised in a highly positive fashion, as an attractive figure with a sweet odour and distinctive ornaments (LL.II.15.1440-1444); the caracal also played a role as the aardvark's 'heart's child' (LL.V.6.4345-4349) because of her sweet !kummi playing, and even the contemporary caracal was a 
handsome animal that was a 'small eater', satisfied with a small piece of meat (LL.V.6.4349').

Ikui; n. 'gambro', 'kambro', a kind of cucumber (Bleek, 1956:324). C. Smith (1966:218, 272, 273, 481) gives this term (and several variants, gameru, gameroo, kambaroo, kambaroe, kamkoo, baroe) as a name applied to several Asclepiadaceae genera, commonly Fockea spp. (especially F. edulis) but also Brachystelma spp. and Pachypodium spp, with large, sweet, and watery rootstocks. Their vernacular names often contain a prefix differentiating the preferred habitat of the species (e.g. bergkamaroo, veldbaroe, C. Smith, 1966) or highlights the importance of the species in indigenous subsistence practices (e.g. Hotnotswaatlemoen for F. edulis, C. Smith, 1966:273). These species were adopted by the colonists, and contemporary Karoo farmers continue to use them in the production of preserves.

Ikwamman'a, $n$. name of the Mantis' son-in-law, a being seen in the rainbow, the husband of the Porcupine (Bleek, 1956:330); he was also referred to with a shortened name (|kwa: Bleek, 1956:328). Although he did not have a specific animal identity, he was related to the meerkats and lions (Lewis-Williams, 1997:203); this connection goes some way to explaining his status as an angry man (LL.II.34.3130-3131). Despite being related to beasts-of-prey, he was more moderate and sensible in his behaviour than |kaggən and listened to his family when they gave him advice (LL.II.34.3146-3147), though he occasionally acted as if he had not been taught (LL.IV.2.4034-4053). See Ikaggən. 


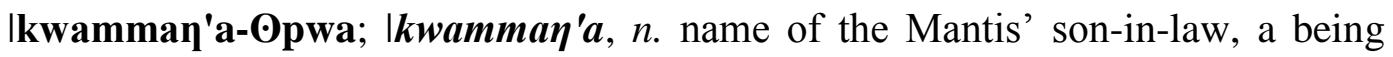
seen in the rainbow, the husband of the Porcupine (Bleek, 1956:330); - Opwa, little, young, $n$. child. In IXam it is only used in the singular, changing in the

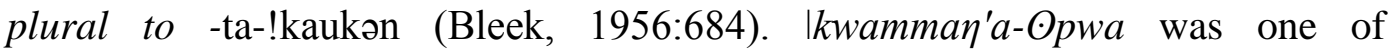
|kwamman'a's children (presumably a different individual to the $\mid n i$ ), and acted along with |kaggən-○pwa to rectify matters after $\|$ khwai-hem (invited by |kaggən to partake in sheep he had stolen from the Ticks) had swallowed down all things (LL.II.34.3120-3149). Like |kaggən-@pwa, he acted in this story as an extension of his father, behaving in an 'angry' fashion, grasping the spear strongly, and slaying \|khwai-hem (LL.II.34.3132-3149). See lhou-!kwi.

|k"a-ka-!k'e; Hartebeest River Bushmen. $\mid \boldsymbol{k}^{\prime \prime} \boldsymbol{a}, n$. riverbed, Hartebeest River (Bleek, 1956:336); -ka, poss. part. following possessor, preceding the thing possessed, often omitted before words denoting relationship or parts of the body, also written -ga, -ta (Bleek, 1956:74); !k'e noun pl. people, men (sing. !kwi; emph. !k'etən) (Bleek, 1956:419). This term denoted IXam populations (otherwise referred to as $\|$ kaitzn-s'o-!k'e:, Bleek, 1956:554) living along the Hartebeest River, flowing north towards the Orange River from the northern margins of Swa-ka-!k'e territories; these populations were considered by Flat Bushman informants as substantially similar to their own groups, speaking the 'other part' of their language (Chapter 7a:219).

Ik"wã:; .v. to seek food, hunt for food, graze (Bleek, 1956:340); |kwã , v. to graze, go to seek food (Bleek, 1956:327). Lloyd (LL.II.36.3296') suggested that it was used 'chiefly used (if not wholly)' for 'woman's hunting', as (what Lloyd 
described as) a figure of speech; descriptions of their 'hunting' and 'killing' of Bushman Rice being based on the fact that these insects were 'their game' (LL.II.36.3296'), The collection of insect resources was also referred to with a more specific term, !kherreja ( $v$. to gather ants' eggs, Bleek, 1956:426), but the practice was identical. Although this subsistence activity was primarily associated with women, men would engage in it if more appropriate antelope resources were scarce (LL.VIII.10.6888'), and for some resources (e.g. the quintessentially masculine $\int o$-|öã, considered very dangerous for women), 'women's hunting' was practised 'prototypically’ by men (LL.II.36.3297). See Jo-löã-ka-!kwi.

Ini; n. ichneumon, Herpestes, a prominent figure in IXam folklore, where he figures as the son of $\mid k w a m m a \eta^{\prime} a$, and the Porcupine (Bleek, 1956:348); often

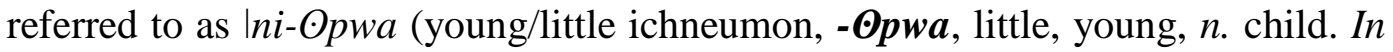
IXam it is only used in the singular, changing in the plural to -ta-!kaukən (Bleek, 1956:684). This latter epithet emphasised Ini-@pwa's most significant role in Early-Race narratives, revolving around his status as the grandson of Ikaggən; he

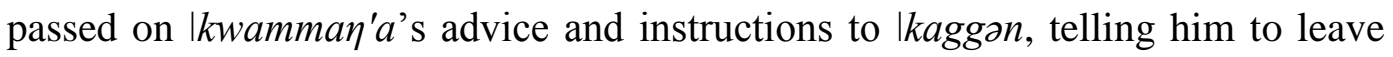
off teasing (LL.II.2.6227-6231) and pitying |kaggən for the injuries his foolishness incurred (LL.III.3.6244-6246). The two occupied opposite positions: where IniOpwa acted with a responsible attitude that was quite beyond his years, |kaggən would not 'grow up leaving off teasing' (LL.VIII.3.6258), and they sometimes appeared as evenly matched, as |kaggan was (at least for a time) able to deceive

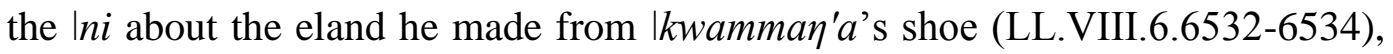
though the Ini eventually outsmarted him. 
Inu-ka-!k'e; |nu, a word signifying dead, departed, spirit, also used as a term of opprobrium (Bleek, 1956:350); -ka, poss. part. following possessor, preceding the thing possessed, often omitted before words denoting relationship or parts of the body, also written -ga, -ta (Bleek, 1956:74); !k'e n. pl. people, men (sing. !kwi; emph. !k'etən) (Bleek, 1956:419); Inu, old, applied to people (Bleek, 1956:351); !nu:, v. to be angry, attack angrily, used also as angry, angrily (Bleek, 1956:482);

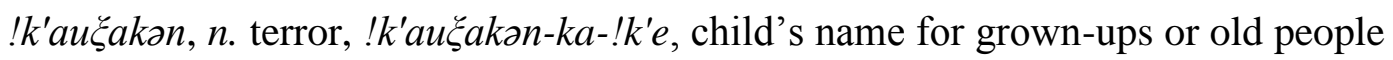
(Bleek, 1956:413). This phrase as a whole may be loosely translated as 'spirit's people', 'departed ones', or 'dead people', and it represented a complex, and rather obscure, category of non-human agents. Ikaggan called the Ticks $\mid n u-! k^{\prime} e$, after being beaten by them (LL.VIII.20.7802), which was translated as 'bad people', with a reverso note stating that Ikaggan was cursing them, a notion repeated elsewhere (LL.VIII.27.8368; LL.VIII.31.8741). Alternatively, these 'spirit-people' were described as 'old people' or 'people which are dead' (!k'e-e|kuka, LL.VIII.26.8309'). Older people could take on a 'terrifying' quality in IXam thought, with young children referring to them as 'terror's people'; $|k ' e-e-| k u k a$ were 'elders' for everyone. The $\mid n u-k a-! k$ 'e behaved in an anti-social manner, travelling in a 'fighting party' $\left(k^{\prime \prime} a: o\right)$ to bring harm and illness that could kill people; they had lost their 'understanding' and therefore acted in a bestial manner (LL.VIII.26.8310-8312). They were also connected particularly with !gi:tən, and an alternative translation in a passage by Dia!kwain (LL.V.22.5735'-5736) described the $\mid n u-! k$ ' $e$ as 'invisible sorcerers' who had died, but whose 'sorcerer's power' (!gi:) continued to go about. See $\| \mathbf{k}^{\prime \prime} \mathbf{a o :}$. 
|Xam-ka-!xoe; |Xam, $n$. Bushman, name of a tribe living south of Orange River (Bleek, 1956:363); -ka, poss. part. following possessor, preceding the thing possessed, often omitted before words denoting relationship or parts of the body, also written -ga, -ta (Bleek, 1956:74); !xoe, n. place, country (Bleek, 1956:500); $\boldsymbol{s}^{\prime} \boldsymbol{o}, \boldsymbol{v}$. to sit, remain (Bleek, 1956:171). |Xam-ka-!xoe thus referred to the 'countryof-the-|Xam', corresponding approximately to the region known today as 'Bushmanland' in the central Nama-Karoo.

!gaue-li:-ta-|kwaלttın; 'Day’s (or Dawn's) Heart Star' (Jupiter); !gaue, $n$. dawn, day, morning, used as tomorrow (Bleek, 1956:379); li:, n. heart (Bleek, 1956:292); -ka, poss. part. following possessor, preceding the thing possessed, often omitted before words denoting relationship or parts of the body, also written -ga, -ta (Bleek, 1956:74); |kwa $\boldsymbol{\xi t t a n , ~} n$. star, cloud (Bleek, 1956:331). During the

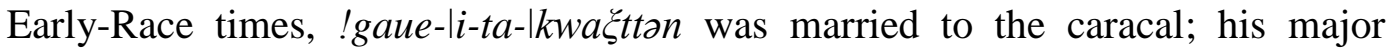
appearance in the archive was in a narrative concerning the 'poisoning' of his wife by the hyena and jackal, which converted her human identity into a 'beast-ofprey’ identity (LL.II.15.1432-1499, LL.II.16.1500-1548; LL.VIII.27.8393-8432). When he discovered this deception and assault, his eyes became large and 'resembled fire' (themselves 'bestial' traits), as his heart became angry (LL.VIII.27.8430-8432).

!gi:; $n$. magic power, sorcery (Bleek, 1956:382). This form of 'magic power' was the prototypical potency associated with the !gi:tan, though it could be associated with other agents (LL.V.21.5706-5707, where swallows send a 'sorcery' into a person). It represented the primary means by which !gi:tan 'worked' their abilities 
to assist (LL.V.22.5758) or to harm (LL.V.4.4179-4180; LL.V.10.4753; LL.V.20.5544-5545); these 'sorcery's doings' could also act independently of the !gi:tən after their deaths (LL.V.11.4801'-4803'), or retain the intentions of !gi:tan who sent it to a 'different place' (LL.V.19.5512-5513) See !gi:xa.

!gi:xa (pl. !gi:tən). The !gi:tən were ritual specialists with particular abilities to influence events beyond those of normal human control. They were by far the most significant ritual specialist in IXam societies, and employed their skills in numerous contexts: most commonly in curing illnesses, influencing the movements of game animals, obtaining rain, and watching over the community in a general sense. The phrase refers literally to a 'possessor-of-!gi:' (magic power, Bleek, 1956:382), and etymologically equivalent concepts are extant among Northern Bushman groups (e.g. nlom k"ausi among the Jul'hoansi, Biesele, 1993). Bleek and Lloyd had some difficulty in deciding on an accurate English equivalent, usually employing sorcerer (spelling it sorceror) or sorceress: witchdoctor (LL.II.1.273), witch (LL.V.4.4199'), wise person (LL.V.3.4131'), doctor (LL.VIII.20.7757), magician (LL.V.15.5079), medicine man or woman (LL.VIII.20.7759), and enchantress (LL.V.10.4740) were also offered in translation. Presumably their choice of 'sorcerer' was governed by the translation offered by the informants themselves (LL.II.1.273): towenaar (Afr. magician, sorcerer). Since sorcery has in anthropology come primarily to refer to 'negative' or socially destructive magic ( $c f$. Stevens, 2006), the Bleek-Lloyd translation is no longer common; although some !gi:tən were seen as anti-social, this was not a consequence of their use of !gi: per se. 'Shaman' (Lewis-Williams, 1992), derived 
from Tungus-speaking reindeer herders of northern Eurasia, has become a widelyused (though not uncontested; Solomon, 2011) alternative.

The term 'shamanism' has a patchy history in terms of its application to religious life in Africa. Eliade's (1972; $c f$. Lewis, 1989, 1997) famous survey of worldwide shamanism omitted the continent with a footnote, and in nineteenth century South Africa (unlike elsewhere; Jones, 2006:10) it was evidently considered an inappropriate translation for indigenous ritual specialists; for the first half of the twentieth century, 'medicine men' was a common gloss (Stow, 1905:125). From the later twentieth century onwards, an increasing awareness of the significance of altered states of consciousness in contemporary Bushman ritual life (L. Marshall, 1962, 1969; Katz, 1982:3-4) underpinned a shift towards the use of 'shaman' as the dominant translation for the 'potency-owners' of northern Bushman populations ( $c f$. Lewis-Williams, 1992:58, for a review of this shift). This shift has been transferred to the Bleek-Lloyd material, through Lewis-Williams' (1981, 1992) observations of the many, detailed analogical details paralleled within the twentieth century ethnographies and the IXam material; this extension has drawn in a series of heuristic tools (e.g. the 'spirit world') that Bleek and Lloyd did not employ in their original collection, and which must therefore be revealed 'darkly' through the narratives themselves.

In addition to the proliferation of English alternatives, the IXam themselves applied various epithets to these specialists: the people who 'possessed their noses' (LL.V.13.5020, referring to the 'snoring power' that empowered !gi:tan to cure illness), |nu-ka-!k'e (LL.V.11.4801', referring to !gi:tən who had died but 
continued to exert an influence through their 'magic power'), as well as a series of categories that referenced the resources they controlled. !khwa-ta-!gi:tan possessed influence over the rain (LL.II.24.2213), Opwai:tən-ta-!gi:tən could control animals (LL.V.11.4801'), and a $\| x i-k a-! g i: x a$ brought illness (LL.V.19.5488-5487'). Despite this differentiation, !gi:tan could readily act in a number of capacities: "kabbo's maternal grandmother controlled the springbok, but also cured illnesses (LL.II.37.3337'). !gi: was an 'understanding' behaviour, which had to be taught to people (LL.V.10.4744'; LL.V.22.5759), and, like hunting, it involved a number of special abilities that allowed !gi:tan to differentiate and distinguish themselves: $\| k \tilde{a} i$ :, ( $v$. to lead out by magic, Bleek, 1956:550) was linked with !khwa-ka-!gi:tan, who needed to entice the rain from its abode (LL.V.12.4990), while !Ahá $\xi$, (v. to sneeze, blowing from the nose a

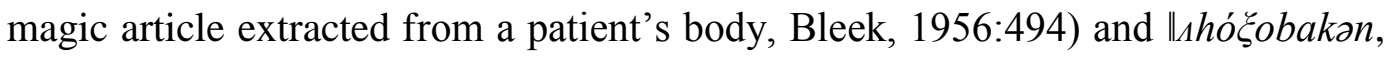
(v. to anoint, rub with perspiration, Bleek, 1956:628) were both connected with curative practices. See !kwi-|a-||ka:n, Jo-|öãa-ka-!kwi, daudáu-ka-!k'e, !khwa:ka-!gi:tən, Ðpwai:tən-ta-!gi:tən.

!gu:; $n$. mat, name of one of the two lions identified with the pointers of the Southern Cross, heroes of some myths (Bleek, 1956:388). !gu: was one of two lions forming the focus of a large number of Early-Race narratives, generally playing a 'buffoonish' role in which their anti-social tendencies were balanced by the consequences attendant on the 'foolish' means by which they attempted to achieve their goals (Guenther, 2002:160). See !haue-ta-łhou. 
!gu!gu; $n$. a non-edible fungus (Bleek, 1956:390). This fungus (also called $\mid k u$ kwai, LL.VIII.11.6945') grew out of the !hakan (Trinervitermes sp.) mound like a 'beacon'. Association with termite mounds is a common trait of Podaxis sp. fungi worldwide ( $c f$. Morse, 1933; Priest and Lenz, 1999); !gu!gu possibly represented Podaxis carcinomalis, a type of buchu (wolf-buchu, C. Smith, 1966). Moffat (1854) recorded this buchu being used by Bushmen from the Doornbergen near Prieska (i.e. on the eastern margins of the IXam territories), noting that it grew out of ant-heaps.

'gwaĩ; $n$. hyena, Hyena villosa, 'strandwolf' (Bleek, 1956:391). The species name Hyena villosa has been superseded by Hyaena brunnea (Mills, 1982:1), and this term thus referred to the brown hyena; this was the 'typical' hyena commented upon in the archives, both in Early-Race and contemporary narratives. The hyena was a prototypical ugly, nocturnal, man-eating 'beast-of-prey' (LL.II.3.420), sitting between the raw power and supra-human abilities of the lion (LL.II.21.1915) and the deceit and social reprehensibility of the jackal (LL.II.2.366). In keeping with this status, the hyena was often in conflict even with other carnivores, as when attempting to kill the lion with boiling soup (LL.II.2.362-366; LL.II.20.1774-1776; the jackal in turn played this same trick on the hyena, LL.V.4.4231-42521/2; LL.V.5.4253-4264). Though they were closely linked with jackals (most obviously in the 'Day's Heart Star' narratives, where they acted in concert as 'angry folk' who poisoned the caracal, LL.II.18.16781679), they would even instigate conflict with these allies and drive them away from meat (LL.II.20.1764-1766). Hyenas behaved 'cunningly' (!khwi, 
LL.II.21.1915), did not properly distribute (!k"wakkan-\|kun, LL.II.21.1915), and in contemporary experience were 'angry' animals that would chase the hunter (LL.IV.2.3539-3542); the deployment of hyena referents in discussing hunters who ate up springbok without sharing (LL.II.20.1793') was a powerful statement of the inappropriate nature of their actions. See Koro, \|kellke, \|khã:.

!gwa $\xi$-!nuntu; $n$. mythical person, a man of the early race, whose grandchild is carried off by elephants and recovered by himself (Bleek, 1956:393); !gwa: $\boldsymbol{\xi}, v$. to strike, beat, hit (Bleek, 1956:390); !nuntu, !nuฑtu, n. ear, hole, sometimes in plural antennae (Bleek, 1956:485). This Early-Race figure shared many traits with |kaggən, and was rebuked by his daughter for acting foolishly and obstinately, generally not in keeping with 'grown up' behaviour (LL.VIII.4.6393; LL.VIII.5.6425); his narrative (LL.VIII.4.6334-6413; LL.VIII.5.6414-6455) paralleled a similar story of the interaction between |kaggan and the Elephants (LL.VI.1.3883-3894; WB.XXV.2416-2434; WB.XXVI.2435-2473).

!gwe:-!kweitən-tu noun name of a mythical being with eyes in its feet, also sometimes called the will-o-the-wisp (Bleek, 1956:392); !gwe, $n$. hail, white lumps on porcupine (Bleek, 1956:392); !kweiton, $n$. penis (Bleek, 1956:464), $n$. black pointed shining stones which only come from the sky when it lightens, they disturb the ground when they fall (Bleek, 1956:464, see !k"abbe); !kwei, $n$. cheek, the rounded flesh above the cheekbone (Bleek, 1956:463); $\boldsymbol{t} u, n$. mouth, hole, opening (Bleek, 1956:239); tu, n. man (Bleek, 1956:239); !kweiton-tu, $n$. flower (Bleek, 1956:464). This was an Early-Race character, otherwise called mumu-!k'o (see \|khã:-ka-mumu) or a 'little fire' that disappeared as one 
approached it (i.e. the will-o'-the-wisp), who appeared in several narratives in conflict with |kaggan (LL.II.9.935-966; LL.V.1.3683-3700; LL.V.2.3793-3861); he had his eyes on his feet (between his big and second toes) and his face above his cheeks was smooth. He may have had some connection with the meerkats (and thus with $\left.\mid k w a m m a \eta^{\prime} a\right)$, as W. Bleek's version of the story began after an episode in which the meerkats had beaten Ikaggan (WB.II.387-397; it was not a direct continuation, but thematic similarities and the evident contiguity in narration suggest a connection). Whether or not this was the case, his identity recalled the anger associated with a 'beast-of-prey' identity; when he fought Ikaggən it was because they were both angry people, who quarrelled (LL.II.9.964-965). In W. Bleek's version, !gwe:-!kweitən-tu even gave |kaggan a reason to fight with him, by hunting ostriches on |kaggan's ground (WB.XXIII.2237'); this was, of course, |kaggən's own report (noted for his untruthfulness, LL.V.2.3808), and \|kabbo remarked that he usually gave a false account of the reasons for his quarrels (LL.II.33.3031'). Their conflicts had the flavour of a contestation between !gi:tən: !gwe:-!kweitzn-tu's ability to avoid attacks (despite his apparent absence of eyes) led |kaggan to question him as to whether he was a !gi:xa (LL.V.1.3692; LL.V.2.3823-3824), and Ikaggan was forced to employ his supra-human skills to escape the beating he received. The mode of Ikaggan's eventual victory, kicking dust into !gwe:-!kweitzn-tu's eyes, strongly recalled the use of this material in other interpersonal conflicts (LL.V.20.5537-5539; LL.V.23.5842'-5843'; LL.V.12.4930), where it brought 'illness' in a manner similar to the $\mid k o: \xi o \xi-d e$ of anti-social !giton: !kh'o, a 'haze' brought illness and made the sun hot 
(LL.V.20.5557; Bleek, 1956:427) was probably the second element in !gwe:!kweitan-tu's alternative name $\left(m u m u-! k^{\prime} o\right)$. See !gi:xa, |ko: $\boldsymbol{g}_{\mathbf{0}} \boldsymbol{\xi}$-de, Ikaggən, |khã:-ka-mumu, !k"abbe.

!gwunni; $n$. a greedy person (Bleek, 1956:393). The 'greedy person' was opposed the !xau: (small-eater), and consumed immoderately, particularly of fat (LL.II.3.418-419; LL.II.15.1478); it was a trait associated with 'ugly' beasts-ofprey, who would carry away and consume whatever they could find (LL.II.20.1843-1847). As a human trait it became particularly unattractive, linked with anger, ugliness, and an absence of generosity (LL.II.14.1318-1320). See !xau:, !kwi-a-|xara.

!haue-ta-łhou; $n$. name of one of the two lions now seen as pointers to the Southern Cross (Bleek, 1956:396). !haueta, v. to tie on, fasten on (Bleek, 1956:396); $¥$ hou, $n$. belt (Bleek, 1956:651). !haue-ta- $¥$ hou was one of two lions forming the focus of a large number of Early-Race narratives, generally playing a 'buffoonish' role in which their anti-social tendencies were balanced by the consequences attendant on the 'foolish' means by which they attempted to achieve their goals (Guenther, 2002:160). See !gu:.

!kakən-!kaka-!kaui struthious Early-Race character. !ka:ka, !ka:kən, v. to go in the dark early (Bleek, 1956:405); !kaui, n. egg (Bleek, 1956:414). This EarlyRace character was described as an ostrich-like bird, which could speak (LL.II.6.667-615; LL.II.7.716-737; LL.II.22.1965-2040). His interactions with |kaggən once again demonstrated the latter's foolishness and, more specifically, 
the dangers of an immoderate appetite; eating up !kakən-!kaka-!kaui's eggs rather than the 'little egg' caused the hair-brush spoon and eggs to stick to Ikaggan's mouth.

!kaui; $n$. wild onion (Bleek, 1956:414); !kouwi, v. to be lean (Bleek, 1956:445); !kouwi, n. a plant, wild onion (?) (Bleek, 1956:445); !khoui, n. a certain bitter foodplant (Bleek, 1956:429); !khou:wi, v. to starve (Bleek, 1956:429). This was an edible bulb with a red outer skin that grew among stones; in the winter, it was bitter and watery, but in the summer it was sweet (LL.IV.3.3735'; LL.IV.4.3903'). The Afrikaans wilde-ui (wild onion) refers to a range of species belonging to the family Liliaceae, in which the bulb recalls that of an onion (C. Smith 1966:507).

!kaukən-ka-!k'e; Mountain Bushmen. !kaukən, !kaugən, n. mountain (Bleek, 1956:413); -ka, poss. part. following possessor, preceding the thing possessed, often omitted before words denoting relationship or parts of the body, also written -ga, -ta (Bleek, 1956:74); !k'e noun pl. people, men (sing. !kwi; emph. !k'etən) (Bleek, 1956:419). This term referred to IXam populations occupying more mountainous, broken territory both to the north and the south of the Flat Bushman territories. Like the Hartebeest River Bushmen, they were considered by Flat Bushman informants to be substantially similar to their own groups, speaking a similar dialect, though with some dietary peculiarities (Chapter 7a:219).

!kau:xu; $n$. hunting ground (Bleek, 1956:417). !kau, $v$. to live, be alive; !k'a $\boldsymbol{u}, n$. earth, dust, ground; $-\boldsymbol{x u}, n$. face, surface, head (Bleek, 1956:261). The translation 'hunting-ground' may reflect the gender bias of the Bleek-Lloyd informants 
(Lewis-Williams, 1997:199), though in the notebooks themselves (LL.VI.1.39533954; LL.VIII.5.6422; LL.VIII.19.7667-7668) !kau:xu was alternatively given as 'veldt' (Afr. veld, presumably a translation offered by the informants themselves). Derived from the Dutch for field, it is a complex term with a substantial colonial history, forming a key component of emergent Afrikaner narratives of European settlement of the subcontinent (e.g. within the plaasroman novels of the early twentieth century, Coetzee, 1986:1). Part of this complexity makes it peculiarly apposite as a translation for !kau:xu, insofar as it combines the concept of wilderness (or anti-society) with creativity and identity-formation ( $c f$. Beningfield, 2006). The !kau:xu both in Early-Race narratives and in daily life was certainly home to anti-social forces, but it was also essential as the source of raw materials vital to the construction of proper social relationships: if the !kau element represented the verb 'to live or be alive', the term would more appropriately be translated as the 'living-place'. Lewis-Williams' (1997:199) observation that regions 'below and above the plane of material life' were 'spiritual realms'

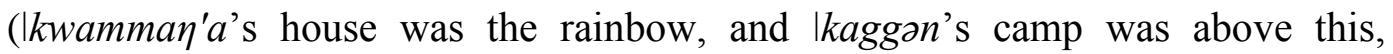
LL.II.22.1974'; LL.VIII.7.6600'-6601') is supported by the IXam term for the sky, !gwaзsxu (Bleek, 1956:391), which recognised some equivalence with the 'hunting-ground' as flat, extensive surfaces $(-x u)$.

!khi:ja; v. to be red (Bleek, 1956:427); !ki:, v. to be red (Bleek, 1956:434). This was a rather ambiguous colour term. It had strong positive connotations as a display of youth, surfeits of good food (LL.II.23.2130; LL.II.24.2149), and access to other desirable resources (to: and $\| k a: \xi$ ), but at the same time might also be 
connected to dangerous forces such as the beasts-of-prey and !khwa: (Chapter $5 c: 164)$.

!khwa:; $n$. water, rain (Bleek, 1956:431). The same term referred to an entity personifying this force, which played a role in numerous Early-Race and contemporary narratives. !khwa: was not a 'character' in the same sense as |kaggən or Ini (with familial relationships with other mythological characters) but rather constituted a predominantly destructive and transformative agency, punishing transgressions of culturally conventional attitudes and behaviours. $\mathrm{He}$ was usually a masculine figure (though !khwa: could have feminine manifestations), and was attracted to the scent of women, especially New Maidens; the smell of $s \tilde{a}:$, the colour of to: and $\| k a: \xi$, and striped patterns (LL.VIII.16.7426; LL.VI.1.3970-3973) all converted his potentially dangerous attentions into a more benign (and possibly beneficial) interest. More than other mythological characters, he played a similar role in nineteenth century IXam society to that in 'mythological' narratives; many contemporaneous 'ritual' practices, particularly those involving the New Maiden, revolved around him (Hewitt, 2008[1986]:59-66). The IXam had a detailed terminology with respect to rain: kaũ, v. to rain, to fall (Bleek, 1956:83); |hakənlhakən, $n$. a weak rain, mist (Bleek, 1956:286); !kwobba, n. fine rain (Bleek, 1956:468); "garakən-\|garaka, v. to make fine, be fine, fall gently (rain) (Bleek, 1956:527); $\|_{\Lambda} h a ́$, $n$. rain liquid (Bleek, 1956:628); Obu:, Obu:kən, v. to rain gently (Bleek, 1956:682); ○pwo○pwõnnu, n. a weak rain (Bleek, 1956:686). This profusion of terms succinctly demonstrates their keen interest in rain that fell 'gently', the female 
rain that was essential for creating 'comfortable' conditions; the creation of these conditions though the encouragement of !khwa: in its feminine aspect was the

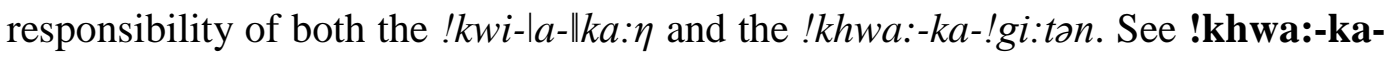
!gi:tən, !kwi-la-\|ka:n.

!khwa:-ka-!gi:tən; rain’s !gi:tən. !khwa:, n. water, rain (Bleek, 1956:431); -ka, poss. part. following possessor, preceding the thing possessed, often omitted before words denoting relationship or parts of the body, also written -ga, -ta (Bleek, 1956:74). These !gi:tən professed control over the rain, and were beseeched to make the place 'comfortable' by bringing 'sweet' water that would make the ground cool, and induce the springbok to return (LL.II.24.2213-2214). The importance of this resource meant that these persons were subject to strong social pressures to behave 'responsibly' (i.e. in accordance to the demands of others); they were exhorted to produce pleasant female rain rather than angry male rain (LL.II.25.2227-2230). In return, these !gi:tən would present their failures as the result of other peoples' lapses in decorum, suggesting that the peoples' fires had caused the rain to disperse (LL.II.24.2220), that the rain would not fall until they brought water for the !khwa:-ta-!gi:xa (LL.II.26.2303-2306a), or that they were deliberately withholding rain because the people were accustomed to do 'evil things' or fight (LL.V.3.4083-4085). They also subjected other !khwa:-ka!gi:tan to their own criticisms, for employing inappropriate or unskilful techniques that might allow the rain-animals to escape (LL.V.3.4086-4121). See !khwa:, !gi:xa. 
!khwa:-ka-!kaukən; Water/rain's children. !khwa:, n. water, rain (Bleek, 1956:431); -ka, poss. part. following possessor, preceding the thing possessed, often omitted before words denoting relationship or parts of the body, also written -ga, -ta (Bleek, 1956:74); -Opwa, little, young, $n$. child. In IXam it is only used in the singular, changing in the plural to -ta-!kaukən (Bleek, 1956:684). These were 'beautiful' or 'handsome' striped creatures about the size of a calf (presumably by comparison with!khwa: often described as a bull or cow). In an Early-Race narrative, a New Maiden killed and ate them, violating the food taboos imposed on her by her xoakan-gu (LL.VI.I.3942-3958); in punishment for this transgression, !khwa: took the girl and her family up in a whirlwind, and transformed them into frogs. See !khwa:.

!khwi; $v$. to be clever, $n$. cunning (Bleek, 1956:433); !khwi!khwi, v. to deceive, cheat, cunningness (Bleek, 1956:433); !khwi!khwisitən, deceiving (Bleek, 1956:433). The term was often translated by the informants with the Afrikaans skelm (LL.II.3.407; LL.II.9.972'), connoting roguishness. I prefer 'cunning/cunningness' as the English translation, for the same reason I prefer 'understanding' as a translation for $\| k w a k k a$ : using 'clever' as a gloss for both of them obscures a division maintained in the IXam. It is, however, important to note that this was not a mistaken conflation: the two were not wholly opposed, as 'cunningness' in some contexts represented an 'understanding' way of dealing with a situation (LL.II.9.973-974). Where agents were in conflict with one another, cunningness and (particularly) deception formed effective ways of achieving victory; this dimension emerged particularly in Early-Race narratives 
involving |kaggan's fights with other Early-Race people, but was also deployed in hunting. Cunningness did not preclude foolish action in the same way that 'understanding' did (LL.II.30.2743-2744), and was associated with characters who often acted in anti-social ways that would be quite inappropriate if linked with the $\| k w a k k a$ persona (e.g. Ikaggan, LL.V.6.4430-4431; jackals, LL.V.5.42594263; the Early-Race sun, who did not share his light with the people, LL.II.35.3157). See Igebbi, \|kwakka, daudáu-ka-!k'e.

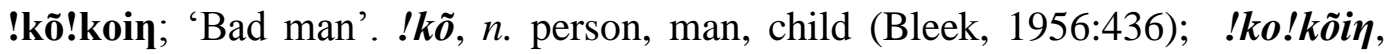

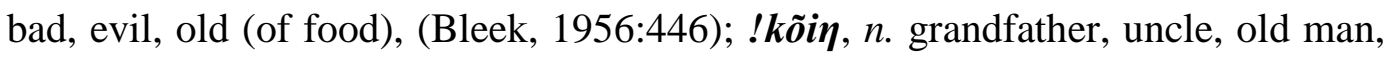
(Bleek, 1956:440). This phrase was used to describe the !gwunni (LL.II.14.1320), and possibly merely represented !ko!kõi rather than epithet associated particularly with this figure: elsewhere, 'bad man' is used as a translation for $\mid n u$ $k a-! k^{\prime} e$ (LL.VIII.18.7620; LL.VIII.29.8593). When !ko!kõi tikan-tikan (things, Bleek, 1956:201) and translated as 'evil things' (LL.V.3.4084), it could refer to people 'not taking care of each other', thus confirming a strong negative moral evaluation of inappropriate participation in resource acquisition and sharing. See Inu-ka-!k'e, \|k"oa:, !gwunni.

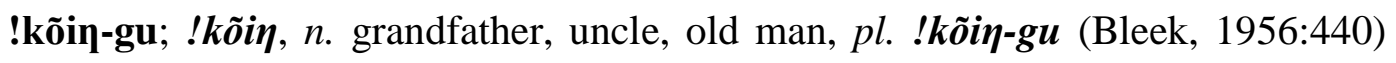
This term was variously translated as grandfathers, uncles, and 'old men': it is perhaps most appropriately considered, in \|kabbo's phrase, as a term used just 'as the Boers use Oud [Afr. old] Oom' LL.II.32.2926'). Oom (Afr. 'uncle') is a respectful or affectionate address for any older man, and while $\|$ kabbo made reference here to men who were actually uncles, the IXam term could include any 
elder males (LL.II.35.3195'; occasionally even including grandmothers, LL.II.13.1266'). In the widow's story (Chapter 6a:169), the widow reminded her brothers that they were her children's !kõi -gu (LL.II.14.1322) in an attempt to persuade them to provision her children with their correct 'portions': the term therefore appears to have encompassed food-sharing responsibilities, and also entailed 'respectful' (!k"werritən) treatment (LL.II.30.2756). See !k"werri:tən, xoakən-gu.

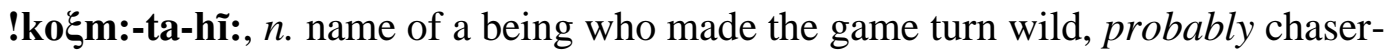
of-food (Bleek, 1956:441); !ko $\boldsymbol{\xi} m m \boldsymbol{m} \boldsymbol{i} \boldsymbol{\eta}$, v. to chase (?), to head (?) (Bleek, 1956:441); !ko $\xi \mathbf{m}:$, v. to plunge, thrust (Bleek, 1956:441); -ka, poss. part. following possessor, preceding the thing possessed, often omitted before words denoting relationship or parts of the body, also written -ga, -ta (Bleek, 1956:74); hĩ:, v. to eat, feed (Bleek, 1956:60) This was an Early-Race character who made the animals 'wild' by beating them; specifically, he made them 'fearful' (!hammi, Bleek, 1956:395) and they could no longer be stroked and handled as domesticated species (LL.V.19.5457-5477). His motivations for so doing were

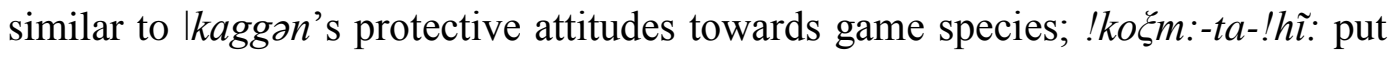
the fear into them so that men would find it difficult to kill them, and would have to hunt them by stealth (LL.V.19.5466-5467, 5474).

!kúi-se-!khwi-|ku; Early-Race lion cub raised as a dog. !kúi, v. to grow, become abundant (Bleek, 1956:449); !kui, n. ashes, meal (Bleek, 1956:449); se, v. to come, often used as v. participle, shall, will, must, may (Bleek, 1956:164); !khwi, n. tail (Bleek, 1956:433); !khwi, $v$. to arrange the bed (Bleek, 1956:433); !khwi, $v$. 
to break, burst, blow hard (wind) (Bleek, 1956:433); |ku:, v. to singe, light (Bleek, 1956:322); |ku:, v. to put, put away, pack, place, set, keep, hide, bow down (with pain), be ill (Bleek, 1956:322); |ku:, n. proteles, 'aardwolf', Proteles cristatus (Bleek, 1956:322); !kúise, $n$. an edible bulb, much eaten (Bleek, 1956:450). This was the name given to a lion cub by a young man of the Early-Race, who attempted to raise it as a dog to assist him in the hunting of gemsbok (LL.II.26.2320-2412； LL.II.27.2413-2504； LL.II.29.2597-2687； LL.II.30.26882779; LL.II.31.2780-2873). !kúi-se-!khwi-|ku behaved in typical leonine fashion, ‘swallowing down' meat (LL.II.26.2359-2360), catching hold of the young man's hand in its mouth and looking as if it would 'throw [him[ down and hold him, in the darkness' (LL.II.26.2366); despite his attempts to 'domesticate' the lion (LL.II.29.2609), it remained a beast-of-prey, and his interactions with it were foolish (LL.II.26.2392-2398).

He was, however, able to successfully hunt gemsbok with his 'dog': lions in the southern Kalahari select for this species (Mills, 1984:281; Hayward and Kerley, 2005:315) and although this is not certain for the Nama-Karoo, it seems a logical extrapolation. Adult gemsbok represent challenging prey, and when faced with a group of predators (Mills, 1990:98-101) will back into thornbushes to defend their rear, slashing with their long horns at animals approaching from the front (Apps, 2000:169). This behaviour renders them vulnerable to humans hunting with dogs, as they can readily be brought to bay through pursuit and intimidation (BlurtonJones and Konner, 1989:28; Ikeya, 1994:123-124). However, these large animals cannot be brought down by the dogs, and gemsbok may inflict serious injuries 
with their horns, which sometimes prove fatal (Ikeya, 1994:127); the attraction of a dog peculiarly suited to killing the gemsbok it tracks is obvious. This narrative explored the tension between the fact that the lion-dog allowed the young hunter to achieve his aims much more successfully (exhibiting his 'cunning', behaviour), and the fact that the strong anti-social tendencies of his 'dog' rendered the products of his hunting extremely dangerous. The lion was constructed as an 'anti-dog', explicitly contrasted with proper canids in terms of physical and behavioural characteristics: its hairy feet (LL.II.26.2339), 'yellow' eyes, 'tearfurrows', short ears (LL.II.29.2597-2598), 'yellow' hair (LL.II.29.2612), long tail (with hair on the tip), black ear 'roots', and head covered over with hair (LL.II.31.2831-2832) were all emblematic of its bestial nature. More abstractly, it was a dog that 'came out of the solitude which it did inhabit', resembling a 'thing of terror' or a 'dog of darkness' (LL.II.30.2759-2761). The young man's choice of this terrifying, nocturnal, anti-social 'dog' as a hunting companion was an apposite emblem of his foolishness, also manifest in his social relations, where he lied to his relatives (LL.II.27.2413, 2454). See Igebbi, \|kwakka, \|ke\|ke, \|khã:.

!kúi:ta (!kúi:tən); v. to be white (Bleek, 1956:450). This form of 'whiteness' formed a partial opposition to notions of kerru; when the bushes were dried or 'old', they became !kúi:ta. This description was, therefore, for the most part a negative one (Chapter 5c:165). Though there was at least one instance of !kúi:ta being associated with beauty (LL.II.6.703), the recurrent connection between whiteness and beauty is an artefact of the translation process. |ko: wa was often linked to an attractive 'paleness', while other more specific terminology 
strengthened the occurrence of this link: $\left\|_{w a}\right\|_{w a t}(v$. to be beautifully white, Bleek, 1956:630), turned up only in a single narrative, describing a distinctly attractive Early-Race character (the $\ddagger$ nuturu, LL.VIII.9.6800). See kerru, Iko:wa, łnuturu.

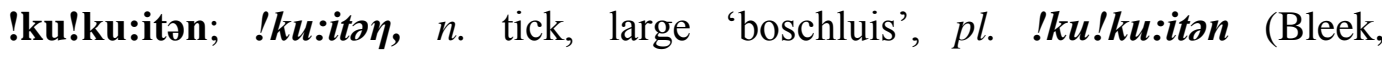
1956:450). The Early-Race Ticks were closely associated with sheep; this clearly made reference to the parasitic relationship between ticks and mammals, as shepherding experiences could hardly have left the informants unaware of the tick load that sheep can acquire. This association was manifested in their settlement pattern, of bushes placed in a circle around the houses, oriented around the sheep kraal (LL.II.33.2998'); their diet consisted of boiled sheep's blood mixed with fat and milk (LL.II.32.2951'-2952). In the Early-Race narratives, they appeared as antagonists; they were an 'angry' murderous people, black and 'bloody-handed' (LL.II.32.2947-2948, 2961), and Ikaggan visited them in order to steal their sheep (LL.II.32.2926-2965; LL.II.33.2966-3044), in one of the rare examples of a 'positive' portrayal of his skills, insofar as he at least acquired desirable resources for his kin (though immediately reverted to foolishness by inviting $\|$ khwai-hem to dine with them). These associations of violence and livestock ownership have formed the basis of proposals that the Early-Race Ticks acted as a cipher for Khoekhoe/Korana populations (Hewitt, 2008[1986]:188); the description of the Ticks as bloody-handed recalled for $\|$ kabbo the nickname of the Koranas (bloodybrowridges), and their violent actions (LL.II.32.2960'). 
!kwe; $n$. orphan, poor person or thing (Bleek, 1956:463). !kwetja, v. to make an orphan, to become an orphan (Bleek, 1956:465); !kwe!kwe, n. scrap of meat, probably liver (Bleek, 1956:465). Becoming an orphan was characterised as a 'great thing' (LL.V.6.4412') rather than a 'light' one; something that was not easy to bear. Dia!kwain suggested that orphaned children would have to rely primarily on the resource acquisition skills they had acquired from their parents (LL.V.6.4410'-4411'), and 'lean one' was an alternative translation for the term (LL.VIII.11.6954). 'Mourning' in general held connections of poverty and deprivation; \|kabbo's translation for the word !goe (want, misfortune (?), Bleek, 1956:385; Lloyd translated this as 'mourn', LL.II.13.1304') was the Afrikaans arm maak (make poor/indigent).

!kwi-a-|xarra; 'Man that (was) different'. !kwi, n. man, person, human (Bleek, 1956:466); a, a:, relative pronoun, who, which, the one who, that which, where, when (Bleek, 1956:4); |xarra, |xarrase, different, differently, separately (Bleek, 1956:363). |xarra or 'difference' was a negative trait, connected with the nonunderstanding beasts-of-prey that ate people and went by night (LL.II.3.421; LL.II.9.914; LL.II.18.1678; LL.II.16.1552), with 'different' odours that smelt badly (LL.II.14.1442); 'monstrous' angry figures including $\| k h w a i-h e m$ were also 'different' (LL.II.33.3083-3084). People did not 'cry over' those who were different (LL.II.11.1183); the man 'who was different' did not maintain amiable social relations, being an angry, ugly, and selfish person (LL.II.14.1317-1320), and 'different' people would not feed orphans (LL.V.6.4410). 'Strangers' would shoot at people (LL.II.22.1981), and less tangible manifestations of antipathy 
were also sent from 'different' places or things (LL.V.19.5512, !gi:tən; LL.V.22.5833, Inu-ka-!k'e); when the rain came violently, the !khwa:-ka-!gi:ton suggested that it had been made by a 'different person' (L.II.23.2283). See Inuka-!k'e, !gwunni.

!kwi-la-\|ka:n; !kwi, n. man, person, human (Bleek, 1956:466); la, feminine ending, probably part of lai:ti (Bleek, 1956:267), lai:ti, $n$. female, woman, wife (Bleek, 1956:268); $\| \boldsymbol{k a}: \boldsymbol{\eta}$, new, fresh, raw, to eat raw (Bleek, 1956:556). The 'New Maiden' is a major figure in the IXam narratives, playing a role both in Early Race stories and in contemporary practice. At the onset of menarche, a girl became a New Maiden and entered a period of ritual seclusion, during which her diet, water consumption, and expressive gestures were subject to a regime of control (Hewitt, 2008[1986]). The New Maiden attracted the interest of the potentially destructive !khwa:; like him her roles in IXam society and in mythological narratives were substantially similar. The New Maiden manifested a 'feminine' (specifically, fertile feminine) sphere of 'competent action', equivalent to that of daudáu-ka-!k'e and !gi:tan. These parallels were made quite explicit by the informants: when becoming a New Maiden, a girl possessed the $\mid k o: \xi o \xi$-de (LL.V.6.4400') that was also employed by !gi:ton and powerful 'new sorcerer'

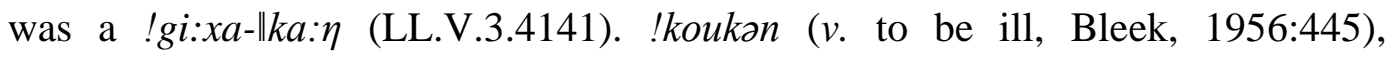
prototypically used to refer to a girl's seclusion at her first menstruation, was identical to the verb!koukan, (to beat, pound, tremble, Bleek, 1956:445), used to describe the 'trembling' that occurred when people incorporated the smell of the !gi:xa blood, or when the !gi:tən set aside their karosses (LL.V.22.5765-5767; 
Lewis-Williams, 1997:209 suggests that might also be translated as 'entering trace').

Many of the characteristic practices of the New Maiden role were parallels of hunting practice: the bush screens that the hunters lay behind when ambushing prey (LL.II.16.1530') were explicitly compared to the 'small house' occupied by the New Maiden. The hunter who shot an eland walked about as if ill (LL.V.6.4417), and when he returned home was physically isolated from the main camp in a hut built for him by the old men (LL.V.17.5338); themes of illness and isolation (as well as the governing role of older men and women) were paramount for New Maidens. Like hunters, New Maidens were said to 'shoot' at things, although in their case the shooting was the result of anger directed at fellow humans and was something to be controlled (LL.V.20.5618-5624), as it would attract the dangerous attentions of !khwa: Although primarily associated with !khwa:, the New Maiden's potency expanded into other domains: she imparted 'wildness' to springbok if she glanced at them (LL.II.28.2521'), and undermined the hunting prowess of dogs (LL.V.20.5593-5601) or efficacy of hunting equipment (LL.II.28.2523), with the 'coolness' of her saliva. To counterbalance these potential problems, she had to obey strictures regarding the sharing of meat with specific individuals, and thus played a direct role in governing hunting success. See Daudáu-ka-!k'e, !gi:xa.

!k"abbe; $n$. storm (?), thunderbolt (?) (Bleek, 1956:507). !k"abbe, v. to be startled (Bleek, 1956:507); !kabbe, v. start, be startled (Bleek, 1956:402); !kweitan, $n$. black pointed shining stones which only come from the sky when it lightens, they 
disturb the ground when they fall (Bleek, 1956:464); !kwerritən, v. to thunder, strike (lighten) (Bleek, 1956:464). This reference was likely connected with general notions concerning the destructive consequences attendant when 'the rain lightened' (i.e. lightning strikes), or threw pointed black stones (LL.V.22.58065808); the striking of such forces was linked with the displeasure of the New Maiden (LL.V.13.4985-4989; LL.V.20.5618-5619), as her identification with

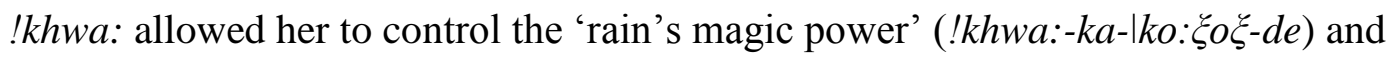

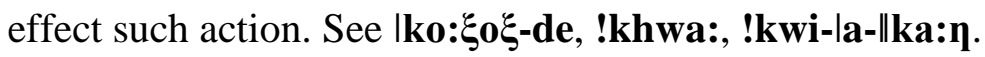

!k"wakka-tukən; 'Foulmouth'. !k"wakkən, !k"wakka v. to be decayed, putrid, n. decayed matter (Bleek, 1956:509); tu, n. mouth, hole, opening, emph. tukən (Bleek, 1956:239); !k"oákkan, v. to decay, smell badly, noun putrid matter (Bleek, 1956:508). In the early notebooks, this referred to an Early-Race character whose name was sometimes translated (LL.II.2.320) as muishond (zorilla, Ictonyx striatus); this may have been based in the similarity of the term !k"wakka (i.e. putridity) to ! $k w^{\prime} a:-k a$ ('of the muishond', Bleek, 1956:457). Later, Lloyd rendered it as 'Bad-smelling-mouth' (LL.VIII.29.8526-8554), a rather 'grotesque' Early-Race man with an 'abominable' smell; putridity and foul smells were indications of angry or anti-social identities. He represented one of the antagonistic Early-Race 'monsters', opposed to the 'human' society of Ikaggən and his family; in this narrative, he fought and stabbed the Blue Crane $\left(! k^{\prime} o\right.$, Tetrapteryx paradisa, the elder sister of |kaggən, LL.VIII.29.8526') with a spear. See !k"wakkən-\|kũn. 
!k"wakkən-\|kũn; 'Decayed Arms'. !k"wakkən, !k"wakka v. to be decayed, putrid, n. decayed matter (Bleek, 1956:509); $\| k \tilde{u}, n$. arm, wing, humerus (Bleek, 1956:590, 593). !k"oákkan, v. to decay, smell badly, $n$. putrid matter (Bleek, 1956:508). This term (sometimes translated as 'stingy') described people who did not properly share or allow access to resources; a man who ate meat with fat while only giving 'lean' food to others was acting in this manner (LL.VIII.25.82168217), while the usual set of anti-social predators (lions, hyenas) were also criticised with the term (LL.II.21.1915). The old man who would not 'dip out' food despite the fact that he 'owned' locusts was behaving as a !k"wakkən-\|kũ $\eta$ (LL.VIII.7.6638-6640). Though not explicitly stated to be a Opwai:tən-ta-!gi:xa, this same 'old man' also owned the rain, and statements of this kind therefore likely represented exhortations for ritual specialists to remember that they should act in the interests of the human community, rather than like 'beasts-of-prey'. See @pwai:tən-ta-!gi:tən, !gwunni.

!k"werri:tən, !k"wirri, !k"wirriton; v. to be ashamed, abashed, to show respect (Bleek, 1956:510); !kworri:tən, v. to be ashamed (Bleek, 1956:469). This was a dimension of interpersonal relationships that manifested propriety; people were supposed to develop 'respectful' attitudes towards appropriate persons in the course of receiving the education that inculcated 'understanding'. It was demonstrated by avoiding mocking or laughing at people, and was supposed to be shown towards !gi:tən (LL.V.10.4756-4757) and !kõi -gu (LL.II.30.2756); this reference to !kõin-gu may instead have been suggesting that this behaviour characterised relationships with affinal kin, as a reverso note (LL.VIII.18.7615') 
gave ! $k$ "wirri as 'shame' or 'respect' directed toward mothers- or fathers-in-law. There was probably a gendered element to the term as well, as (masculine) !khwa: was supposed be 'ashamed for' the women's eyes (LL.VIII.26.8305') and baboons treated a girl's belongings with respect (LL.V.24.5923, 5953); this latter aspect, extending the 'respectful' attitude from a person to their belongings, was a general feature of the term (LL.II.31.2824). See !nanna-se.

!nanna-se; $v$. to show respect, avoid, $n$. hunting observances showing respect (Bleek, 1956:473). This form of respect manifested itself primarily in the avoidance of certain words; girls used the name |khogən rather that $\|$ gauxu to refer to porcupines (LL.V.6.4377'-4378'). By engaging in these respectful behaviours, relationships were created that obliged the non-human partners (usually animals, hence the definition emphasising 'hunting observances') to behave in ways that humans desired: the porcupines addressed as |khogən would come out of their holes where they could be killed (LL.V.7.4379') and if people did not !nanna-se the game they had shot, it was not 'obliged' to die from the poisoned arrow (LL.V.17.5270). Other non-humans could be encompassed by !nanna-se: the 'protection' the New Maiden applied to people to prevent !khwa: killing them with lightning was more properly the creation of a !nanna-se relationship that would make !khwa: morally obliged not to act in this way (LL.V.13.4986), and people used the term $\| k^{\prime \prime} w a n-t u$ to address the moon to prevent it 'going into the sky’ (LL.VIII.28.8442-8443). See !k"werri:tən.

!nu!numma-!kwitən; $n$. name of a monster, probably swallower-of-eggs (Bleek, 1956:486); !num:, v. to take, put, hold in the mouth (Bleek, 1956:484); !kuiton, 
!kúi:ta, n. pl. eggs (Bleek, 1956:450). Though the name appears to literally translate as 'to take eggs in the mouth', |hanłkass'o glossed it as wit-mond or 'white-mouth' from the colour of the eggs he ate. He was an Early-Race figure mentioned by this name only in one short note (LL.VIII.29.8555-8560), described as a 'beast-of-prey' that swallowed down eggs and great pieces of meat in a form of 'monstrous' consumption typical of the Early-Race grotesques (see \|khwaihem, !k"wakka-tukən). This figure was used as a bogeyman (a term in folklore studies referring specifically to frightening figures used to coerce children into good behaviour; Simpson and Roud, 2000); Ihanłkass'o recalled his grandfather impersonating !nu!numma-!kwitən saying 'I kill children who cry!', to induce them to stop screaming and calling out (\|kanalkana, LL.VIII.29.8556'). His alternative name was !kotta-koe (LL.VIII.29.8554'-8555'; given because he 'swallowed down eggs'); in this guise he appeared in another narrative (LL.VIII.28.8486-8506), where he managed to fool a group of Koranas, intent on violence, into leaving him alone.

!xau:; $n$. a small eater, a rich man (Bleek, 1956:498). This formed a counterpart to the !gwunni, and was considered an attractive state: the fact that the Early-Race lynx was a small-eater contributed to her 'sweetness' (LL.V.3.4349'), or handsomeness (LL.II.3.419). This reverso note explained its alternative definition as 'rich man' as the result of a person being satisfied with only a small piece of meat; in particular, they would leave fat and be satisfied with meat alone. This would leave this desirable resource for others, or allow them to apply it to the skin as part of the process of becoming handsome. See !gwunni, to:. 
!xoe-s'o-!k'e; !xoe, $n$. place, country (Bleek, 1956:500); s'o, v. to sit, remain (Bleek, 1956:171); !k'e noun pl. people, men (sing. !kwi; emph. !k'etən) (Bleek, 1956:419). This term translated approximately as the 'people belonging to (particular) places'; the !xoe were territories, usually based around permanent water sources, that were associated with particular individuals (broadly corresponding with Jul'hoansi n!ore territories and their $k^{\prime \prime}$ ausi owners, Lee, 1976). This connection with territory endowed people with rights of access to specific resources (honey and ostrich eggs 'belonged' to the !xoe, LL.II.14.13601363; LL.II.22.2029') and created obligations to take of and treat 'nicely' these resources (LL.II.32.2894-2896). The identity associated with water sources does not appear to have been unequivocally positive: people who 'possessed' named water-linked locales were sometimes referred to as $\| k^{\prime \prime}$ oakan - people (they could be men or women, LL.VIII.23.8071'). This was given in translation as 'star's man', possibly representing a connection with $\| k^{\prime \prime}$ oagu (a name for Canopus, Bleek, 1956:606); shooting-stars were supposed to fall into waterholes (LL.V.19.5478). It also likely referred to 'cursing', 'badness', or 'making unfortunate' (Bleek, 1956:606); the stars that fell into waterholes were the hearts of !gi:ton after they died (LL.V.19.5483-5484). Although this may also have had shamanic connotations ( $c f$. Lewis-Williams, 2002:60, 79-80), these non-living !gi:tən were strongly associated with anti-social, illness-causing activities, as after death their hearts longed to 'take away' the people whom they had loved (LL.V.19.5485); $\| k^{\prime \prime} a o$ : or cursing was also linked with bring a 'death influence' down on people. This series of beliefs about permanent water sources plausibly 
underpinned the avoidance practices associated with their 'owners': the names of such people were subject to avoidance terms (LL.VIII.23.8071'), likely an attempt to prevent drawing the unwanted and potentially negative attention of these 'fallen hearts'.

!xwe:-\|na-s'o-!k'e; $n$. 'First-there-sitting-people' (Bleek, 1956:504); 'xwe:, first, in front, early, leading (Bleek, 1956:504); \|na, v. to stay, be with, dwell, stand, often used as here, there, in, at, with, or simply as emphasis (Bleek, 1956:611); $\boldsymbol{s}^{\prime} \boldsymbol{o}, \boldsymbol{v}$. to sit, remain (Bleek, 1956:171); !k'e noun pl. people, men (sing. !kwi; emph. !k'etən) (Bleek, 1956:419). W. Bleek gave this as 'First Bushmen' (WB.II.378'), while Lloyd usually translated it as the 'Early-Race'. Though there were hints of a rather inchoate concept of a group of people who occupied the |Xam-ka-!xoe before the !xwe:-\|na-s'o-!k'e (\|khwai-hem's distinctive calls were a remnant of their 'very old language', WB.XXIII.2160'), the !xwe:-\|na-s'o-!k'e were usually referred to as the first occupants of the IXam territories (LL.VIII.10.6885'); they too had their own language (LL.II.35.3197'), and many of them spoke IXam in unusual ways (LL.VIII.11.6990' for the Early-Race Baboons' speech; LL.II.37.3356-3357, for the manner in which the jackal, moon, lion, hyena, and tortoise spoke IXam; LL.II.34.3118'-3119' for Ikaggən's speech). Some the Early-Race people were the forerunners of the animals of the contemporary IXam world (being transformed by the 'cursing' of the aardvark and caracal into their animal forms, see $\| \mathbf{k}^{\prime \prime} \mathbf{a o :}$ ), but others were distinctive, often quite monstrous, characters without 'mundane' correspondences (see Ikhwaihem, !k"wakka-tukən, !nu!numma-!kwitən); the 'first family' of the Early- 
Race times consisted of the various characters to whom Ikaggən was related (see |kaggən, |kwamman'a). The Early-Race people behaved 'childishly' on a grand scale; where IXam children were characterised as foolish because they had yet to receive the narratives that inculcated understanding (see $\| \mathbf{k w a k k a ) , ~ t h e ~ E a r l y - ~}$ Race people had yet to generate the very experiences that underpinned this state. Much of their behaviour, then, was 'improper' and contrary to contemporary IXam practice. They hunted (in a 'foolish' fashion), killed, and ate lions (LL.VIII.18.7572', 7582), an avoidance of which was observed by IXam generally (Chapter 5b:151). The Early-Race !ka $\xi u$ (lizard, Agama sp. Bleek, 1956:413, probably A. atra) and !kwa: (muishond, Bleek, 1956:458; Ictonyx striatus) cut their own flesh to bring as food, rather than hunting properly (LL.VIII.12.71147116; LL.VIII.13.7157'); other Early-Race people acted in a 'stingy' manner by not sharing meat (LL.VIII.25.8216), or conversely, 'ate up' portions not intended for their consumption (LL.VIII.27.8351-8352). These inappropriate forms of sociality (i.e. incorrect methods of dealing with resources) were mirrored in the violent dimensions of their interpersonal relations; they were 'angry people' who cursed one another (LL.VIII.15.7307'), who ate people (LL.VIII.12.7036') and killed their in-laws (LL.VIII.25.8198). By shooting the rain (in the form of an eland) (LL.VIII.16.7461) or disobeying prohibitions of the xoagən-gu and consuming the water-child (LL.VIII.17.7473-7521), the actions of the Early-Race characters dramatically portrayed the consequences of subverting the normative mechanisms of contemporary |Xam society; many of the Early-Race narratives were deliberately educational, told to children in attempts to inculcate desirable 
attitudes (LL.VIII.17.7520'). See \|khwai-hem, !k"wakka-tukən, !nu!numma!kwitən, |kaggən, |kwamman'a, \|kwakka, \|k"ao:.

\|gwatton, \|gwatta; $n$. cat (Bleek, 1956:537). In the Early-Race narratives, the Cat formed one of the many adversaries of Ikaggan (LL.II.5.547-565; LL.II.9.965985), where he behaved in a manner reminiscent of !gwe:-!kweitan-tu (LL.II.9.965-985 was a conflict fought immediately after he had encountered !gwe:-!kweitan-tu); |kaggan could not defeat the Cat because it placed its head inside the earth where he could not strike it, and it was only with the advice of IniOpwa that he managed to defeat it. The Cat was not a particularly 'anti-social' predator, and in these conflicts behaved in a quiet or still fashion until |kaggən provoked him (LL.II.22.1986). See !gwe:-!kweitən-tu.

\|hara; $n$. a certain stone which is said to be both hard and soft (Bleek, 1956:540). This 'soft' black stone was mixed with fat and rubbed onto the head to make the hair grow long and abundant, creating a handsome appearance that was without parallel. "hara was said to sparkle or shimmer, and was found in association with to: (haematite); it thus probably represented specularite, or specular haematite (LL.VIII.14.7272'-7276').See $\| \mathbf{k a :} \xi$, to:.

$\|$ ka: $\xi ; n$. haematite, 'rooiklip' (Bleek, 1956:547). Like to:, this was a form of haematite and it was used in a similar fashion, producing a red pigment that placated !khwa: when strewn on water (LL.VI.1.3970-3971), and that was painted onto the skin in stripes to deflect his anger (LL.VI.1.3972'-3973). Unlike to:, it 
was painted on the ostrich-feather sticks that were used to direct the springbok in game drives (LL.VIII.23.8072'-8073). See \|hara, to:

\|kau\|kaugən, (\|kau\|kaukən, $\|$ kau\|kauukən): n. pl. thoughts, thinking strings. (Bleek, 1956:564); $\|k h o u\| k h o ́ u g ə n, n$. thoughts, great throat artery, thinking strings (Bleek, 1956:577); $\neq \tilde{\imath}, v$. to think, remember, desire, $n$. thoughts (Bleek, 1956:652; these two terms were considered equivalents, LL.II.6.689'). This pair of 'thinkingstrings' ran along both sites of the front of the neck (LL.II.6.689'); the 'grown-up' person who possessed his 'thinking-strings' was a person who understood (LL.V.10.4783). When a person became angry, his thinking-strings 'went astray' (LL.V.23.5871); if one's 'thoughts' were closed, then one behaved foolishly or aggressively (LL.II.30.2754, 2763; LL.VIII.26.8311). These thoughts were also the means by which !gi:tan worked their !gi; this was the reason they might continue to affect people after they died, as they continued to think about the people they knew in life (consequently their 'magic power' might assault these people, LL.V.19.5484-5485). See \|kwakka, !gi:xa.

$\|$ ke\|ke; $n$. beast of prey, wild beast (Bleek, 1956:571). Though the term described carnivores in general, including smaller species such as bat-eared foxes; the lion and hyena best typified the category. Beasts-of-prey displayed a series of physical traits, most notably hairiness (LL.II.2.333; LL.II.30.2693-2694; LL.V.3.41274128) and a kai:nja ('yellow') colour (LL.II.18.1657), especially about the eyes (LL.II.18.1653'-1654); more specific traits such as hair that covered the feet and 'closed in' claws (LL.II.18.1655-1656) and a 'great' stomach (LL.II.18.1670) were linked with superlative 'bestial' identities (lions). This distinctive physicality 
was matched by an equally characteristic morally-dubious personality that governed their interactions with people and with one another. They were 'different' creatures (LL.II.9.914) that came by night (LL.II.9.914-915; LL.II.18.1660), 'greedy' animals (LL.II.20.1844-1846) that 'swallowed down' food in an immoderate fashion (LL.II.20.1862; LL.II.30.2695; 8554'), and angry folk (LL.II.30.2731; LL.II.30.2767) that wanted to chase and bite people (LL.V.3.4128). They therefore did not act 'nicely’ (łhannũwa) or do 'good’ $(a: k ə n)$ in their relationships with people, by eating the game that the latter had killed (LL.II.23.2121) or, in perhaps the most apposite demonstration of their status, attempting to kill and eat people (LL.II.26.2339'-2340). Their attacks were part of a series of 'misfortunes' (see !k"ao:); if a beast-of-prey were going to bite a person, his hunting would remain unsuccessful until the attack had occurred and the blood 'come out' (LL.V.11.4854-4957). Similarly, their attacks could take the form of punishments for the transgression of respectful behaviours; if they looked at the Moon as the game lay dying, beasts-of-prey would eat it LL.V.21.56445645). Their unusual modes of behaviour empowered them with particular suprahuman abilities: a result of their nocturnal activities, they (like !gi:tan) could learn things that ordinary Bushmen could not (LL.V.8.4616'-4617'). In other cases, their actions were equated with familiar domains of human action, but set up in opposition to human society: like successful human hunters (Chapter 5a:145), they drew upon control of winds when hunting, making the wind blow strongly so that they could approach the huts without alerting the dogs (LL.V.23.5847-5856). This 'parallel' identity was also employed in reverse characterisations, and when 
involved in hunting activities the IXam addressed one another as 'beasts of prey' (LL.II.30.2699'; LL.VIII.14.7235, 7238). The same address was mobilised when attempting to cajole people into undertaking tasks within the context of specific relationships. Women used it when asking their husbands to fetch wood (LL.II.30.2699'), and men addressed their wives as $\|k e\| k e$ when asking them to 'work nicely' the houses, covering them with bushes (LL.VIII.23.8017'-8018'); people also called !gi:tan 'beasts of prey' when asking them to alleviate uncomfortable conditions (LL.VIII.7.6639'-6640'; LL.VIII.26.8305'). Such associations may have mobilised the generally 'irresponsible' stereotype of these bestial characters, reminding people that acting in a manner reminiscent of the $\|k e\| k e$ was not appropriate in their relationships with their 'fellows'. See $\| \mathbf{k h a ̃}:$, !gwaĩ, la:, \|k"ao:.

$\|$ ken; $n$. magic, sorcery (Bleek, 1956:569); $\| k e: \eta:, v$. to stab, stick in, prick, pierce, sting, dig (Bleek, 1956:569). This was another term for the 'magic power' possessed by !gi:tən: it was a rather 'disembodied' power that manifested when the !gi:xa went about on nocturnal 'magical expeditions' (|xãu:, Bleek, 1956:363) to discover things that were happening at a distance (5501-5505; 5534-5535), and was also the name for a type of dance 'danced by sorcerers' (LL.4744'-4745). It often took on an antagonistic character as the means by which !gi:tən would try to 'snatch away' people, killing them (LL.V.3.4138'-4139; 5485; 5515-5516; 5548); in this case, Lloyd initially translated the term as 'witchcraft', but was corrected by Dia!kwain who informed her that it referred to 'tornar manier' (probably Afr. tovenaar manier, 'sorcerer's way'). !kweitən-ta-\|kəך received her name because 
of the 'sorcery' $(\| k \partial \eta)$ that 'killing, took away [her mother's] people' (LL.V.10.4759), and the similar term for 'stabbing' or 'sticking in' further recall the methods whereby anti-social forces attacked human groups, shooting at them with 'magic arrows': this connection was made more explicit on a note on nonhuman figure called !gwonni, who sat at the 'bee's hole' and 'bewitched people to

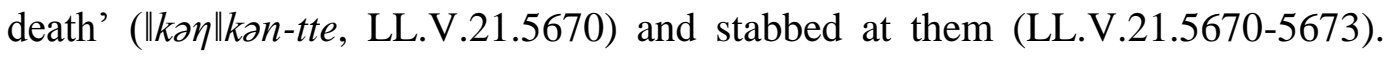
See lko: $\xi_{0} \xi$-de, !gi:, !gi:xa.

\|khã:; $n$. lion (Bleek, 1956:572). $\| k w a \xi m m a$, noun lion, name used by early race

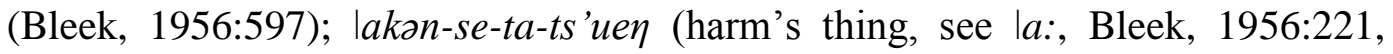
238), !kui (for many lions together), !ku-tu-|i, a lion with great paws (LL.II.30.2754'). Lions were the premier 'beast-of-prey' in IXam thought, both in physical characteristics (see !kúi-se-!khwi-|ku; lions with black hair on their tails were 'fearful', LL.II.22.1949-1951) and temperament: they were the quintessential 'angry people' (LL.II.20.1775). This anger governed their treatment in contemporary practice, where they were referred to obliquely (as in the 'harm's thing', above); children were not supposed to mention the name of lions ( $\| k h \tilde{a}:)$, instead calling them 'Hair' (|kukən, Bleek, 1956:323) in an attempt to avoid drawing their attention (LL.V.8.4563-4568). Lions that killed people displayed a lack of understanding (LL.VIII.23.8074'), and they would even behave cannibalistically, killing and eating one another (LL.II.16.1551'). This characterisation of their behaviour (as |gebbi) determined their common depiction in Early-Race narratives, where they took on a buffoonish quality somewhat reminiscent of Ikaggan; as they went about 'seeking fights' they (usually 
inadvertently) managed to cause themselves a great deal of trouble (see !gu, !haue-ta-łhou). Their problems in Early-Race narratives were often caused by their inappropriate attitudes when dealing with food: eating each other's flesh (LL.II.2.314-315), refusing to share (WB.XIV.1367-1368), or swallowing down food (LL.II.1.256'-258') all caused them difficulties. See \|ke\|ke, !gu, !haue-tałhou, !gwaĩ, koro, !kúi-se-!khwi-lku.

\|khã:-ka-mumu; 'spectre lion'. \|khãa, $n$. lion (Bleek, 1956:572); -ka, poss. part. following possessor, preceding the thing possessed, often omitted before words denoting relationship or parts of the body, also written -ga, -ta (Bleek, 1956:74); momo, $n$. court round the moon (lunar rainbow) (Bleek, 1956:138); mumu, $n$. halo, ghost, spectre (Bleek, 1956:139); !kh'o, n. a blue mist, haze (Bleek, 1956:427); !ho:, $n$. mirage (?) (Bleek, 1956:397). This highly idiosyncratic term (mentioned only once by $\|$ kabbo, WB.XXIII.2190') probably denoted an optical illusion of some kind. Though the Dictionary definitions do not identify mumu and momo with precision, they seemingly connoted shimmering, hazy, or miragelike visual effect: the 'court around the moon' (probably a $22^{\circ}$ halo or a corona) was supposed to be the hazy dust raised by a dancing party, while mumu also formed part of the name of the will-o'-the-wisp (see !gwe:-!kweitən-tu). Mists and hazes were sometimes given an antagonistic character, carrying illness to kill people (LL.V.20.5551-5561); this 'mistiness' would thus be in keeping with the anti-social nature of lions, which could also manifest another form of visual deception with their |hulhunta (n. image (?) shadow (?) reflection (?), Bleek, 1956:291) that came into sight before lion itself arrived (LL.VIII.6.6576'; this may 
represent lhanłkass'o's conception of the 'spectre lion'). See !khã:, !gwe:!kweitən-tu.

\|khwai-hem; $n$. name of the father of the porcupine, perhaps All-devourer (Bleek, 1956:578); \|khwaik, v. to chew (Bleek, 1956:578); hem, v. to devour, consume (Bleek, 1956:59). The name of this monstrous Early-Race character was usually rendered as 'All-Devourer': he was the 'devourer' or 'eater-up' of things (WB.2162-2163), also referenced in his alternative name, !kautu-!kui:ja (stomach-great, Bleek, 1956:416, 450), translated into Afrikaans by the informant as dik-pens (thick-stomach, LL.II.34.3118'). He spoke in a distinctive manner, giving characteristic exclamations such as !kabba: (Bleek, 1956:402) and !bubbu (WB.XXIII.2160'). He was the 'biological' father of |kaggən's adopted daughter (the Porcupine), and consequently appeared in narratives concerning Ikaggən's doings; he was usually introduced as part of a contiguous extension of 'The Mantis takes the Tick’s Sheep' stories (see !ku!ku:itən), as Ikaggən invited him to partake of the stolen livestock. As his name suggests, this character demonstrated an unparalleled capacity for 'swallowing things down'; his visits led to him first consuming the sheep |kaggan had provided, and eventually to his eating the trees, bushes, pots, and people (LL.II.34.3139-3141). He was affiliated with fire, making a large shadow that resembled that of clouds, putting out a long tongue that 'swallowed in' the trees, and where he touched with this tongue was 'like fire’ (LL.II.32.2950'-2951'). See !nu!numma-!kwitən.

\|kwakka $(\| k w a \xi k k a n) ; \quad v$. to understand, be wise, clever, cunning (Bleek, 1956:596); "k"wakka, v. to understand, do understandingly (Bleek, 1956:609); ki:- 
se, v. to teach or advise (Bleek, 1956:93) $\| k h o u, v$. to instruct (Bleek, 1956:577); $\|x a:\| x a:$ v. to teach, learn (Bleek, 1956:634); $\| k a m m a, v$. to counsel, advise,

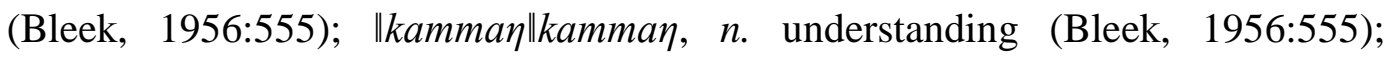
$d d a b b-i$ to educate, make non-foolish (LL.VIII.26.8308'). The notion of 'understanding' or 'doing understandingly' was crucial to IXam constructions of adult responsibility; it was inculcated through tum: (to hear, listen, or understand, Bleek, 1956:241): this referred to receiving and paying attention to information, transmitted in kum (story, talk, history, news, Bleek, 1956:106). Actions that were done in an 'understanding' manner were subject to positive evaluations as $a: k a n$ or twai: $\tilde{l}$, which made reference to the skills required in their performance (this 'competence' aspect of $\| k w a k k a$ closely paralleled !khwi). It was antithetical to foolishness (|gebbi), a trait that was linked to childhood: as 'understanding' states were developed over time, they were naturally absent in younger people (who nonetheless displayed understanding with specific, circumscribed domains; even infants knew to look to their mother's breasts for food, LL.II.9.991). These actions took place in the context of the 'ordered' desirable social relationships that existed between adults who 'possessed their thinking-strings', and was particularly opposed to angry action (see Chapter 6a: 173, 175) . See a:kən, twai:ĩ, |gebbi, !khwi, \|kau\|kaugən.

$\| \mathbf{k}$ "ao:; v. to curse, $n$. cursing, predictions of evil (Bleek, 1956:603); $\| k^{\prime \prime} a u$; $\left\|k^{\prime \prime} a u\right\| k " a u, v$. to curse, $n$. cursing, predictions of evil (Bleek, 1956:604); $\| k^{\prime \prime}$ oakən, $v$. to curse (?), make unfortunate (?), $n$. badness (?) (Bleek, 1956:606); $¥ k^{\prime \prime} a \xi k k a n$, $\neq k^{\prime \prime} a \xi k k a, \neq k^{\prime \prime} a \xi \neq k^{\prime \prime} a \xi b b a \xi k \partial n, v$. to scold, curse, speak angrily (Bleek, 1956:668); 


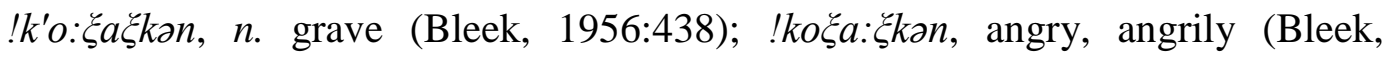
1956:438); !k"au:gən, n. death news, misfortune, death influence (Bleek, 1956:508); !k"aukan, $k^{\prime \prime} a u: k a, v$. possibly to mourn, or to be under the death influence of (Bleek, 1956:508); !k"auokən, n. destruction (Bleek, 1956:508); $\| a u:$, v. to curse (Bleek, 1956:518); \|kha, v. to curse, used as an exclamation of anger, fie! (?) (Bleek, 1956:572); $\left\|k^{\prime \prime} a o \xi w a,\right\| k^{\prime \prime} a \|^{\prime \prime} a o$, bitter, bitterness (Bleek, 1956:603). This collection of terms loosely grouped around 'cursing' formed a domain of anti-social interaction comparable with 'fighting' ( $\mid a$ :, which was itself sometimes translated as 'curse', Bleek, 1956:267), and like fighting, cursing was a type of 'angry action': people cursed a person when his or her actions were 'not agreeable' (LL.V.24.5913'). It usually referred to less-tangible forms of assault; the New Maiden could 'curse' people, causing !khwa: to strike at them with lightning (LL.V.13.4987-4988) or causing ! $k$ "abbe to 'get into their flesh' (LL.V.20.5608-5609), and the 'curse' of the baboon and hyena killed by the hunter would sit in his bow (unless incisions were made in it, LL.V.24.59125915). The IXam's 'modes of cursing' commonly drew on idioms of violent, homicidal death (LL.VIII.31.8741-8743), and related 'cursed' states could be induced by close contact with death; if a companion had died, springbok hunting would become unfortunate because of this 'death influence' (LL.VIII.14.72817282). It was through the medium of 'cursing' that the Early-Race times came to end; when the aardvark and the caracal started to 'curse' on another in their conflict over the springbok-child, they instigated the process of defining their 
bestial qualities and codified their new status as animals (LL.VIII.20.7593'; LL.VIII.29.8600). See la:,

$\| x e: ; ~ n . ~ c h r y s a l i d e s$ of ants, 'Bushman rice' (Bleek, 1956:635). This term definitely denoted a species of eusocial insect, but the term 'ant' is sufficiently ambiguous in colloquial epithets to render species identifications difficult. Reflecting convergence on a eusocial lifestyle, the phrase 'white ant' remains a common gloss for termite species despite the considerable differences between true ants and termites: Marais (1937) refers to the termite as the 'white ant', and in the original Afrikaans (Marais, 1934), it lacked even the descriptive epithet. With respect to the translation of 'Bushman rice', the most important of these differences is that termites (unlike ants) are hemimetabolous; they do not pupate and references to the 'chrysalides of ants' must be erroneous if these 'ants' are, in fact, termites. !hakən, a similar resource, was also translated as 'chrysalides of ants', and as this term almost certainly referred to Trinervitermes sp. (Chapter 7c:254), $\| x e$ : likely also represented a termite species: \|kabbo noted that it was consumed by aardvarks (LL.II.2.338-339) and bat-eared foxes (LL.II.3.471). These species favour harvester termites (Hodotermitidae), but it is not possible to confirm the species on these grounds, as these mammals also consume other termite species as well as ants (Taylor, Lindsey and Skinner, 2002; Clark, 2005). The term Ikoro evidently referred to the emerging reproductive flying adults, and was applied to a range of insects with similar behaviours: Lloyd found it difficult to ascertain the 'proper name' of these insects (LL.II.37.3344'). 
$\ddagger$ gou $(\ddagger g o u w a) ; v$. to be silent, quiet, at peace, often used as adv., $n$. $\ddagger g a u w a$, peace (Bleek, 1956:648); kwe:, v. to be quiet, be still, leave, let alone, often used as quietly (Bleek, 1956:111) [LL.1630, 1636]; s'-up, still, silent (Bleek, 1956:174); $\| k o w a, v$. to be still (Bleek, 1956:589); $\| o u$, still, quiet (Bleek, 1956:626) $\ddagger g a u$, łgau:wa, v. to be gently, silent, (Bleek, 1956:645). This complex of terms combined notions of peacefulness in the sense of remaining still in mind and body with an opposition to 'angry action': the 'angry' foolish |kaggen fought the Cat, who was still or at peace (LL.II.22.1986), and being 'still' was placed in opposition to angry scolding (LL.II.25.2264'). This opposition to anger underpinned a strongly positive, socially desirable aspect to these characteristics, which were also signifiers of 'comfortable' conditions: when the clouds were 'shady' rather than 'dark' (which made the bushes green), it was łgouwa rather than 'a fight' (LL.II.24.2226), and chasing off lions allowed people to walking in peace on 'good' ground (LL.II.17.1619-1621). See Dakrrakən, kerru.

łho; touchingly (?) (Bleek, 1956:651). This word was used extremely infrequently; Lloyd's reverso note (LL.V.6.4348') glosses it as 'play[ing] so that one got haartsair, was verdriet.' The latter two terms represent the Afrikaans, hartseer (lit. heart-sore) and verdriet (grief, sadness); D. Bleek (1956:651) has only this sentence as an example, and suggests the term may have referred specifically to the ability to play the $\neq k u m m i$ instrument well. The ability to make people 'heart-sore' was commonly encountered as a constituent of relationships between Early-Race characters, where it motivated individuals to attempt to force others to live with them (LL.V.6.4350). See twai:ĩ. 
łkwerre; $n$. a bad shot, a person who shoots badly (Bleek, 1956:667). This term referred to a method dealing with resources; Ihanłkass'o used it to discuss the correct way to treat locusts (LL.VIII.7.6671-6672). He used the phrase $\neq k w e ́ r r e t z n-d d i$, a 'thing that shoots badly' and provided a further clarification that it described a man who was swak (Afr. weak, infirm, delicate, feeble): Lloyd rendered this as a 'weakling' or 'feebleminded'. It was contrasted with $\ddagger x i-\ddagger x i$ (sensible thing/strong), a term not present in the Dictionary: it was possibly a reduplication of $\ddagger x i$;, to shoot (Bleek, 1956:680), while the notebook translation (contrasting sense and 'feeblemindedness') implies that it may have been a variation on $\neq \tilde{\imath}$ (thoughts, Bleek, 1956:652). The reverso note (LL.VIII.7.6671') gives it as an equivalent to $\mid g i-g i$, (strong Bleek, 1956:270), and the pair of terms can usefully be combined with $\mid$ kerre-gwai and $\mid i$ :-sa as part of the construction of competent, admirable identities. See Ikerre-gwai, li:-sa.

łnuturu; $n$. weevil, Cleonis glacialis (?) (Bleek, 1956:674). The łnuturu was the focus of a single Early-Race narrative (LL.VIII.9.6786-6857); she was a singularly attractive person described with the term $\left\|_{w a}\right\|_{w a t}$ (to be beautifully white, Bleek, 1956:630; LL.VIII.9.6800). In consequence of her great beauty, the returning hunters gave her the fat springbok breasts, withholding these choice morsels from their wives (LL.VIII.9.6802-6804); after her ruse was discovered (the antennae she removed when the hunters were approaching), they only gave her 'meat scraps' to gnaw at, repenting of their former generosity (LL.VIII.9.6814-6815). The hunters' earlier behaviour was criticised as foolish, childish behaviour (LL.VIII.9.6857), and one of the themes of this story thus 
revolved around the correct 'understanding' behaviour to be deployed in conjugal relationships.

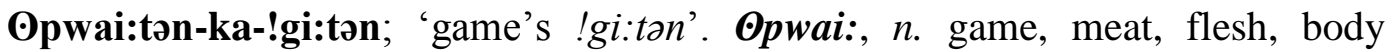
(Bleek, 1956:685); -ka, poss. part. following possessor, preceding the thing possessed, often omitted before words denoting relationship or parts of the body, also written -ga, -ta (Bleek, 1956:74). These !gi:tən were people supposed to possess powers influencing the movement or presence of certain resources; primarily these were larger animals such as bovids (springbok's !gi:tən, LL.V.10.4739') and ostriches (LL.V.10.4778), but locusts too were 'sent about' by !gi:tan (LL.V.21.5719; LL.V.22.5720). Their powers continued after death, when they became 'spirit people' who could be entreated to send game, beating a stone on the ground and reminding them of their social obligations (LL.V.11.4801'-4806'). See !gi:xa. 


\section{Appendix E}

\section{Tables of IXam terms}

The following tables collate IXam terminology for the fauna, flora, and mineral resources with which the informants were familiar. The tables primarily reference the notebooks themselves, with Dictionary references providing explanatory supplements where appropriate. The tables are intended to provide modern species identifications for the biota recognised and divided in IXam taxonomies, as much of the information recorded by Bleek and Lloyd on this topic is scattered throughout the archive, and makes reference to obsolete scientific names. Some species (particularly plants) cannot be securely assigned to species or even genus; in these cases the data presented by the informants is included in a summarised form, with appropriate notebook references being provided. 
Table 1: IXam terms for mammals, with scientific and common names

\begin{tabular}{|c|c|c|c|}
\hline Common Name & Scientific Name & IXam name, reference & Further notes \\
\hline \multicolumn{4}{|c|}{ Bovidae } \\
\hline Buck & $N \mid A$ & !koen (WB.XXV.2384) & Generic term for buck \\
\hline Bushbuck & Tragelaphus scriptus & !koen (WB.II.366) & $\begin{array}{c}\text { Named at museum (?the } \\
\text { generic term) }\end{array}$ \\
\hline Cattle & $\begin{array}{l}\text { Bos primigenius taurus } \\
\text { (various subspecies) }\end{array}$ & $\begin{array}{c}\text { Xoro (LL.VIII.1.6049; Bleek, } \\
\text { 1956: 260) }\end{array}$ & |ka:xa, bull (Bleek, 1956:305) \\
\hline ?Common Duiker & ?Sylviacapra grimmia & Inãu, !khi (LL.II.2.291) & $\begin{array}{l}\text { "Duiker"!ka:, duiker buck } \\
\text { (Bleek, 1956:401) }\end{array}$ \\
\hline Eland & Taurotragus oryx & $\begin{array}{c}\text { Zza, ssa (WB.II.366'; } \\
\text { LL.VIII.1.6047) }\end{array}$ & $\begin{array}{c}\text { Women use } \neq k o u k a n-k h u r u \\
\text { (LL.VIII.27.8435) }\end{array}$ \\
\hline Gemsbok & Oryx gazella & $\begin{array}{l}\text { !kwai, !khwai (LL.II.2.291; } \\
\text { Bleek, 1956:431) }\end{array}$ & \\
\hline Goat & Caprus hircus & Burri (LL.VIII.1.6048) & \\
\hline Grysbok & $\begin{array}{c}\text { Raphicerus }(=\text { Calotragus }) \\
\text { melanotis }\end{array}$ & !koen (WB.XXV.2382) & $\begin{array}{l}\text { Named at museum (?the } \\
\text { generic term) }\end{array}$ \\
\hline Hartebeest & Alcelaphus buselaphus caama & $\begin{array}{c}! k w a \\
\text { (LL.VIII.1.6047;LL.VIII.7838) }\end{array}$ & $\begin{array}{l}\text { Women use } \| \text { kuerritan } \\
\text { (LL.VIII.27.8435) }\end{array}$ \\
\hline Klipspringer & Oreotragus oreotragus & $\| k o$ (LL.I.2.186; Bleek, 1956:582) & \\
\hline
\end{tabular}




\begin{tabular}{|c|c|c|c|}
\hline Kudu & Tragelaphus strepsiceros & $\begin{array}{l}\text { !xau (LL.VIII.1.6047), ! kau } \\
\text { (LL.II.2.288) }\end{array}$ & $\begin{array}{c}\text { !nshá, } n . \text { kudu (Bleek, } \\
\text { 1956:486) }\end{array}$ \\
\hline Rhebok & Pelea capreolus & $\begin{array}{l}k h i \text { (LL.VIII.21.7839); } d z z a \\
\text { (LL.VIII.1.6047) }\end{array}$ & \\
\hline Sheep & Ovis aries & $\begin{array}{l}\text { !gei (LL.VIII.1.6048; Bleek, } \\
\text { 1956:381) }\end{array}$ & \\
\hline Sheep (different breed) & Ovis aries & !koa (LL.VIII.1.6048) & $\begin{array}{c}\text { 'Vaderland' or 'moff' breed } \\
\text { (Bleek, 1956:508) }\end{array}$ \\
\hline Springbok & Antidorcas marsupialis & $\begin{array}{l}\text { Whai,(LL.VIII.1.6047), } \| k a k a n \\
\text { ('other' name, LL.VIII.27.8432') }\end{array}$ & $\begin{array}{l}\text { !gwara!gwara, a white } \\
\text { springbok (Bleek, 1956:391); } \\
\text { other name used by all }\end{array}$ \\
\hline Steenbok & Raphicerus campestris & $\begin{array}{c}\text { !koin, !koen (LL.VIII.1.6047; } \\
\text { Bleek, 1956:439) }\end{array}$ & Named at museum (?generic) \\
\hline Black Wildebeest & Connochaetes gnou & $\begin{array}{c}\text { !nu (WB.I.189), !kou-|ko } \\
\text { (LL.VIII.1.6051) }\end{array}$ & $\begin{array}{l}\text { !aulko blue wildebeest } \\
\text { (Bleek, 1956:372) }\end{array}$ \\
\hline Blue Wildebeest & Connochaetes taurinus & $\begin{array}{c}\text { !nu (WB.I.189), !kou-|ko } \\
\text { (LL.VIII.1.6051) }\end{array}$ & $\begin{array}{l}\text { !nu, black wildebeest (Bleek, } \\
1956: 483)\end{array}$ \\
\hline \multicolumn{4}{|c|}{ Other artiodactyla (Giraffidae, Hippopotamidae, Suidae) } \\
\hline Giraffe & Giraffa camelopardalis & !nui (LL.II.2.288) & \\
\hline Hippopotamus & Hippopotamus amphibius & $\lg a($ LL.VIII.21.7837) & \\
\hline Pig & ?Sus scrofa & $\begin{array}{c}\text { Haggu (LL.II.1.34), Iho (Bleek, } \\
\text { 1956:288) }\end{array}$ & $\begin{array}{c}\text { ?Cape warthog Phacochoerus } \\
\text { aethiopicus aethiopicus }\end{array}$ \\
\hline \multicolumn{4}{|c|}{ Perissodactyla (Rhinocerotidae, Equidae) } \\
\hline Horse & Equus ferus caballus & $\begin{array}{c}\text { Barra (LL.VIII.1.6048), hara } \\
\text { (LL.IV.1.3448) }\end{array}$ & $\begin{array}{l}\text { !ai:tan, white-blazed horse } \\
\text { (Bleek, 1956:370) }\end{array}$ \\
\hline Quagga & Equus quagga quagga & Ddeou, $\| k^{\prime \prime} w i$ (LL.VIII.21.7837) & $\begin{array}{c}\| k w i:, n . \text { quagga (Bleek, } \\
1956: 600)\end{array}$ \\
\hline
\end{tabular}




\begin{tabular}{|c|c|c|c|}
\hline Rhinoceros & $\begin{array}{c}\text { Diceros bicornis/ } \\
\text { Ceratotherium simum }\end{array}$ & $\begin{array}{c}\text { \|xuagan, \|kuagan (Bleek, } \\
\text { 1956:638) }\end{array}$ & $\begin{array}{l}\text { Historically, both species } \\
\text { were present. }\end{array}$ \\
\hline Zebra & Equus quagga burchelli & $\begin{array}{c}\text { Douw, } \| k^{\prime \prime} u i, \mid h a b b a \\
\text { (LL.VIII.21.7837) }\end{array}$ & $\begin{array}{c}\text { |habba: striped quagga, zebra } \\
\text { (Bleek, 1956:286) }\end{array}$ \\
\hline \multicolumn{4}{|c|}{ Primates (Cercopithedcidae) } \\
\hline Baboon & Papio ursinus ursinus & $\begin{array}{c}\text { |hu|hu (LL.7836); } \| x e t t a n \\
\text { 'unusual name' (Bleek, } \\
\text { 1956:635) }\end{array}$ & $\begin{array}{l}\text { 'The people who sit on their } \\
\text { heels' (LL.VIII.11.6978), } \\
\text { euphemistic term }\end{array}$ \\
\hline Vervet Monkey & Chlorocebus pygerythrus & $\begin{array}{c}\| k^{\prime \prime} \text { warre (LL.VIII.18.7615'; } \\
\text { Bleek, 1956:609) }\end{array}$ & $\begin{array}{l}\text { Primate with a long tail, } \\
\text { smaller than baboon }\end{array}$ \\
\hline \multicolumn{4}{|c|}{$\begin{array}{c}\text { Carnivora }- \text { Felidae } \\
\end{array}$} \\
\hline $\begin{array}{l}\text { Cheetah (Hunting pard, } 19^{\text {th }} \\
\text { Century term) }\end{array}$ & Acinonyx jubata & $\begin{array}{c}\mid k w a \eta-x u, \text { and variants } \\
\text { (WB.XXV.2409) }\end{array}$ & $\begin{array}{c}\text { Men use } ! k^{\prime \prime} \text { auru (Bleek, } \\
1956: 508)\end{array}$ \\
\hline Leopard & Panthera pardus & $\begin{array}{c}\| \text { kaue, and variants } \\
\text { (LL.VIII.1.6047) }\end{array}$ & \\
\hline Lion & Panthera leo & $\begin{array}{c}\| k h \tilde{a}: \text { (LL.VIII.1.6047), lakən-ta- } \\
\text { tcheun ('harm's thing) } \\
\text { (LL.II.30.2754') }\end{array}$ & $\begin{array}{c}k u-t u, \text { 'great pawed' lion } \\
\text { (LL.II.30.2754') Early race } \\
\text { call } \| k w a m m a \text { (Bleek, } \\
1956: 597)\end{array}$ \\
\hline Caracal (Lynx, Rooicat) & Caracal caracal & $\begin{array}{c}\mid k u-! n w a i n, ! k u-g-! n w i n \\
\text { numerous variants (LL.II.2.290) }\end{array}$ & $\begin{array}{l}\text { "Name of the female lynx" } \\
\text { (Bleek, 1956:439) }\end{array}$ \\
\hline Wild Cat & Felis silvestris cafra & $\begin{array}{l}\ddagger g \text { watten, varying initial click } \\
\text { (LL.II.2.290) }\end{array}$ & $\begin{array}{l}\text { May refer to Felis nigrepes } \\
\text { (Black-footed cat) }\end{array}$ \\
\hline \multicolumn{4}{|c|}{ Carnivora - Canidae } \\
\hline Bat-eared Fox & $\begin{array}{c}\text { Otocyon megalotis }(=\text { caffer } \\
\text { lalandi) }\end{array}$ & $\begin{array}{c}\| u a \text { (LL.VIII.21.7817), } \| " o a \\
\text { (WB.XXV.2425) }\end{array}$ & $\begin{array}{l}\text { Delalande's fox, Loffelhund } \\
\text { (Bleek, 1956:629). Often } \\
\text { translated as \|aitchies }\end{array}$ \\
\hline
\end{tabular}




\begin{tabular}{|c|c|c|c|}
\hline & & & (LL.II.21.1880) \\
\hline Black-backed Jackal & $\begin{array}{l}\text { Canis mesomelas }(= \\
\text { variegatoides })\end{array}$ & $\begin{array}{c}\text { Koro (WB.XXV.2381), !gwiton } \\
\text { and variants (LL.VIII.21.7817) } \\
\| k w a(\text { WB.I.290) }\end{array}$ & $\begin{array}{c}\text { Bleek (1956:101) gives koro } \\
\text { as 'official', but evidently } \\
\text { some overlap in use }\end{array}$ \\
\hline $\begin{array}{l}\text { Cape Fox, Caama Fox } \\
\text { (Silver Jackal) }\end{array}$ & Vulpes chama & $\begin{array}{l}\text { !gwitan and variants (Bleek, } \\
1956: 393)\end{array}$ & $\begin{array}{l}\text { "Crosser of the spoor" } \\
\text { (WB.XXIII.2157') }\end{array}$ \\
\hline Dog & Canis lupus familiaris & !khuin (LL.II.2.291), !kuin & \\
\hline Wild Dog & Lycaon pictus & $\begin{array}{l}\text { |xerru (LL.VIII.21.7837) !gwitan } \\
\text { (LL.VIII.25.8216) }\end{array}$ & $\begin{array}{c}\text { Bleek (1956:363) gives as } \\
\text { |xarru }\end{array}$ \\
\hline \multicolumn{4}{|c|}{$\begin{array}{c}\text { Carnivora - Hyaenidae } \\
\end{array}$} \\
\hline Aardwolf & Proteles cristatus & $\mid k u($ Bleek, 1956:322) & Also |gipp, gipp \\
\hline Brown Hyena & Hyaena brunnea $(=$ villosa $)$ & $\begin{array}{l}\text { !gwai (LL.II.2.288) !goai } \\
\text { (WB.II.367') }\end{array}$ & $\begin{array}{c}\text { Bleek (1956: 391): 'Strand' } \\
\text { or 'Hairy' Hyena; "Black- } \\
\text { haired” Hyena }\end{array}$ \\
\hline Spotted Hyena & Crocuta crocuta $(=$ maculata $)$ & $\begin{array}{c}\text { !kau-!(k)annu, and variants } \\
\text { (LL.II.2.289) \|naui and variants } \\
\text { (Bleek, 1956:617) }\end{array}$ & \\
\hline "Striped Hyena" & Hyaena hyaena & $\begin{array}{c}|k u(\mathrm{WB} . X X V .2425)| g i p p \\
\text { (WB.II.367) }\end{array}$ & $\begin{array}{l}\text { Striped hyena proper is not } \\
\text { found in South Africa; the } \\
\text { term refers to the aardwolf }\end{array}$ \\
\hline \multicolumn{4}{|c|}{ Other small carnivora (Musteloidae, Viverridae, Herpestidae) } \\
\hline $\begin{array}{l}\text { Egyptian Mongoose } \\
\text { (Ichneumon) }\end{array}$ & Herpestes ichneumon & lni: (LL.II.27.2425) & \\
\hline Meerkat & $\begin{array}{c}\text { Suricata suricatta suricatta } \\
\text { (=zenick) }\end{array}$ & $\begin{array}{l}\text { !kao (LL.VIII.21.7817); !hai } \\
\text { (LL.VIII.21.inset) }\end{array}$ & $\begin{array}{c}\text { xa:ra, mierkat (Bleek, } \\
1956: 257)\end{array}$ \\
\hline ?Mongoose & $\begin{array}{l}\text { Description suggests Suricata } \\
\text { suricatta; 'Bushy-tailed }\end{array}$ & |hai (WB.1.191) & $\begin{array}{c}\text { Sits in small hole, eats } \\
\text { termite eggs, stands on two }\end{array}$ \\
\hline
\end{tabular}




\begin{tabular}{|c|c|c|c|}
\hline & meerkat' Cynictis penicillata & & legs, runs on four (WB.I.191) \\
\hline ?Genet & $\begin{array}{c}\text { Genetta genetta most likely } \\
\text { species }\end{array}$ & $\begin{array}{l}\text { !nuerre and variants } \\
\text { (LL.VIII.2.6144) }\end{array}$ & $\begin{array}{l}\text { Smaller than a cat, with a } \\
\text { pointed face, long tail, and } \\
\text { spots: "tiger-cat", genet } \\
\text { (WB.II.367') }\end{array}$ \\
\hline Ratel (Honey Badger) & Mellivora capensis & !khou (LL.VIII.21.7817) & $\begin{array}{l}\text { ?Name derives from 'honey' } \\
\text { (favoured food) }\end{array}$ \\
\hline Yellow Mongoose & Cynictis penicillata & $\begin{array}{l}\text { !hai (LL.VIII.21.7817), !kau } \\
\text { (LL.II.2.289) }\end{array}$ & $\begin{array}{c}\text { Bushy-tailed meerkat (Bleek, } \\
1956: 394)\end{array}$ \\
\hline Zorilla (Muishond) & Ictonyx (= Zorilla) striatus & $\begin{array}{l}k w^{\prime} a \text { and variants } \\
\text { (LL.VIII.21.7817) }\end{array}$ & Striped Muishond, polecat \\
\hline \multicolumn{4}{|c|}{ Lagomorpha and Rodentia } \\
\hline Hare & $\begin{array}{c}\text { Lepus sp. (Cape Hare, Lepus } \\
\text { capensis; Shrub hare, Lepus } \\
\text { saxatilis) }\end{array}$ & $\begin{array}{c}\ddagger n a b b e, \neq n a u(\text { WB.I.249) |"oa } \\
\text { (LL.II.2.290) }\end{array}$ & $\begin{array}{c}\text { 'White-tailed' } \\
\text { (WB.XXV.2383) Same term } \\
\text { for 'rabbit' (LL.II.2.290) }\end{array}$ \\
\hline Hare & Not Given & $\operatorname{lnu-xa(\text {WB.I.192)}}$ & "Great hare" (LL.II.4.448) \\
\hline Cape Porcupine & Hystrix africaeaustralis & $\begin{array}{c}! k o, \text { and variants } \\
(\text { LL.II.33.3043'), \|gauxo, and } \\
\text { variants (WB.XXV.2383) }\end{array}$ & $\begin{array}{c}\text { Girls use } \mid \text { khogan } \\
\text { (LL.V.6.4377')!koa } \\
\text { (LL.VIII.2.6199'), 'used by } \\
\text { all' }\end{array}$ \\
\hline Fieldmouse & Not Given & $\begin{array}{c}k^{\prime \prime} \text { waitan, } ! k h w i \text { (Bleek, } \\
1956: 340,433)\end{array}$ & \\
\hline $\begin{array}{c}\text { Four Striped Grass } \\
\text { Rat/Mouse }\end{array}$ & Rhabdomys (= Mus) pumilio & $\|$ khou (LL.VIII.21.7836) & $\begin{array}{c}\text { "Common striped field } \\
\text { mouse" }\end{array}$ \\
\hline Mice & Not Given & $\begin{array}{l}\text { Ikonde (Bleek, 1956:319; } \\
\text { LL.VIII.21.7829) }\end{array}$ & Applied to mice in general \\
\hline Mouse or shrew & Not Given & !kabbe (Bleek, 1956:403) & Species unclear \\
\hline
\end{tabular}




\begin{tabular}{|c|c|c|c|}
\hline $\begin{array}{c}\text { South African Ground } \\
\text { Squirrel }\end{array}$ & Xerus (= Sciurus) inauris & $\begin{array}{l}\text { |kwerre, } \| \text { ken-|khu } \\
\text { (LL.VIII.2.6144) }\end{array}$ & $\begin{array}{l}\| k e n-\mid k h u=\text { "digger of } \\
\text { cucumber" }\end{array}$ \\
\hline Springhare & Pedetes capensis $(=$ cafer $)$ & $\ddagger g o$ (LL.VIII.21.7817) & \\
\hline & Not Given & !ne-sara (LL.V.4.4235') & 'Small mouse' (?shrew) \\
\hline \multicolumn{4}{|c|}{ Other small mammals (Macroscelidea, Soricomorpha, etc.) } \\
\hline $\begin{array}{c}\text { Cape Elephant Shrew (Cape } \\
\text { Sengi) }\end{array}$ & $\begin{array}{c}\text { Elephantulus } \\
\text { (=Macroscelides) edwardii }\end{array}$ & $\begin{array}{l}\text { lebbukan (WB.XXV.2341) |kiten- } \\
\text { koro (LL.VIII.21.7828') }\end{array}$ & $\begin{array}{c}\text { A mouse, also called } \\
\text { lebbukan (Bleek, 1956:316) }\end{array}$ \\
\hline Mole & $\begin{array}{l}\text { ?Chrysochloridae 'golden } \\
\text { mole') sp. }\end{array}$ & $\| k h u($ LL.VIII.14.7216') & $\begin{array}{c}\text { Found at Mowbray, not in } \\
\text { Bushmanland }\end{array}$ \\
\hline Short-eared Elephant Shrew & $\begin{array}{c}\text { Macroscelides proboscideus } \\
\text { (=typicus) }\end{array}$ & $\begin{array}{c}\text { !hennixa (WB.XXV.2342; Bleek, } \\
\text { 1956:397) }\end{array}$ & "African Jumping Shrew" \\
\hline Shrew & Sorex $\mathrm{sp}$ & !kabbe (LL.II.26.2341) & \\
\hline \multicolumn{4}{|c|}{ Miscellaneous (Procaviidae, Elephantidae, Orycteropodidae, Chiroptera) } \\
\hline $\begin{array}{c}\text { Aardvark (Cape Anteater; } \\
\text { Antbear) }\end{array}$ & Orycteropus afer (= capensis) & $\begin{array}{c}k u k e n-t u \text { and variants } \\
\text { (LL.VIII.21.7836), } \neq \text { go-xumm } \\
\text { ('other name’, all use, } \\
\text { LL.VIII.16.7432') }\end{array}$ & $\begin{array}{l}\text { Shares a special name (hurru- } \\
\text { si-tuturriten) with a bird } \\
\text { (Bleek, 1956: 65); }\end{array}$ \\
\hline Bat & $\begin{array}{c}\text { Not Given (several potential } \\
\text { species) }\end{array}$ & \|khettan (LL.V.6.4378) & $\begin{array}{c}\text { Bleek,(1956:385): !gogan; } \\
\text { usually Uxettan }\end{array}$ \\
\hline Elephant & Loxodonta africana & $\neq x o a$ and variants (LL.II.2.288) & \\
\hline Hyrax, Dassie & Procavia capensis & |kun, |hun (Bleek, 1956:290) & $\begin{array}{c}\text { !árroxu, !árruxu, !kauru-wo } \\
\text { (Bleek, 1956:371, 416; } \\
\text { "Mantis' wife") }\end{array}$ \\
\hline \multicolumn{4}{|c|}{ Ambiguous information/generic categories } \\
\hline ?Gemsbok & Not Given & $\mid k w a i$ (LL.II.1.205') & $\begin{array}{l}\text { Chiansbok - 'horns turn } \\
\text { back' (mishearing/odd } \\
\text { pronunciation of gemsbok?) }\end{array}$ \\
\hline
\end{tabular}




\begin{tabular}{|c|c|c|c|}
\hline ?Smith's Red Rock Hare & ?Pronolagus rupestris & $\ddagger$ nabbe (Bleek, 1956:669) & "White-tailed rock rabbit" \\
\hline Not Given & Not Given & $\mid$ kaulkarru (WB.I.191) & Cat-sized, black hair \\
\hline Not Given & Not Given & $\|$ kain-|kui, $\mid$ kuarrei (WB.I.193) & $\begin{array}{c}\text { Small; white underbelly, } \\
\text { 'green' back, white tail }\end{array}$ \\
\hline Beast-of-prey, wild beast & $N \mid A$ & $\|k h e\| k h e$ (Bleek, 1956:571) & Generic term for predators \\
\hline
\end{tabular}


Table 2: IXam terms for birds, with common and scientific names

\begin{tabular}{|c|c|c|c|}
\hline Common name & Scientific name & IXam name with reference & Further notes \\
\hline \multicolumn{4}{|c|}{ Passerines } \\
\hline $\begin{array}{l}\text { Crow, 'Korenland Kraai' } \\
\text { (Afr. corn-country crow) }\end{array}$ & Corvus segetum & \|hoe (LL.VIII.21.7842) & $\begin{array}{l}\text { Applied in museum to } \\
\text { Australian Raven } \\
\text { (WB.XXV.2350) }\end{array}$ \\
\hline Pied Crow & Corvus albus (= scapulatus) & !kagən (LL.VIII.2.6142) & $\begin{array}{c}\text { "Bonte [Afr. spotted] kraai" } \\
\text { (Bleek, 1956:403) }\end{array}$ \\
\hline White-necked Raven & Corvus albicollis & $\| x u r u$ (LL.VIII.21.7842) & $\begin{array}{l}\text { Applied in museum to } \\
\text { Common Raven } \\
\text { (WB.XXV.2350) }\end{array}$ \\
\hline ?Rook & Not Given & $\begin{array}{l}\text { !kwarra-k"anni } \\
\text { (WB.XXV.2342) }\end{array}$ & $\begin{array}{l}\text { Bleek (1956:461) gives as } \\
\text { rook or 'black bird'; see } \\
\text { below. }\end{array}$ \\
\hline Shrike & Lanius collaris & $\begin{array}{c}\text { Kuru-\|kaitau (LL.VIII.2.6143; } \\
\text { WB.I.191) ?Kuru-\|kaitən } \\
\text { (LL.VIII.23.8072) }\end{array}$ & $\begin{array}{l}\text { Butcher Bird, Fiscal. Latter } \\
\text { part of name means to climb } \\
\text { or ascend (Bleek, 1956:553) }\end{array}$ \\
\hline ?Shrike species & Not Given & !kain (LL.VIII.30.8668) & Resembles Lanius collaris \\
\hline ?Glossy Starling & $\begin{array}{c}\text { ?Lamprotornis phanciopteris; } \\
\text { Lamprotornis }(=\text { Juida })\end{array}$ & $\begin{array}{c}\text { !kwuea- } k^{\prime \prime} \text { erri } \\
\text { (WB.XXV.2349)!kuaro-k"ani }\end{array}$ & $\begin{array}{c}\text { "Koranna bird" } \\
\text { (WB.XXV.2349) k"ani or }\end{array}$ \\
\hline
\end{tabular}




\begin{tabular}{|c|c|c|c|}
\hline & australis & (LL.VIII.21.7842) & $\begin{array}{l}\text { k"erri,'bird'; !kwara, Korana } \\
\text { (Bleek, 1956:119, 461). }\end{array}$ \\
\hline ?Wattled Starling & Creatophora cinerea & !kerri (LL.VIII.10.6858) & $\begin{array}{c}\text { 'Locust Bird' (Bank, } \\
2006: 268)\end{array}$ \\
\hline Capped Wheat-ear & $\begin{array}{l}\text { Oenanthe }(=\text { Saxicola } \\
\text { Campicola) pileata }\end{array}$ & $\begin{array}{l}\text { Kuirri-kuirri (LL.VIII.21.7843) } \\
\text { |ko-ka-toitoi (LL.VIII.21.7842) }\end{array}$ & $\begin{array}{l}\text { 'To travel the sky running } \\
\text { gently' (Bleek, 1956: 207, } \\
\text { 317) }\end{array}$ \\
\hline ?Chat & $\begin{array}{l}\text { Saxicola castor. ?Karoo chat } \\
(\text { Cercomela schegelii })\end{array}$ & |ka-kau (LL.VIII.21.7843) & \\
\hline Grey-Backed Grass Warbler & Drymoica subrufica & $\|k h o u\| k h o u$ (LL.VIII.21.7843) & $\begin{array}{c}\| \text { khou, to fly (Bleek, } \\
1956: 576)\end{array}$ \\
\hline $\begin{array}{c}\text { Yellow-Backed (-breasted) } \\
\text { Bush Warbler }\end{array}$ & Drymoica flavida & $\begin{array}{l}\text { !kaukan!kaukan } \\
\text { (LL.VIII.21.7843) }\end{array}$ & $\begin{array}{c}\text { See Blue korhaan; !kaukən, } \\
\text { cry as a child, Bleek, } \\
1956: 414)\end{array}$ \\
\hline $\begin{array}{c}\text { ?Alario Finch } \\
\text { ?Black-headed Canary }\end{array}$ & $\begin{array}{c}\text { Crithologus and Amadina } \\
\text { alario (?Alario/Serinus alario) }\end{array}$ & $\begin{array}{l}\text { |konnin, |kuonnin } \\
\text { (WB.XXV.2349; }\end{array}$ & |kon-ni:n (Bleek, 1956:319) \\
\hline Sparrow & Not Given & 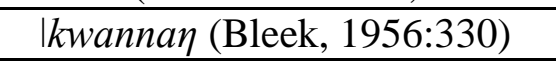 & Linked with above \\
\hline ?Weaver Bird & Not Given & $\neq k a i: n j a-t a r a$ (LL.VIII.21.7388) & $\begin{array}{l}\text { Small and yellow, numerous. } \\
\text { Lit. 'yellow-old woman' }\end{array}$ \\
\hline Bokmakierie (Bushshrike) & $\begin{array}{c}\text { Telophonus sp. (= Telophorus } \\
\text { sp.) }\end{array}$ & $\begin{array}{l}\text { !korokan!korokan } \\
\text { (LL.V.25.6014) }\end{array}$ & $\begin{array}{c}\text { !koro } \text {; to look afar off, or } \\
\text { backwards/upside down } \\
\text { (Bleek, 1956:443) }\end{array}$ \\
\hline Swallows & Not Given & $\begin{array}{c}\text { !kuerri-nan (LL.V.21.5699), } \\
\text { !kwerri-Inan (Bleek, 1956:464) }\end{array}$ & $\begin{array}{c}\text { !kwerriten, lightning strike; } \\
\text { !kweri, to strike dead, Bleek, } \\
\text { 1956:464). Inan, head (Bleek, } \\
1956: 342)\end{array}$ \\
\hline
\end{tabular}




\begin{tabular}{|c|c|c|c|}
\hline \multicolumn{4}{|c|}{ Near-Passerines } \\
\hline White-backed Mousebird & Colius colius (= capensis) & $\begin{array}{c}\| k u, \neq k^{\prime \prime} i \text { (LL.VIII.21.7842; } \\
\text { Bleek, 1956:668) }\end{array}$ & $\begin{array}{l}\text { Original gives as 'Speckled } \\
\text { Mousebird' (Colius striatus) }\end{array}$ \\
\hline Wild Dove, or Pigeon & Not Given & !khi-!khi, !nu-uru & Bleek, 1956:316, 434 \\
\hline \multicolumn{4}{|c|}{ Galliformes } \\
\hline $\begin{array}{l}\text { ?Greywing Partridge } \\
\text { ?Red-necked Francolin }\end{array}$ & $\begin{array}{c}\text { Francolinus sp. } \\
\text { Francolinus afer (Bleek, } \\
\text { 1956:390) }\end{array}$ & $\begin{array}{c}\text { 'gu!gubbi (WB.XXV.2342), } \\
\text { kurri-kurri (LL.I.2.185) }\end{array}$ & $\begin{array}{l}\text { Europeans shoot and eat } \\
\text { them. Kurri, broad, wide, } \\
\text { ball, Bleek, 1956:107) }\end{array}$ \\
\hline Partridge & Not Given & Kottan (LL.II.1.192) & $\begin{array}{l}\text { Europeans shoot and eat } \\
\text { them. }\end{array}$ \\
\hline ?Partridge/Quail & See below & !kabbi (Bleek, 1956:403) & Has white legs \\
\hline ?Partridge/Quail & $\begin{array}{l}\text { ?Cotumis communis; ?C. } \\
\text { coturnix? }\end{array}$ & $\begin{array}{c}\text { !kebbi (WB.XXV.2342; Bleek, } \\
\text { 1956:420) }\end{array}$ & \\
\hline Helmeted Guineafowl & Numida meleagris & $\begin{array}{l}\| k h o, \text { and variants } \\
\text { (LL.VIII.2.6142) }\end{array}$ & \\
\hline \multicolumn{4}{|c|}{ 'Birds of Prey' (Falconiformes and Strigiformes) } \\
\hline African Fish Eagle & Haliatus vocifer & $\| k^{\prime \prime} o($ LL.VIII.2.6142) & Probably same as below \\
\hline Hawk (?generic) & Not Given & $\neq k^{\prime} o$ (LL.I.2.185) & Probably same as above \\
\hline Eagle (?Bateleur Eagle) & $\begin{array}{c}\text { Aquila ecauda (?Terathopius } \\
\text { ecaudata) }\end{array}$ & !hou (LL.VIII.2.6142) & \\
\hline White-bellied Eagle & Aquila thoracica & $\begin{array}{c}\text { !kabbu!kabbu-k"as-|ka-!koen } \\
\text { (LL.I.2.186) }\end{array}$ & $\begin{array}{l}\text { Kills steinbok (name was lit. } \\
\text { 'springs-to kill-buck') }\end{array}$ \\
\hline ?Rock Kestrel & $\begin{array}{c}\text { Tinnunculus rapicoloides } \\
\text { (=ruficolus?) }\end{array}$ & $\begin{array}{l}\text { !kuerre-!kuerre } \\
\text { (LL.VIII.9.6774) } \\
\end{array}$ & $\begin{array}{c}\text { Eats lizards, flutters wings } \\
\text { (LL.VIII.7.6596) }\end{array}$ \\
\hline Bearded Vulture & Gypatus barbartus & $\begin{array}{l}\text { kui (LL.VIII.9.6774); ; kui } \\
\text { (WB.XXV.2342) }\end{array}$ & Bleek, 1956:334 \\
\hline Cape Griffon Vulture & Gyps coprotheres kolbii & \|hau-k"o (LL.VIII.9.6774; & A white vulture \\
\hline
\end{tabular}




\begin{tabular}{|c|c|c|c|}
\hline & (Vulture kolbii) & Bleek, 1956:543) & (LL.VIII.23.8070') \\
\hline Vulture & Not Given & \|naukan (LL.VIII.30.8616) & 'White' vulture \\
\hline Secretary Bird & Sagittarius serpentarius & $\begin{array}{c}\text { Opuorrisse (LL.VIII.21.7837) } \\
\text { !goхи: } \eta \text {, (Bleek, 1956:387) }\end{array}$ & $\begin{array}{c}\text { !uhai- } \mid k u \text { (LL.II.26.2350) or } \\
\text { 'carry-feathers' (Bleek, } \\
\text { 1956:686) }\end{array}$ \\
\hline Owl & Not Given & !hu!hu (LL.VIII.1.6048) & \\
\hline \multicolumn{4}{|c|}{ Gruiformes, Ciconiiformes and Phoenicopteriformes } \\
\hline Ibis & Tantalus sp. & $\begin{array}{c}\text { !uhattan - !kwomm } \\
\text { (LL.VIII.9.6773) }\end{array}$ & See Stork \\
\hline Flamingo & $\begin{array}{c}\text { Phoenicopterus roseus, (poss. } \\
\text { P. minor) }\end{array}$ & Ikein (LL.IV.1.3448) & \\
\hline Great Flamingo & $\begin{array}{c}\text { Phoenicopterus roseus }(= \\
\text { antiquorum })\end{array}$ & !xu-!gei (Bleek, 1956:503) & \\
\hline Stork & Not Given & $\begin{array}{l}\text { !uhatten-!kwomm } \\
\text { (LL.VIII.29.8579') }\end{array}$ & See Ibis \\
\hline Hamerkop & Scopus umbretta & !hi:n (Bleek, 1956:397) & \\
\hline Blue Crane & Tetrapteryx paradisa & $\begin{array}{c}\text { !k'o (LL.VIII.9.6773) } \\
\text { (WB.XXV.2342) }\end{array}$ & \\
\hline Blue Korhaan/Karoo Bustard & $\begin{array}{c}\text { Eupodotis }(=\text { Otis }) \\
\text { caerulescens, E. vigorsii }\end{array}$ & $\begin{array}{c}\text { !kaukən (LL.VIII.9.6773; } \\
\text { LL.I.2.185) }\end{array}$ & \\
\hline $\begin{array}{c}\text { Southern Black } \\
\text { Korhaan/Black Bustard }\end{array}$ & Eupodotis (= Otis) afra & Kwakwara (LL.VIII.9.6774) & \\
\hline Kori Bustard & Ardeotis ( = Otis) kori & $\begin{array}{c}|k i-| n a \text { (LL.VIII.2.6142; } \\
\text { LL.I.2.185) }\end{array}$ & Afr. Gompou \\
\hline Bustard & Not Given. & $\begin{array}{l}\|k o a,\| \text { koa-gwai, !karru } \\
\text { (LL.VIII.2.6142) }\end{array}$ & $\begin{array}{l}\text { Paauw, pou (Afr. bustard). } \\
\text { Latter } 2 \text { names for male birds. }\end{array}$ \\
\hline
\end{tabular}




\begin{tabular}{|c|c|c|c|}
\hline \multicolumn{4}{|c|}{ Seabirds (Procellariiformes) } \\
\hline Wandering Albatross & Diomedea exulans & $\begin{array}{c}k u i \text { (WB.XXV.2384; Bleek, } \\
\text { 1956:324) }\end{array}$ & \\
\hline \multicolumn{4}{|c|}{$\begin{array}{c}\text { Ratites (Struthioniformes) } \\
\end{array}$} \\
\hline Ostrich & Struthio camelus & $\begin{array}{c}K^{\prime \prime} \text { arru, toi (WB.XXV.2341) } \\
\text { |khui (LL.VIII.10.6859') }\end{array}$ & $\begin{array}{l}\text { Latter name used for ostrich } \\
\text { with chicks. First is probably } \\
\text { mistranslated !karru, bustard }\end{array}$ \\
\hline \multicolumn{4}{|c|}{ Non-real birds/insufficient information provided } \\
\hline 'Duiker bird' & Not Given & $\begin{array}{c}\text { !karritan (LL.VIII.3.6304; } \\
\text { Bleek, 1956:410) }\end{array}$ & Duiker (Afr. 'diver') \\
\hline 'Duiker bird', resembles the & Not Given & $\begin{array}{c}\text { !kwai-!kwai (LL.VIII.3.6304; } \\
\text { Bleek, 1956:460) }\end{array}$ & $\begin{array}{c}\text { Black with white beak, same } \\
\text { size as 'duiker' }\end{array}$ \\
\hline Not Given & Not Given & $\begin{array}{l}\text { Nerru (LL.VIII.28.8525) } \\
\text { ‡nerru (LL.VIII.29.8529; } \\
\text { Bleek, 1956:672) }\end{array}$ & $\begin{array}{l}\text { Male plumage resembles } \\
\text { ostrich. Large, communal } \\
\text { grass nests. Small with short } \\
\text { beak }\end{array}$ \\
\hline Not Applicable & Not Applicable & $\begin{array}{c}\text { !kakən-!kaka-!kaui } \\
\text { (LL.II.22.1965) }\end{array}$ & $\begin{array}{c}\text { See Glossary !kakən-!kaka- } \\
\text { !kaui }\end{array}$ \\
\hline Not Applicable & Not Applicable & $\ddagger$ kagara (Bleek, 1956:654) & $\begin{array}{c}\text { Bird, features as the } \\
\text { protagonist of a story }\end{array}$ \\
\hline Not Given & Not Given & $\begin{array}{c}\text { \|nuo\|nuobboko (Bleek, } \\
\text { 1956:622) }\end{array}$ & $\begin{array}{l}\text { Small with a long tail } \\
\text { (?mousebird) }\end{array}$ \\
\hline Not Given & Not Given & |kittan|kittan (Bleek, 1956:316) & Black or dark plumage \\
\hline Not Given & Not Given & $\| k a n($ LL.VIII.21.7828) & $\begin{array}{l}\text { Yellow/white plumage. Duck- } \\
\text { like bill. }\end{array}$ \\
\hline Not Given & Not Given & |harritan (LL.VIII.15.7310') & $\begin{array}{c}\text { Eats locusts, flesh. Crow } \\
\text { sized. }\end{array}$ \\
\hline
\end{tabular}




\begin{tabular}{|c|c|c|c|}
\hline Not Given & Not Given & $\begin{array}{c}\text { gerritzn-ti (LL.VIII.8.6709); } \\
\text { the 'son of the wind' }\end{array}$ & $\begin{array}{c}\text { Lit. Feathered-thing; a large } \\
\text { bird (Bleek (1956: 530) }\end{array}$ \\
\hline $\begin{array}{c}\text { Not Given; same IXam name } \\
\text { as flamingo }\end{array}$ & Not Given & !xu-!gei (LL.VIII.23.8070') & $\begin{array}{c}\text { Large, white, black wings; } \\
\text { eats millipedes/locusts }\end{array}$ \\
\hline
\end{tabular}


Table 3: IXam terms for reptiles and amphibians, with common and scientific names

\begin{tabular}{|c|c|c|c|}
\hline Common Name & Scientific Name & |Xam Name, reference & Further notes \\
\hline \multicolumn{4}{|c|}{ Amphibians } \\
\hline Tadpole & Not Given & $\begin{array}{c}\text { !kwai-!kwai (LL.VIII.11.6999'), } \\
\text { thun-tthun (LL.V.10.4750') }\end{array}$ & $\begin{array}{c}\text { Former is same as 'singly, } \\
\text { separating' (Bleek, 1956:460) }\end{array}$ \\
\hline Frog & Not Given & "|kain (LL.VIII.11.6999') & Young frog \\
\hline Frog & Not Given & $\begin{array}{l}\text { !ga (LL.VIII.11.6999'; } \\
\text { LL.VIII.21.7837) }\end{array}$ & 'Huge frog' (LL.I.2.187) \\
\hline Toad & Not Given & ! $g a$ (LL.VIII.21.7837) & \\
\hline \multicolumn{4}{|c|}{ Testudines } \\
\hline Tortoise & Not Given & $\neq g o$ (LL.I.2.187) & General term \\
\hline Turtles & Not Given & !kerru (LL.I.2.187) & General term \\
\hline ?Geometric Tortoise & $\begin{array}{c}\text { ?Psammobates geometrica } \\
\text { (N.B. this species is restricted } \\
\text { to Western Cape) }\end{array}$ & $\begin{array}{l}\text { !goe (LL.VIII.21.7837); !koe } \\
\text { (LL.IV.1.3451) }\end{array}$ & $\begin{array}{l}\text { 'Little.' Bleek (1956:385) } \\
\text { gives as Testudo geometrica, } \\
\text { and also as tortoiseshell. The } \\
\text { same word signified want, or } \\
\text { misfortune }\end{array}$ \\
\hline Leopard Tortoise & Stigmochelys pardalis & $\| g o($ Bleek, 1956:531) & "Water tortoise" \\
\hline $\begin{array}{c}\text { Angulate or Bowsprit } \\
\text { Tortoise (Rooipens, Afr. red- }\end{array}$ & Chersina angulata & $\begin{array}{c}\text { lgolgokan (LL.VIII.21.7843; } \\
\text { LL.VIII.25.8236) }\end{array}$ & $\begin{array}{l}\text { Bleek (1956:282) gives as } \\
\text { 'water tortoise' }\end{array}$ \\
\hline
\end{tabular}




\begin{tabular}{|c|c|c|c|}
\hline stomach) & & & \\
\hline Tortoise, unspecified & Not Given & !khou (LL.VIII.21.7837) & $\begin{array}{l}\text { 'a certain water tortoise; } \\
\text { (Bleek. 1956:428) }\end{array}$ \\
\hline Tortoise, unspecified & Not Given & $\begin{array}{c}k^{\prime \prime} \text { ammi, } \mid k^{\prime \prime} \text { emmi (Bleek, } \\
\text { 1956:337) } \mid \text { kummi } \\
\text { (LL.IV.1.3451) }\end{array}$ & $\begin{array}{l}\text { "Large round schildpad." } \\
\text { Perhaps related to Korana, } \\
\text { |kammip (used by łkasin) }\end{array}$ \\
\hline ?Tent Tortoise & ?Psammobates tentorius & |kappəm (Bleek, 1956:301) & $\begin{array}{l}\text { D. Bleek gives as 'Testudo } \\
\text { geometrica tentoria' }\end{array}$ \\
\hline \multicolumn{4}{|c|}{ Squamates (Lacertilia) } \\
\hline $\begin{array}{l}\text { ?Keeled Indian Mabuya (from } \\
\text { museum) }\end{array}$ & $\begin{array}{c}\text { Eutropis carinata }(=\text { Euprepes } \\
\text { carinatus })\end{array}$ & $\begin{array}{l}\text { !guatton (LL.VIII.21.7846) } \\
\text { !gwatton (Bleek, 1956:391) }\end{array}$ & $\begin{array}{l}\text { Presumably name refers a } \\
\text { similar Karoo Mabuya sp. }\end{array}$ \\
\hline Gecko, 'the Common Gecko' & $\begin{array}{c}\text { Not Given -Pachydactylus } \\
\text { capensis (Common Cape } \\
\text { Gecko) or other Pachydactylus } \\
\text { sp. likely } \\
\end{array}$ & $\begin{array}{c}\text { |hai-Opua (LL.VIII.31.8791) } \\
\text { |haitวn (Bleek, 1956: 286) }\end{array}$ & \\
\hline Agama & $\begin{array}{l}\text { Agama sp. (probably Agama } \\
\text { atra, Southern Rock Agama, } \\
\text { largest of Karoo agamas) }\end{array}$ & $\begin{array}{l}\text { !khou (LL.VIII.32.8822)!khau } \\
\text { (Bleek, 1956:425) }\end{array}$ & $\begin{array}{l}\text { 'Kochelman' !guamma gerri } \\
\text { (derisive name for large male } \\
\text { agama, LL.VIII.23.8068') } \\
\text { 'Ugly' (LL.VIII.32.8858') }\end{array}$ \\
\hline Agamid Lizard & $\begin{array}{c}\text { Several potential species: } \\
\text { Agama aculeata, A.anchietae, } \\
\text { A.hispida. }\end{array}$ & $\begin{array}{l}\| \text { horu, (Bleek, 1956:543) } \| \text { hou } \\
\text { (LL.VIII.32.8822) }\end{array}$ & $\begin{array}{l}\text { Smaller than (and resembles) } \\
\text { agama, eaten by children }\end{array}$ \\
\hline Chameleon & $\begin{array}{l}\text { ?Chamaeleo namaquensis } \\
\text { (arid adapted, turns black } \\
\text { when stressed) }\end{array}$ & $\begin{array}{c}\text { \|khua-setan (LL.VIII.21.7837) } \\
\neq k w a-s e t a n \text { (WB.I.195) }\end{array}$ & $\begin{array}{l}\text { 'Black' chameleon. } \\
\text { Derivation unclear. }\end{array}$ \\
\hline Chameleon & Not Given & $\begin{array}{c}\text { kuru (Bleek, 1956:326; } \\
\text { LL.V.3.4071') }\end{array}$ & $\begin{array}{l}\text { ?Derived from !Ora (!kurup, } \\
\text { lizard). "Veldt vorschleg" }\end{array}$ \\
\hline
\end{tabular}




\begin{tabular}{|c|c|c|c|}
\hline & & & chameleon \\
\hline Chameleon & Not Given & ‡nau-itan (WB.I.195) & $\begin{array}{l}\text { 'White' chameleon. May } \\
\text { connect with } \ddagger \text { nai-i, to kick or } \\
\text { scrape with the feet (Bleek, } \\
\text { 1956:670), this behaviour is } \\
\text { characteristic of chameleon } \\
\text { egg-burying }\end{array}$ \\
\hline Lizard & Not Given & |gi(LL.VIII.32.8858') & $\begin{array}{l}\text { Has stripes, smaller than } \\
\text { agama, 'beautiful' and 'red.' }\end{array}$ \\
\hline Lizard & $\begin{array}{c}\text { Not Given - possibly a } \\
\text { burrowing gecko? (Ptenopus } \\
\text { sp.?) }\end{array}$ & Tt'ain (LL.VIII.23.8068') & $\begin{array}{l}\text { Found in the holes of } \\
\text { porcupines. Paws 'hold fast' } \\
\text { to the rock }\end{array}$ \\
\hline Lizard & Not Given & $\begin{array}{l}\text { taз̧rru-gu (Bleek, 1956: 193) } \\
\text { t'urugukən (Bleek, 1956:242) }\end{array}$ & $\begin{array}{c}\text { The 'ackeldos' (Afr. akkedis, } \\
\text { lizard) a red lizard about the } \\
\text { length of a man's hand } \\
\text { (LL.VIII.17.7537') }\end{array}$ \\
\hline \multicolumn{4}{|c|}{ Squamates (Serpentes) } \\
\hline Snake & Not Given & \|kerritan-ti (LL.VIII.21.7838) & General term \\
\hline Snake & Not Given & $\begin{array}{l}\neq k h e i t a n \text { (LL.I.2.186) } \| \text { keitan } \\
\text { (WB.I.190) }\end{array}$ & $\begin{array}{c}\text { Used for boa (LL.I.2.186), } \\
\text { and a thick, long, large- } \\
\text { headed poisonous snake } \\
\text { (WB.I.190) }\end{array}$ \\
\hline Snake & ?Python sp. & $\begin{array}{c}\| x e t \partial n \text { (Bleek, 1956:635) } \| \text { ketan } \\
\text { (Bleek, 1956:571) !nuin (Bleek, } \\
\text { 1956:486) } \| k e-t \text { tan (WB.I.197) }\end{array}$ & $\begin{array}{c}\text { May be the same referent as } \\
\text { above. }\end{array}$ \\
\hline Cape Cobra & Naja nivea & \|gerri (LL.I.2.187) & $\begin{array}{c}\text { 'Egyptian Cobra' (in } \\
\text { Museum); Naja haje (not a }\end{array}$ \\
\hline
\end{tabular}




\begin{tabular}{|c|c|c|c|}
\hline & & & South African species) \\
\hline Snake & Not Given & łgerri (LL.VIII.23.8070') & $\begin{array}{l}\text { Long, slender and not } \\
\text { poisonous. Constrictor; feeds } \\
\text { on mice and lizards. }\end{array}$ \\
\hline Snake & Not Given & |geri (WB.I.197) & Very small \\
\hline $\begin{array}{l}\text { 'Viper': Common Night } \\
\text { Adder; Puffadder; Common } \\
\text { Eggeater } \\
\end{array}$ & $\begin{array}{l}\text { Causus rhombeatus; Bitis } \\
\text { arietans; Dasypeltis scaber }\end{array}$ & $\begin{array}{c}\text { 'gukan (LL.I.2.187) !gukən } \\
\text { (LL.VIII.23.8039'), lgukən } \\
\text { (WB.I.189) }\end{array}$ & $\begin{array}{l}\text { Most commonly applied to the } \\
\text { puffadder. }\end{array}$ \\
\hline Python & $\begin{array}{c}\text { Not Given (presumably Python } \\
\text { sebae) }\end{array}$ & $\begin{array}{l}\neq x u \text { (LL.VIII.32.8875'); } \neq x u \\
\text { (Bleek, 1956:680) }\end{array}$ & In one of Stow's pictures \\
\hline Snake & Not Given & $\neq k u$ (Bleek, 1956: 664) & $\begin{array}{l}\text { Poisonous snake; term can } \\
\text { also be used to refer to } \\
\text { 'poison' generically }\end{array}$ \\
\hline $\begin{array}{l}\text { ?Spotted Harlequin Snake (at } \\
\text { museum) }\end{array}$ & $\begin{array}{c}\text { Homoroselaps lacteus }(= \\
\text { Elaps hygiae })\end{array}$ & $\begin{array}{l}\text { łgannang (LL.I.2.187) |garran } \\
\text { (WB.XXV.2360) }\end{array}$ & $\begin{array}{l}\text { Term appears to be connected } \\
\text { with below }\end{array}$ \\
\hline $\begin{array}{l}\text { Blind snake, or an } \\
\text { amphisbaenian }\end{array}$ & $\begin{array}{c}\text { Several potentials: } \\
\text { Rhinotyphlops lalandii; } R . \\
\text { schinzi; Monopeltis capensis; } \\
\text { Zygaspis quadrifrons } \\
\end{array}$ & łgãnnuך (Bleek, 1956:645) & $\begin{array}{l}\text { Like a snake, goes into the } \\
\text { earth }\end{array}$ \\
\hline Bronzeback (at museum) & Dendrophis picta & $\begin{array}{l}\text { "kanga whai (WB.I.193; } \\
\text { LL.I.2.187) }\end{array}$ & $\begin{array}{l}\text { 'Long' (WB.I.193) or 'short' } \\
\text { (WB.I.197). Whai, } \\
\text { 'springbok'. } \| \text { kang-a, is 'to } \\
\text { tear a hole' (Bleek, 1956:556) }\end{array}$ \\
\hline Coral Snake (at museum) & Elaps corallins & łhumm (LL.I.2.187) & \\
\hline Snake & Not Given & |hem (WB.I.197) & $\begin{array}{c}\text { Little snake; 'boute' (possibly, } \\
\text { boud, Afr. fearless) }\end{array}$ \\
\hline Snake & Not Given & |hũ (Bleek, 1956:290) & Short, found near Cape Town \\
\hline
\end{tabular}




\begin{tabular}{|c|c|c|c|}
\hline Cobra & Not Given & $\begin{array}{c}\| k w i(\text { WB.I.189) } \| k u i \\
\text { (WB.I.197), } \| k h w i(\text { Bleek, } \\
\text { 1956: 579) }\end{array}$ & $\begin{array}{l}\text { 'a long snake'; yellow snake; } \\
\text { May reflect a connection with } \\
\text { 'quagga' (cobra scale patterns } \\
\text { can be similar) }\end{array}$ \\
\hline Snake & Not Given & $\begin{array}{c}\text { koiri (WB.I.189) } \mid \text { kuri } \\
\text { (WB.I.97)) }\end{array}$ & $\begin{array}{l}\text { Long, black; like the } \\
\text { puffadder, large }\end{array}$ \\
\hline 'Spitting cobra' & $\begin{array}{l}\text { Haemachatus hemachatus or } \\
\text { Naja nigricollis }\end{array}$ & Tssou (LL.VIII.22.7946') & $\begin{array}{l}\text { Long, black, spits at people, } \\
\text { sounds like bees }\end{array}$ \\
\hline Snake & Not Given & Tts'ann (WB.I.197) & $\begin{array}{c}\text { Black; probably related to the } \\
\text { above }\end{array}$ \\
\hline $\begin{array}{l}\text { ?Blind snake or } \\
\text { amphisbaenian }\end{array}$ & $\begin{array}{c}\text { Several potentials: } \\
\text { Rhinotyphlops lalandii; } R . \\
\text { schinzi; Monopeltis capensis; } \\
\text { Zygaspis quadrifrons }\end{array}$ & Okuara (WB.I.197) & $\begin{array}{l}\text { Little; "sits inside the ground" } \\
\text { (see } \neq \text { gannun, above: may be } \\
\text { another amphisbaenian?) }\end{array}$ \\
\hline $\begin{array}{l}\text { ?Blind snake or } \\
\text { amphisbaenian }\end{array}$ & $\begin{array}{c}\text { Several potentials: } \\
\text { Rhinotyphlops lalandii; } R . \\
\text { schinzi; Monopeltis capensis; } \\
\text { Zygaspis quadrifrons }\end{array}$ & Opwana (WB.I.189) & $\begin{array}{c}\text { Little; this is probably the } \\
\text { 'correct' version of the above } \\
(\odot \text { is not commonly combined } \\
\text { with '-k') }\end{array}$ \\
\hline Snake & Not Given & $\neq k o \ddagger k o r o x i$ (WB.I.197) & $\begin{array}{c}\text { Little snake; 'boute' (see } \\
\text { above) }\end{array}$ \\
\hline Snake & Not Given & $\mid k^{\prime \prime} a u$ (Bleek, 1956:338) & Black \\
\hline
\end{tabular}


Table 4: IXam terms for invertebrates, with common and scientific names

\begin{tabular}{|c|c|c|c|}
\hline Common Name & Scientific Name & IXam Name with reference & Further Notes \\
\hline \multicolumn{4}{|c|}{ Insecta } \\
\hline \multicolumn{4}{|c|}{ Orthoptera } \\
\hline Common Field Cricket; Kritjie & Gryllus capensis & $\neq$ kuirri-ti-tikan (LL.VIII.11.6999'); & Poss. 'staggering's things' \\
\hline ?Koringkrieke & $\begin{array}{c}\text { Anostostomatidae, } \\
\text { Bradyporidae }\end{array}$ & $\mid k^{\prime \prime}$ ururu (LL.IV.1.3515) & Chapter 7c:250 \\
\hline Grasshopper - 'small' & Not Given & łen-ko-\|nein (LL.VIII.21.7846) & Poss. 'small-nest-maker' \\
\hline ?Cricket & Hetrodes sp. & $\begin{array}{l}k^{\prime \prime} \text { erritan (LL.VIII.21.7820); } \\
\text { |karritan (LL.IV.2.3530') }\end{array}$ & \\
\hline ?Cricket|?Locust|?Grasshopper & Acridium $\mathrm{sp}$ & $\neq e n-k o-\| n e i n$ (LL.VIII.21.7821) & Poss. 'small-nest-maker' \\
\hline ?Cricketl?Locustl?Grasshopper & Phymateus morbillosus & |k"arritan, !na (WB.XXV.2431) & \\
\hline $\begin{array}{c}\text { Locust, Common Cape Locust, } \\
\text { Sprinkaan (Afr.) }\end{array}$ & $\begin{array}{c}\text { Acridium ruficorne } \\
\text { (Acanthacris ruficornis } \\
\text { ruficornis) }\end{array}$ & "kabba-kha (LL.VIII.7.6660) & $\begin{array}{l}\text { Named after resemblance } \\
\text { to a bone forming part of } \\
\text { an arrow (link shaft) }\end{array}$ \\
\hline \multicolumn{4}{|c|}{ Coleoptera } \\
\hline African Ground Weevil & $\begin{array}{c}\text { Brachycerus sp. } \\
\text { (Brachycerus obesus) }\end{array}$ & $\begin{array}{c}\text { !nu!nurussi (LL.VIII.21.7819) } \\
\text { (Bleek, 1956:486) }\end{array}$ & Poss. 'angry crawler' \\
\hline Longhorn & Zographus scaber & !nwa-!ka (LL.VIII.21.7819) & !nwa, reed/arrow \\
\hline Blister beetle & Mylabris sp. & !nwa-!ka (LL.VIII.21.7819) & !nwa, reed/arrow \\
\hline
\end{tabular}




\begin{tabular}{|c|c|c|c|}
\hline Toktokkie (Afr.) & Moluris sp. & !kwubbo (LL.VIII.21.7819) & $\begin{array}{c}\text { !kwubbi:tan, to lift up the } \\
\text { ground or sprout (Bleek, } \\
1956: 469 \text { ) }\end{array}$ \\
\hline Tiger Beetle & Manticora sp. & $\|$ "ko\|korosi, $\|$ go (LL.VIII.21.7819) & $\begin{array}{c}g o, \text { outer skin (Bleek, } \\
\text { 1956:531) } \| k o: \text { ro, horns } \\
\text { (Bleek, 1956:587) }\end{array}$ \\
\hline Ground Beetle & $\begin{array}{c}\text { Anthia thoracica, Manticora } \\
\text { sp. }\end{array}$ & $\begin{array}{l}\| \text { ko\|korosi (LL.VIII.21.7819), } \\
\| \text { ka\|kurrassi (WB.XXV.2431), } \| \text { go } \\
\text { (WB.XXV.2431) }\end{array}$ & $\begin{array}{l}\| g o, \text { outer skin (Bleek, } \\
\text { 1956:531) } \| k o: \text { ro, horns } \\
\text { (Bleek, 1956:587) }\end{array}$ \\
\hline Jewel Beetle & Julodis sp. & Bara-xam (LL.VIII.21.7819) & $\begin{array}{c}\text { Eaten by Bushmen. } \\
\text { Barabara, flat (Bleek, } \\
\text { 1956:15) }\end{array}$ \\
\hline Dung Beetle & Ateuchus sp. & Zshu (LL.VIII.21.7819) & $\begin{array}{c}\text { To spring, hop, fly (Bleek, } \\
1956: 265)\end{array}$ \\
\hline ?Woolly chafer & $\begin{array}{c}\text { Leocaeta }(=\text { Sparrmannia }) \\
\text { sp. }(\text { probably S. flava })\end{array}$ & !nubbu-juaiton (LL.VIII.21.7819) & \\
\hline Weevil & Cleonus glacialis & $\neq$ пиturu (LL.VIII.21.7819) & $\begin{array}{l}\ddagger n u, \text { to be black (Bleek, } \\
1956: 674) ! n u: r u, \text { horns }\end{array}$ \\
\hline Leaf Beetle & Chrysomela sp. & !kauru (LL.VIII.21.7820) & $\begin{array}{l}\text { !kauru, 'bitterbolle' plant } \\
\text { (Bleek, 1956:416) }\end{array}$ \\
\hline Chafer & Cetonia sp. & Zshu (LL.VIII.21.7821) & $\begin{array}{c}\text { To spring, hop, fly (Bleek, } \\
1956: 265)\end{array}$ \\
\hline Tenebrionid, 'Darkling' Beetle & Epiphysa sp. & $\|$ kaxai-|xu (LL.VIII.21.7821) & $\begin{array}{c}\text { "kaxai, sister (Bleek, } \\
\text { 1956:564) }\end{array}$ \\
\hline \multicolumn{4}{|c|}{ Lepidoptera } \\
\hline Tiger moth & $\begin{array}{c}\text { Aloa }(=\text { Rhodogastria }) \\
\text { amasis }\end{array}$ & $\begin{array}{l}\| k h \tilde{a}:-k a-\mid g o r o(\text { LL.VIII.21.7842), } \\
\text { !num!num (Bleek, 1956:485) }\end{array}$ & $\begin{array}{l}\text { Lit. 'Lion's moth'; !num, to } \\
\text { take, hold, put in the }\end{array}$ \\
\hline
\end{tabular}




\begin{tabular}{|c|c|c|c|}
\hline & & & mouth (Bleek, 1956:484) \\
\hline Lime Swallowtail & Papilo demoleus & $\begin{array}{l}\text { !gwe-ta-dadabassi } \\
\text { (LL.VIII.21.7819) }\end{array}$ & $\begin{array}{c}\text { Moth, butterfly (Bleek, } \\
\text { 1956:20) "hail's butterfly"; } \\
\text { presumably a pattern } \\
\text { (!gwe, white markings of } \\
\text { porcupine) }\end{array}$ \\
\hline Moth ('Heady Maiden') & Syntomia $(=$ Amata $)$ cerbera & Dadabasi (Bleek, 1956:20) & $\begin{array}{c}\text { Moth, butterfly (Bleek, } \\
1956: 20)\end{array}$ \\
\hline Butterflies & Not Given & Dadabasi (LL.VIII.9.6771) & $\begin{array}{c}\text { Moth, butterfly (Bleek, } \\
1956: 20)\end{array}$ \\
\hline $\begin{array}{l}\text { "Owl Moth" (White Witch } \\
\text { moth) }\end{array}$ & Thysnia agrippina & |gu:ro (WB.XXV.2344; WB.I.184) & \\
\hline ?Death's Head Hawk Moth & $\begin{array}{l}\text { Acherontis atropos; also } \\
\text { Attacus attus (India) }\end{array}$ & $\begin{array}{l}\| k h \tilde{a}:-k a-\text { dadabasi; } \| k h \tilde{a}:-k a-\mid \text { goro } \\
\text { (LL.VIII.9.6771) }\end{array}$ & Lit. 'Lion's moth' \\
\hline Moth (a hawkmoth) & Sphynx sp. & \|khã:-ka-|goro (WB.I.188) & Lit. 'Lion's moth' \\
\hline Butterfly & Not Given & !gwe (WB.I.187') & $\begin{array}{c}\text { Presumably related to } \\
\text { Papilio demoleus, above. }\end{array}$ \\
\hline Noctuiid moth & $\begin{array}{l}\text { Celoena renisigma (poss. } \\
\text { Synpa renisigma) }\end{array}$ & |go:ro (Bleek, 1956:282) & \\
\hline \multicolumn{4}{|c|}{ Larvae } \\
\hline Caterpillar & Not Given & !nwruken (LL.VIII.21.7845) & $\begin{array}{c}\text { !nu:ru, to crawl or crouch } \\
\text { (Bleek, 1956:485) }\end{array}$ \\
\hline Hide Beetle larvae & Dermestes sp. (?maculata) & 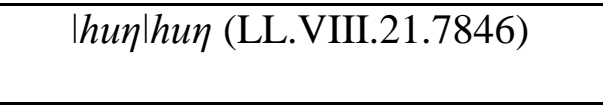 & $\begin{array}{c}\text { "a beetle which eats } \\
\text { skins" }\end{array}$ \\
\hline Caterpillar & Not Given & !kukan-|u-|ипи (LL.VIII.15.7311) & \\
\hline Caterpillar of Garden Acraea & Acraea horta & $\begin{array}{l}\text { Resembles '!kuari-a-wai’ } \\
\text { (LL.VIII.21.7841) }\end{array}$ & See below \\
\hline
\end{tabular}




\begin{tabular}{|c|c|c|c|}
\hline Caterpillar of Noctuiid moth & Not Given & !kwana-wai (LL.VIII.31.8790') & $\begin{array}{l}\text { !kwana, to bleat (Bleek, } \\
\text { 1956:461), wai, springbok } \\
\text { (Bleek, 1956:251) }\end{array}$ \\
\hline Caterpillar, 'pietje canary' & Acraea horta & !nummin (Bleek, 1956:485) & $\begin{array}{l}\text { Inum, to take, hold, put in } \\
\text { the mouth (Bleek, } \\
1956: 484)\end{array}$ \\
\hline $\begin{array}{c}\text { Worms (which make holes in } \\
\text { trees) }\end{array}$ & Not Given & $\neq k u n u$ (LL.V.10.4761') & \\
\hline White maggots (in the ground) & Not Given & !kuin (Bleek, 1956: 450) & \\
\hline \multicolumn{4}{|c|}{ Diptera } \\
\hline Flies & Not Given & $\neq$ łaukanłhaukən (WB.I.210) & $\begin{array}{c}\text { fhau, to spring, jump, } \\
\text { dance (Bleek, 1956:650) } \\
\text { ?transliteration error for } \\
\text { !haukan (Bleek, 1956:396) }\end{array}$ \\
\hline Long-proboscid fly & $\begin{array}{c}\text { Nemestrina }(= \\
\text { Moegistorhynchus }) \\
\text { longinostrus }\end{array}$ & !kum-@тиi (LL.VIII.21.7821) & $\begin{array}{l}\text { !kum, to plunge or dip in } \\
\text { (Bleek, 1956:451) }\end{array}$ \\
\hline Biting Fly & Pangonia sp. & !kwomm (Bleek, 1956:468) & $\begin{array}{l}\text { !kwomma, a large swelling } \\
\text { (Bleek, 1956:468). }\end{array}$ \\
\hline Tsetse Fly & Glossina morsitans & !kum-○тиі (LL.VIII.9.6772) & $\begin{array}{l}\text { !kum, to plunge or dip in } \\
\text { (Bleek, 1956:451) }\end{array}$ \\
\hline Mosquito & ?Anopheles sp. & Tssutto (LL.XIII.1.6108') & \\
\hline Flies - feed on blood & $\begin{array}{c}\text { Musca } \text { sp. (? Stomoxys } \\
\text { calcitrans) }\end{array}$ & $\begin{array}{c}\text { !houkan-!houkan, !kwara } \\
\text { (LL.VIII.9.6772)!kwara (Bleek, } \\
\text { 1956:461) }\end{array}$ & $\begin{array}{c}\text { Several poss.: !hau, } \\
\text { follow, be together in } \\
\text { numbers, frustrate or } \\
\text { bewitch (Bleek, 1956:395- } \\
\text { 396) }\end{array}$ \\
\hline
\end{tabular}




\begin{tabular}{|c|c|c|c|}
\hline Robberflies & Asilus sp. & $\mid g u-! x a$ (LL.VIII.9.6772) & $\begin{array}{c}\text { Igu, bruise or blister } \\
\text { (Bleek, 1956:282); } ! x a, \\
\text { upper part of the spine or } \\
\text { back of neck (Bleek, } \\
\text { 1956:496) }\end{array}$ \\
\hline Beefly species & Bombylius analis & !kum-Отиі (LL.VIII.9.6772) & $\begin{array}{l}\text { !kum, to plunge or dip in } \\
\text { (Bleek, 1956:451) }\end{array}$ \\
\hline "Cape Winged Horse Tick" & $\begin{array}{c}\text { Hippobroca sp. - Probably } \\
\text { Hippobosca equine }\end{array}$ & !kam (LL.VIII.9.6772) & $\begin{array}{l}\text { Same word as for 'ostrich } \\
\text { louse' (Bleek, 1956:406) }\end{array}$ \\
\hline \multicolumn{4}{|c|}{ Odonata } \\
\hline Dragonfly (Hawker) & Aeshna sp. & $\begin{array}{c}\neq k a k k a n-\|k h o-t u \tilde{n u}, \neq k a k k a n-\| k h o: a- \\
\text { t'orru (Bleek, 1956:655) }\end{array}$ & \\
\hline \multicolumn{4}{|c|}{ Hemiptera } \\
\hline Plant Bug & Lygaeus sp. & $\neq$ natta-xam (LL.VIII.21.7821) & $\begin{array}{c}\text { (LL.VIII.7.6770); } \\
\text { contrasts with 'bara-xam', } \\
\text { unpleasant smell }\end{array}$ \\
\hline Cicada & $\begin{array}{c}\text { (?Quintillia sp.; Karoo } \\
\text { cicadas) }\end{array}$ & |nuattən-|nuattən(LL.VIII.21.7821) & \\
\hline Long-legged Plant Bug & $\begin{array}{c}\text { Mygdonia }(=\text { Elasmopoda }) \\
\text { valga }\end{array}$ & łnatta-xam (LL.VIII.9.6771) & Contrasts with 'bara-xam' \\
\hline \multicolumn{4}{|c|}{ Hymenoptera } \\
\hline Honeybee & Apis mellifera? ?scutellata & $\begin{array}{c}\text { lkau (WB.I.210) !khou (Bleek, } \\
1956: 413 \text { ) }\end{array}$ & Insect and honey \\
\hline Giant Cape Sand Wasp & Mygnimia capensis & !kou (LL.VIII.9.6771) & $\begin{array}{c}\text { ?Related to !khou, } \\
\text { honey/bee }\end{array}$ \\
\hline Mason Wasp & Lyrops sp? Thynnus brownii & $\begin{array}{l}\text { !gabbakan!gabbakan } \\
\text { (LL.VIII.21.7846) }\end{array}$ & $\begin{array}{c}\text { !gabba, to nod (Bleek, } \\
1956: 374)\end{array}$ \\
\hline
\end{tabular}




\begin{tabular}{|c|c|c|c|}
\hline Ant & Not Given & !gu!gú (Bleek, 1956:390) & See Glossary !gu!gú \\
\hline 'Red ant' & Not Given & $\|k o k a n\| k o k a n$ & $\begin{array}{l}\text { A response to an image in } \\
\text { a book }\end{array}$ \\
\hline Ants & Not Given & !kwain!kwain (Bleek, 1956:330) & \\
\hline Sandwasp & Mygnimia capensis & !khou (Bleek, 1956:429) & ?Related to, honey/bee \\
\hline $\begin{array}{l}\text { Wild bee or wasp; 'kapater bee' } \\
\text { (LL.V.3.4071') }\end{array}$ & $\begin{array}{c}\text { ?Carpenter bee (Xylocopa } \\
\text { sp.) }\end{array}$ & $z u:($ Bleek, 1956:264) & $\begin{array}{c}\text { Insect and honey. } \\
\text { Afr. Kapaterby, } \\
\text { bumblebee or drone; } \\
\text { houtkapperby, carpenter } \\
\text { bee }\end{array}$ \\
\hline Small bee or wasp & Not Given & $\begin{array}{l}\text { !ka-kagaua (Bleek, 1956:405); } \\
\text { !akka-!gaua (Bleek, 1956: 370) }\end{array}$ & $\begin{array}{l}\text { Insect and honey ('sweet } \\
\text { food') (Bleek, 1956:370). }\end{array}$ \\
\hline Small bee or wasp & $\begin{array}{l}\text { Not Given (Halicitiid or } \\
\text { Anthrophorid bees?) }\end{array}$ & $\begin{array}{c}\text { Opwai (Bleek, 1956:685; } \\
\text { LL.II.27.2416') }\end{array}$ & $\begin{array}{c}\text { Opwai:, meat or game } \\
(\text { Bleek, 1956:685) }\end{array}$ \\
\hline Bee or wasp & Not Given & | doro-|e-\|kau (LL.VIII.32.8811') & $\begin{array}{l}\text { Lit. 'over/above- } \\
\text { tinderbox-fire' }\end{array}$ \\
\hline \multicolumn{4}{|c|}{ Blattodea } \\
\hline Cockroach & Not Given & $\begin{array}{l}\text { !kukan!kukan-xutara } \\
\text { (LL.VIII.21.7820) }\end{array}$ & $\begin{array}{c}\text { !kukan!kukan-xu-; leave } \\
\text { off putting on shoes } \\
\text { (Bleek, 1956:451, 260) }\end{array}$ \\
\hline ?Harvester Termite & Hodotermes sp. & $\| x e$ (Bleek, 1956:635) & $\begin{array}{c}\text { 'Bushman rice', 'ant } \\
\text { chrysalides/eggs' }\end{array}$ \\
\hline ?Snouted termite & Trinervitermes sp. & !hakən, !ha:gən (Bleek, 1956:394) & Eaten by aardwolf \\
\hline ?Termite & Not Given & !kuin & Similar to $\| x e$ and !hakan. \\
\hline \multicolumn{4}{|c|}{ Neuroptera } \\
\hline Antlion & Not Given & $\begin{array}{l}\text { !ka!kabbo-ta-!kwa } \\
\text { (LL.VIII.21.7821) }\end{array}$ & $\begin{array}{l}\text { Poss. 'to be startled (by) } \\
\text { rain' }\end{array}$ \\
\hline
\end{tabular}




\begin{tabular}{|c|c|c|c|}
\hline $\begin{array}{l}\text { Cape Short-horned (Spotted } \\
\text { Veld) Antlion }\end{array}$ & Palpares speciosus & $\neq k a k k a n-\| k h o-t u n u$ (LL.VIII.9.6770) & See Dragonfly \\
\hline \multicolumn{4}{|c|}{ Mantodea } \\
\hline Mantis & Empusa sp. & |kaggən (LL.VIII.9.6770) & \\
\hline $\begin{array}{l}\text { Withered-Leaf Insect (?Ghost } \\
\text { Mantis) }\end{array}$ & $\begin{array}{c}\text { Phyllomorpha } \text { (probably }= \\
\text { Phyllocrania) paradoxa }\end{array}$ & $\begin{array}{c}\text { \|khoa-!uhaitan-!uhaitan-|guakan } \\
\text { (LL.VIII.9.6771) }\end{array}$ & \\
\hline \multicolumn{4}{|c|}{ Phasmatodea } \\
\hline Walking Stick Insect & $\begin{array}{c}\text { Palophus haworthii }(= \\
\text { Bactrododema hecticum })\end{array}$ & $\begin{array}{c}\text { \|narra-\|khukan (LL.VIII.9.6770); } \\
\| \text { naxawakən } \\
\text { (WB.XXV.2344;LL.VIII.21.7819); } \\
\text { |kaggan (WB.XXV.2382) }\end{array}$ & $\begin{array}{l}\text { \#nara, rattling, going back } \\
\text { and forth' (Bleek, } \\
\text { 1956:614, 615) } \| k h u k a n, \\
\text { urine (Bleek, 1956:577) }\end{array}$ \\
\hline \multicolumn{4}{|c|}{ Siphonaptera } \\
\hline Flea & Not Given & !ai -tama (Bleek, 1956:370) & $\begin{array}{c}\text { Nama, !ai-dab (Bleek, } \\
1956: 370) \\
\end{array}$ \\
\hline \multicolumn{4}{|c|}{ Myriapoda } \\
\hline $\begin{array}{l}\text { Millipede - large, black, } \\
\text { reddish legs, 9" }\end{array}$ & $\begin{array}{l}\text { Probably Archispirostreptus } \\
\text { gigas }\end{array}$ & $\begin{array}{l}\text { !kummin-!kummin } \\
\text { (LL.VIII.17.7513') }\end{array}$ & Derivation obscure \\
\hline Millipede, resembles the & Not Given & !nu-!kattan-tsi (LL.VIII.17.7513') & $\begin{array}{l}\text { - to trot (Bleek, 1956:410); } \\
\text { - to bite (Bleek, 1956:215) }\end{array}$ \\
\hline Giant Centipede, Centipede & Scolopendra sp. & $\begin{array}{c}\| k e r r i-s s i-! k a u \text { (LL.VIII.21.7820; } \\
\text { LL.VIII.17.7514';LL.VIII.31.8791)) }\end{array}$ & $\begin{array}{l}\text { 'Rain's caterpillar' } \\
\text { (LL.VIII.1.6074), } \\
\text { poisonous. \|kerri, exclaim, } \\
\text { cry (Bleek, 1956:570) }\end{array}$ \\
\hline \multicolumn{4}{|c|}{ Arachnida } \\
\hline Spider & Not Given & $\begin{array}{l}\text { |hamminitti (WB.I.209) |hommain- } \\
\text { dde (Bleek, 1956:289) }\end{array}$ & $\begin{array}{l}\text { Generic 'spider' (Bleek, } \\
\text { 1956). Ihomm, dawn or } \\
\text { twilight. Poisonous. }\end{array}$ \\
\hline
\end{tabular}




\begin{tabular}{|c|c|c|c|}
\hline Spider & Not Given & !uhi-!khe-!koa (LL.IV.2.3530') & $\begin{array}{c}\text { Yellow and black. } \\
\text { ?Related to !kwi-!khe-!kwa }\end{array}$ \\
\hline Spider (Crab Spider) & Misumena sp. & $\mid k h u($ Bleek, 1956: 322) & \\
\hline Spider & Not Given & !ka-ka-|khwetən (Bleek, 1956:405) & $\begin{array}{l}\text { Lit. 'sticks of the !ka-bush' } \\
\text { (Bleek, 1956:405) }\end{array}$ \\
\hline Spider & Not Given & !kwi:-!khe-!kwa: (Bleek, 1956:468) & $\begin{array}{l}\text { Crossing-continues- } \\
\text { travelling (Bleek, } \\
\text { 1956:495). Poisonous. }\end{array}$ \\
\hline Ground Spider & Not Given & $\mid k u$ (LL.VIII.21.7820) & Presume related to $\mid k h u$ \\
\hline Scorpion Spider & Galeodes sp. & !huru (LL.VIII.21.7820) & \\
\hline ?Scorpion Spider & Not Given & $\begin{array}{c}\text { Ttui ttui ttuappam! } \\
\text { (LL.VIII.127072') "the !huru which } \\
\text { is large." }\end{array}$ & $\begin{array}{l}\text { Lit. 'Go away let go!'; an } \\
\text { exclamation (Bleek, } \\
\text { 1956:240) }\end{array}$ \\
\hline Scorpion & Not Given & $\begin{array}{c}\text { !kanna (LL.VIII.21.7820, 7841), } \\
\text { kukukulkhe (LL.VIII.21.7841) }\end{array}$ & \\
\hline Tick & Ixodes sp. & $\begin{array}{c}\text { \|happam (LL.VIII.31.8790'); !khoa- } \\
\text { ka-s'o (LL.VIII.21.7820) }\end{array}$ & $\begin{array}{c}\text { Latter name; } \\
\text { "remains/sits-(at)-water" } \\
\text { (Bleek, 1956:171, 341) } \\
\end{array}$ \\
\hline "Ostrich louse" & ?Struthiolipeurus struthionis & !kamm (Bleek, 1956:406) & $\begin{array}{c}\text { Poss. Hippobosca } \\
\text { struthionis (ostrich louse- } \\
\text { fly) } \\
\end{array}$ \\
\hline \multicolumn{4}{|c|}{ Miscellanea } \\
\hline $\begin{array}{l}\text { Insect - poisonous, in the } \\
\text { ground }\end{array}$ & Not Given & $\begin{array}{c}\text { !kukan!kukan-xutara } \\
\text { (LL.VIII.21.7817) }\end{array}$ & See cockroach, above \\
\hline Insect - 'bite is poison' & Not Given & |kuatton (LL.VIII.21.7817) & \\
\hline Insect - 'creeping' & Not Given & $\neq n u: t u:$ (Bleek, 1956:674) & see $\neq$ nuturu \\
\hline Spider's egg bags & Not Given & |hommin-dde-ka-!kuitən-ka-\|ho & Lit. 'spider-egg's-bag' \\
\hline
\end{tabular}




\begin{tabular}{|c|c|c|c|}
\hline & & (LL.VIII.21.7829') & \\
\hline Crab & Not Given & $\begin{array}{l}\text { \|nouttan (WB.II.367); } \| \text { nuerritan } \\
\text { (LL.VIII.23.8039') }\end{array}$ & \\
\hline A freshwater mollusc & Helix. sp. & tutun (Bleek, 1956:242) & $\begin{array}{l}\text { Tutunnu 'a, to be wet } \\
\text { (Bleek, 1956:242) }\end{array}$ \\
\hline
\end{tabular}


Table 5: IXam terms for plants, their uses, and identifications

\begin{tabular}{|c|c|c|c|}
\hline IXam name & Identification & Descriptive notes, additional use(s) & Reference \\
\hline ma:乡̧in & Bulb & Found in water (similar to below). Eaten & LL.VIII.29.8613' \\
\hline !uherritan & Bulb & Found in water (similar to above). Eaten & LL.VIII.29.8613' \\
\hline !hwin & Root & $\begin{array}{l}\text { Scented. Eaten as meal, roasted, or raw. Red } \\
\text { flowered plant. }\end{array}$ & $\begin{array}{l}\text { LL.II.28.2516'; } \\
\text { LL.VIII.27.8395' }\end{array}$ \\
\hline !koa & Root/tuber & Bitter, hairy root. Grows in clay. Eaten. & LL.V.13.5006' \\
\hline !nutu & Root/tuber & Resemble potatoes; not deep. Eaten, & LL.VIII.11.6945' \\
\hline$\| g w i$ : & Root/tuber & 'Like a sweet potato'. Eaten. & LL.V.10.4745' \\
\hline \|kerri, !kerri & ??? & $\begin{array}{l}\text { Seed/berries eaten in summer; 'sweet' root } \\
\text { eaten in winter (LL.VIII.18.7599) }\end{array}$ & LL.VIII.18.7598 \\
\hline$\|k u\| k u m$ & Root/tuber & $\begin{array}{l}\text { Green, 'flag-like'. Red flowers. Root called } \\
\text { Inunu-soakan. Eaten }\end{array}$ & LL.VIII.18.7552' \\
\hline xarru-ssi & Bulb & Uintjies (Afr. 'little onions'). Eaten & LL.VIII.26.8271' \\
\hline |khurukan & Bulb & $\begin{array}{l}\text { Green leaves, bulbs. Resembles \|garakan. } \\
\text { 'Feared' }\end{array}$ & LL.V.24.5992' \\
\hline !kouwi, !kauwi & ?Cyanella hyacinthoides & Uintjies ('little onions'). Eaten. & LL.IV.3.3735' \\
\hline !khoui & ?Cyanella hyacinthoides & "Bitter". See !kouwi. ?Eaten & LL.VIII.1.6106' \\
\hline !kuisi, !kuise & Bulb & Eaten & LL.V.7.4498' \\
\hline \|ga६rakan & Bulb & Eaten & LL.V.21.5661 \\
\hline
\end{tabular}




\begin{tabular}{|c|c|c|c|}
\hline !kuitən & Moraea (Fockea) sp.; ?M. edulis & Non-poisonous Moraea sp.? Eaten & LL.VIII.18.7553' \\
\hline \|kwerre & Moraea collina & A poisonous 'tulp' & LL.VIII.11.7552' \\
\hline |kui & Fockea angustifolia, or F. edulis & $\begin{array}{l}\text { Kambaroe, baroe (Smith, 1966:218, 272). } \\
\text { Eaten }\end{array}$ & LL.IV.3.3706' \\
\hline $\operatorname{ta\xi n:}$ & Cucumis spp. & Cucumber. Eaten. & Bleek, 1956:191 \\
\hline !nwanna & Cucumis spp. & Sweet gherkin. Eaten. & LL.VIII.1.6106' \\
\hline$\| k w a \xi k k \partial n$ & Cucumis sp.; ?C. africanus & $\begin{array}{l}\text { "axoort" (LL.VIII.18.7597') Afr. agurk } \\
\text { (gherkin). Eaten. }\end{array}$ & LL.VIII.1.6106' \\
\hline gara & Rhus sp.; ?R. lancea & Berries of 'krie boom' (kareeboom). Eaten. & WB.XXIII.2154' \\
\hline !kauru & Citrullus lanatus (bitter) & Bitterboela, bitter melon. Eaten. & LL.VIII.10.6944' \\
\hline Opwerre & ?Grewia flava & Fruit resembling an acorn, boiled and eaten & LL.VIII.21.7835 \\
\hline !kou, !khou & Acacia sp., ?A. karroo & Made into flour; flowers eaten; tinder. & LL.V.10.4761' \\
\hline gui & Acacia sp. & Gum arabic. Eaten. & LL.VIII.18.7551' \\
\hline$! k h i$ & Boscia sp., ?B. albitrunca & Witgatboom/umbrella-top tree. Fruit eaten. & LL.VIII.29.8526 \\
\hline !horro & ?Generic 'cereal' & Corn & Bleek, 1956:398 \\
\hline gwa: & $? ? ?$ & Thorny. Also used medicine/charm. Eaten. & LL.VIII.21.7830 \\
\hline$s \tilde{a}:$ & Prob. Agathosoma spp. & 'Buchu' (see Glossary, sã:), charm/medicine & LL.VIII.14.7282 \\
\hline Tabbaka, $\|$ keitənsi & Nicotiana sp. & fkun (Bleek, 1956:665), 'oil of tobacco’' & LL.II.6.648 \\
\hline$\| k w a r r i \mid k w a: \xi e$ & $? ? ?$ & Adhesive; used to make a shooting target & $\begin{array}{l}\text { LL.VIII.26.8292 } \\
\text { LL.VIII.8.6687' }\end{array}$ \\
\hline$\neq k a b b u k a n$ & Bush & Resin; tinder. & LL.VIII.21.7833 \\
\hline !gauß̆ukən & ??? & Informant 'did not know' this plant & LL.IV.1.3476 \\
\hline !goe:, !gwe: & Böophane distichia & Malkop-gif(Afr. madness poison) & LL.IV.1.3472 \\
\hline !kanna & Bulb & Bulb had a white interior & LL.IV.1.3479 \\
\hline$\neq k u(\| k a o)$ & $? ? ?$ & A 'vegetable juice' used as poison & LL.VIII.26.8328 \\
\hline !koa: $\xi$ & Bush & Bush used to stop up earths when hunting & LL.VIII.6.6582' \\
\hline$! k^{\prime \prime} w a ! k^{\prime \prime} w a:$ & Tree in mountains & ?Tinder. Branches used for digging sticks & Bleek, 1956:509 \\
\hline
\end{tabular}




\begin{tabular}{|c|c|c|c|}
\hline $\int o-\mid \tilde{o} \ddot{a}$ & Root & Root, medicine/charm. & LL.II.36.3242 \\
\hline$\| k h \tilde{a}:-k a-\odot h o$ & Root (prob. of bush) & Lit. 'Lion's plant'. Medicine/charm & LL.IV.1.inset \\
\hline !gaue-ta-@ho & Root (prob. of bush) & 'Morning's plant'. Medicine/charm & LL.IV.1.inset \\
\hline łherri & Root of bush/tree & $\begin{array}{l}\text { Tree } \text { not } \text { used in bow manufacture } \\
\text { (LL.VIII.23.8059'). Medicine/charm }\end{array}$ & LL.IV.1.inset \\
\hline |hun-ta-@ho & Root (prob. of bush) & Lit. 'Dassie's plant'. Medicine/charm. & LL.IV.1.3425 \\
\hline Kottan-ka-@ho & ?Bush & Lit. 'Partridge's plant'. Medicine/charm. & LL.IV.1.3427 \\
\hline$! k^{\prime \prime}$ wain & Sceletium tortuosum & !k"wain, anger. Kougoed (Afr.) & LL.IV.1.3431 \\
\hline !kurri-|ka-tchuen & Seeds of tree & $\begin{array}{l}\text { !kurrukan, to be dry, hot; !kurri, riverbed; } \\
\text { seeds of a large tree used as medicine/charm }\end{array}$ & LL.IV.1.3436 \\
\hline$\neq i$-tanna & Root & $\begin{array}{l}\text { Lit. 'thoughts/thinking', 'to beg/ask for'. } \\
\text { (Bleek, 1956: 191,652). Medicine/charm. }\end{array}$ & LL.IV.1.3439 \\
\hline Koro-ka-@ho & Bush & $\begin{array}{l}\text { Part above ground used. Lit. Jackal's bush. } \\
\text { Medicine/charm, }\end{array}$ & LL.VIII.21.7830 \\
\hline !nabba, Inabba & Rhigozum sp. ?R. ovobatum & $\begin{array}{l}\text { Driedoorn. Not used for bows } \\
\text { (LL.VIII.23.8059') }\end{array}$ & Bleek, 1956:472 \\
\hline !kwaggan & ?Cadaba aphylla & Swartstorm (Afr. black-storm) tree & Bleek, 1956:458 \\
\hline$\| k^{\prime \prime} e r r i$ & ?Rhamnus sp. & $\begin{array}{l}\text { Dwasdoorn. 'Dwaas' (fool) or 'dwars' } \\
\text { (cross) (Afr.) Branches used in houses. }\end{array}$ & $\begin{array}{l}\text { Bleek, 1956:605 } \\
\text { (LL.II.22.2039') }\end{array}$ \\
\hline !kwerri ( $\ddagger$ ke $\ddagger$ hann $)$ & Acacia giraffae & $\begin{array}{l}\text { Great-thorn (Afr. grootdoring) tree (and its } \\
\text { flower) }\end{array}$ & Bleek, 1956:660 \\
\hline$\neq k u$ k"omm ka|kagən & Dimorphotheca sp. & Large, white daisies. $-k a-\mid k a g \partial n=$ 'women's' & LL.II.33.3023' \\
\hline$\ddagger k u \ddagger k " a m$ & Dimorphotheca annua & Cape Daisy, Ox-Eye, Cape Marigold & Bleek, 1956:665 \\
\hline Nanannase: & Pharnaceum dichotomum & Small white flower & LL.VIII.31.8789' \\
\hline ka & Cotula turbinata & Ganskos (Afr. goose-food), a yellow flower & Bleek, 1956:297 \\
\hline 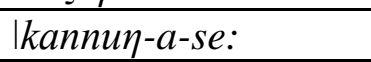 & Poaceae & 'Bank grass' & Bleek, 1956:301 \\
\hline 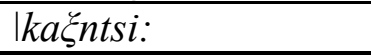 & Tribulus terrestris & Caltrop & Bleek, 1956:301 \\
\hline
\end{tabular}




\begin{tabular}{|c|c|c|c|}
\hline |keinjau, |kainyau & ?Stobaea spp. & 'Little thornbush' & Bleek, 1956:308 \\
\hline$|k u: i t \partial n| k u: i t \partial n$ & 'Bushman bush' & & Bleek, 1956:324 \\
\hline kũ kwai, !gu!gu & ?Podaxis sp. & Not eaten. & Bleek, 1956:325 \\
\hline$\|$ khou & Fungus & Belonged to !khwa:, smelled strongly. & LL.VIII.16.7447 \\
\hline$\neq$ karro & ?Acacia mellifera subsp. detinens & $\begin{array}{l}\text { Haakboom, Hakkis doorn (LL.VIII.23.8059'). } \\
\text { Not used for bows }\end{array}$ & Bleek, 1956:657 \\
\hline$! k a u: \xi a$ & Salsola sp. & $\begin{array}{l}\text { Lye-bush (Afr. loogbos) ash-bush (Afr. } \\
\text { asbos) }\end{array}$ & Bleek, 1956:413 \\
\hline !khuirri & ?Rhigozum sp. & $\begin{array}{l}\text { Grenad-boom; ?wildegranaat (Afr. wild } \\
\text { pomegranate) }\end{array}$ & Bleek, 1956:430 \\
\hline !kui, !khui & Rhigozum trichotomum & Driedoorn; ostrich/sheep eat flowers (\|goa) & LL.VIII.16.7379' \\
\hline !kho!kho & Corycium orobanchoides & Monkshood orchid (Afr. kapotjie) & Bleek, 1956:429 \\
\hline \|xwara & Euphorbia aria & & WB.II.335 \\
\hline !gabba & Brunsvigia josephinae & Josephine's Lily, Candelabra lily & WB.II.335 \\
\hline$\|a\|$ ara & ??? & Belonged to !khwa:, appeared after rainfall & LL.VIII.1.6126 \\
\hline !khau-xu-\|koa & ?Mesembryanthum; ?Lithops & Adheres to stones; 'kruis' (Afr. cross) & LL.VIII.25.8260' \\
\hline$\| k^{\prime \prime}$ ari & ?Acacia sp. & 'Great thorn tree' & WB.V.597' \\
\hline $\operatorname{lga}$ & ?Algae & ' $g a$ ' relates to water (frog, hippopotamus) & WB.XXVII.2538 \\
\hline$\| k w a b b a$ & Aloe sp. & & WB.I.177 \\
\hline !nu!uarase & Harpagophytum procumbens & 'Devil's Claw' & Bleek, 1956:486 \\
\hline$\| n a:$ & $\begin{array}{l}\text { Searsia sp. S. lancea ?S. pyroides, } \\
\text { discolour }\end{array}$ & $\begin{array}{l}\text { Taaibos (Afr. tough-bush). Used to make } \\
\text { stick supports of matjieshuise, and bows. }\end{array}$ & LL.VIII.23.8059' \\
\hline $\begin{array}{l}\text { !nou ssi, } \\
\text { !kauro!kauro }\end{array}$ & Mesembryanthemum glaucum & & LL.VIII.31.8789' \\
\hline$\| x O$ & Mesembryanthemum sp. & & 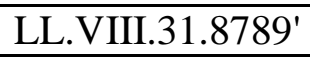 \\
\hline$\|k o a\| k o a$ & Bush & Small, flat, flowered; visited by bees & LL.II.23.2044 \\
\hline
\end{tabular}




\begin{tabular}{|l|l|l|l|}
\hline$!$ kwan-|gerre & Poaceae & Grass. Eaten by springbok & Bleek, 1956:461 \\
\hline |ke:, |khe:, $\mid x e$ & Poaceae & Grass. 'Bread' (Bleek, 1956:306) & Bleek, 1956:306 \\
\hline Tabara, debbi & Tree & 'Cooi-cass-boom' & LL.V.20.5618' \\
\hline
\end{tabular}


Table 6: IXam lithic terminology, translation and uses

\begin{tabular}{|c|c|c|c|}
\hline IXam Name & Reference & Translation of IXam, and likely material & Further information \\
\hline !kue!kuerritan & LL.VIII.27.8433' & $? ? ?$ & $\begin{array}{l}\text { Soft white stone in } \\
\text { Bushmanland; not used for } \\
\text { 'stone knives' }\end{array}$ \\
\hline !kuire & LL.VIII.21.7834 & $? ? ?$ & $\begin{array}{l}\text { White stone, found in IXam- } \\
\text { ka-!xoe }\end{array}$ \\
\hline$S s a-! n w a-\mid k a$ & LL.VIII.21.7834 & ?Eland-arrow-stone. ?Hornfels & $\begin{array}{l}\text { Black stone, found in } \mid X a m-k a- \\
\text { lxoe }\end{array}$ \\
\hline |xwomma & LL.VIII.21.7834 & $? ? ?$ & $\begin{array}{l}\text { Powdered and used for } \\
\text { dressing skins, found in } \\
\text { Bushmanland }\end{array}$ \\
\hline Tto: & LL.VIII.14.7273 & Red haematite & \\
\hline Uhara & LL.VIII.14.7273 & Probably specularite & $\begin{array}{l}\text { Black. With tto:, 'both hard } \\
\text { and soft.' }\end{array}$ \\
\hline$\| k \ddot{a} \xi$ & LL.V.22.5574 & Red haematite & \\
\hline !goa & LL.VIII.1.6086;6085' & Quartz; 'wit-klip' (Afr. white stone) & \\
\hline !gwa!gwara & LL.V.22.5574-5575 & Quartz; brown, rose, crystal. & $\begin{array}{l}\text { Namaqualand and } \\
\text { Bushmanland samples }\end{array}$ \\
\hline !kwa!kwara & LL.VIII.31.8728' & ?Quartz (see above) & 'Shining white stone', \\
\hline
\end{tabular}




\begin{tabular}{|c|c|c|c|}
\hline & & & (Beaufort West) \\
\hline !kou & LL.VIII.31.8728' & 'Like quartz' & $\begin{array}{l}\text { Brown stone spotted with } \\
\text { white. Sample. }\end{array}$ \\
\hline !xwa & LL.V.25.5993' & Boulder & $\begin{array}{l}\text { 'Great stone', hard, round, and } \\
\text { smooth }\end{array}$ \\
\hline !khwa!kweitan & LL.V.22.5806' & Lit. 'Rain's stones' (fell in thunderstorms) & $\begin{array}{l}\text { Black, pointed, and shining } \\
\text { stones }\end{array}$ \\
\hline |kalkaggan & LL.V.21.5639' & 'Blue stones', ?slate or hornfels & $\begin{array}{l}\text { Found on the hill (?hornfels); } \\
\text { 'slate' (Bleek, 1956:305) }\end{array}$ \\
\hline |koulkoutan & LL.V.21.5639' & 'Flat stones', ?slate or shale & $\begin{array}{l}\text { "Like slates"; used for } \\
\text { sharpening arrows }\end{array}$ \\
\hline !karu & LL.V.22.5574 & $\begin{array}{l}\text { Magnetite; copper sulphate (purple copper } \\
\text { ore) }\end{array}$ & In response to samples shown \\
\hline Doro-ka-!kauokan & LL.V.22.5574 & $\begin{array}{l}\text { Lit. 'tinderbox-stones'?Agates; ?fibrous } \\
\text { silica }\end{array}$ & $\begin{array}{l}\text { ?strike-a-lights. In response to } \\
\text { samples shown }\end{array}$ \\
\hline Toi-|xoa-ka-!kauogən & LL.V.22.5574 & $\begin{array}{l}\text { Lit. 'Ostrich stomach's stones', agates, } \\
\text { chalcedonies }\end{array}$ & $\begin{array}{l}\text { From Diamond Drift and Free } \\
\text { State. ?gizzard stones }\end{array}$ \\
\hline Koro-|urru-!ka & LL.V.3.4155' & Jackal-?rectum -?to be stiff/?bone & Red stone; $! k a=? \| k a$ \\
\hline !kui & LL.IV.4.3896' & $\begin{array}{l}\text { Bui-klip (Afr. bui, squall, storm, caprice, } \\
\text { fit) }\end{array}$ & $\begin{array}{l}\text { Used in arrow manufacture, } \\
\text { heat-treated }\end{array}$ \\
\hline$\| k u r r u$ & LL.IV.1.3481 & Quartz, 'flints' & $\begin{array}{l}\text { White or red stones. Word also } \\
\text { meant 'arrowhead/knife' }\end{array}$ \\
\hline
\end{tabular}




\section{Bibliography}

\section{Archival Sources}

Western Cape Archives and Record Service

(Cape Town, South Africa)

Anthing, L. 1863. L. Anthing to the Honourable the Colonial Secretary, 21/04/1863, enclosure in 'Message from His Excellency the Governor, with Enclosures, relative to Affairs in the North-Western Districts of the Colony'. [Printed Government Report]. Cape Town (South Africa). Western Cape Archives: AMPT PUBS CCP 1/2/1/12 [A39], 446-460.

Anthing, L. 1868. L. Anthing to Colonial Secretary, forwarding memorial from Victoria West and Fraserburg inhabitants regarding disturbances at the Orange River, 26/07/1868. [MS. Letter]. Cape Town (South Africa). Western Cape Archives: CO 4150 [41].

Anthing, L. 1868a. L. Anthing 10/08/1868 to Colonial Secretary reporting on raids into the Country at Hartebeest River, by Korannas, 10/08/1868. Cape Town (South Africa). Western Cape Archives: CO 4150 [45].

Bleek, W. H. I. 1873. REPORT of DR. BLEEK concerning his RESEARCHES into the BUSHMAN LANGUAGE and CUSTOMS presented to the Honourable the HOUSE OF ASSEMBLY by command of HIS EXCELLENCY THE GOVERNOR. [Printed Government Report]. Cape Town (South Africa). Western Cape Archives: AMPT PUBS CCP 1/2/1/25 (A17) 
Bleek, W. H. I. 1875. "SECOND REPORT concerning BUSHMAN RESEARCHES with a short account of Bushman Folklore by W.H.I. BLEEK, PhD., Curator of the Grey Library, Foreign Member of the Royal Bavarian Academy of Sciences, \&c." [Printed Government Report] Cape Town (South Africa). Western Cape Archives: AMPT PUBS CCP 1/2/1/27.

Collins, R. 1808. Major Collins' report respecting the Bosjesmans, papers despatched to the Secretary of State, London: General Despatches. [MS.] Cape Town (South Africa). Western Cape Archives: GH 23/2.

Collins, R. 1808a. Journal of a tour to the Northeastern Boundary of the Colony, the Orange River and the Storm Mountains in 'Major Collins' report respecting the Bosjesmans, papers despatched to the Secretary of State, London: General Despatches.' [MS.] Cape Town (South Africa). Western Cape Archives: GH 23/2.

Death Notices. 1849. Avontuur, a Bushman and a shepherd. Death notice. [MS.] Cape Town (South Africa). Western Cape Archives: MOOC 6/9/49 [473]

Death Notices. 1849a. Avontuur, a Bushman. Inventory of the Effects. [MS.] Cape Town (South Africa). Western Cape Archives: MOOC 7/1/199 [137]

Death Notices. 1857. Carolus, a Bushman. Death Notice. [MS.] Cape Town (South Africa). Western Cape Archives: MOOC 6/9/80 (4800)

Garcia, A. 1879. Total amount brought to charge on war account in consequence of the disturbances on the northern border and the Morosi campaign. [Printed Government Report]. Cape Town (South Africa). Western Cape Archives: AMPT PUBS CCP 1/2/1/41 [A27].

House of Assembly. 1880. Report of the Commission appointed to enquire intro and report upon the losses sustained by farmers and other residents on the Northern Border during the late war and rebellion, presented to both Houses of Parliament by command of His Excellency the Governor, 1880, Cape Town: Saul Solomon and Co. [Printed Government Report]. Cape Town (South Africa). Western Cape Archives: AMPT PUBS CCP 1/2/1/40 [G68].

House of Assembly. 1888. Reports and Correspondence relating to Affairs on the Northern Border of the Colony of the Cape of Good Hope. [Printed Government Report]. Cape Town (South Africa). Western Cape Archives: AMPT PUBS CCP 1/2/1/72 [G60].

Jackson, M. J. 1879. Enclosure No. 159, M. J. Jackson, Special Commissioner, to Colonial Secretary, 10/03/1879, in "Disturbances on the Northern Border. Telegrams, Correspondence and other papers" [Printed Government Report] 
Cape Town (South Africa). Western Cape Archives: AMPT PUBS CCP 1/2/1/40 [G61].

Jackson, M. J. 1879a. Annexure No. 5. M. J. Jackson report to the Colonial Secretary, 11/10/1869, in Report on and Papers Connected with affairs on the Northern Border of the Colony of the Cape of Good Hope presented to both Houses of Parliament by Command of His Excellency the Governor. Cape Town: Saul Solomon and Co. [Printed Government Report]. Cape Town (South Africa). Western Cape Archives: AMPT PUBS CCP 1/2/1/40 [G61].

Layton. 1879. Annexure No. 172, 10/04/1878, Under-Colonial Secretary and His Excellency Colonel Layton, in "Disturbances on the Northern Border. Telegrams, Correspondence and other papers" [Printed Government Report] Cape Town (South Africa). Western Cape Archives: AMPT PUBS CCP 1/2/1/40 [G61].

Memorials Received. 1846. Inhabitants of the ward of Onder-Roggeveld to His Excellency the Governor Sir Peregrine Maitland, 02/07/1846. [MS. Letter] Cape Town (South Africa). Western Cape Archives: CO 4030 [566]

Nightingale, P. 1888. Percy Nightingale, inspecting Civil Commissioner to Under Colonial Secretary, 08/07/1887, in Reports and Correspondence relating to Affairs on the Northern Border of the Colony of the Cape of Good Hope. [Printed Government Report]. Cape Town (South Africa). Western Cape Archives: AMPT PUBS CCP 1/2/1/72 [G60].

Redelinghuis, J. N. 1834. Jacobus Nicolaas Redelinghuis commandant of the Northern Frontier, to Governor Benjamin D'Urban. [MS. Letter] Cape Town (South Africa). Western Cape Archives: CO 3973 [95]

Ryneveld. 1846. Report from the Civil Commissioner's office and Clan William, 27/07/1846, in Memorials Recieved. [MS. Letter] Cape Town (South Africa). Western Cape Archives: CO 4030 [566]

Ryneveld. 1846. Ryneveld to Veldcornet Nel, 25/06/1846, in Memorials Recieved, annexure no.3 [MS. Letter] Cape Town (South Africa). Western Cape Archives: CO 4030 [566]

Smit, J. 1823. Memorial of Jacobus Smit to Governor H. Somerset, 13/06/1823 [MS. Letter]. Cape Town (South Africa). Western Cape Archives: CO 3924 [204].

Upington, T. 1879. Report on and Papers Connected with affairs on the Northern Border of the Colony of the Cape of Good Hope presented to both Houses of Parliament by Command of His Excellency the Governor. Cape Town: Saul 
Solomon and Co. [Printed Government Report]. Cape Town (South Africa). Western Cape Archives: AMPT PUBS CCP 1/2/1/40 [G61].

Wade, I. 1832. Lieutenant Colonel I. Wade to Right Honourable E. G. Stanley, 10/12/1832. [MS. Letter]. Cape Town (South Africa). Western Cape Archives: $\mathrm{GH} 23 / 10$ [11A]

Wodehouse, H. P. E. 1868. Letter to Duke of Buckingham from H.P.E Wodehouse, from Cape Town 08/10/1868. [MS. Letter]. Cape Town (South Africa). Western Cape Archives: GH 23/30 [92].

\section{Published Historical Sources (Eighteenth and Nineteenth}

\section{Century)}

Bird, W.W. and Colebrooke, H.T. 1823. State of the Cape of Good Hope in 1822. London: John Murray.

Bleek, W.H.I. 1874. A few Remarks on Orpen's "A Glimpse into the Mythology of the Maluti Bushmen". Cape Monthly Magazine 9: 10-13.

Bleek, W.H.I. 1874a. On Resemblances in Bushman and Australian Mythology, with Preliminary Remarks. Cape Monthly Magazine 8: 98-102.

Bleek, W.H.I. 1875. Bushman Researches. Cape Monthly Magazine 11: 104-115, 150-155.

Bleek, W.H.I. 1875a. A Brief Account of Bushman Folk-lore and other Texts. Second Report Concerning Bushman Researches Presented to Both Houses of Parliament of the Cape of Good Hope. Cape Town: Juta.

Bleek, W.H.I. 1864. Reynard the Fox in South Africa; or, Hottentot Fables and Tales, Chiefly Translated from Original Manuscripts in the Library of His Excellency Sir George Grey, K.C.B. London: Trübner and Co.

Burchell, W.J. 1822. Travels in the Interior of Southern Africa, Volume 1. London: Longman, Hurst, Rees, Orme and Brown.

Burchell, W.J. 1824. Travels in the Interior of Southern Africa, Volume 2. London: Longman, Hurst, Rees, Orme and Brown. 
Daniell, S. 1804-1805. African Scenery and Animals. London: S. Daniell.

Dunn, E.J. 1872. Through Bushmanland (Part 1). Cape Monthly Magazine Volume V, No. 30: 374-384.

Dunn, E.J. 1873. Through Bushmanland (Part 2). Cape Monthly Magazine Volume VI, No. 31: 31-42.

Kicherer, J. 1804. Rev. Mr. Kicherer's Narrative of his Mission to the Hottentots. In Transactions of the Missionary Society (1804) containing The Rev. Mr. Kicherer's Narrative of his Mission to the Hottentots and Boschemen: with a General Account of the South African Missions. Number 1: Volume II. London: Bye and Law. Pp. 1-48.

Le Vaillant, F. 1790. Travels into the Interior Parts of Africa, 2 vols. London: Robinson.

Lichtenstein, H. 1812. Travels in Southern Africa, in the years 1803, 1804, 1805, and 1806 Volume I. (translated from the German, by A. Plumptre). London: Henry Colburn.

Livingstone, D. 1857. Missionary travels and researches in South Africa: including a sketch of sixteen years' residence in the interior of Africa, and a journey from the Cape of Good Hope to Loanda on the west coast; thence across the continent, down the River Zambesi, to the eastern ocean. London: John Murray.

Lloyd, L.C. 1889. A short account of further Bushman material collected. Third report concerning Bushman Researches, present to both House of the Parliament of the Cape of Good Hope, by command of His Excellency the Governor. London: David Nutt.

Lloyd, L.C. 1880. Bushman folklore. Folklore Journal II: III: 39-43.

Locke, J. 1764 [1689]. Two treatises of government. London: A. Millar, M. Woodfall, I. Whiston and B. White, I. Rivington, L. Davis and C. Reymers, R. Baldwin, Hawes Clark and Collins; W. Johnston; W. Owen, I. Richardson, S. Crowder, T. Longman, B. Law, C. Rivington, E. Dilly, R. Withy, C. and R. Ware, S. Baker, T. Payne, A. Shuckburgh, L. Hinxman.

Moffat, R. 1858. Journey from Colesberg to Steinkopf in 1854-5. Journal of the Royal Geographical Society of London, Vol. 28: 153-173. 
Orpen, J.M. 1874. "A glimpse into the mythology of the Maluti Bushmen". Cape Monthly Magazine 9: 1-13.

Sparrman, A.E. 1785. A Voyage to the Cape of Good Hope, towards the Antarctic Polar Circle, and Round the World, but chiefly into the Country of the Hottentots and Caffres, from the year 1772, to 1776 Volume I. London: G.G.J. and J. Robinson.

Thomson, A.T. 1832. Elements of Materia Medica and Therapeutics, including the recent discoveries and analyses of medicines (Volume 1) London: Longman, Rees, Orme, Brown, Green, and Longman.

Tylor, E.B. 1871. Primitive Culture: researches into the development of mythology, philosophy, religion, are, and custom (Volume 1). London: John Murray.

\section{Unpublished Theses and Reports}

Besten, M.P. 2006. Transformation and reconstitution of Khoe-San identities: A.A.S. le Fleur 1, Griqua identities and post-apartheid Khoe-San revivalism (1894-2004). Unpublished $\mathrm{PhD}$ thesis, Universiteit Leiden.

Challis, W. 2008. The impact of the horse on the Amatola 'Bushmen': new identity in the Maloti-Drakensberg mountains of southern Africa. Unpublished D. Phil. thesis, University of Oxford.

Low, C. 2004. Khoisan healing: understandings, ideas and practices. Unpublished D.Phil. thesis, University of Oxford.

McKay, A. 2009. History and Selection in the Late Pleistocene archaeology of the Western Cape, South Africa. Unpublished $\mathrm{PhD}$ thesis, Australian National University.

(http://anu.academia.edu/AlexMacKay/Books/171380/History_and_selection_in_t he_late_Pleistocene_archaeology_of_the_Western_Cape_South_Africa_Volume_ 1_Text_) (accessed 26/08/2011). 
Morris, D. 2002. Driekopseiland and 'the rain's magic power': history and landscape in a new interpretation of a Northern Cape rock engraving site. Unpublished Masters Thesis, University of the Western Cape.

Parsons, I. 2006. Later Stone Age socio-economic variability during the last 2000 years in the Northern Cape, South Africa. Unpublished PhD thesis, University of Cambridge.

Wiessner, P. 1977. Hxaro, a regional system of reciprocity for reducing risk among the !Kung San. Unpublished PhD Thesis, University of Michigan.

\section{Published Sources}

Acocks, J.P.H. 1953. Veld types of South Africa. Memoirs of the Botanical Survey of South Africa, No. 28: 1-192. Pretoria: Botanical Research Institute.

Acocks, J.P.H. 1975. Veld Types of South Africa. Memoirs of the Botanical Survey of South Africa, No. 40: 1-128. Pretoria: Botanical Research Institute.

Anderson, E. 1987. A History of the Xhosa of the Northern Cape, 1795-1879. Centre for African Studies Communications No. 12. Cape Town: University of Cape Town.

Anderson, V.D. 2004. Creatures of Empire: How Domestic Animals Transformed Early America. Oxford: Oxford University Press.

Appadurai, A. (ed.) 1986. The Social Life of Things: Commodities in Cultural Perspective. Cambridge: Cambridge University Press.

Apps, P. 2000. Wild Ways: Field Guide to the Behaviour of Southern African Mammals ( ${ }^{\text {nd }}$ Edition). Cape Town: Struik Publishers.

Archer, S. 2000. Technology and Ecology in the Karoo: A Century of Windmills, Wire and Changing Farming Practice. Journal of Southern African Studies 26:4: 675-696.

Arkush, B.S. 1987. Historic Northern Paiute Winter Houses in Mono Basin, California. Journal of California and Great Basin Anthropology 9:2:174-187. 
Arkush, B.S. 1990. The Protohistoric Period in the Western Great Basin. Journal of California and Great Basin Anthropology 12:1: 28-36.

Armstrong Oma, K. 2010. Between Trust and Domination: Social Contracts between Humans and Animals. World Archaeology 42:2:175-187.

Arnold, J.E. 1996. The Archaeology of Complex Hunter-gatherers. Journal of Archaeological Method and Theory 3:1:77-126.

Bailey, R.C. 1988. The Significance of Hypergyny for Understanding Subsistence Behaviour among Contemporary Hunters and Gatherers. In Kennedy, B.V. and LeMoine, G.M. (eds.) Diet and Subsistence: Current Archaeological Perspectives. Calgary: University of Calgary Archaeological Association. Pp. 5765.

Bank, A. 2000. Evolution and Racial Theory: The Hidden Side of Wilhelm Bleek. South African Historical Journal 43:1: 163-178.

Bank, A. 2006. Bushmen in a Victorian World: The Remarkable Story of the Bleek-Lloyd Collection of Bushman Folklore. Cape Town: Double Storey.

Barnard, A. (ed.) 2004. Hunter-Gatherers in History, Archaeology, and Anthropology. Oxford: Berg.

Barnard, A. 1992. Hunters and Herders of Southern Africa: a Comparative Ethnography of the Khoisan Peoples. Cambridge: Cambridge University Press.

Barnard, A. 2002. The Foraging Mode of Thought. In Stewart, H., Barnard, A. and Omura, K. (eds.) Self- and other images of hunter-gatherers. SENRI Ethnological Studies 60: 5-24.

Barnard, A. 2008. Ethnographic Analogy and the Reconstruction of Early Khoekhoe Society. Southern African Humanities 20: 61-75.

Barnard, A. 1992. The Kalahari Debate: A Bibliographic Essay. University of Edinburgh: Centre of African Studies, Occasional Paper No. 35.

Bauman, R. 1992. Folklore, Cultural Performances, and Popular Entertainments. Oxford: Oxford University Press.

Beaumont, P.B. and Vogel, J.C. 1989. Patterns in the Age and Context of Rock Art in the Northern Cape. South African Archaeological Bulletin 44:73-81. 
Beaumont, P.B. and Vogel, J.C. 2006. On a Timescale for the Past Million Years of Human History in Central South Africa. South African Journal of Science 102: 217-228.

Beaumont, P.B., Smith, A.B., and Vogel, J.C. 1995. Before the Einiqua: The Archaeology of the Frontier Zone. In Smith, A.B. (ed.) Einiqualand: Studies of the Orange River Frontier. Cape Town: University of Cape Town. Pp. 236-264.

Beck, R.B. 2000. A History of South Africa. Westport: Greenwood.

Beinart, W. 1990. Empire, Hunting and Ecological Change in Southern and Central Africa. Past \& Present 128: 162-186.

Beinart, W. 1998. The Night of the Jackal: Sheep, Pastures and Predators in the Cape. Past and Present 158:1: 172-206.

Beinart, W. 2003. The Rise of Conservation in South Africa: Settlers, Livestock, and the Environment 1770-1950. Oxford: Oxford University Press.

Bellesiles, M. 1996. The Origins of Gun Culture in the United States, 1760-1865. The Journal of American History 83:2: 425-455.

Bellesiles, M. 2002. Arming America: the Origins of a National Gun Culture. Brooklyn: Soft Skull Press.

Beningfield, J. 2006. The Frightened Land: Land, Landscapes and Politics in South Africa in the Twentieth Century. Oxford: Routledge.

Berlin, I. and Kay, P. 1969. Basic Color Terms: their Universality and Evolution. Berkeley: University of California Press.

Bettinger, R. 1991. Hunter-gatherers: Archaeological and Evolutionary Theory. New York: Plenum Press.

Biesele, M. 1993. Women like Meat: the Foraging and Folklore Ideology of the Kalahari Jul'hoan. Bloomington: University of Indiana Press.

Binford, L. 1980. Willow Smoke and Dogs' Tails: Hunter-gatherer Settlement Systems and Archaeological Site Formation. American Antiquity 45:1: 4-20.

Bird, D.W., Bird, R.B. and Parker, C.H. 2005. Aboriginal Burning Regimes and Hunting Strategies in Australia's Western Desert. Human Ecology 33:4: 443-464.

Bird-David, N. 1990. The Giving Environment: Another Perspective on the Economic System of Gatherer-hunters. Current Anthropology 31: 183-196. 
Bird-David, N. 1999. "Animism" Revisited: Personhood, Environment, and Relational Epistemology. Current Anthropology 40, Supplement: S67-S91

Birmingham, J. 2000. Resistance, Creolization or Optimal Foraging at Killalpaninna Mission, South Australia. In Torrence, R. and Clarke, A. (eds.) The Archaeology of Difference: Negotiating Cross-cultural Engagements in Oceania. London: Routledge. Pp. 368-414.

Blackburn, R.H. 1982. In the Land of Milk and Honey: Okiek Adaptations to their Forests and Neighbours. In Leacock, E. and Lee, R. Politics and History in Band Societies. Cambridge: Cambridge University Press. Pp. 283-306.

Bleek, D.F. 1923. The Mantis and His Friends: Bushman Folklore from Material Collected by the Late Dr. W. H. I. Bleek and the Late Dr. Lucy C. Lloyd. Cape Town: Maskew Miller.

Bleek, D.F. 1924. Bushman Terms of Relationship. Bantu Studies 2:2: 57-70.

Bleek, D.F. 1927. The Distribution of Bushman Languages in South Africa. In Boas, F., Dempwolff, O.H., Panconcelli-Calzia, G. (eds.) Festschrift Meinhof. Glückstadt and Hamburg: J.J. Augustin. Pp. 55-64.

Bleek, D.F. 1931. Customs and Beliefs of the !Xam Bushmen, 1: Baboons. Bantu Studies 5: 167-179.

Bleek, D.F. 1932. Customs and Beliefs of the /Xam Bushmen, 2: the Lion. Bantu Studies 6: 47-63.

Bleek, D.F. 1932a. Customs and Beliefs of the /Xam Bushmen, 3: Game Animals. Bantu Studies 6: 233-249.

Bleek, D.F. 1932b. Customs and Beliefs of the /Xam Bushmen, 4: Omens, Windmaking, Clouds. Bantu Studies 6: 323-342.

Bleek, D.F. 1933. Customs and Beliefs of the /Xam Bushmen, 5: the Rain. Bantu Studies 7: 297-312.

Bleek, D.F. 1933a. Beliefs and Customs of the /Xam Bushmen, 6: Rain-making. Bantu Studies 7: 375-392.

Bleek, D.F. 1935. Customs and Beliefs of the /Xam Bushmen, 7: Sorcerors. Bantu Studies 9:1: 1-47.

Bleek, D.F. 1936. Customs and Beliefs of the /Xam Bushmen, 8: More About Sorcerors and Charms. Bantu Studies 10: 131-162. 
Bleek, D.F. 1936a. Special Speech of Animals and Moon used by the /Xam Bushmen. Bantu Studies 10: 163-199.

Bleek, D.F. 1936b. Notes on the Bushman Photographs. Bantu Studies 10: 201203.

Bleek, D.F. 1956. A Bushman Dictionary (American Oriental Series 41). New Haven; American Oriental Society.

Bleek, D.F. 2011 [1929]. Comparative Vocabularies of Bushman Languages. Cambridge: Cambridge University Press.

Bleek, W.H.I. and Lloyd, L.C. 1911. Specimens of Bushman Folklore. London: George Allan.

Blurton-Jones, N. and Konner, M.J. 1989. !Kung Knowledge of Animal Behaviour. In Johannes, R. E. (ed.) Traditional Ecological Knowledge: a Collection of Essays. Cambridge: IUCN. Pp. 21-30.

Bollong, C.A., Sampson, C.G., and Smith, A.B. 1997. Khoikhoi and Bushman Pottery in the Cape Colony: Ethnohistory and Later Stone Age Ceramics of the South African Interior. Journal of Anthropological Archaeology, 16:3:269-299.

Boonzaier, E., Berens, P., Malherbe, C., and Smith. A. 1996. The Cape Herders: a History of the Khoikhoi of Southern Africa. Cape Town: David Philip.

Bosch, C.H. 2004. Cucumis africanus. In Grubben, G. J. H. and Denton, O.A. (eds.) Plant Resources of Tropical Africa 2: Vegetables. Wageningen: PROTA Foundation and CTA; Leiden: Backhuys Publishers. Pp. 237-238.

Boshoff, A.F. and Kerley, G.I.H. 2010. Historical Mammal Distribution Data: How Reliable are Written Records? South African Journal of Science 106:1-2:2633.

Bottomley, A.M. and Fuller, C. 1921. The Fungus Foods of Certain Termites. The South African Journal of Natural History III:1: 139-144.

Bourdieu, P. 1977 [1972]. Outline of a Theory of Practice (translated by R. Nice). Cambridge: Cambridge University Press.

Bousman, B. 2005. Coping with Risk: Later Stone Age Technological Strategies at Blydefontein Rock Shelter, South Africa. Journal of Anthropological Archaeology 24:193-226. 
Boycott, R.C. and Bourquin, O. 1988. The South African Tortoise Book: a Guide to South African tortoises, Terrapins and Turtles. Johannesburg: Southern Book.

Brightman, R. 1996. The Sexual Division of Foraging Labor: Biology, Taboo, and Gender Politics. Comparative Studies in Society and History 38: 687-729.

Brink, Y.L. 1990. The Voorhuis as a Central Element in Early Cape Houses. Social Dynamics: a Journal of African Studies 16:1: 38-54.

Bronstein, J.L., Izhaki, I, Nathan, R., Tewksbury, J.J., Spiegel, O., Lotan, A., and Altstein, O. 2007. Fleshy-fruited Plants and Frugivores in Desert Ecosystems. In Dennis, A.J., Schupp, E.W., Green, R.A. and Westcott, D.A. (eds.) Seed Dispersal: Theory and its Application in a Changing World. Wallingford: CAB International. Pp. 148-177.

Brown, K. 2008. Frontiers of Disease: Human Desire and Environmental Realities in the Rearing of Horses in Nineteenth and Twentieth Century South Africa. African Historical Review 40:1: 30-57.

Browne, J. 2009. The Voyages of Charles Darwin: Travels in South America. ReVista: Harvard review of Latin America May 2009: 6-10.

Burke, A. 2002. Properties of Soil Pockets on Arid Nama Karoo Inselbergs - the Effect of Geology and Derived Landforms. Journal of Arid Environments 50:219234.

Campbell, I. 1995. The Indigenous Sheep and Goat Breeds of South Africa. Bloemfontein: Dreyer Printers and Publishers.

Catling, P.M. 2001. Extinction and the Importance of History and Dependence in Conservation. Biodiversity 2: 2-13.

Challis, W. 2009. Taking the Reins: The Introduction of the Horse in the Nineteenth-Century Maloti-Drakensberg and the Protective Medicine of Baboons. In Mitchell, P. and Smith, B. (eds.) The Eland's People: New Perspectives in the Rock Art of the Maloti-Drakensberg Essays in Memory of Patricia Vinnicombe. Johannesburg: Wits University Press. Pp. 104-107.

Chapman, A.M. 1982. Drama and Power in a Hunting Society: the Selk'nam of Tierra del Fuego. Cambridge: Cambridge University Press.

Chapman, A.M. n.d. Economic and Social Structure of Selk'nam Society. http://www.thereedfoundation.org/rism/chapman/ecosocial.htm. (Accessed on 08/02/2011). 
Charlton, A. 2004. Medicinal Uses of Tobacco in History. Journal of the Royal Society of Medicine 97:6: 292-296.

Chazan, M., Ron, H., Matmon, A., Porat, N., Goldberg, P., Yates, R., Avery, M., Sumner, A., \& Horwitz, L.K. 2008. Radiometric Dating of the Earlier Stone Age Sequence in Excavation I at Wonderwerk Cave, South Africa: Preliminary Results. Journal of Human Evolution 55:1-11.

Chevallier, N. and Ashton, B. 2006. A Report on the Porcupine Quill Trade in South Africa. Report commissioned by Internation Fund for Animal Welfare. http://www.ifaw.org/Publications/Program_Publications/Wildlife_Trade/Campaig n_Scientific_Publications/asset_upload_file1000_12480.pdf. (Downloaded 03/11/2011).

Claeys, G. Imperial Sceptics: British Critics of Empire, 1850-1920. Cambridge: Cambridge University Press.

Clark, H.O. 2005. Otocyon megalotis. Mammalian Species 766: 1-5.

Clark, N. 2007. Animal Interface: the Generosity of Domestication. In Cassidy, R. and Mullin, M. (eds.) Where the Wild Things are Now. Oxford: Berg. Pp. 49-70.

Clutton-Brook, J. 1995. Origins of the Dog: Domestication and Early History. In Serpell, J. (ed.) The Domestic Dog: its Evolution, Behaviour and Interactions with People. Cambridge: Cambridge University Press. Pp. 7-20.

Coetzee, J.M. 1986. Farm Novel and "Plaasroman" in South Africa. English in Africa 13:2:1-19.

Comaroff, J. and Comaroff, J. 1991. Of Revelation and Revolution: Christianity, Colonialism, and Consciousness. Chicago: University of Chicago Press

Conklin, H.C. 1955. Hanunóo Color Categories. Southwestern Journal of Anthropology 11:4: 339-344.

Coombes, A. (ed.) 2005. Rethinking Settler Colonialism: History and Memory in Australia, Canada, New Zealand and South Africa. Manchester: Manchester University Press.

Cooper, R.G., Horbańczuk, J.O., Villegas-Vizcaíno, R., Sebei, S.K., Mohammed, A.E.F., Mahrose, K.M.A. 2010. Wild Ostrich (Struthio camelus) Ecology and Physiology. Tropical Animal Health and Production 42:363-373.

Crane, E. 2009. Bee Products. In Schowalter, T. D. (ed.) Insect Ecology: an Ecosystem Approach (2 ${ }^{\text {nd }}$ Edition). Burlington: Academic Press. Pp. 71-75. 
Crosby, A.W. 2004. Ecological Imperialism: the Biological Expansion of Europe, 900-1900 (2 ${ }^{\text {nd }}$ Edition). Cambridge: Cambridge University Press.

Cunningham, A.B. and Davis, G.W. 1997. Human Use of Plants. In Cowling R.M., Richardson, D.M. and Pierce, S.M. (eds.) Vegetation of Southern Africa. Cambridge: Cambridge University Press.

Curtis, B.A. 1985. Temperature Tolerances in the Namib Desert Dune Ant, Camponotus detritus. Journal of Insect Physiology 31:6: 463-466.

Dane, F. and Liu, J. 2007. Diversity and Origin of Cultivated and Citron Type Watermelon (Citrullus lanatus). Genetic Resources and Crop Evolution 54: 12551265 .

de Castro, E.V. 1998. Cosmological Deixis and Amerindian Perspectivism. Journal of the Royal Anthropological Institute 4:3: 469-488.

de Foliart, G.R. 1992. Insects as Human Food. Crop Protection 11:395-399.

de Jongh, M. 2002. No Fixed Abode: the Poorest of the Poor and Elusive Identities in Rural South Africa. Journal of Southern African Studies 28:2: 441460 .

de Prada-Samper, J.M. 2007. The Plant Lore of the IXam San: \|kabbo and łkasin's Identification of 'Bushman Medicines'. Culturas populares. Revista Electrónica 4: $1-17$.

Deacon, H. 1998. Midwives and Medical Men in the Cape Colony before 1860. The Journal of African History 39:2: 271-292.

Deacon, H. 2004. The Cape Doctor and the Broader Medical Market, 1800-1850. In Deacon, H., Philips, H., and van Heyningen, E. (eds.) The Cape Doctor in the Nineteenth Century: a Social History. New York: Rodopi B.V. Pp. 45-84.

Deacon, H.J. 1993. Planting an Idea: an Archaeology of Stone Age Gatherers in South Africa. South African Archaeological Bulletin 48:86-93.

Deacon, H.J. and Deacon, J. 1999. Human Beginnings in South Africa: Uncovering the Secrets of the Stone Age. Claremont: David Philip Publishers.

Deacon, J. 1984. The Later Stone Age of Southernmost Africa (Cambridge Monographs in African Archaeology No. 12). Oxford: British Archaeological Reports. 
Deacon, J. 1986. 'My Place is the Bitterpits': the Home Territories of Bleek and Lloyd's IXam Informants. African Studies 45:2: 135-155.

Deacon, J. 1988. The Power of a Place in Understanding Southern San Rock Engravings. World Archaeology 20:1: 129-140.

Deacon, J. 1992. Arrows as Agents of Belief Amongst the XXam Bushmen. (Margaret Shaw Lecture 3). Cape Town: South African Museum.

Deacon, J. 1996. The /Xam Informants. In Deacon, J. and Dowson, T.A. Voices from the Past: XXam Bushmen and the Bleek and Lloyd Collection. Johannesburg: Witwatersrand University Press. Pp. 11-39.

Deacon, J. 1996a. Archaeology of the Flat and Grass Bushmen. In Deacon, J. and Dowson, T.A. (eds.) Voices from the Past: /Xam Bushmen and the Bleek and Lloyd Collection. Johannesburg: Witwatersrand University Press. Pp. 245-270.

Deeming, D.C. and Bubier, N.E. 1999. Behaviour in Natural and Captive Environments. In Deeming, D. C. (ed.) The Ostrich: Biology, Production and Health. Wallingford; CABI Publishing. Pp. 83-104.

Dégh, L. 2001. Legend and Belief: Dialectics of a Folklore Genre. Bloomington: University of Indiana Press.

Denham, T. 2008. Traditional Forms of Plant Exploitation in Australia and New Guinea: the Search for Common Ground. Vegetation History and Archaeobotany: 17: $245-248$.

Denham, T., Donohue, M., and Booth, S. 2009. Horticultural Experimentation in Northern Australia Reconsidered. Antiquity 83: 634-648.

Descola, P. 1992. Societies of Nature and the Nature of Society. In Kuper, A (ed.) Conceptualizing Society. London: Routledge.

Descola, P. 1996. Constructing Natures: Symbolic Ecology and Social Practice. In Descola, P. Pálsson, G. (eds.) Nature and Society: Anthropological Perspectives. London: Routledge. Pp. 82-102.

Descola, P. 2010. From Wholes to Collectives. In Otto, T. and Bubandt, N. (eds.) Experiments in Holism: Theory and Practice in Contemporary Anthropology. Oxford: Blackwell Publishing. Pp. 209-226.

Desmet, P.G. and Cowling, R.M. 1999. The Climate of the Karoo - a Functional Approach. In Dean, W.R.J. and Milton, S.J. (eds.) The Karoo: Ecological Patterns and Processes. Cambridge: Cambridge University Press. Pp. 3-16. 
Deumert, A. 2004. Language Standardization and Language Change: the Dynamics of Cape Dutch. Amsterdam: John Benjamin B.V.

Dewar, G., Halkett, D., Hart, T., Orton, J., and Sealy, J. 2006. Implications of a Mass Kill Site of Springbok (Antidorcas marsupialis) in South Africa: Hunting Practice, Gender Relations and Sharing. Journal of Archaeological Science 33: 1266-1275.

Digby, A. 2005. Self-medication and the Trade in Medicine within a Multi-ethnic Context: a Case Study of South Africa from the Mid-nineteenth to the Midtwentieth Centuries. Social History of Medicine 18:3: 439-457.

Dirks, N.B. 1992. Introduction. In Dirks, N.B. (ed.) Colonialism and Culture. Michigan: University of Michigan Press. Pp. 1-26.

Donlan, C.J., Berger, J., Bock, C.E., Bock, J.H., Burney, D.A., Estes, J.A., Foreman, D., Martin, P.S., Roemer, G.W., Smith, F.A., Soulé, M.E., and Greene, H.W. 2006. Pleistocene Rewilding: an Optimistic Agenda for Twenty-first Century Conservation. The American Naturalist 168:5: 660-681.

Dowson, T.A. 1989. Dots and Dashes: Cracking the Entoptic Code in Bushman Rock Paintings. South African Archaeological Society Goodwin Series 6: 84-94.

Dowson, T.A. 1992. Rock Engravings of Southern Africa. Johannesburg: University of Witwatersrand Press.

Dowson, T.A. 2007. Debating Shamanism in Southern African Rock Art: Time to Move on ... . South African Archaeological Bulletin 62:185: 49-61.

Dowson, T.A. 2009. Re-animating Hunter-gatherer Rock Art Research. Cambridge Archaeological Journal 19: 378-387.

Draper, P. 1978. The Learning Environment for Aggression and Anti-social Behaviour among the !Kung. In Montagu, A. (ed.) Learning Non-aggression: the Experience of Non-literate Societies. Oxford: Oxford University Press. Pp. 31-53.

duBois, L. 2005. Avengers of the New World: the Story of the Haitian Revolution. Cambridge: Harvard University Press.

Elbourne, E. 2002. Blood Ground: Colonialism, Missions, and the Contest for Christianity in the Cape Colony and Britain, 1799-1853. Montreal \& Kingston: McGill-Queen's University Press.

Eliade, M. 1972. Shamanism: Archaic Techniques of Ecstasy. New York: Routledge \& Kegan Paul. 
Ellis, F. and Lambrechts, J.J.N. 1986. Chapter 2: Soils. In Cowling, R.M., Roux, P.W., and Pieterse, A.J.H. (eds.) The Karoo Biome: a Preliminary Synthesis. Part I - Physical Environment (South African National Scientific Programmes Report No. 124). Pretoria: CSIR. Pp. 18-38.

Elphick, R. 1977. Kraal and Castle: Khoikhoi and the Founding of White South Africa. New Haven: Yale University Press.

Elphick, R. and Giliomee, H. (eds.) 1979. The Shaping of South African Society: 1652-1820. Cape Town: Longman Penguin Southern Africa.

Engelbrecht, J.A. 1936. The Korana: An Account of their Customs and their History with Texts. Cape Town: Maskew Miller.

Estes, R.D. 1991. The Behaviour Guide to African Mammals Including Hoofed Mammals, Carnivores, Primates. Berkeley and Los Angeles: University of California Press.

Etherington, N. 2001. The Great Treks: the Transformation of Southern Africa 1815-1855. Harlow: Longman.

Fauvelle-Aymar, F-X. 2008. Against the 'Khoisan paradigm' in the Interpretation of Khoekhoe Origins and History: a Re-evaluation of Khoekhoe Pastoral Traditions. Southern African Humanities 20: 77-92.

Feinstein, C.H. 2005. An Economic History of South Africa. Cambridge: Cambridge University Press.

Finzsch, N. 2005. 'It is Scarcely Possible to Conceive that Human Beings could be so Hideous and Loathsome': Discourses of Genocide in Eighteenth- and Nineteenth-century America and Australia. Patterns of Prejudice 39:2:97-115.

Foucault, M. 1975. The Birth of the Clinic: an Archaeology of Medical Perception (English ed.) New York: Vintage Books.

Foucault, M. 1977. Discipline and Punish: the Birth of the Prison (English ed.) London: Allen Lane.

Foucault, M. 2001 [1964]. Madness and Civilisation: a History of Insanity in the Age of Reason. London: Routledge.

Gammage, B. 2005. “...far more happier than we Europeans”: Aborigines and Farmers (London Papers in Australian Studies No. 12). London: Menzies Centre for Australian Studies, King's College London. 
Geist, V. 1985. On Pleistocene Bighorn Sheep: Some Problems of Adaptation, and Relevance to Today's American Megafauna. Wildlife Society Bulletin 13:3: 351-359.

Georges, R.A and Jones, M.O. 1995. Folkloristics: an Introduction. Bloomington: Indiana University Press.

Guelke, L. 1985. "The Making of Two Frontier Communities: Cape Colony in the Eighteenth Century." Historical Reflections/Reflexions Historiques 12:3: 419-48

Gifford-Gonzalez, D. 2000. Animal Disease Challenges to the Emergence of Pastoralism in Sub-Saharan Africa. African Archaeological Review 17:3: 95-139.

Giliomee, H. 1979. The Eastern Frontier, 1770-1812. In Elphick, R. and Giliomee, H. (eds.) The Shaping of South African Society: 1652-1820. Cape Town: Longman Penguin Southern Africa.

Given, M. 2004. The Archaeology of the Colonized. London: Routledge.

Glazer, J.L. 2005. Management of Heatstroke and Heat Exhaustion. American Family Physician 71:11: 2133-2140.

Glen, A.S. and Dickman, C.R. 2005. Complex Interactions among Mammalian Carnivores in Australia, and their Implications for Wildlife Management. Biological Reviews 80: 387-401.

Goodwin, J. 1945. Some Historical Bushman Arrows. South African Journal of Science 41: 429-443.

Gordon, D. 1996. From Rituals of Rapture to Dependence: the Political Economy of Khoikhoi Narcotic Consumption, c. 1487-1870. South African Historical Journal 35:1:62-88.

Gordon. R. 1992. The Bushman Myth: the Making of a Namibian Underclass. Oxford: Westview.

Görgens, A.H.M. and Hughes, D.A. 1986. Chapter 4: Hydrology. In Cowling, R.M., Roux, P.W., and Pieterse, A.J.H. (eds.) The Karoo Biome: a Preliminary Synthesis. Part I - Physical Environment (South African National Scientific Programmes Report No. 124). Pretoria: CSIR. Pp. 53-83.

Gosden, C. 2004, Archaeology and Colonialism: Cultural Contact from 5000 BC to the Present. Cambridge: Cambridge University Press. 
Greer, S., Harrison, R., and McIntyre-Tamwoy, S. 2002. Community-based Archaeology in Australia. World Archaeology 34:2: 265-287.

Grootenhuis, J.G. 2000. Wildlife, Livestock and Animal Disease Reservoirs. In Prins, H. H.T., Grootenhuis, J.G., and Dolan, T.T. (eds.) Wildlife Conservation by Sustainable Use (Conservation Biology Series). Dordrecht: Kluwer Academic Publishers. Pp. 81-114.

Guelke, L. 1985. The Making of Two Frontier Communities: Cape Colony in the Eighteenth Century. Historical Reflections/Reflexions Historiques 12:3: 419-48.

Guenther, M.G. 1986. The Nharo Bushmen of Botswana: Tradition and Change. Hamburg: Helmut Buske Verlag.

Guenther, M.G. 1989. Bushman Folktales: Oral Traditions of the Nharo of Botswana and the /Xam of the Cape. Stuttgart: Franz Steiner Verlag.

Guenther, M.G. 1999. Tricksters and Trancers: Bushman Religion and Society. Bloomington: Indiana University Press.

Guenther, M.G. 2002. The Bushman Trickster: Protagonist, Divinity and Agent of Creativity. Marvels and Tales: Journal of Fairy-Tale Studies 16 (1): 13-28

Guha, R. 1996. The Small Voice of History. In Amin, S. and Chakrabarty, D. (eds.) Subaltern Studies IX. Delhi: Oxford University Press. Pp. 1-12.

Güldemann, T. 2006. Changing Profile when Encroaching on Hunter-gatherer Territory?: Towards a History of the Khoe-Kwadi Family in Southern Africa, paper presented at the conference on Historical Linguistics and Hunter-gatherer Populations in Global Perspective, Max Planck Institute for Evolutionary Anthropology. http://email.eva.mpg.de/ gueldema/projects.html. (Downloaded 14/09/2011).

Güldemann, T. 2006a. Structural Isoglosses between Khoekhoe and Tuu: the Cape as a Linguistic Area. In Matras, Y., McMahon, A. and Vincent, N. (eds.) Linguistic Areas: Convergence in Historical and Typological Perspective. Basingstoke: Palgrave Macmillan. Pp. 99-134.

Güldeman, T. 2008. A Linguist's View: Khoe-Kwadi Speakers as the Earliest Food-producers of Southern Africa. Southern African Humanities 20: 93-132.

Haacke, W.H.G. 2008. Linguistic Hypotheses on the Origins of Namibian Khoekhoe Speakers. Southern African Humanities 20: 163-177. 
Haakonssen, K. 1996. Enlightenment and Religion: Rational Dissent in Eighteenth-Century Britain. Cambridge: Cambridge University Press.

Hack, M.A and Lorenzen, E. 2008. Equus quagga. In: IUCN 2011. IUCN Red List of Threatened Species. Version 2011.2. www.iucnredlist.org. (Downloaded 27/03/2012).

Hall, M. and Markell, A. 1993, Introduction: Historical Archaeology in the Western Cape. South African Archaeological Society Goodwin Series 7: 3-7

Hamilton, W.J. and Seely, M.K. 1976. Fog-basking by the Namib Desert Beetle, Onymacris unguicularis. Nature 262: 284-285.

Harley, E.H., Knight , M.H., Lardner, C., Wooding, B., and Gregor, M. 2009. The Quagga Project: Progress over 20 years of Selective Breeding. South African Journal of Wildlife Research 39:2:155-163.

Harlow, V and Madden, F. 1953. British Colonial Developments 1774-1834: Select Documents. Oxford: Clarendon Press.

Harrison, R. 2002. Australia's Iron Age: Aboriginal Post-contact Metal Artefacts from Old Lamboo Station, Southeast Kimberley, Western Australia. Australasian Historical Archaeology 20: 67-76.

Harrison, R. 2002a. Shared Histories and the Archaeology of the Pastoral Industry in Australia. In Harrison, R. and Williamson, C. (eds.) After Captain Cook: the Archaeology of the Recent Indigenous Past in Australia. Walnut Creek: Altamira Press. Pp. 38-58.

Harrison, R. 2004. Contact Archaeology and the Landscapes of Pastoralism in the North-west of Australia. In Murray, T. (ed.) 2004. The Archaeology of Contact in Settler Societies. Cambridge: Cambridge University Press. Pp. 109-143.

Hart, J.A. 1978. From Subsistence to Market: a Case Study of the Mbuti Net Hunters. Human Ecology 6:3: 325-353.

Harvey, G. 2010. Animism rather than Shamanism: New Approaches to what Shamans do (for other Animists). In Schmidt, B. and Huskinson, L. (eds.) Spirit Possession and Trance: New Interdisciplinary Perspectives. London: Continuum International Publishing Group. Pp. 16-34.

Haskin, V. and Maynard, J. 2005. Sex, Race and Power. Australian Historical Studies 36:126: 191-216. 
Haskins, D.R. and Bell, F.G. 1995. Drakensberg Basalts: their Alteration, Breakdown and Durability. Quarterly Journal of Engineering Geology 28:287302.

Hayward, M.W. and Kerley, G.I.H. 2005. Prey Preferences of the Lion (Panthera leo). Journal of Zoology, London 267: 309-322.

Head, L. 2000. Second Nature: the History and Implications of Australia as Aboriginal Landscape. Syracuse: Syracuse University Press.

Headland, T.N. and Reid, L.A. 1989. Hunter-gatherers and their Neighbors from Prehistory to the Present. Current Anthropology 30:1: 43-66.

Heath, D. and de Bod, D. 2000. Hunting the Gemsbok. African Hunter 6:4:10-13.

Henderson, Z. 2002. A Dated Cache of Ostrich Eggshell Flasks from Thomas' Farm, Northern Cape Province, South Africa. South African Archaeological Bulletin 57: 38-40.

Hetem, R.S., de Witt, B.A., Fick, L.G., Fuller, A., Kerley, G.I.H., Meyer, L.C.R., Mitchell, D., and Maloney, S.K. 2009. Body Temperature, Thermoregulatory Behaviour and Pelt Characteristics of Three Colour Morphs of Springbok (Antidorcas marsupialis). Comparative Biochemistry and Physiology Part A, 152: 379-388.

Hewitt, R. 2008 [1986]. Structure, Meaning, and Ritual in the Narratives of the Southern San ( $2^{\text {nd }}$ ed.) Johannesburg: Wits University Press.

Hobsbawm, E. 1962. The Age of Revolution: Europe 1789-1848. London: Weidenfled \& Nicholson.

Hobsbawm, E. 1975. The Age of Capital: 1848-1875. London: Weidenfeld \& Nicholson.

Hobsbawm, E. 1987. The Age of Empire: 1875-1914. London: Weidenfeld \& Nicholson.

Hodgson, F.D.I. 1986. Chapter 5: Geohydrology. In Cowling, R.M., Roux, P.W., and Pieterse, A.J.H. (eds.) The Karoo Biome: a Preliminary Synthesis. Part I physical environment (South African National Scientific Programmes Report No. 124). Pretoria: CSIR.

Hoernlé, A.W. 1918. Certain Rites of Transition and the Conception of !Nau among the Hottentots. Harvard African Studies 2: 65-82. 
Hoff, A. 1997. The Water Snake of the Khoekhoen and the IXam. South African Archaeological Bulletin 52: 21-37.

Hoff, A. 1998. The Water Bull of the IXam. South African Archaeological Bulletin 53: 109-124.

Hoffman, T. 1996. Bushmanland Upper Nama Karoo. In Low, A.B. and Rebelo, T.G. (eds.) Vegetation of South Africa, Lesotho and Swaziland. Pretoria: Department of Environmental Affairs and Tourism. Pp. 53.

Hoffman, T. 1996a. Upper Nama Karoo. In Low, A.B. and Rebelo, T.G. (eds.) Vegetation of South Africa, Lesotho and Swaziland. Pretoria: Department of Environmental Affairs and Tourism. Pp.53-54.

Hoffman, T. 1996b. Orange River Nama Karoo. In Low, A.B. and Rebelo, T.G. (eds.) Vegetation of South Africa, Lesotho and Swaziland. Pretoria: Department of Environmental Affairs and Tourism. Pp. 54-55.

Hoffmann, M.T. and Cowling, R.M. 1987. Chapter 1: Plant Physiognomy, Phenology and Demography. In Cowling, R.M. and Roux, P.W. (eds.) The Karoo Biome: a Preliminary Synthesis. Part 2 - vegetation and history. (South African National Scientific Programmes Report No. 142). Pretoria: CSIR. Pp. 1-34.

Hoffmeyer, J. 2008. The Semiotic Niche. Journal of Mediterranean Ecology 9:530 .

Hoffmeyer, J. 2010. Semiotics of nature. In Cobley, P. (ed.) The Routledge Companion to Semiotics. Abingdon, Oxon.: Routledge. Pp. 29-42.

Hollmann, J.C. 2001. 'Big Pictures': Insights into Southern African San Rock Paintings of Ostriches. South African Archaeological Bulletin 56:173/174: pp. 6275.

Hollman, J.C. (ed.) 2004. Customs and Beliefs of the Kam Bushmen. Johannesburg: Wits University Press.

Hollon, W. E. 1974. Frontier Violence: Another Look. Oxford: Oxford University Press.

Holm, J. 2000. An Introduction to Pidgins and Creoles. Cambridge: Cambridge University Press.

Honko, L. 1964. Memorates and the Study of Folk Beliefs. Journal of the Folklore Institute 1:1/2: 5-19. 
Horsburgh, K.A. 2008. Wild or Domesticated: An Ancient DNA Approach to Canid Species Identification in South Africa's Western Cape Province. Journal of Archaeological Science 35: 1474-148.

Humphreys, A.J.B. 1975. Burchell's Shelter: the History and Archaeology of a Northern Cape Rock Shelter. South African Archaeological Bulletin 30:117/118: 3-18.

Humphreys, A.J.B. 1989. The Archaeological Setting of Genadendal, the First Mission Station in South Africa. The Digging Stick 6:3: 2-4.

Humphreys, A.J.B. 2007. Behavioural Ecology and Hunter-gatherers: from the Kalahari to the Later Stone Age. South African Archaeological Bulletin 62:186: 98-103.

Humphreys, A.J.B., and Thackeray, A.I. 1983. Ghaap and Gariep: Later Stone Age studies in the Northern Cape (South African Archaeological Society Monograph Series No. 2). Cape Town: South African Archaeological Society.

Hynes, W.J. 1993. Mapping the Characteristics of Mythic Tricksters: A Heuristic Guide. In Hynes, W.J. and Doty, W.G. (eds.) Mythical Trickster Figures: Contours, Contexts and Criticisms. Tuscaloosa: University of Alabama Press. Pp. 33-45.

Ikeya, K. 1994. Hunting with Dogs among the San in the Central Kalahari. African Study Monographs 15:3:119-134.

Ingold, T. 1993. The Temporality of the Landscape. World Archaeology 25:2: 152-174.

Ingold, T. 1999. On the Social Relations of the Hunter-gatherer Band. In Lee, R. B. and Daly, R.H. (eds.) The Cambridge Encyclopedia of Hunters and Gatherers. Cambridge: Cambridge University Press. Pp. 399-410.

Ingold, T. 2000. The Perception of the Environment: Essays on Livelihood, Dwelling and Skill. London: Routledge.

Ingold, T. 2008. Bindings against Boundaries: Entanglements of Life in an Open World. Environment and Planning A 40:8: 1796-1810.

Ingold, T., Riches, D., and Woodburn, J. 1988. Hunters and Gatherers: History, Evolution and Social Change, volume 1 (Explorations in Anthropology). Oxford: Berg. 
Ingold, T., Riches, D., and Woodburn, J. 1988a. Hunters and Gatherers: Property, Power and Ideology, volume 2 (Explorations in Anthropology). Oxford: Berg.

James, A. 2001. The First Bushman's Path: Stories, Songs and Testimonies of the IXam of the Northern Cape. Pietermaritzburg: University of kwaZulu-Natal Press.

Janzen, D.H. and Martin, P.S. 1982. Neotropical Anachronisms: the Fruits the Gomphotheres ate. Science (New Series) 215:4256: 19-27.

Jesser, P., Markula, A., and Csurhes, S. 2008. Pest Animal Risk Assessment: Water Buffalo (Bubalus bubalis). Brisbane: The State of Queensland, Department of Primary Industries and Fisheries.

Johnson, C. 2006. Australia's Mammal Extinctions: a 50000 Year History. Cambridge: Cambridge University Press.

Johnson, C.N. and Wroe, S. 2003. Causes of Extinction of Vertebrates during the Holocene of Mainland Australia: Arrival of the Dingo, or Human Impact? The Holocene 13:6: 941-948.

Johnson, W.E., Eizirik, E., Pecon-Slattery, J., Murphy, W.J., Antunes, A., Teeling, E. and O'Brien, S.J. 2006. The Late Miocene Radiation of Modern Felidae: A Genetic Assessment. Science 311: 73-77.

Jones, P.N. 2006. Shamanism: an Inquiry into the History of the Scholarly Use of the term in English-speaking North America. Anthropology of Consciousness 17:2: 4-32.

Jordan, P. 2008. Hunters and Gatherers. In Chippindale, C., Bentley, R.A., and Maschner, H.D.G. (eds.) Handbook of Archaeological Theories. Plymouth: Altamira Press. Pp. 447-466.

Kallaway, P. 1982. Danster and the Xhosa of the Gariep: towards a Political Economy of the Cape Frontier, 1790-1820. African Studies Journal 41:1: 143160 .

Kane, R.P. 2009. Periodicities, ENSO Effects and Trends of some South African Rainfall Series: an Update. South African Journal of Science 105:199-207.

Katz, R. 1982. Boiling Energy: Community Healing among the Kalahari Kung. Cambridge: Harvard University Press. 
Kelly, I.T. and Fowler, C.S. 1986. Southern Paiute. In D'Azevedo, W.L. (ed.) Volume 11: Great Basin. (Handbook of North American Indians, general editor W.C. Sturtevant). Washington: Smithsonian Institution. Pp. 368-397

Kent, S. 1993. Sharing in an Egalitarian Kalahari Community. Man (New Series) 28:3: 479-514.

Kiberd, P. 2001. Bundu Farm, a Middle and Later Stone Age Pan Site, Northern Cape, South Africa: Preliminary Results of Fieldwork, 1998-2000. Nyame Akuma 55:51-55.

Kiberd, P. 2006. Bundu Farm: A Report on Archaeological and Palaeoenvironmental Assembalges from a Pan Site in Bushmanland, Northern Cape, South Africa. South African Archaeological Bulletin 61:184: 189-201.

Kilani, M. 1999. The Biology of the Honey-bee. In Colin, M.E., Ball, B., Kilani, M. (eds.) Bee Disease Diagnosis (Option Méditerranéennes Series B: Etudes et Recherches 25), Zaragoza: CIHEAM. Pp. 9-24.

Kinahan, J. 1991. Pastoral Nomads of the Central Namib Desert: the People History Forgot. Windhoek: New Namibia Books.

Kinahan, J. 1996. The Archaeology of Social Rank among Eighteenth-century Nomadic Pastoralists in Southern Namibia. African Archaeological Review 13:4: 225-245.

Knack, M.C. 1995. The Dynamics of Southern Paiute Women's Roles. In Klein, L.F. and Ackerman, L.A. (eds.) Women and Power in Native North America. Norman: University of Oklahoma Press. Pp. 146-158.

Knack, M.C. 2001. Boundaries Between the Southern Paiutes, 1775-1995. Lincoln: University of Nebraska Press.

Knappett, C. and Malafouris, L. (eds.) 2008. Material Agency: Towards a Nonanthropocentric Approach. New York: Springer.

Köhler, A. 2005. Money Makes the World go Round? Commodity Sharing, Gifting and Exchange in the Baka (Pygmy) Economy. In Widlok, T. and Gossatadesse, W. (eds.) Property and Equality (Volume II): Encapsulation, Commercialisation Discrimination. Oxford: Berghahn Books. Pp. 32-55

Kramer, P. and Malan, A. (eds.) 2007. Preliminary List of Sites. Vernacular Architecture Society of South Africa Journal 17: 30-61. 
Kramer, P., Kramer, J., Burger, C.S., and Maguire, J. 2008. VASSA Corbelled Building Survey. Vernacular Architecture Society of South Africa Journal 20: 179.

Krog, A. 2004. The Stars Say 'Tsau': |Xam poetry of Dia!kwain, Kweiten-ta-\|ken, $\mid A !$ kunta, |Hanłkass'o, and $\|$ Kabbo. Roggebaai: Kwela Books.

Kuntzsch, V. and Nel, J.A.J. 1992. Diet of Bat-eared foxes (Otocyon megalotis) in the Karoo. Koedoe 35:2: 37-48.

Lamar, H. and Thompson, L. (eds). 1981. The Frontier in History: North America and Southern Africa New Haven: Yale University Press.

Lamar, H.R. and Truett. S. 1996. Chapter 10: The Greater Southwest and California from the Beginning of European Settlement to the 1880s. In Trigger, B.G. And Wilcomb, E.W. (eds.) The Cambridge History of the Native Peoples of the Americas (Volume 1: Part 2). Cambridge: Cambridge University Press. Pp. 57-115.

Layton, R.H. 2001. Hunter-gatherers, their Neighbours, and the Nation State. In Panter-Brick, C., Layton, R.H., Rowley-Conwy, P. (eds.) Hunter-gatherers: an Interdisciplinary Perspective (Biosocial Society Symposium Series). Cambridge: Cambridge University Press. Pp. 292-321.

Lee, R.B. 1969. Eating Christmas in the Kalahari. Natural History 78:14-22, 6063.

Lee, R.B. 1976. !Kung Spatial Organization: an Ecological and Historical Perspective. In Lee, R. B. and de Vore, I (eds.) Kalahari Hunter-gatherers: Studies of the !Kung San and Their Neighbours. Cambridge: Harvard University Press. Pp. 73-97.

Lee, R.B. 1979. The !Kung San: Men, Women and Work in a Foraging Society. Cambridge: Cambridge University Press.

Lee, R.B. 2003. Indigenous Rights and the Politics of Identity in Post-apartheid Southern Africa. In Dean, B. and Levi, J.M. (eds.) At the Risk of Being Heard: Identity, Indigenous Rights, and Postcolonial States. Ann Arbor: University of Michigen Press. Pp. 80-111.

Lee, R.B. and deVore, I. (eds.) 1968. Man the Hunter. Chicago: Aldine.

Lee, R.B. and deVore, I. 1968a. Problems in the Study of Hunters and Gatherers. In Lee, R. B. and deVore, I. (eds.) Man the hunter. Chicago: Aldine. Pp. 3-12. 
Lee, R.B. and deVore, I. (eds.) 1976. Kalahari Hunter-gatherers: Studies of the !Kung San and their Neighbours. Cambridge: Harvard University Press.

Lee, R.B. and Guenther, M. 1993. Problems in Kalahari Historical Ethnography and the Tolerance of Error. History in Africa 20: 185-235.

Lee, R.B. and Hitchcock, R.K. 2001. African Hunter-gatherers: Survival, History, and the Politics of Identity. African Study Monographs, Supplement 26: 257-280.

Lefebvre, H. 1991 [1974]. The Production of Space. (translated by Donald Nicholson-Smith). Oxford: Blackwell Publishing Ltd.

Legassick, M. 1979. The Northern Frontier to 1820: The Emergence of the Griqua People. In Elphick, R. and Giliomee, H. (eds.) The Shaping of South African Society: 1652-1820. Cape Town: Longman Penguin Southern Africa. Pp. 243290.

Legassick, M. 2006. From Prisoners to Exhibits: Representations of 'Bushmen' of the Northern Cape, 1880-1990. In Coombes, A. (ed.) Rethinking Settler Colonialism: History and Memory in Australia, Canada, New Zealand and South Africa (Studies in Imperialism). Manchester: Manchester University Press. Pp. 6385 .

Legassick, M. C. 2010 [1969]. The Politics of a South African Frontier: The Griqua, the Sotho-Tswana and the Missionaries, 1780-1840. Basel: Basler Afrika Bibliographien.

Leonard, J.A., Wayne, R.K., Wheeler, J., Valadez, R., Guillén, S., and Vilà, C. 2002. Ancient DNA Evidence for Old World Origin of New World Dogs. Science 298: 1613-1616.

Lewis, I.M. 1989. South of North: Shamanism in Africa: a Neglected Theme. Paideuma 35, Afrika-Studien I: 181-188.

Lewis, I.M. 1997. The Shaman's Quest in Africa (À la Recherche d'un Chamanisme Africain) Cahiers d'Études Africaines 37:145: 119-135.

Lewis-Williams, D. 1977. Ezeljagdspoort Revisited: New Light on an Enigmatic Rock Painting. South African Archaeological Bulletin 32:129:165-159.

Lewis-Williams, D. 1981. Believing and Seeing: Symbolic Meanings in Southern San Rock Painting. London: Academic Press. 
Lewis-Williams, D. 1981a. The Thin Red Line: Southern San Notions and Rock Paintings of Supernatural Potency. South African Archaeological Bulletin 36:133: 5-13.

Lewis-Williams, D. 1983. The Rock Art of Southern Africa. Cambridge: Cambridge University Press.

Lewis-Williams, D. 1992. Ethnographic Evidence Relating to 'Trance' and 'Shamans' among Northern and Southern Bushmen. South African Archaeological Bulletin 47:155: 56-60.

Lewis-Williams, D. 1996. A Visit to the Lion's House: The Structures, Metaphors and Sociopolitical Significance of a Nineteenth-Century Bushman Myth. In Deacon, J. and Dowson, T.A. Voices from the Past: Xam Bushmen and the Bleek and Lloyd Collection. Johannesburg: Witwatersrand University Press. Pp. 122141.

Lewis-Williams, D. 1997. The Mantis, the Eland and the Meerkats: Conflict and Mediation in a Nineteenth-century San Myth. African Studies 56:2: 195-216.

Lewis-Williams, D. 2000. Stories that Float from Afar: Ancestral Folklore of the San of Southern Africa. College Station: Texas A\&M University Press.

Lewis-Williams, D. 2002. A cosmos in stone: interpreting religion and society through rock art. Walnut Creek: Altamira Press.

Lewis-Williams, D. 2002a. The Mind in the Cave: Consciousness and the Origins of Art. London: Thames and Hudson.

Lewis-Williams, D. 2010. The Imagistic Web of San Myth, Art and Landscape. Southern African Humanities 22: 1-18.

Lewis-Williams, D. and Biesele, M. 1978. Eland Hunting Rituals among Northern and Southern San Groups: Striking Similarities. Africa: Journal of the International African Institute 48:2: 117-134.

Lewis-Williams, D. and Challis, W. 2011. Deciphering Ancient Minds: the Mystery of San Bushman Rock Art. London: Thames and Hudson.

Lewis-Williams, D. and Dowson, T. 1988. Signs of All Times: Entoptic Phenomena in Upper Palaeolithic Art. Current Anthropology 29: 201-245.

Lewis-Williams, D. and Dowson, T. 1989. Images of Power: Understanding Bushman Rock Art. Johannesburg: Southern Book Publishers. 
Lewis-Williams, D. and Pearce, D. 2004. San Spirituality: Roots, Expressions and Social Consequences. Cape Town: Double Storey.

Liebenberg, L. 1990. The Art of Tracking: the Origin of Science. Claremont: David Philip Publisher.

Liebenberg, L. 2006. Persistence Hunting by Modern Hunter-Gatherers. Current Anthropology 47:6: 1017-1026.

Lindholm, K.J. 2006. Wells of Experience: a Pastoral Land-use History of Omaheke, Namibia (Studies in Global Archaeology 9). Uppsala: Universitetstryckeriet.

Lönnberg, A. 1981. The Digger Stereotype in California. Journal of California and Great Basin Anthropology 3:2: 215-223.

Lott, D.F. 1991. American Bison Socioecology. Applied Animal Behaviour Science 29: 135-145.

Lovegrove, B.G. 1999. Animal Form and Function. In Dean, W.R.J. and Milton, S.J. (eds.) The Karoo: Ecological Patterns and Processes. Cambridge: Cambridge University Press. Pp. 145-163.

Lovegrove, B.G. 1993. The Living Deserts of Southern Africa. Cape Town: Fernwood Press.

Low, A.B. and Rebelo, T.G. (eds.) 1996. Vegetation of South Africa, Lesotho and Swaziland. Pretoria: Department of Environmental Affairs and Tourism.

Low, C. 2007. Different Histories of Buchu: Euro-American Appropriation of San and Khoekhoe Knowledge of Buchu Plants. Environment and History 13: 333361.

Low, C. 2007a. Khoisan Wind: Hunting and Healing. in Low, C. and Hsu, E. (eds.) Wind, Life, Health: Anthropological and Historical Perspectives, Journal of the Royal Anthropological Institute 13: S71-S90.

Lyons, C.L., and Papadopoulos, J.K. (eds.) 2002.The Archaeology of Colonialism. Los Angeles: Getty Research Institute.

MacCorquodale, S.M. and DiGiacomo, R.F. 1985. The Role of Wild North American Ungulates in the Epidemiology of Bovine Brucellosis: A Review. Journal of Wildlife Diseases 21:4: 351-357. 
MacKenzie, J.M. 1988. The Empire of Nature: Hunting, Conservation and British Imperialism. Manchester: Manchester University Press

MacLaury, G., Paramei, V. and Dedrick, D. (eds.) 2007. Anthropology of Color: Interdisciplinary Multilevel Modeling. Amsterdam: John Benjamins.

Maguire, J. 2007. Nieuweveld Farms that tell a Story. Vernacular Architecture Society of South Africa Journal 17: 27-29.

Maingard, L.F. 1932. Studies in Korana History, Customs and Language. Bantu Studies 6:1: 103-162.

Malan, A. 1998. Beneath The Surface, Behind The Doors: Historical Archaeology Of Households In Mid-Eighteenth Century Cape Town. Social dynamics 24:1:88118.

Malan, A. 2002. South Africa. In Olser, C.E. (ed.) Encyclopedia of Historical Archaeology. London: Routledge. Pp. 510-513.

Mann, G.K.H., O’Riain, M.J.O., and Hofmeyr, M.D. 2006. Shaping up to Fight: Sexual Selection Influences Body Shape and Size in the Fighting Tortoise (Chersina angulata). Journal of Zoology 269:373-379.

Marais, E.N. 1934. Die Siel van die Mier. Pretoria: J. L. van Schaik.

Marais, E.N. 1937. The Soul of the White Ant. London: Methuen.

Markell, A., Hall, M., and Schrire, C. 1995. The Historical Archaeology of Vergelegen, an Early Farmstead at the Cape of Good Hope. Historical Archaeology 29:1: 10-34.

Marks, S. 1972. Khoisan Resistance to the Dutch in the Seventeenth and Eighteenth Centuries. The Journal of African History 13:1: 55-80.

Marshall, J. 1973. Hunting among the Kalahari Bushmen. In Skinner, E. P. (ed.) Peoples and Cultures of Africa: an Anthropological Reader. New York: The Doubleday/Natural History Press. Pp. 106-121.

Marshall, L. 1961. Sharing, Talking and Giving: the Relief of Social Tensions among the !Kung Bushmen. Africa 31:231-249.

Marshall, L. 1962. !Kung Bushman Religious Beliefs. Africa: Journal of the International African Institute 32:3: 221-252. 
Marshall, L. 1969. The Medicine Dance of the !Kung Bushmen. Africa: Journal of the International African Institute 39:4: 347-381.

Marshall, L. 1976. The !Kung of Nyae Nyae. Cambridge: Harvard University Press.

Marshall, L. 1976a. Sharing, Talking and Giving: Relief of Social Tensions among the !Kung. In Lee, R.B. and DeVore, I. (eds.) Kalahari Hunter-gatherers. Cambridge: Harvard University Press. Pp. 349-371.

Martin, D.L. 2011. Curious Visions of Modernity: Enchantment, Magic, and the Sacred. Cambridge: Massachusetts Institute of Technology.

Martin, P.S. 2005. Twilight of the Mammoths: Ice Age Extinctions and the Rewilding of America. Berkeley: University of California Press.

McKnight, T. 1976. Friendly Vermin: a Survey of Feral Livestock in Australia (University of California publications in Geography, Volume 21). Berkeley: University of California Press.

McShane, C. and Swart, S. 2011. Designing Equids in South African and North America. Safundi: the Journal of South African and American Studies. 12:2: 203227.

Meadows, M.E. and Watkeys, M.K. 1999. Palaeoenvironments. In Dean, W.R.J. and Milton, S.J. (eds.) The Karoo: ecological patterns and processes. Cambridge: Cambridge University Press. Pp. 27-41.

Meinhof, Carl. 1930. Der Koranadialekt des Hottentottischen. Berlin: Dietrich Reimer.

Mills, M.G.M. 1982. Hyaena brunnea. Mammalian Species 194:1-5.

Mills, M.G.M. 1984. Prey Selection and Feeding Habits of the Large Carnivores in the Southern Kalahari. Koedoe (Supplement) 27: 281-294.

Mills, M.G.M. 1990. Kalahari Hyaenas: Comparative Behavioural Ecology of Two Species. London: Unwin Hyman.

Milton, K. 1987. Primate Diets and Gut Morphology: Implications for Hominid Evolution. In Harris, M. and Ross, E.B. (eds.) Food and Evolution: Towards a Theory of Human Food Habits. Philadelphia: Temple University Press. 
Milton, S.J. 1992. Plants Eaten and Dispersed by Adult Leopard Tortoises Geochelone pardalis (Reptilia: Chelonii) in the Southern Karoo. South African Journal of Zoology 27: 45-49.

Mitchell, L.J. 2009. Belongings: Property and Identity in Colonial South Africa (an Exploration of Frontiers, 1725 - c.1830). New York: Columbia University Press.

Mitchell, P.J. 2002. The Archaeology of Southern Africa. Cambridge: Cambridge University Press.

Mitchell, P.J. 2008. The Canine Connection: Dogs and People in Southern African Hunter-gatherer Societies. In Badenhorst, S., Mitchell, P.J. and Driver, J.C. (eds.) Animals and People: Archaeozoological Papers in Honour of Ina Plug. Oxford: British Archaeological Reports. Pp. 104-116.

Moir, R.W. and Sampson, C.G. 1993. European and Oriental Ceramics from Rock Shelters in the Upper Seacow Valley. Southern African Field Archaeology 2:1: $35-43$.

Monadjem, A., Reside, A., Cornut, J., and Perrin, M.R. (2009) Roost Selection and Home Range of an African Insectivorous Bat Nycteris thebaica (Chiroptera, Nycteridae). Mammalia, 73:4: 353-359.

Moran, S. 2001. Specimens of 'Bushman' studies. Wasafiri 16:34: 46-51.

Moran, S. 2009. Representing Bushmen: South Africa and the Origin of Language. Rochester: University of Rochester Press.

Morphy, H. 1993. Colonialism, History and the construction of place: the politics of landscape in Northern Australia. In B. Bender (ed.) Landscape: politics and perspectives. Oxford: Berg. Pp. 205-243

Morris, D. 1988. Engraved in Place and Time: a Review of Variability in the Rock Art of the Northern Cape and Karoo. South African Archaeological Bulletin 43:109-121.

Morris, D. 2002a. Another Spouted Ostrich Eggshell Container from the Northern Cape. South African Archaeological Bulletin. 57: 41.

Morris, D. 2005. Further Evidence of Spouts on Ostrich Eggshell Containers from the Northern Cape, with a Note on the History of Anthropology and Archaeology at the MacGregor Museum, Kimberley. South African Archaeological Bulletin 60:182: 112-114. 
Morris, D. 2008. Driekopseliand Rock Engraving Site, South Africa: A Precolonial Landscape Lost and Re-membered. In Smith, A.P. and GazinSchwartz, A. (eds.) Landscapes of Clearance: Archaeological and Anthropological Perspectives. Walnut Creek: Left Coast Press. Pp. 87-111.

Morris, D. and Beaumont, P. 1991. !Nawabdanas: Archaeological Sites in Renosterkop, Kakamas District, Northern Cape. South African Archaeological Bulletin 46:115-124.

Morse, E.E. 1933. A Study of the Genus Podaxis. Mycologia 25:1: 1-33.

Mucina, L, and Rutherford, M.C. 2006. Vegetation Types of South Africa, Lesotho and Swaziland. Strelitzia 19. Pretoria: South African National Biodiversity Institute.

Mulvaney, D.J. 1989. Encounters in Place: Outsiders and Aboriginal Australians 1606-1985. St Lucia: University of Queensland Press.

Musselman, E.G. 2003. Plant Knowledge at the Cape: A Study in African and European Collaboration. The International Journal of African Historical Studies 36:2: 367-392.

Netz, R. 2004. Barbed Wire: an Ecology of Modernity. Middletown: Wesleyan University Press.

Neville, D., Sampson, B.E. and Sampson, C.G. 1994. The Frontier Wagon Track System in the Seacow River Valley, North-eastern Cape. South African Archaeological Bulletin 49: 65-72.

Newton-King, S. 1999. Masters and Servants of the Cape Eastern Frontier 17601803. Cambridge: Cambridge University Press.

Nienaber, G.S. 1963. Hottentots. Pretoria: J.L. van Schaik Beperk.

Noyes, J.K. 2001. Nomadic Landscapes and the Colonial Frontier: the Problem of Nomadism in German South West Africa. In Russell, L. (ed.) Colonial Frontiers: Indigenous-European Encounters in Settler Societies. Manchester: Manchester University Press. Pp. 134-150

Orton, J. 2003. Superficial Comparisons and Reality: a Reassessment of Dunefield Midden and the Swartkop Industry. Southern African Field Archaeology 11/12: 64-67.

Orton, J. 2008. A Late Pleistocene Microlithic Later Stone Age Assemblage from Coastal Namaqualand, South Africa. Before Farming 2008/1 article 3: 1-9 
Osterhammel, J. 2005. Colonialism: A Theoretical Overview ( $2^{\text {nd }}$ Edition; translated by S. Frisch). Princeton: Markus Wiener Publishers.

Ouzman, S. 2005. The Magical Arts of a Raider Nation: Central South Africa's Korana Rock Art. South African Archaeological Society Goodwin Series 9: 101113.

Owen-Smith, N. 1987. Pleistocene Extinctions: The Pivotal Role of Megaherbivores. Paleobiology 13:3: 351-362.

Palmer, A.R., Novellie, P.A. and Lloyd, J.W. 1999. Community Patterns and Dynamics. In Dean, W.R.J. and Milton, S.J. (eds.) The Karoo: Ecological Patterns and Processes. Cambridge: Cambridge University Press. Pp. 208-223.

Parkington, J. and Hall, M. 1987. Pattering in Recent Radiocarbon Dates from Southern Africa as a Reflection of Prehistoric Settlement and Interaction. Journal of African History 28: 1-25.

Parkington, J., Morris, D. and Rusch, N. 2008. Karoo Rock Engravings. Clanwilliam: Krakadouw Trust.

Parsons, I. 2003. Lithic Expressions of Later Stone Age Lifeways in the Northern Cape. South African Archaeological Bulletin 58:177: 33-37.

Parsons, I. 2007. Hunter-gatherers or Herders? Reconsidering the Swartkop and Doornfontein Industries, Northern Cape Province, South Africa. Before Farming 2007:4: article 3: 1-10.

Paterson, A. 2005. Hunter-gatherer Interactions with Sheep and Cattle Pastoralists from the Australian Arid Zone. In Veth, P.M., Smith, M.A., and Hiscock, P. Desert Peoples: Archaeological Perspectives. Oxford: Blackwell Publishing. Pp. 276-292.

Pedersen, M.A. 2001. Totemism, Animism and North Asian Indigenous Ontologies. Journal of the Royal Anthropological Institute 7:3: 411-427.

Peires, J. 1981. The House of Phalo: A History of the Xhosa People in the Days of their Independence. Johannesburg: Raven Press.

Peires, J. 1989. The Dead will Arise: Nongqawuse and the Great Xhosa CattleKilling Movement of 1856-7. Johannesburg: Jonathon Ball Publishers.

Pels, P. 1997. The Anthropology of Colonialism: Culture, History and the Emergence of Western Governmentality. Annual Review of Anthropology 26: 163-183. 
Penn, N. 1995. The Orange River Frontier Zone, c. 1700-1805. In Smith, A.B. (ed.) Einiqualand: Studies of the Orange River Frontier. Cape Town: University of Cape Town Press. Pp. 21-109.

Penn, N. 1999. Rogues, Rebels and Runaways: Eighteenth-century Cape Characters. Cape Town: David Philips Publishers.

Penn, N. 2005. The Forgotten Frontier: Colonist and Khoisan on the Cape's Northern Frontier in the $18^{\text {th }}$ Century. Cape Town: Double Storey.

Pettman, C. 1913. Africanderisms: a Glossary of South African Colloquial Words and Phrases and of Place and Other Names. London: Longmans, Green and Co.

Phelps, R.J., Struthers, J.K. and Moyo, S.J.L. 1975. Investigations into the Nutritive Value of Macrotermes falciger (Isoptera: Termitidae). Zoologica Africana 10:2: 123-132.

Picker, M., Griffiths, C. and Weaving, A. 2004. Field Guide to the Insects of South Africa. Cape Town: Struik Publishers.

Pluciennik, M. 2002. The Invention of Hunter-gatherers in Seventeenth-century Europe. Archaeological Dialogues 9:2: 98-118.

Pluciennik, M. 2004. The Meaning of 'Hunter-Gatherers' and Modes of Subsistence: A Comparative Historical Perspective. In Barnard, A. (ed.) Huntergatherers in history, archaeology, and anthropology. Oxford: Berg.

Plug, I. and Sampson, C.G. 1996. European and Bushman Impacts on Karoo Fauna in the Nineteenth century: An Archaeological Perspective. South African Archaeological Bulletin 51: 26-31.

Poland, M. and Hammond-Tooke, D. 2003. The Abundant Herds: A Celebration of the Cattle of the Zulu People. Simonstown: Fernwood Press.

Price, R.E. and Brown, H.D. 2000. A Century of Locust Control in South Africa. In Cheke, R.A., Rosenberg, L.J. and Kieser, M.E. (eds.) Workshop on Research Priorities for Migrant Pests of Agriculture in Southern Africa, Plant Protection Research Institute, Pretoria, 24-26 March 1999. Chatham: Natural Resources Institute. Pp. 37-49.

Price, T.D. and Brown, J.A. 1985. Aspects of Hunter-Gatherer Complexity. In Price, T.D. and Brown, J.A. (eds.) Prehistoric Hunter-Gatherers: the Emergence of Cultural Complexity. San Diego: Academic Press. Pp. 3-20. 
Priest, M.J. and Lenz, M. 1999. The Genus Podaxis (Gasteromycetes) in Australia with a Description of a New Species from Termite Mounds. Australian Systematic Botany 12:1: 109-116.

Raper, P.E. and Boucher, M. (eds.) 1988. Robert Jacob Gordon: Cape Travels, 1777 to 1786 . Houghton: Brenthurst Press.

Reason, C.J.C. and Rouault, M. 2005. Links between the Antarctic Oscillation and winter rainfall over western South Africa. Geophysical Research Letters 32, L07705, doi:10.1029/2005GL02241: 1-4.

Reichel-Dolmatoff, G. 1975. The Shaman and the Jaguar: A Study of Narcotic Drugs among the Indians of Coloumbia. Philadelphia: Temple University Press.

Reynolds, H. 2006 [1981]. The Other Side of the Frontier: Aboriginal Resistance to the European Invasion of Australia. Sydney: University of New South Wales Press.

Richardson, P.R.K. 1987. Aardwolf: The Most Specialized Myrmecophagous Mammal. South African Journal of Science 83:10: 643-646.

Riley, E. 2007. The Hunting Ground's Doings: IXam Narratives of Animals, Hunting and the Veld. In Skotnes, P (ed.) Claim to the Country: The Archive of Wilhelm Bleek and Lucy Lloyd. Johannesburg: Jacana. Pp. 291-311

Rival, L. 1993. The Growth of Family Trees: Understanding Huaorani Perceptions of the Forest. Man (New Series) 28:4: 635-652.

Rival, L. 2002. Trekking Through History: The Huaorani of Amazonian Ecuador. New York: Columbia University Press.

Roche, C. 2008. 'The Fertile Brain and the Inventive Power of Man': Anthropogenic Factors in the Cessation of Springbok Treks and the Disruption of the Karoo Ecosystem, 1865-1908. Africa: Journal of the Internation African Institute 78:2: 157-188.

Rohde, R., Hoffmann, M.T., and Allsopp, N. 2003. Hanging on a Wire: A Historical and Socio-economic Study of Paulshoek Village in the Communal Area of Leliefontein, Namaqualand (Research Report no. 17). Cape Town: Programme for Land and Agrarian Studies.

Roper, J. 2007. 'Thoms and the Unachieved "Folk-Lore of England" Folklore 118:2: 203-216. 
Rose, D.B. 2002. Country of the Heart: An Indigenous Australian Homeland. Canberra: Aboriginal Studies Press.

Ross, R. 1975. The !kora Wars on the Orange River, 1830-1880. Journal of African History 16:4: 561-576.

Ross, R. 2008. A Concise History of South Africa (2 ${ }^{\text {nd }}$ Edition). Cambridge: Cambridge University Press.

Rouault, M. and Richard, R. 2003. Intensity and Spatial Extension of Drought in South Africa at Different Time Scales. Water SA 29:4: 489-500.

Roux, P.W. and Opperman, D. P. J. 1986. Chapter 6: Soil Erosion. In Cowling, R.M., Roux, P.W., and Pieterse, A.J.H. (eds.) The Karoo biome: a Preliminary Synthesis. Part I - Physical Environment (South African National Scientific Programmes Report No. 124). Pretoria: CSIR. Pp. 92-111.

Rubenstein, D.R., Rubenstein, D.I., Sherman, P.W., and Gavin, T.A. 2006. Pleistocene Park: Does Re-wilding North America Represent Sound Conservation for the $21^{\text {st }}$ Century? Biological Conservation 132: 232-238.

Russell, L. (ed.) 2001. Colonial Frontiers: Indigenous-European Encounters in Settler Societies. Manchester: Manchester University Press.

Rutherford, M.C. 1997. Categorization of Biomes. In Cowling, R. M., Richardson, D.M. and Pierce, S.M. (eds.) The Vegetation of Southern Africa. Cambridge: Cambridge University Press. Pp. 91-98.

Rutherford, M.C. and Westfall, R.H. 1986. Biomes of Southern Africa - An Objective Categorization. Memoirs of the Botanical Survey of South Africa No. 54. Pretoria: Government Printer. Pp. 1-98.

Sadler, K., Kerven, C., Calo, M., Manske, M., and Catley, A. 2010. The Fat and the Lean: Review of Production and Use of Milk by Pastoralists. Pastoralism 1:2: 291-324.

Sadr, K. 1997. Kalahari Archaeology and the Bushman Debate. Current Anthropology 38:1: 104-112.

Sadr, K. 2003. The Neolithic of Southern Africa. The Journal of African History 44:2:195-209.

Sadr, K. 2004. Feasting on Kasteelberg? Early Herders on the West Coast of South Africa. Before Farming 2004/3: article 2: 167-83. 
Sadr, K. 2005. Hunter-gatherers and Herders of the Kalahari During the Late Holocene. In Veth, P., Smith, M., and Hiscock, P. (eds.) Desert peoples: Archaeological Perspectives. Oxford: Blackwells.

Sadr, K. and Sampson, C.G. 1999. Khoekhoe Ceramics of the Upper Seacow River Valley. South African Archaeological Bulletin 54:3-15.

Sahlins, M.D. 1995. How Natives Think, About Captain Cook, for example. Chicago: University of Chicago Press.

Saitowitz, S.J. and Sampson, C.G. 1992. Glass Trade Beads from Rock Shelters in the Upper Karoo. South African Archaeological Bulletin 47: 94-103.

Saladin D'Anglure, B. 1994. Nanook, Super-male: The Polar Bear in the Imaginary Space and Social Time of the Inuit of the Canadian Arctic. In Willis, R. (ed.) Signifying Animals: Human Meaning in the Natural World. London: Routledge. Pp. 178-195.

Sales, J. 1975. Mission Stations and the Coloured Communities of the Eastern Cape, 1800-1852. Cape Town: Balkema.

Sampson, C.G. 1974. The Stone Age Archaeology of Southern Africa. New York: Academic Press.

Sampson, C.G. 1984. A Prehistoric Pastoralist Frontier in the Upper Zeekoe Valley, South Africa. In Hall, M.J., Avery, G., Avery, D.M., Wilson, M.L., and Humphreys, A.J.B. (eds.) Frontiers: Southern African Archaeology Today. Oxford: British Archaeological Reports International Series 207. Pp. 94-220.

Sampson, C.G. 1985. Atlas of Stone Age Settlement in the Seacow Valley, Memoirs of the National Museum (Bloemfontein) 20: 1-116.

Sampson, C.G. 1986. Model of a Prehistoric Herder-Hunter Contact Zone: A First Approximation. South African Archaeological Society Goodwin Series 5: 50-56.

Sampson, C.G. 1988. Stylistic Boundaries among Mobile Hunter-foragers. Washington: Smithsonian Institution Press.

Sampson, C.G. 1993 'Zeer grote liefhebbers van tobak': Nicotine and Cannabis Dependency of the Seacow River Bushmen. The Digging Stick 10:1: 2-6.

Sampson, C.G. 1994. Ostrich Eggs and Bushmen Survival on the North-east Frontier of the Cape Colony, South Africa. Journal of Arid Environments 26: 383399. 
Sampson, C.G. 1995. Acquisition of European Livestock by the Seacow River Bushmen between AD 1770-1890 South African Field Archaeology 4: 30-36.

Sampson, C.G. 1996. Spatial Organization of Later Stone Age Herders in the Upper Karoo. In Pwiti, Soper, G. and Soper, R. (eds.) Aspects of African Archaeology. Harare, Zimbabwe: University of Zimbabwe Press. Pp.317-326.

Sampson, C.G. 2010. Chronology and Dynamics of Later Stone Age Herders in the Upper Seacow valley, South Africa, Journal of Arid Environments 74: 842848.

Sampson, C.G. and Plug, I. 1993. Late Holocene and Historical Bone Midden Density in Rock Dhelters of the Upper Seacow River Valley. Southern African Field Archaeology 2:2: 59-66.

Sampson, C.G. and Sadr, K. 1999. On the Size and Shape of Later Stone Age Fibre-tempered Vessels from the Upper Seacow River Valley. Southern African Field Archaeology 8:1: 3-16

Sampson, C.G. and Vogel, J.C. 1995. Radiocarbon Chronology of Later Stone Age Pottery Decorations in the Upper Seacow Valley. Southern African Field Archaeology 4: 84-94.

Sampson, C.G. and Vogel, J.C. 1996. Fibre Temper in Later Stone Age Veramics from the Upper Karoo. South African Archaeological Bulletin 51: 99-105.

Sampson, C.G., Bailiff, I., and Barnett, S. 1997. Thermoluminescence Dates from Later Stone Age Pottery on Surface Sites in the Upper Karoo. South African Archaeological Bulletin 52: 38-42.

Sampson, C.G., Hart, T., Wallsmith, D.L., and Blagg, J.D. 1989. The Ceramic Sequence in the Upper Seacow Valley: Problems and Implications. South African Archaeological Bulletin 44: 3-16.

Sampson, C.G., Sampson, B.E. and Neville, D. 1994. An Early Dutch Settlement Pattern on the North East Frontier of the Cape Colony. South African Archaeological Bulletin 3: 74-81.

Schaffer, K. 2001. Handkerchief diplomacy: E. J. Eyre and Sexual Politics on the South Australian Frontier. In Russell, L. (ed.) Colonial Frontiers: IndigenousEuropean Encounters in Settler Societies. Manchester: Manchester University Press. Pp. 134-150. 
Schapera, I. 1930. The Khoisan Peoples of South Africa: Bushmen and Hottentots. London: Routledge and Kegan Paul Ltd.

Scheper-Hughes, N. and Bourgois, P. (eds.). 2004. Violence in War and Peace: An Anthology. Oxford: Blackwells.

Schieffelin, E.L. 1996. On Failure and Performance: Throwing the Medium Out of the Seance. In Laderman, C. and Roseman, M. (eds.) The Performance of Healing. New York: Routledge. Pp. 59-89.

Schieffelin, E.L. 1998. Problematizing Performance. In Hughes-Freeland, F. (ed.) Ritual, Performance, Media (ASA Monographs 35). London: Routledge. Pp. 199212.

Schoeman, K. 1993. Die Londense Sendinggenootskap en die San: Die Stasies Toornberg en Hephzibah, 1814-1818. South African Historical Journal 28: 221234.

Schoeman, K. 1993a. Die Londense Sendinggenootskap en die San: Die Stasies Ramah, Konnah en Philippolis, 1816-1828. South African Historical Journal 29: 132-152.

Schoeman, K. (ed.) 1996. Griqua Records: The Philippolis Captaincy, 18251861. Goodwood: Van Riebeeck Society.

Schoeman, K. 1996a. J. J. Kicherer en die vroeë sending, 1799-1806. Cape Town: Suid-Afrikaanse Biblioteek.

Schrire, C. 1980. An Enquiry into the Evolutionary Status and Apparent Identity of San Hunter-gatherers. Human Ecology 8:1: 9-32.

Schrire, C. 1988. The Historical Archaeology of the Impact of Colonialism in $17^{\text {th }}$-century South Africa. Antiquity 62:235: 214-225.

Schrire, C., Cruz-Uribe, K. and Klose, J. 1993. The Site-history of the Historical Site at Oudepost I, Cape. South African Archaeological Bulletin Goodwin Series 7: 21-32.

Schultze, L. 1928. Zur Kenntnis des Körpers der Hottentotten und Buschmänner. Jena: Gustav Fischer.

Sealy, J. and Yates, R. 1994. The Chronology of the Introduction of Pastoralism to the Cape, South Africa. Antiquity 68: 58-67.

Seboek, T.A. 1963. Communication in Animals and Men. Language 39: 448-466. 
Shaw, E.M., Woolley, P.L. and Rae, F.A. 1963. Bushman Arrow Poisons. Cimbebasia 7:2: 2-41.

Short, J. and Smith, A. 1994. Mammal Decline and Recovery in Australia. America Society of Mammalogists 75:2: 288-297.

Shoshani, J, Goldman, C.A. and Thewissen, J. G. M. 1988. Orycteropus afer. Mammalian Species No. 300:1-8.

Silberbauer, G.B. 1981. Hunter and Habitat in the Central Kalahari Desert. Cambridge: University of Cambridge Press.

Simpson, J. and Roud, S. 2000. A Dictionary of English folklore. Oxford: Oxford University Press.

Skead, C.J. 1980. Historical Mammal Incidence in the Cape Province, vol. 1. The Western and Northern Cape. Cape Town: Chief Directorate Nature and Environmental Conservation.

Skead, C.J. 1987. Historical Mammal Incidence in the Cape Province, vol. 2. The Eastern half of the Cape Province, including the Ciskei, Transkei and East Griqualand. Cape Town: Chief Directorate Nature and Environmental Conservation.

Skotnes, P. (ed.) 1991. Sound from the Thinking Strings: A Visual, Literary, Archaeological and Historical Interpretation of $19^{\text {th }}$ Century IXam Life. Cape Town: Axeage Private Press.

Skotnes, P. (ed.) 1996. Miscast: Negotiating the Presence of the Bushmen. Cape Town: University of Cape Town Press.

Skotnes, P. 2001. "Civilised Off the Face of the Earth": Museum Display and the Silencing of the /Xam. Poetics Today 22: 2: 299-321.

Skotnes, P. (ed.) 2007. Claim to the Country: The Archive of Wilhelm Bleek and Lucy Lloyd. Johannesburg: Jacana.

Smith, A.B. 1990. On Becoming Herders: Khoikhoi and San Ethnicity in Southern Africa. African Studies 49:2: 51-73.

Smith, A.B. 1999. Hunters and Herders in the Karoo Landscape. In Dean, W.R.J. and Milton, S.J. (eds.) The Karoo: Ecological Patterns and Processes. Cambridge: Cambridge University Press. Pp. 243-256. 
Smith, A. 2000. The Origins of the Domesticated Animals of Southern Africa. In Blench, R.M. And MacDonald, K.C. The Origins and Development of African Livestock: Archaeology, Genetics, Linguistics and Ethnography. London: Routledge. Pp. 222-238.

Smith, C.A. 1966. Common Names of South African Plants (Department of Agricultural Technical Services, Botanical Survey Memoir No 35). Pretoria: Government Printer.

Smith, M., Veth, P., Hiscock, P., and Wallis, L.A. 2005. Global Deserts in Perspective. In Veth, P.M., Smith, M.A., and Hiscock, P. Desert Peoples: Archaeological Perspectives. Oxford: Blackwell Publishing. Pp. 1-14.

Sofaer, J.R. 2006. The Body as Material Culture: A Theoretical Osteoarchaeology. Cambridge: Cambridge University Press.

Solomon, A. 2011. Writing San Histories: The IXam and 'Shamanism' Revisited. Journal of Southern African Studies 37:1: 99-117.

Solway, J.S. and Lee, R.B. 1990. Foragers, Genuine or Spurious? Situating the Kalahari San in History. Current Anthropology 31:2: 109-142.

Sparke, M. 2011. A Map that Roared and an Original Atlas: Canada, Cartography, and the Narration of a Nation. In Dodge, M., Kitchin, R., and Perkins, C. (eds.) The Map Reader: Theories of Mapping Practice and Cartographic Representation. Oxford: Wiley and Sons. Pp. 430-439.

Spielmann, K.A. and Eder, J.F. 1994. Hunters and Farmers: Then and Now. Annual Review of Anthropology 23: 303-323.

Spivak, G.C. 1988. Can the Subaltern Speak? In Nelson, C. and Grossberg, L. (eds.) Marxism and the Interpretation of Culture. Basingstoke: Macmillan Education. Pp. 271-313.

Stein, G.J. (ed.) 2005. The Archaeology of Colonial Encounters: Comparative Perspectives. Santa Fe: School of American Research Press.

Stevens, C.E. and Hume, I.D. 1995. Comparative Physiology of the Vertebrate Digestive System ( $2^{\text {nd }}$ Edition). Cambridge: Cambridge University Press.

Stevens, Jr., P. 2006. Sorcery. In Birx, H.J. (ed.) Encyclopedia of Anthropology.. Thousand Oaks: Sage Publications. Pp. 2117-2120 
Steward, J.H. 1936. The Economic and Social Basis of Primitive Bands. In Lowie, R.H. (ed.) Essays in Anthropology in Honor of Alfred Louis Kroeber. Berkeley: University of California Press. Pp. 331-350.

Stewart, G.G. 1967. A History of the Medicinal Uses of Tobacco, 1492-1860. Medical History 11:3: 228-268.

Steyn, D.G. 1962. Grasshopper Poisoning in a Bantu Child. South African Medical Journal 36: 822-823.

Stoffle, R.W., Halmo, D.B., and Austin, D.E. 1997. Cultural Landscapes and Traditional Cultural Properties: A Southern Paiute View of the Grand Canyon and Colorado River. American Indian Quarterly 21:2: 229-249.

Storey, W.K. 2008. Guns, Race, and Power in Colonial South Africa. Cambridge: Cambridge University Press.

Stow, G.W. 1905. The Native Races of South Africa: A History of the Intrusion of the Hottentots and Bantu into the Hunting Grounds of the Bushmen, the Aborigines of the Country. London: Swan Sonnenschien \& Co., Limited.

Strauss, T. 1979. War along the Orange: the Korana and the Northern Border Wars of 1868-9 and 1878-9 (Centre for African Studies, Communication 1). Cape Town: University of Cape Town Press.

Strong, P. T. 1986. Fathoming the Primitive: Australian Aborigines in Four Explorers' Journals, 1697-1845. Ethnohistory 33:2: 175-194.

Swart, S. 2007. 'But where's the bloody horse?': Textuality and Corporeality in the 'Animal Turn'. Journal of Literary Studies 23:3: 271-292.

Taylor, W.A., Lindsey, P.A., and Skinner, J.D. 2002. The Feeding Ecology of the Aardvark Orycteropus afer. Journal of Arid Environments 50: 132-152.

Terashima, H. 1998. Honey and Holidays: The Interactions Mediated by Honey between Efe Hunter-gatherers and Lese Farmers in the Ituri Forest. African Study Monographs, Supplement 25: 123-135.

Testart, A. 1982. The Significance of Food Storage Among Hunter-Gatherers: Residence Patterns, Population Densities, and Social Inequalities. Current Anthropology 23:5: 523-537.

Thomas, E.M. 1959. The Harmless People. New York: Knopf. 
Tonkinson, R. 2000. Gender role transformation among Australian Aborigines. In Schweitzer, P.P., Biesele, M. and Hitchcock, R.K. (eds.) Hunters and Gatherers in the Modern World: Conflict, Resistance, and Self-determination. New York: Berghahn Books. Pp. 343-360.

Traill, A. 1995. The Khoesan Languages of South Africa. In Mesthrie, R. (ed.) Language and Social History: Studies in South African Sociolinguistics. Cape Town: David Philip Publishers. Pp. 1-18.

Traill, A. 2002. The Khoesan Languages. In Mesthrie, R. (ed.) Language in South Africa. Cambridge: Cambridge University Press. Pp. 27-49.

Traill, A. 2007. !Khwa-ka hhouiten hhouiten 'The Rush of the Storm': The Linguistic Death of Ixam. In Skotnes, P. (ed.) Claim to the Country: The Archive of Wilhelm Bleek and Lucy Lloyd. Johannesburg: Jacana. Pp. 130-147.

Trigger, D.S. 1992. 'Whitefella comin': Aboriginal Responses to Colonialism in Northern Australia. Cambridge: Cambridge University Press.

Trigger, D.S. 2008. Indigeneity, Ferality, and what 'belongs' in the Australian Bush: Aboriginal Responses to 'Introduced' Animals and Plants in a Settlerdescendant Society. Journal of the Royal Anthropological Institute (N.S.) 14: 628646.

Tuan, Y. 1974. Topophilia: A Study of Environmental Perception, Attitudes, and Values. Englewood Cliffs: Prentice-Hall.

Turner, V. 1967. The Forest of Symbols: Aspects of Ndembu Ritual. Ithaca: Cornell University Press.

van Sittert, L. 1998. "Keeping the enemy at bay": The Extermination of Wild Carnivora in the Cape Colony, 1889-1910. Environmental History 3:3: 333-356.

van Sittert, L. 2005. Bringing in the Wild: The Commodification of the Wild Animals in the Cape Colony/Province c. 1850-1950. Journal of African History 46: 269-291.

van Wyk, B.E. 2008. A Review of Khoi-San and Cape Dutch Medical Ethnobotany. Journal of Ethnopharmacology 119:3:28: 331-341.

van Wyk, B. and van Wyk, P. 1997. Field Guide to Trees of Southern Africa. Cape Town: Struik Publishers

van Wyk, B. E., van Oudtshoorn, B., and Gerike, N. 1997. Medicinal Plants of South Africa. Pretoria: Briza Publications. 
Vaughan, T.A., Ryan, J.M., and Czaplewski, N.J. 2011. Mammalogy (5 ${ }^{\text {th }}$ Edition). Sudbury: Jones and Bartlett Learning.

Venter, J.A. and Watson, L.H. 2008. Feeding and Habitat use of Buffalo (Syncerus caffer caffer) in the Nama-Karoo, South Africa. South African Journal of Wildlife Research 38:1: 42-51.

Venter, J.M., Mocke, C. and de Jager, J.M. 1986. Chapter 3: Climate. In Cowling, R.M., Roux, P.W., and Pieterse, A.J.H. (eds.) The Karoo Biome: A Preliminary Synthesis. Part I - Physical environment (South African National Scientific Programmes Report No. 124). Pretoria: CSIR. Pp. 39-52.

Veracini, L. 2010. Settler Colonialism: A Theoretical Overview. Houndmills: Palgrave Macmillan.

Veracini, L. 2011. Introducing Settler Colonial Studies. Settler Colonial Studies 1: $1-12$.

Vercamman, P. and Mason, D.R. 1993. The Warthogs (Phacochoerus africanus and P.aethiopicus). In Oliver, W.L.R. (ed.) Pigs, Peccaries and Hippos: Status Survey and Conservation Action Plan. Gland: International Union for Conservation of Nature and Natural Resources. pp. 75-84.

Vernon, C.J. 1999. Biogeography, Endemism and Diversity of Animals in the Karoo. In Dean, W.R.J. and Milton, S.J. (eds.) The Karoo: Ecological Patterns and Processes. Cambridge: Cambridge University Press. Pp. 57-85.

Visser, J.N.J. 1986. Chapter 1: Geology. In Cowling, R.M., Roux, P.W., and Pieterse, A.J.H. (eds.) The Karoo Biome: A Preliminary Synthesis. Part I Physical Environment (South African National Scientific Programmes Report No. 124). Pretoria: CSIR. Pp. 1-17.

Voigt, E.A., Plug, I. and Sampson, C.G. 1995. European Livestock from Rock Shelters in the Upper Seacow River Valley. Southern African Field Archaeology 4:1: 37-49.

von Till Warren, E. 2007. Southern Paiutes in Las Vegas History. In Roberts, H., von Till Warren, E., and Eskenazi, S. (eds.) Coyote Named this Place Pakonapanti: Corn Creek National Register Archaeological District, Desert National Wildlife Refuge, Clark County, Nevada. HRA, Inc. Conservation Archaeology. Pp. 87-100.

von Uexküll, J. 1957 [1934]. A Stroll Through the Worlds of Animals and Men: A Picture Book of Invisible Worlds. In Schiller, C. H. (ed. and trans.) Instinctive 
Behavior: The Development of a Modern Concept. New York: International Universities Press, Inc.

von Wielligh, G.R. 1919. Boesmans-stories, deel I: Mitologie en Legendes. Cape Town: De Nasionale Pers.

von Wielligh, G.R. 1920. Boesmans-stories, deel II: Dierstories en ander Verhale. Cape Town, De Nasionale Pers.

von Wielligh, G.R. 1921. Boesman-stories, deel III: die Boesman Self, sy Sedes, Gewoontes en Bekwaamhede. Cape Town: De Nasionale Pers.

von Wielligh, G.R. 1921a. Boesman-stories, deel IV: Gemengde Vertellings, mees van 'n Avontuurlike Aard. Cape Town: De Nasionale Pers

Vorster, M and Roux, P.W. 1983. Veld of the Karoo Areas. Proceedings of the Annual Congresses of the Grassland Society of Southern Africa 18:1: 18-24.

Voss, A.E. 1987. The Image of the Bushman in South African English Writing of the Nineteenth and Twentieth Centuries. English in Africa 14:1: 21-40.

Wadley, L. 2001. Who Lived in Mauermanshoek Shelter, Korannaberg, South Africa? African Archaeological Review 18:3: 153-179.

Waldman, L. 2003. Houses and the Ritual Construction of Gendered Homes in South Africa. Journal of the Royal Anthropological Institute (New Series) 9: 675679.

Waldman, L. 2007. The Griqua Conundrum: Political and Socio-cultural Complexity in the Northern Cape, South Africa. Bern: Peter Lang AG.

Wallace, W.J. 1980. Death Valley Indian Farming. Journal of California and Great Basin Anthropology 2:2: 269-272.

Walton, J. 1975. Wolwehokke. Africana Notes and News 21:5: 184-187.

Walton, J. 2007 [1989]. Corbelled Buildings in South Africa. Vernacular Architecture Society of South Africa Journal 17: 15-26.

Watkeys, M.K. 1999. Soils of the Arid South-western Zone of Africa. In Dean, W.R.J. and Milton, S.J. (eds.) The Karoo: Ecological Patterns and Processes. Cambridge: Cambridge University Press. Pp.17-26.

Watson, S. 1991. Song of the Broken String: after the IXam Bushmen: Poems from a Lost Oral Tradition. Riverdale-on-Hudson: Sheep Meadow Press. 
Webley, L. 1997. Jakkalsberg A and B: The Cultural Material from Two Pastoralist Sites in the Richtersveld, Northern Cape. Southern African Field Archaeology 6: 3-19.

Webley, L. 2005. Hideworking among Descendants of Khoekhoen Pastoralists in the Northern Cape, South Africa. In Frink, L. and Weedman, K. (eds.) Gender and Hide Production. Walnut Creek: Altamira Press. Pp. 153-173.

Wehmeyer, A.S. 1986. Edible Wild Plants of Southern Africa: Data on the Nutrient Contents of over 300 Species. Pretoria: CSIR National Food Research Institute.

Weissner, P. 1983. Style and Social Information in Kalahari San Projectile Points. American Antiquity 48: 253-276.

Werger, M.J.A. 1978. The Karoo-Namib Region. In Werger, M.J.A. (ed.) Biogeography and ecology of southern Africa (Volume 1). The Hague: W. Junk. Pp. 231-300.

Werger, M.J.A. 1986. The Karoo and Southern Kalahari. In Evenari, M., NoyMeir, I. and Goodall, D.W. (eds.) Hot Deserts and Arid Shrublands. Amsterdam: Elsevier.

Wessels, M. 2007. The Discursive Character of the IXam texts: A Consideration of the IXam "Story of the Girl of the Early Race, who made stars" Folklore 118:3: 307-324.

Wessels, M. 2010. Bushman Letters: Interpreting /Xam Narrative. Johannesburg: Witwatersrand University Press.

Westbury, W. and Sampson, C.G. 1993. To Strike the Necessary Fire: Acquisition of Guns by the Seacow Valley Bushmen. South African Archaeological Bulletin 48: 26-31.

Whitaker, R. and Andrews, H. V. 1996. Snake Capture and Venom Extraction in Tamil Nadu, India. In Prescott-Allen, R. and Prescott-Allen, C. (eds.) Assessing the Sustainability of Uses of Wild Species: Case Studies and Initial Assessment Procedure (Occasional paper of the IUCN Species Survival Commission No. 12). Gland and Cambridge: IUCN. Pp. 40-46.

White, R. and Cronon, W. 1988. Ecological Change and Indian-White Relations. In Washburn, W.E. (ed.) Volume 4: History of Indian-White Relations (Handbook of North American Indians, general editor W. C. Sturtevant). Washington: Smithsonian Institution. Pp. 417-429. 
Whitley, D.S. 1988 Bears and Baskets: Shamanism in North American Rock Art. In Dowson, T.A. (ed.) The State of the Art: Advances in World Rock Art Research. Johannesburg: University of the Witwatersrand, Rock Art Research Unit. Pp. 34-42.

Wiessner, P. 1982. Risk, Reciprocity, and Social Influence on !Kung San Economics. In Leacock, E. and Lee, R. (eds.) Politics and History in Band Societies. Cambridge: Cambridge University Press. Pp. 61-84.

Wiessner, P. 1983. Style and Social Information in Kalahari San Projectile Points. American Antiquity 48:2: 253-276.

Wiessner, P. 1994. Pathways of the Past: !Kung San Hxaro Exchange and History. In Bollig, M. and Klees, F. (eds.) Überlebensstrategien in Afrika. Colloquium Africanum 1 Cologne: Heinrich-Barth Institut. Pp. 101 - 124.

Willerslev, R. 2004. Not Animal, Not Not-animal: Hunting, Imitation and Empathetic Knowledge among the Siberian Yukaghirs. Journal of the Royal Anthropological Institute 10:3: 629-652.

Willerslev, R. 2007. Soul Hunters: Hunting, Animism and Personhood among the Siberian Yukaghirs. Berkeley: University of California Press.

Willerslev, R. 2011. Frazer Strikes Back from the Armchair: A New Search from the Animist Soul. Journal of the Royal Anthropological Institute 17:3: 504-526.

Williams, V.L., Raimondo, D., Brueton, V.J., Crouch, N.R., Cunningham, A.B., Scott-Shaw, C.R., Lötter, M. \& Ngwenya, A.M. 2008. Boophone disticha (L.f.) Herb. National Assessment: Red List of South African Plants version 2011.1. http://redlist.sanbi.org/species.php?species=2076-2 (Accessed on 01/11/2011).

Wilman, M. 1933. The Rock Engravings of Griqualand West and Bechuanaland, South Africa. Cambridge: Deighton Bell.

Wilmsen, E.N. 1989. Land Filled with Flies: A Political Economy of the Kalahari. Chicago: University of Chicago Press.

Wilson, E.O. 1971. The Insect Societies. Cambridge: Harvard University Press.

Winterhalder, B. 2001. The Behavioural Ecology of Hunter-gatherers. In PanterBrick, C., Layton, R.H., and Rowley-Conwy, P. (eds.) Hunter-gatherers: An Interdisciplinary Perspective. Cambridge: Cambridge University Press. Pp. 12-38.

Wolfe, P. 1997. History and Imperialism: A Century of Theory, from Marx to Postcolonialism. The American Historical Review 102:2: 388-420. 
Woodburn, J. 1982. Egalitarian societies. Man (New Series) 17:3: 431-451.

Woodroffe, R. and Ginsberg, J.R. 2005. King of the Beasts? Evidence for Guild Redundancy among Large Mammalian Carnivores. In Ray, J.C., Redford, K.H., Steneck, R.S., and Berger, J. (eds.) Large Carnivores and the Conservation of Biodiversity. Washington: Island Press. Pp. 154-176.

Woodward, S.L. and Quinn, J.A. 2011. Encyclopedia of Invasive Species from Africanized Honey Bees to Zebra Mussels: Volume 1 Animals. Santa Barbara: Greenwood (ABC-CLIO).

Wright, J.B. 1971. Bushman Raiders of the Drakensberg, 1840-1870: A Study of their Conflict with Stock-keeping Peoples in Natal. Pietermaritzburg: University of Natal Press.

Wright, J.B. 2007. Bushman Raiders Revisited. In Skotnes, P. (ed.) Claim to the country: The Archive of Wilhelm Bleek and Lucy Lloyd. Johannesburg: Jacana. Pp. 118-29.

Wroe, S., Myers, R.T., and Gillespie, A. 1999. Estimating the Weight of the Pleistocene Marsupial Lion, Thylacoleo carnifex (Thylacoleonidae: Marsupialia): Implications for the Ecomorphology of a Marsupial Super-predator and Hypotheses of Impoverishment of Australian Marsupial Carnivore Faunas. Australian Journal of Zoology 47:5: 489-498.

Wuras, C.F. 1920. Vokabular der Korana-Sprache. Berlin: Dietrich Reimer.

Youngblood, D. 2004. Identification and Quantification of Edible Plant Foods in the Upper (Nama) Karoo, South Africa. Economic Botany 58 (Supplement): S43S65.

Youngblood, D. 2008. Edible Plant Foods in South Africa: New Light from Ancient Dwellers. In Peter, K.V. (ed.) Underutilized and Underexploited Horticultural Crops: Volume 4. New Delhi: New India Publishing Agency. 


\section{Image Attributions}

Berniedup (Photographer). 2009. Lion, Panthera leo. [Photograph]. Retrieved from

http://www.flickr.com/photos/berniedup/6041671298/sizes/o/in/photostream/

Licensed under Creative Commons License: Attribution-NonCommercialShareAlike 2.0 Generic (CC BY-NC-SA 2.0). [Downloaded 18/03/2012].

Berniedup (Photographer). 2011. Brown hyena, Parahyaena brunnea. [Photograph]. Retrieved from http://www.flickr.com/photos/berniedup/6472939035/sizes/o/in/photostream/ Licensed under Creative Commons License: Attribution-NonCommercialShareAlike 2.0 Generic (CC BY-NC-SA 2.0). [Downloaded 18/03/2012].

Custer, A. 2006. Tsetse fly (Glossina morsitans) head and proboscis. [Photograph]. Retrieved from Wikimedia Commons http://commons.wikimedia.org/wiki/File:Tsetse_head-proboscis.jpeg. Licensed under Creative Commons License: Attribution-ShareAlike 3.0 Unported (CC BYSA 3.0). [Downloaded 23/08/2011].

Barz, R. 2008. 'Beefly (Bombylius major)'. Retrieved From Wikimedia Commons http://commons.wikimedia.org/wiki/File:Grosser_Wollschweber_Bombylius_maj or.jpg. Photography taken by R. Bartz (Own work). Licensed under Creative Commons License: Attribution-ShareAlike 2.5 Generic (CC BY-SA 2.5). [Downloaded 23/08/2011]. 